\title{
Quantitative analysis of New Zealand- Antarctica plate motions during the Paleogene and Late Cretaceous
}

\author{
by
}

Amandine Iris Chambord

Thesis submitted to Victoria University of Wellington in fulfilment of the requirements for the degree of Doctor of Philosophy

Victoria University of Wellington

2017 



\section{ABSTRACT}

Quantifying past motions of tectonic plates in the southwest Pacific is important because the Pacific-Antarctic ridge is the only non-destructive boundary between the Pacific plate and other major plates. However, formation of sea-ice near Antarctica impairs the collection of magnetic anomaly data that are necessary to calculate plate rotations. A detailed analysis of all ship-track magnetic data available in the southwest Pacific (61 cruises, 153 profiles, including several cruises collected after 1995) is presented here. Four different sources of uncertainty are quantified: (1) confidence of magnetic anomaly identification, (2) magnetic reversal location picking precision, (3) ship navigation precision, and (4) magnetic data quality. Finite plate rotations are calculated for the southwest Pacific (42.5 to $79 \mathrm{Ma}$ ) using the resulting magnetic anomaly database (1528 magnetic reversal data). Finite rotations were calculated using the Hellinger criterion, or by a new method presented here that assumes orthogonality between fracture zones and ridge segments. The new method requires less parameters and is hence able better estimate rotations in cases with an uneven distribution of sparse magnetic data. Rotations and formal uncertainties are calculated for thirty-one chrons (c20y to c33o). They confirm the existence of a three plate system (Pacific, Marie Byrd Land, Bellingshausen) in the southwest Pacific from before c31o (68.7 Ma) until c28y (62.5 Ma). After c28y, the Bellingshausen and Marie Byrd Land plates moved as a single plate. 



\section{TABLE OF CONTENTS}

Table of contents

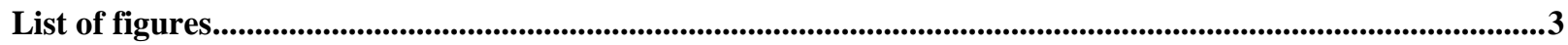

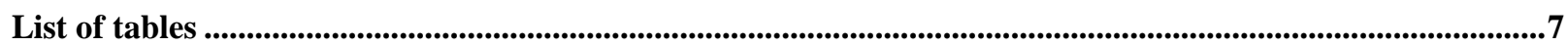

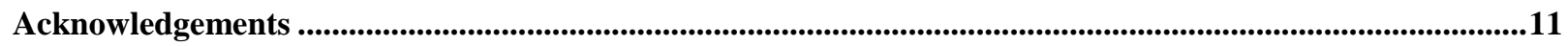

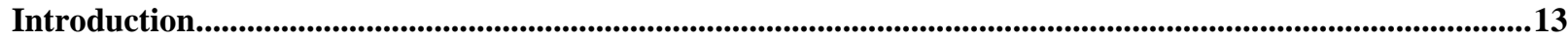

Chapter I Previous interpretations of the tectonic history of the Southwest Pacific .....................................19

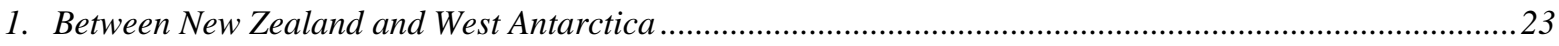

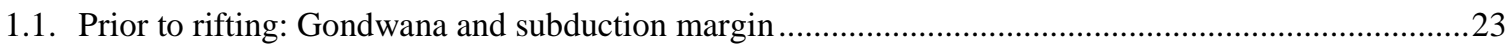

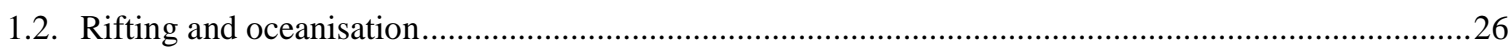

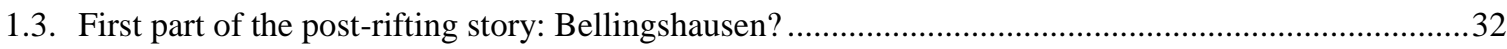

1.4. Second part of the post-rifting story: the eastern Southwest Pacific ...................................................37

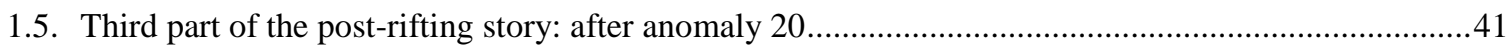

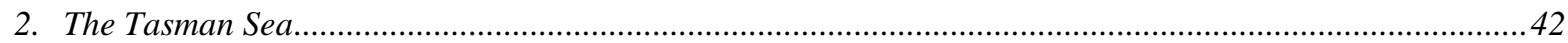

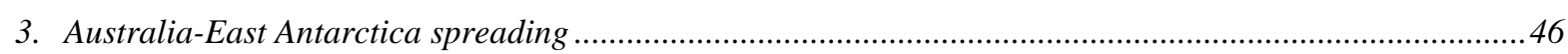

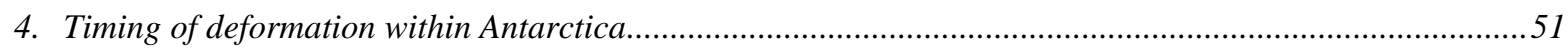

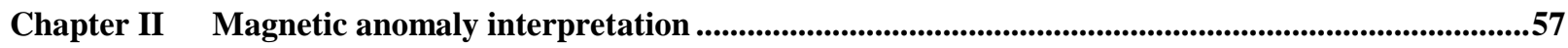

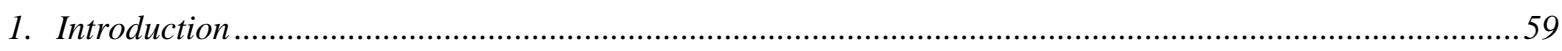

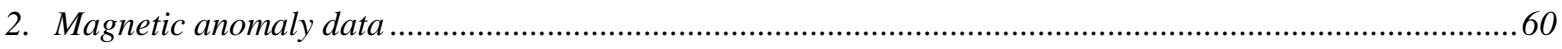

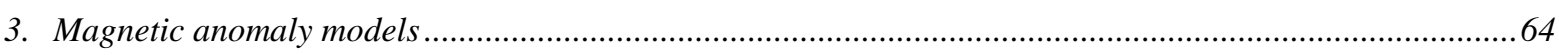

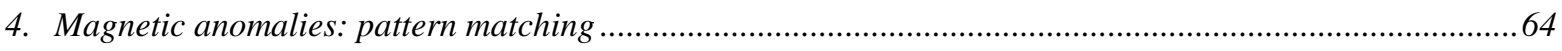

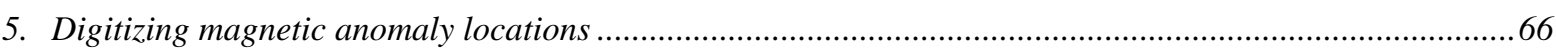

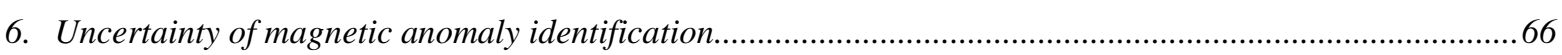

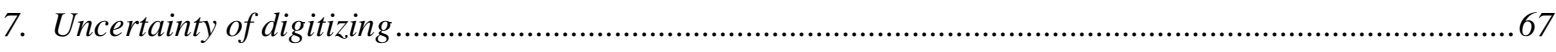

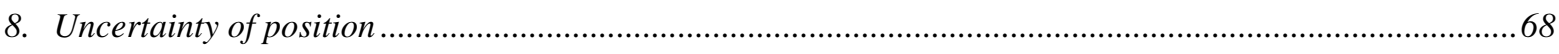

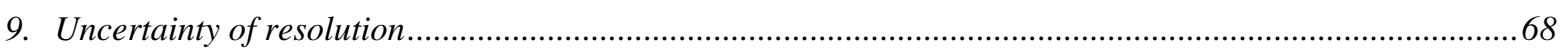

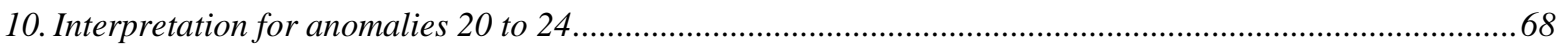

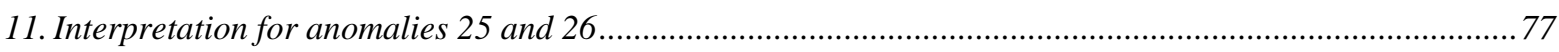

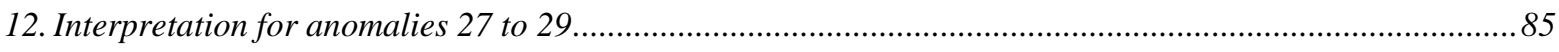

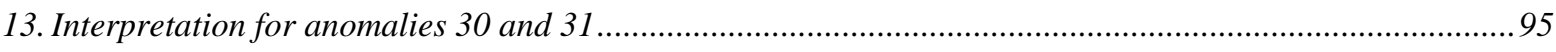

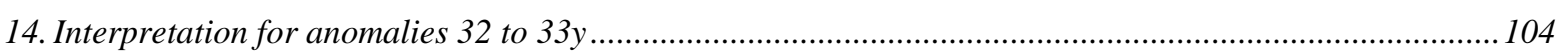

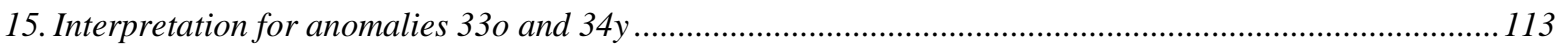

Chapter III Tectonic interpretation of seafloor morphology and gravity anomalies................................123

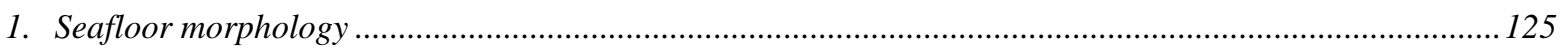

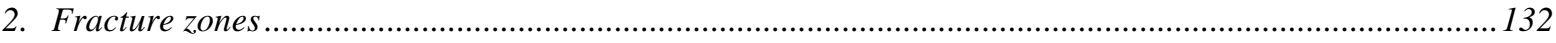

Chapter IV Plate reconstruction: methods.....................................................................................141

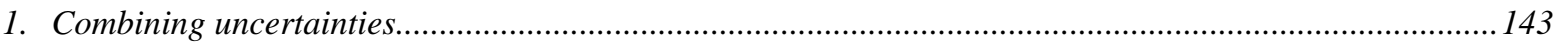

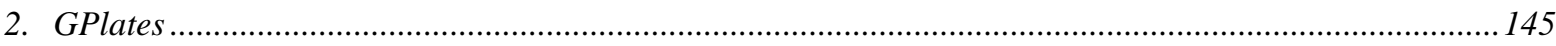




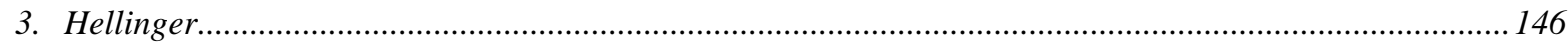

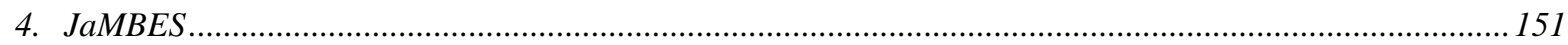

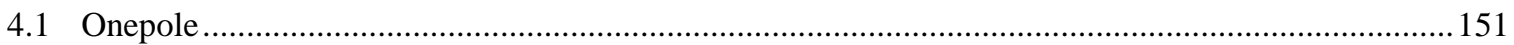

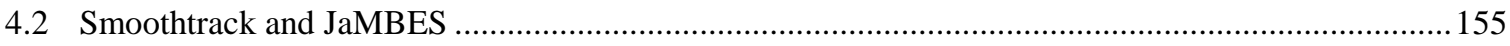

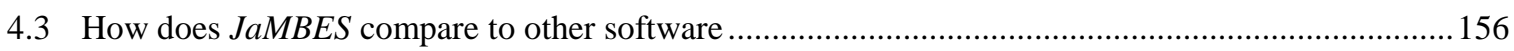

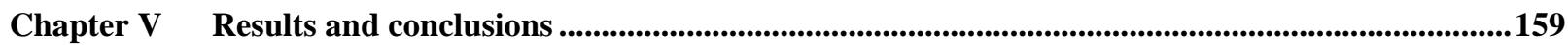

1. Inclusion of data based on identification uncertainty .................................................................. 161

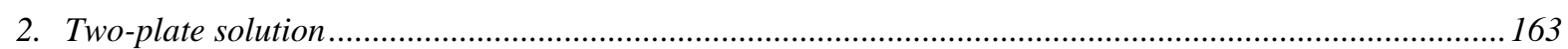

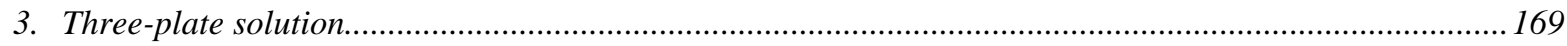

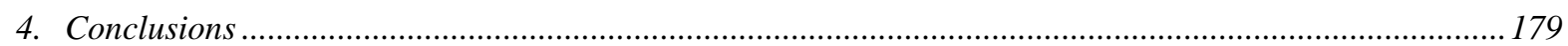

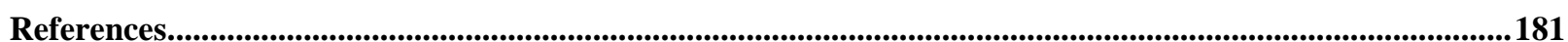




\section{LIST OF FIGURES}

Figure I-1: Location of the main features, plates and areas discussed in this thesis................23

Figure I-2: Formation of the Hikurangi-Manihiki-Otong-Java plateau ................................. 24

Figure I-3: The Hikurangi plateau reaches the Gondwana subduction zone .........................25

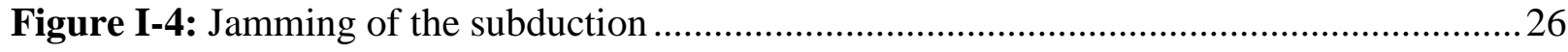

Figure I-5: Just before the breakup, opening of the Great South Basin ................................2 28

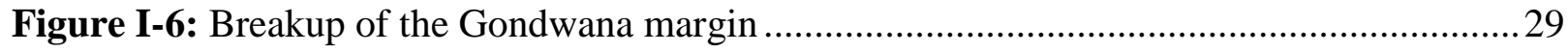

Figure I-7: Asymmetric spreading on two segments with asymmetry in opposite directions ... 30

Figure I-8: Rifting and seafloor spreading around the Bollons Seamount ............................ 31

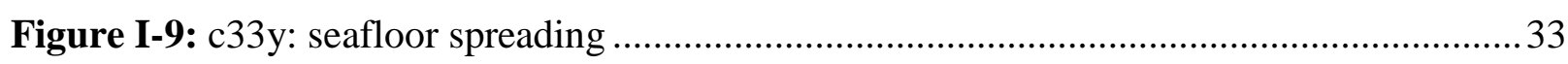

Figure I-10: Interpretation of a seismic reflection profile from [Gohl et al., 1997] ................. 35

Figure I-11: c27o: cessation of independent Bellingshausen motion ..................................... 37

Figure I-12: c24o: seafloor spreading stops in the Tasman Sea ......................................... 38

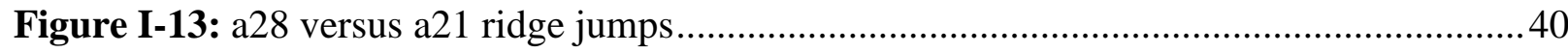

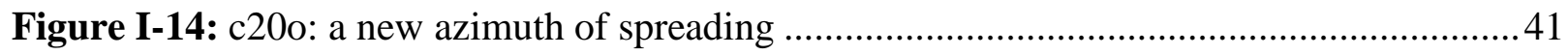

Figure I-15: Continental blocks and magnetic anomalies in the Tasman Sea ....................... 44

Figure I-16: Satellite gravity in the Australia-Antarctica basin ........................................... 47

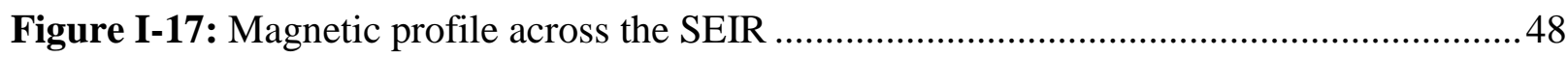

Figure I-18: Misfit between rotated Balleny fracture zone crossings and their conjugates.......51

Figure I-19: Seafloor between New Zealand and the Ross Sea .........................................5 52

Figure I-20: Configuration of plate boundaries at c27, c24 and c21. ..................................53

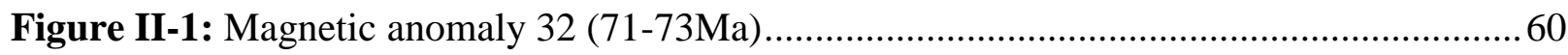

Figure II-2: Schematic illustration of two effects on the magnetic signal ............................63

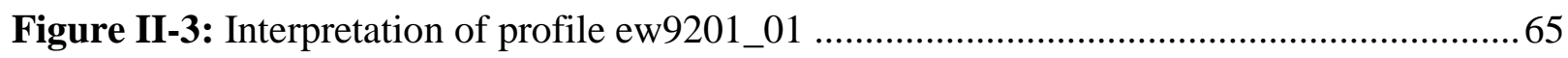

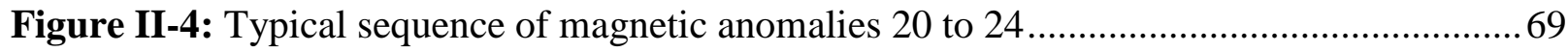

Figure II-5: Anomalies 20 to 24 for the Pacific sector ...................................................... 70

Figure II-6: Anomalies 20 to 24 for the Antarctica sector................................................... 73

Figure II-7: Interpreted profiles and magnetic reversals identified for c20y-c24o..................77

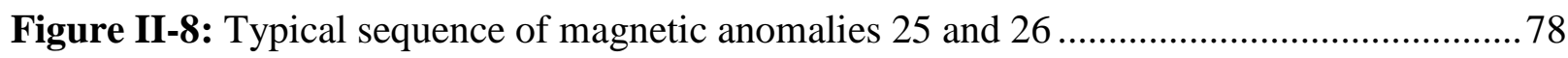

Figure II-9: Anomalies 25 and 26 for the Pacific sector..................................................... 79

Figure II-10: Anomalies 25 and 26 for the Antarctica sector .......................................... 82

Figure II-11: Interpreted profiles and magnetic reversals identified for c25y-c26o.................85 
Figure II-12: Typical sequence of magnetic anomalies 27 to 29 86

Figure II-13: Anomalies 27 to 29 for the Pacific sector ...................................................... 87

Figure II-14: Anomalies 27 to 29 for the Antarctica sector ................................................ 91

Figure II-15: Interpreted profiles and magnetic reversals identified for c27y-c29o .............. 94

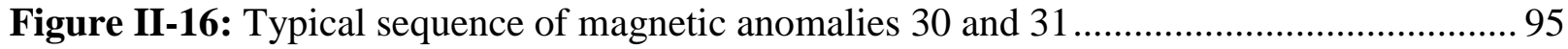

Figure II-17: Anomalies 30 and 31 for the Pacific sector ................................................. 97

Figure II-18: Anomalies 30 and 31 for the Antarctica sector............................................ 100

Figure II-19: Interpreted profiles and magnetic reversals identified for c30y-c31o .............. 104

Figure II-20: Typical sequence of magnetic anomalies 32.1 to $33 \mathrm{y}$..................................... 104

Figure II-21: Anomalies 32.1 to 33y for the Pacific sector, first part .................................. 105

Figure II-22: Anomalies 32.1 to 33y for the Pacific sector, second part............................... 106

Figure II-23: Anomalies 32.1 to 33y for the Antarctica sector .......................................... 109

Figure II-24: Interpreted profiles and magnetic reversals identified for c32.1y-c33y ........... 113

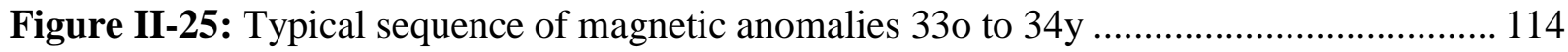

Figure II-26: Anomalies 33o and 34y for the Pacific sector, first part................................. 115

Figure II-27: Anomalies 33o and 34y for the Pacific sector, second part ............................ 116

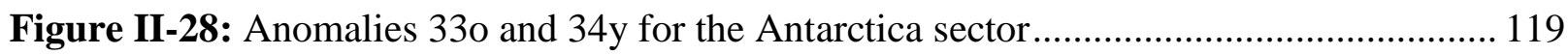

Figure II-29: Interpreted profiles and magnetic reversals identified for c33o-c34y .............. 122

Figure III-1: Bathymetric profile of the Southwest Pacific............................................... 129

Figure III-2: Multibeam swath bathymetry from the area surrounding the Pitman FZ ......... 131

Figure III-3: Gravity anomaly corresponding to a fracture zone ....................................... 131

Figure III-4: Fracture zones used to calculate the reconstructions...................................... 133

Figure III-5: Bathymetric profiles and gravity anomaly across the main fracture zones........ 139

Figure IV-1: Basic concept of finite pole calculation.................................................... 143

Figure IV-2: Finite rotation poles for the Southwest Pacific, obtained using GPlates........... 145

Figure IV-3: 3D schematic representation of the Hellinger criterion ................................. 147

Figure IV-4: Fracture zone and magnetic lineation segments .......................................... 148

Figure IV-5: Finite rotation poles for the Southwest Pacific, obtained using hellinger1 ........ 150

Figure IV-6: Comparison between hellingerl and JaMBES ........................................ 152

Figure IV-7: Comparison between onepole, smoothtrack and JaMBES ............................ 153

Figure IV-8: Comparison between hellingerl and onepole .............................................. 157 
Figure V-1: onepole finite rotation poles for different identification uncertainty categories .. 162

Figure V-2: JaMBES finite rotation poles (two plate system) .......................................... 165

Figure V-3: onepole confidence ellipses and residual distribution ....................................... 166

Figure V-4: Tectonic reconstructions for the Southwest Pacific (two plate system).............. 168

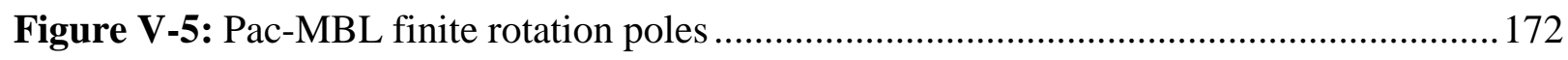

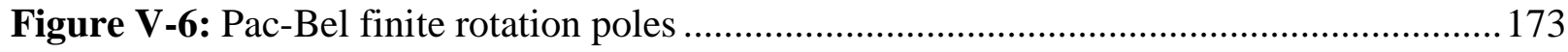

Figure V-7: Tectonic reconstructions for the Southwest Pacific using a three plate system ... 176

Figure V-8: Comparison between Pac-MBL and Pac-Bel rotation poles .............................. 178 


\section{LIST OF TABLES}

Table II-1: Cruises used to digitize magnetic anomalies ..................................................63

Table II-2: Spreading and asymmetry rates used for ew9201_01 ......................................65

Table II-3: Values for the digitizing uncertainties, and equivalent in kilometres.....................67

Table II-4: Positioning systems used on cruises and their precision. .....................................68

Table II-5: Profiles presented in Figure II-5 and Figure II-6 ........................................... 71

Table II-6: Profiles presented in Figure II-9 and Figure II-10 ........................................... 80

Table II-7: Profiles presented in Figure II-13 and Figure II-14 ......................................... 89

Table II-8: Profiles presented in Figure II-17 and Figure II-18 _........................................ 98

Table II-9: Profiles presented in Figure II-21 and Figure II-22 ........................................ 107

Table II-10: Profiles presented in Figure II-23 f................................................................ 110

Table II-11: Profiles presented in Figure II-26 and Figure II-27 ........................................ 117

Table II-12: Profiles presented in Figure II-28 ........................................................... 119

Table IV-1: Value of $p j$ for each category of identification uncertainty. ............................. 144

Table V-1: Finite rotation poles for a two plate model. ..................................................... 164

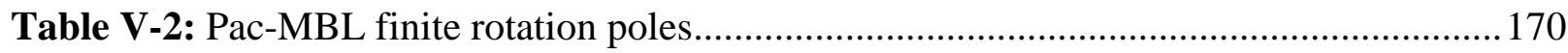

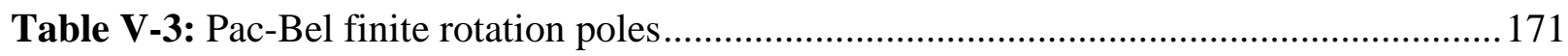




\section{LIST OF APPENDICES}
A: Magnetic profiles and their interpretation
B: Location of magnetic profiles used
C: Magnetic anomaly and fracture zone data
D: Magnetic timescale used
E: Finite rotation tables
F: Documentation for JaMBES and associated software
G: Figures from the main text with $95 \%$ confidence regions
H: Full finite rotation results from alternate runs of hellingerl
A0 map showing distribution of data used (electronic version only) 


\title{
ACKNOWLEDGEMENTS
}

\author{
“- Can you promise that I will come back? \\ - No, and if you do, you will not be the same."
}

JRR Tolkien

What a journey it has been!

First of all, I would like to thank the examiners for giving some of their time to review my thesis and to attend my defence.

I wish to thank my supervisors Rupert Sutherland and Euan Smith for giving me the opportunity to do this $\mathrm{PhD}$, and especially Euan Smith, whose support never wavered in spite of everything I put him (and his family) through. I would never have been able to finish without his compassion, dedication, open-mindedness and professionalism. His wise comments always put me back on track, and I cannot thank him enough. I wish him a happy, well deserved retirement.

In a similar line of thought, I thank Victoria University of Wellington for granting me a submission scholarship and for the various supports they provided me over the years: the Student Health and Counselling services, the Student Learning Support group, the Hardship fund, the Disability services. I particularly thank Sharon Renfro, Deborah Laurs, Maria Rorke, and of course Patricia Stein and Shona De Sain from the faculty of Sciences. Sharon's controversial counselling helped me understand who I am and become more confident in life. I don't know where I would be without her.

How could I not thank GNS? GNS Science granted me the Sarah Beanland scholarship, provided me with an office space and outstanding IT support, and more importantly were very patient in letting me finish this $\mathrm{PhD}$. This is truly an exceptional place to work. I do not forget the GNS Social Club where I had a lot of fun and learned a lot, nor the many friends and colleagues I made over the years (special wink to Jenny and Leanne). 
I thank Karsten Gohl, Florian Wobbe and the Alfred Wegener Institute for the opportunity to go on the Polarstern cruise and acquire new data, and the subsequent collaborations. I thank Peter Molnar and the staff and crew of the MOANA cruise (2010), for the opportunity to gather new data and the interesting discussions we had.

My friends and family I thank profusely, but I will tell them privately.

I thank New Zealand for welcoming me in their beautiful country. I only wish I could have enjoyed it more.

I thank Hell Pizza Lower Hutt (especially David Bryce) for providing me with the means to survive financially and for opening my eyes to a different world.

Summarizing seven years in a few lines is never easy, and I hope I did not forget anyone. 
INTRODUCTION 
The region between New Zealand, Australia and Antarctica plays a key role in global tectonic reconstructions. Because the region includes the only non-destructive boundary linking the Pacific plate to the rest of the world [Gordon, 2000; Steinberger et al., 2004], it is essential to understand its past history, and especially its kinematics. A small error in the calculations of past relative movements south of New Zealand can have a major impact on estimation of past motions of the Pacific plate relative to other plates [Gordon, 2000; Steinberger et al., 2004].

The breakup of Gondwana after $90 \mathrm{Ma}$ led to the separation of New Zealand from Australia and Antarctica, and of Australia from Antarctica [Cande and Mutter, 1982; Cande et al., 1982; Eagles et al., 2004a; Gaina et al., 1998; Larter et al., 2002; Mayes et al., 1990; Molnar et al., 1975; Veevers and Li, 1991; Weissel and Hayes, 1977; Weissel et al., 1977; Whittaker et al., 2007]. Several boundary reorganizations, both at small and larger scales, occurred during the first stages of spreading (several in the southwest Pacific, complex rifting in the Tasman Sea). They are discussed in Chapter 1. Meanwhile, some boundaries (AustraliaAntarctica Basin, motion between East and West Antarctica) do not show undisputable evidence of seafloor spreading or extension ${ }^{1}$ between 85 and 40 Ma.

The region between New Zealand, Australia and Antarctica has been the subject of many studies since the 1960s. At first they were region-wide. Then as technology improved, research focused on smaller regions and/or time periods. Studies (in publication order) include: first evidence of seafloor spreading in the Tasman sea [Hayes and Ringis, 1973]; seafloor spreading between New Zealand and Antarctica since breakup [Molnar et al., 1975]; seafloor spreading in the Tasman Sea since the breakup [Weissel and Hayes, 1977]; compilation of data from previous studies and tectonic reconstructions for the area between New Zealand, Australia and Antarctica since breakup [Weissel et al., 1977]; magnetic anomalies older than anomaly 20 between Australia and Antarctica [Cande and Mutter, 1982]; seafloor spreading in the southern Pacific ocean, with a focus on the Eltanin fracture zone (FZ) and anomalies 21 to 34 [Cande et al., 1982]; tectonic reconstructions between New Zealand, Australia and Antarctica since the

\footnotetext{
${ }^{1}$ Seafloor spreading occurs at mid-ocean ridges and produces new lithosphere. Rifting (with or without volcanic activity) usually precedes the formation of new oceanic lithosphere and its result is the thinning of continental lithosphere, up to the creation of an ocean-continent boundary (named COB thereafter). Both seafloor spreading and rifting are forms of extension.
} 
breakup, focusing on defining uncertainties [Stock and Molnar, 1982]; introduction of the Bellingshausen plate in the plate circuit [Stock and Molnar, 1987]; use of satellite altimetry in the study of the southwest Pacific since Gondwana breakup [Mayes et al., 1990]; tectonic reconstructions focused on Australia for the last 160 Ma [Veevers et al., 1991]; extension between Australia and Antarctica, in the Tasman Sea and surrounding basins, since $150 \mathrm{Ma}$ [Veevers and Li, 1991]; Pitman FZ, since c27y [Cande et al., 1995]; seafloor surrounding the Macquarie Ridge [Sutherland, 1995]; eastern boundary of the Bellingshausen plate [Gohl et al., 1997]; Emerald FZ for times older than c24o [Marks and Stock, 1997]; South Tasman Rise and surrounding areas, from the breakup to anomaly 13 [Royer and Rollet, 1997]; spreading in the Tasman Sea from its inception to its cessation [Gaina et al., 1998]; western boundary of the Bellingshausen plate [Heinemann et al., 1999]; underwater extension of New Zealand terranes [Sutherland, 1999]; seafloor spreading between Australia and Antarctica, from the breakup to c18[Tikku and Cande, 1999]; Adare Trough, Nella Dan and Scott rifts, between anomalies 29 and 9 [Cande et al., 2000]; eastern boundary of the Bellingshausen plate, new data [Cunningham et al., 2002]; southern Pacific tectonic reconstructions between 105 and 45 Ma, including new data [Larter et al., 2002]; Cenozoic Pacific-Australia tectonics in the Southeast Tasman Sea [Keller, 2003]; introducing the Macquarie plate [Cande and Stock, 2004]; compilation of data for the Bellingshausen plate for times older than anomaly 27 [Eagles et al., 2004b]; reconstructions for the southwest Pacific between 90 and $45 \mathrm{Ma}$ [Eagles et al., 2004a]; Bollons Seamount from 85 to $70 \mathrm{Ma}$ [Davy, 2006]; Adare Trough sedimentation since $40 \mathrm{Ma}$ [Whittaker and Mueller, 2006]; structure of the Bounty Trough [Grobys et al., 2007]; AustraliaAntarctica spreading before anomaly 18 [Whittaker et al., 2007]; Menard FZ [Croon et al., 2008]; Hikurangi Plateau between 125 and 85 Ma [Davy et al., 2008]; structure of the Campbell Plateau and Great South Basin [Grobys et al., 2009]; intersections between ridge segments and fracture zones along the Pacific-Antarctica present spreading ridge [Croon et al., 2010]; cruise focusing on seafloor older than c20y close to Antarctica [Gohl and participants, 2010].

A major unresolved issue arising when looking at the past history of the region is the postulated existence of the Bellingshausen plate, its lifespan and location. The Bellingshausen plate was first introduced in the plate circuit by [Stock and Molnar, 1987], as a way to account for differing azimuths of magnetic lineations adjacent to the Antipodes FZ. However, several studies have since focused on the Bellingshausen plate, without being able to ascertain the precise location of its boundaries [Cunningham et al., 2002; Eagles et al., 2004a; b; Larter et al., 2002]. 
One factor impairing research in the Southwest Pacific is the difficult climatic conditions of Antarctica and its surrounding oceans. High quality data in reasonable quantity and with a good geographic distribution has so far been necessary to compile a detailed tectonic history of the region using traditional methods. Because of the sea-ice forming close to Antarctica, shiptrack magnetic data in the southern half of the region are sparse and clustered in three or four narrow zones.

In addition to the uneven distribution of data, the large timespan (five decades) during which they were acquired led some authors [Cande et al., 1995; Eagles et al., 2004b] to put aside the oldest data (imprecise location due to the pre-GPS navigation). In other words, by increasing the quality of the data, those authors became blind to important information about the region.

In order to calculate new, more accurate tectonic reconstructions for the southwest Pacific, it is necessary to take as much magnetic data as possible into account. To do so, a systematic analysis of all the magnetic profiles available in the region has to be done, magnetic reversals have to be identified on these profiles, and a system of weights has to be put into place to take into account the quality and reliability of each datum (Chapter II). Fracture zone data also have to be collected from satellite gravity and bathymetry in order to calculate finite plate rotations (Chapter III).

Another way to improve existing models is by using a reconstruction method that does not rely on data that is only of good quality and quantity. By going back to the original theory of plate tectonics (i.e. by imposing that the ridge and fracture zone segments be at right angle) and calculating finite rotations by successive approximations, with a suitable measure of fit (least-squares method), it is possible (to an extent) to compensate for the lack of magnetic data on the Antarctica side (method described in Chapter IV and results in Chapter V). 


\section{Chapter I}

PREVIOUS INTERPRETATIONS OF THE TECTONIC HISTORY OF THE SOUTHWEST PACIFIC 
The area studied during this $\mathrm{PhD}$ encompasses the oldest half of the seafloor formed between the New Zealand continental margin and its Antarctic equivalent. To understand the history of formation of this seafloor, it is also necessary to look at surrounding areas, like the Tasman Sea, the Australia-Antarctica basin, and the seafloor located between New Zealand and the Ross Sea (Figure I-1).

At present, the Pacific plate drifts away from Marie Byrd Land, creating new seafloor along the Pacific-Marie Byrd Land spreading ridge. Similarly, the Southeast Indian Ridge (SEIR) accommodates extension between Australia and East Antarctica. New Zealand is "cut in half': the North Island and part of the South Island move with the Australia plate, while the rest of the South Island moves with the Pacific plate. The Alpine Fault represents the boundary between the two parts of New Zealand. The nature of the Australia-Pacific boundary changes along its length: north of the Cook Strait the Pacific plate is obliquely subducted southwest beneath the North Island; south of Milford Sound the plate boundary is under northeast compression along the Macquarie Ridge Complex, with the Australia plate starting to be subducted obliquely beneath the Pacific plate; the Alpine Fault itself is an active transpressive fault. South of the Macquarie Ridge Complex, the subduction of Australian lithosphere is more orthogonal to the margin along the Hjort Trench. Southeast of the Hjort Trench, a series of short en échelon spreading segments marks one extremity of the Pacific-Marie Byrd Land spreading system. 


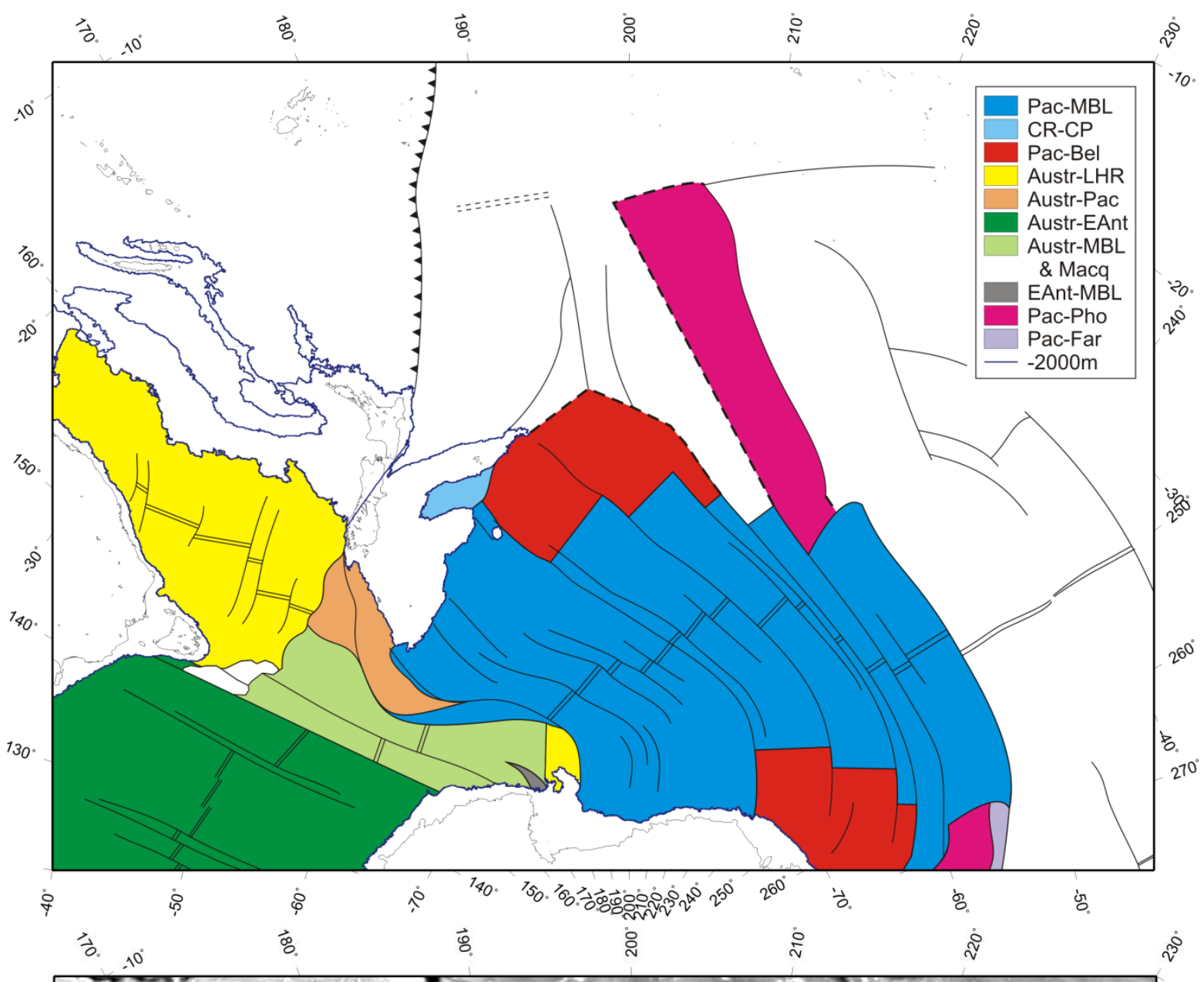

a)

$10^{\circ}$

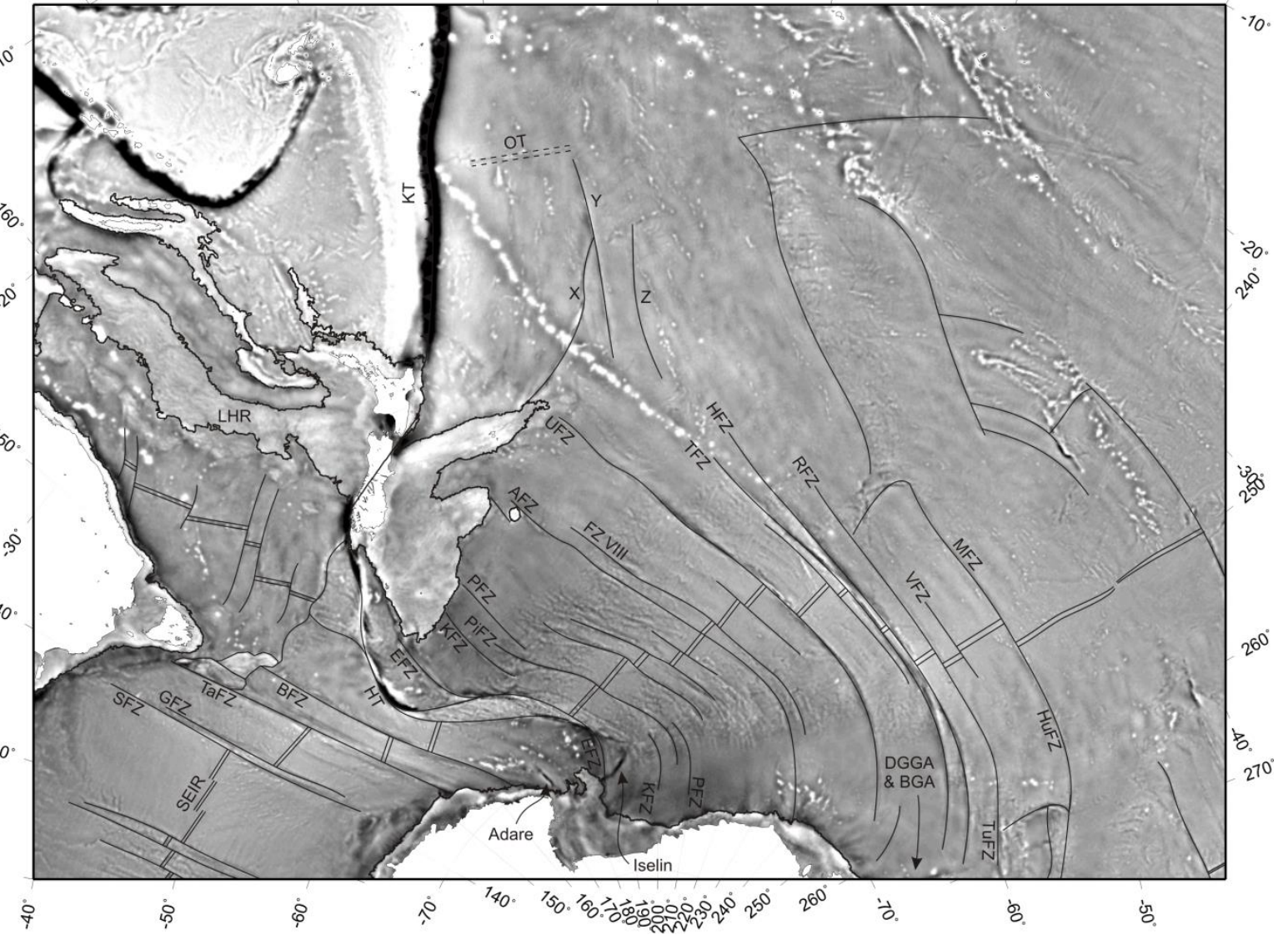


Figure I-1: Present-day location of the main features, plates and areas discussed in this thesis. a) Seafloor formed on different ridges are colour-coded: Pac-MBL=Pacific-Marie Byrd Land, $\mathrm{CR}-\mathrm{CP}=\mathrm{Chatham}$ Rise-Campbell Plateau (Bounty trough), Pac-Bel=Pacific-Bellingshausen, Austr-LHR=Australia-Lord Howe Rise (Tasman Sea), Austr-Pac=Australia-Pacific (Macquarie Ridge complex area), Austr-EAnt=Australia-East Antarctica, Austr-MBL=Australia-Marie Byrd Land (before c8), Macq=Macquarie microplate, EAnt-MBL=East Antarctica-Marie Byrd Land (Adare Trough and Iselin Rift), Pac-Pho=Pacific-Phoenix and Pac-Far=Pacific-Farallon. The $-2000 \mathrm{~m}$ contour is used to represent the continental margins. Dashed bold black lines are used to indicate unconfirmed boundaries. From Cande et al. (1982), Cande et al. (2000), Croon et al. (2008), Marks \& Stock (1997) and Sutherland (1995). b) Fracture zones, plates and main tectonic features. The same abbreviations are used throughout the text. Adare: Adare Trough; AFZ: Antipodes fracture zone; BFZ: Balleny fracture zone; BGA: Bellingshausen gravity anomaly; DGGA: De Gerlache gravity anomaly; EFZ: Emerald fracture zone; GFZ: George V fracture zone; HFZ: Heezen fracture zone; HT: Hjort trench; HuFZ: Humboldt fracture zone; Iselin: Iselin Rift; KFZ: Kohiku fracture zone; KT: Kermadec trench; LHR: Lord Howe Rise; MFZ: Meinard fracture zone; OT: Osbourn trough; PFZ: Pahemo fracture zone; PiFZ: Pitman fracture zone; RFZ: Raitt fracture zone; SEIR: South-East Indian Ridge; SFZ: Spencer fracture zone; TFZ: Tharp fracture zone; TaFZ: Tasman fracture zone; TuFZ: Tula fracture zone; UFZ: Udintsev fracture zone; VFZ: Vacquier fracture zone; $\mathrm{X}, \mathrm{Y}$ and $\mathrm{Z}$ : lineaments $\mathrm{X}, \mathrm{Y}$ and $\mathrm{Z}$. Gravity background from Sandwell \& Smith (1997) (version 20).

\section{Between New Zealand and West Antarctica}

\subsection{Prior to rifting: Gondwana and subduction margin}

120 million years ago, the Pacific plate and the Phoenix paleoplate were being subducted beneath the Gondwana margin (present-day New Zealand, Chatham Rise, Mary Bird Land and Antarctic Peninsula) (Davy et al., 2008; Eagles et al., 2004a). Around that time (125-120Ma; Taylor, 2006), the large igneous province (henceforth abbreviated as LIP) made of present-day Otong-Java, Manihiki and Hikurangi plateaus erupted (Figure I-2). Its creation has been attributed to the birth of the Louisville hotspot (Courtillot et al., 2003). Shortly after its formation, it began separating into the three distinct basaltic plateaus (Davy et al., 2008). East of the area, it has been proposed that the Pacific-Phoenix spreading ridge connected with the Farallon paleoplate on the ridge-ridge-ridge Tongareva triple junction (McCarron \& Larter, 1998). 


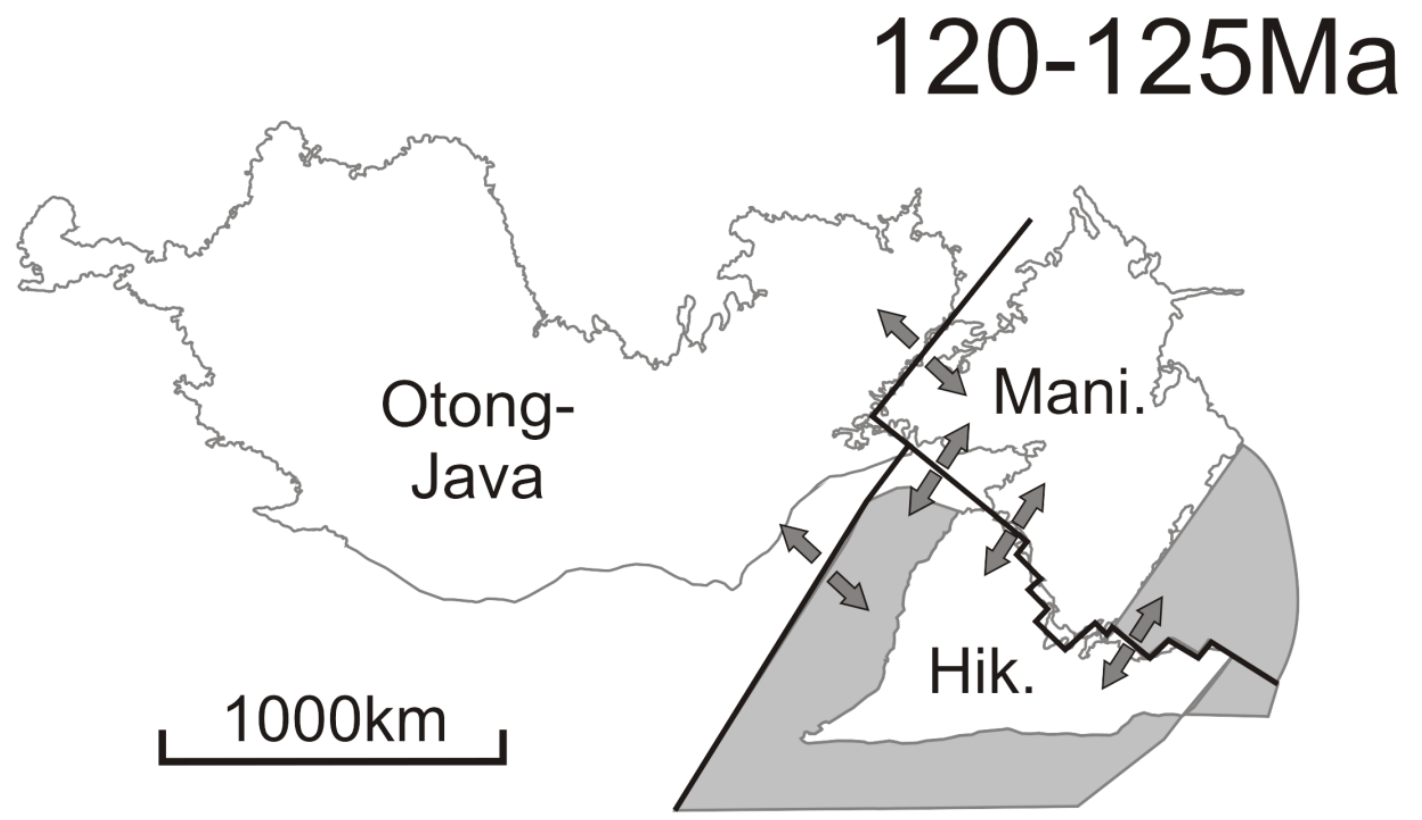

Figure I-2: Formation of the Hikurangi-Manihiki-Otong-Java plateau followed by its breakup. Bold black lines mark the supposed location of the initial breakup of the plateau. Shaded areas represent an estimate of parts of the initial plateau that have since been subducted. Mani.: Manihiki plateau; Hik.: Hikurangi plateau. Figure modified from Davy et al. (2008).

Following its separation from the rest of the LIP, the Hikurangi plateau started moving southwards with the Pacific plate, towards the Gondwana subduction (Davy et al., 2008). It is estimated the plateau's southern tip reached the trench around $110 \mathrm{Ma}$ (Davy, 2014; Figure I-3). The arrival at the trench of the buoyant crust increased compression within the accretionary prism, which led to the uplift of the Chatham Rise from previously accreted terranes (Davy et al., 2008).

Around $100 \mathrm{Ma}$, the Pacific lithosphere surmounted by the Hikurangi Plateau entered the trench, increasing the friction along the subduction interface, and the subduction became jammed (Davy et al., 2008). As the motion between the Pacific plate and what was the Gondwana forearc became null, spreading ceased on the Osbourn trough and was progressively transferred southward, opening the Bounty Trough (Davy et al., 2008; Larter et al., 2002; McCarron \& Larter, 1998; Figure I-3 and Figure I-4). There is also evidence of widespread volcanism between 99 and 89 Ma from the Chatham Rise to the South Island of New Zealand (Davy et al., 2008; Hoernle et al., 2006; Hoernle et al., 2010). By 96 Ma, the Pacific slab below the Chatham Rise was detached from the rest of the plate, initiating the uplift of the Chatham 
Rise and a regional tectonic reorganisation, leading to the breakup of the Gondwana margin (Davy et al., 2008; Davy, 2014).

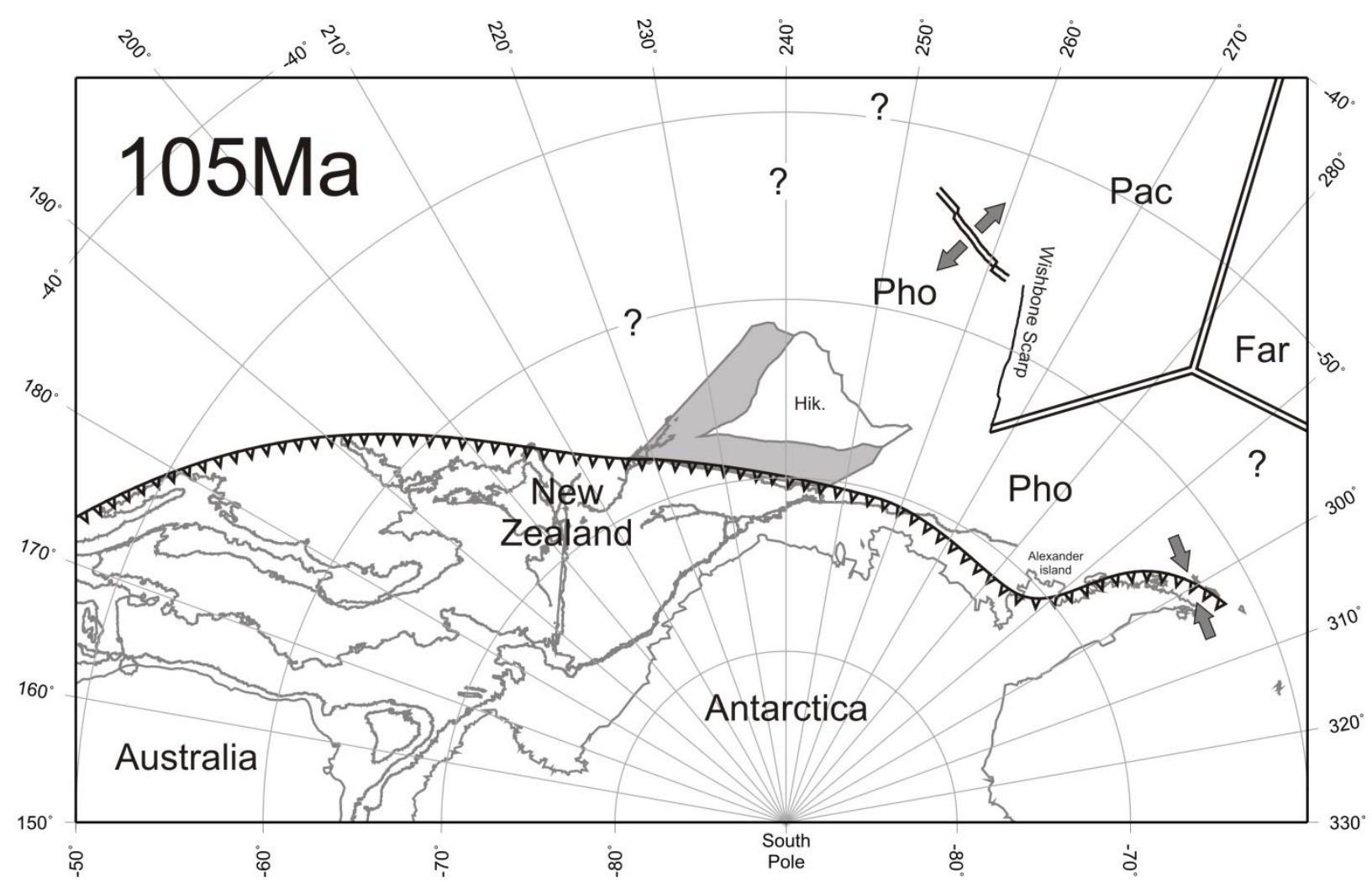

Figure I-3: The Hikurangi plateau reaches the Gondwana subduction zone. Location for the Pacific-Phoenix-Farallon triple junction from McCarron \& Larter (1998). There is a lack of consensus on the position of the Pacific-Phoenix-Farallon triple junction in Late Cretaceous times. Uninterrupted double lines mark the location of active ridge segments. Bold grey arrows indicate the direction of movement between two plates (extension or compression). Question marks were added where no reliable information exists. Far; Farallon plate; Pac: Pacific plate; Pho: Phoenix plate. Bathymetric contours from Smith \& Sandwell (1997). Rotations used to calculate the paleoposition of the margins are from Keller (2003) (Lord Howe Rise-Campbell Plateau), Sutherland (1995) (Australia-Antarctica), this study and visual fitting. Antarctica is fixed. 


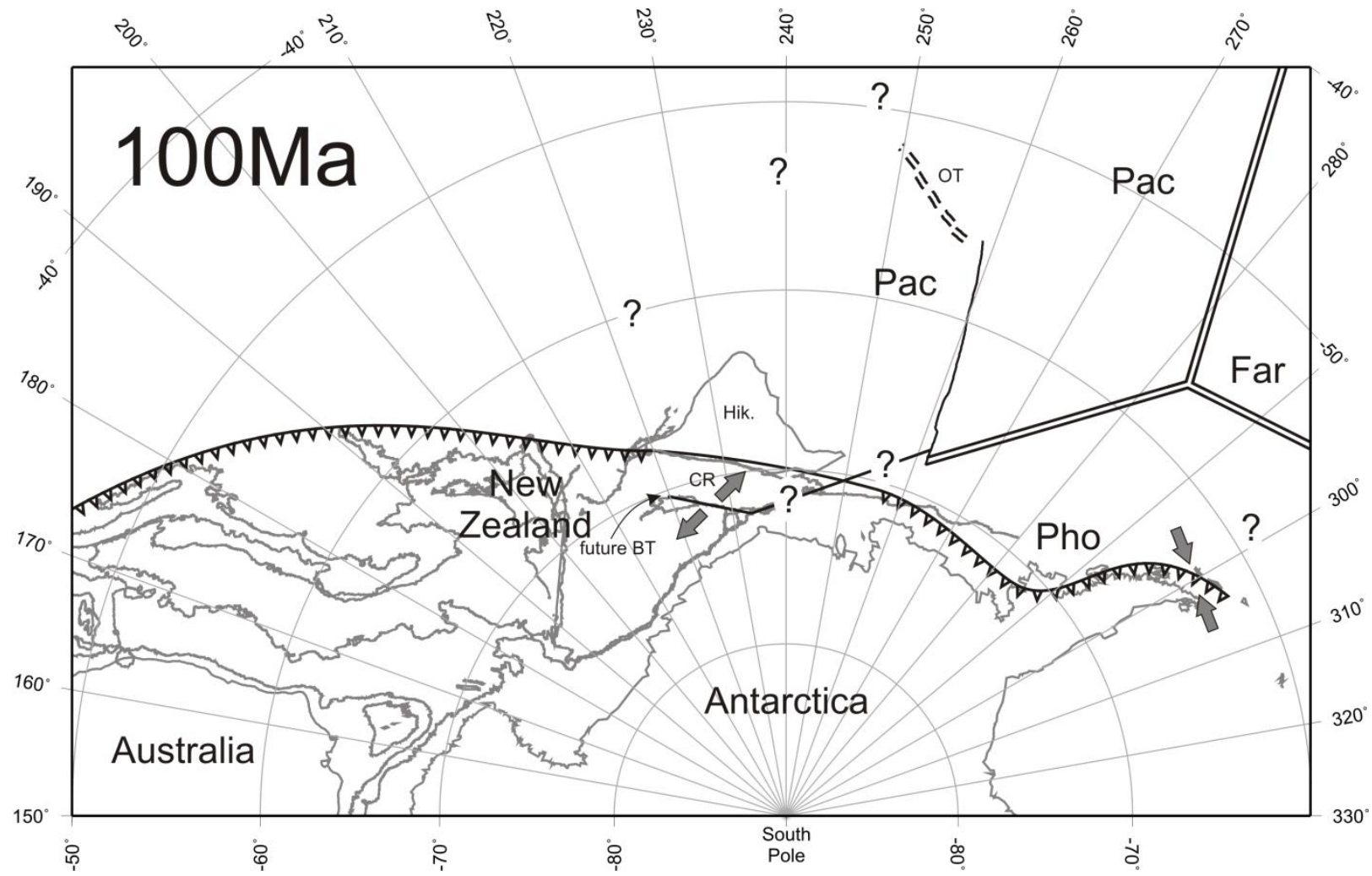

Figure I-4: Jamming of the subduction and transfer of extension from the Osbourn Trough (OT) to the Bounty Trough. The Pacific-Phoenix ridge is getting closer to the margin. The connection between the Pacific-Phoenix ridge and the newly created Bounty Trough is unclear. The area west of the Hikurangi Plateau and the Osbourn Trough has since been subducted. BT: Bounty Trough; CR: Chatham Rise. Bathymetric contours from Smith \& Sandwell (1997). Legend and rotations as in Figure I-3.

\subsection{Rifting and oceanisation}

The first episode of rifting of the southwest Pacific set off the opening of the Bounty Trough. A recent study by Grobys et al. (2007) combines several geophysical datasets. According to Grobys et al. (2007), the Bounty trough is a failed rift arm and can be divided into three zones (Grobys et al., 2007):

- the outer Bounty Trough presents clear magnetic anomalies ${ }^{2}$, indicating that extension in that area reached the seafloor spreading stage;

\footnotetext{
${ }^{2}$ Magnetic anomalies are departures from the chosen reference magnetic field on a magnetic profile. Magnetic anomalies can be either positive (normal) or negative (reverse). Magnetic chrons are the point of inversion between a positive and a negative anomaly. Each chron corresponds to a well-defined date (the timescale by Cande \& Kent (1995) is used throughout this thesis). Magnetic lineations are the trend made on a map view by linking magnetic chrons of a same age between two fracture zones. Each magnetic lineation reflects the orientation of one paleo-ridge segment. Each magnetic lineation can be considered an isochon.
} 
- the inner Bounty Trough is mostly composed of thinned continental crust intruded by volcanics;

- the centre part of the Bounty Trough is intermediate between the inner and outer Bounty Trough, with numerous magmatic intrusions in thinned continental crust.

Grobys et al. (2007) mention the possible existence of a triple junction at the mouth of the Bounty Trough. Although the interpretation by Schellart et al. (2006) of the Bounty Trough spreading as back-arc extension would explain the later change of spreading direction (see below), their model also dates the collision of the Hikurangi plateau with the Gondwana margin at about $82 \mathrm{Ma}$, and links it to the cessation of spreading in the Bounty Trough around 83Ma.

Gravity modelling by Grobys et al. (2007), by analogy with the spreading fabric of the Gulf of Aden, suggests the presence of "oblique magmatic cells",3 at depth under the Bounty trough, related to incipient seafloor spreading. Grobys et al. (2007) interpret these cells as a sign of oblique or en échelon rifting, which indicates that the extension was oblique to the Bounty Trough by the time it moved further south. The stretching factors Grobys et al. (2007) calculated suggest the location of rifting jumped shortly after the inception of seafloor spreading within the outer Bounty Trough.

${ }^{3}$ I understand this to mean the magmatic cells are oblique to the ridge segments. 


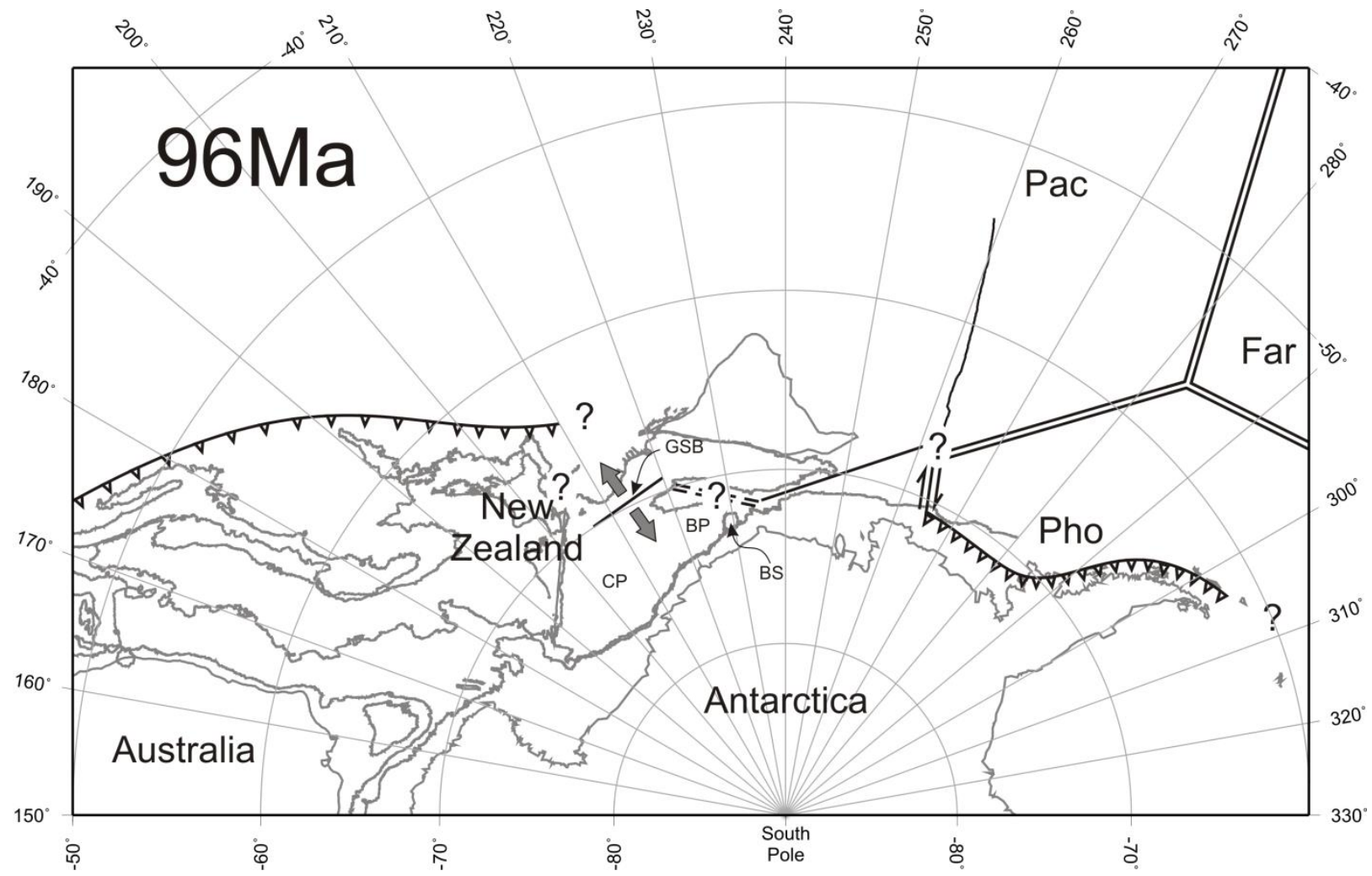

Figure I-5: Just before the breakup, opening of the Great South Basin. Bathymetric contours from [Smith and Sandwell, 1997]. BP: Bounty plateau; BS: Bollons seamount; CP: Campbell plateau; GSB: Great South Basin. Legend and rotations as in Figure I-3.

Grobys et al. (2009) propose that the Campbell plateau and surrounding areas were subject to regional underplating and extension in the late Jurassic to Early Cretaceous. The Great South Basin is composed of stretched continental crust (about 13km thick, [Grobys et al., 2009]). The sedimentation in the Great South Basin, as evidenced by seismic reflection profiles interpretation, indicates that extension stopped in Piripauan time (86.5-84 Ma) [Grobys et al., 2009]: the Hoiho sedimentary group (108.4-83.5 Ma) is faulted and presents syn-rift depositional features, whereas the Pakaha sedimentary group is not faulted but shows signs of rapid subsidence. These results are consistent with a transfer of the location of extension from the Bounty Trough and Great South Basin to the south shortly before magnetic reversal c34y (83 Ma) [Davy, 2014].

It is generally accepted that at that time the extension jumped to its current location (between the Campbell plateau, the Chatham Rise and the Antarctica margin), with the exception of the Bollons Seamount (Figure I-6). Davy (2006) found that the fit of Bollons Seamount against the margin of the Campbell Plateau is good, as long as he applied a 15 degrees rotation to the Bollons Seamount in his reconstruction. Using high quality bathymetric, magnetic and gravity data, Davy (2006) also dated the ridge jump mentioned at the beginning 
of this paragraph at 83.7 Ma. His model includes a ridge jump from north of Bollons Seamount to south of it at approximately 78.5 Ma (Figure I-8). According to Davy (2006), the jump was due to the distance between "Bollons Seamount" and the "Bounty Plateau" ridge segments becoming too great: prior to the ridge jump, the seafloor spreading on each segment was asymmetric (Figure I-7), in opposite directions, resulting in the distance between the segments increasing with time.

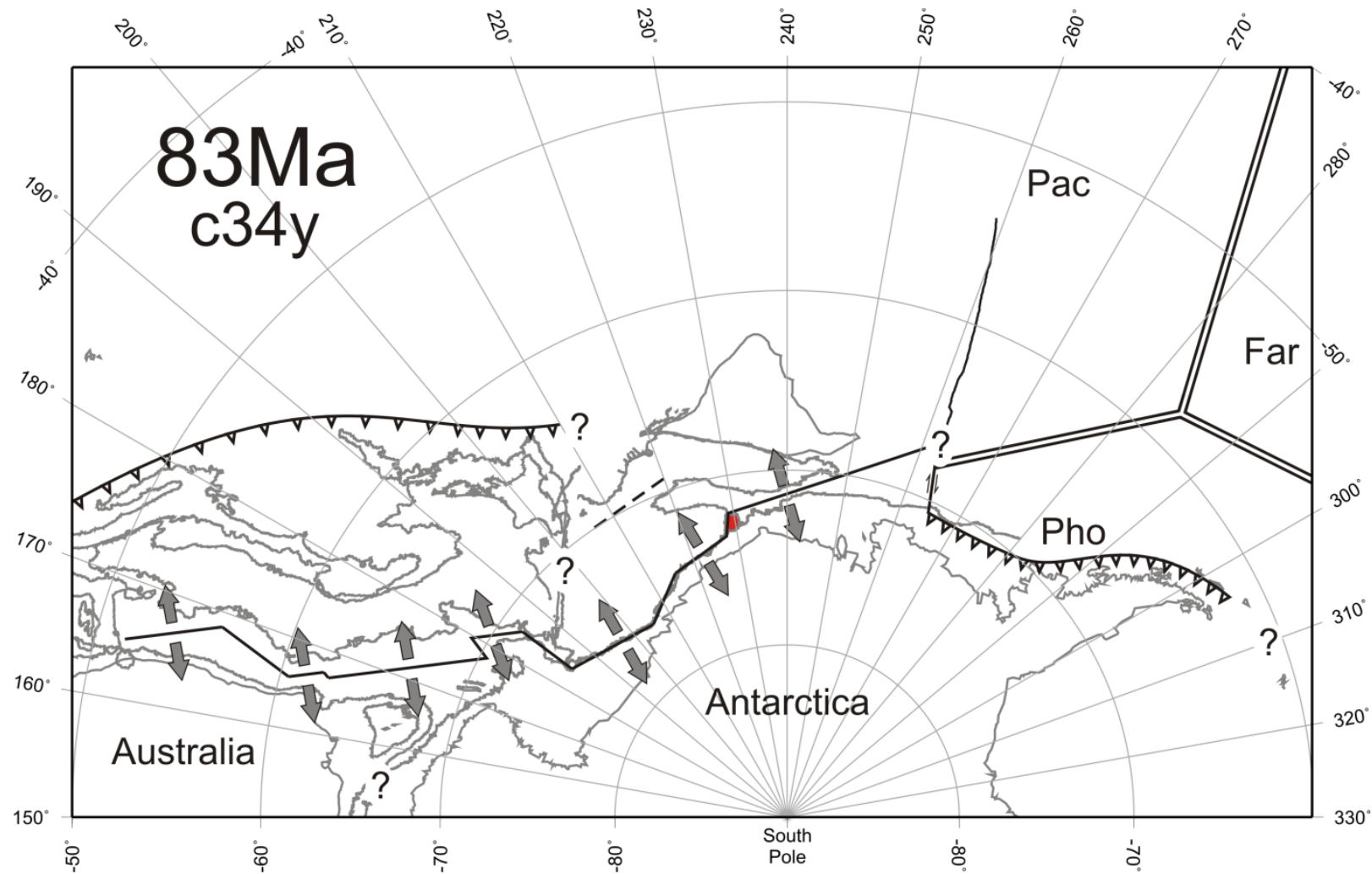

Figure I-6: Transfer of extension from the Great South Basin to south of the Campbell Plateau and breakup of the Gondwana margin. The Bollons Seamount is highlighted in red. Bathymetric contours from [Smith and Sandwell, 1997]. Rotations from [Keller, 2003] (Lord Howe Rise-Campbell Plateau), [Sutherland, 1995] (Australia-Antarctica), this study and visual reconstruction. Legend as in Figure I-3. 

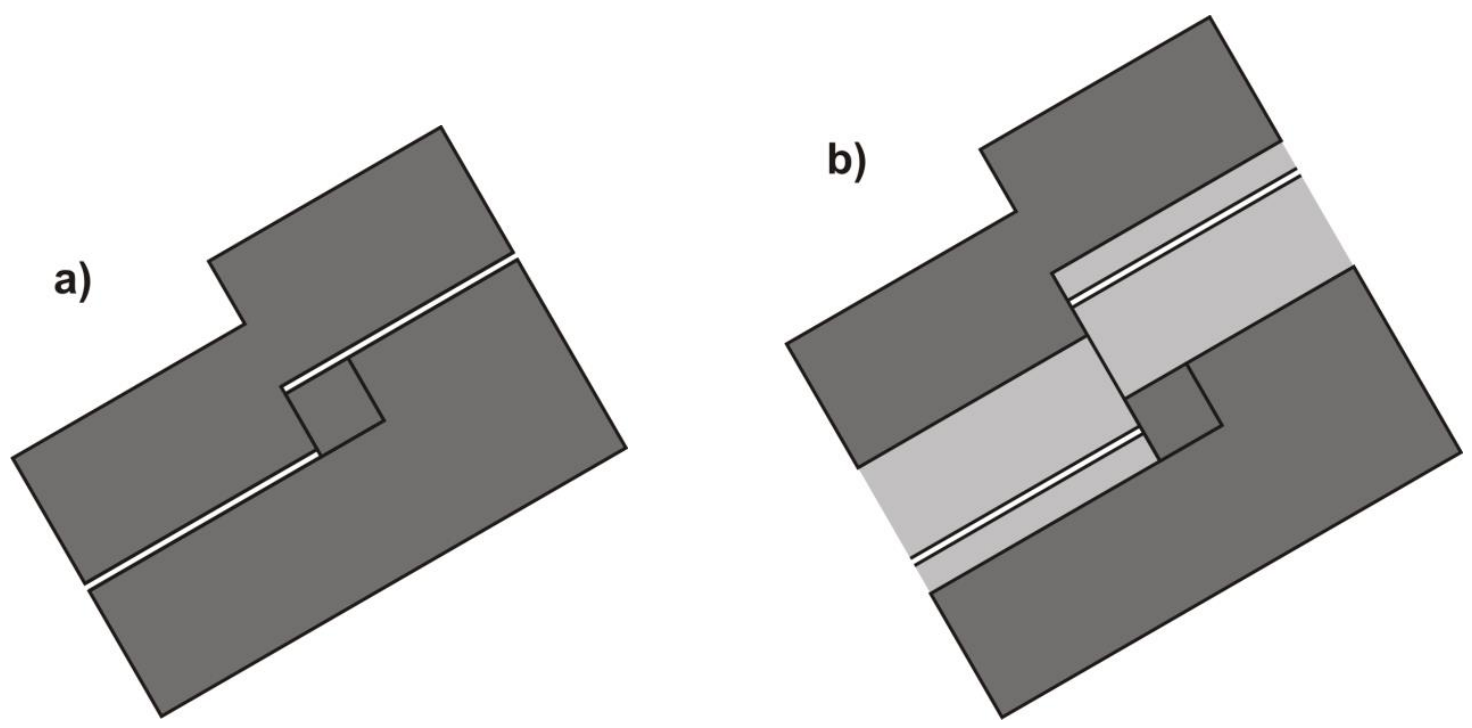

Figure I-7: Asymmetric seafloor spreading on two segments with asymmetry in opposite directions. Dark grey: continental blocks; light grey: seafloor formed at the spreading segments; black double line: active spreading segment. a) $t_{0}$ : before any asymmetric spreading. b) $t_{1}$ : after a period of asymmetric spreading; spreading between $t_{0}$ and $t_{1}$ was faster to the North of the western spreading segment and to the South of the eastern one.

Following the 78.5 Ma ridge jump, the offset between the "Bounty Plateau" and the "Chatham Rise" spreading segments caused the formation of the Antipodes FZ (see Figure I1b). The bathymetric signature of the oldest parts of the Antipodes FZ indicates the fracture zone was under stress at the time of its formation [Davy, 2006]. As Pacific fracture zones in general are relatively weak, they can accommodate changes in plate motions by undergoing deformation [Hall and Gurnis, 2005]. In the case of the Antipodes FZ, it shows obvious signs of convergence then divergence at the time of its formation [Davy, 2006]. Davy (2006) suggests deformation of the Antipodes FZ was a precursor to separation of the Bellingshausen plate (see below). 
(a)

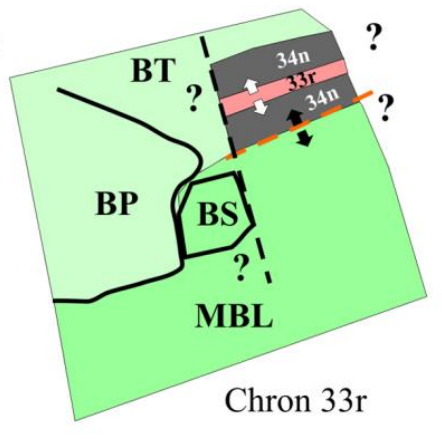

(c)

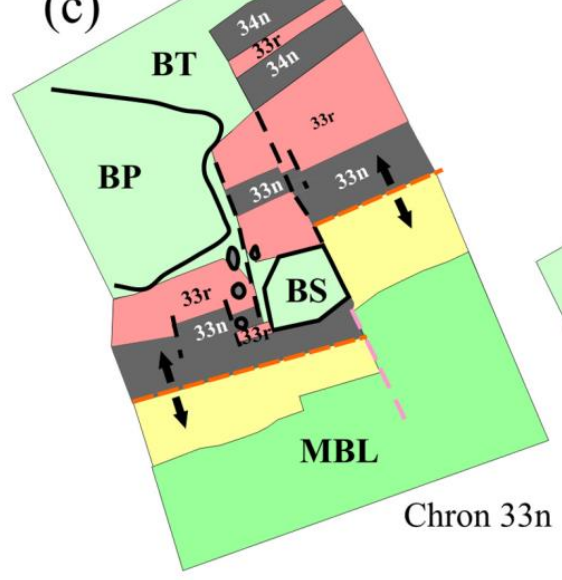

(b)

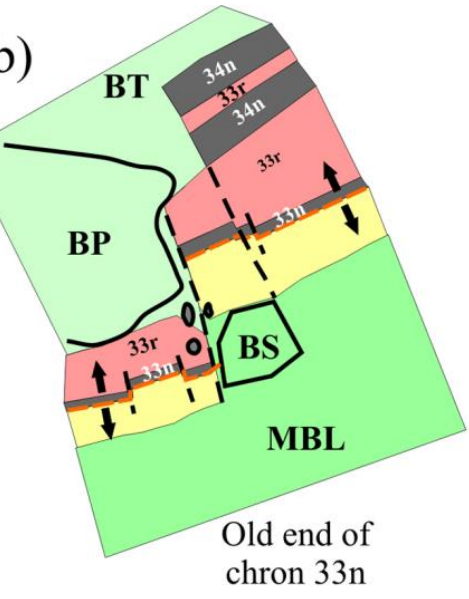

(d)

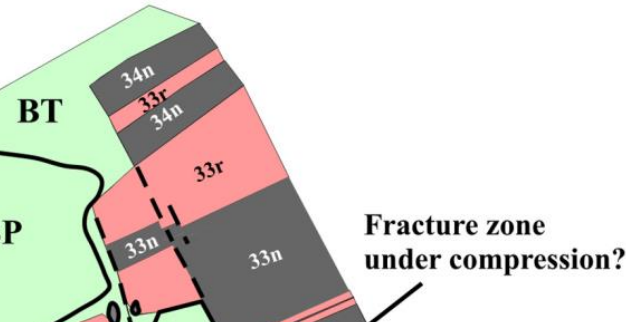

BP

(e)

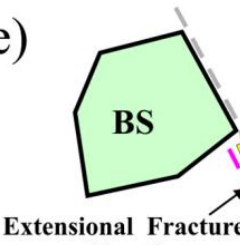

Ridge-end MBL
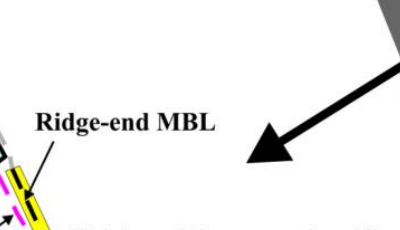

I Thickened (compressional?)

Extensional Fra
Zone?

Thickened (compressiond
Fracture Zone

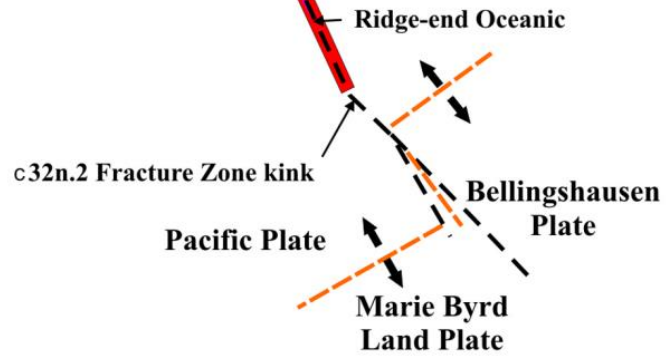

MBL

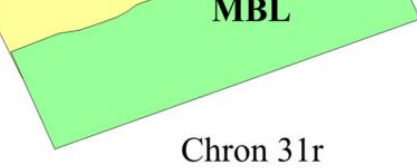

Chron 31r

Figure I-8 (previous page): Rifting and seafloor spreading around the Bollons Seamount. BS: Bollons Seamount; BP: Bounty Plateau; BT: Bounty Trough; MBL: Marie Byrd Land. Orange dashed line: spreading segment; black dashed line: fracture zone; violet dashed line: possible extension of the fracture zone; yellow fill: already formed Marie Byrd Land oceanic crust. a) 83.7Ma: southward ridge jump following the initial rifting in the mouth of the Bounty Trough during anomaly 34 . b) $79 \mathrm{Ma}$ (c33o): seafloor spreading prior to the second ridge jump; the Bollons Seamount "belongs" to Marie Byrd Land. c) 76 Ma: the seafloor spreading has jumped south of the Bollons Seamount, which now "belongs" to the Pacific plate. d) about 70 Ma: the Antipodes FZ is under positive and negative normal stress. The northern ridge segment has cleared the Bollons Seamount. e) Schematic drawing focused on the Pacific-Marie Byrd Land-Bellingshausen triple junction and the deformations observed on the Antipodes FZ; snapshot at about 70Ma. After Davy (2006), his figure 7. 


\subsection{First part of the post-rifting story: Bellingshausen?}

The Bellingshausen plate is a paleoplate that was postulated to exist south of the PacificAntarctica ridge in the Late Cretaceous and Early Tertiary, just east of the Marie Byrd Land plate (Figure I-9). It was first introduced by Stock and Molnar (1987) who included it in their tectonic reconstructions, following the observation that magnetic lineations north and south of the Antipodes FZ are not parallel to each other [Molnar et al., 1975]. Stock and Molnar (1987) found that reconstructions involving a three-plate system (Pacific, Marie Byrd Land and Bellingshausen) gave a better fit than those using a two-plate system (Pacific and Marie Byrd Land only), even when considering oblique spreading. These conclusions were confirmed [Stock et al., 1996] ${ }^{4}$, and it was noted that there was a difference in spreading rates either side of the Antipodes FZ (faster on the western side) and fan-shaped magnetic lineations east of it (opening to the east on the Bellingshausen side of the ridge, to the west on the Pacific side, see their figure 1). Davy (2006), after Cande et al. (1995), also uses the fact that in the Early Tertiary, fracture zones west of the Antipodes FZ were left-stepping, whereas fracture zones east of it were right-stepping, as a confirmation that each group of fracture zones was formed along a different plate boundary.

The precise dating of the start and cessation of independent Bellingshausen movement is still unresolved, despite recent improvements. Stock and Molnar (1987) estimate the transfer of Bellingshausen seafloor to Marie Byrd Land between c18y and c21o (linking it to a major plate reorganisation in the southwest Pacific) by noting, around that time, changes in the roughness of the seafloor, in the direction, step and number of fracture zones and in the orientation of magnetic lineations. Subsequent works [Mayes et al., 1990] and [Stock et al., 1996] date cessation to just after c25y and between anomalies 27 and 28 , respectively ${ }^{5}$.

\footnotetext{
${ }^{4}$ Although this article was never published, I decided to use it for my study as it contains valuable information about the magnetic data and finite rotations associated with the Bellingshausen plate, and it is cited in numerous publications.

${ }^{5}$ Although the study by [Mayes et al., 1990] is valuable because it was one of the first to use satellite altimetry to look at the South Pacific seafloor, its use of magnetic data is disputable. As they explain in their paragraph 16, they do not make any distinction between the different parts of any magnetic anomaly. For instance, a magnetic pick labelled c27 in [Mayes et al., 1990] could in fact correspond to chrons c27o, c27y or to the maximum point of the anomaly, c27. This simplification can lead to inaccuracies of more than $50 \mathrm{~km}$.
} 


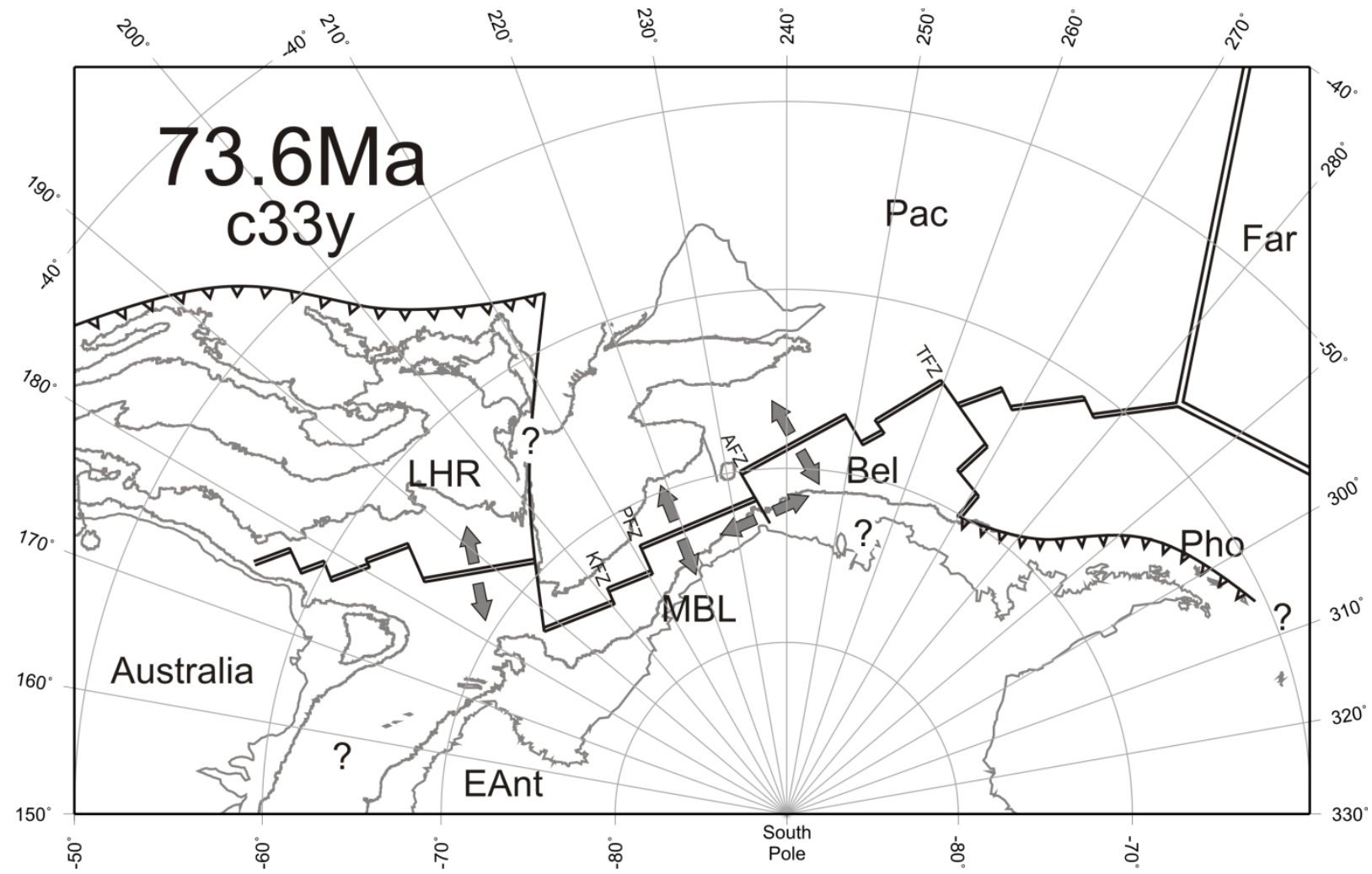

Figure I-9: Snapshot at c33y: seafloor spreading is in place in the South Pacific and the Tasman Sea. Bathymetric contours from [Smith and Sandwell, 1997]. Bel: Bellingshausen plate; EAnt: East Antarctica; LHR: Lord Howe Rise; MBL: Marie Byrd Land. Rotations from [Gaina et al., 1998; Stock et al., 1996]. The motion between Australia and East Antarctica is not directly constrained. Legend as in Figure I-3.

More recent studies tend to agree on a cessation of independent Bellingshausen movement around anomaly 27. Cunningham et al. (2002) and Larter et al. (2002) link that event to a more global plate boundary reorganisation involving termination of the wedge that produced the Bellingshausen Gravity Anomaly $\left(94^{\circ} \mathrm{W}, 69.5^{\circ} \mathrm{S}\right.$; see below) and the start of extension of the structure causing the De Gerlache Gravity Anomaly $\left(91.5^{\circ} \mathrm{W}, 65^{\circ} \mathrm{S}\right.$, see Figure I-1b). Eagles et al. (2004b) propose that the failure to initiate subduction on the Bellingshausen Gravity Anomaly could be the reason for the circa anomaly 27 plate reorganisation. Davy (2006) uses the change from left- to right-stepping offset of fracture zones south of the Antipodes FZ to date the event around c27. A detailed study (combining swath bathymetry, high resolution gravity and magnetic data) of the Pitman FZ finds that it appeared instantaneously during anomaly 26r as a right-stepping fracture zone [Cande et al., 1995]. The authors suggest a link with demise of the Bellingshausen plate, dating that demise at anomaly 27. 
The precise date of the start of independent Bellingshausen motion is an unresolved issue. Dates of c29 [Mayes et al., 1990] and c31 [Stock and Molnar, 1987] have been suggested, and ages put forward by other authors vary from anomaly c32.1 [Davy, 2006] to just after c33o [Eagles et al., 2004b]. Davy (2006) bases his dating on a kink in the strike of the Antipodes FZ, whereas Eagles et al. (2004b) find a third plate is needed after c33o in their detailed tectonic reconstruction calculations. Eagles et al. (2004b) also suggest excessive stress on some fracture zones could have caused the Bellingshausen plate to start moving independently. Stock et al. (1996) agree with both studies mentioned above and estimate the "birth" of the Bellingshausen plate to be between anomaly c32.1 and c33o. Finally, Larter et al. (2002) and Cunningham et al. (2002) place the start of independent Bellingshausen motion at c33y for three reasons:

- spreading segments on the Pacific-Bellingshausen ridge underwent a counter-clockwise rotation after c33y [Stock et al., 1996];

- c33y corresponds to a change in the azimuth of several fracture zones (Udintsev and Eltanin, plus the kink in the Antipodes FZ dated at c32.1 by [Davy, 2006]);

- a change from a two to a three plates system at c33y in their tectonic reconstructions gives the best fit.

The precise location of the western, southern and eastern boundaries of the Bellingshausen plate is a more controversial issue. Although most studies [Cande et al., 1982; Davy, 2006; Eagles et al., 2004b; Molnar et al., 1975; Stock and Molnar, 1987; Stock et al., 1996] consider the Antipodes FZ to be the western boundary of Bellingshausen, only one study looks into the question in detail [Heinemann et al., 1999]. Using recently collected seismic reflection profiles, on two adjacent profiles they image a graben oriented $\mathrm{N} 17^{\circ} \mathrm{W}$ cantered at about $233.65^{\circ} \mathrm{E}, 68.45^{\circ} \mathrm{S}$ (just east of the Antipodes FZ) [Heinemann et al., 1999]. The graben is associated with a gravity low which cannot be mistaken for the signature of a fracture zone (amplitude too high, [Heinemann et al., 1999]). They interpret the graben to be a short paleospreading ridge segment, fragment of the boundary between Bellingshausen and Marie Byrd Land, and limited to the north and south by fracture zones. They also note that seafloor east of the two profiles is systematically rougher, indicative of slower spreading on a different ridge [Heinemann et al., 1999].

Regarding the southern boundary of the Bellingshausen plate, it is not known whether the Bellingshausen plate was entirely oceanic or whether some part of it was made of continental lithosphere [Eagles et al., 2004b]. A scenario has been proposed in which the Bellingshausen 
plate was entirely oceanic [Stock et al., 1996], and could be the result of the evolution of a fragment of the Phoenix plate; by analogy with the Rivera plate, offshore Mexico, which evolved from a fragment of Farallon. However, it is equally possible that the southern boundary of the Bellingshausen plate was situated in continental crust: reconstructions [Larter et al., 2002] suggest it could either have been a diffuse boundary, or it could have jumped over time. The Peacock Gravity Anomaly $\left(106.5^{\circ} \mathrm{W}, 72.5^{\circ} \mathrm{S}\right)$, the Noville Gravity Lineation $\left(103^{\circ} \mathrm{W}\right.$, $\left.70.2^{\circ} \mathrm{S}\right)$ and the margin north of Thurston Island $\left(99^{\circ} \mathrm{W}, 72.2^{\circ} \mathrm{S}\right)$ may have been part of that boundary at some point in time [Larter et al., 2002].

Defining the eastern boundary of the Bellingshausen plate is more complex, as it involves interactions with several plates (Phoenix, Charcot, Pacific, Marie Byrd Land) and was not stable over time. One main feature of this boundary is the Bellingshausen Gravity Anomaly [Cunningham et al., 2002]. Located near the Antarctica margin at approximately $270^{\circ} \mathrm{E}$, the Bellingshausen Gravity Anomaly results from an accretionary wedge buried under more recent sediments (Figure I-10), with Late Cretaceous basement dipping eastward beneath more elevated oceanic basement [Cunningham et al., 2002; Gohl et al., 1997]. Both basements probably formed on the Pacific-Charcot ridge during the Cretaceous magnetic superchron (c34) [Cunningham et al., 2002]. The location of the Bellingshausen Gravity Anomaly wedge was probably determined by the presence of a zone of weakness such as a fracture zone or a ridge segment [Cunningham et al., 2002]. The Bellingshausen Gravity Anomaly wedge may be the result of Early Tertiary oblique subduction of the Bellingshausen plate beneath the Phoenix plate [Gohl et al., 1997]. The sedimentary sequences on several seismic profiles indicate the Bellingshausen Gravity Anomaly may have been active shortly after c34y [Cunningham et al., 2002].

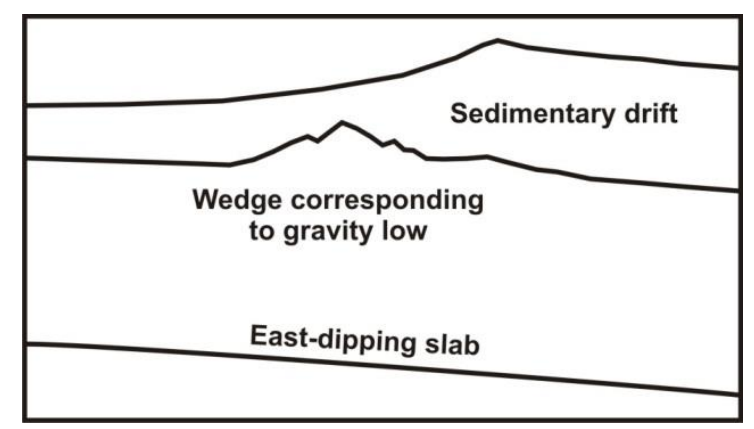

Figure I-10: Interpretation of a seismic reflection profile across the Bellingshausen Gravity Anomaly copied from [Gohl et al., 1997], their figure 2. 
There is evidence that, prior to formation of the Bellingshausen Gravity Anomaly, the Eltanin $\mathrm{FZ}^{6}$ was the site of Charcot/Marie Byrd Land/Bellingshausen-Phoenix interactions [Cande et al., 1982; Davy, 2006; Larter et al., 2002; Mayes et al., 1990] (Figure I-11). Davy (2006) notes a large difference in spreading rates between the mouth of the Bounty Trough and the seafloor east of the Heezen FZ where Late Cretaceous spreading is faster. That difference can be explained if the seafloor east of the Heezen FZ was formed on the Pacific-Phoenix ridge rather than on the Pacific-Marie Byrd Land/Bellingshausen ridge. Cande et al. (1982) also reach that conclusion. Initial interpretation of satellite altimetry data [Mayes et al., 1990] is that the Eltanin FZ system represented the boundary between the Bellingshausen and Phoenix plates during anomaly 29, but that boundary propagated eastward around anomaly 28. Larter et al., (2002) propose the boundary jumped west to the modern-day De Gerlache Gravity Anomaly when Bellingshausen became part of Marie Byrd Land. Moreover, Larter et al. (2002) identify lineament Z (Figure I-1) as the trace of a triple junction: Pacific-Phoenix-Charcot ${ }^{7}$ before $\mathrm{c} 34 \mathrm{y}$, then Pacific-Phoenix-Marie Byrd Land until c33y (or whenever the Bellingshausen plate starts moving independently), then Pacific-Phoenix-Bellingshausen until anomaly 27.

To sum up, although authors now agree on the existence of a Bellingshausen plate, the resolution of its boundaries, start and end dates still requires further work.

Following the demise of the Bellingshausen plate, a regional reorganisation of the PacificMarie Byrd Land spreading system led to the appearance of new fracture zones, such as the Pitman FZ ([Cande et al., 1995], see above). Stock and Molnar (1987) were first to identify these changes, but their dating of the event ( c18, no doubt due to the scarcity of data available at the time) made them relate it to the Hawaii-Emperor bend (see below). Cande et al. (1995) present an "excellent fit" of calculated flowlines ${ }^{8}$ with each corresponding fracture zone, as do the rotations calculated by [Molnar et al., 1975]. However, it is worth noting thatCande et al.

\footnotetext{
${ }^{6}$ The Eltanin fracture zone system is formed of the Heezen FZ and the Tharp FZ, separated (over time) by one or more small ridge segment(s).

${ }^{7}$ Charcot is a microplate that separated from the Phoenix plate when the Pacific-Charcot active spreading ridge reached the Gondwana subduction [Larter et al., 2002]. Nowadays, remnants of the Charcot microplate are located east of the Bellingshausen Gravity Anomaly [Eagles et al., 2004a].

${ }^{8}$ A flowline is the calculated trajectory, using a set of relative rotations between two plates, of a point situated on the boundary between the two plates. If the point is chosen at the intersection of a ridge segment and a fracture zone, and the rotations are accurate, the flowline should match the fracture zone exactly.
} 
(1995) found a $75 \mathrm{~km}$ discrepancy with an earlier analysis of flowlines [Mayes et al., 1990] and the corresponding fracture zones.

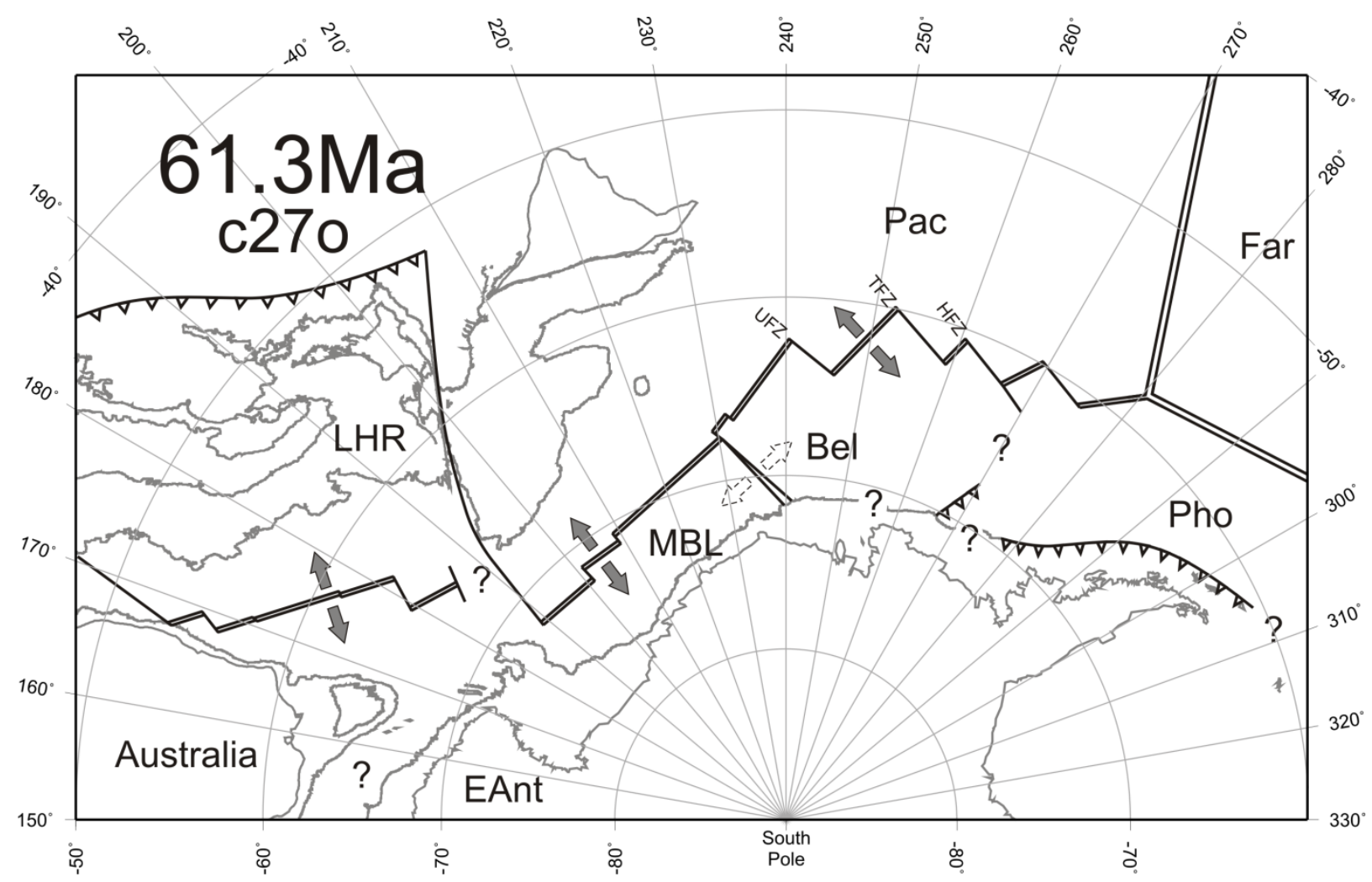

Figure I-11: Snapshot at c27o (61.3 Ma): cessation of independent Bellingshausen motion. Rotations from [Cande et al., 1995] (Pacific-Marie Byrd Land), [Keller, 2003] (Pacific-Lord Howe Rise), [Gaina et al., 1998] (Lord Howe Rise-Australia), and this study. The motion between Australia and East Antarctica is not directly constrained. Legend as in Figure I-3.

Because of their proven good quality, Eagles et al. (2004a) use Cande et al. (1995) rotations in their high resolution tectonic reconstructions. They find that the postBellingshausen reorganisation of the Pacific-Marie Byrd Land fracture zones was over by anomaly 26 (57.7 Ma). They [Eagles et al., 2004a] suggest a causal link between the decrease in Pacific-Marie Byrd Land spreading rate between anomaly 27 and anomalies 25-24 and the increase in the number of fracture zones on the ridge; and they also propose that the increase in amplitude of the gravity signal of the Udintsev and Eltanin FZs could result from the fracture zones being under transpression during the reorganisation.

\subsection{Second part of the post-rifting story: the eastern Southwest Pacific}

The Hawaii-Emperor bend ( $\mathrm{H}$ bend) is a famous sharp eastward change of direction in the track of the Hawaii hotspot. It is of interest in this study because it marks a major event in the tectonic history of the Pacific plate. The $\mathrm{H}$ bend also appears on the Louisville hotspot track as 
a more progressive curve [Richards and Lithgow-Bertelloni, 1996]. Such a change of azimuth of the track is indicative of a change of motion of the plate the seamounts formed on, the Pacific plate. Until recently, the $\mathrm{H}$ bend was dated at $\sim 33 \mathrm{Ma}$ (chron 20), which does not match the observed changes in fracture zone orientation on the Pacific plate [Stock, 2006], and cannot be linked to any major change in plate configuration around that time: the $\sim 43 \mathrm{Ma}$ subduction of the Kula-Pacific ridge would have made the Pacific plate move northward instead of the observed westward movement [Richards and Lithgow-Bertelloni, 1996]. A recent study [Sharp and Clague, 2006] pushes the date of the $\mathrm{H}$ bend back to $50 \mathrm{Ma}$ (chron c22r), which corresponds to several plate configuration changes (mainly the initiation of new subductions: Aleutians, Izu-Bonin-Marianas, Tonga-Kermadec) and better fits the observed change of orientation of the Pacific fracture zones identified previously [Stock, 2006] (Figure I-12).

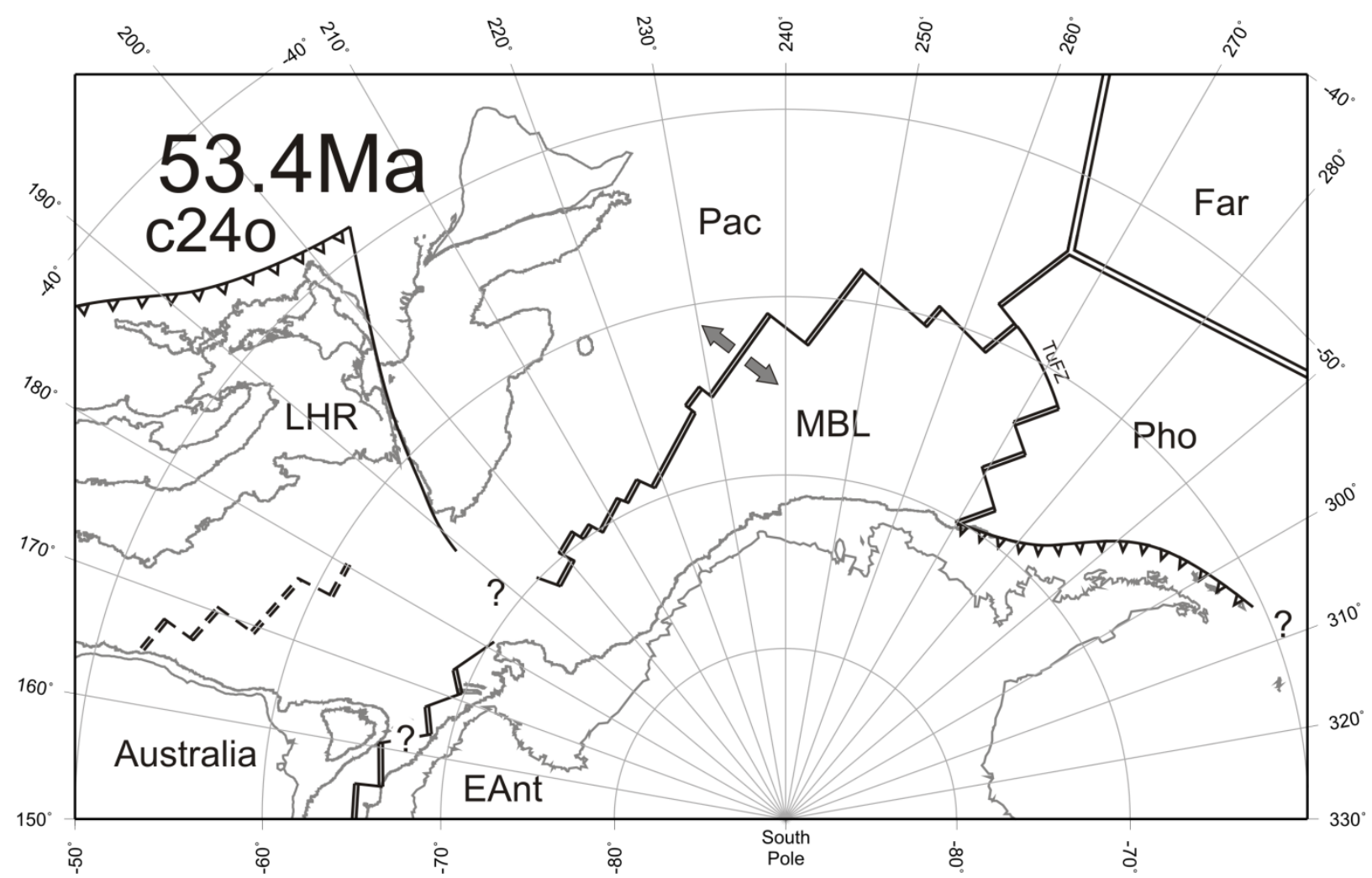

Figure I-12: Snapshot at c24o: seafloor spreading stops in the Tasman Sea. Rotations from [Keller, 2003] (Pacific-Lord Howe Rise), and this study. The motion between Australia and East Antarctica is not directly constrained and the location of the SEIR is indicative only. Legend as in Figure I-3.

However, no consensus exists regarding what caused the $\mathrm{H}$ bend. Several studies mention the initiation of the Izu-Bonin-Mariana subduction ( 48 Ma, [Richards and Lithgow-Bertelloni, 1996]) closely followed by that of the Tonga-Kermadec subduction [Faccenna et al., 2012] as the most likely cause of a change in Pacific motion, but subduction initiation could be the result 
of a change in plate motions or forces at plate boundaries rather than its cause [Stock, 2006]. The collision of continental India with Asia has also been considered as the main cause of a change in Pacific motion [Richards and Lithgow-Bertelloni, 1996] but recent work argues the resultant force transmission across plate boundaries is not sufficient [Faccenna et al., 2012]. Other possible causes for the $\mathrm{H}$ bend include [Faccenna et al., 2012]:

- the subduction of the Pacific-Kula ridge (60-50 Ma);

- an avalanche of subducted material through the upper-lower mantle transition zone;

- a major plume event;

- the sudden change of motion on the Pacific-Australia boundary from strike-slip to subduction ( 50 Ma, [Schellart et al., 2006]).

The westernmost segment of the Marie Byrd Land-Phoenix ridge reached the Antarctic Peninsula subduction East of $270^{\circ} \mathrm{E}$ around anomaly 22 [Weissel et al., 1977] (Figure I-12). This event is one possible reason for the anomaly 21 plate boundary reorganisation that happened in the eastern Southwest Pacific [Cande et al., 1982]. Around anomaly 21, the easternmost segment of the Pacific-Marie Byrd Land ridge propagated through crust formed on the Pacific-Phoenix ridge, resulting in the formation of the Henry and Hudson Troughs and the capture of a fragment of the Pacific plate by Marie Byrd Land [Cande et al., 1982] ${ }^{9}$ (Figure I-13). The fragment captured by the Marie Byrd Land plate around anomaly 21 is limited to the North by the Humboldt FZ, also interpreted to be the trace of the Pacific-Farallon-Phoenix triple junction [Cande et al., 1982] (Figure I-14). By the time the tip of the Pacific-Marie Byrd Land ridge segment reached the Humboldt FZ, the Tula FZ (to the south) was extinct, and the ridge segments north of the Humboldt FZ, formerly accommodating Pacific-Phoenix movement, now belonged to the Pacific-Marie Byrd Land ridge. Propagation of the PacificMarie Byrd Land ridge could have exploited a Pacific-Farallon fracture zone (zone of weakness) [Eagles et al., 2004a].

\footnotetext{
${ }^{9}$ Cande et al. (1982) make a point in differentiating the anomaly 21 'progressive ridge jump' from the anomaly 28 'ridge decomposition' (see Figure I-13).
} 

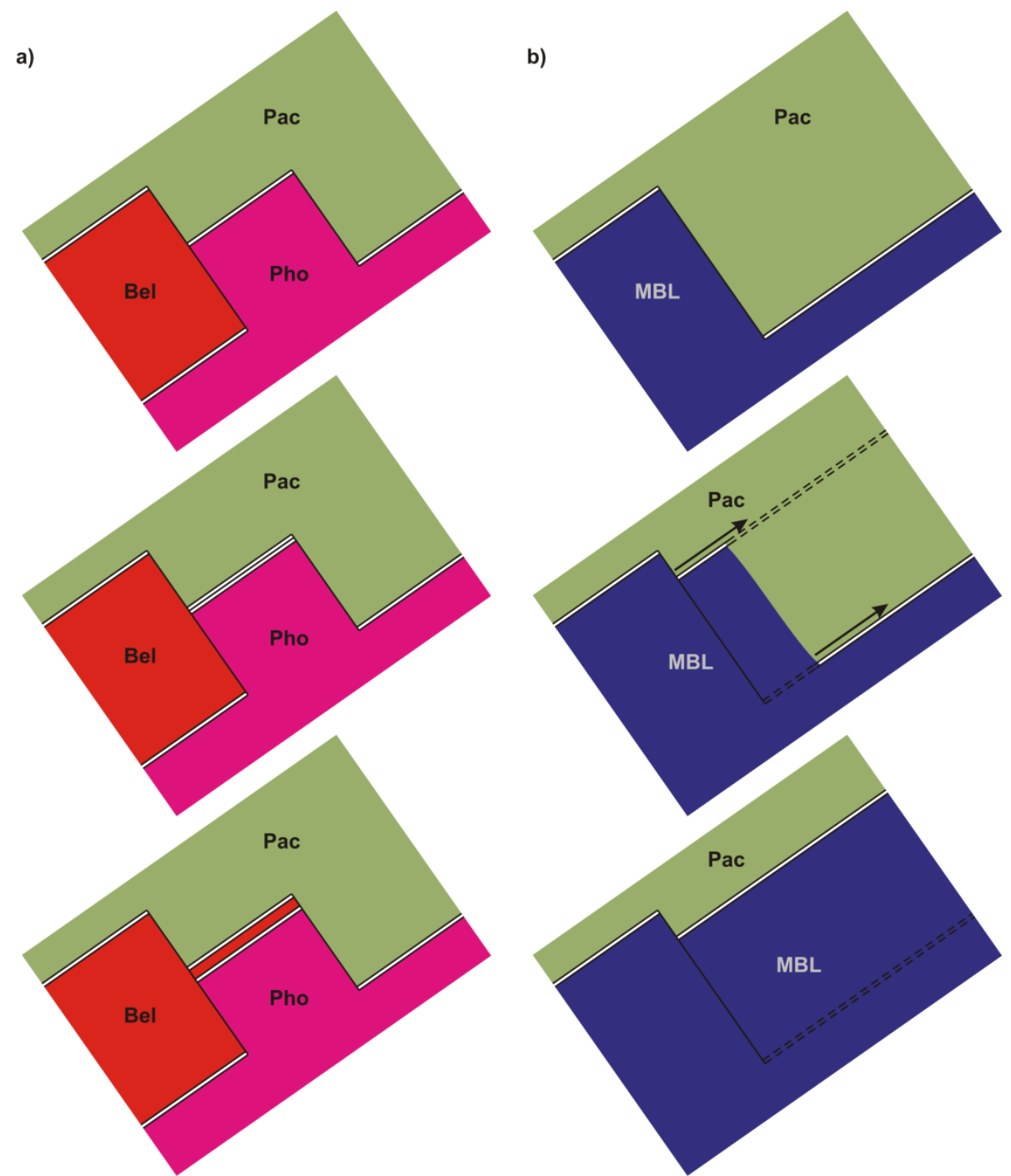

Figure I-13: anomaly 28 versus anomaly 21 ridge jumps. a) anomaly 28 ridge jump (before and after). One fast spreading segment (Pacific-Phoenix, 160mm/yr) "decomposes itself" into two slower ones (Pacific-Bellingshausen, $80 \mathrm{~mm} / \mathrm{yr}$; Bellingshausen-Phoenix, $80 \mathrm{~mm} / \mathrm{yr}$ ). b) Anomaly 21 ridge jump (before and after). Before the ridge jump, the offset between the two active spreading segments is lengthening. When the offset length reaches a threshold (over $1000 \mathrm{~km}$ in that case), the southern spreading segment starts propagating northward at a rate of about $100 \mathrm{~mm} / \mathrm{yr}$ [Cande et al., 1982] while the northern segment becomes progressively extinct. The result is the transfer of part of the Pacific lithosphere to the Marie Byrd Land plate. This transfer is not instantaneous, but takes approximately one magnetic reversal $(\sim 1.5 \mathrm{Myr})$ to complete [Cande et al., 1982]. 


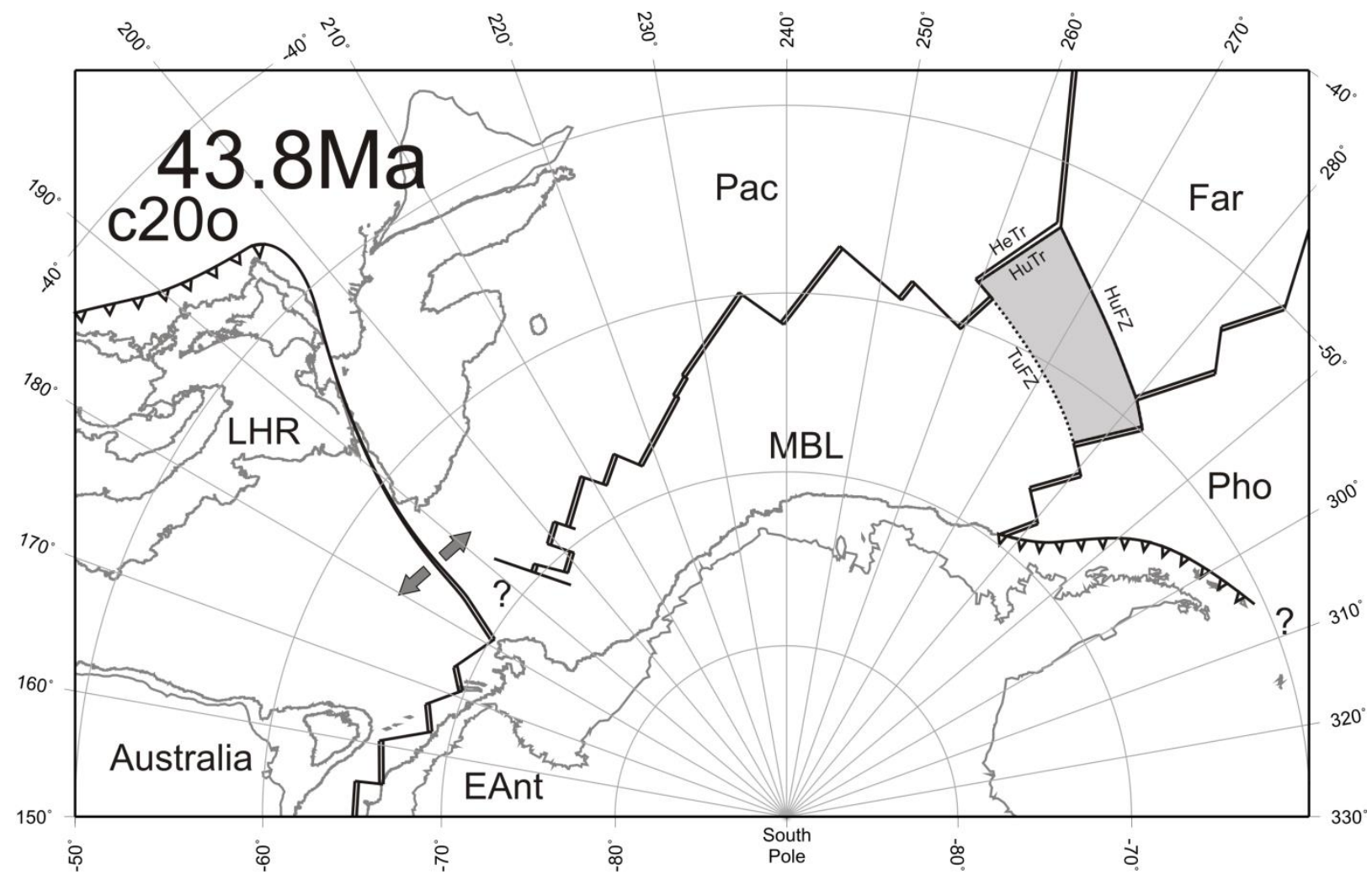

Figure I-14: Snapshot at c20o: a new azimuth of spreading. The area filled in light grey was formed on the Pacific plate, then transferred onto Marie Byrd Land during the formation of the Hudson and Henry Troughs. At the same time, the northeastern boundary of the Marie Byrd land plate moves from the Tula fracture zone to the Humboldt fracture zone. HeTr: Henry trough; HuFZ: Humboldt fracture zone; HuTr: Hudson trough; TuFZ: Tula fracture zone. Rotations from Pacific-Marie Byrd Land [Croon et al., 2008], Pacific-Lord Howe Rise [Keller, 2003], and this study. The motion between Australia and East Antarctica is not directly constrained and the location of the SEIR is indicative only. Legend as in Figure I-3.

\subsection{Third part of the post-rifting story: after anomaly 20}

The Emerald FZ has been used to study the evolution of the Pacific-Marie Byrd Land boundary following the anomaly 21 reorganisation: its proximity to the pole of rotation means it recorded every change of spreading direction on the ridge [Croon et al., 2008]. Other fracture zones were affected by such changes in a similar but less pronounced way [Croon et al., 2010]. [Croon et al., 2008] use the rotations they obtain using high resolution data from several cruises focused on the Menard FZ to look at the Emerald FZ. These new data highlight changes in Pacific-Marie Byrd Land motion around c20o-c21y (appearance of the Menard FZ) and c5y (change of spreading direction initiating transtension on the fracture zone which led to the formation of en échelon spreading segments) [Croon et al., 2008].

The anomaly 21 reorganisation induced a $30^{\circ}$ counter-clockwise rotation of the Emerald FZ, leading to formation of a transform fault between the Macquarie triple junction (Australia- 
Pacific-Antarctica) and the Terror FZ [Croon et al., 2008]. That transform fault lengthened between $\mathrm{c} 20 \mathrm{o}$ and $\mathrm{c} 13 \mathrm{o}$, before being placed under extension (c13o-c10y) by a progressive $20^{\circ}$ clockwise rotation of the spreading direction [Croon et al., 2008]. There is no evidence that this episode of extension led to the formation of en échelon spreading segments [Croon et al., 2008]. c10y marked the beginning of another period of counter-clockwise rotation $\left(20^{\circ}\right)$, leading to the reappearance of the transform fault by anomaly $6 \mathrm{C}$ and its lengthening until c5y [Croon et al., 2008]. At c5y (9.8 Ma), a clockwise rotation of almost $30^{\circ}$ (associated with the migration of the rotation pole) put the Emerald FZ under extension again, this time leading to the formation of 15 en échelon spreading segments [Croon et al., 2008], highly recognisable on [Smith and Sandwell, 1997]'s satellite bathymetry. This last change of spreading direction affected other fracture zones in a similar way, especially the Heirtzler FZ [Croon et al., 2008].

Other fracture zones show signs of more recent changes of spreading direction. The Pitman FZ has rotated about $15^{\circ}$ clockwise since formation of anomaly $4 \mathrm{~A}$ (a third of which happened before c3Ao), transforming it into a "broad diffuse boundary" [Croon et al., 2010]. This change is also visible in the seafloor spreading, with several ridge jumps occurring since c2Ay (2.6 Ma) in the vicinity of the fracture zone [Croon et al., 2010].

The last notable event in the tectonic history of the Southwest Pacific ocean was the transfer of the last part of the Phoenix plate to Antarctica during anomaly c2A ( 3.3 Ma, [Eagles, 2004]).

\section{The Tasman Sea}

The Tasman Sea was first described as an oceanic basin [Hayes and Ringis, 1973] after magnetic anomalies were identified on shiptrack magnetic profiles, and it has been considered a back-arc basin of the Tonga subduction system [Veevers and Li, 1991]. A tectono-geodynamic model [Schellart et al., 2006] places the opening of the Tasman Sea in a context of slab rollback initiated after the decrease of Pacific-Australia relative motion orthogonally to the trench (99 Ma: switch from "head-on subduction" to oblique sinistral subduction). Although, "the nature of the boundary East of the Lord Howe Rise since the Late Cretaceous and prior to about 45 Ma remains uncertain and is currently a matter of debate" [Schellart et al., 2006]. At 
approximately $70 \mathrm{Ma}$, no Pacific slab was being actively subducted ${ }^{10}$ in the Tonga trench [Faccenna et al., 2012]. This means that all the Pacific-Australia relative motion was being accommodated by slab rollback and back-arc opening (Tasman Sea) [Schellart et al., 2006].

Gaina et al. (1998) identify thirteen continental blocks actively involved in the rifting history of the Tasman Sea (Figure I-15): mainland Australia (including the Marion Plateau), the Lord Howe Rise (three blocks: North, centre and the Challenger Plateau), the Dampier Ridge (four blocks: North, centre North, centre South and South), the Chesterfield Plateau, the South Tasman Rise (two blocks: East and West), the East Tasman Plateau and the Gilbert Seamount Complex. Although the Dampier Ridge was first identified as being created by oceanic volcanism [Hayes and Ringis, 1973], dredging has since proved it to be continental in nature, like most of the other blocks [Gaina et al., 1998].

The rifting history of the Tasman Sea started around $90 \mathrm{Ma}$ and involved several jumps of the plate boundary [Gaina et al., 1998]. At that time, Tasmania was in its current position relative to mainland Australia [Gaina et al., 1998] and slow seafloor spreading had started between Australia and East Antarctica [Gaina et al., 1998]. Rifting in the Tasman Sea started in the South around $90 \mathrm{Ma}$, with the separation of the Challenger Plateau from the rest of the Lord Howe Rise, forming the Bellona Trough through transtension [Gaina et al., 1998]. Shortly after, the Challenger Plateau (now moving with the central block of the Lord Howe Rise) started rifting away from Australia [Gaina et al., 1998]. Around $84 \mathrm{Ma}$, following short-lived strike-slip motion between the Gilbert Seamount Complex and the eastern South Tasman Rise, the two blocks began to move apart [Gaina et al., 1998]. Around the same time, extension started between the Dampier Ridge and the northern part of the Lord Howe Rise, creating the Lord Howe and Middleton basins [Gaina et al., 1998].

\footnotetext{
${ }^{10}$ I refer to active subduction as subduction driven by slab pull forces. In this case it means the Pacific slab pull forces are null.
} 


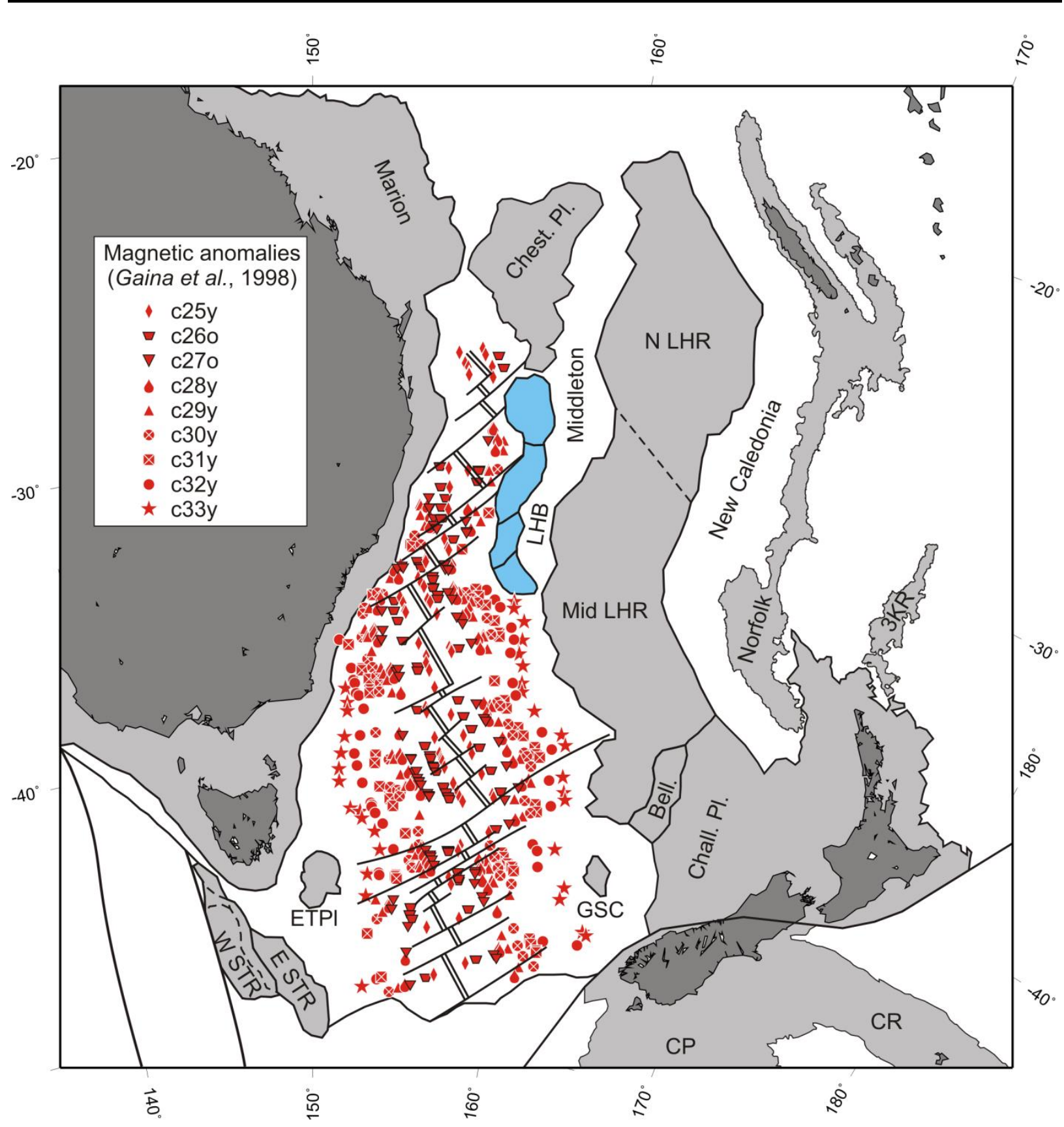

Figure I-15: Continental blocks and magnetic anomalies in the Tasman Sea. 3KR $=$ Three Kings Ridge; Bell = Bellona trough; $\mathrm{CP}=$ Campbell plateau; Chall $\mathrm{Pl}=$ Challenger plateau; $\mathrm{CR}$ $=$ Chatham Rise; Chest $\mathrm{Pl}=$ Chesterfield plateau; $\mathrm{ETPl}=$ East Tasman plateau; $\mathrm{GSC}=$ Gilbert Seamount Complex; LHB = Lord Howe basin; Mid LHR = Lord Howe Rise, middle segment; $\mathrm{N}$ LHR = Lord Howe Rise, northern segment; W STR = West South Tasman Rise. The Dampier Ridge fragments are highlighted in pale blue. Location of tectonic structures (limits of continental blocks and seafloor spreading areas, fracture zones, active or fossil ridge segments, plate boundaries...) were interpreted from the satellite gravity and bathymetry of [Smith and Sandwell, 1997] and [Sandwell and Smith, 1997]. Magnetic anomalies from [Gaina et al., 1998].

Gaina et al. (1998) find evidence that seafloor spreading was active by 83 Ma between the Gilbert Seamount Complex and the Challenger Plateau. By 79 Ma, the southernmost part of the Dampier Ridge became attached to the northern part of the Lord Howe Rise, which led to 
transtension between the two blocks forming the southern half of the Dampier Ridge [Gaina et $a l ., 1998]$. Before c33y, the eastern part of the South Tasman Rise started separating from the East Tasman Plateau [Royer and Rollet, 1997]. By this time (73.6 Ma), extension between the Gilbert Seamount Complex and the Challenger Plateau had stopped [Gaina et al., 1998] ${ }^{11}$. Around $72 \mathrm{Ma}$, after a short period where transtensive deformation jumped from north of the southernmost fragment of the Dampier Ridge to south of its northernmost fragment, the whole Dampier Ridge became fixed relative to the Lord Howe Rise, putting an end to the stretching of continental lithosphere in the Lord Howe and Middleton basins [Gaina et al., 1998]. By about $70 \mathrm{Ma}$, the eastern South Tasman Rise was in its present-day location relative to Australia [Gaina et al., 1998]. According to Gaina et al. (1998), the Chesterfield and Marion plateaus showed signs of transpressive deformation at c31y (67.7 Ma), lasting until c29y (64 Ma). Just before (c30y, $\approx 65 \mathrm{Ma}$ ), motion between the eastern South Tasman Rise and the East Tasman Plateau ceased [Royer and Rollet, 1997]. By c28 ( $\approx 63 \mathrm{Ma})$, a stable spreading system was established in the Tasman Sea as far north as between the Chesterfield and Marion plateaus, and the Lord Howe Rise behaved as one single continental block [Gaina et al., 1998].

The seafloor spreading history of the Tasman Sea is more simple than its rifting history. While the spreading direction averaged northeast-southwest, slight changes in the azimuth of fracture zones indicate moderate variations in the spreading direction [Gaina et al., 1998]. A counter-clockwise rotation of the direction of spreading occurred between c33y and c29y (73.664.0 Ma), followed by a clockwise change from c29y to c27o (61.3 Ma), and finally a counterclockwise rotation after c27o [Gaina et al., 1998]. That latest rotation of the spreading direction strongly intensified just before the cessation of spreading (around c25, 56.1 Ma) [Gaina et al., 1998]. Common evidence for these changes is the compressive deformation identifiable on several fracture zones around the time the direction underwent counter-clockwise rotations [Gaina et al., 1998]. The half-spreading rate also evolved in the Tasman Sea, increasing from $3.1 \mathrm{~mm} / \mathrm{yr}(\mathrm{c} 34 \mathrm{y}-\mathrm{c} 33 \mathrm{o}, \approx 84-79 \mathrm{Ma})$ to $20 \mathrm{~mm} / \mathrm{yr}$ (c33o-c25y), before slowing down to 16 $\mathrm{mm} / \mathrm{yr}$ after c25y (55.9 Ma) [Gaina et al., 1998].

\footnotetext{
11 According to Gaina et al. (1998), at c33y (73.6 Ma) the Gilbert Seamount Complex is transferred to the Challenger Plateau, but seafloor spreading between the Gilbert Seamount Complex and the Challenger Plateau only occurs between 83 and 77Ma. I understand this to mean that between the Gilbert Seamount Complex and the Challenger Plateau seafloor spreading stops at $77 \mathrm{Ma}$, but extension continues until 73.6Ma.
} 
Between c33y and c27o the seafloor spreading was asymmetric, with more lithosphere being produced west of the ridge [Gaina et al., 1998]. The Coral Sea (considered by Gaina et al. (1998) as the northernmost part of the Tasman Sea spreading system) opened between c27o and c24y (61.3-53.3 Ma) [Veevers and Li, 1991]. [Gaina et al., 1998] suggest the changes in the spreading direction in the Tasman Sea at c27o were linked to the changes happening in the Pacific-Antarctica spreading system around that time (see I.1.3). In my opinion, changes in the Tasman Sea around c27o could also be linked to contemporaneous changes in the AustraliaAntarctica spreading system (see below). For instance, [Royer and Rollet, 1997] note that the cessation of spreading in the Tasman Sea coincided with the onset of spreading south of the Tasman Sea (anomaly 24).

\section{Australia-East Antarctica spreading}

The ocean basin separating Australia from Antarctica formed on what is now called the South-East Indian Ridge (SEIR, see Figure I-16). Although the younger part of the story of the formation of the Australia-Antarctica basin (AAB) is well defined, seafloor older than anomalies 18 to 21 (39-47 Ma) is more difficult to interpret. The younger part of the seafloor displays "many closely spaced fracture zones with small offsets" [Stock and Molnar, 1982] and was formed by fast spreading ([Cande and Mutter, 1982] in [Veevers and Li, 1991]). Cande and Mutter (1982) identify three stages of spreading in the AAB: slow from c34y to c20o, intermediate during anomaly 20 (42.5-43.8 Ma), and fast for times younger than c20y. Royer and Rollet (1997) modify that scenario slightly and calculate full spreading rates of $8 \mathrm{~mm} / \mathrm{yr}$ before chron 20, $30 \mathrm{~mm} / \mathrm{yr}$ between chrons 20 and 18 (43-39 Ma), and $46 \mathrm{~mm} / \mathrm{yr}$ for times younger than chron 18. Royer and Rollet (1997) also see a connection between the major reorganisation of tectonic boundaries in the Indian ocean about c18 and the change of spreading rate at that time in the AAB. Last but not least, Veevers and Li (1991) suggest 10 to 30\% of asymmetry for seafloor spreading in the $\mathrm{AAB}$, but a more recent study does not support this interpretation [Tikku and Cande, 1999]. 


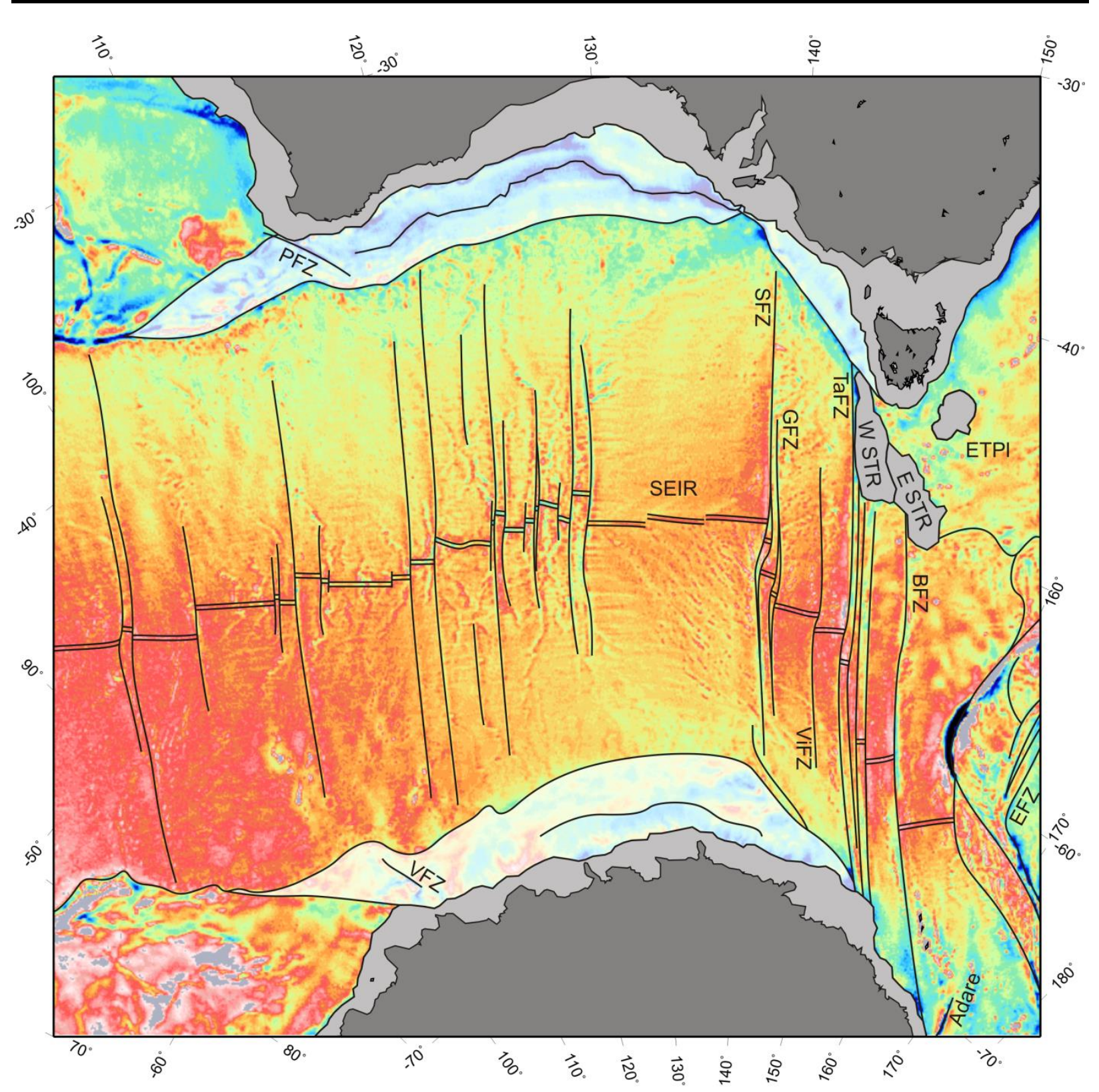

Figure I-16: Satellite gravity in the Australia-Antarctica basin. The area covered with a white transparent layer corresponds to slow to ultraslow seafloor spreading (see text). The SEIR's active ridge segments and fracture zones are indicated. Adare: Adare Trough; E STR: East South Tasman Rise; ETPl: East Tasman Plateau; GFZ: George V fracture zone; PFZ: Perth fracture zone; SFZ: Spencer fracture zone; TaFZ: Tasman fracture zone; VFZ: Vincennes fracture zone; ViFZ: Vincent fracture zone; W STR: West South Tasman Rise.

The date of breakup and oceanisation in the AAB has been debated (see Figure I-17 for the raw data). Le Pichon and Heirtzler (1968) identified anomaly 13 (33.3 Ma) as the oldest magnetic anomaly in the basin. A few years later, Weissel and Hayes (1972) recognized anomaly 22 on their profiles, giving seafloor spreading between Australia and Antarctica an average half-spreading rate of $25 \mathrm{~mm} / \mathrm{yr}$. Hayes and Ringis (1973) find a similar date of 60-50 Ma, but Cande and Mutter (1982) identify anomaly 34 on their profiles. The continent-ocean 
boundary has an age of about 95 Ma [Veevers, 1986], but this is questioned by Tikku and Cande (1999), who note that the edge of the magnetic quiet zone (QZB for Quiet Zone Boundary) does not necessarily mark the location of the continent-ocean boundary.

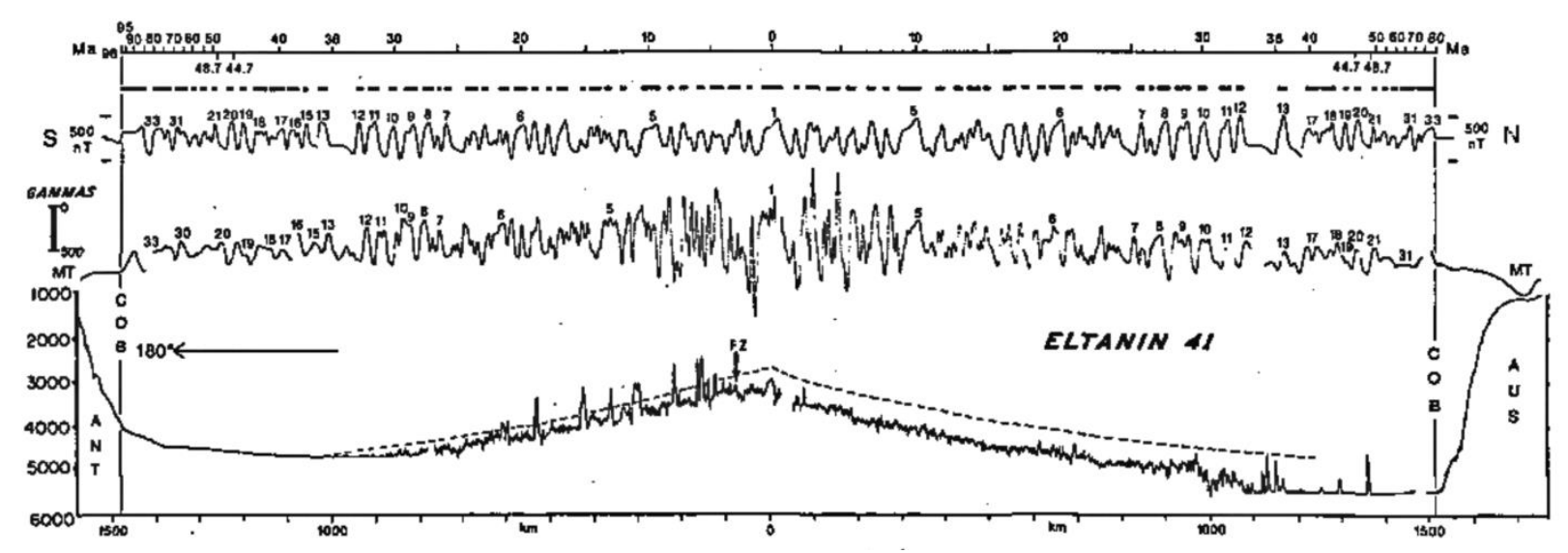

Figure I-17: Magnetic profile across the SEIR. From top to bottom: timescale in millions of years, block model used (see Chapter II), synthetic magnetic profile, shiptrack magnetic profile, bathymetric profile, distance along track in $\mathrm{km}$. FZ: fracture zone; MT: magnetic trough. From [Veevers and Li, 1991] (their figure 5).

As scientists began to agree on a Late Cretaceous date for the initiation of spreading, the early direction of extension became clearer through the use of satellite altimetry. The eastsoutheast trend of the continent-ocean boundary east of $131^{\circ} \mathrm{E}$ [Veevers and Li, 1991] and the identification of the Perth and Vincennes FZs as conjugates [Tikku and Cande, 1999] are evidence of the initial direction of Australian motion relative to Antarctica. The breakup progressed from West to East [Mutter et al., 1985], with the tip of the SEIR propagating through continental crust that was being stretched at the same rate as the SEIR was spreading [Veevers and Li, 1991]. Tikku and Cande (1999) suggest the QZB could be a marker of the propagation of the SEIR. Veevers and Li (1991) identify a ridge jump east of the Spencer FZ towards Australia 84 million years ago.

At approximately anomaly 33, the azimuth of seafloor spreading changes from eastsoutheast to almost north-south [Royer and Rollet, 1997]. Following this reorientation, seafloor spreading started at a slow rate $\left(\mathrm{v}_{1 / 2}=5 \mathrm{~mm} / \mathrm{yr}\right)$ until anomaly 31 , then decreases to $1.5 \mathrm{~mm} / \mathrm{yr}$ (c24o-c31o, ultraslow spreading) [Tikku and Cande, 1999]. The half-spreading rate then increases again to $6.5 \mathrm{~mm} / \mathrm{yr}(\mathrm{c} 21 \mathrm{y}-\mathrm{c} 24 \mathrm{o})$ then $10 \mathrm{~mm} / \mathrm{yr}(\mathrm{c} 18 \mathrm{o}-\mathrm{c} 21 \mathrm{y})$, before accelerating to fast spreading [Tikku and Cande, 1999; Veevers and Li, 1991]. Evidence of a period of ultraslow spreading can be found in the bathymetry, as the seafloor in the Diamantina zone is 
unusually rough [Tikku and Cande, 1999]. Furthermore, the absence of fracture zones south of the Australian Bight is consistent with ultraslow spreading [Dick et al., 2003].

"Our knowledge of the transition between slow spreading crustal accretion and crustal extension is poor" [Tikku and Cande, 1999]. In practical terms, it means that the magnetic anomaly identified by [Tikku and Cande, 1999] as c27y-c31o, which is extremely narrow, could represent a temporary cessation of spreading (hiatus) and its subsequent re-initiation around anomaly 27, possibly due to a change in regional tectonics (anomaly 27 reorganisation in the Southwest Pacific, see above). During the brief period when no new crust was formed, extension between Australia and Antarctica would have been accommodated through deforming existing crust [Tikku and Cande, 1999]. Indeed, there is evidence of normal faulting for oceanic crust located between the QZB and the c27y-c31o magnetic lineation [Tikku and Cande, 1999]. These observations and interpretations lead Tikku and Cande (1999) to suggest that some magnetic "anomalies [...] may not be isochrons", but could reflect either the deformation mentioned above, or the existence of magnetized structural features (dykes), or they could be the relics of propagating rifts. Ultraslow spreading affects our ability to interpret geophysical data, as “individual anomalies are not well resolved on marine magnetic records and are difficult to identify confidently", [Stock and Molnar, 1982].

The West South Tasman Rise is a highly deformed continental fragment located south of Tasmania. When the Australia-Antarctica breakup started, the West South Tasman Rise was attached to Antarctica and moved along the western margin of Tasmania, along a N140-145 $\mathrm{E}$ sinistral strike-slip fault [Royer and Rollet, 1997]. By the time the eastern edge of the West South Tasman Rise reached the East South Tasman Rise around anomaly 27, the boundary between the two jumped progressively westward, until it reached the location of what is now the Tasman FZ [Royer and Rollet, 1997]. This progressive transfer of the West South Tasman Rise from Antarctica to Australia had as a consequence the faulting of the West South Tasman Rise [Royer and Rollet, 1997].

Although it represents less than a third of the total surface of the AAB, several studies have focused on the seafloor created east of the Tasman FZ [Cande and Stock, 2004; Royer and Rollet, 1997; Stock and Molnar, 1982; Tikku and Cande, 1999]. Seafloor spreading south of the Tasman Sea did not start until c27, with rates faster than further west [Royer and Rollet, 1997; Tikku and Cande, 1999], prompting the suggestion that it resulted from Australia-West 
Antarctica (Marie Byrd Land) relative motion [Royer and Rollet, 1997]. Intraplate deformation could be responsible for the impossibility of matching conjugate magnetic anomalies 18 to 13 on both sides of the Tasman FZ [Stock and Molnar, 1982].

After $6 \mathrm{Ma}$ (c3Ay), conjugate magnetic lineations east of the Balleny FZ cannot be reconstructed using rotations calculated with data west of the Tasman FZ: the lineations are offset along strike by up to $50 \mathrm{~km}$ (Figure I-18) [Cande and Stock, 2004]. Using the same rotations, Cande and Stock (2004) calculate flowlines for fracture zones east of $140^{\circ} \mathrm{E}$ and find that they deviate significantly from tracks of the Tasman (middle and eastern splays) and Balleny FZs, but not once the flowlines have been 'offset' to start before c3Ay. These observations, along with evidence of active deformation of the Australian plate East of $140^{\circ} \mathrm{E}$ (seismicity), imply the existence of an independent Macquarie plate since c3Ay (6 Ma) [Cande and Stock, 2004]. The Australia-Macquarie rotation poles for c3Ay and c2Ay are located within the deformation zone defined by the seismicity, which is typical of rigid subplates (Capricorn microplate, [Cande and Stock, 2004]). The western boundary of the Macquarie microplate could not be defined through the analysis of seismic reflection profiles (not enough data) or by the systematic comparison of Australia-Macquarie flowlines with fracture zone tracks (the azimuths of Australia-Macquarie and Australia-Antarctica flowlines West of $140^{\circ} \mathrm{E}$ are too similar) [Cande and Stock, 2004]. The "onset of deformation in the South Tasman sea and the development of an independent Macquarie plate may have been triggered by the buoyancy of young oceanic crust entering the southern Hjort trench" [Cande and Stock, 2004]. 


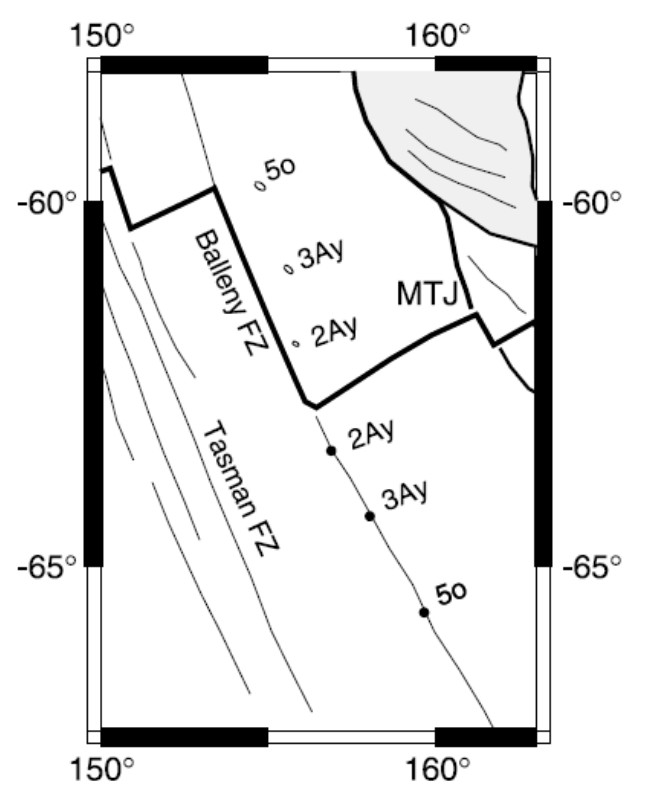

Figure I-18: Misfit between rotated Balleny fracture zone crossings and their conjugates [Cande and Stock, 2004]. Three points on the Antarctic side of the SEIR (black dots) correspond to the intersection of the Balleny fracture zone with the 2Ay, 3Ay and 5 magnetic lineations. The points have been rotated back to the Australian plate (small ellipses). The rotated points fall up to $50 \mathrm{~km}$ east of the fracture zone. MTJ: Macquarie triple junction.

\section{Timing of deformation within Antarctica}

The junction of the Pacific-Marie Byrd Land ridge with the SEIR has been the subject of several studies. Following the demise of the Tasman Sea ridge, the spreading rates on both the Pacific-Marie Byrd Land ridge and the SEIR were too different to be part of the same spreading system [Stock and Molnar, 1982]. This means a third plate boundary was needed, but that boundary could have been either between the Pacific and Australia plates, or between East Antarctica and Marie Byrd Land [Hayes and Ringis, 1973; Stock and Molnar, 1982]. Likely candidates for the "missing link" include the Adare Trough, sedimentary basins in the Ross Sea, the Iselin Rift, and the seafloor surrounding the present-day boundary between Pacific and Australia, the Macquarie Ridge [Cande et al., 2000; Hayes and Ringis, 1973; Marks and Stock, 1997; Sutherland, 1995; 1999] (Figure I-19). 


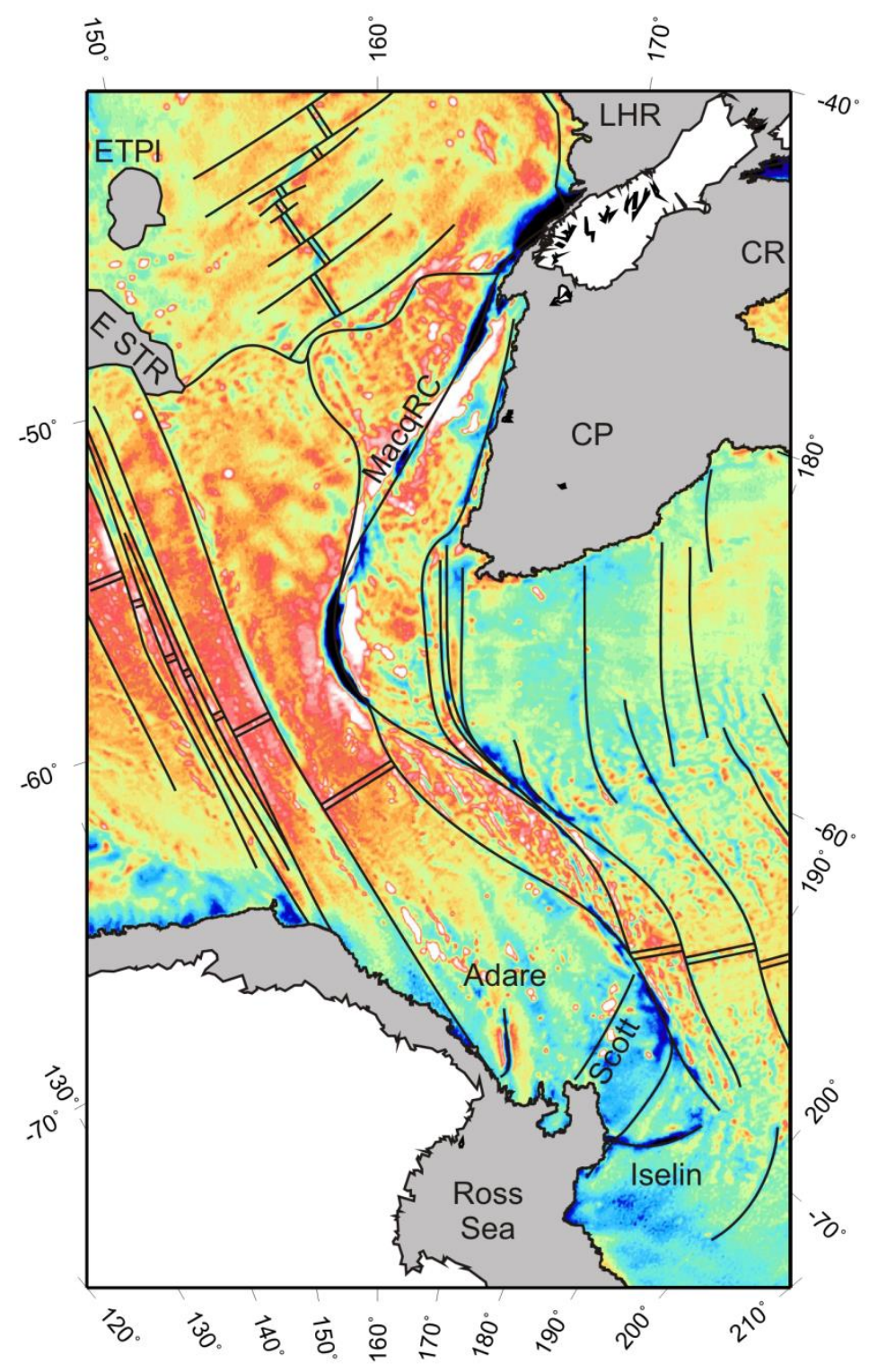

Figure I-19: Seafloor between New Zealand and the Ross Sea. Satellite gravity [Sandwell and Smith, 1997] (version 20). The -2000m depth contour is used to represent the continental slope for Antarctica and New Zealand. Fracture zones and ridge segments are indicated for the Tasman Sea, the SEIR and the Pacific-Antarctica ridge. Adare: Adare Trough;CP: Campbell Plateau; CR: Chatham Rise; E STR: East South Tasman Rise; ETPl: East Tasman Plateau; Iselin: Iselin Trough; LHR: Lord Howe Rise; MacqRC: Macquarie Ridge Complex; Scott: Scott Rift.

Prior to anomaly 27, the SEIR propagated from West to East (see I-3). At approximately anomaly 26, the tip of the SEIR propagated eastward past the Tasman FZ, forming the Nella Dan and Scott rifts [Cande et al., 2000]. The SEIR's propagation probably followed a zone of crustal weakness [Marks and Stock, 1997]: seismic profiles crossing the southern boundary of the Tasman Sea reveal it was the location of major shearing between 80 and $60 \mathrm{Ma}$ [Hayes and Ringis, 1973]. As the SEIR kept on propagating eastwards, it reached the Tasman ridge shortly 
before anomaly 24, which may have led to the cessation of spreading on the Tasman Sea ridge at approximately c24o [Marks and Stock, 1997]. This change in plate boundary configuration caused a small fragment of the Australian seafloor formed on the Tasman Sea ridge to be transferred to Antarctica, and the part of the Tasman Sea ridge south of the SEIR to become a transform fault linking it to the Pacific-Marie Byrd Land ridge [Marks and Stock, 1997] (Figure I-20). After the SEIR reached the Tasman Sea ridge prior to anomaly 24, it continued its eastward propagation along the edge of the Campbell Plateau, causing formation of the Resolution Ridge [Marks and Stock, 1997].
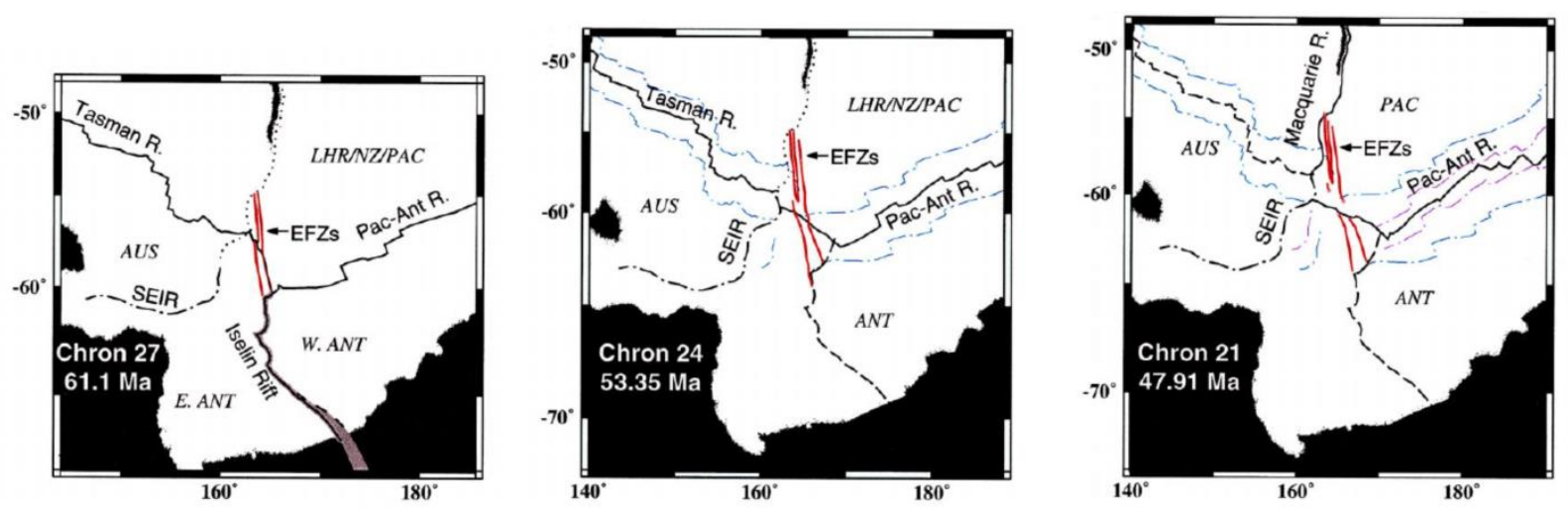

Figure I-20: Configuration of plate boundaries at c27, c24 and c21 [Marks and Stock, 1997] (their figure 2). EFZs: Emerald FZ splays. Pac-Ant R.: Pacific-Marie Byrd Land ridge. Blue and violet dashed lines are c27 and c24 isochrons respectively. The location of the SEIR west of the Tasman Sea ridge is not well constrained. The region of predicted extension south of the Iselin Rift is indicative only.

Although Stock and Molnar (1982) find no evidence of Lord Howe Rise-Campbell Plateau seafloor spreading prior to anomaly 18, Cande et al. (2000) date initiation of spreading south of the Resolution Ridge at anomaly 20 (43.2 Ma), and Marks and Stock (1997) suggest spreading started at approximately anomaly 24 , while the spreading ridge was still propagating. Combining the identification of anomaly 18 south of the Resolution Ridge with the history of sedimentation south of New Zealand [Sutherland, 1995] gives an age of $45 \pm 5 \mathrm{Ma}$ for the inception of Lord Howe Rise-Campbell Plateau (Australia-Pacific) spreading [Sutherland, 1995]. Spreading on the ridge continued until at least anomaly 8 (26 Ma, [Cande et al., 2000]) or $20 \mathrm{Ma}$ (anomaly 6, [Sutherland, 1995]). Seafloor younger than $20 \mathrm{Ma}$ has since either been subducted in the Hjort Trench, or its gravity signature is obscured by that of the subduction [Sutherland, 1995]. No magnetic anomaly can be identified east of the Macquarie Ridge, making traditional plate tectonic reconstructions impossible (no conjugate anomalies) [Sutherland, 1995]. The direction of spreading was orthogonal to the ridge until about $30 \mathrm{Ma}$ 
(c11o), then it became progressively more and more oblique [Cande and Stock, 2004; Cande et al., 2000; Sutherland, 1995]. After 20 Ma (anomaly 8, [Cande et al., 2000]), the AustraliaPacific motion evolved into strike-slip then compression [Cande and Stock, 2004; Cande et al., 2000]. The Alpine Fault and Macquarie-Hjort subduction zone are the modern-day manifestations of the Australia-Pacific motion. The Alpine Fault is estimated to have accommodated $850 \mathrm{~km}$ of displacement since Eocene time [Sutherland, 1999].

The Lord Howe Rise-Campbell Plateau boundary is only one of two possible locations for accommodating the difference of motion between the Australia-East Antarctica and the PacificMarie Byrd Land spreading systems after the demise of the Tasman Sea ridge. Intra-Antarctic deformations are proposed by [Cande et al., 2000; Gaina et al., 1998; Hayes and Ringis, 1973; Marks and Stock, 1997; Stock and Molnar, 1982; Sutherland, 1999] as an alternative to Lord Howe Rise-Campbell Plateau extension. The Ross Sea is a good candidate for East AntarcticaMarie Byrd Land deformation [Cande et al., 2000; Hayes and Ringis, 1973; Marks and Stock, 1997; Sutherland, 1995; 1999], as it harbours several sedimentary basins [Cande et al., 2000] and is located just west of the Emerald FZ. The Ross Sea is also neighboured by two extensional features, the Adare Trough and the Iselin Rift.

The Iselin Rift is a prominent northeast-southwest gravity low located North of Marie Byrd Land, East of the Emerald FZ. As no fracture zone or magnetic anomaly can be identified in the Iselin Rift, it is likely the extension in that location never evolved enough to produce new seafloor [Marks and Stock, 1997]. The necessity of the existence of an additional plate boundary between the Emerald and Kohiku FZs is demonstrated [Marks and Stock, 1997], and plate reconstructions for chrons 27 and 24 (61.1 \& 52.9 Ma) show the Emerald FZ conjugate segments are offset in the absence of extension on the Iselin Rift. It is estimated that 25 to 50 $\mathrm{km}$ of extension took place within the Iselin Rift between anomaly 27 and anomaly 24 [Cande et al., 2000], at which time the SEIR reached the Tasman Sea ridge, changing the plate configuration of the region [Marks and Stock, 1997]. Although Marks and Stock (1997) consider extension on the Iselin Rift to be between East and West Antarctica, Cande et al. (2000) disagree, as it would imply a $90^{\circ}$ rotation of the direction of extension around anomaly 24 (when extension is thought to have jumped from the Iselin Rift to the Adare Trough, see below). 
The Adare Trough is a north-south oriented graben structure north of the Ross Sea. It is aligned with several sedimentary basins within the Ross Sea, which makes it a good candidate for being the result of East-West Antarctica deformation [Cande et al., 2000]. Magnetic anomalies 18 to 9 are identified within the Adare Trough, with a relatively constant total spreading rate of $12.5 \mathrm{~mm} / \mathrm{yr}$ and slight asymmetry [Cande et al., 2000]. Cande et al. (2000) tentatively identify anomaly 24 on several magnetic profiles, but their lack of confidence in the identification makes pinpointing formation of the Adare Trough difficult. Noting the discrepancy between the start of uplift of the Transantarctic Mountains (55-50 Ma) and the initiation of seafloor spreading in the Adare Trough (43 Ma), they propose the period between 55 and 43 Ma corresponds to rifting of the Adare Trough.

To summarize, global plate tectonics models require deformation between Australia and Pacific and/or East Antarctica and Marie Byrd Land after the extinction of the Tasman Sea ridge in order to accommodate the difference in spreading rates between the SEIR and the Pacific-Marie Byrd Land spreading systems. The Nella Dan and Scott rifts are the result of the eastward propagation of the SEIR past the Tasman FZ after anomaly 27 (61.1 Ma). A fragment of crust located east of the Scott Rift formed on the Tasman Sea ridge then was transferred onto Antarctica. Between anomalies 27 and 24, while the SEIR propagated eastwards between the Tasman FZ and the Tasman Sea ridge, East-West Antarctica extension formed the Iselin Rift, although no new crust was created. Around anomaly 24, the SEIR reached the Tasman Sea ridge, changing the plate configuration. Between anomalies 24 and 18 the SEIR continued to propagate eastwards, forming the Resolution Ridge and initiating Lord Howe Rise-Campbell Plateau spreading. It is also possible that the Adare Trough was rifting at that time. Between anomalies 18 and 9 (39.3-27.5 Ma), East-West Antarctica motion was accommodated by seafloor spreading in the Adare Trough. Between about 45 and $30 \mathrm{Ma}$, Lord Howe RiseCampbell Plateau spreading was orthogonal to the ridge. The spreading direction progressively rotated clockwise until the motion between Australia and Pacific became parallel to the plate boundary around $20 \mathrm{Ma}$. After $20 \mathrm{Ma}$, the Pacific-Australia motion changed to transpression along the Macquarie Ridge and the Alpine Fault and compression (subduction) on the Hjort Trench. 
Chapter II

MAGNETIC ANOMALY INTERPRETATION 


\section{Introduction}

The rocks of oceanic crust are mostly basalts and diabase dykes in the upper part, and gabbros and ultramafic cumulates in the lower part. During their cooling, they record Earth's magnetic field at the time of their formation (remanent magnetisation). It is possible to identify the magnetic anomalies resulting from past inversions of the geomagnetic field on magnetic profiles. Once identified, the set of correlative magnetic anomalies can be interpreted as isochrons ${ }^{12}$, which are then used to calculate the paleoposition of the plates they belong to at the time of the isochron. The sequence of magnetic reversals is well known for times younger than about $158 \mathrm{Ma}$ [Cande and Kent, 1995; Channell et al., 1995].

My re-interpretation of magnetic anomalies in the South Pacific in terms of isochrons represents the primary significant body of work carried out during preparation of this thesis. I focused on identifying isochrons older than c20y (42.5 Ma) and younger than c34y (83 Ma). This time interval includes the final Gondwana breakup through to the time of major plate reorganisation in the Pacific that includes the Hawaii-Emperor bend (47 Ma, [Croon et al., 2008; Gordon, 2000; Steinberger et al., 2004; Weissel et al., 1977] or 50 Ma [Sharp and Clague, 2006]). The younger period of South Pacific seafloor spreading has previously been thoroughly analysed and documented [Cande et al., 1995; Croon et al., 2008].

In this study, my primary interpretation of magnetic anomalies is based on the identification of patterns that are distinctive in realistic synthetic models. Magnetic anomaly 32 (71-73 Ma) represents a recognizable sequence (Figure II-1), as do anomalies 29-28-27 and anomalies 26-25-24r. After specific identification of an anomaly sequence by pattern matching, the location of each isochron pick is measured. Uncertainties exist during both the pattern matching and location steps, and this is discussed below (II-4 and II-5). To avoid plate model interpretations at this stage, I refer to the Pacific sector as all crust that formed north of the paleo-spreading ridge. South of the ridge is the Antarctica sector, which lies adjacent to Antarctica.

\footnotetext{
${ }^{12}$ Isochrons are abbreviated chron, and further abbreviated $c$, followed by an isochron number, and sometimes $n$ (for anomalies of normal polarity), $r$ (for anomalies of reverse polarity), $y$ (for the young edge of the anomaly), $o$ (for the old edge). Similarly, magnetic anomalies are abbreviated $a$, followed by an anomaly number (e.g. a20). See II-10 to II-15
} 


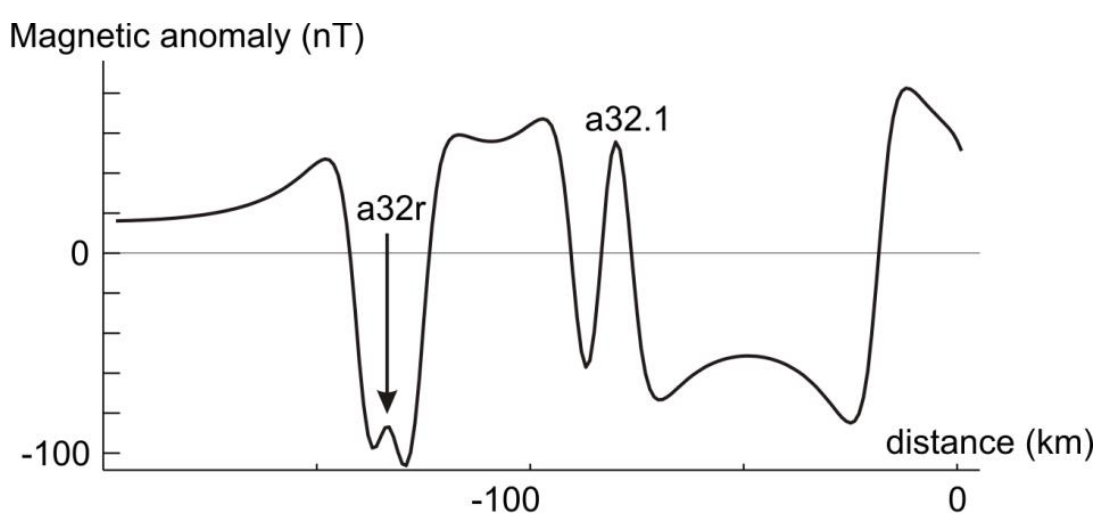

Figure II-1: Synthetic magnetic anomaly profile for anomaly $32(71-73 \mathrm{Ma})$ for a halfspreading rate of $50 \mathrm{~km} / \mathrm{Myr}$.

\section{Magnetic anomaly data}

Most magnetic anomaly data used in this study are from shiptrack magnetic profiles. These profiles are publicly available as part of the GEODAS database (www.ngdc.noaa.gov). They were acquired during cruises around New Zealand from the 1960s to the present. Additional data are available from recent cruises and airborne data (Table II-1). Two cruises ( $R V$ Melville 2009, PIs Hager \& Molnar; RV Polarstern 2010, PI Gohl) were planned in consultation with myself in order to help advance this $\mathrm{PhD}$ study. The magnetic data were incorporated into a geophysical database at GNS Science, and have been corrected by staff at GNS Science using a geomagnetic reference field (IGRF10, [Maus et al., 2005]). 


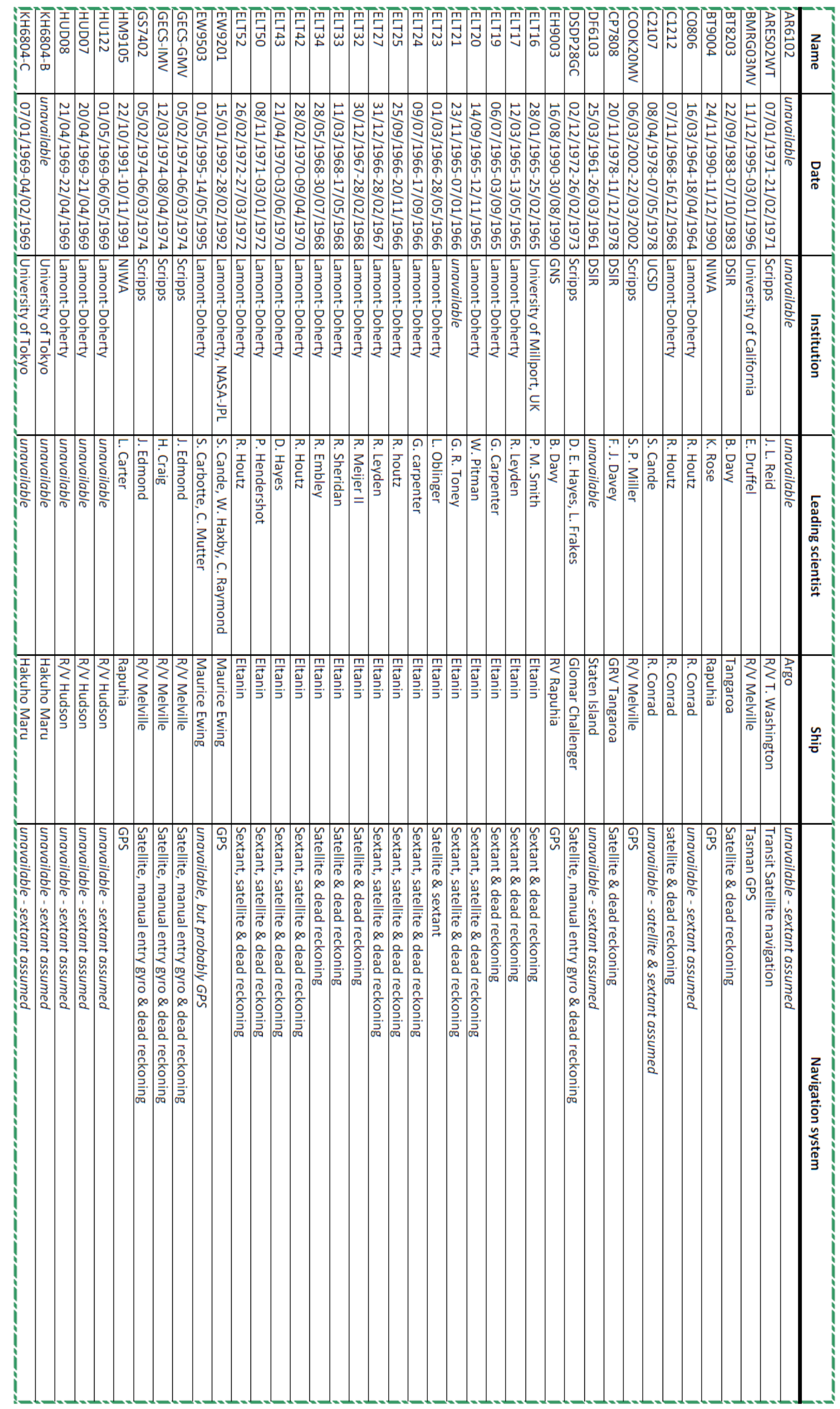




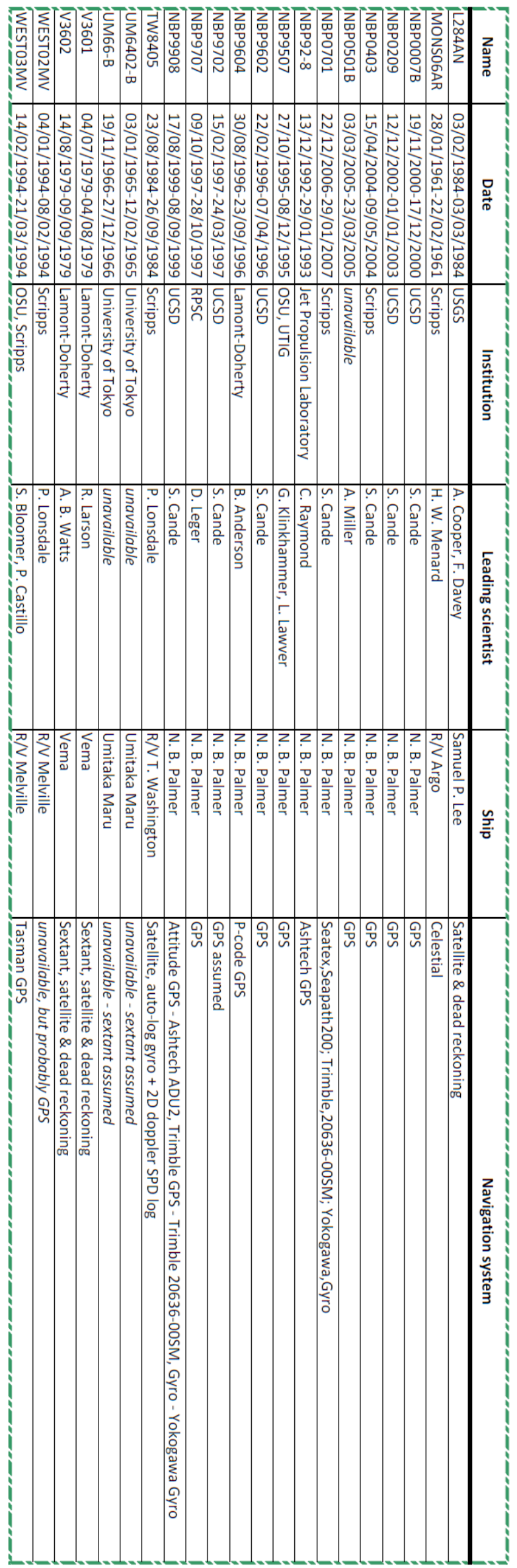


Table II-1 (previous page): Cruises used to digitize magnetic anomalies. Technical information about each cruise is noted where available.

When a ship records a sequence of magnetic anomalies on a profile, the angle the shiptrack makes with the orthogonal to the magnetic lineations affects the appearance of the anomalies: the greater the angle, the more the anomalies will be spread out along the profile (Figure II-2). However, as the MODMAG software (see II-3) takes that angle into account when calculating a synthetic profile, I used a non-reprojected version of the profiles to digitize magnetic reversals.

a)

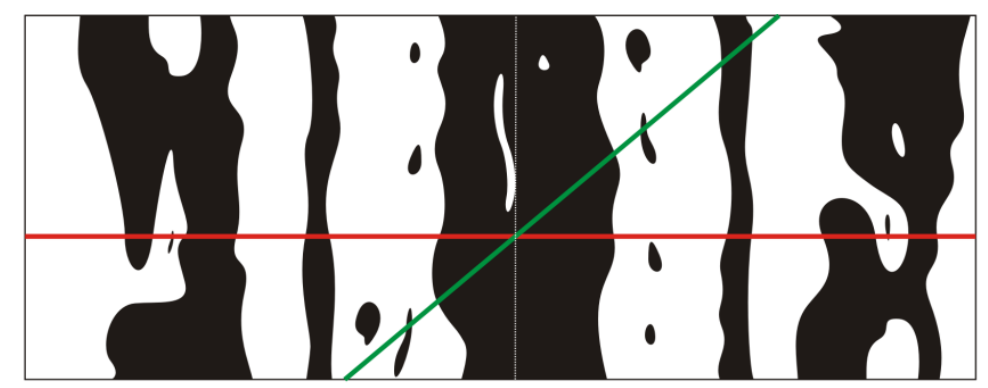

b)
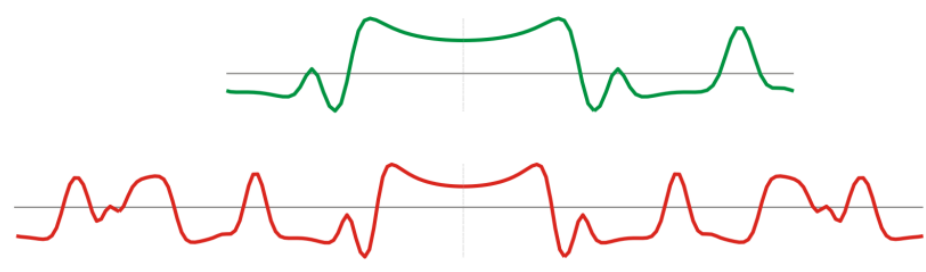

c)

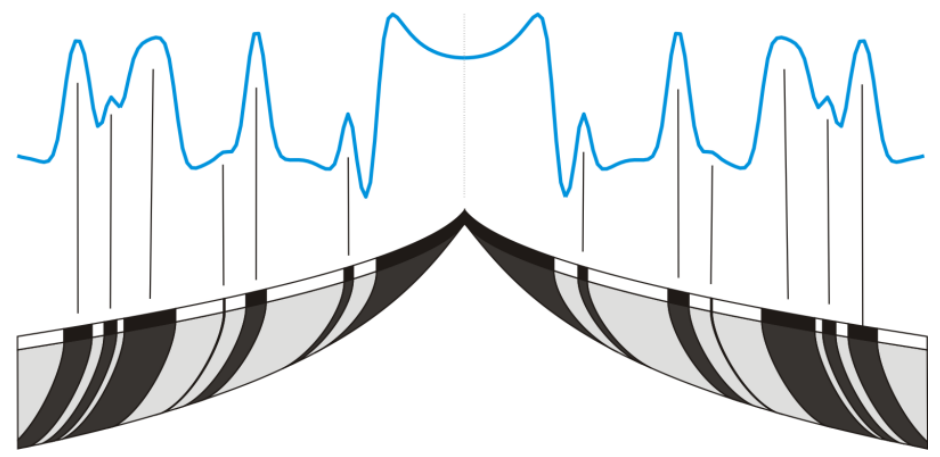

Figure II-2: Schematic illustration of two effects on the magnetic signal: angle between the azimuth of the profile and that of the seafloor spreading, and magnetic source contamination. a) Map view of hypothetical magnetic anomalies. Positive (normal) anomalies are in black; negative (reverse) anomalies are in white. Profiles red and green intersect over the spreading ridge. The red profile crosses the ridge at right angle, whereas the green profile crosses it with an angle of 50 degrees. b) Magnetic anomaly signal (post-processing) recorded along the red and green shiptracks. The difference in apparent spreading rate between the two profiles is due to the difference in azimuth of the profiles compared to the paleo-spreading direction. c) Synthetic magnetic profile that best fits the red profile. The block model (succession of magnetic blocks of alternating polarity of varying size) used to calculate the synthetic profile is 
shown in d). d) Cross section of the magnetic sources along the red profile. The top magnetic layer (black and white) is commonly simulated by a block model. Below the top layer is represented the corresponding pattern of paleo Curie point curves. Because the curves are not vertical, some contamination of the magnetic signal occurs. The coefficient of contamination depends on the spreading rate (the faster the spreading, the larger the coefficient and the less vertical the Curie point curve). The software I used to calculate synthetic models (MODMAG, see II-3) takes a constant, user defined contamination coefficient into account.

\section{Magnetic anomaly models}

I used the MODMAG software [Mendel et al., 2005] to generate a synthetic magnetic model along each trackline for which magnetic anomaly data exist. Comparisons between model and observed data were used as the basis for primary interpretations of the magnetic anomalies and hence the locations of isochrons.

Bathymetric data from the trackline database and from interpolation using satellite altimetry data [Smith and Sandwell, 1997] were used to determine the depths to magnetic sources during creation of synthetic magnetic anomaly profiles (see Figure II-3).

To calculate a synthetic magnetic profile, MODMAG makes assumptions about the paleo-orientation of the ridge and about the magnetization and depth of the magnetic sources. It uses a reversal timescale and requires, for each timestep, a total spreading rate and an asymmetry rate. I decided to use the reversal timescale by [Cande and Kent, 1995] because it is considered "the optimum representation of Cenozoic and Late Cretaceous polarity history" [Lowrie and Kent, 2004] and because it is the timescale used by [Cande et al., 1995; Cande et al., 2000] and other prominent studies, making the comparison of my work with theirs more direct.

\section{Magnetic anomalies: pattern matching}

I chose to use profile ew9201_01 $1^{13}$ as a reference (Figure II-3) because it is precisely located (GPS navigation), was recorded at high sample frequency (an average of about 4 points per kilometre after projection) with high data quality, has corresponding bathymetric swath data

\footnotetext{
${ }^{13}$ Hereafter each profile is named as such: name of the cruise (e.g. ew9201) followed by a profile number (e.g. 01). When a long profile crosses a fracture zone or other discontinuity, or its azimuth changes noticeably, $a$ and $b$ are added to the profile number of each profile fragment, or $N$ and $S$ (for North and South).
} 
along it, is more than $2500 \mathrm{~km}$ long and follows the Pitman FZ from the Campbell continental margin almost to the Antarctic margin, and has previously been analysed [Cande et al., 1995]. It provides a valuable reference profile for comparison with other data.

a)

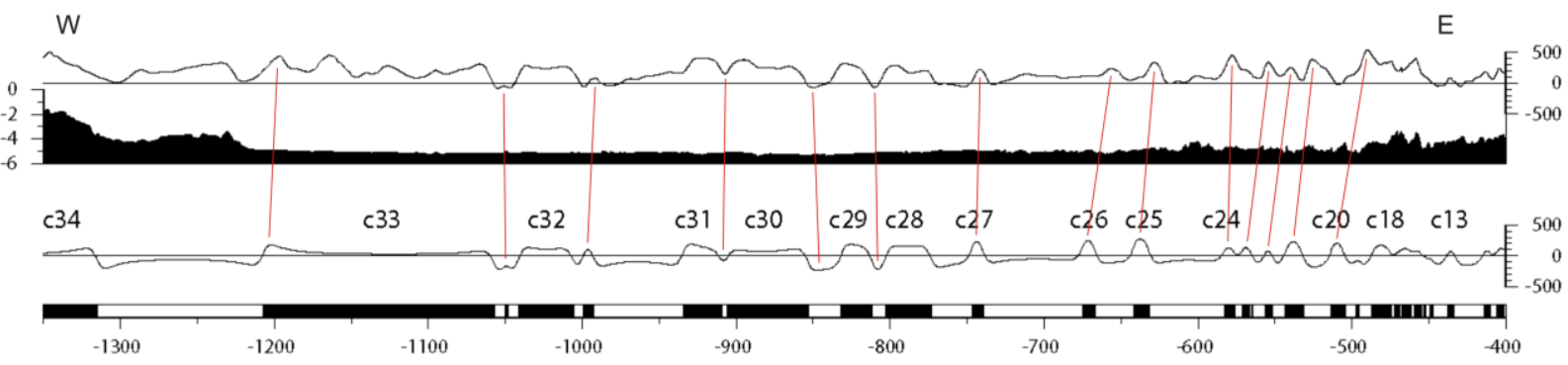

b)

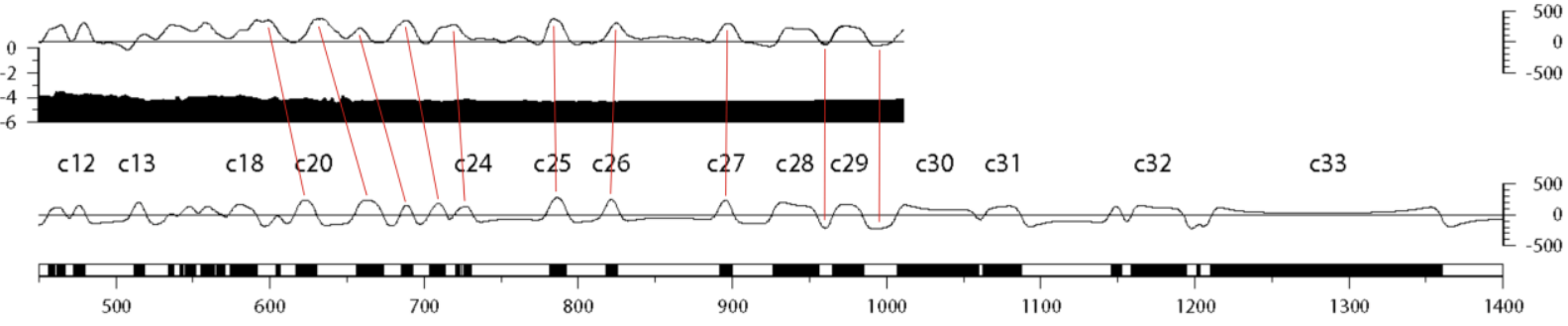

Figure II-3: Interpretation of profile ew9201_01. (a) Pacific sector. (b) Antarctica sector. From top to bottom for each: magnetic anomaly, depth profile, synthetic magnetic profile and spreading model for ew9201_01. Horizontal distance in $\mathrm{km}$ to the ridge, depth in $\mathrm{km}$, magnetic anomaly in $\mathrm{nT}$. The spreading direction is $\mathrm{N} 170^{\circ}$. The spreading rates used to calculate the synthetic model are listed in Table II-2. Declination $55^{\circ}$; inclination $-78.5^{\circ}$; magnetic layer $500 \mathrm{~m}$ thick; magnetization of the blocks of $10 \mathrm{~A} / \mathrm{m}$ (on axis) and $7 \mathrm{~A} / \mathrm{m}$ (off axis); contamination coefficient of 0.7. Please refer to the A0 map at the end of this thesis for the location of this profile (profile $\mathrm{n}^{\circ} 32$ ).

\begin{tabular}{|c|c|c|c|}
\hline Time interval (Ma) & Chron interval & Total spreading rate (km/Ma) & Asymmetry rate (\%) \\
\hline $0-30.3$ & $\mathrm{c} 1 \mathrm{n}-\mathrm{c} 11 \mathrm{r}$ & 29.00 & 7.00 \\
\hline $30.3-34.4$ & $\mathrm{c} 11 \mathrm{r}-\mathrm{c} 13 \mathrm{r}$ & 24.00 & 25.00 \\
\hline $34.4-53.8$ & $\mathrm{c} 13 \mathrm{r}-\mathrm{c} 24 \mathrm{r}$ & 17.75 & 18.30 \\
\hline $53.8-62.5$ & $\mathrm{c} 24 \mathrm{r}-\mathrm{c} 28 \mathrm{y}$ & 43.50 & 1.10 \\
\hline $62.5-67.6$ & $\mathrm{c} 28 \mathrm{y}-\mathrm{c} 30 \mathrm{o}$ & 52.00 & 0.00 \\
\hline $67.6-73.4$ & $\mathrm{c} 30 \mathrm{o}-\mathrm{c} 32 \mathrm{r}$ & 50.00 & 0.00 \\
\hline $73.4-90$ & $\mathrm{c} 32 \mathrm{r}-\mathrm{c} 34 \mathrm{n}$ & 55.00 & 0.00 \\
\hline
\end{tabular}

Table II-2: Spreading and asymmetry rates used for the profile shown in Figure II-3. To calculate the half-spreading rates, the following formula was used: $v_{1 / 2}=\frac{v_{t o t}}{2}(a+1)$, where $a$ is the absolute value of the asymmetry rate and $v_{1 / 2}$ is the fastest half-spreading rate. A positive asymmetry means the spreading is faster on the Antarctica side.

The first interpretation of each profile was made independently, by comparison with its corresponding synthetic profile. I created each synthetic model based on profile ew9201_01, using the MODMAG software ([Mendel et al., 2005]) and the magnetic reversal timescale of [Cande and Kent, 1995] (see III-3). 
Description and interpretation of specific magnetic anomaly sequences are given in more detail in sections below. The detailed interpretation of each profile is documented in Appendix A. The full magnetic reversals database (including the detailed calculation of the uncertainties) is documented in Appendix C.

Once a profile has been interpreted with MODMAG, one should check that interpretation is coherent with that of surrounding profiles by plotting the location of the magnetic anomalies on a map (see Figure II-7a and similar).

A synthetic magnetic profile represents an ideal case, but reality is inevitably more complex. Interpretation can be complicated by fracture zones or seamounts along the profile, as well as fragments of continental crust, dike intrusions, traces of ridge propagators or ridge jumps, uncertainties in the dip of deep magnetic layers due to the position of the Curie isotherm at depth during cooling, post-spreading deformations, and possible remagnetization. In some circumstances, more than one primary interpretation of the magnetic data is possible. Only preferred interpretations are shown on figures in the main text (unless documented), but alternative interpretations are documented in Appendix A. The implications of the uncertainty on plate reconstructions derived from such interpretations are discussed more fully below (II-6).

\section{Digitizing magnetic anomaly locations}

Once a spreading model is created and anomaly patterns have been matched to the model, it is possible to digitize the location of the change in seabed magnetic polarity through direct comparison with the model. The MODMAG software extracts geographic coordinates for each magnetic reversal digitized. The software also allows for a digitizing uncertainty to be assigned to each magnetic data point.

\section{Uncertainty of magnetic anomaly identification}

In most previous studies, uncertainties in the interpretation of magnetic anomaly identifications have not been documented. It was assumed that primary interpretations were correct, thus alternative interpretations were ignored. Estimated a priori uncertainties in plate motion analyses generally only reflect the precision associated with navigation. It became clear to me during this study that some anomaly identifications are not certain and there may be 
plausible alternative interpretations, and so an identification uncertainty classification scheme was developed.

The definition for each category is as follows:

1. the magnetic anomaly lies within a distinctive sequence of anomalies that is unequivocally matched to a realistic synthetic model;

2. the magnetic anomaly is not within a distinctive sequence of anomalies, but is adjacent to a sequence of category 1 anomalies and reasonable spatial correlation predicts the anomaly location;

3. the interpretation is plausible, and preferred if there are alternate possibilities, based upon a consideration of surrounding anomalies;

4. the magnetic anomaly interpretation is plausible, but tentative. It is possible that the magnetic anomaly is not caused by a seafloor-spreading magnetic reversal.

A value of magnetic anomaly identification uncertainty is assigned to each magnetic reversal digitized (see II-10 to II-15, see also Table IV-1). There is a clear need for caution when using uncertain anomaly interpretations and hence isochron locations to compute plate motions.

\section{Uncertainty of digitizing}

The "digitizing uncertainty" quantifies the uncertainty linked to digitising each magnetic reversal on the profiles. The scale for this uncertainty ranges from 1 to 3 in steps of 0.5 , and the values are first evaluated in pixels. The uncertainty is then converted to kilometres (Table II-3). So that the transformation to kilometres of the digitising uncertainty would be consistent for every profile, the horizontal scale was kept the same for every profile during the digitizing procedure. It follows that the digitizing uncertainty is linked to the identification uncertainty (shape of the anomaly) and the resolution uncertainty (spatial resolution of the profile).

\begin{tabular}{|c|c|c|}
\hline Uncertainty scale & Number of pixels & Value in kilometres \\
\hline 1.0 & 1 & 0.5 \\
\hline 1.5 & 2 & 1.0 \\
\hline 2.0 & 6 & 3.0 \\
\hline 3.5 & 17 & 8.5 \\
\hline
\end{tabular}

Table II-3: Values for the digitizing uncertainties, and equivalent in kilometres. 


\section{Uncertainty of position}

The navigation system used by the ship which acquired the magnetic data is generally considered the largest or most likely source of spatial uncertainty. For instance, [Royer and Rollet, 1997] observe crossover errors of 15 to $20 \mathrm{~km}$ when correlating profiles from different cruises.

The data I used were acquired over a period of fifty years since 1961, and hence a wide range of positioning systems were used (sextant, gyroscope, Loran-C, navsat (also known as satellite and satnav), and GPS; see Table II-1). The nominal precision of each system is shown in Table II-4.

\begin{tabular}{|c|c|}
\hline Positioning system & Estimated uncertainty \\
\hline GPS & $500 \mathrm{~m}$ \\
\hline sextant & $20 \mathrm{~km}$ \\
\hline navsat & $10 \mathrm{~km}$ \\
\hline $\begin{array}{c}\text { satellite \& sextant or } \\
\text { satellite \& dead reckoning }\end{array}$ & $15 \mathrm{~km}$ \\
\hline
\end{tabular}

Table II-4: Positioning systems used on cruises and their precision.

\section{Uncertainty of resolution}

The identification of the anomalies and the localisation of magnetic reversals depend on the spatial resolution of the data on the profiles. The spatial resolution of the profiles available varies from more than six points per kilometre (nbp9602) to less than one point every five kilometres (elt50). It follows that the shape of the anomalies is less recognizable and the resolution uncertainty greater on profiles with a larger spatial datum separation interval.

The number of points per kilometre, averaged per profile, is used to quantify this uncertainty.

\section{Interpretation for anomalies 20 to 24}

This sequence of five magnetic anomalies of variable width over a relatively narrow strip (c. $100 \mathrm{~km}$ ) of ocean floor creates a distinctive pattern (Figure II-4). Anomaly 20 is 
recognizable as a distinct positive peak followed by a broader negative interval (in some places with a minor peak on the edge furthest from the spreading ridge) and preceded by a low amplitude peak and another peak (anomalies 19 and 18 respectively). Anomalies 21 to 24 follow anomaly 20 . Anomaly 21 is of similar size to anomaly 20 . It is followed closely by anomaly 22 , which is of smaller amplitude and width. Anomaly 23 is very similar to anomaly 22 , though slightly broader on some profiles. Anomaly 24 is what I like to call a "double" anomaly: [Cande and Kent, 1995] define anomaly 24 as caused by a sequence of closely spaced short-lived magnetic reversals, whose magnetic signals combine to form two intertwined picks. I was able to identify without doubt this sequence on a number of profiles in both the New Zealand and the Antarctica sectors.
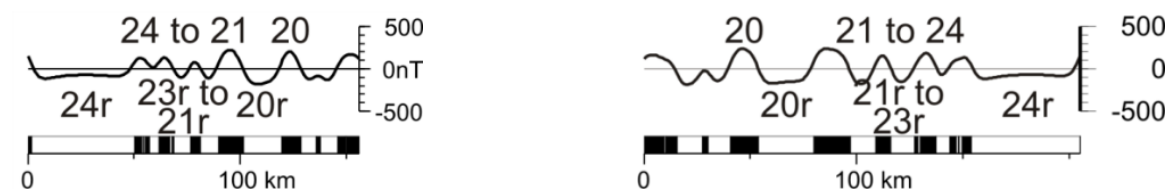

Figure II-4: Typical sequence of magnetic anomalies 20 to 24 . The total spreading rate used for this model is $17.75 \mathrm{~km} / \mathrm{My}$, with an asymmetry of $18.3 \%$ (spreading faster on the Antarctica side).

I reprojected all profiles with an azimuth differing from the expected direction of spreading by 15 degrees or more along a small circle following the estimated flowline to create Figure II-5 and Figure II-6 and their equivalent for older anomalies. What follows is a description of my interpretations, ordered by increasing identification uncertainty (see Table II-5 and similar). Three profiles (ew9201_01o, kh6804-C_02 and nbp9507_01) display a c20yc24o sequence of magnetic anomalies that fits the synthetic model exemplarily (Figure II-5). Such anomalies are assigned a category 1 identification uncertainty (u1). 


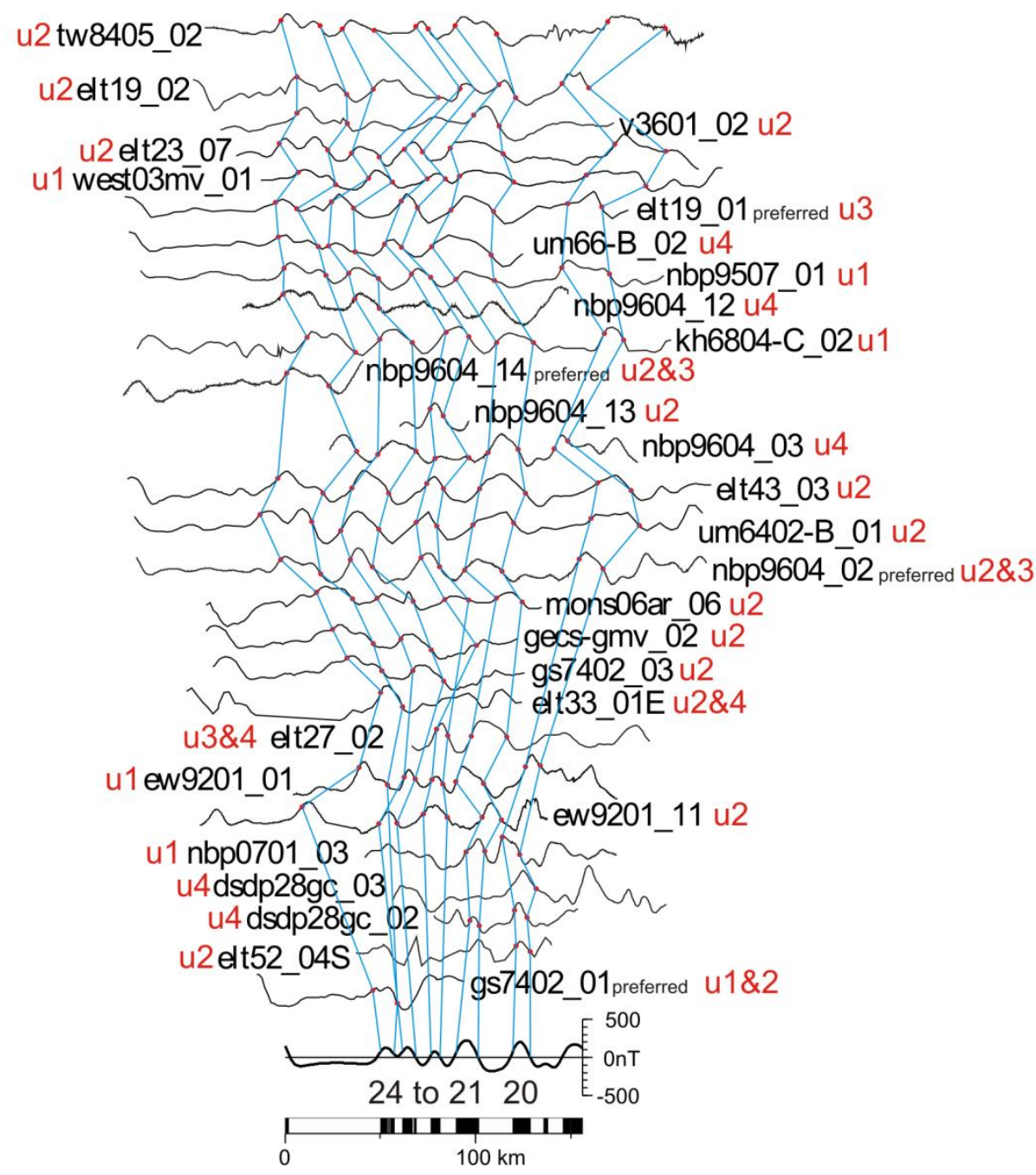

Figure II-5: Interpreted magnetic profiles showing anomalies 20 to 24 for the Pacific sector. Profiles elt52_04S, dsdp28gc_02, dsdp28gc_03, nbp0701_03, elt27_02, nbp9604_02, nbp9604_03, nbp9604_13, nbp9604_14, kh6804-C_02, nbp9507_01, v3601_02, elt19_02 and tw8405_02 were reprojected onto a line parallel to the nearest recognized fracture zone, due to an azimuth difference of more than $15^{\circ}$ from the expected flow line. The synthetic model (bottommost profile) is from Figure II-4. The identification uncertainty of the sequence for each profile is indicated in red (see Appendix A for more detail). 


\begin{tabular}{|c|c|}
\hline Identification uncertainty & profile \\
\hline \multirow{5}{*}{ u1 } & nbp0701_03 \\
\hline & ew9201_01 \\
\hline & kh6804-C_02 \\
\hline & nbp9507_01 \\
\hline & west03mv_01 \\
\hline u1 \& u2 & gs7402_01 \\
\hline \multirow{12}{*}{ u2 } & elt52_04S \\
\hline & ew9201_11 \\
\hline & gs740203 \\
\hline & gecs-gmv_02 \\
\hline & mons06ar_06 \\
\hline & um6402-B_01 \\
\hline & elt43_03 \\
\hline & nbp9604_13 \\
\hline & elt23_07 \\
\hline & v3601_02 \\
\hline & elt19_02 \\
\hline & tw8405_02 \\
\hline \multirow{2}{*}{ u2 \& u3 } & nbp9604_02 \\
\hline & nbp9604_14 \\
\hline $\mathrm{u} 2$ \& u4 & elt33_01E \\
\hline u3 & elt19_01 \\
\hline u3 \& u4 & elt27_02 \\
\hline \multirow{5}{*}{ u4 } & dsdp28gc_02 \\
\hline & dsdp28gc_03 \\
\hline & nbp9604_03 \\
\hline & nbp9604_12 \\
\hline & um66-B 02 \\
\hline
\end{tabular}

\begin{tabular}{|c|c|}
\hline Identification uncertainty & profile \\
\hline \multirow{3}{*}{ u1 } & ew9201_01 \\
\hline & nbp92-8 02 \\
\hline & nbp92-8_03 \\
\hline u1 \& u2 & elt20_03 \\
\hline \multirow{6}{*}{ u2 } & helimag_12 \\
\hline & helimag_08 \\
\hline & helimag_07 \\
\hline & helimag_09 \\
\hline & elt33_05 \\
\hline & elt21_01 \\
\hline \multirow{2}{*}{ u2 \& u4 } & polarstern3 \\
\hline & ew9201_11 \\
\hline u3 & nbp9507 02 \\
\hline
\end{tabular}

Table II-5: Profiles presented in Figure II-5 and Figure II-6, sorted by identification uncertainty for the sequence of magnetic anomalies 20-24.

Although the sequence a20-a24 on profile nbp0701_03 is very narrow, a20 is almost identical to the model, making the interpretation a category u1. Anomalies 22 to 24 on profile west03mv_01 were identified independently (u1). On profile gs7402_01 the negative anomaly $24 \mathrm{r}$ was first identified, with confidence (u1). The position of anomalies 20 to 24 was deduced from that of anomaly $24 \mathrm{r}$.

The identification of anomaly 20 on profile elt52_04S was made by spatial correlation with the position of a25, assuming little variation in spreading rate. Though the older part of profile ew9201_11o fits the model very well, a localised lack of data after c24o makes correlation with surrounding profiles necessary for identifying the position of anomalies 23 to 20. Profile nbp9604_13 is a short profile. The identification of anomalies 20 to 24 on this profile was done based on surrounding profiles and satellite gravity data ${ }^{14}$. Each anomaly of the

\footnotetext{
${ }^{14}$ Please refer to Appendix A for a more detailed interpretation of each profile mentioned in this chapter.
} 
sequence was then tested against those of surrounding profiles (spatial correlation). The c20yc24o sequence was identified on six profiles (elt52_04S, mons06ar_06, nbp9604_02, nbp9604_14, v3601_02 and tw8405_02) based on spatial correlation with older parts of the profile and little variation of the spreading rate. Some profiles show a good fit with the model (elt52_04S, tw8405_02); others have an irregular signal (mons06ar_06, v3601_02). On profile tw8405_02 the magnetic signal gets rougher towards a20. This is an artefact due to the change of azimuth of the profile: on a short portion of the profile its azimuth differs from the spreading direction more than on the rest of the profile.

Profiles nbp9604_02 and nbp9604_14 were both interpreted based on the identification of a25, leading to alternative interpretations for part of the sequence. Assuming little or no variation of spreading rate favours one interpretation. The identification of anomaly 24 on profile elt33_01E is a typical example of category $\mathrm{u} 4$ : only if we assume that the remanent magnetisation of the crust is the only factor influencing the signal can anomaly 24 be identified, based on spatial correlation with the rest of the profile and little variation of spreading rate.

As profiles dsdp28gc_02 and dsdp28gc_03 are two consecutive segments of the same cruise, both were interpreted at the same time. Anomalies 20 and 21 were identified on profile dsdp28gc_02 by spatial correlation with surrounding profiles and satellite gravity data. The interpretation of profile dsdp28gc_03 was deduced from that of its neighbouring profile, though with little confidence in the identification (u4). The anomalies identified on profiles nbp9604_03 and um66-B_02 were assigned a category 4 identification uncertainty. The magnetic anomalies on profile nbp9604_03 are very similar to the model but the geographic location of the profile suggests the reversals are younger than $\mathrm{c} 20$. The low spatial resolution of magnetic data on profile um66-B_02 makes the signal unclear. Profile nbp9604_12's interpretation is inferred from that of neighbouring profile kh6804-C_02, although the profile's short length makes the identification of the anomalies uncertain $(u 4)$.

In the Antarctica region, the profiles east of the AFZ are generally harder to interpret for the sequence a20 to a24, as the anomalies are less regular (Figure II-6). 


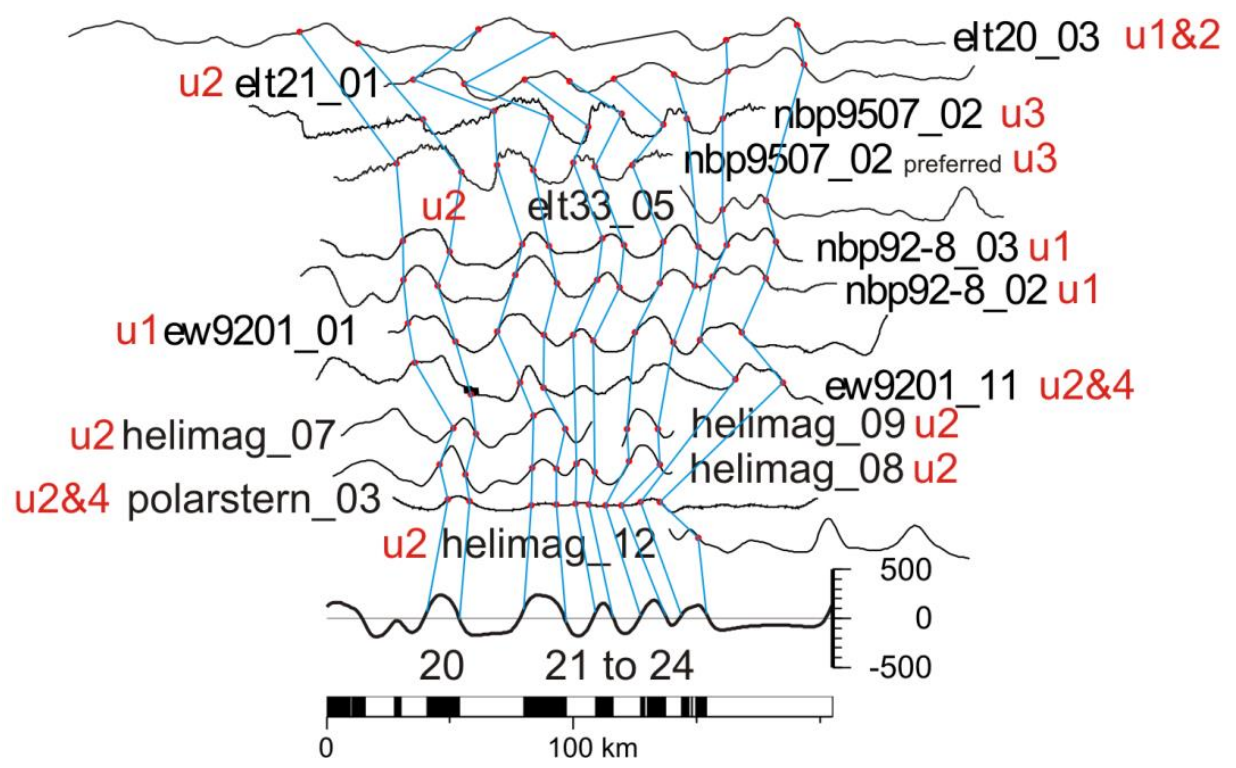

Figure II-6: Interpreted magnetic profiles showing anomalies 20 to 24 for the Antarctica sector. Profiles nbp92-8_02, elt33_05, nbp9507_02, nbp9507_03, elt21_01, elt23_02, elt20_03 and tw8405_03W were reprojected onto a line parallel to the nearest recognized fracture zone, due to an azimuth difference of more than $15^{\circ}$ from the expected flow line. The synthetic model (bottommost profile) is from Figure II-4. The identification uncertainty of the sequence for each profile is indicated in red (see Appendix A for more detail).

Profile ew9201_01 is the reference profile for the Antarctica sector. Two other profiles also show a perfect fit with the model (u1): nbp92-8_02 and nbp92-8_03. Most profiles were interpreted based on ew9201_01 and other surrounding profiles: polarstern3, elt33_05 and elt21_01 were assigned $u 2$. The azimuth of profile elt23_06 (not on Figure II-6) is very oblique to the direction of spreading.

I analysed the aeromagnetic data collected during the Polarstern cruise (2010, ANTXXVI/3). Profile helimag_08 shows a very good fit with the synthetic model, despite a21 being of smaller amplitude than expected. Profiles helimag_07 and helimag_09 were interpreted by spatial correlation with profile helimag_08. The anomalies identified on both profiles were assigned category 2 identification uncertainties. c24o was identified on profile helimag_12 based on the rest of the profile.

Profile ew9201_11 was interpreted by spatial correlation with the rest of the profile and with its adjacent profile, ew9201_01. The interpretation of profile nbp9507_02 is made by spatial correlation with surrounding profiles and a20, for which two possible locations exist.

A comparison between satellite gravity data in the South Pacific region and the new magnetic identifications (Figure II-7) shows that the seafloor becomes suddenly rough during the reverse period $24 \mathrm{r}$ (53.3 to $55.9 \mathrm{Ma}$ ). The region of ocean crust formed between c20y and 
c24o (42.5 to $53.4 \mathrm{Ma}$ ) is characterised by bifurcation and local reorganisation of fracture zones, and low spreading rates (less than $20 \mathrm{~km} / \mathrm{Myr}$ along the Pitman FZ according to the synthetic model) which are known to result in high local spreading asymmetry [Dick et al., 2003; Gac et al., 2003]. This explains the poor fit of some profiles with the model (see profile gs7402_03, Figure II-5), and my interpretation of those profiles is less confident. The apparent change of spreading rate on profile dsdp28gc_03 (Figure II-5) could be due to post-spreading deformation. 
ㅎ․

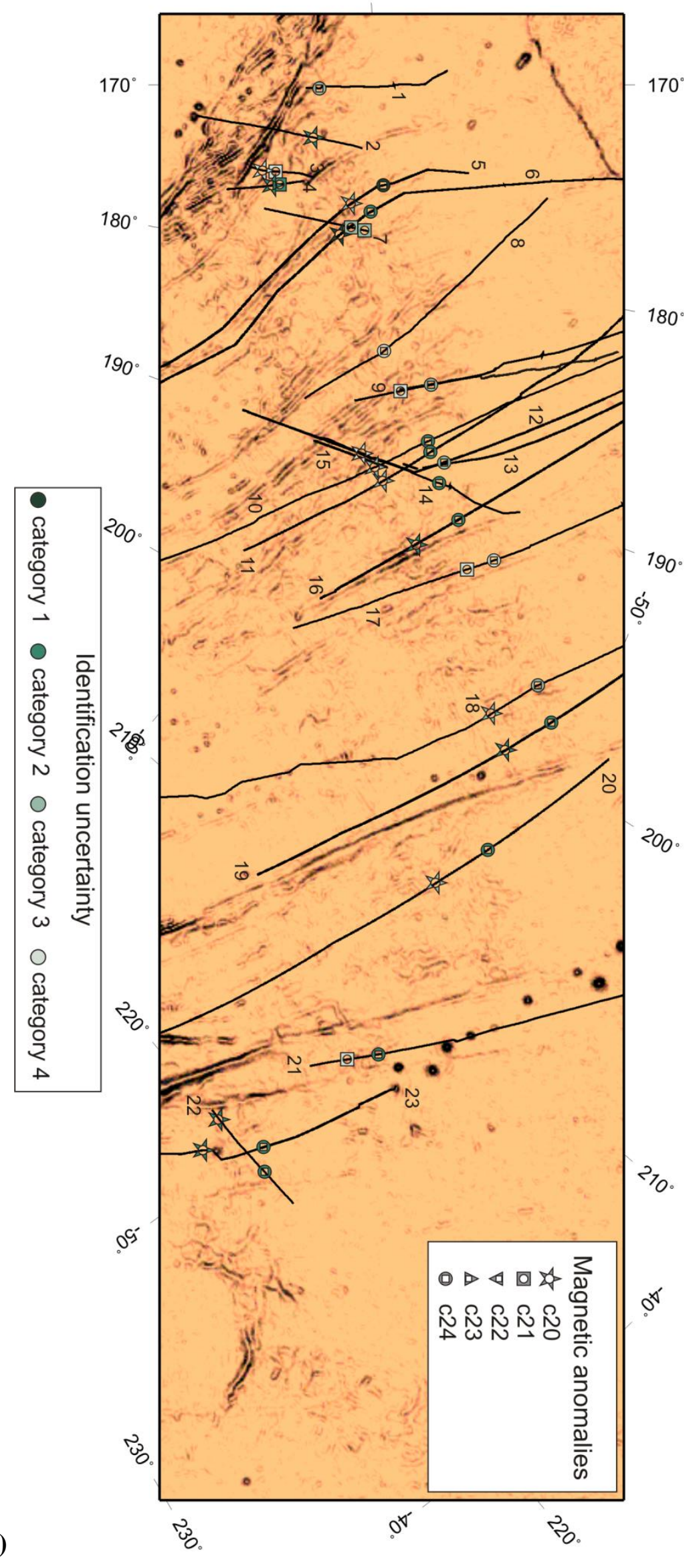




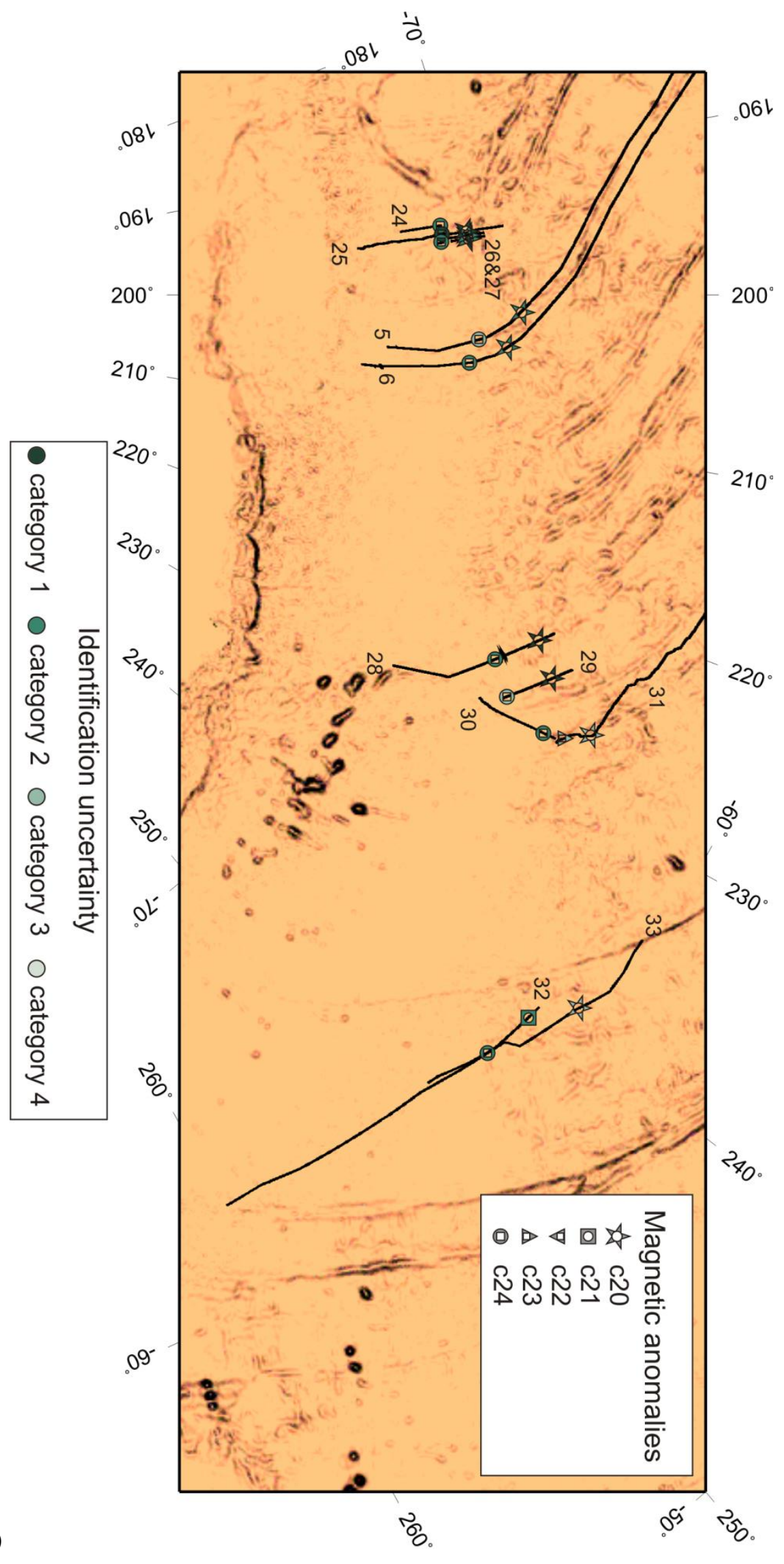




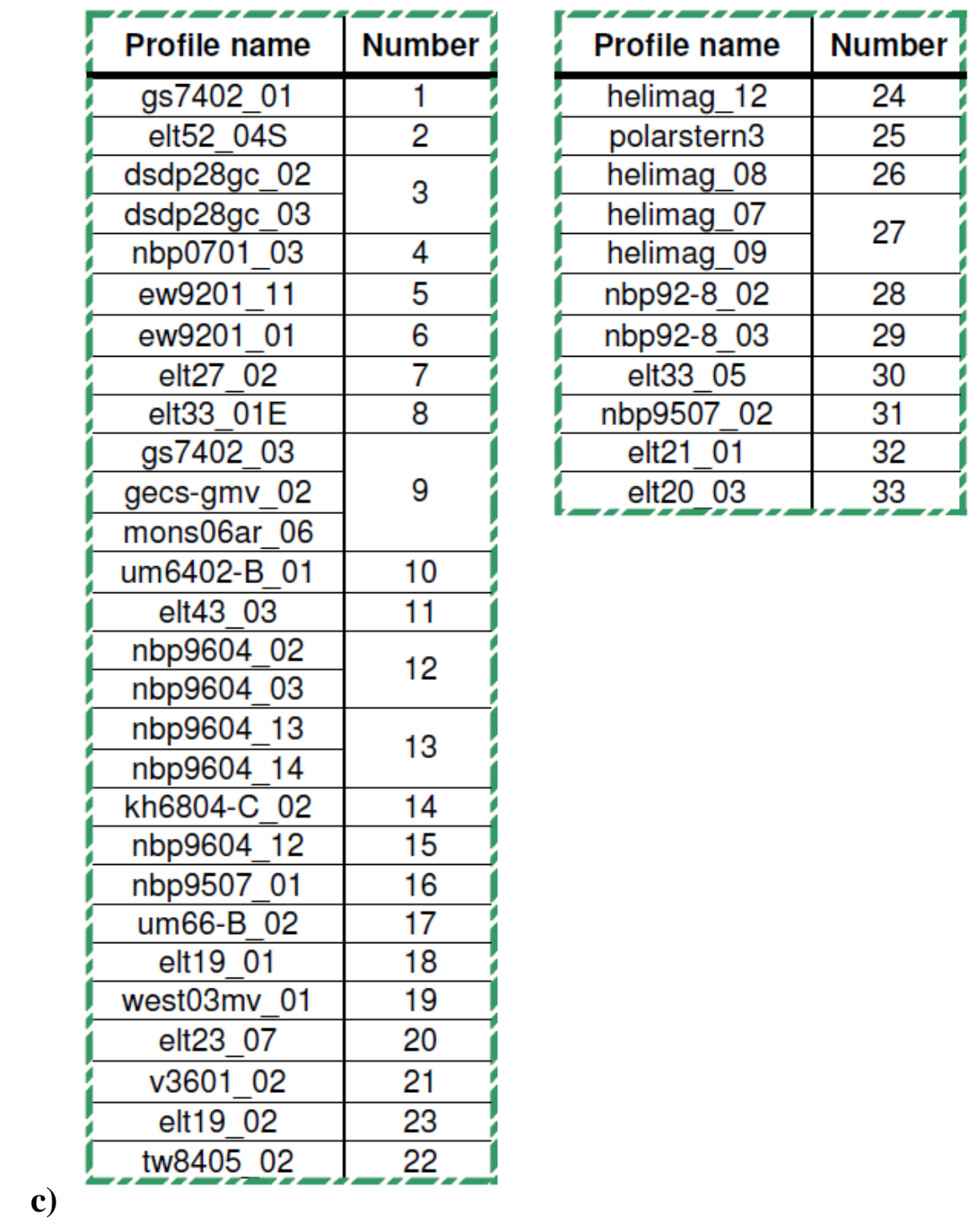

Figure II-7: Interpreted profiles and magnetic reversals identified, with identification uncertainty. Horizontal gravity gradient computed using GMT from [Sandwell and Smith, 1997] (version 18) in the background. a) Map for the Pacific sector. b) Map for the Antarctica sector. c) Profiles and corresponding numbers on the maps.

\section{Interpretation for anomalies 25 and 26}

This sequence of two magnetic anomalies over a width of 50 to $100 \mathrm{~km}$ of ocean floor produces a distinctive pattern (Figure II-8). Both a25 and a26 are recognizable as relatively high amplitude positive peaks, separated by a narrow negative interval. The sequence is framed by two broad negative intervals (a24r and a26r), a26r being the broader of the two. I identified this sequence on numerous profiles in both the Pacific and the Antarctica sectors. 

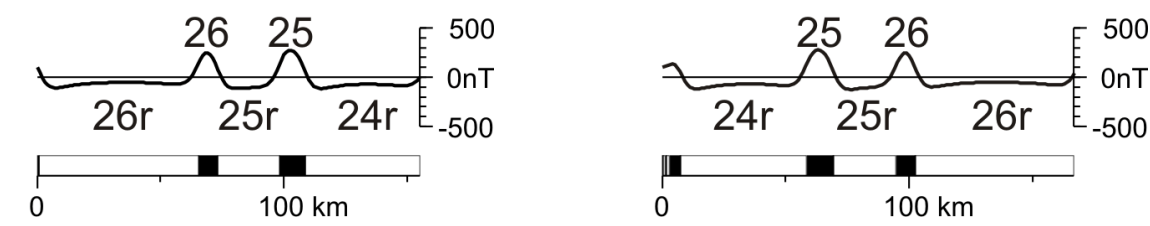

Figure II-8: Typical sequence of magnetic anomalies 25 and 26. The total spreading rate used for this model is $43.5 \mathrm{~km} / \mathrm{My}$, with an asymmetry of $1.1 \%$ (spreading faster on the Antarctica side).

In the Pacific sector, nineteen profiles present a sequence of magnetic anomalies 25 and 26 almost identical to the synthetic model (Figure II-9, u1). This interpretation is further supported by the identification of sequence a27-a29 farther north along the same profiles (Table II-6). The apparent absence of a25 on profile gecs-gmv_01 is due to missing data. 


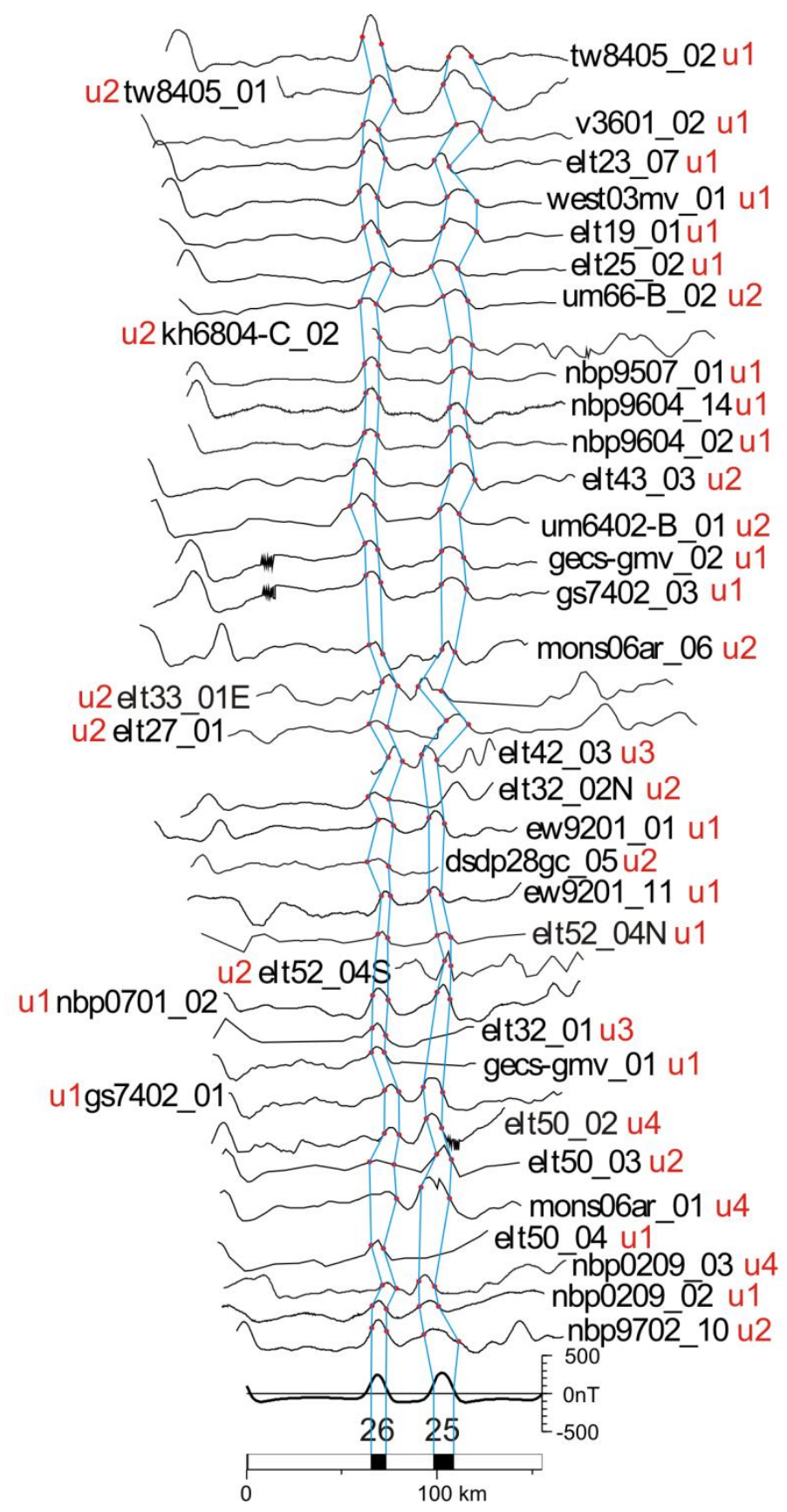

Figure II-9 : Interpreted magnetic profiles showing anomalies 25 and 26 for the Pacific sector. Profiles nbp0209_03, elt52_04S, elt42_03, elt27_01, nbp9604_02, nbp9604_14, kh6804-C_02, v3601_02, tw8405_02 and tw8405_01 were reprojected onto a line parallel to the nearest recognized fracture zone, due to an azimuth difference of more than $15^{\circ}$ from the expected flowline. The synthetic model (bottommost profile) is from Figure II-8. The identification uncertainty is indicated in red (see Appendix A for more detail). 

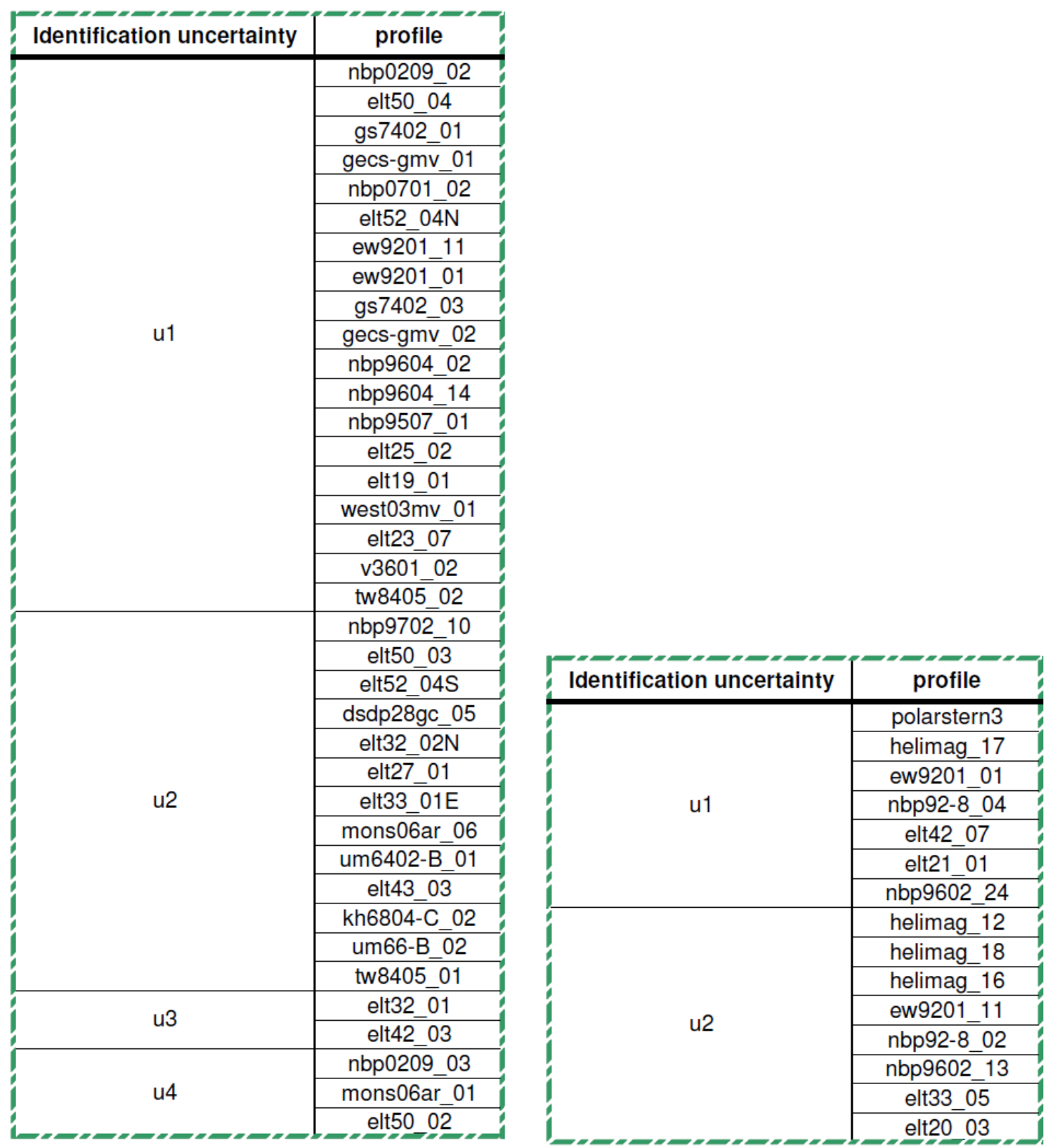

Table II-6: Profiles presented in Figure II-9 and Figure II-10, sorted by identification uncertainty for the sequence of magnetic anomalies 25-26.

Profile nbp9702_10 displays a clear a26, next to a wide rough positive anomaly. Encouraged by the roughness of the seafloor below the second anomaly, I interpreted it as a25 (u2). The same process was applied to profiles um6402-B_01, elt43_03, um66-B_02 and tw8405_01. The high amplitude bathymetric anomalies on profile tw8405_01 are seamounts associated with the Louisville hotspot.

On profile elt52_04S, the identification of a 25 was made in the context of a close spatial correlation to surrounding profiles, so it was assigned to uncertainty category u2 (Figure II-9). 
Because they are at the end of profile kh6804-C_02, a25 and a26 were identified by spatial correlation with the a20-a24 sequence (u2). On profiles elt50_03, dsdp28gc_05, elt32_02N, elt27_01, elt33_01E and mons06ar_06 the sequence a27-a29 was used as a reference to identify younger anomalies by spatial correlation (u2).

On profile elt32_01, a positive anomaly south of c27y can be interpreted as either a25 or a26 (u3), depending on the spreading rate. Anomaly 26 is slightly more likely, but it is not possible to distinguish between the two interpretations based solely on profile interpretation. Similarly, two locations for anomaly 25 were found on profile elt42_03, the one closer to anomaly 26 being preferred. Profile elt42_03 was interpreted by spatial correlation with surrounding profiles (u3).

On several profiles, the sequence a25-a26 can only be interpreted by spatial correlation with other parts of the profile (often the sequence a27-a29) and/or neighbouring profiles. The southern end of profile elt50_02 shows a wide high amplitude magnetic signature, next to two magnetic anomalies that resemble the sequence a25-a26. The identity of the sequence (u4) is confirmed by spatial correlation with the rest of the profile. Other profiles, like mons06ar_01, do not show a clear sequence a25-a26. However, a clear sequence a27-a29 and the assumption of geographically consistent spreading rates leads to tentative identification of anomalies 25 to 26 (u4). Because profile nbp0209_03 crosses numerous fracture zones, each section of the profile separated by fracture zones is too short to be interpreted without the help of surrounding profiles.

Similarly to the Pacific side, several profiles on the Antarctica side of the ridge present a sequence of a25-a26 almost identical to the synthetic model (Figure II-10 and Table II-6, u1). 


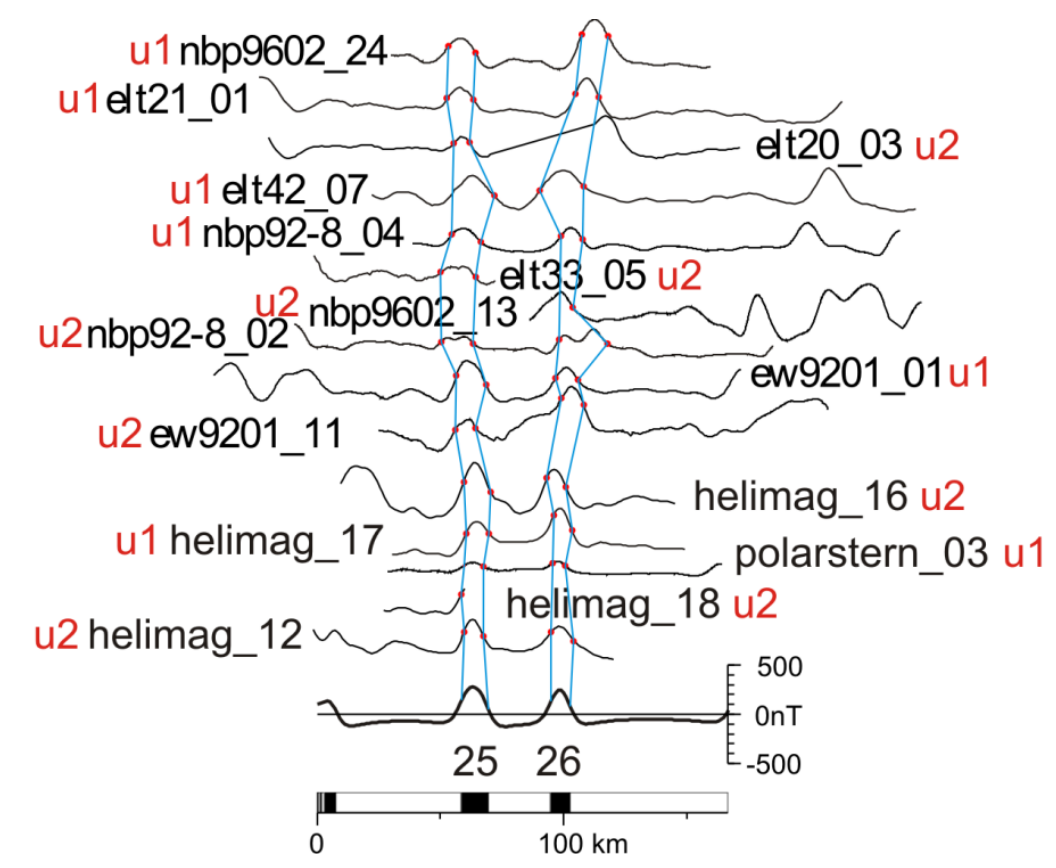

Figure II-10: Interpreted magnetic profiles showing anomalies 25 and 26 for the Antarctica sector. Profiles nbp92-8_02, elt33_05, elt42_07, nbp9507_03, elt23_06, elt20_03, elt21_01, elt23_02 and nbp9604_24 were reprojected onto a line parallel to the nearest recognized fracture zone, due to an azimuth difference of more than $15^{\circ}$ from the expected flowline. The synthetic model (bottommost profile) is from Figure II-8. The identification uncertainty is indicated in red (see Appendix A for more detail).

Neighbouring sequences a20-a24 and a27-a29 were used to help identify the sequence a25-a26 by spatial correlation on profiles ew9201_11, nbp92-8_02 and nbp9602_13 (u2). The sequence was identified on profile elt33_05 with the help of profiles nbp92-8_02 and nbp928_04. Profiles helimag_12, helimag_18 and helimag_16 were interpreted based on surrounding profiles. The identifications of sequence a25-a26 were assigned an identification uncertainty $\mathrm{u} 2$.

Profile nbp9702_01 does not appear on Figure II-10 because my preferred interpretation for this profile does not include the sequence 25-26. Two alternative interpretations include this sequence (see Appendix A): if the older part of the profile is interpreted as the sequence a27a29, the younger part displays magnetic anomalies that could be either the sequence a25-a26, or a25 proceeded by a spurious anomaly.

Comparing the location of the c25y-c26o reversals with the regional satellite gravity data (Figure II-11) shows a correlation between c25y and the limit between rough and smooth seafloor. 


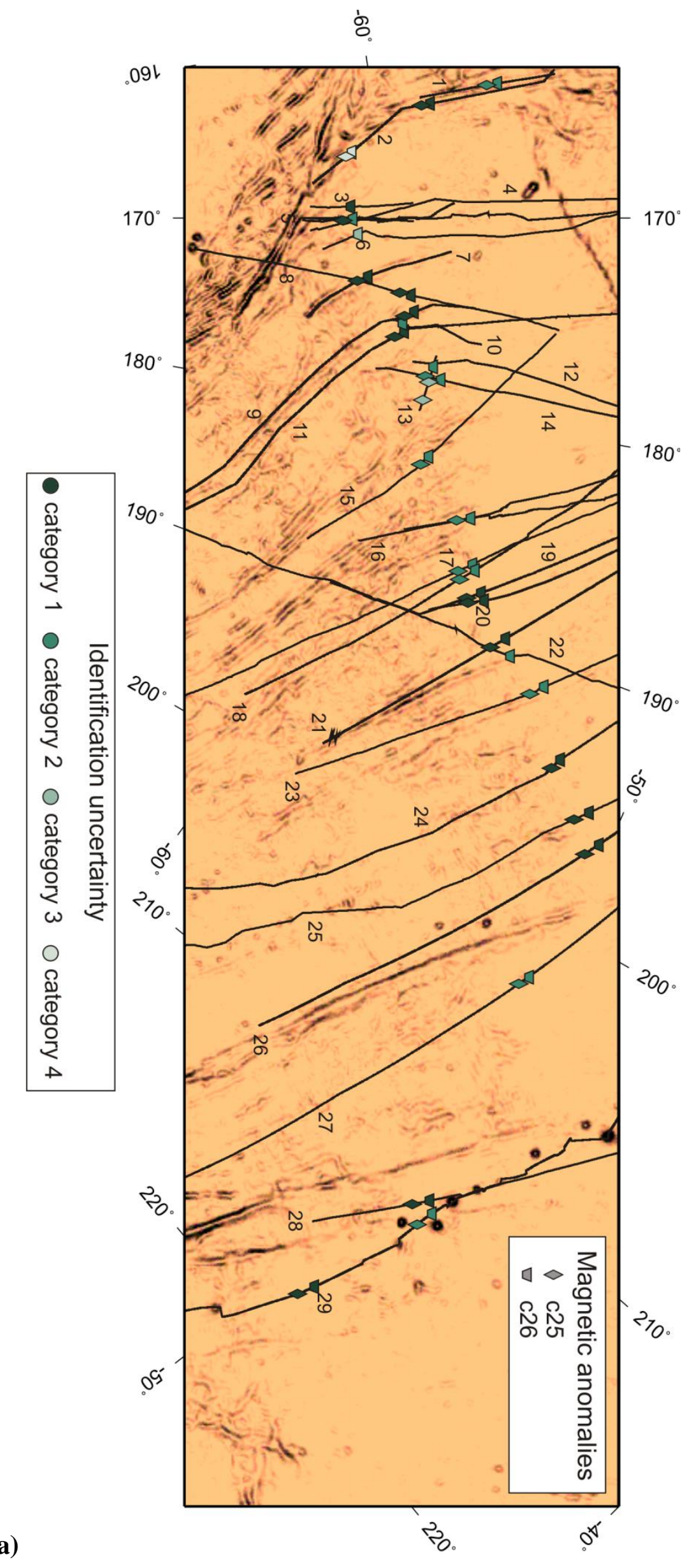




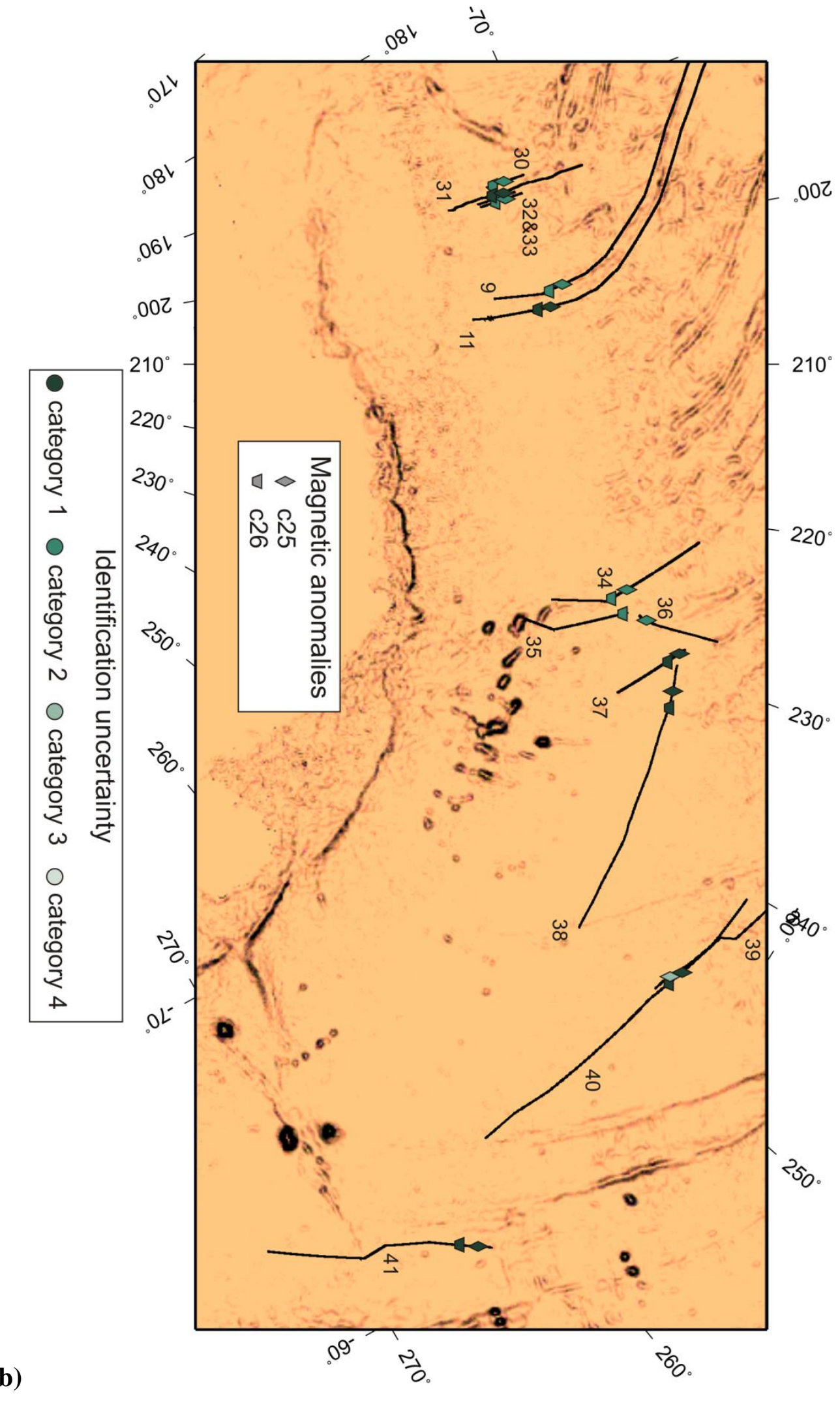




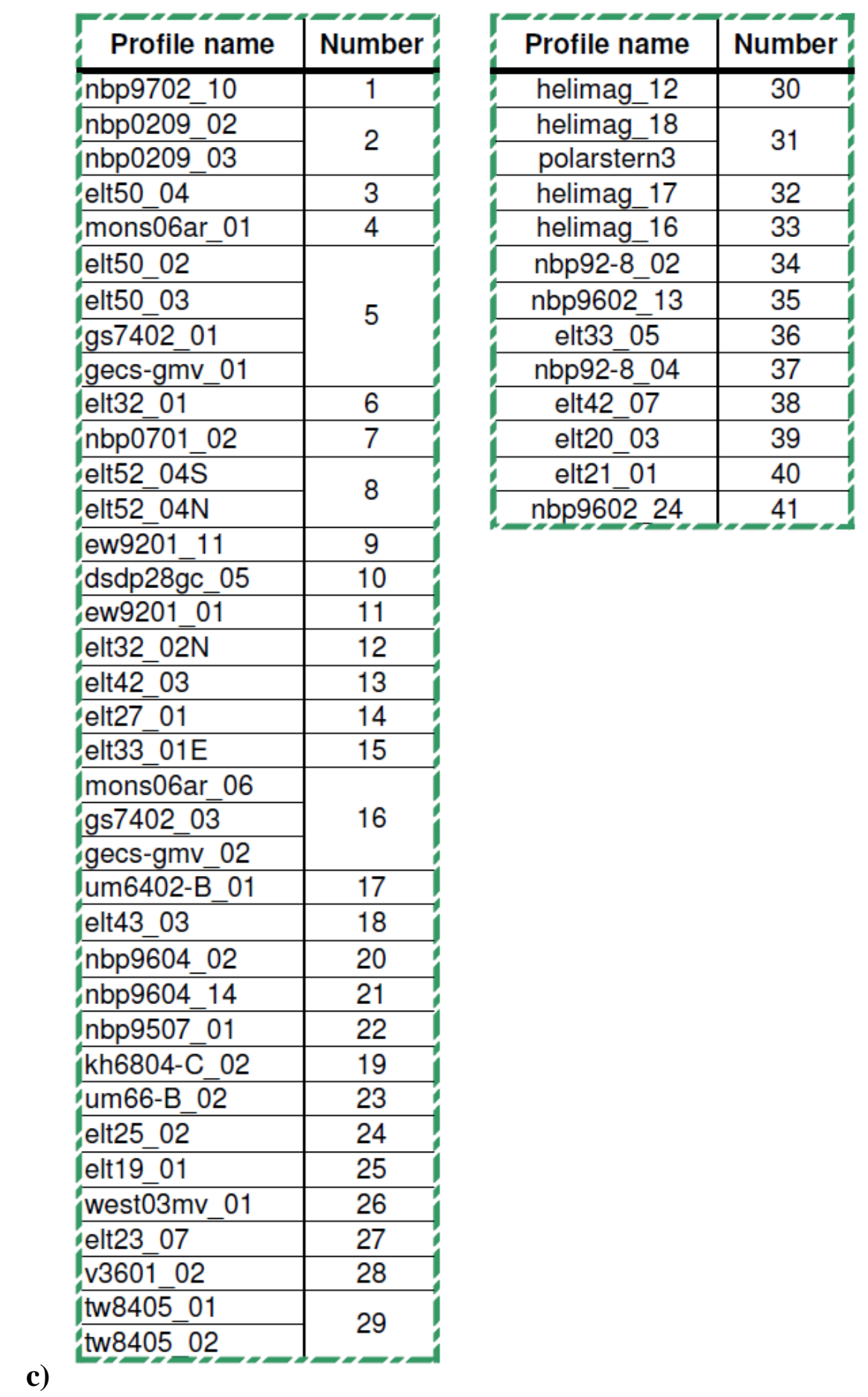

Figure II-11: Interpreted profiles and magnetic reversals identified, with identification uncertainty. Horizontal gravity gradient computed using GMT from [Sandwell and Smith, 1997] (version 18) in the background. a) Map for the Pacific sector. b) Map for the Antarctica sector. c) Profiles and corresponding numbers on the maps.

\section{Interpretation for anomalies 27 to 29}

The sequence of anomalies 27, 28 and 29 (Figure II-12) is one of the most distinctive and hence easily recognisable. Anomaly 27 is a well-defined positive anomaly, preceded by a 
moderate negative anomaly (27r), and followed by a broad one (26r). Anomalies 28 and 29 are moderate-amplitude positive anomalies, separated by a narrow negative anomaly (28r) adjacent to a slightly broader one (29r).
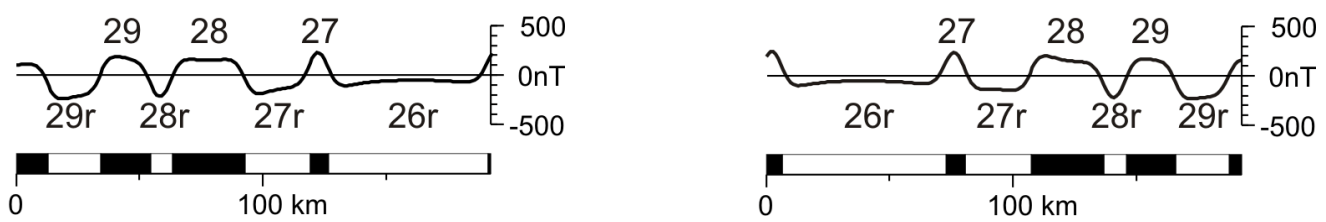

Figure II-12: Typical sequence of magnetic anomalies 27 to 29. The total spreading rate used for this model is $43.5 \mathrm{~km} / \mathrm{My}$, with an asymmetry of $1.1 \%$ (spreading faster on the Antarctica side) during anomaly 27, and 52km/My with no asymmetry during anomalies 28 and 29.

Because of its particular shape, I used the sequence c27-c29 as a starting point for interpreting most of the profiles, and more particularly the reference profile, ew9201_01. Profiles nbp9702_10, nbp9702_09, ares02wt_01, gs7402_01, gecs-gmv_01, mons06ar_01, elt32_02N, mons06ar_06, gs7402_03, gecs-gmv_02, um6402-B_01, elt43_03, nbp9604_02, nbp9604_14, nbp9507_01, um66-B_02, elt25_02, elt19_01, west03mv_01, elt23_08 and elt23_07 show a very good fit with the synthetic model (Figure II-13 and Table II-7, u1). Profile elt27_01 displays a sequence of anomalies that can be easily identified but is incomplete due to missing data.

I have no doubt regarding the identification of the a27-a29 sequence on profiles elt50_04 and elt50_03 (u1), despite the large distance between each data point. On profile nbp0701_02 only the younger half of the sequence can be identified (u1) due to the presence of a fracture zone. On profile v3601_02, the part of a28 farthest from the ridge is affected by the local bathymetry. On profile dsdp28gc_05, the identification of a28 and a29 (u2) is deduced from that of a27 (u1). For profile tw8405_01 the identification of the sequence is based on that of a29 (u1). 


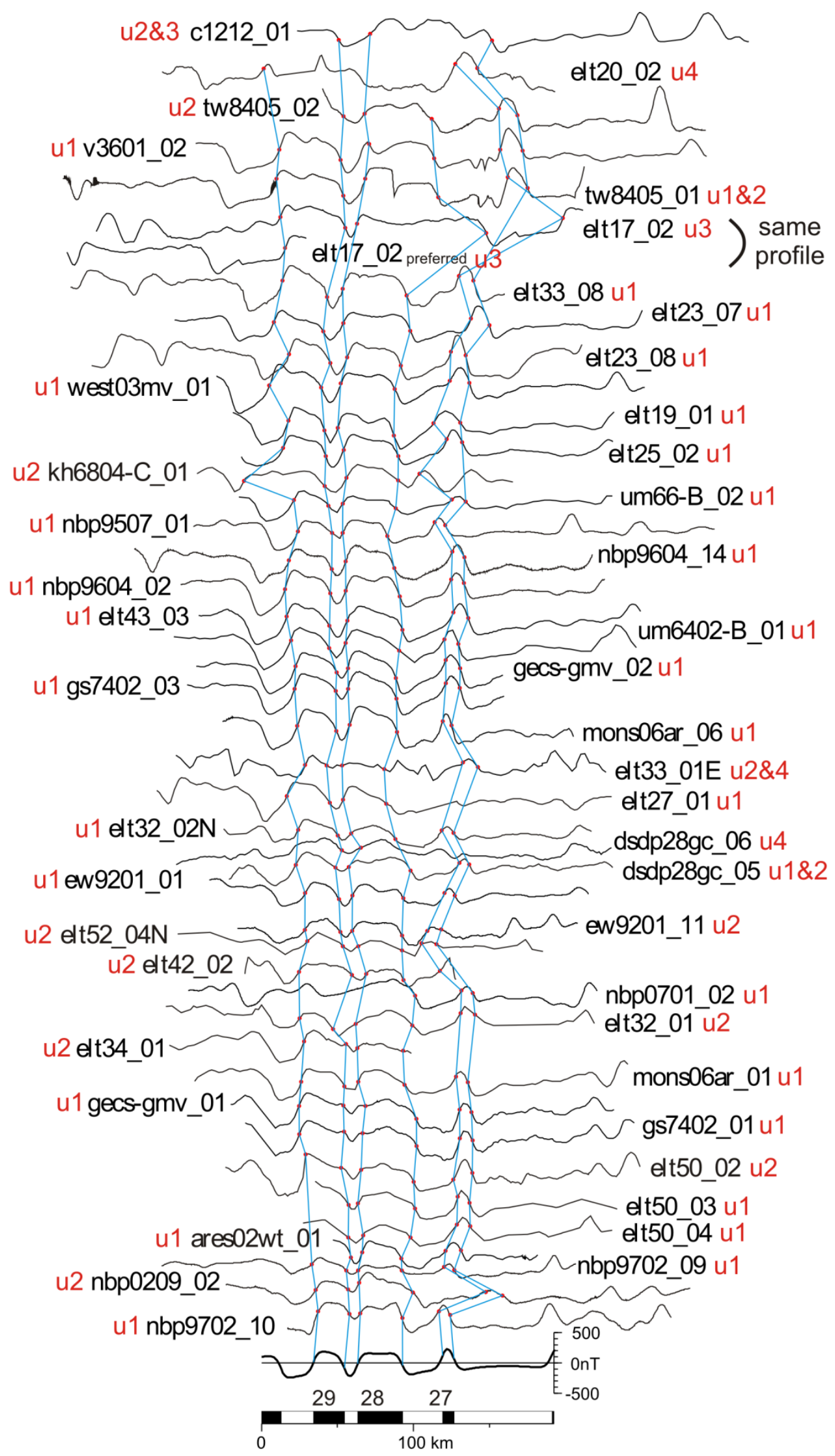

Figure II-13: Interpreted magnetic profiles showing anomalies 27 to 29 for the Pacific sector. Profiles nbp9707_10, nbp0209_02, nbp9702_09, elt50_04, elt50_03, elt50_02, mons06ar_01, elt34_01, elt32_01, elt42_02, elt32_02N, elt27_01, nbp9604_02, nbp9604_14, nbp9507_01, 
kh6804-C_01, elt23_08, elt33_08, tw8405_01, v3601_02, tw8405_02 and elt20_02 were reprojected onto a line parallel to the nearest recognized fracture zone, due to an azimuth difference of more than $15^{\circ}$ from the expected flowline. Profiles c2107_01, c2107_03 and c2107_04 do not appear on this figure because they display crust formed on the Pacific-Phoenix spreading center. The synthetic model (bottommost profile) is from Figure II-12. The identification uncertainty of the sequence for each profile is indicated in red (see Appendix A for more detail).
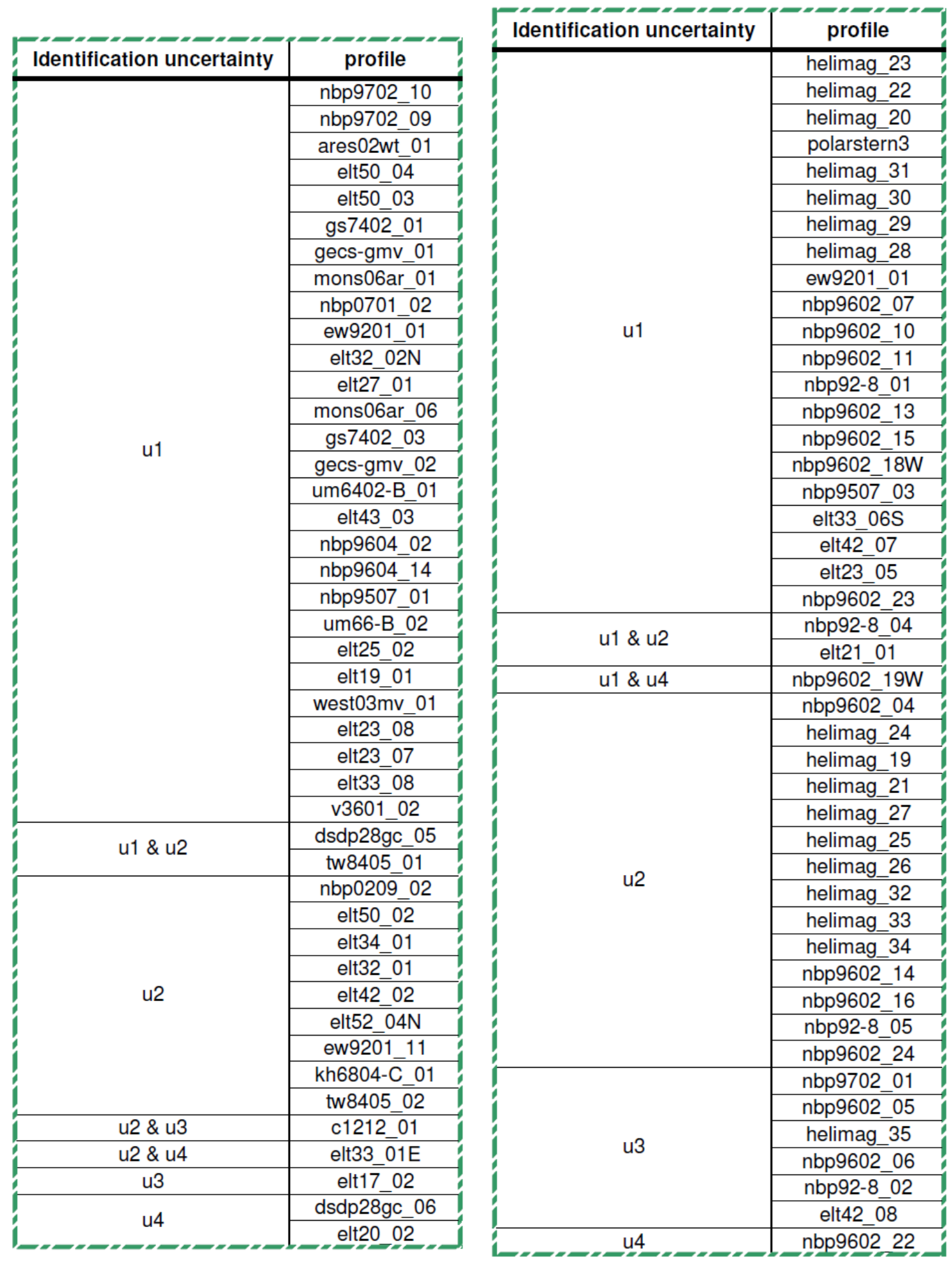
Table II-7 (previous page): Profiles presented in Figure II-13 and Figure II-14, sorted by identification uncertainty for the sequence of magnetic anomalies 27-29.

For several profiles the identification of a 27 to a29 is done by spatial correlation with another part of the same profile. That is the case for profiles nbp0209_02, elt50_02, elt34_01, elt52_04N, ew9201_11, kh6804-C_01 and tw8405_02 (u2). Profiles elt32_01 and elt42_02 were interpreted in spatial correlation with surrounding profiles. The identification of a27 on profile tw8405_03E is based on the profile's younger anomalies on the same profile, but what appears to be a28 might be a spurious anomaly due to the bathymetry ${ }^{15}$. On profile c1212_01 a28r can be identified by spatial correlation with surrounding profiles (u2) but what I chose to identify as c27y could also be c28y (u3). The identification of a27 on profile elt33_01E is tentative (u4) and based solely on the identification of younger and older anomalies on the profile.

The sequence of three anomalies on profile elt17_02 can be interpreted as either c27yc29o or c29y-c31o. The short length of the profile allows the two possibilities (u3).

Profile dsdp28gc_06 was interpreted by spatial correlation with surrounding profiles and a28r could be identified, but its lack of resemblance to the model makes the identification very uncertain (u4). The a27-a29 sequence was tentatively identified (u4) on profile elt20_02 by spatial correlation with older anomalies on the profile, but its shape doesn't match that of the model.

On the Antarctica side, twenty-one profiles (helimag_23, helimag_22, helimag_20, polarstern_03, helimag_31, helimag_30, helimag_29, helimag_28, ew9201_01, nbp9602_07, nbp9602_10, nbp9602_11, nbp92-8_01, nbp9602_13, nbp9602_15, nbp9602_18W, nbp9507_03, elt33_06S, elt42_07, elt23_05 and nbp9602_23) show a c27y-c29o sequence that is almost identical to the synthetic model (Figure II-14, u1). For profiles nbp92-8_04 and elt21_01, a27 was identified first (u1). The rest of the sequence was deduced from that identification $(\mathrm{u} 2)$. Although anomalies 27 and 28 on profile nbp9602_19W are almost identical to the synthetic model (u1), c29o cannot be identified with certainty (u4).

\footnotetext{
15 Profile tw8405_03E is not represented on Figure II-13 because its seafloor formed on the Pacific-Phoenix spreading system.
} 


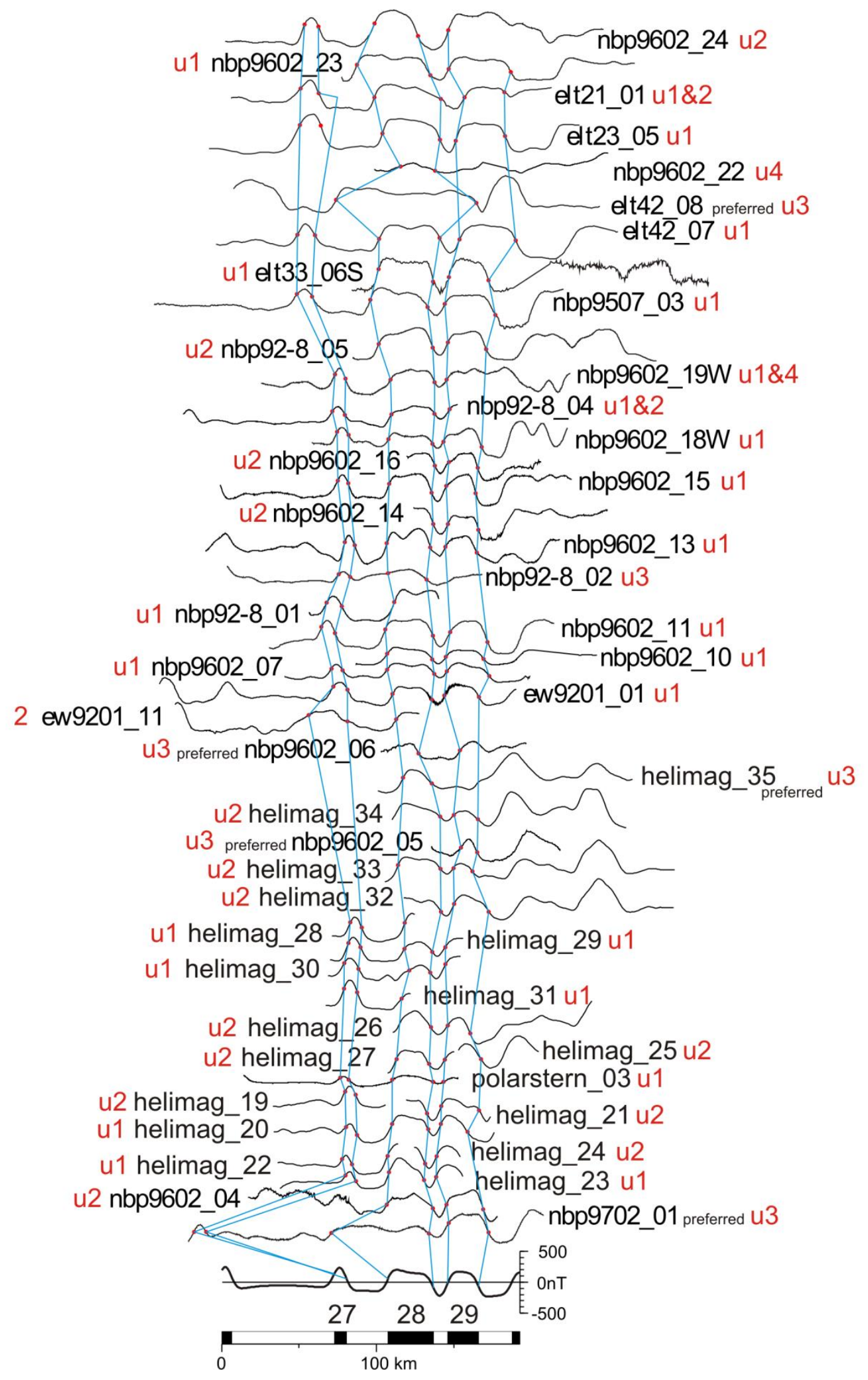


Figure II-14 (previous page): Interpreted magnetic profiles showing anomalies 27 to 29 for the Antarctica sector. Profiles nbp92-8_02, nbp9507_03, elt42_07, elt33_06S, elt42_08, elt23_05, elt21_01, nbp9602_23 and nbp9602_24 were reprojected onto a line parallel to the nearest recognized fracture zone, due to an azimuth difference of more than $15^{\circ}$ from the expected flowline. The synthetic model (bottommost profile) is from Figure II-12. The identification uncertainty of the sequence for each profile is indicated in red (see Appendix A for more detail).

The intersection of profile nbp9602_04 with the Iselin Trough makes its interpretation difficult. As a result, the identification of a27 to a29 on that profile is made by spatial correlation with surrounding profiles (u2). Profile ew9201_11 was interpreted by spatial correlation using its proximity to the reference profile. Surrounding profiles were used to identify the a27-a29 sequence (u2) on thirteen profiles (helimag_24, helimag_19, helimag_21, helimag_27, helimag_25, helimag_26, helimag_32, helimag_33, helimag_34, nbp9602_14, nbp9602_16, nbp92-8_05 and nbp9602_24) because of their short length.

Profile nbp9702_01's sequence of anomalies is interrupted by the presence of the Iselin Trough, leading me to consider several interpretations for it (u3). The northernmost anomaly of profile helimag_35 can be interpreted as either a28 or a29 (u3), due to the relatively broad negative anomaly that separates it from a30. Profiles nbp9602_05 and nbp9602_06 both present two interpretations (u3). I selected one of those as the most likely based on my interpretation of surrounding profiles. I propose two interpretations for profile nbp92-8_02 (u3): the first is based on the shape of the anomalies (a32), the second is based on spreading rates not varying along the profile (a27 and a28). I was unable to select one interpretation over the other. Anomaly 28 is identified on profile elt42_08 as an alternative to the interpretation involving two sequences 32 separated by an extinct ridge. It is my preferred interpretation because it does not require a ridge jump.

Anomaly 28 on profile nbp9602_22 is interpreted based on its shape, but it is assigned an identification uncertainty category 4 because it does not match the interpretation of surrounding profiles. 


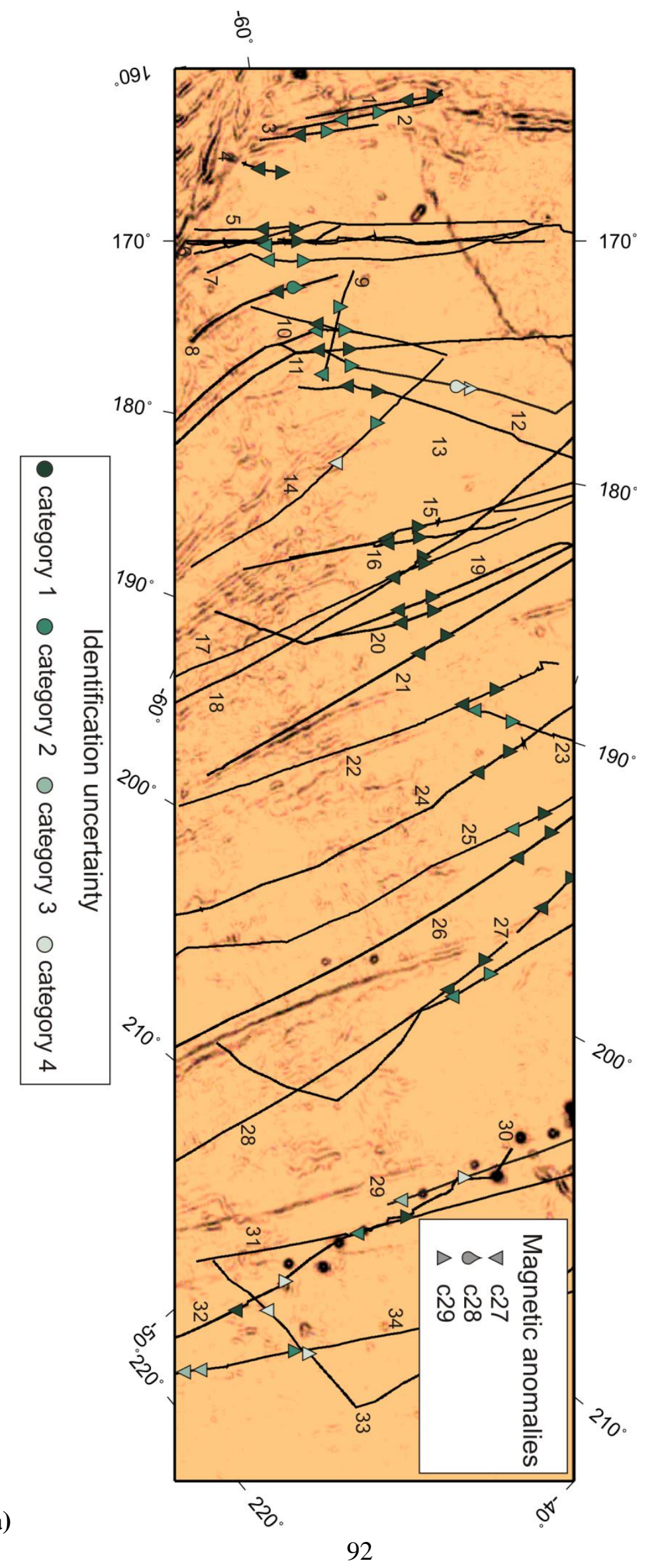




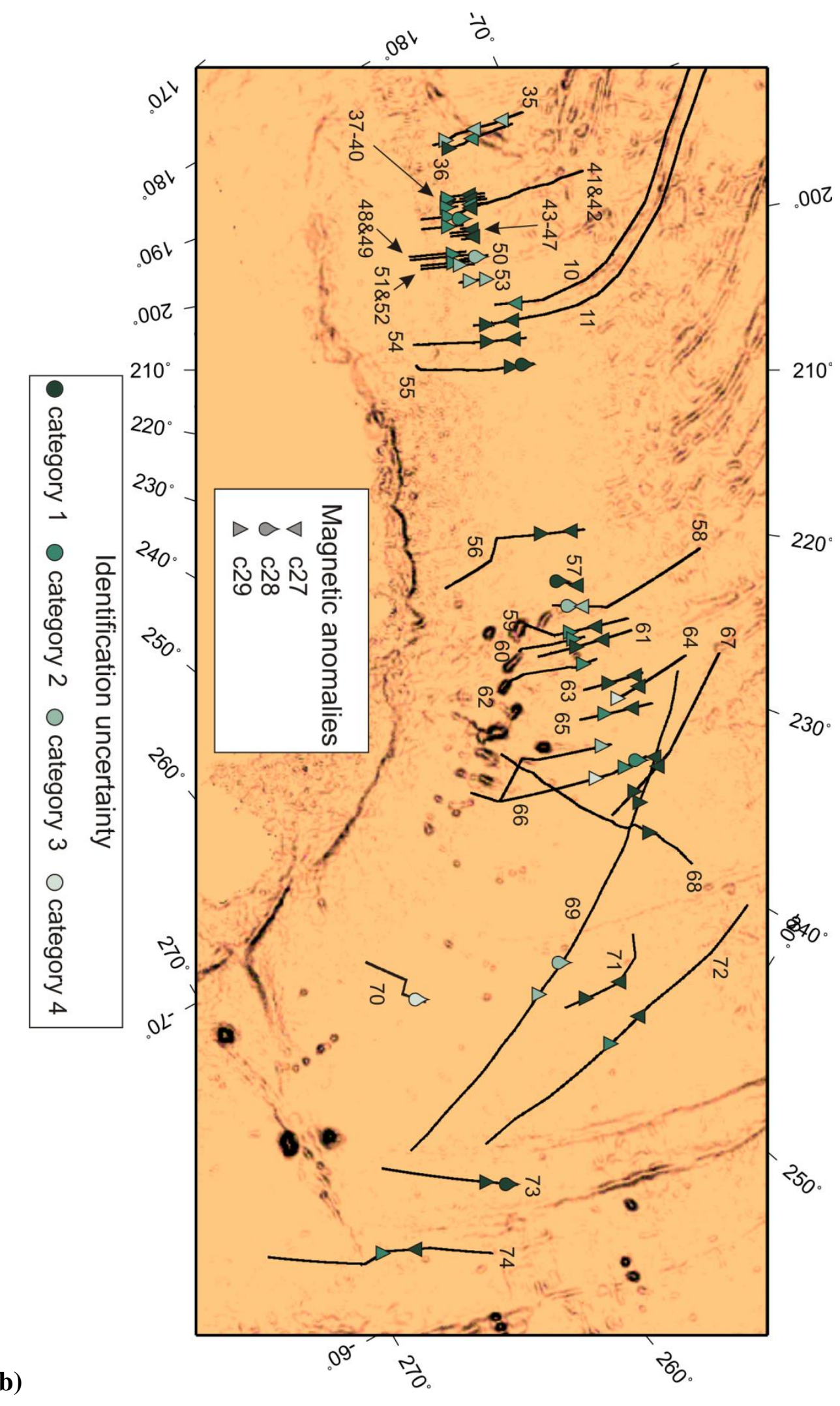




\begin{tabular}{|c|c|}
\hline Profile name & Number \\
\hline nbp9702_10 & 1 \\
\hline nbp0209 02 & 2 \\
\hline nbp9702_09 & 3 \\
\hline ares02wt_01 & 4 \\
\hline elt50_04 & 5 \\
\hline elt50_03 & \multirow{6}{*}{6} \\
\hline elt50_02 & \\
\hline gs7402_01 & \\
\hline gecs-gmv_01 & \\
\hline mons06ar_01 & \\
\hline elt34_01 & \\
\hline elt32 01 & 7 \\
\hline nbp0701_02 & 8 \\
\hline elt42_02 & 9 \\
\hline elt52_04N & \multirow{2}{*}{10} \\
\hline ew9201_11 & \\
\hline ew9201_01 & \multirow{3}{*}{11} \\
\hline dsdp28gc 05 & \\
\hline dsdp28qc 06 & \\
\hline elt32_02N & 12 \\
\hline elt27 01 & 13 \\
\hline elt33_01E & 14 \\
\hline mons06ar_06 & 15 \\
\hline gs7402_03 & \multirow{2}{*}{16} \\
\hline gecs-gmv_02 & \\
\hline um6402-B_01 & 17 \\
\hline elt43_03 & 18 \\
\hline nbp9604_02 & 19 \\
\hline nbp9604_14 & 20 \\
\hline nbp9507_01 & 21 \\
\hline um66-B_02 & 22 \\
\hline kh6804-C_01 & 23 \\
\hline elt25_02 & 24 \\
\hline elt19_01 & 25 \\
\hline west03mv_01 & 26 \\
\hline elt23_08 & \multirow{2}{*}{27} \\
\hline elt23_07 & \\
\hline elt33_08 & 28 \\
\hline elt17 02 & 29 \\
\hline tw8405_01 & 30 \\
\hline v3601 02 & 31 \\
\hline tw8405_02 & 32 \\
\hline $\mathrm{c} 1212 \quad 01$ & 33 \\
\hline elt20 02 & 34 \\
\hline
\end{tabular}

\begin{tabular}{|c|c|}
\hline Profile name & Number \\
\hline nbp9702_01 & 35 \\
\hline nbp9602 04 & 36 \\
\hline helimag_23 & 37 \\
\hline helimag_22 & \multirow{2}{*}{38} \\
\hline helimag_24 & \\
\hline helimag_20 & 39 \\
\hline helimag_19 & \multirow{2}{*}{40} \\
\hline helimag_21 & \\
\hline polarstern3 & 41 \\
\hline helimag_27 & \multirow{2}{*}{42} \\
\hline helimag_25 & \\
\hline helimag_26 & 43 \\
\hline helimag_31 & 44 \\
\hline helimag_30 & 45 \\
\hline helimag_29 & 46 \\
\hline helimag_28 & 47 \\
\hline helimag 32 & 48 \\
\hline helimag_33 & 49 \\
\hline nbp9602_05 & 50 \\
\hline helimag_34 & 51 \\
\hline helimag_35 & 52 \\
\hline nbp9602_06 & 53 \\
\hline nbp9602_07 & 54 \\
\hline nbp9602 10 & 55 \\
\hline nbp9602_11 & 56 \\
\hline nbp92-8_01 & 57 \\
\hline nbp92-8_02 & 58 \\
\hline nbp9602 13 & 59 \\
\hline nbp9602_14 & 60 \\
\hline nbp9602_15 & 61 \\
\hline nbp9602_16 & 62 \\
\hline nbp9602_18W & 63 \\
\hline nbp92-8_04 & 64 \\
\hline nbp9602 19W & 65 \\
\hline nbp92-8_05 & 66 \\
\hline nbp9507_03 & 67 \\
\hline elt33_06S & 68 \\
\hline elt42_07 & \multirow{2}{*}{69} \\
\hline elt42 08 & \\
\hline nbp9602_22 & 70 \\
\hline elt23_05 & 71 \\
\hline elt21_01 & 72 \\
\hline nbp9602_23 & 73 \\
\hline nbp9602 24 & 74 \\
\hline
\end{tabular}

Figure II-15: Interpreted profiles and magnetic reversals identified, with identification uncertainty. Horizontal gravity gradient computed using GMT from [Sandwell and Smith, 1997] (version 18) in the background. a) Map for the Pacific sector. b) Map for the Antarctica sector. c) Profiles and corresponding numbers on the maps. 


\section{Interpretation for anomalies 30 and 31}

This sequence of magnetic anomalies has a similar shape to the sequence a28-a29, though the anomalies are broader (Figure II-16). The sequence is separated from a29 by a short negative anomaly (a29r) of width slightly narrower than that of a29. A broad negative anomaly separates a31 and a32.
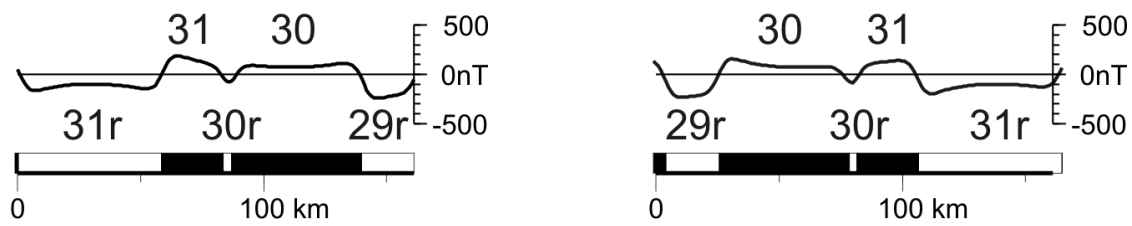

Figure II-16: Typical sequence of magnetic anomalies 30 and 31. The total spreading rate used for this model is $52 \mathrm{~km} / \mathrm{Myr}$ during anomaly 30 and $50 \mathrm{~km} / \mathrm{Myr}$ during anomaly 31, with no asymmetry.

Several profiles display anomalies 30 and 31 that are a good match to the synthetic model (Table II-8, u1). Profile mons06ar_01 is missing data at the expected location of a30, but a31 is clearly recognizable (u1). Profile elt33_01E presents an almost perfect a30-a31 sequence (u1) but for a localised high amplitude magnetic low within anomaly 31. This narrow negative magnetic anomaly is not linked to the presence of a seamount or other bathymetric feature or to a change in azimuth of the profile. Profile mons06ar_06 is also missing data at the expected location of a30r, though the sequence is still clearly recognizable (u1). Anomalies a30y and a31o were digitized on this profile. Profile elt25_02 also presents an almost perfect magnetic sequence except for a31o, where the ship doubled back before resuming its course. Although the match between profiles v3601_01, nbp0007B_02, nbp0501B_02 and nbp9707_02 and the synthetic model presented in Figure II-17 appears rough, the apparent distortion is due to the difference in spreading rates between the two. 


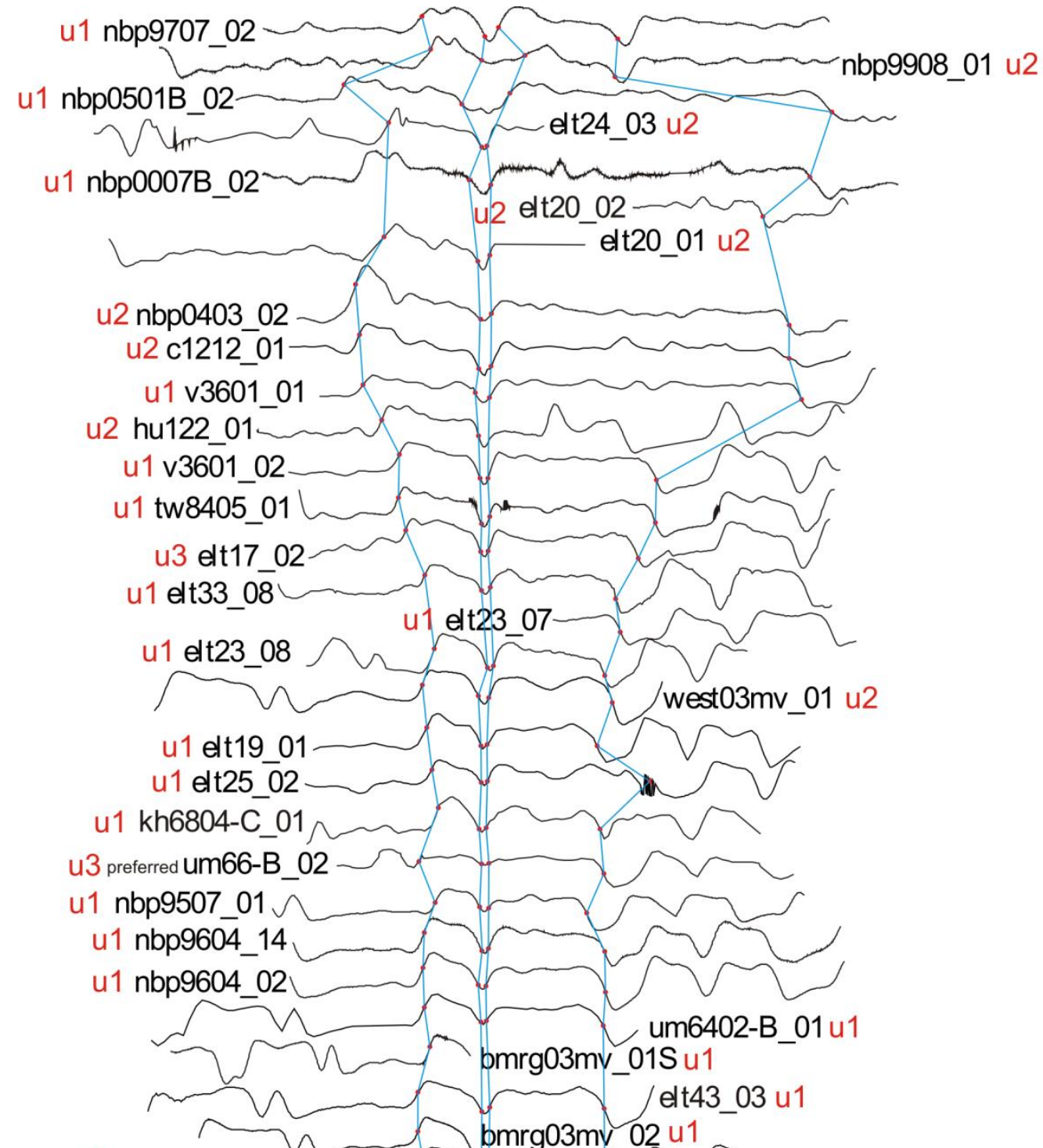

u1 gecs-gmv_02

u1 gs7402_03

u1 mons06ar_06

u1 elt27_01

u2 elt32_02N
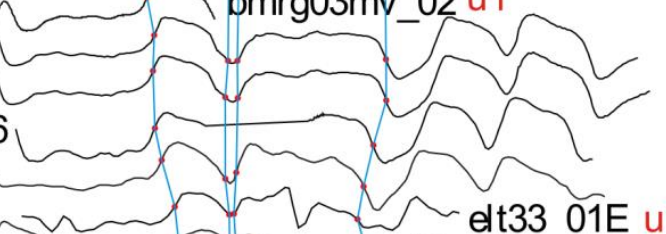

elt33_01E u1

u4 dsdp28gc 05

u1 ew9201_01
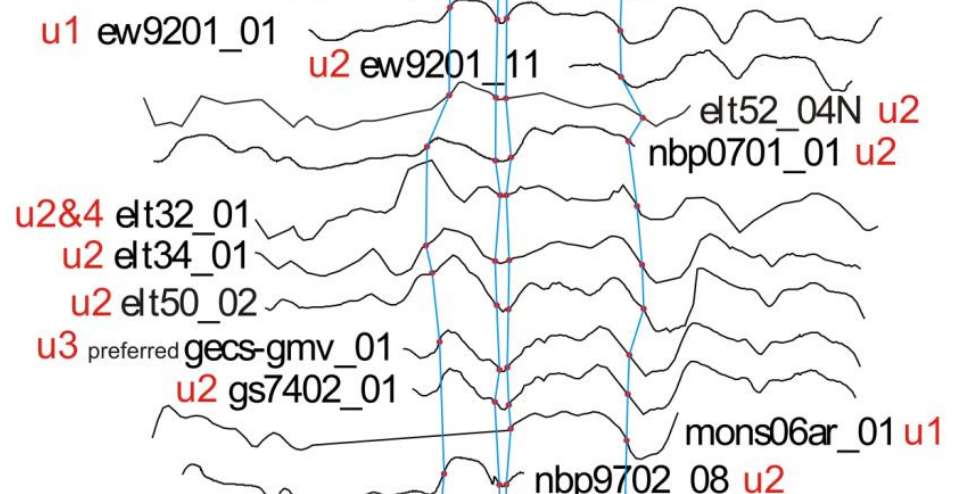

u2 nbp9702 09

u2 nbp0209_02.

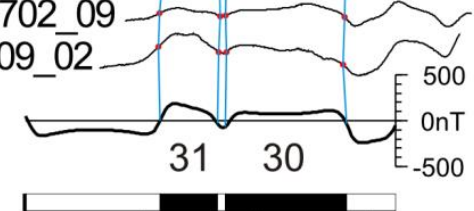


Figure II-17 (previous page): Interpreted magnetic profiles showing anomalies 30 and 31 for the Pacific sector. Profiles dsdp28gc_05, elt27_01, bmrg_03mv_01S, nbp9604_02, nbp9604_14, nbp9507_01, kh6804-C_01, elt23_08, elt33_08, elt17_02, tw8405_01, v3601_02, hu122_01, v3601_01, nbp0403_02, elt20_01, elt20_02, nbp0007B_02, elt24_03, nbp0501B_02, nbp9908_01 and nbp9707_02 were reprojected onto a line parallel to the nearest recognized fracture zone, due to an azimuth difference of more than $15^{\circ}$ from the expected flowline. The synthetic model (bottommost profile) is from Figure II-16. The identification uncertainty of the sequence for each profile is indicated in red (see Appendix A for more detail).

The interpretation of sequence a30-a31 on eighteen profiles (nbp0209_02, nbp9702_09, nbp9702_08, gs7402_01, elt50_02, elt34_01, nbp0701_01, elt52_04N, ew9201_11, elt32_02N, west03mv_01, hu122_01, c1212_01, nbp0403_02, elt20_01, elt20_02, elt24_03 and nbp9908_01) is done by spatial correlation with other sequences on the same profiles $(u 2)$. The interpretation of sequence a30-a31 on profiles nbp0701_01, nbp9908_01 and hu122_01 is also confirmed by that of surrounding profiles (u2). Although I assigned them an identification uncertainty of category 2, a30 and a31 on profiles elt50_02, elt34_01 and west03mv_01 have a shape similar enough to those of the synthetic model that they could almost have been assigned a category $u 1$. Regarding profile elt32_01, the approximate location of sequence a30-a31 can be correlated spatially from the interpretation of the rest of the profile, but pinpointing the exact location of the reversals is difficult due to the mismatch in shape between the data and the synthetic profile. Consequently, although the sequence (and c30y) is assigned a category 2 identification uncertainty, c31y and c30o are assigned a category 4 identification uncertainty. 

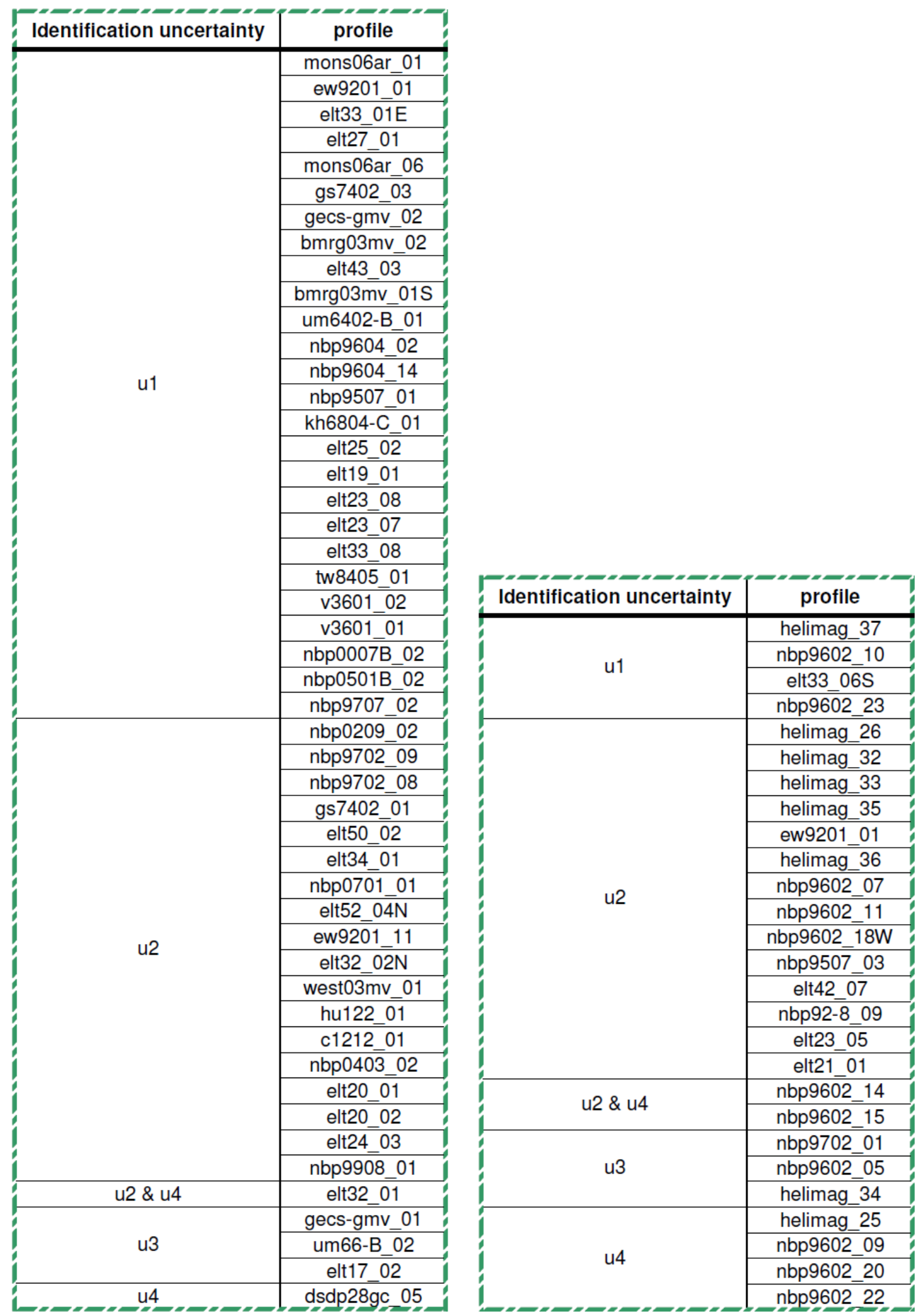

Table II-8: Profiles presented in Figure II-17 and Figure II-18, sorted by identification uncertainty for the sequence of magnetic anomalies 30-31. 
The identification of a31 on profile gecs-gmv_01 is uncertain (u3) due to the lack of data east of it that would confirm its identity. What I identify here as anomaly 31 could also be anomaly 32.1 (see Figure II-21). On profile um66-B_02, the sequence a30-a31 is identified with confidence (c30y is assigned a category 1 identification uncertainty), but the exact location of anomaly 31 presents two alternatives (see Figure II-17). Consequently c30o, c31y and c31o are assigned a category 3 identification uncertainty. On profile elt17_02 because the magnetic anomalies are close to one end of the profile, the sequence identified as a29-a31 could also be identified as a27-a29 (u3, see Figure II-13). Surrounding profiles suggest the a29-a31 interpretation is more likely.

c30y is tentatively identified on profile dsdp28gc_05 based on the rest of the profile. The location of the reversal near the end of the profile makes the identification a category $u 4$.

On the Antarctica side, on four profiles (helimag_37, nbp9602_10, elt33_06S and nbp9602_23) the sequence a30-a31 is almost identical to that of the synthetic profile (Figure II-18). Consequently these reversals are assigned a category u1 identification uncertainty.

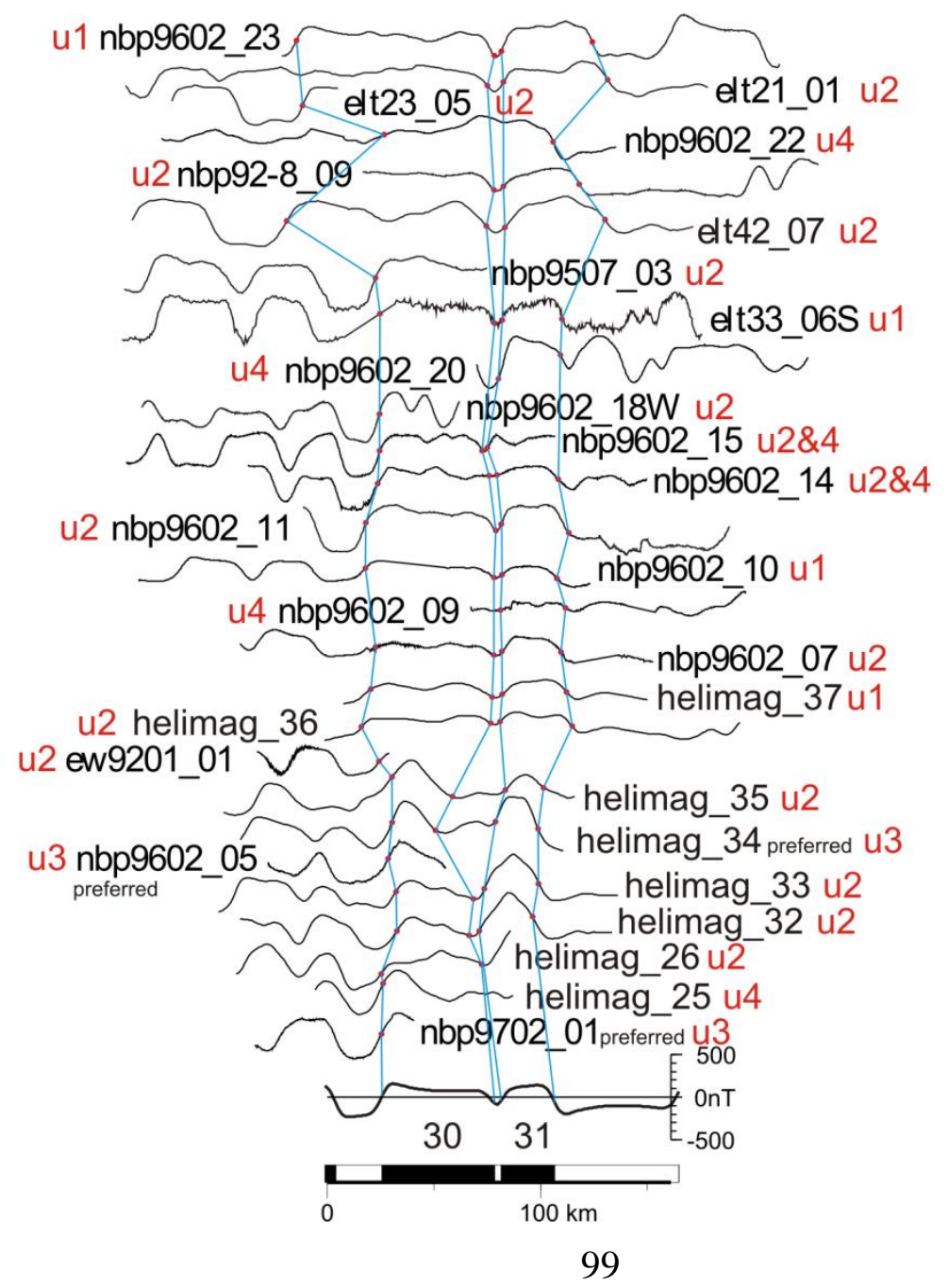


Figure II-18 (previous page): Interpreted magnetic profiles showing anomalies 30 and 31 for the Antarctica sector. Profiles nbp9602_11, elt33_06S, nbp9507_03, elt42_07, nbp9602_22, elt21_01 and nbp9602_23 were reprojected onto a line parallel to the nearest recognized fracture zone, due to an azimuth difference of more than $15^{\circ}$ from the expected flowline. The synthetic model was (bottommost profile) is from Figure II-16. The identification uncertainty of the sequence for each profile is indicated in red (see Appendix A for more detail).

The identification of the sequence on fourteen profiles (helimag_26, helimag_32, helimag_33, helimag_35, ew9201_01, helimag_36, nbp9602_07, nbp9602_11, nbp9602_18W, nbp9507_03, elt42_07, nbp92-8_09, elt23_05 and elt21_01) is done by spatial correlation with the rest of the profiles. The identification of c30y on profiles nbp9602_14 and nbp9602_15 is also done by spatial correlation. The identification of the rest of the a30-a31 sequence is tentative $(\mathrm{u} 4)$, based on the assumption of little variation of spreading rates.

Spatial correlation with other anomalies on profile helimag_34 made me identify c30o at two different locations (u3). Please refer to Chapter II-12 regarding the interpretation of profiles nbp9702_01 and nbp9602_05.

I tentatively identified c30y on profile helimag_25 because of its proximity to c29o. A bathymetric trough and the short length of the profile account for c30y being a category 4 identification uncertainty. The identification of the sequence on profile nbp9602_22 is done by spatial correlation with surrounding profiles (see Chapter II-12 for more detail). 


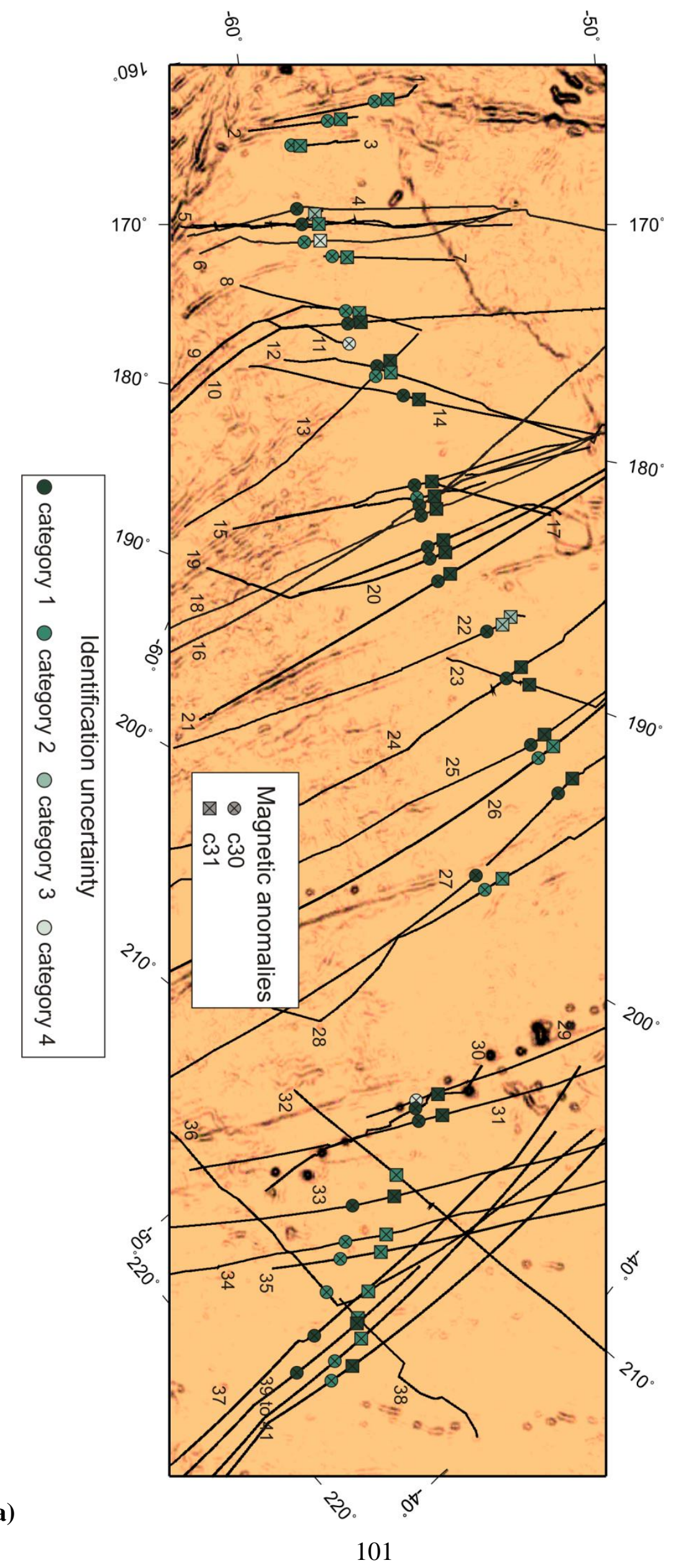




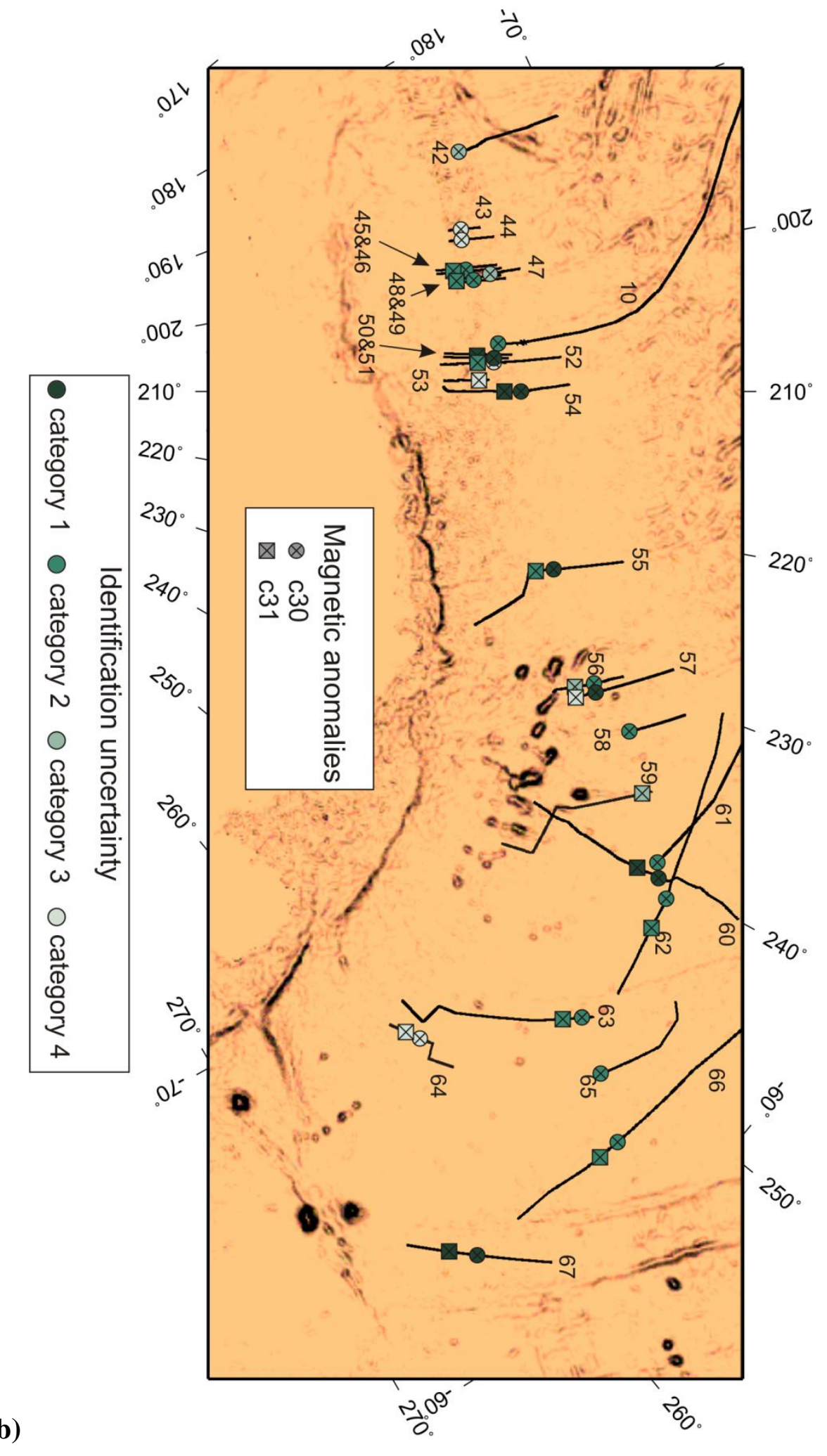




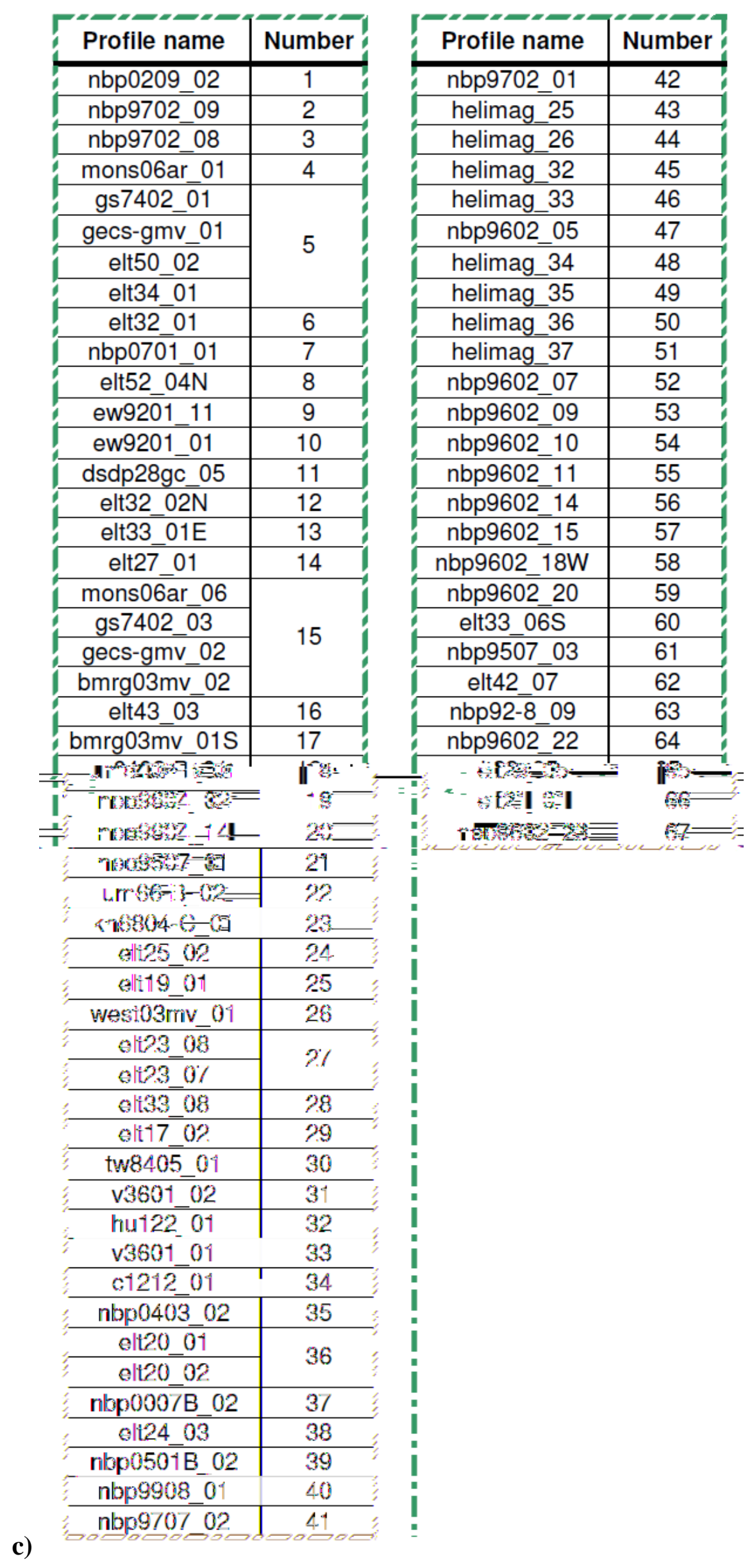


Figure II-19: Interpreted profiles and magnetic reversals identified, with identification uncertainty. Horizontal gravity gradient computed using GMT from [Sandwell and Smith, 1997] (version 18) in the background. a) Map for the Pacific sector. b) Map for the Antarctica sector. c) Profiles and corresponding numbers on the maps.

\section{Interpretation for anomalies 32 to $33 y$}

The sequence of anomalies $32.1 \mathrm{y}$ to $33 \mathrm{y}$ is the most easily identifiable succession of reversals of the period studied (Figure II-20). It is expressed by a narrow positive peak (32.1), closely followed by a much broader magnetic anomaly (32.2). The negative anomaly between 32.2 and $33 y$ is slightly narrower than the length of 32.2. It also features occasionally a narrow length local maximum, identified as c32r.1n in the literature [Cande and Kent, 1995] and as a32r hereafter.
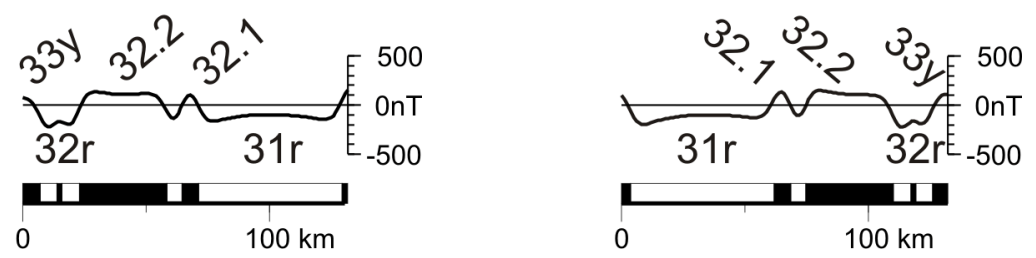

Figure II-20: Typical sequence of magnetic anomalies 32.1 to $33 \mathrm{y}$. The total spreading rate used for this model is $50 \mathrm{~km} / \mathrm{My}$ up to anomaly $32 \mathrm{r}$ and $55 \mathrm{~km} / \mathrm{My}$ during anomaly $33 \mathrm{y}$, with no asymmetry.

The quasi-similarity between the synthetic model and the data for a32 on twenty-eight profiles (nbp9702_08, cp7808_06, cp7808_05, elt42_01, mons06ar_01, ar6102_01, elt34_01, elt32_01, elt52_04N, ew9201_01, elt33_01E, mons06ar_06, bmrg03mv_02, gs7402_03, gecsgmv_03, gecs-gmv_02, um6402-B_01, bmrg03mv_01S, nbp9604_02, nbp9604_14, nbp9507_01, elt25_02, kh6804-C_01, elt19_01, west03mv_01, elt33_08, v3602_01 and nbp0501B_01) makes the identification of the reversals a category 1 identification uncertainty (Figure II-21, Figure II-22 and Table II-9). On profiles elt52_04N and elt32_01 the large distance between data points (about $5.4 \mathrm{~km}$ ) makes the shape of the anomalies angular, though I have no doubt as to the identification of the anomalies (u1). Profiles cp7808_05 and mons06ar_01 do not show an a32r positive anomaly. The short wavelength wiggles on profile gs7402_03 are an artefact due to the ship's change of course being projected. On profile nbp0501B_01 the identification of a32r and a32.1 is unequivocal (u1). The presence of a ridge jump within a32.2 would explain its length. Similarly, the interpretation of profile nbp9908_01 is based on a32r and a32.2 could be affected by two ridge jumps. On profiles elt50_02, elt43_03 and nbp9707_02 a32.1 was identified first (u1) and the rest of the sequence was 
deduced from its location (u2). On profile nbp0403_02 the sequence was identified through $\mathrm{a} 32 \mathrm{r}$ but there are several possibilities as to the location of a32.1.

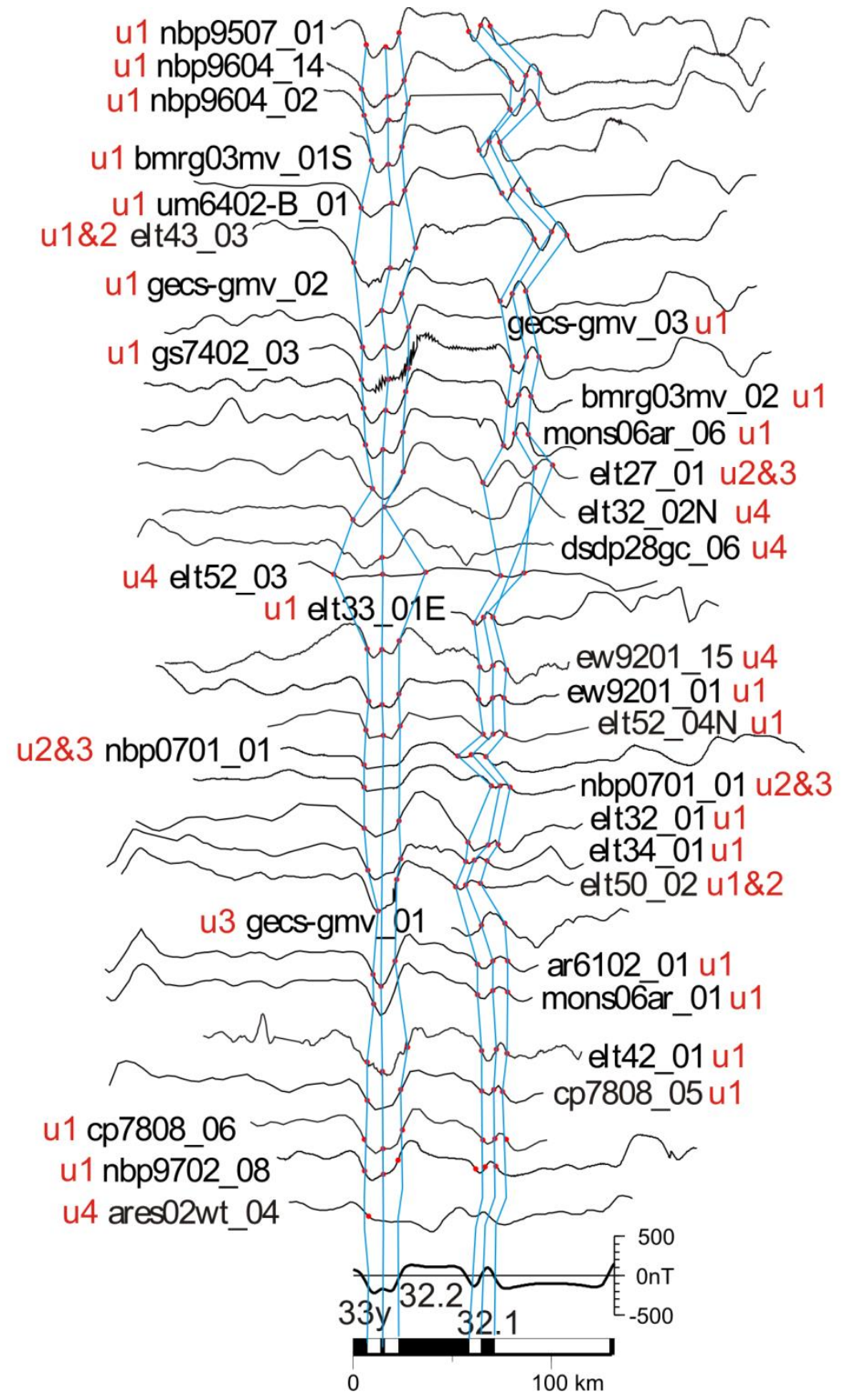

Figure II-21: Interpreted magnetic profiles showing anomalies 32.1 to $33 y$ for the Southern Pacific sector. Profiles elt16_03, elt42_01, elt52_03, bmrg03mv_01S, nbp9604_02 and nbp9602_14 were reprojected onto a line parallel to the nearest recognized fracture zone, due to an azimuth difference of more than $15^{\circ}$ from the expected flowline. The synthetic model (bottommost profile) is from Figure II-20. The identification uncertainty of the sequence for each profile is indicated in red (see Appendix A for more detail). 


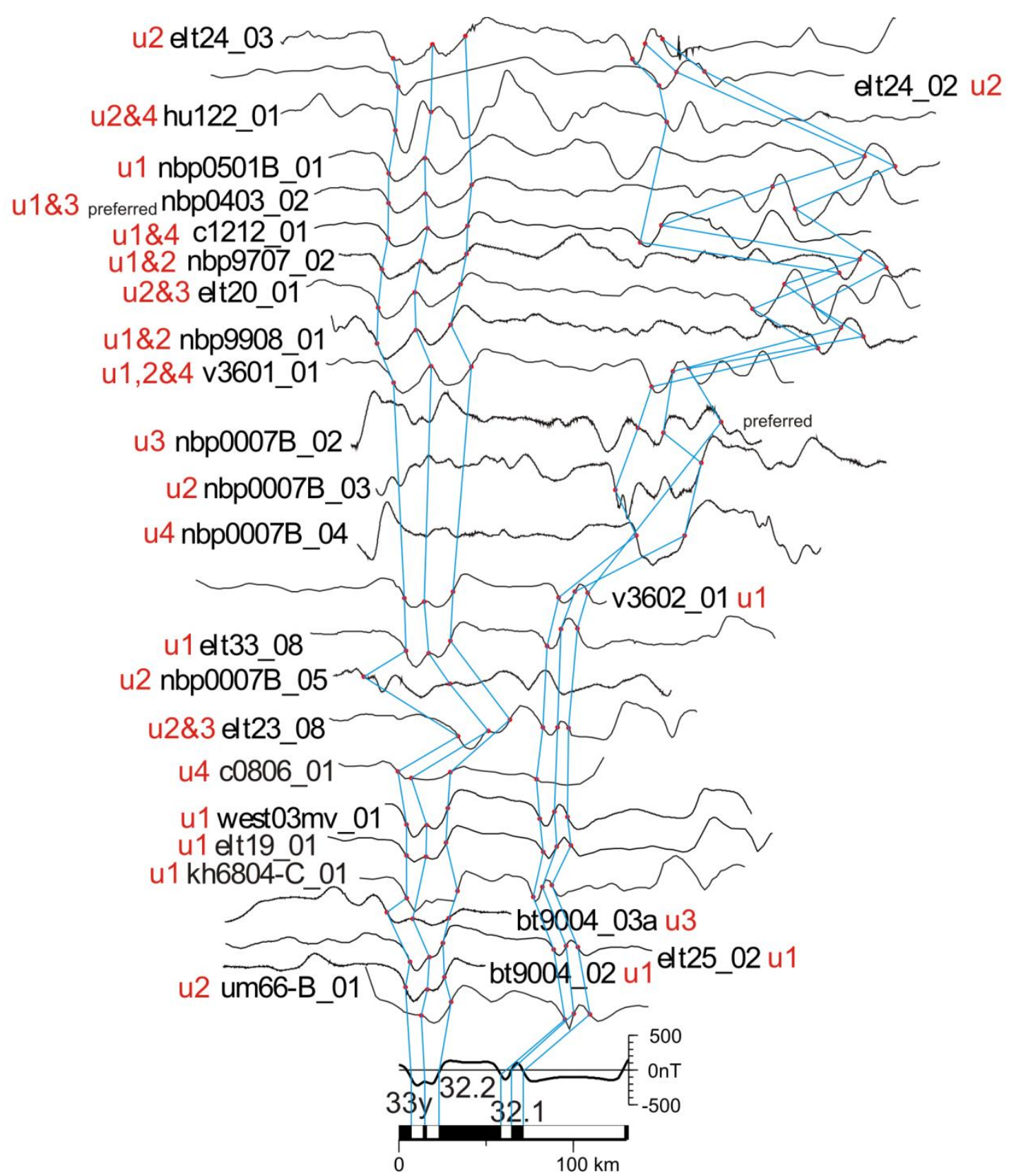

Figure II-22: Interpreted magnetic profiles showing anomalies 32.1 to $33 y$ for the Northern Pacific sector. Profiles um66-B_01, kh6804-C_01, c0806_01, elt23_08, kh6804-B_02, elt33_08, v3602_01, v3601_01, nbp0007B_05, nbp0007B_04, nbp0007B_03, nbp0007B_02, nbp9908_01, elt20_01, nbp9707_02, nbp0403_02, nbp0501B_01, hu122_01, elt24_02 and elt24_03 were reprojected onto a line parallel to the nearest recognized fracture zone, due to an azimuth difference of more than $15^{\circ}$ from the expected flowline. The synthetic model (bottommost profile) is from Figure II-20. The identification uncertainty of the sequence for each profile is indicated in red (see Appendix A for more detail). 


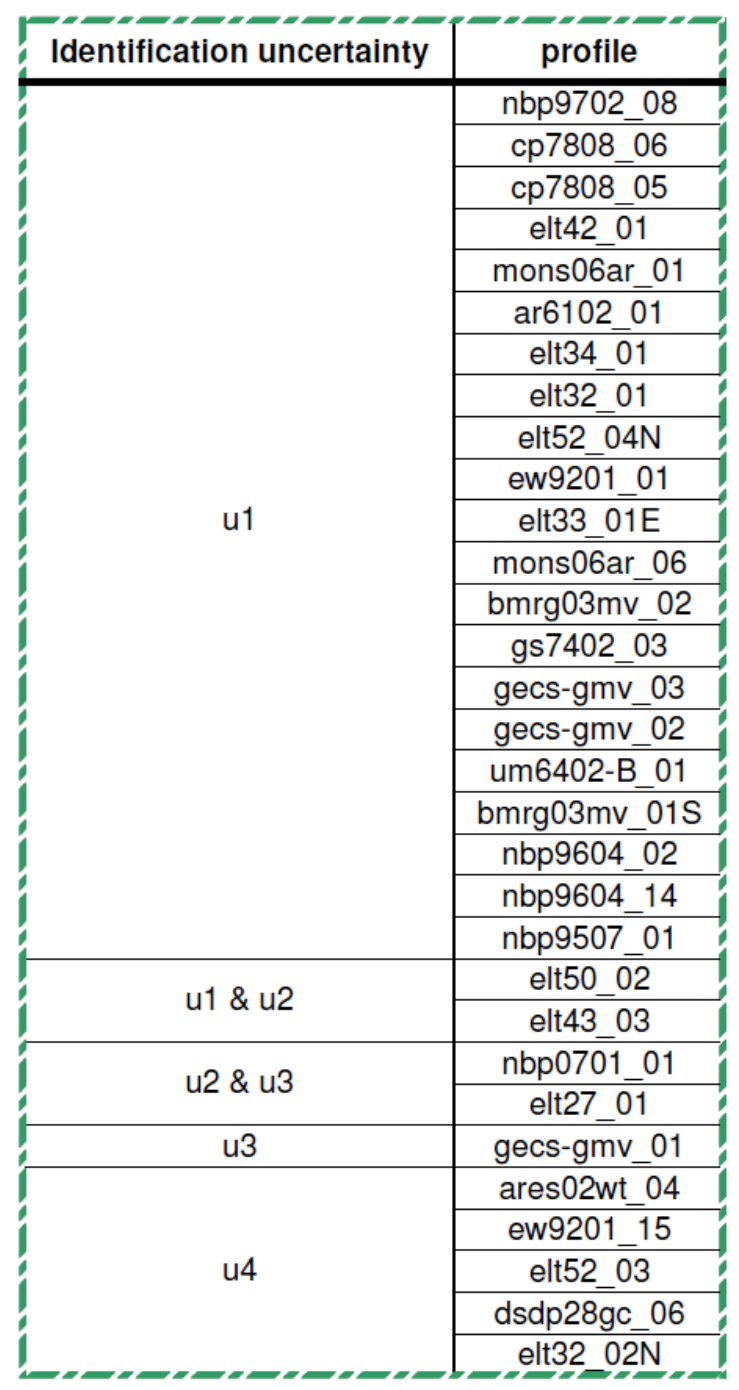

\begin{tabular}{|c|c|}
\hline Identification uncertainty & profile \\
\hline \multirow{8}{*}{ u1 } & bt9004_02 \\
\hline & elt25_02 \\
\hline & kh6804-C_01 \\
\hline & elt19_01 \\
\hline & west $03 \mathrm{mv} 01$ \\
\hline & elt33 08 \\
\hline & v3602_01 \\
\hline & nbp0501B_01 \\
\hline \multirow{2}{*}{ u1 \& u2 } & nbp9908_01 \\
\hline & nbp9707_02 \\
\hline u1, u2 \& u4 & v3601_01 \\
\hline u1 \& u3 & nbp0403_02 \\
\hline $\mathrm{u} 1 \mathrm{u} u 4$ & $c 1212 \_01$ \\
\hline \multirow{5}{*}{ u2 } & um66-B_01 \\
\hline & nbp0007B_05 \\
\hline & nbp0007B_03 \\
\hline & elt24_02 \\
\hline & elt24_03 \\
\hline \multirow{2}{*}{ u2 \& u3 } & elt23_08 \\
\hline & elt20_01 \\
\hline u2 \& u4 & hu122_01 \\
\hline \multirow{2}{*}{ u3 } & bt9004_03a \\
\hline & nbp0007B_02 \\
\hline $\mathrm{u} 4$ & $\mathrm{c} 0806 \quad 01$ \\
\hline
\end{tabular}

Table II-9: Profiles presented in Figure II-21 and Figure II-22, sorted by identification uncertainty for the sequence of magnetic reversals c32.1y-33y (Pacific side).

Six profiles (um66-B_01, nbp0007B_05, nbp0007B_03, hu122_01, elt24_02 and elt24_03) were interpreted by spatial correlation with surrounding profiles (u2). Part of a32.2 is missing on profile elt 24 _02 due to a gap in the data.

The a32 sequence was identified on profile nbp0701_01 by spatial correlation (u2). Two locations for a32.1 are possible (see Figure II-21, u3). The identification of the sequence on profiles elt27_01 and elt23_08 is based on the identification of other anomalies (u2). Anomaly 32.1 has two possible locations on these profiles (u3). The identification of the sequence as a whole on profile elt20_01 is based on the identification of younger anomalies on the profile, but it presents three possibilities for the precise location of anomaly 32.1 (u3), the most likely of which is shown on Figure II-22. The identification of sequence 32 on profile hu122_01 is confirmed by spatial correlation (c33o and a31). 
The anomaly identified as a32.1 on profile gecs-gmv_01 could also be a31 (see II-13). I interpreted profile bt9004_03 in two different ways, one of which is presented here, the other in III-15 (see Appendix A). On profile nbp0007B_02 the approximate location of the sequence is based on surrounding profiles but its exact position is uncertain. Two alternative interpretations are presented in Figure II-22 (u3).

The identification of sequence 32 on profiles ares02wt_04, dsdp28gc_06 and elt32_02N is tentative (u4) and the result of spatial correlation with surrounding profiles. The intersection of profile ares02wt_04 with a fracture zone complicates its interpretation, but correlation with surrounding profiles predicts the approximate location of c33y. Only the older part of the sequence (c32.2o, c32r and c33y) is recognizable on profile dsdp28gc_06. The identification of the sequence on profile elt32_02N is based on spatial correlation with younger anomalies and surrounding profiles. The identification of sequence 32 of profile ew9201_15 is based on the identification of c33o on the same profile (see II-15). The identification of sequence 32 on profile elt52_03 is based on that of c33o on that profile (see II-15). Anomaly 32r on profile c0806_01 may not be due to seafloor spreading remanent magnetisation but could be a spurious anomaly linked to the large distance between each data point (about $4 \mathrm{~km}$ ).

On the Antarctica side, nine profiles (helimag_36, helimag_37, nbp9602_07, nbp9602_11, nbp9602_12, nbp9602_19E, nbp9602_20, nbp92-8_09 and elt42_08) display remarkable similarity with the synthetic model (Figure II-23 and Table II-1, u1). Sadly, several profiles were too short for me to locate more than a few reversals: helimag_36, helimag_37, and nbp9602_12.

The identification of sequence 32 on nine profiles (nbp9602_08, nbp9602_09, nbp9602_10, polarstern2, helimag_46, polarstern1, helimag_47, nbp9602_18E and elt23_03) derives from spatial correlation with of surrounding profiles. A lacuna in the data along profile nbp9602_10 makes the identification of a32.1 impossible on this profile. The identification of the sequence on profile elt33_06S is based on that of younger anomalies. The presence of a seamount at the expected location of $\mathrm{c} 32.2 \mathrm{y}$ affects the interpretation. 


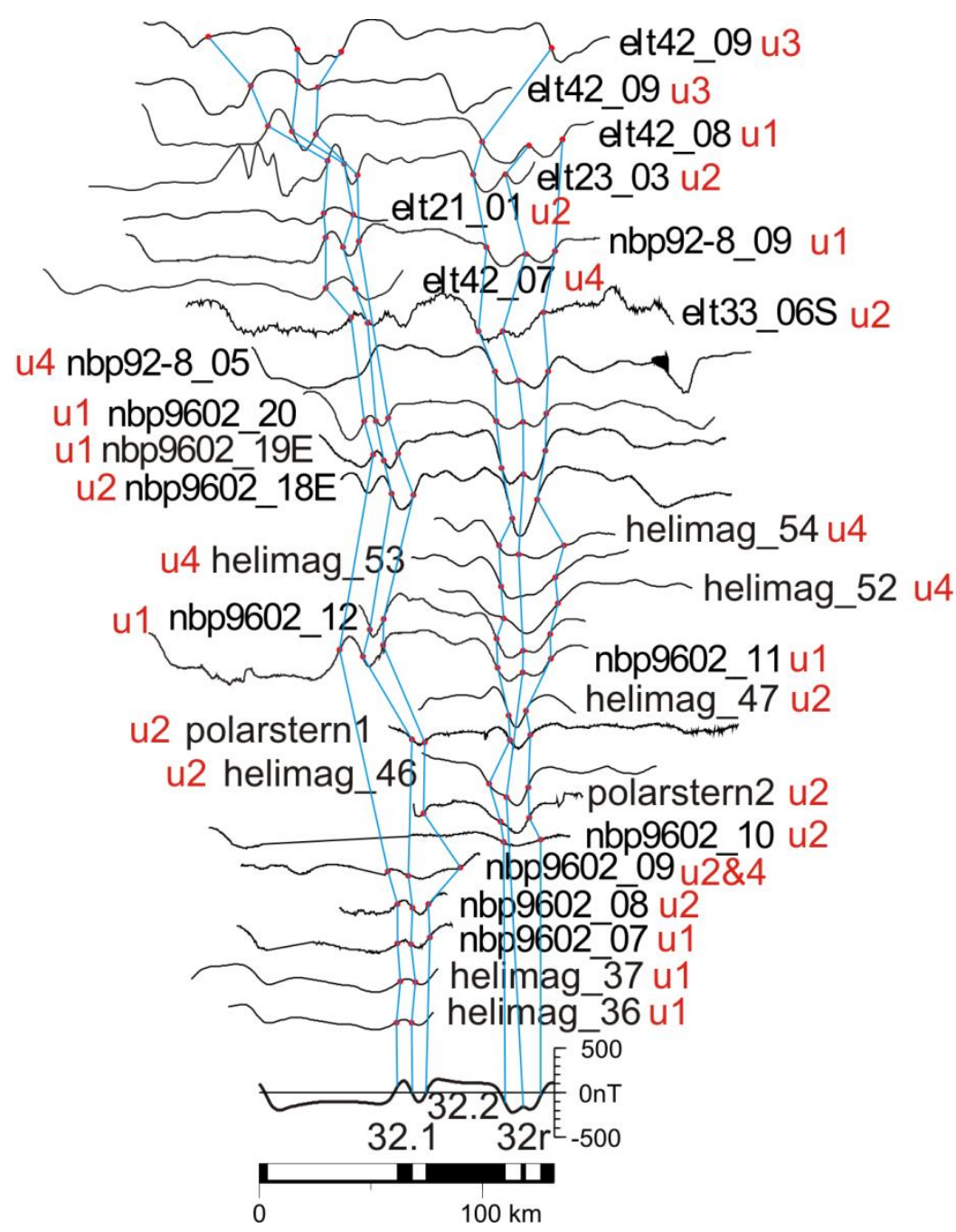

Figure II-23: Interpreted magnetic profiles showing anomalies 32.1 to $33 y$ for the Antarctica sector. Profiles nbp9602_11, nbp9602_12, nbp9602_20, elt33_06S, elt42_07, elt23_03 and elt21_01 were reprojected onto a line parallel to the nearest recognized fracture zone, due to an azimuth difference of more than $15^{\circ}$ from the expected flowline. The synthetic model (bottommost profile) is from Figure II-20. The identification uncertainty of the sequence for each profile is indicated in red (see Appendix A for more detail). 


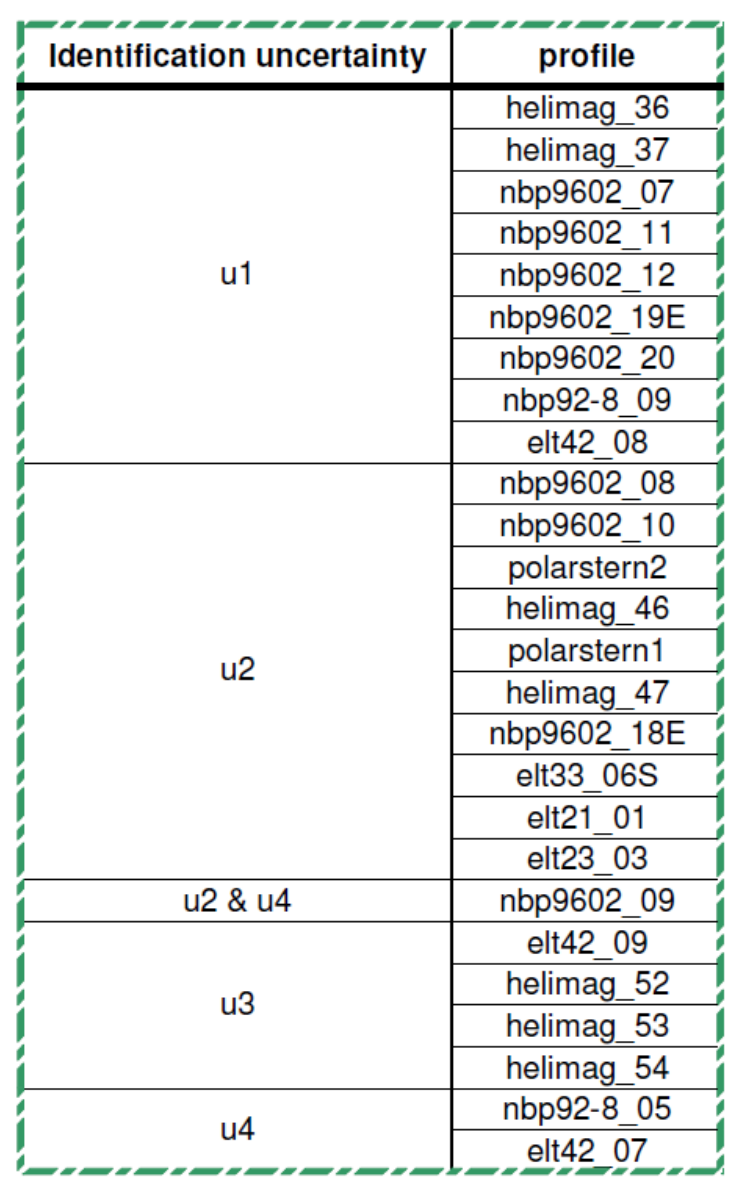

Table II-10: Profiles presented in Figure II-23, sorted by identification uncertainty for the sequence of magnetic reversals c32.1y-33y (Antarctica side).

Although I identify a32 on profile nbp92-8_02 based on its shape, the alternative interpretation (a27 and a28) is more likely: it does not require any major decrease in spreading rate and/or a ridge jump, and it matches the interpretation of surrounding profiles better. The identification of sequence 32 on profile elt42_09 is done by spatial correlation with surrounding profiles. The exact locations of the magnetic reversals are unsure (Figure II-23, u3).

The identification of the 32 sequence on profile nbp92-8_05 (u4) is based on the interpretation of the rest of the profile and on the similarities in shape between the data and the synthetic model. Anomaly 32.1 is identified in profile elt42_07 as a category 4 identification uncertainty because of its proximity to the Udintsev FZ. The tentative identification (u4) of c32.1y, c32.1o and c32.2y on profile elt21_01 is based on younger anomalies. 


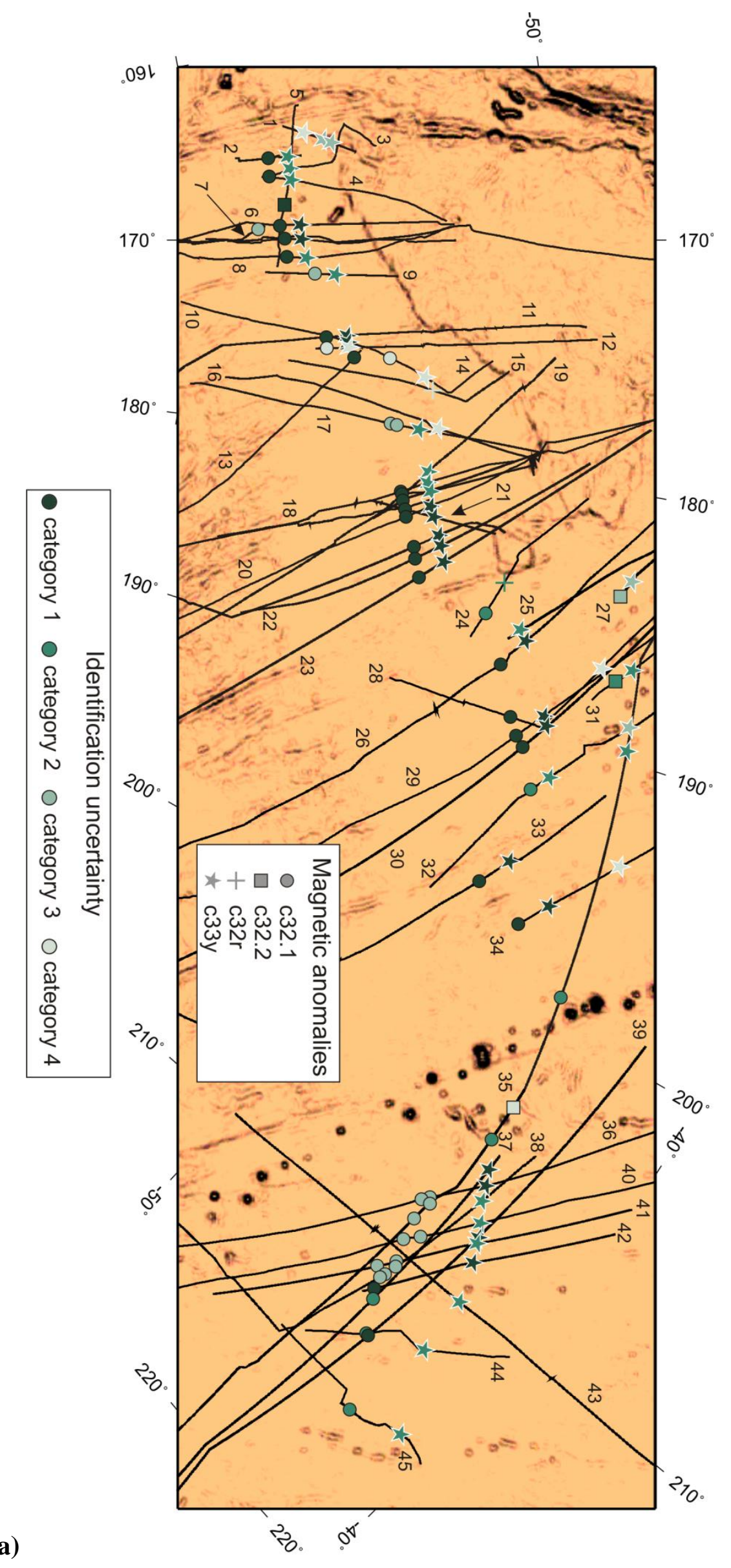




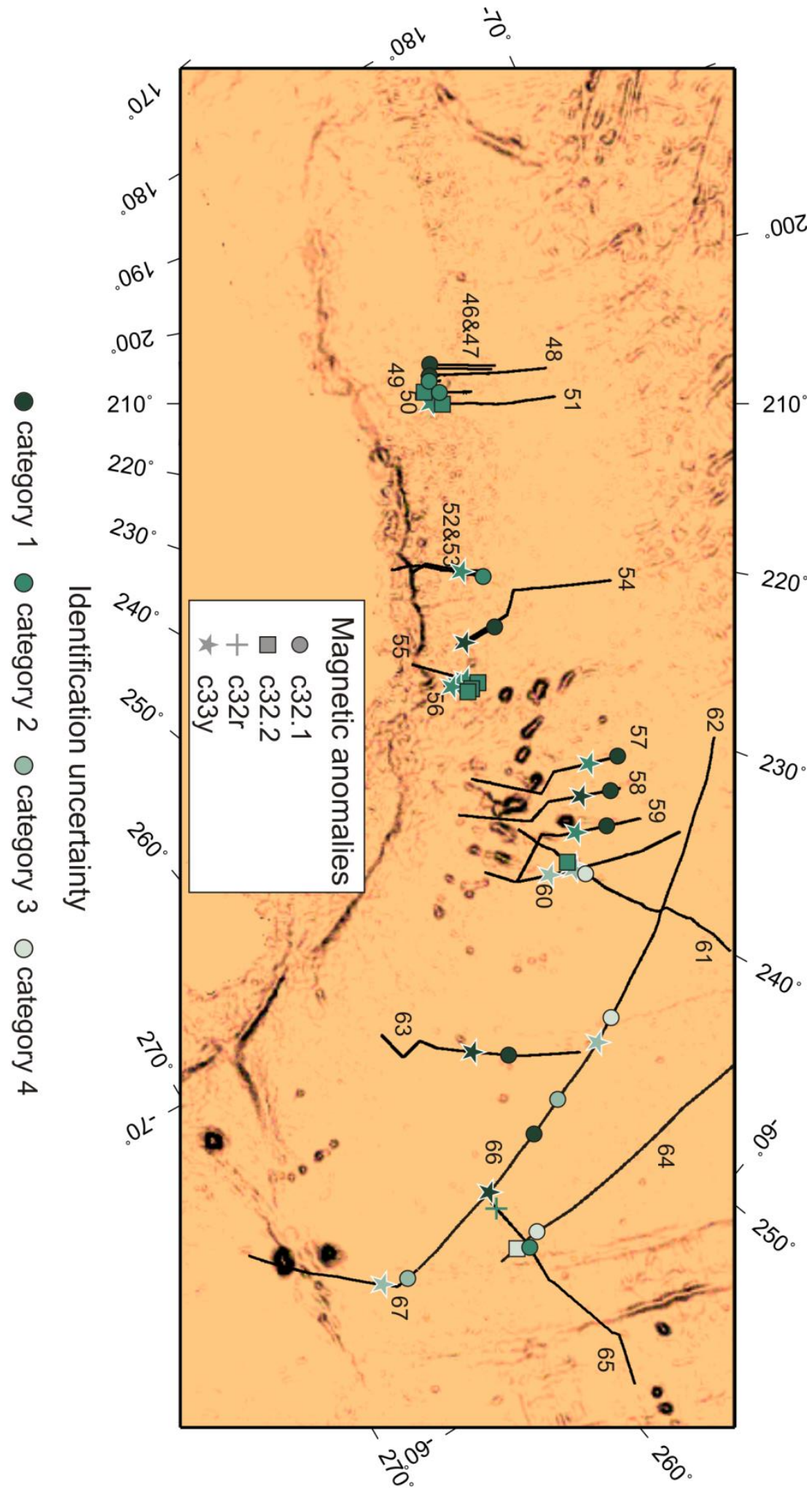




\begin{tabular}{|c|c|c|c|}
\hline Profile name & Number & Profile name & Number \\
\hline ares02wt_04 & 1 & um66-B_01 & 24 \\
\hline nbp9702_08 & 2 & bt9004_02 & 25 \\
\hline cp7808_06 & 3 & elt25_02 & 26 \\
\hline cp7808 05 & 4 & bt9004 03a & 27 \\
\hline elt42_01 & 5 & kh6804-C_01 & 28 \\
\hline mons06ar_01 & \multirow{2}{*}{6} & elt19_01 & 29 \\
\hline ar6102_01 & & west03mv_01 & 30 \\
\hline gecs-gmv_01 & \multirow{3}{*}{7} & c0806 01 & 31 \\
\hline elt50_02 & & elt23_08 & 32 \\
\hline elt34_01 & & elt33_08 & 33 \\
\hline elt32_01 & 8 & v3602_01 & 34 \\
\hline nbp0701 01 & 9 & nbp0007B 05 & \multirow{4}{*}{35} \\
\hline elt52_04N & 10 & nbp0007B_04 & \\
\hline ew9201_01 & 11 & nbp0007B_03 & \\
\hline ew9201_15 & 12 & nbp0007B_02 & \\
\hline elt33_01E & 13 & v3601_01 & 36 \\
\hline elt52_03 & 14 & nbp9908_01 & 37 \\
\hline dsdp28gc 06 & 15 & elt20 01 & 38 \\
\hline elt32 02N & 16 & nbp9707 02 & 39 \\
\hline elt27_01 & 17 & c1212_01 & 40 \\
\hline mons06ar_06 & \multirow{5}{*}{18} & nbp0403_02 & 41 \\
\hline bmrg03mv_02 & & nbp0501B_01 & 42 \\
\hline gs7402_03 & & hu122_01 & 43 \\
\hline gecs-gmv_03 & & elt24_02 & 44 \\
\hline gecs-gmv_02 & & elt24 03 & 45 \\
\hline elt43_03 & 19 & & \\
\hline um6402-B_01 & 20 & & \\
\hline bmrg03mv_01S & 21 & & \\
\hline nbp9604_02 & \multirow{2}{*}{22} & & \\
\hline nbp9604_14 & & & \\
\hline nbp9507 01 & 23 & & \\
\hline
\end{tabular}

\begin{tabular}{|c|c|}
\hline Profile name & Number \\
\hline helimag_36 & 46 \\
\hline nelimag_37 & 47 \\
\hline nbp9602_07 & 48 \\
\hline nbp9602_08 & 49 \\
\hline nbp9602_09 & 50 \\
\hline polarstern2 & \\
\hline helimag_46 & 510 \\
\hline polarstern1 & $52 \& 53$ \\
\hline helimag_47 & \\
\hline nbp9602_11 & 54 \\
\hline nbp9602_12 & 54 \\
\hline helimag_52 & 55 \\
\hline helimag_53 & 56 \\
\hline helimag_54 & 57 \\
\hline nbp9602_18E & 57 \\
\hline nbp9602_19E & 58 \\
\hline nbp9602_20 & 59 \\
\hline nbp92-8_05 & 60 \\
\hline elt33_06S & 61 \\
\hline elt42_07 & 62 \\
\hline nbp92-8_09 & 63 \\
\hline elt21_01 & 64 \\
\hline elt23_03 & 65 \\
\hline elt42_08 & 66 \\
\hline elt42_09__- & 67 \\
\hline
\end{tabular}

Figure II-24: Interpreted profiles and magnetic reversals identified, with identification uncertainty. Horizontal gravity gradient computed using GMT from [Sandwell and Smith, 1997] (version 18) in the background. a) Map for the Pacific sector. b) Map for the Antarctica sector. c) Profiles and corresponding numbers on the maps.

\section{Interpretation for anomalies 330 and $34 y$}

The edge of a33 farthest from the spreading ridge is the oldest chron identified in both sectors with confidence. Anomaly 33 is a very broad positive anomaly. The edges of anomaly 33 are not always well defined, hence the precise location of c33o can be difficult to determine. The negative interval between anomalies 33 and 34 is also relatively broad (Figure II-25). 


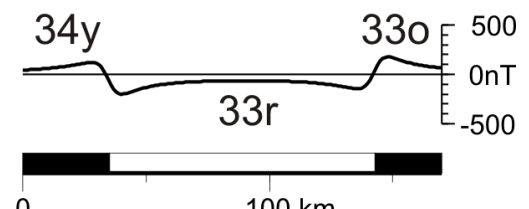

$100 \mathrm{~km}$

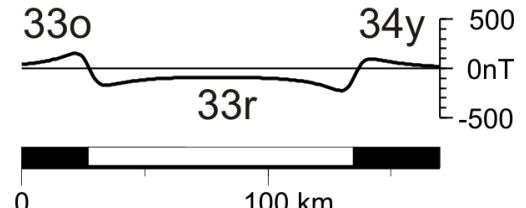

0

$100 \mathrm{~km}$

Figure II-25: Typical sequence of magnetic anomalies 33o to 34y. The total spreading rate used for this model is $55 \mathrm{~km} / \mathrm{Myr}$ with no asymmetry.

Because the number of identifiable magnetic reversals diminishes away from the ridge, a33o and a34y are harder to identify with confidence than younger anomalies. Hence only profiles elt50_02, bt9004_02, west03mv_01 and elt33_08 present a category u1 magnetic reversal (c33o). The location of c34y on elt50_02 and west03mv_01 is deduced from that of a33o and a34y is assigned a category 4 identification uncertainty.

For most profiles on the Pacific side of the ridge (Figure II-26, Figure II-27 and Table II-11), a33o and a34y were identified based on younger anomalies and/or surrounding profiles. On thirteen profiles (cp7808_06, cp7808_05, mons06ar_01, nbp0701_01, ew9201_01, elt43_03, bmrg03mv_02, kh6804-C_01, elt19_01, nbp0403_02, nbp0501B_01, hu122_01 and v3601_01) a33o was identified based on the identification of younger anomalies. Although I assigned a category 2 identification uncertainty to the a33o reversal identified on profile ew9201_01 because of its proximity to the margin (possible contamination of the signal), I have no doubt as to its identity. The anomaly identified as a34y on profile elt19_01 may not be due solely to seafloor spreading remanent magnetisation, but rather to its proximity to a seamount (u4). 


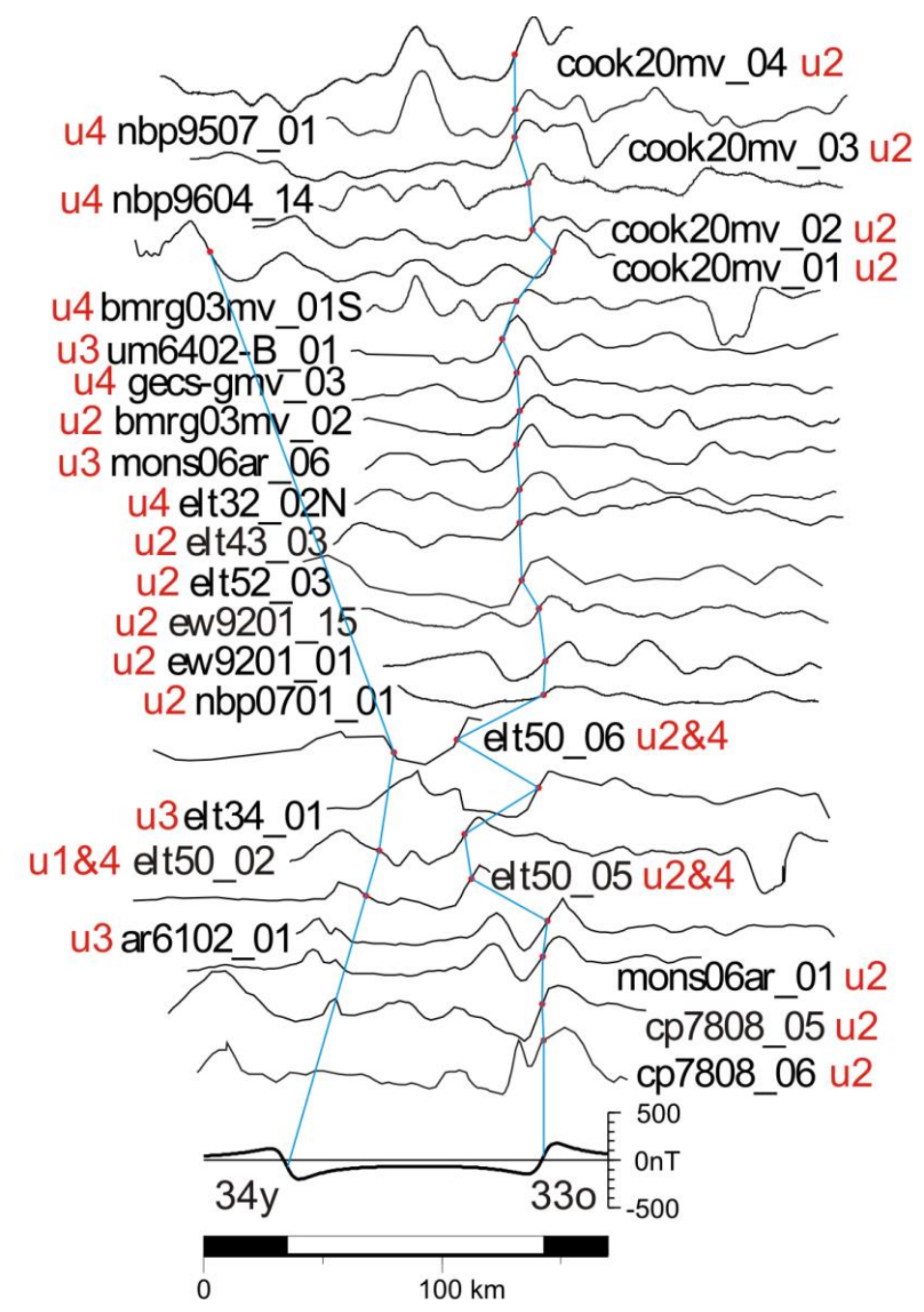

Figure II-26: Interpreted magnetic profiles showing anomalies 33o and 34y for the Southern Pacific sector. Profiles elt52_03, bmrg03mv_01S, nbp9604_14 and nbp9507_01 were reprojected onto a line parallel to the nearest recognized fracture zone, due to an azimuth difference of more than $15^{\circ}$ from the expected flowline. The synthetic model (bottommost profile) is from Figure II-25. The identification uncertainty of the sequence for each profile is indicated in red (see Appendix A for more detail). 


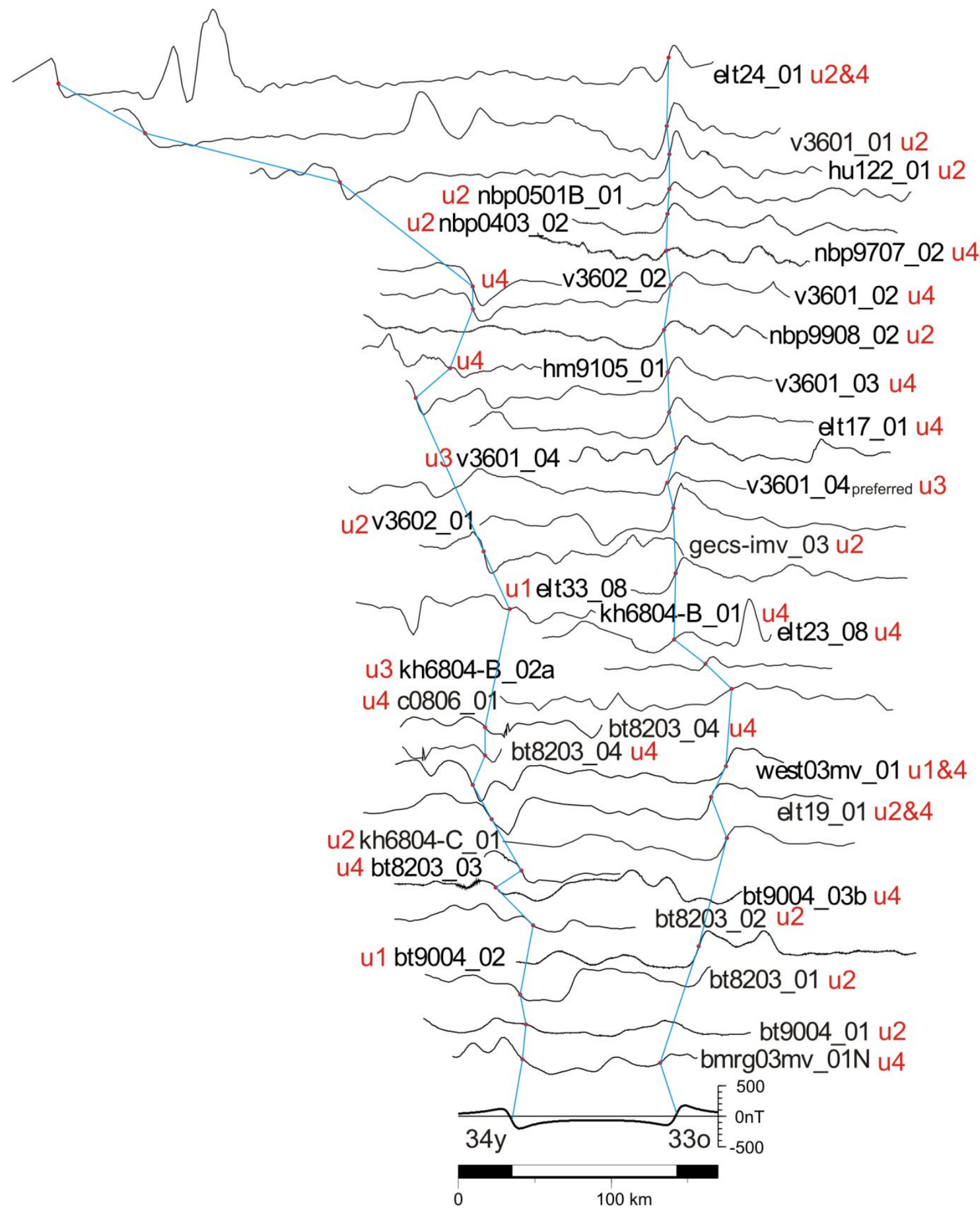

Figure II-27: Interpreted magnetic profiles showing anomalies 33o and 34y for the Northern Pacific sector. Profiles kh6804-C_01, bt8203_04, c0806_01, kh6804-B_02, elt23_08, kh6804B_01, elt33_08, gecs-imv_03, v3602_01, v3601_04, elt17_01, v3601_03, hm9105_01, nbp9908_02, v3601_02, v3602_02, nbp9707_02, v3601_01, nbp0403_02, nbp0501B_01 and hu122_01 were reprojected onto a line parallel to the nearest recognized fracture zone, due to an azimuth difference of more than $15^{\circ}$ from the expected flowline. The synthetic model (bottommost profile) is from Figure II-25. The identification uncertainty of the sequence for each profile is indicated in red (see Appendix A for more detail). 


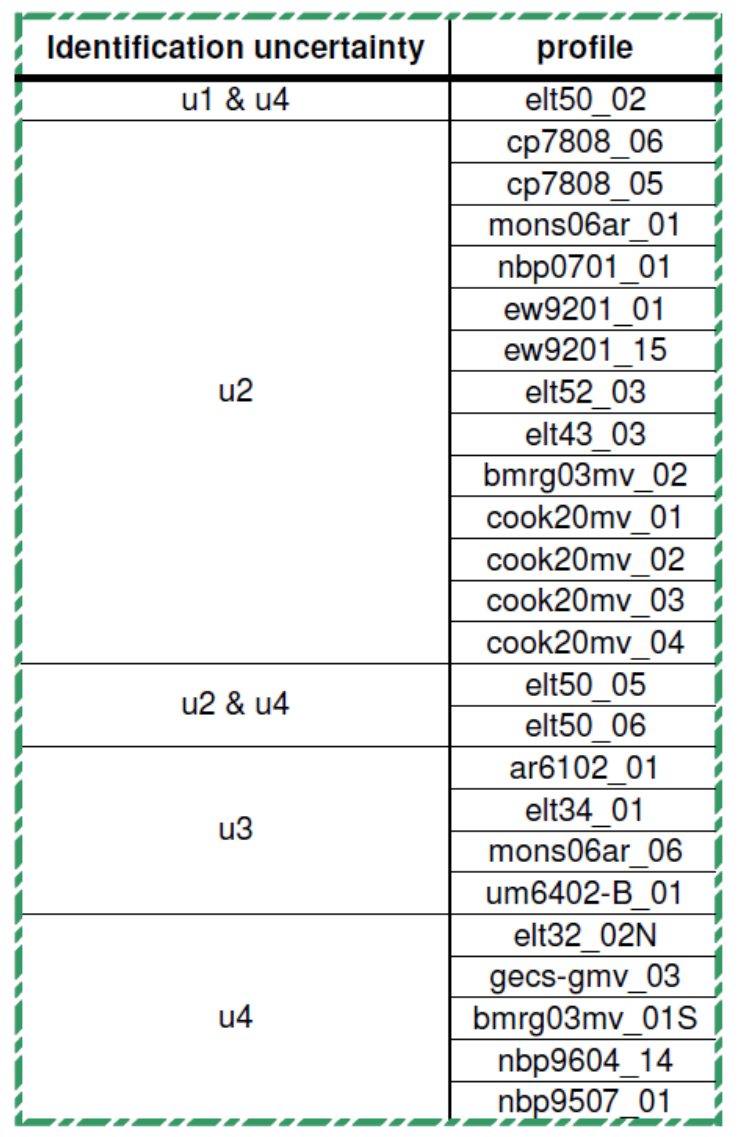

\begin{tabular}{|c|c|}
\hline Identification uncertainty & profile \\
\hline \multirow{2}{*}{ u1 } & bt9004_02 \\
\hline & elt33_08 \\
\hline u1 \& u4 & west03mv_01 \\
\hline \multirow{11}{*}{$\mathrm{u} 2$} & bt9004_01 \\
\hline & bt8203_01 \\
\hline & bt8203_02 \\
\hline & kh6804-C_01 \\
\hline & gecs-imv_03 \\
\hline & v3602_01 \\
\hline & nbp9908_02 \\
\hline & nbp0403_02 \\
\hline & nbp0501B_01 \\
\hline & hu122_01 \\
\hline & v3601_01 \\
\hline \multirow{2}{*}{ u2 \& u4 } & elt19_01 \\
\hline & elt24_01 \\
\hline \multirow{2}{*}{ u3 } & kh6804-B_02 \\
\hline & v3601_04 \\
\hline \multirow{12}{*}{ u4 } & bmrg03mv_01N \\
\hline & bt9004_03b \\
\hline & bt8203_03 \\
\hline & bt8203_04 \\
\hline & c0806_01 \\
\hline & kh6804-B_01 \\
\hline & elt17_01 \\
\hline & v3601_03 \\
\hline & hm9105_01 \\
\hline & v3601_02 \\
\hline & v3602_02 \\
\hline & nbp9707 02 \\
\hline
\end{tabular}

Table II-11: Profiles presented in Figure II-26 and Figure II-27, sorted by identification uncertainty for the sequence of magnetic reversals c33o-c34y (Pacific side).

Fifteen profiles (elt50_05, elt50_06, ew9201_15, elt52_03, cook20mv_01, cook20mv_02, cook20mv_03, cook20mv_04, cook20mv_10, bt9004_01, bt8203_01, bt8203_02, gecs-imv_01, nbp9908_02 and elt24_01) were interpreted from spatial correlation with surrounding profiles. The identification of a34y on profile elt50_05 and a33o on profile elt50_06 is only tentative (u4). The interpretation of profile cook20mv_01 was made by spatial correlation with surrounding profiles and by testing different spreading rates combinations through the use of synthetic models. My interpretation of other profiles of the cook20mv cruise derives from that analysis. Profiles bt9004_01, bt8203_01, bt9004_02 and bt8203_02 are located in the Bounty Trough. On profile bt9004_02 two ridge jumps can be interpreted between the two c34y reversals (see Appendix A). On profile elt24_01, a33o was identified by spatial correlation with surrounding profiles $(\mathrm{u} 2)$, but the distance (about $700 \mathrm{~km}$ ) between a33o and a34y on this profile and the presence of a seamount (Louisville hotspot track) between the two could mask 
the existence of a ridge jump or an irregularity in the fabric of the seafloor. Consequently I assigned a34y on elt24_01 a category 4 identification uncertainty.

Although I consider my identification of c33o on profile ar6102_01 based on younger anomalies to be without doubt, I note two possibilities for its exact location (u3). Profiles elt34_01, mons06ar_06 and um6402-B_01 can be interpreted in two different ways based on the identification of younger anomalies. In the case of profile um6402-B_01, the distance between a33o and a33y is either longer or shorter than that predicted by the synthetic model. Profile bt9004_03 can be interpreted in two different ways (see II-14) based on spatial correlation. The second alternative, presented here, places a34y on either side of an extinct ridge segment. Profile kh6804-B_02 can also be interpreted in two different ways, based on spatial correlation with surrounding profiles: c32.1o-c33o or c33o-c34y. Both possibilities are shown on Figure II-27. Profile v3601_04 shows two possible locations for a33o from spatial correlation with surrounding profiles (Figure II-27, u3).

I identified c33o on profile gecs-gmv_03 as a category 4 identification uncertainty because its location coincides with a moderate bathymetric feature and the associated positive magnetic anomaly is more symmetrical than the synthetic model predicts. The interpretation is based on that of the rest of the profile. Reversals identified on several other profiles are assigned a category 4 identification uncertainty (elt32_02N, bmrg03mv_01S, nbp9604_14, nbp9507_01, bmrg03mv_01N, bt8203_03, bt8203_04, c0806_01, kh6804-B_01, elt17_01, v3601_03, hm9105_01, v3601_02, v3602_02 and nbp9707_02). The interpretation was made based on younger anomalies (elt32_02N, bmrg03mv_01S, nbp9604_14, nbp9507_01, c0806_01 and v3601_02) or by spatial correlation with surrounding profiles (bmrg03mv_01N, bt8203_03, bt8203_04, kh6804-B_01, elt17_01, v3601_03, hm9105_01 and v3602_02). c33o on profile nbp9604_14 is assigned a category 4 identification uncertainty because its shape barely matches that of the synthetic model. a33o on profile nbp9507_01 is assigned a category 4 identification uncertainty because of the possible presence of a ridge jump on the profile. Profiles bt8203_03 and bt8203_04 are located in the Bounty Trough. Profile bt8203_04 has two interpretations (both $\mathrm{u} 4$ ). Although the shape of a33o on profile elt17_01 matches that of the synthetic model, it is assigned a category 4 because the azimuth of the profile is very oblique to the spreading direction. Although the shape of profile v3601_03 matches that of the model, a33o and a34y are assigned a category 4 identification uncertainty because the interpretation is done by spatial correlation with surrounding profiles with category 4 anomalies. The absence of 
a recognizable sequence 32 on profile v3601_02 makes the identification of older anomalies (a33 and a34) highly uncertain. Profile nbp9707_02 was interpreted based on younger anomalies.

On the Antarctica side of the spreading ridge, a33o was identified on two profiles, based on younger anomalies: nbp9602_19E and nbp9602_20. They were assigned a category 4 uncertainty. Anomalies a33o and a34y were tentatively identified (u4) on profile nbp92-8_11 based on surrounding profiles (spatial correlation) and literature. Other profiles shown on Figure II-28 are located in the Bounty Trough (formed on the Antarctic side, before a ridge jump transferred them to the Pacific side- my interpretation and that of $\operatorname{Davy}(2006))$.

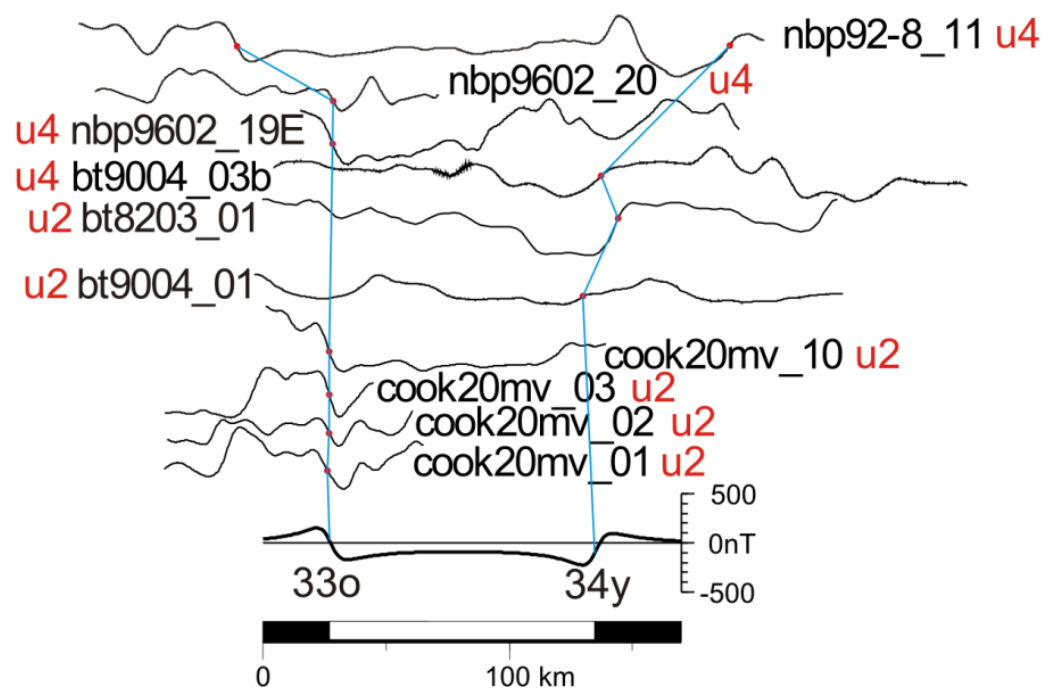

Figure II-28: Interpreted magnetic profiles showing anomalies $33 \mathrm{o}$ and $34 \mathrm{y}$ for the Antarctica sector. Profile nbp9602_20 was reprojected onto a line parallel to the nearest recognized fracture zone, due to an azimuth difference of more than $15^{\circ}$ from the expected flowline. The synthetic model (bottommost profile) is from Figure II-25. The identification uncertainty of the sequence for each profile is indicated in red (see Appendix A for more detail).

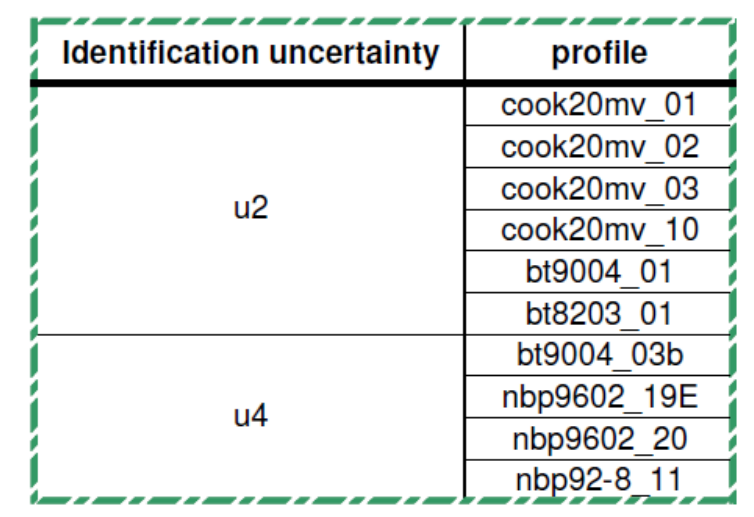

Table II-12: Profiles presented in Figure II-28, sorted by identification uncertainty for the sequence of magnetic reversals c33o-c34y (Antarctica side). 
iั

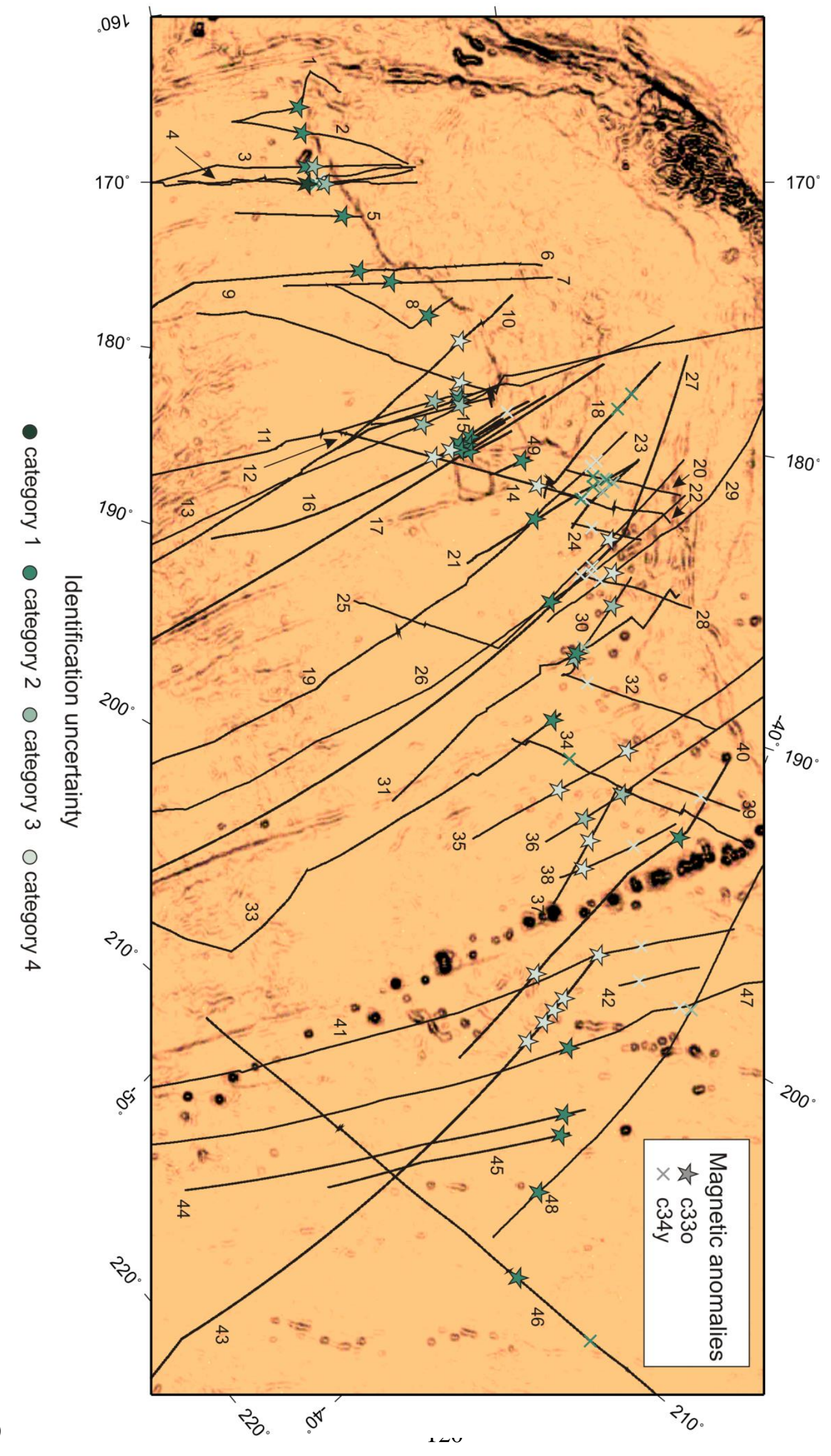




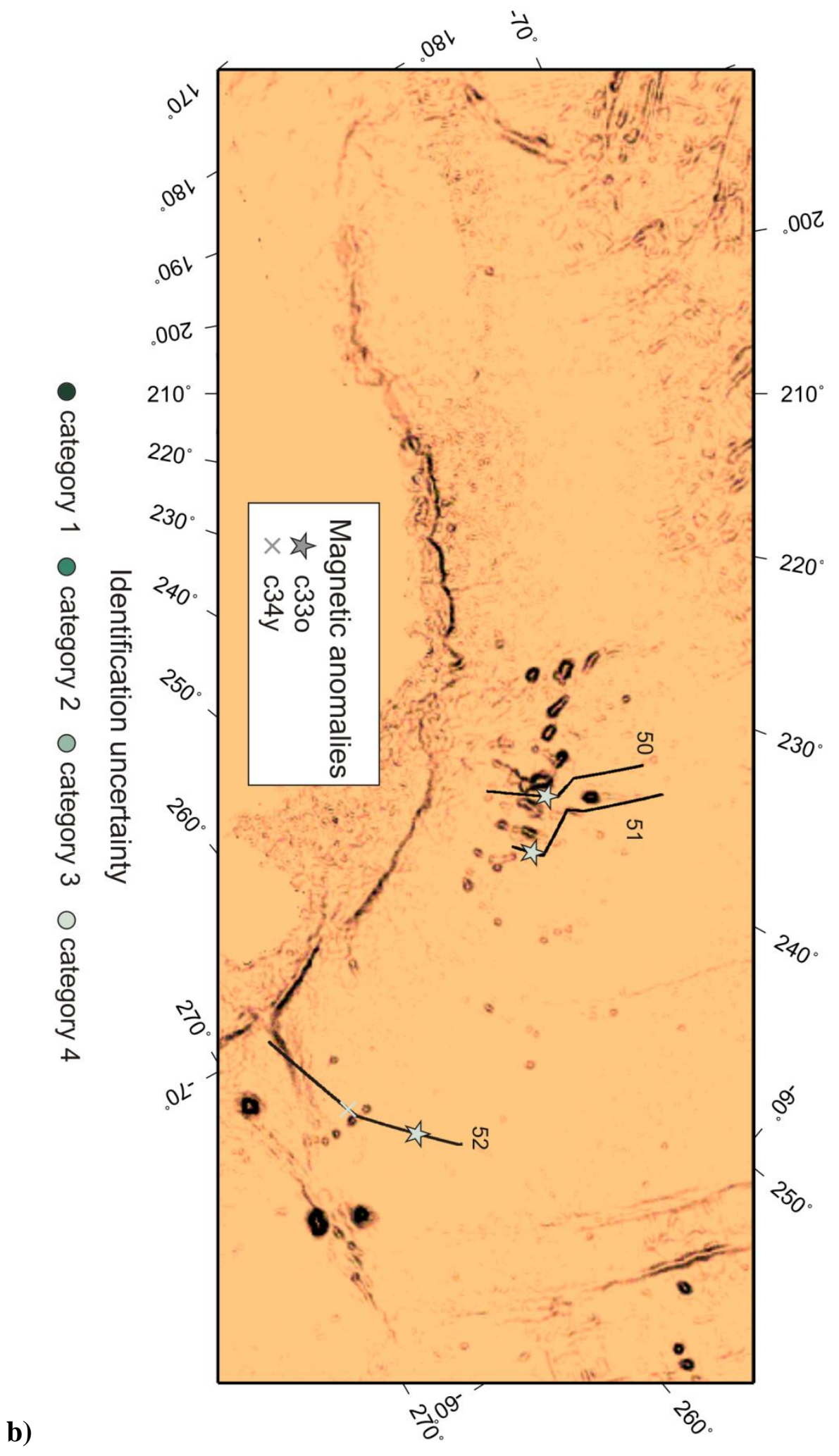




\begin{tabular}{|c|c|}
\hline Profile name & Number \\
\hline cp7808_06 & 1 \\
\hline cp7808_05 & 2 \\
\hline mons06ar_01 & \multirow[b]{2}{*}{3} \\
\hline \multirow{2}{*}{$\frac{a r 6102 \_01}{\text { elt50_05 }}$} & \\
\hline & \multirow{4}{*}{4} \\
\hline elt50_02 & \\
\hline elt34_01 & \\
\hline elt50 06 & \\
\hline nbp0701_01 & 5 \\
\hline lew9201_01 & 6 \\
\hline ew9201_15 & 7 \\
\hline elt52_03 & 8 \\
\hline elt43_03 & 9 \\
\hline elt32_02N & 10 \\
\hline mons06ar_06 & 11 \\
\hline bmrg03mv_02 & \multirow{2}{*}{12} \\
\hline gecs-gmv 03 & \\
\hline um6402-B_01 & 13 \\
\hline \multirow{2}{*}{ bmrg03mv_01S } & \multirow{2}{*}{14} \\
\hline & \\
\hline cook20mv_01 & \multirow{4}{*}{15} \\
\hline cook20mv_02 & \\
\hline \multirow{2}{*}{ cook20mv_03 } & \\
\hline & \\
\hline nbp9604_14 & 16 \\
\hline nbp9507 01 & 17 \\
\hline
\end{tabular}

c)

\begin{tabular}{|l|c|c|c|}
\hline Profile name & Number & Profile name & Number \\
\hline bt9004_01 & 18 \\
\hline elt25_02 & 19 \\
\hline bt8203_01 & 20 \\
\hline$b t 9004 \_02$ & 21 & inbp9602_19E & 50 \\
\hline inbp9602_20 & 51 \\
\hline
\end{tabular}

Figure II-29: Interpreted profiles and magnetic reversals identified, with identification uncertainty. Horizontal gravity gradient computed using GMT from [Sandwell and Smith, 1997] (version 18) in the background. a) Map for the Pacific sector. b) Map for the Antarctica sector. c) Profiles and corresponding numbers on the maps. 


\section{Chapter III}

TECTONIC INTERPRETATION OF SEAFLOOR MORPHOLOGY

AND GRAVITY ANOMALIES 
Chapter II described the acquisition of magnetic data, which are used to calculate relative plate motions: they are interpreted as chrons and hence to determine age. Other important constraints on the calculation of a rotation pole are fracture zone crossings. In this study, fracture zone crossings were acquired from analysis of satellite altimetry data, shiptrack depth readings and swath bathymetry. This chapter will first shortly describe the seafloor between New Zealand and Antarctica, then it will detail the process of picking fracture zone crossings and the issues/uncertainties associated with this process.

\section{Seafloor morphology}

Satellite altimetry is commonly used to analyse the morphology of the seafloor (Figure III-1). Most often satellite altimetry data are interpreted in the shape of gravity anomaly (difference between the geoid and the gravity field derived from the satellite altimetry data). Here the satellite gravity signal is used to identify post-spreading structures (seamounts, rifts, seafloor deformations ...) as well as to collect information about seafloor spreading and ridge geometry (fracture zone tracks, abyssal hills, curvature near fracture zones, propagators ...). 


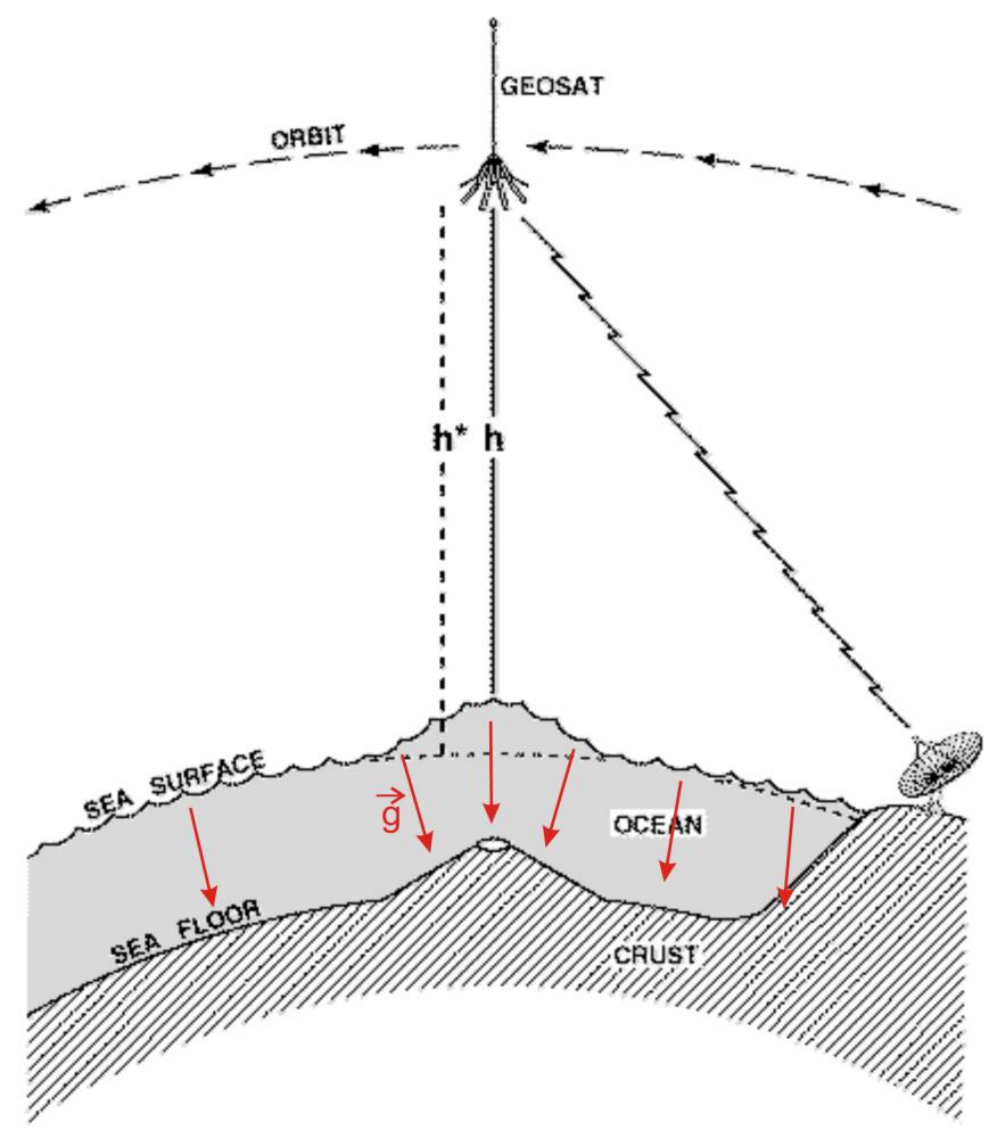

Figure III-1: Schematic representation of the principles of satellite altimetry. The satellite measures its distance to the surface of the ocean. The difference between that distance $h$ and the distance to the equipotential corresponding to the 0 sea level is proportional to the topography of the seafloor underneath. $\vec{g}$ are the local gravity vectors. Image modified from www.ngdc.noaa.gov.

The seafloor between New Zealand and Antarctica was formed by accretion of material at ridge segments (see Chapter II). Once the seafloor is formed it can be affected by deformation and/or volcanism and/or sedimentation. Around $\left(190^{\circ} \mathrm{E}, 72^{\circ} \mathrm{S}\right)$ a sharp, deep northeastsouthwest oriented low can be identified on the satellite gravity (Figure III-2). That low has previously been described as the Iselin Trough [Marks and Stock, 1997]. Close to Antarctica, along longitude $270^{\circ} \mathrm{E}$ are two similar signals, the northern one being more subdued. From West to East these features can be described as a deep gravity low followed by a gravity high. The two linear features are identified [Cunningham et al., 2002] as the Bellingshausen Gravity Anomaly and the De Gerlache Gravity Anomaly (see Chapter I for an extended description of deformation of the zone). 


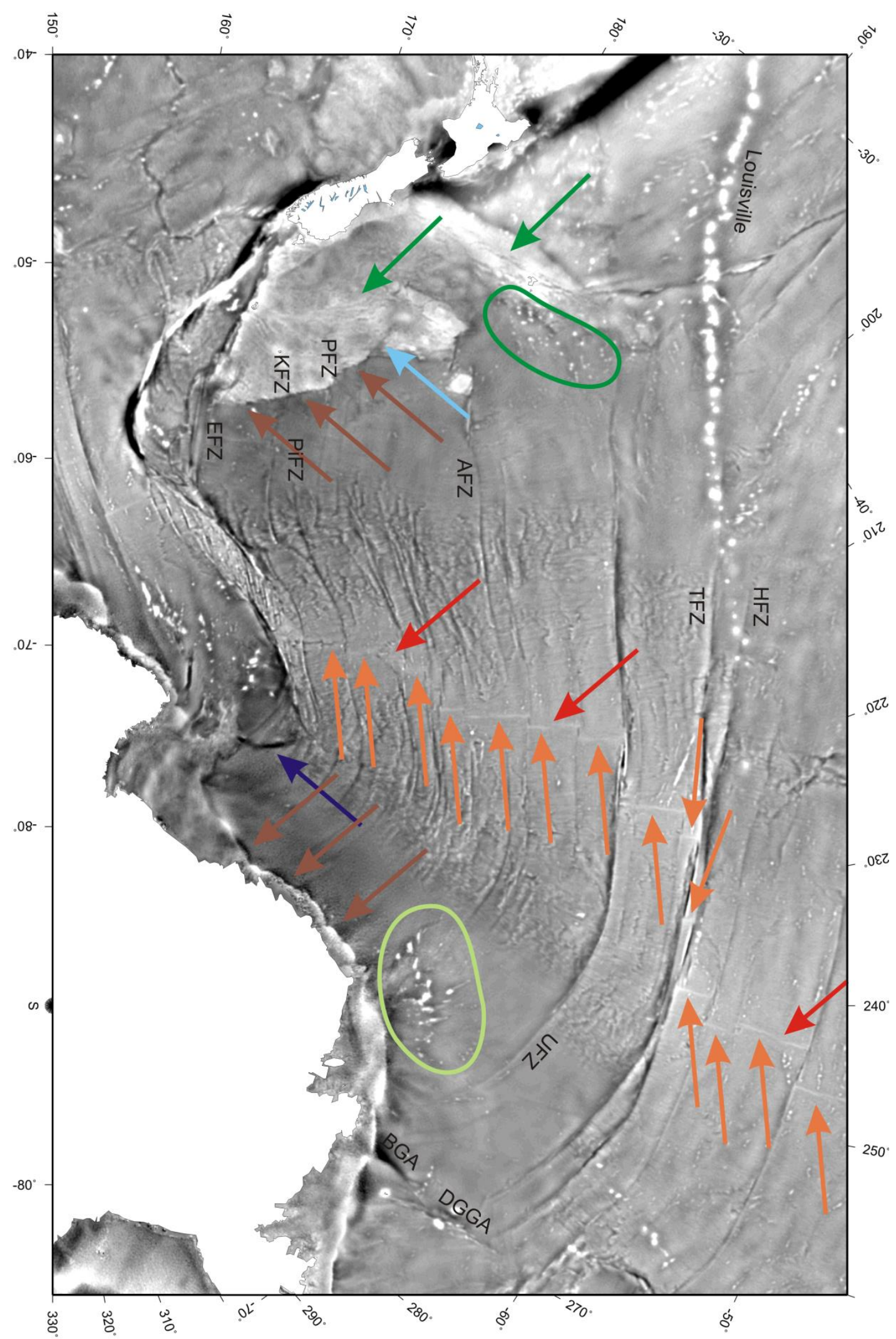

Figure III-2: Syn- and post-spreading structures in the Southwest Pacific. Light green: Marie Byrd Seamounts; dark green: extensive deformation from the rifting phase; brown: steep continental slope; light blue: site of possible Campbell Plateau-Bounty Platform boundary; dark blue: Iselin Trough; orange: currently active ridge segments; red: overlapping spreading centre (north and centre) and propagator (south). Gravity background [Sandwell and Smith, 1997]. 
Between $\left(186^{\circ} \mathrm{E}, 30^{\circ} \mathrm{S}\right)$ and $\left(213^{\circ} \mathrm{E}, 48^{\circ} \mathrm{S}\right)$, an alignment of gravity highs surrounded by gravity lows is typical of the gravity signature of a hotspot seamount chain. This chain is identified as the Louisville seamounts. Between $225^{\circ} \mathrm{E}$ and $250^{\circ} \mathrm{E}$ and centred on latitude $70^{\circ} \mathrm{S}$, a cluster of elongated steep gravity highs is referred to as the Marie Byrd Seamounts in literature. Kipf et al. (2014) dated the seamounts between 65 and 52 Ma, making their formation post-spreading. Unfortunately the seamounts are situated over the expected location of part of the Bellingshausen-Marie Byrd Land boundary, which means that seismic reflection profiles are necessary to ascertain the precise location of that boundary [Cunningham et al., 2002].

In general, the seafloor is smoother and higher near Antarctica than it is on the conjugate side of the southwest Pacific (Figure III-3). That difference is mostly due to a thicker sediment cover on the Antarctica side. Such a thick cover makes the localisation of fracture zones more difficult near Antarctica, as their gravity signal is more subdued. 

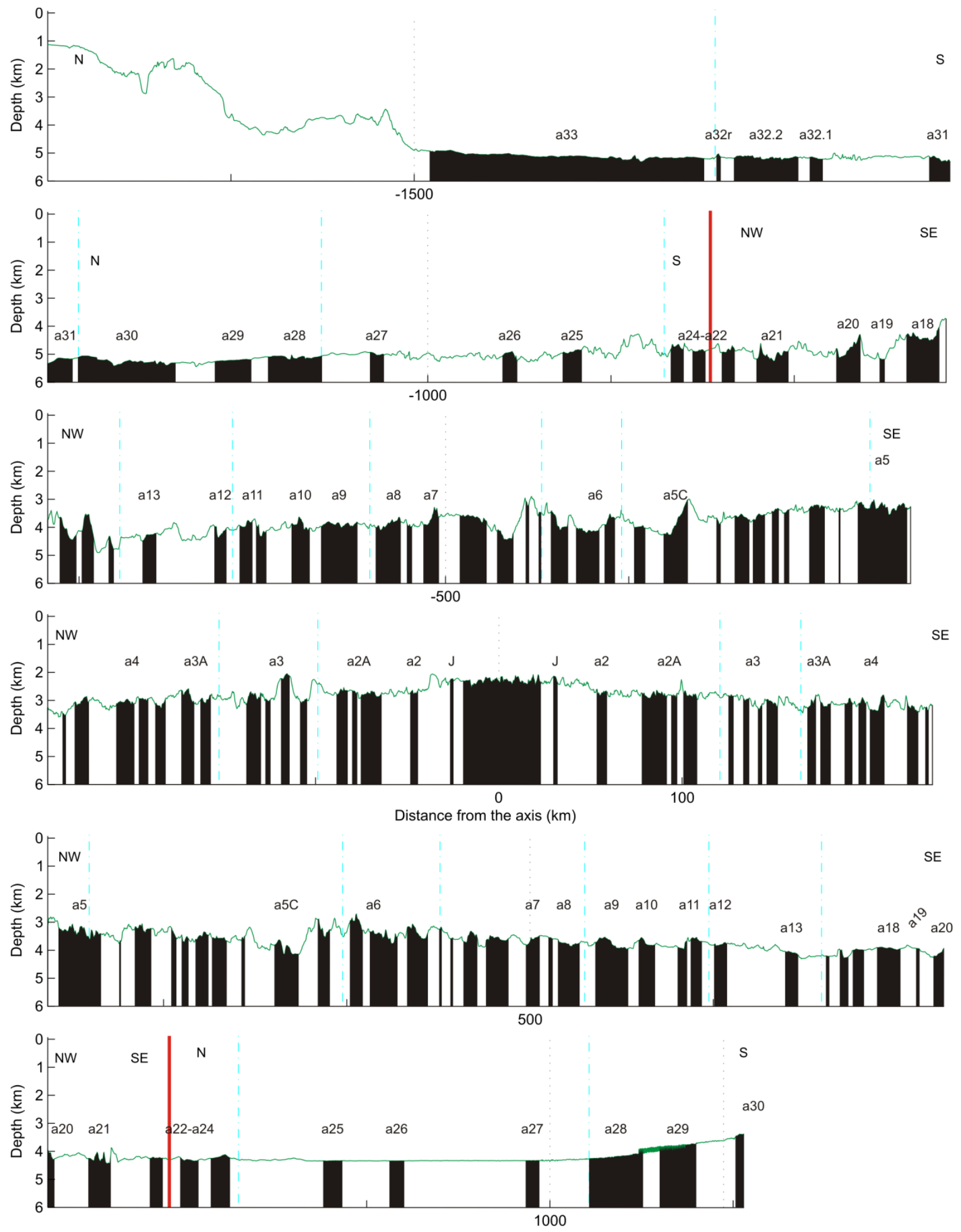

Figure III-3: Bathymetric profile of the Southwest Pacific just north of the Pitman FZ. Shiptrack bathymetry (shown here in several sections) from the ew9201 cruise is projected in the direction of spreading: $120^{\circ}$ between the two red lines and $170^{\circ}$ on either side of the red lines. The magnetic block model used to interpret the magnetic anomalies on profile ew9201_01 is added as a guide to date the seafloor. Horizontal distance from the current spreading segment and depth are in $\mathrm{km}$. Please refer to the A0 map at the end of this thesis for the location of this profile (profile $\mathrm{n}^{\circ} 32$ ). 
The continental slope on both sides of the oceanic basin is relatively steep $\left(6 \% \approx 3.4^{\circ}\right)$, indicative of a rapid breakup, possibly connected to pre-existing faulting. The Chatham Rise is expressed as a gravity high displaying east-west lineations, a relic of its formation as the forearc of the Gondwana subduction (see Chapter I). Along its southeastern edge, small scale gravity highs can be interpreted as continental fragments separated from the Chatham Rise margin during the first stages of spreading in the Southwest Pacific (B. Davy, personal communication, 2008). The gravity signature of the Campbell Plateau is that of continental lithosphere. Like the Chatham Rise, it shows signs of compressive deformation linked to the Gondwana subduction [Sutherland, 1999]. The Bounty Platform, although similar to the Campbell Plateau, is separated from it by a linear, approximately north-south gravity low. Rupert Sutherland (personal communication, 2008) suggests the Bounty Platform separated from the Campbell Plateau during the early stages of rifting and rotated. Grobys et al. (2007) suggest the Bounty Plateau separated from the Chatham Rise via rifting to its present position. Bollons Seamount is easily identifiable as a gravity high.

The spreading rate on a ridge segment is linked to the tectonic regime of that segment (magmatic or amagmatic, fast or slow, [Cordier, 2006]). As a result, the spreading rate at the time of formation of the seafloor determines its morphology [Cordier, 2006]. As a general rule, the slower the spreading rate, the rougher the seafloor (for instance the smooth East Pacific Rise is the fastest active spreading ridge on Earth, while seafloor surrounding the Southwest Indian Ridge is rough). Between c25y and c24o, the bathymetric signature of the seafloor changes radically, from smooth and flat (anomalies 32 to 25 ) to rough, broken and irregular (anomaly 23 to after anomaly 20, see Figure III-3, around 870km, and Figure III-6). This change in seabed morphology has been previously shown to correspond to a rapid reduction in spreading rate during chrons 24 to 20 [Cande et al., 1995; Croon et al., 2008].

Fracture zones have a linear gravity (and bathymetric) signal that varies depending on the spreading rate at the time of their formation ([Gaina et al., 1998], see Figure III-4). 

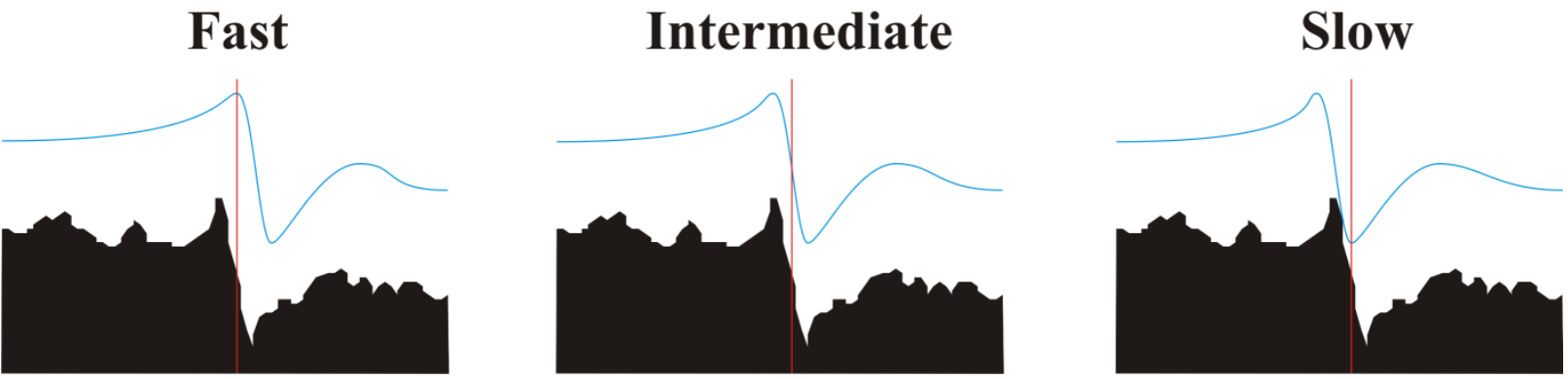

Figure III-4: Gravity anomaly corresponding to a fracture zone. The precise location of the fracture zone with regards to the geoid anomaly depends on the spreading rate: gravity low for slow spreading ridge segments, gravity high for fast spreading, and highest gravity gradient for intermediate spreading rates. After [Mayes et al., 1990].

Swath bathymetry from recent cruises (ew9201, nbp9604, west02mv) enables detailed observation of the seafloor on and around fracture zones (Figure III-5). These data image the curvature of abyssal hills ${ }^{16}$ close to fracture zones, as described in [Croon et al., 2010].

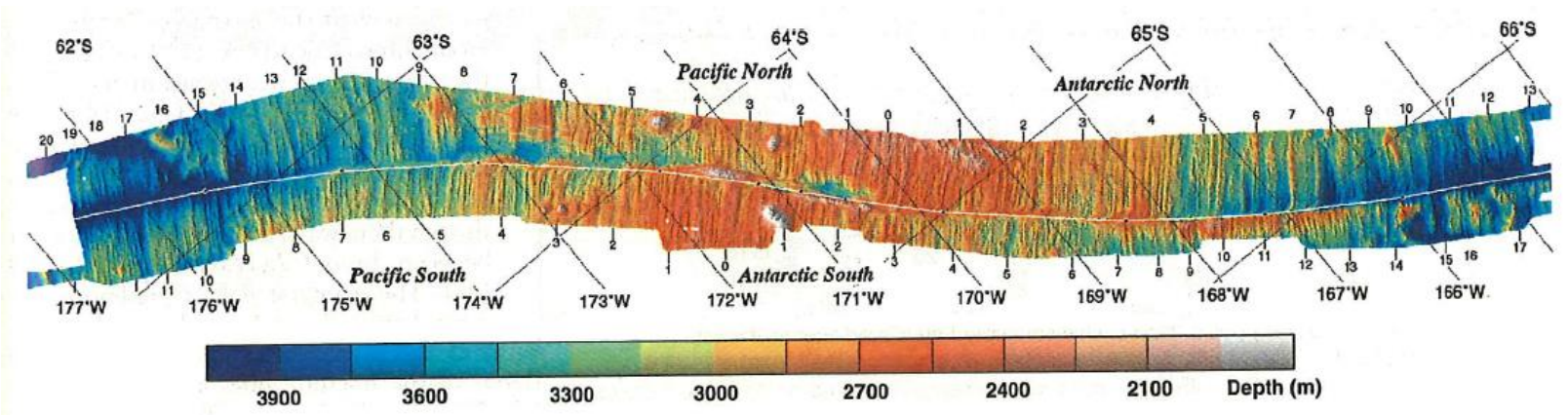

Figure III-5: Multibeam swath bathymetry from the area surrounding the Pitman FZ. Numbers above and below the image indicate the age of the crust in millions of years [Cande and Kent, 1995]. The white line is a synthetic flowline [Cande et al., 1995].

16 Abyssal hills are positive relief features created during the formation of the seafloor by the switch between magmatic and a-magmatic phases of the spreading cycle. They are parallel to the ridge. 


\section{Fracture zones}

In this study, fracture zone crossing locations were picked on shiptrack profiles based on bathymetry (Figure III-6, III-6 and Figure III-8). These crossings were then used in mapping software (ArcGIS) to pick the location of major fracture zones in the southwest Pacific by spatial correlation using the gravity grid [Sandwell and Smith, 1997]. 


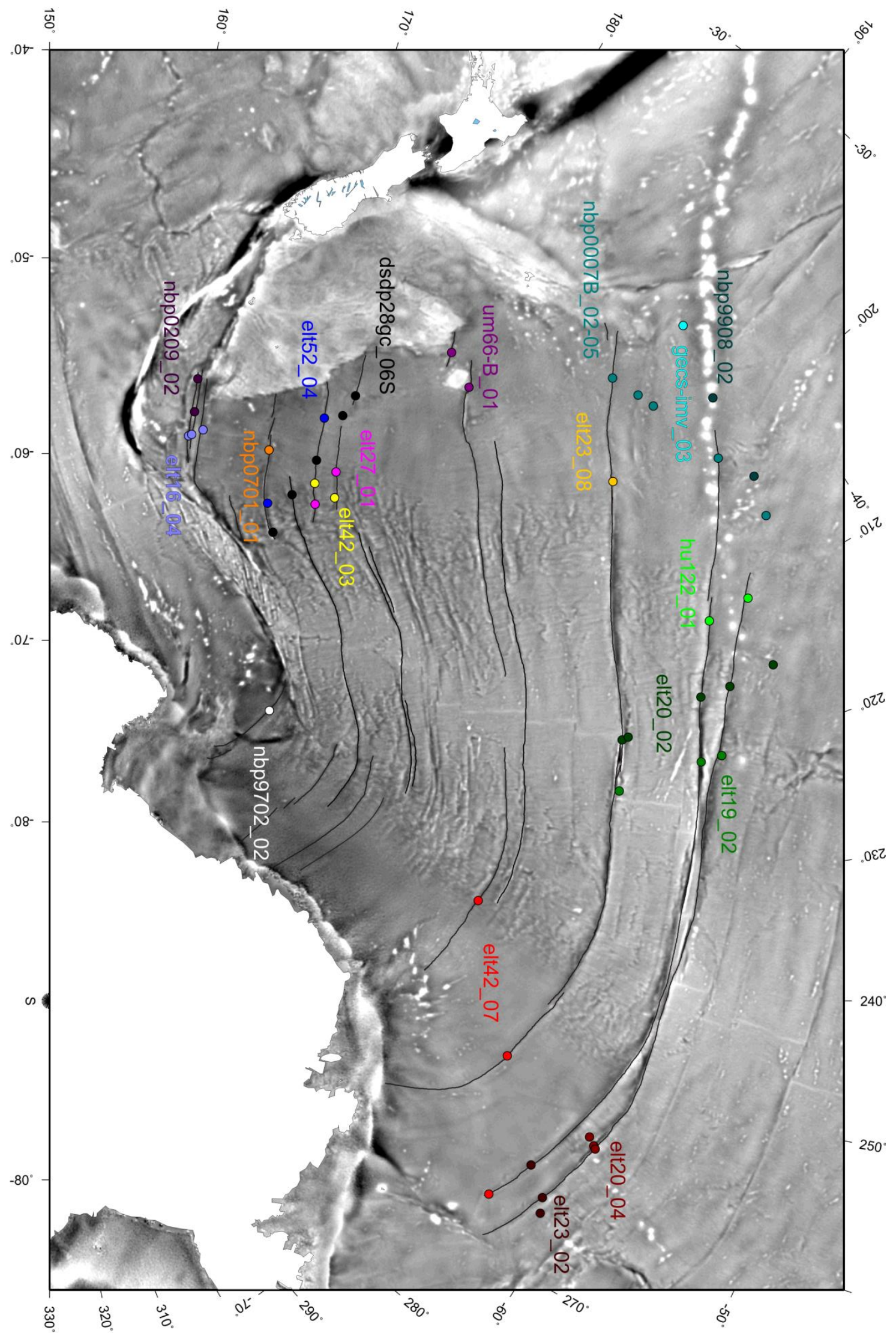

Figure III-6: Fracture zones used to calculate the reconstructions. Fracture zone crossings are colour-coded by profile. Gravity background by [Sandwell and Smith, 1997]. Stereographic projection. 
Once each fracture zone was digitized, each of its points was assigned an age interval corresponding to the time range those points were on the transform fault part of the fracture zone (i.e. when they were "active", see Figure III-7). This was achieved by interpolating the magnetic lineations to the fracture zones. Because the uncertainty associated with each fracture zone datapoint is linked to the uncertainty of each magnetic datapoint, I only considered magnetic points with an identification uncertainty of $\mathrm{u} 1$ or $\mathrm{u} 2$ (see Chapter II-6).

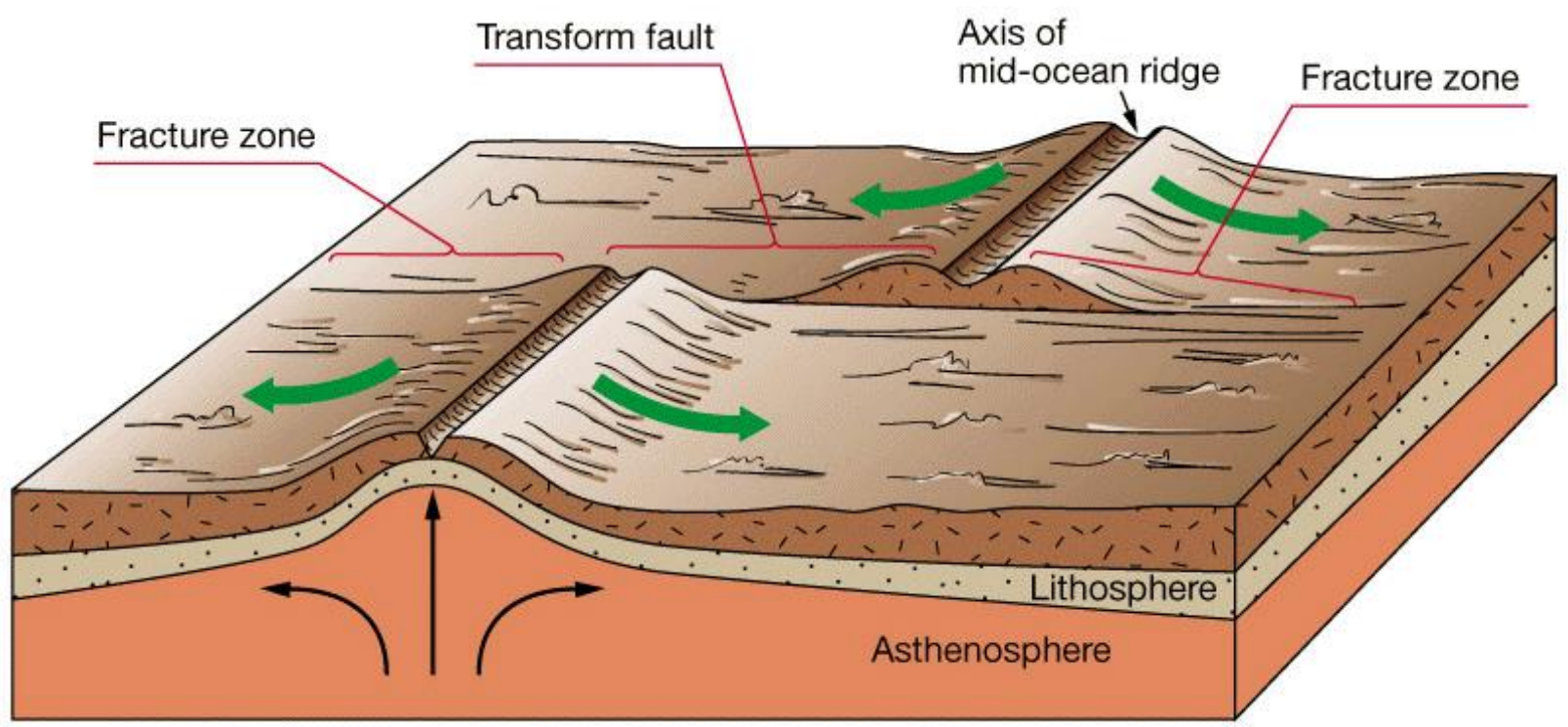

Figure III-7: Segmentation of an active spreading ridge. Ridges are segmented, with the ridge segments being separated most often by fracture zones. The term fracture zone applies to the extinct or non-moving part of the boundary between two ridge segments. The active part is called the transform fault as it accommodates the transform motion between the lithosphere formed on either side. 3D drawing from http://wps.prenhall.com.

Figure III-8 shows examples of shiptrack fracture zone crossings for the main fracture zones of the Southwest pacific. Correlating the gravity signal [Sandwell and Smith, 1997] with the bathymetry allows one to characterize the gravity signature of each fracture zone in different places. Most fracture zones in the southwest Pacific are expressed as a gravity low (Emerald FZ, Kohiku FZ, Pitman FZ, Pahemo FZ, Antipodes FZ, Tharp FZ and Heezen FZ), with the exception of the Heezen FZ crossing shown on profile elt19_02 (gravity gradient). 


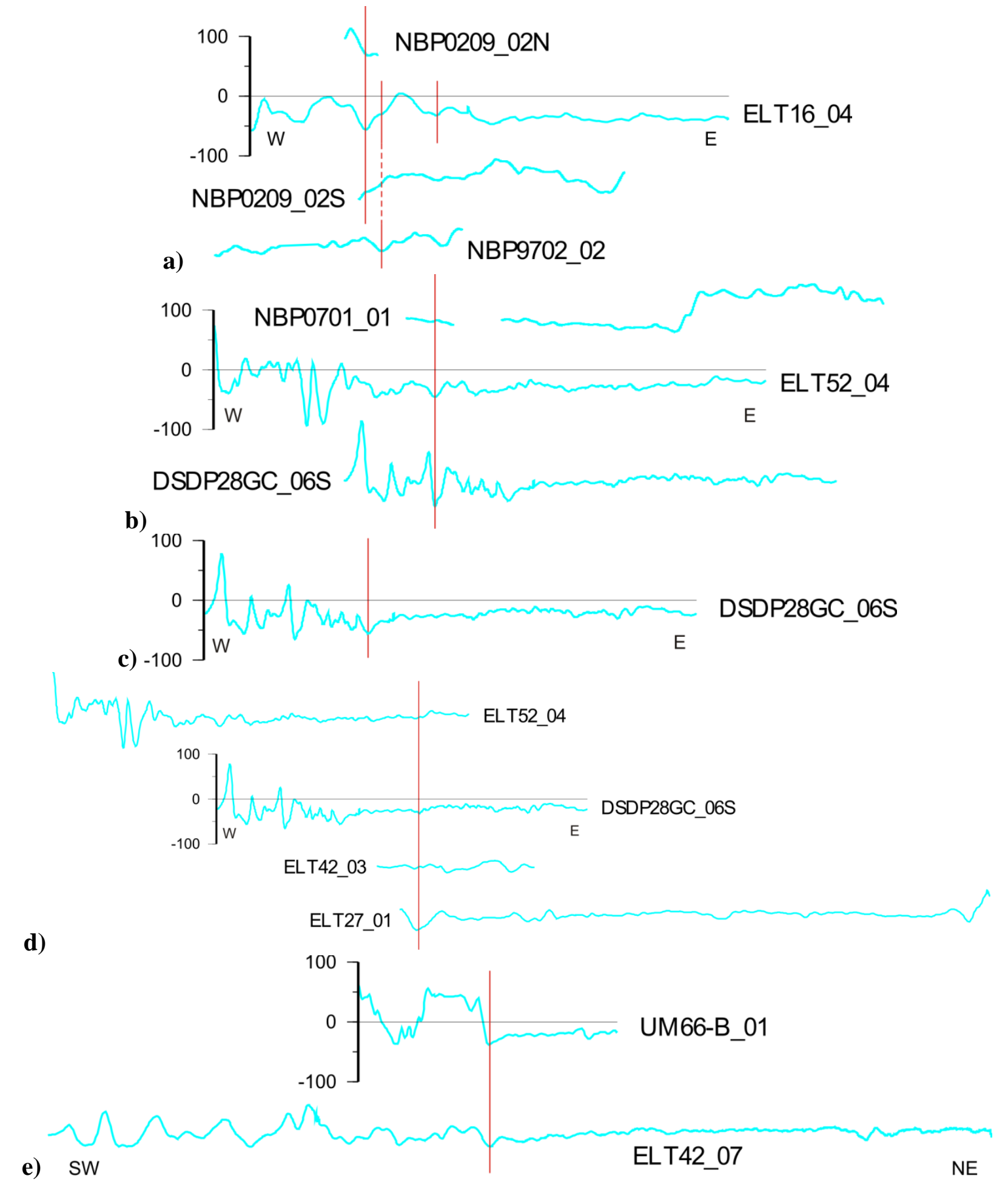




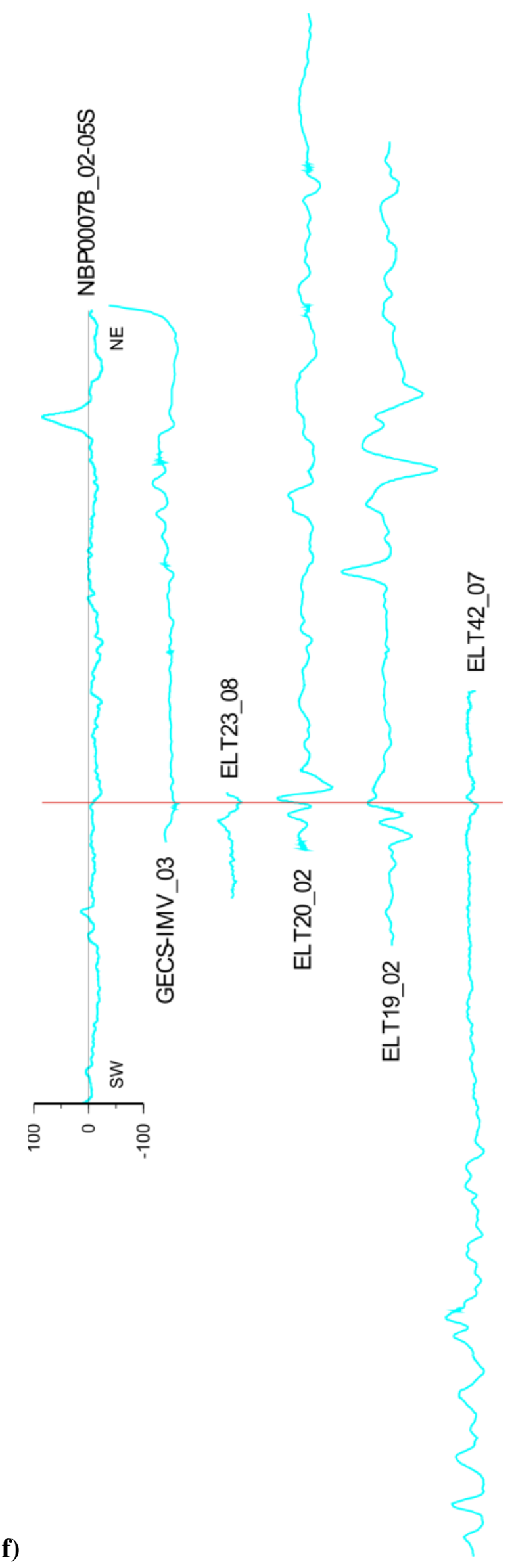




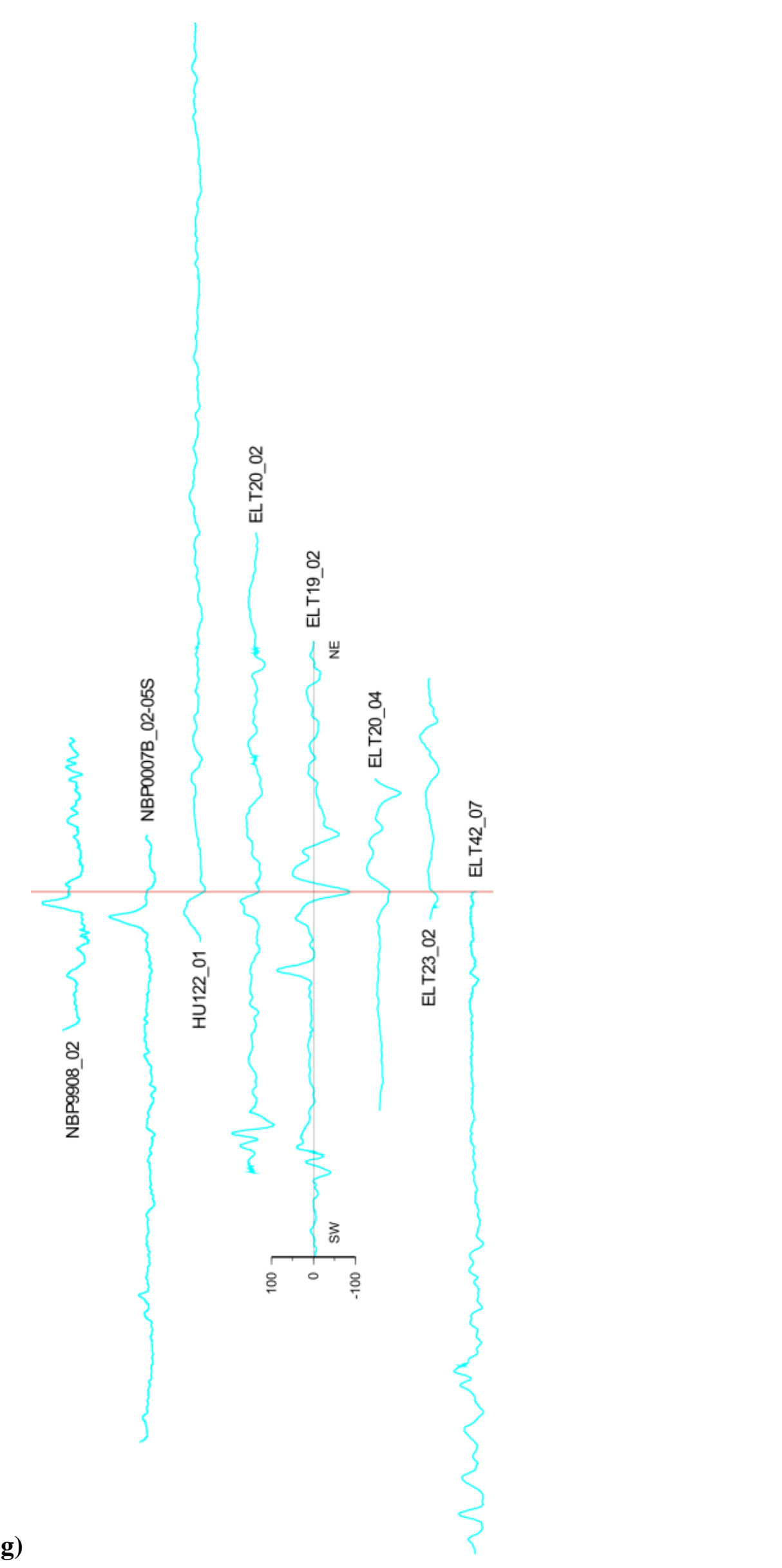




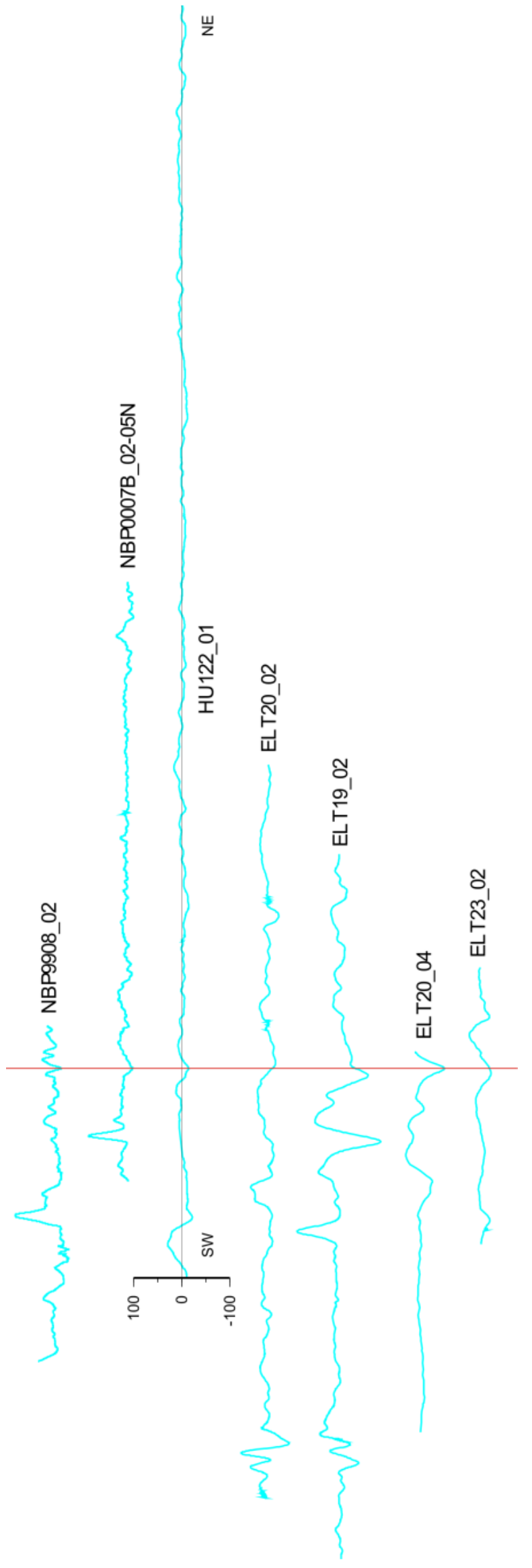


Figure III-8 (previous pages): Bathymetric profiles and gravity anomaly across the main fracture zones of the Southwest Pacific. Each shiptrack bathymetric profile is projected orthogonally to the azimuth of spreading. Satellite gravity [Sandwell and Smith, 1997] is extracted along the track, then projected (cyan curve). Red lines indicate the fracture zone picking of interest for each figure. Gravity anomalies in $\mathrm{mGal}$, depth and horizontal distance in km. a) Emerald FZ. b) Kohiku FZ. c) Pitman FZ. d) Pahemo FZ. e) Antipodes FZ. f) Udintsev FZ. g) Tharp FZ. h) Heezen FZ.

The gravity expression of the Udintsev FZ changes along track. Although the seafloor's sedimentary cover closest to the margin is too thick to confirm the location of the fracture zone in the bathymetry (Figure III-8f, profiles nbp0007B_02-05S and elt42_07), profiles farther offshore show a correlation between the Udintsev FZ and a gravity low (gecs-imv_03, elt23_08). Close to the ridge however, the bathymetric trace of the fracture zone can be matched to a gravity high (elt19_02). Profile elt20_02 displays an intermediate state of the fracture zone being expressed as a steep gravity gradient, which could represent a transition between two seafloor spreading regimes.

Track elt19_02 shows a gravity low for the Tharp FZ near the ridge, so it seems to be an exception to the trend of gravity lows at slow spreading and gravity highs at fast spreading segments. This is especially curious because it is so close the Heezen FZ.

The location of the Tharp FZ on profile nbp9908_02 is uncertain due to the presence of a seamount from the Louisville hotspot chain. However, satellite altimetry confirms the seamount coincides with the location of the fracture zone. In other words the fracture zone lies under the seamount, but the seamount hides the precise location of the fracture zone. 
Chapter 3: Magnetic anomaly interpretation 


\section{Chapter IV}

PLATE RECONSTRUCTION: METHODS 
Chapter IV: Plate reconstructions: methods 
Euler's theorem states that any movement on a sphere can be quantified by a rotation around an axis whose intersection with the surface of the sphere is called a pole. To calculate the rotation of one tectonic plate relative to another, one uses the coordinates of conjugate fracture zones (small circles on the sphere in the reference frame of the rotation) and conjugate magnetic lineations (great circles that cross at the location of the poles) (Figure IV-1).

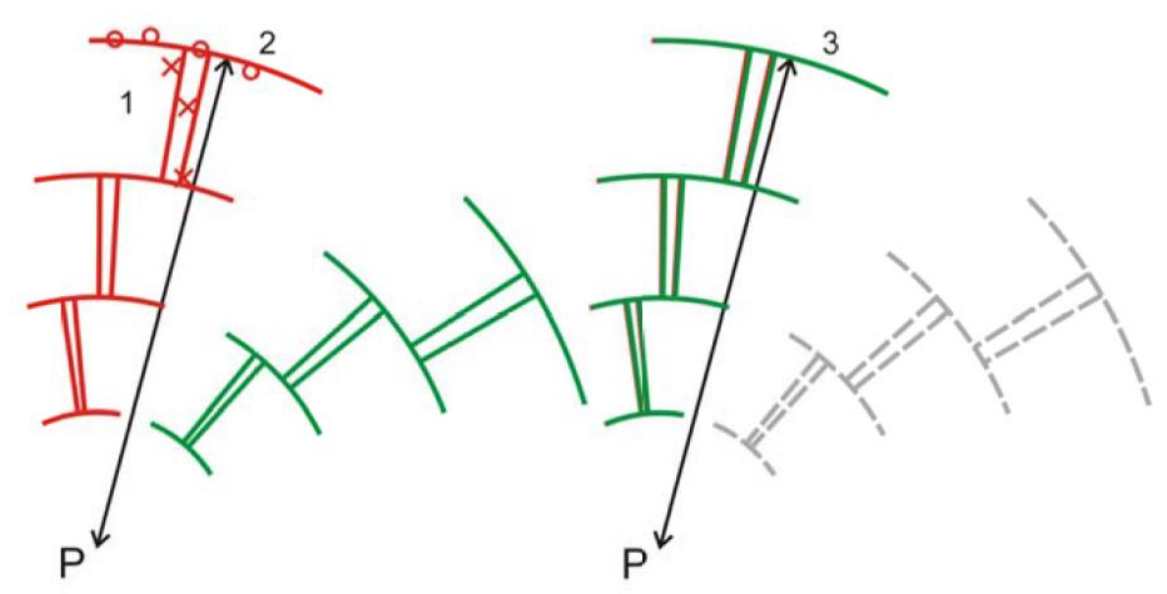

Figure IV-1: Basic concept of finite pole calculation. 1) Alignment of the magnetic anomalies of each segment on each plate with a best-fitting great circle through the rotation pole. 2) Alignment of the points of each fracture zone on both plates with a small circle centered on the rotation pole. 3) The rotation angle is calculated by matching the conjugate weighted great and small circles defined in 1) and 2).

In this chapter I detail my efforts to calculate rotation poles for the southwest Pacific as accurately and precisely as possible, by testing three different computer codes and comparing their output.

\section{Combining uncertainties}

In Chapter II I detailed the different types of uncertainty associated with the identification and picking of magnetic reversals on shiptrack magnetic profiles. In order to use the magnetic reversals to calculate finite plate rotations, the four different types of uncertainty have to be combined into one total uncertainty value $\sigma_{\text {tot }}$. 
For each datapoint, the variance of the total error, $\sigma_{1}^{2}$, is given by: $\sigma_{1}^{2}=\sigma_{\text {res }}^{2}+\sigma_{\text {location }}^{2}+\sigma_{\text {picking }}^{2}$ where $\sigma_{\text {res }}$ is the resolution uncertainty, $\sigma_{\text {location }}$ is the location uncertainty and $\sigma_{\text {picking }}$ is the digitising uncertainty associated with picking the location.

If there are multiple interpretations, then the total uncertainty $\sigma_{t o t}^{2}$ is found as follows. There are four identification uncertainty categories ( $\mathrm{u} 1$ to $\mathrm{u} 4$ ). For each category, $\sigma_{1}^{2}$ is modified by a multiplier $m_{j}$, reflecting the increased uncertainty of the less reliable interpretations. For category $\mathrm{u} 1, m_{1}=1$.

For multiple interpretations (u3), for each datapoint the total uncertainty is:

$$
\sigma_{t o t}^{2}=p_{j} \sigma_{1}^{2}+\left(1-p_{j}\right) m_{j} \sigma_{1}^{2}
$$

where $p_{j}$ is the probability that the interpretation is correct (Table IV-1).

\begin{tabular}{|c|c|c|}
\hline \multirow{2}{*}{ Identification uncertainty } & Probability that the identification is true \\
\hline \multirow{2}{*}{ u1 } & 1 & 1.0 \\
\cline { 2 - 3 } & 2 & 0.9 \\
\hline \multirow{2}{*}{$\mathrm{u} 3$} & 3 & 0.8 \\
\hline
\end{tabular}

Table IV-1: Value of $p_{j}$ for each category of identification uncertainty ${ }^{17}$.

Hence, for each magnetic anomaly: $\sigma_{t o t}^{2}=\sigma_{1}^{2}\left(p_{j}+\left(1-p_{j}\right) m_{j}\right)$

so that if $p_{j}=1 \quad \sigma_{\text {tot }}^{2}=\sigma_{1}^{2}$

${ }^{17}$ Initially I had defined six categories of identification uncertainty. It was decided at a later stage that reducing the number of categories in the text would make for an easier read. Table IV-1 shows the correspondence between the system with four categories (used in the text and figures) and that with six categories (used in the database). 


\section{GPlates}

Gplates is open-source software created by geophysicists as an easy-to-use tool to display data on a globe [Gurnis et al., 2012]. Its plate tectonic reconstruction facility allowed me to calculate a first approximation of finite rotation poles for the Southwest Pacific, which are in broad agreement with the standard finite rotation poles of ([Cande et al., 1995], Figure IV-2).

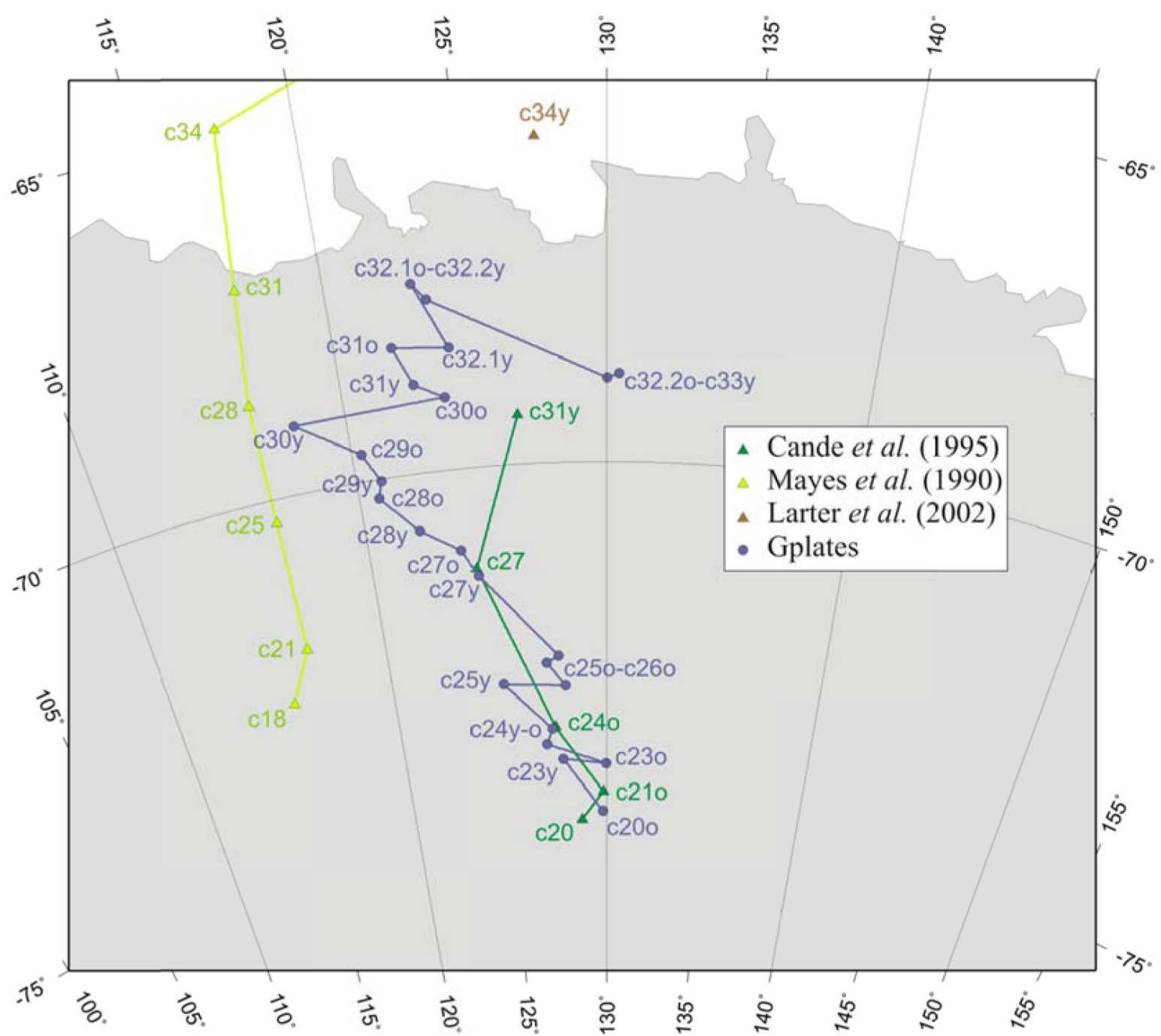

Figure IV-2: Finite rotation poles for the Southwest Pacific, obtained using GPlates.

To determine finite rotations of one plate relative to another on Gplates, one uses the mouse to change the coordinates of the rotation pole and the rotation angle, while seeing in real time the effect on the position of the mobile plate. 
Because for each chron the rotation is determined visually, GPlates does not provide a confidence region or any quantitative measure of fit, which makes it unsatisfactory for this work. For that reason, I decided to try and calculate finite rotations for the Southwest Pacific using the software used in most studies: hellingerl [Kirkwood et al., 1999].

\section{Hellinger}

The Hellinger criterion [Hellinger, 1981] has been widely used for decades for calculating finite rotations. It is a measure of fit for the calculation of finite rotation poles. Let us consider a cluster of points (magnetic reversals or fracture zone crossings), each defined by a unit vector $v_{i j k}$ ( $i$ being the plate number, $j$ the segment number, and $k$ the datapoint identifier) (Figure IV-3). If a rotation $R$ is applied to the points of segment $J$ on plate 2 , the rotated vectors $v_{2 J k}$ become $R v_{2 J k}=v_{2 J k}^{\prime}$. If we then define the great circle that best fits both sets of $v_{1 J k}$ and $v_{2 J k}^{\prime}, \sigma_{1 J k}$ and $\sigma_{2 J k}^{\prime}$ are the standard deviations for $v_{1 J k}$ and $v_{2 J k}^{\prime}$ respectively. The rotation that best fits all data is that for which the squared sum of weighted deviations $\left(\sum\left(\frac{\sigma_{1 J k}}{\bar{\sigma}}\right)^{2} \sum\left(\frac{\sigma_{\prime \prime} J k}{\bar{\sigma}}\right)^{2}\right.$ where $\bar{\sigma}$ is the overall standard deviation of error) is minimum. 


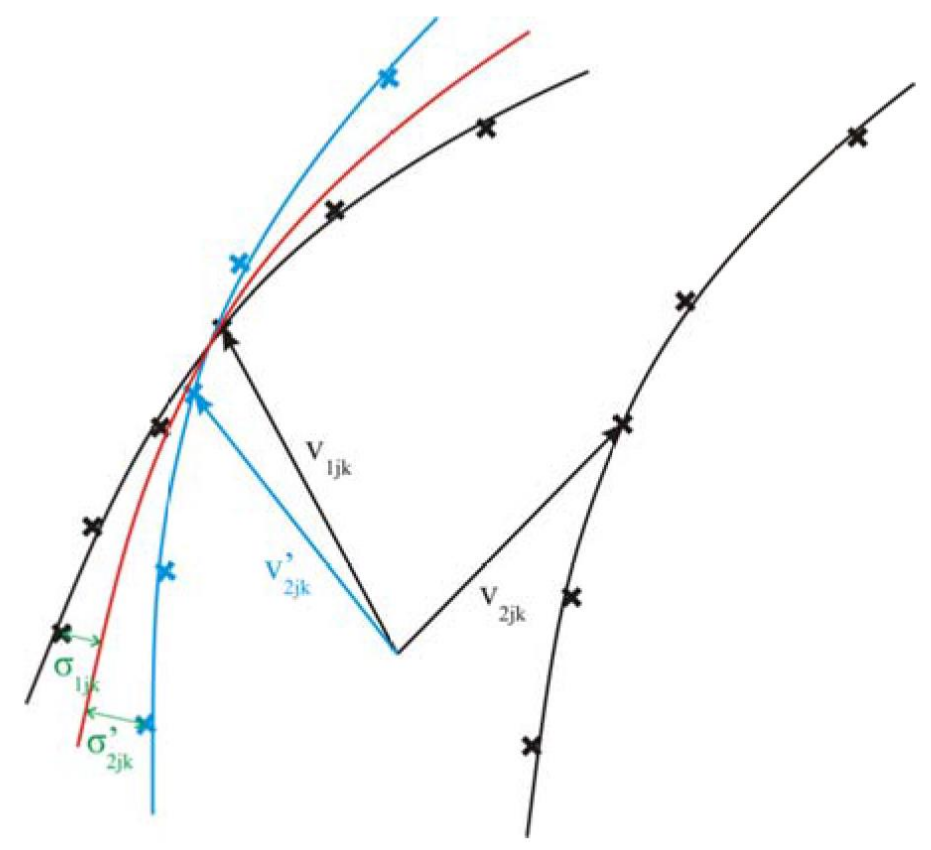

Figure IV-3: 3D schematic representation of the Hellinger criterion. $v_{1 J k}, v_{2 J k}$ and $v_{2 J k}^{\prime}$ are unit vectors (see text). $\sigma_{1 J k}$ and $\sigma_{2 J k}^{\prime}$ are the standard deviations (residuals) for $v_{1 J k}$ and $v_{2 J k}^{\prime}$ respectively. Black lines $=$ great circles that best fit the cluster of $v_{1 J k}$ and $v_{2 J k}$; cyan line $=$ great circle that best fits the cluster of $v^{\prime}{ }_{2 J k}$; red line $=$ great circle that best fits the cluster of both $v_{1 J k}$ and $v^{\prime}{ }_{2 J k}$.

The FORTRAN code hellingerl [Kirkwood et al., 1999], is based on the Hellinger criterion [Hellinger, 1981] using a statistical approach [Chang, 1988]. For each chron, it uses magnetic anomaly locations and fracture zone crossings as input data. Each fracture zone represents a "fracture zone segment"; "magnetic lineation segments" are similarly defined as groups of magnetic datapoints located between two fracture zone segments (Figure IV-1). For simplicity of data management I assigned odd numbers to fracture zone segments and even numbers to magnetic lineation segments (Figure IV-4). 
a)

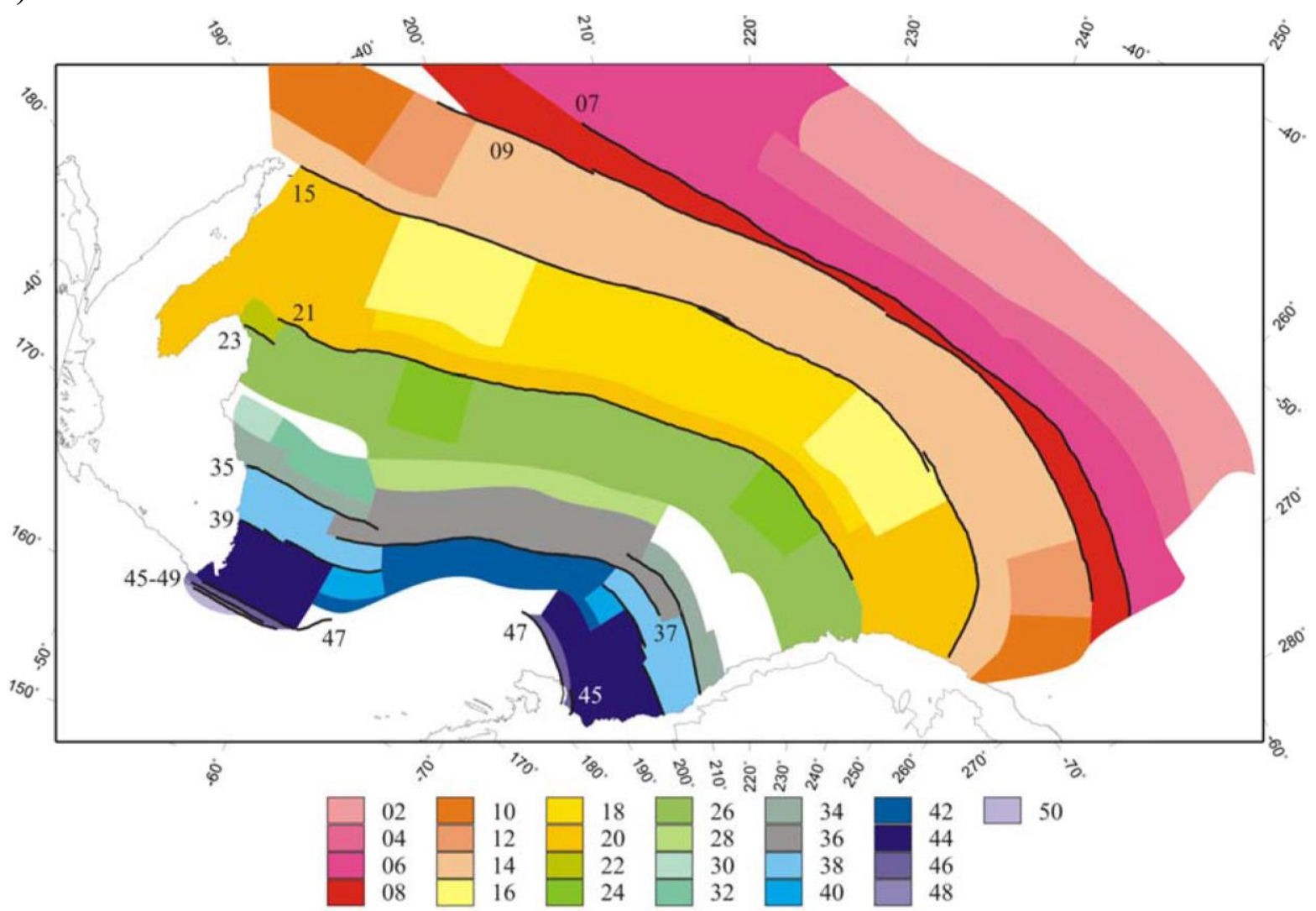

b)

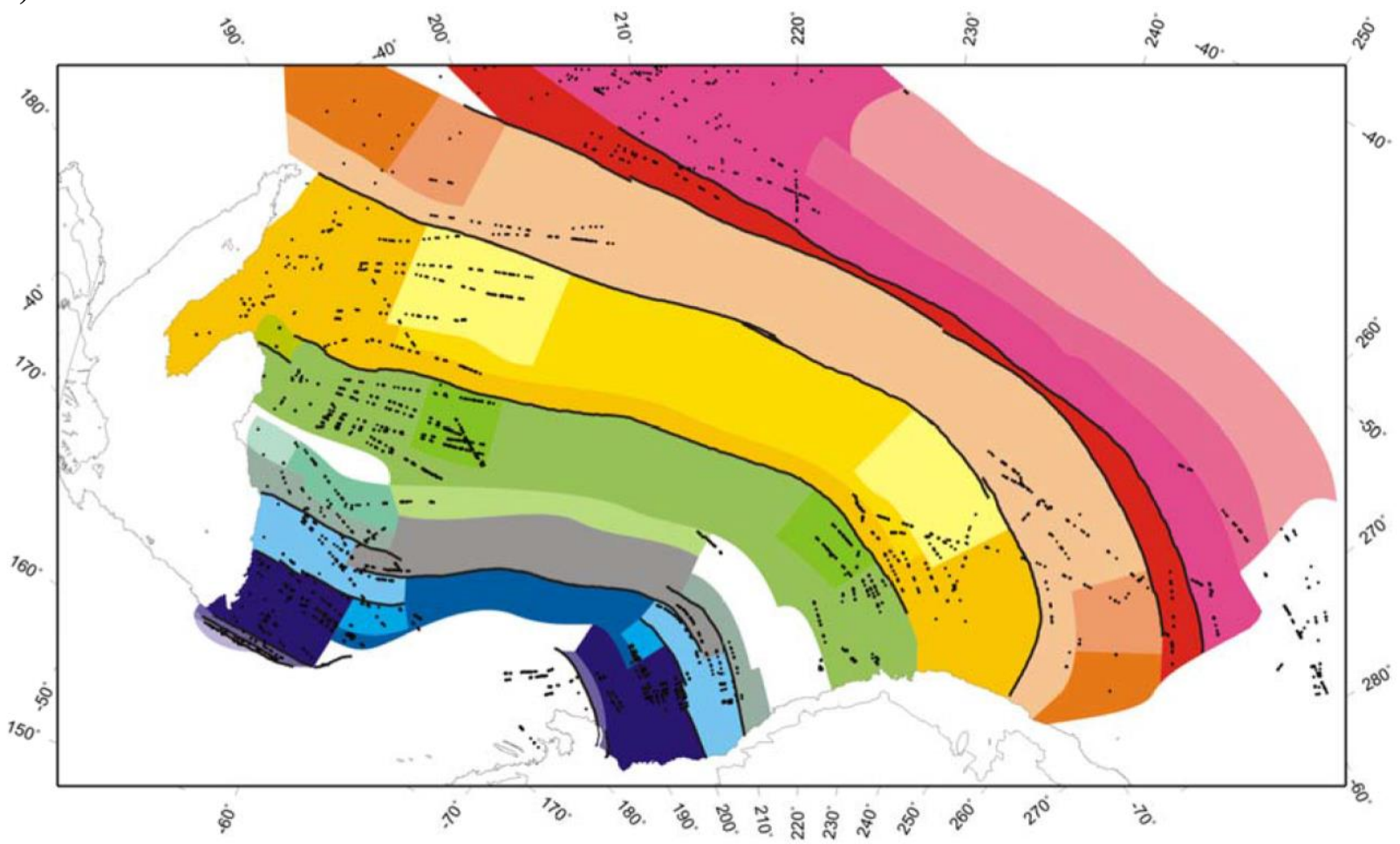

Figure IV-4: Fracture zone and magnetic lineation segments. a) Segment numbers are indicated on the map (fracture zones) or in the legend (magnetic data, colour-coded). b) The magnetic data have been added. See Appendix B for more information about the age of the magnetic data. Same colour scheme as in a). 
hellingerl defines a unit-length vector orthogonal to each segment (magnetic lineation or fracture zone) and to its conjugate and tries to match the direction of one vector to that of its conjugate after reconstructions. hellingerl uses a downhill simplex method (subroutine amoeba) to search for the best-fitting rotation pole. Residuals are calculated for each data point. The rotation pole corresponding to the lowest cumulated squared residuals is considered the best-fitting pole. The software does not make any distinction between fracture zone segments and magnetic lineation segments.

For the Hellinger program to produce stable results, I found that the input data must be abundant and spatially well distributed. Because the program considers each fracture zone datum as independently determined, it takes each of these points into account when calculating the best-fitting rotation. In a region like the Southwest Pacific, where fracture zone crossings are much more numerous than magnetic reversals, this gives too much weight to the fracture zone data compared to the magnetic anomaly data.

Consequently, several of the rotation poles calculated by hellingerl from the database I created (Chapter II and Chapter III) are unrealistic and inconsistent with previous studies (Figure IV-5). 


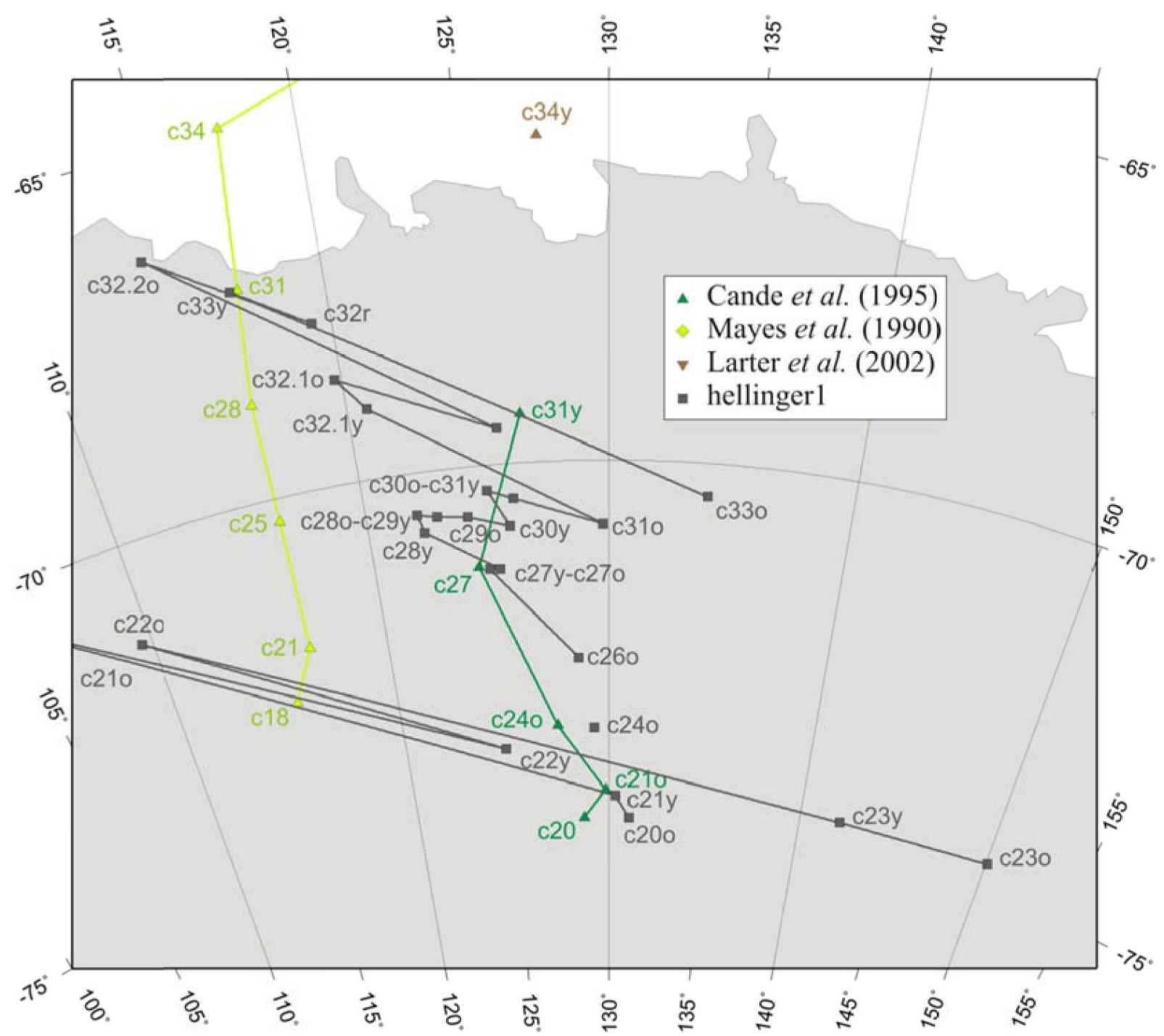

Figure IV-5: Finite rotation poles for the Southwest Pacific, obtained using hellingerl. Rotation poles from previous studies are also represented. hellingerl's rotation poles for c24y, c $25 \mathrm{y}$ and c25o are out of frame (latitudes vary from $-74.91^{\circ}$ to $-66.43^{\circ}$, longitudes from $-171.01^{\circ}$ to $\left.177.99^{\circ}\right)$.

hellingerl assumes the uncertainties assigned to each datapoint follow a von Misses distribution (see their $\hat{\kappa}$ ). However, because in the present situation an uncertainty represents a small portion of the sphere, the difference between a von Mises distribution and a bivariate Gaussian distribution is negligible.

Because the rotations calculated with hellingerl are sometimes unsatisfactory, I decided to try a different method to calculate finite rotations. In collaboration with my supervisor Pr. Euan Smith the software suite JaMBES was created. 


\section{JaMBES}

In 1965, Bullard, Everett \& Smith [Bullard et al., 1965] introduced the idea of calculating, by successive approximation, with a measure of fit (least-squares), the closure between two conjugate sets of points. [Bullard et al., 1965] applied Euler's theorem to fit the coasts of Africa and South America, closing the southern Atlantic Ocean. Although their method was developed for fitting continental margins, it can be applied to any set of points defining conjugate contours, such as magnetic anomalies and fracture zones.

[Morgan, 1968] describes the foundation of the theory of plate tectonics: in a stable spreading system, the ridge segments are roughly at right angles to the neighbouring fracture zones, which are parallel to the direction of spreading. Hence, fracture zones and ridge segments (or magnetic lineations) can be assimilated to small and great circles respectively of a sphere whose poles are the Euler rotation poles of the plates system.

JaMBES' basic principle is derived from the ideas introduced by [Bullard et al., 1965] and [Morgan, 1968]. The software suite is made of three Matlab scripts, which must be run in order: poledatain, onepole and JaMBES. poledatain reads the input data (magnetic lineations, fracture zones and other parameters) and organizes them into a convenient format, to be used by onepole and JaMBES.

\subsection{Onepole}

Both hellingerl and onepole define magnetic lineations and fracture zones as great and small circles. Issues arise with hellingerl when the density of datapoints is low (see IV-3). onepole adds a further constraint (each magnetic lineation being orthogonal to the adjacent fracture zones) as a way to compensate for the lack of abundant data (Figure IV-6). 

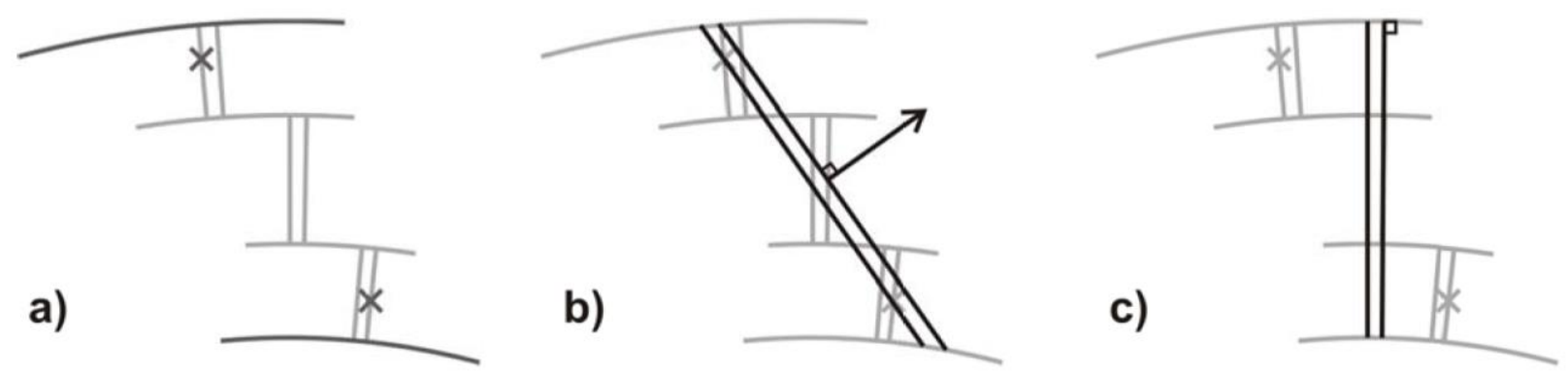

Figure IV-6: Comparison between the hellingerl software and our JaMBES Matlab software: definition of the segments. a) Real configuration : between two major fracture zones, one major ridge segment is in fact made of three second-order segments. The two crosses are the magnetic anomalies available from shiptrack data. The two intervening fracture zones are not known. b) Ridge segment as defined by hellingerl: the orientation of the best-fit great circle is not parallel to the real ridge segment. hellingerl cannot obtain a good fit on both the fracture zones and the ridge segment. c) Ridge segment as defined by JaMBES: the orientation of the best-fit great circle is much closer to the real one. When calculating a reconstruction, the finite rotation pole will be accurately determined, the misfit being absorbed by the rotation angle uncertainty.

onepole calculates Euler poles for each of a series of consecutive chrons in a two plates system. The poles are calculated independently for each chron. For each chron, onepole does a grid search for the best-fitting pole by computing the misfit at each grid point. The total misfit has three contributions:

- the magnetic lineation mismatch is the cumulative squared distance between each magnetic datapoint and its segment's great circle;

- the fracture zone segment mismatch is the cumulative squared angular distance between each fracture zone datapoint and its fracture zone's small circle;

- the rotation angle misfit quantifies the misclosure from back-rotation.

Because the misfit is calculated for each grid point, 95\% confidence regions are extracted directly from the pole calculation for each chron (Figure IV-7). 


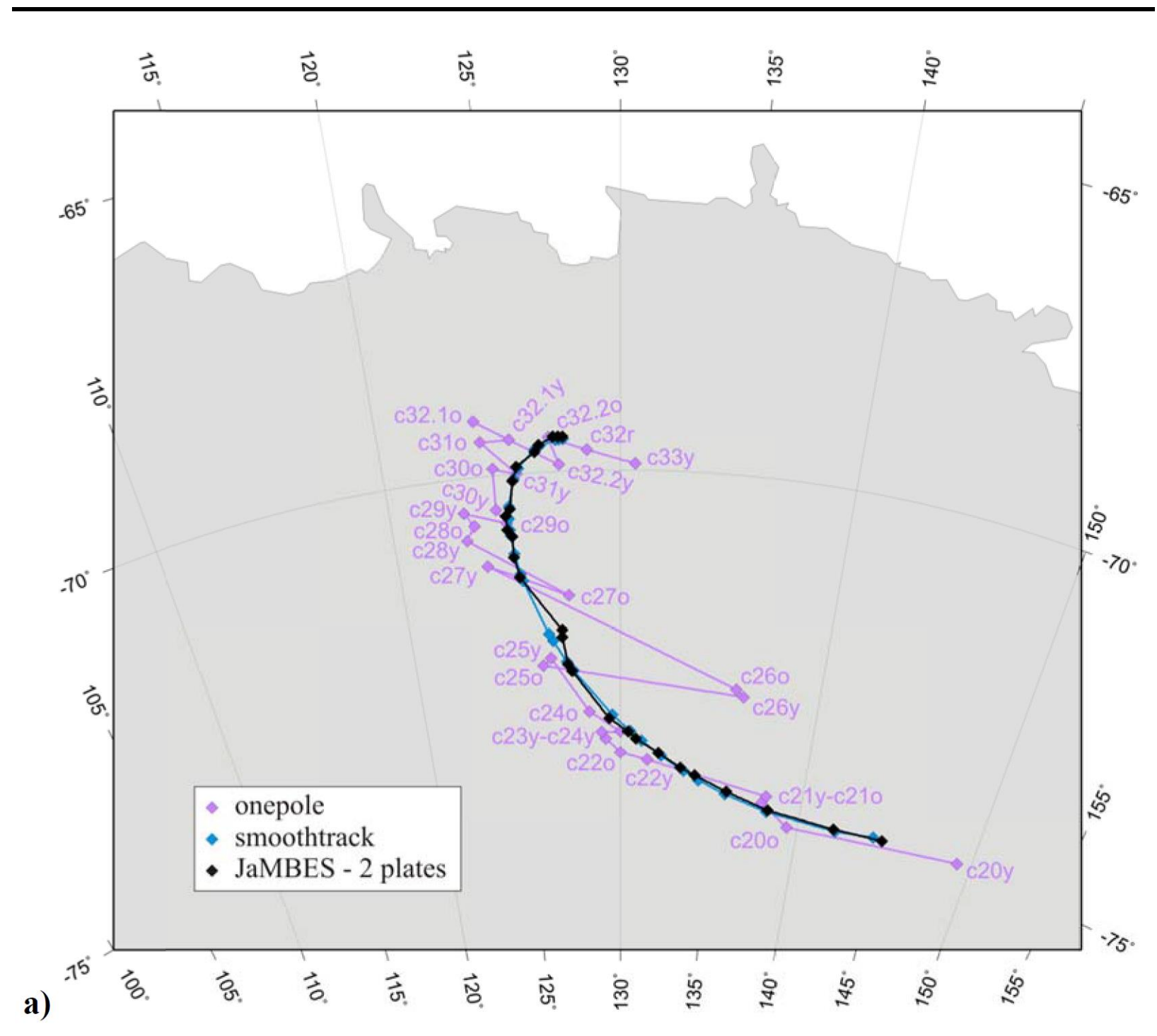




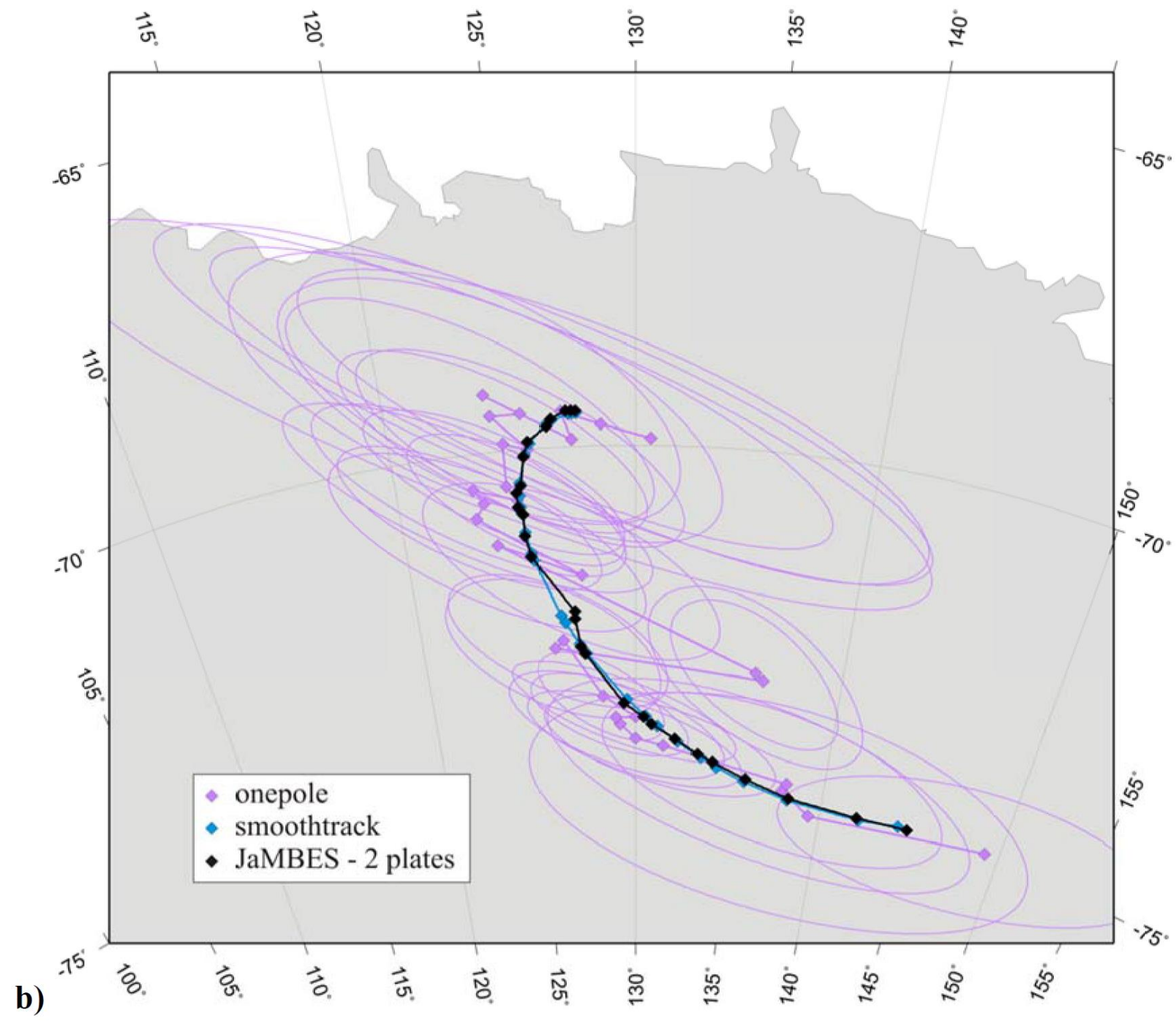

Figure IV-7: Comparison between the finite rotation poles calculated by onepole, smoothtrack and JaMBES. 95\% confidence regions are included for onepole calculations on b). Chron labels are left off $J a M B E S$ ' poles for clarity but they can be identified by tracing in order from onepole.

onepole uses the same segmentation (i.e. ridge and fracture zone numbers) as hellingerl. The user can choose not to use specific data (e.g. magnetic data from segment 6 is excluded from the calculation of Pacific-Antarctica rotations because the seafloor formed on the PacificPhoenix ridge).

onepole does the grid search three times, each time re-centering the grid on the bestfitting pole of the previous iteration. The search grid spacings for the first and final searches are set by the user, so as to increase the precision of the calculation while minimizing the computing time. 
In order to minimize the contribution of possible misidentified magnetic data (without annulling it), Jeffrey's weighting can be applied to the calculations during the second and third iterations. Jeffrey's method calculates the probability each residual has of being part of a Gaussian distribution with a variance determined by the central part of the residual distribution. It then uses that probability to assign weights to each datapoint. This has the effect of weighting extreme outliers to zero, while retaining data (with lowered weight) which could be valid. The user chooses whether to use Jeffrey's weights. It was found to be unnecessary to apply Jeffrey's weighting to fracture zone datapoints because their residuals seldom had outliers.

\subsection{Smoothtrack and JaMBES}

One commonly encountered problem with plate tectonic reconstructions is the lack of fluidity of movement of the plates. This is evident in the roughness of the rotation poles track (e.g. zigzag versus smooth curve). In an effort to produce more realistic plate tectonic evolution illustrations, JaMBES was developed ${ }^{18}$.

JaMBES is a two-step code. The first step consists of calculating a smooth equivalent to the track of Euler poles calculated by onepole (Figure IV-7). To do that, JaMBES calculates the polynomial function that best fits the onepole track. The smooth track thus obtained is a function of time, longitude and latitude. The polynomial order is user-defined. A polynomial order of 3 was chosen in the present case (Figure IV-7). JaMBES' second step is to calculate a "compromise", between the smooth track and the "true" Euler pole track (onepole, Figure IV7). To do that, JaMBES calculates a maximum likelihood estimate of the rotation poles (JaMBES results in). The likelihood function is a product of two Gaussian functions: one for the onepole uncertainties and the other for the smooth track uncertainties. The outcome is a sequence of finite rotation poles that allows continuous movement of the plates while remaining as close as possible to the "true" poletrack.

\footnotetext{
${ }^{18} \mathrm{I}$ am aware of the release of the new software REDBACK in 2014. However, due to the lack of examples in the published version of the software [Iaffaldano et al., 2014], I decided to use JaMBES. Moreover, REDBACK was released after most of the work on designing JaMBES had been done.
} 


\subsection{How does JaMBES compare to other software?}

Because onepole and hellingerl are similar in method they are comparable (Figure IV-8). Some rotation poles calculated with hellingerl and plotted on Figure IV-8 are consistent with the onepole ones. However, some of the Euler poles calculated with hellingerl are hundreds of kilometers away from each other (i.e. c22o-c23y) and from [Cande et al., 1995]'s poles, whereas those calculated with onepole are all within reasonable distance from [Cande et al., 1995]'s rotation poles (Figure V-2) ${ }^{19}$. This shows that onepole (and to a larger extent JaMBES) is more reliable than hellingerl when calculating rotations in areas where the magnetic (and/or fracture zone) data are not abundant and/or uniformly spatially distributed.

19 I use [Cande et al., 1995] as a comparison because it is to my knowledge the most recent finite rotation calculation available for the whole Southwest Pacific, because it is cited by numerous papers and because the flowlines calculated from their rotations fit the Pitman fracture zone well. 


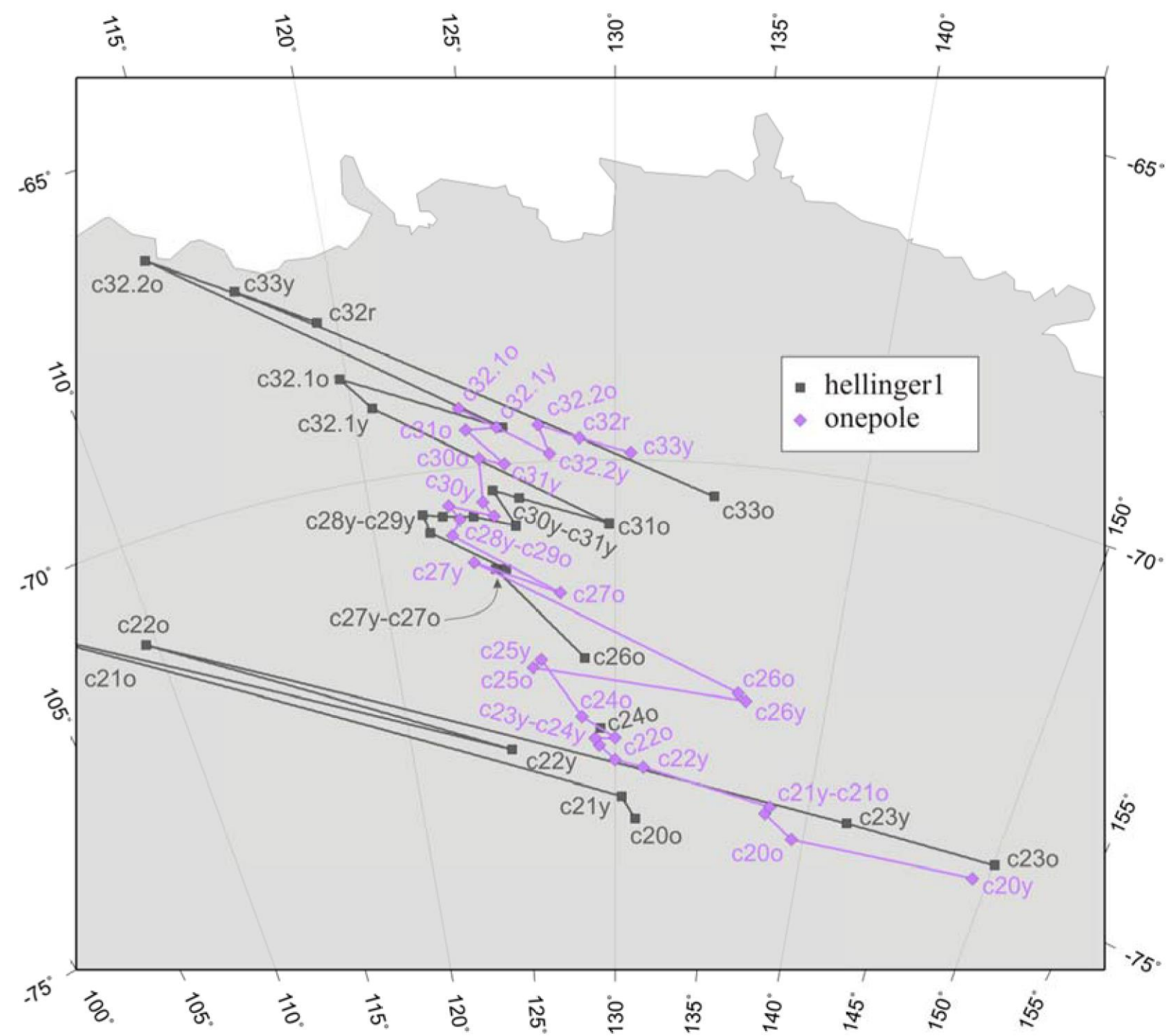

Figure IV-8: Comparison between the finite rotation poles calculated by hellingerl and onepole. hellingerl's rotation poles for $\mathrm{c} 24 \mathrm{y}, \mathrm{c} 25 \mathrm{y}$ and $\mathrm{c} 25 \mathrm{o}$ are out of frame (latitudes vary from $-74.91^{\circ}$ to $-66.43^{\circ}$, longitudes from $-171.01^{\circ}$ to $177.99^{\circ}$ ).

In summary, adding the constraint of orthogonality between ridge segments and fracture zones does efficiently compensate for localized lacks of data (onepole). 
Chapter IV: Plate reconstructions: methods 


\section{Chapter V}

RESULTS AND CONCLUSIONS 
In this final chapter I present quantitative plate reconstruction results and I use those results to test the Bellingshausen plate hypothesis. Finite rotations were calculated with the JaMBES method which is appropriate for the sparse distribution of magnetic data in the southwest Pacific. I start by addressing the issue of data quality versus quantity, because the spatial distribution of data is a primary concern for this analysis.

\section{Inclusion of data based on identification uncertainty}

Most plate rotation studies [e.g. Cande et al., 1995; Eagles et al., 2004b; Molnar et al., 1975; Stock et al., 1996; Weissel et al., 1977] do not take into account the uncertainty of identification of magnetic reversals or anomalies when calculating rotations. The decision of whether to include or reject a datum in the analysis is a complex one. As detailed in Chapter II, a systematic cataloguing of identification uncertainty was made when I interpreted each magnetic profile.

To investigate the impacts of including higher levels of identification uncertainty, I calculated rotations for a two plate Pacific-Antarctica system using progressive inclusion of higher uncertainty categories. The rotation poles obtained from these calculations are displayed in Figure V-1. 


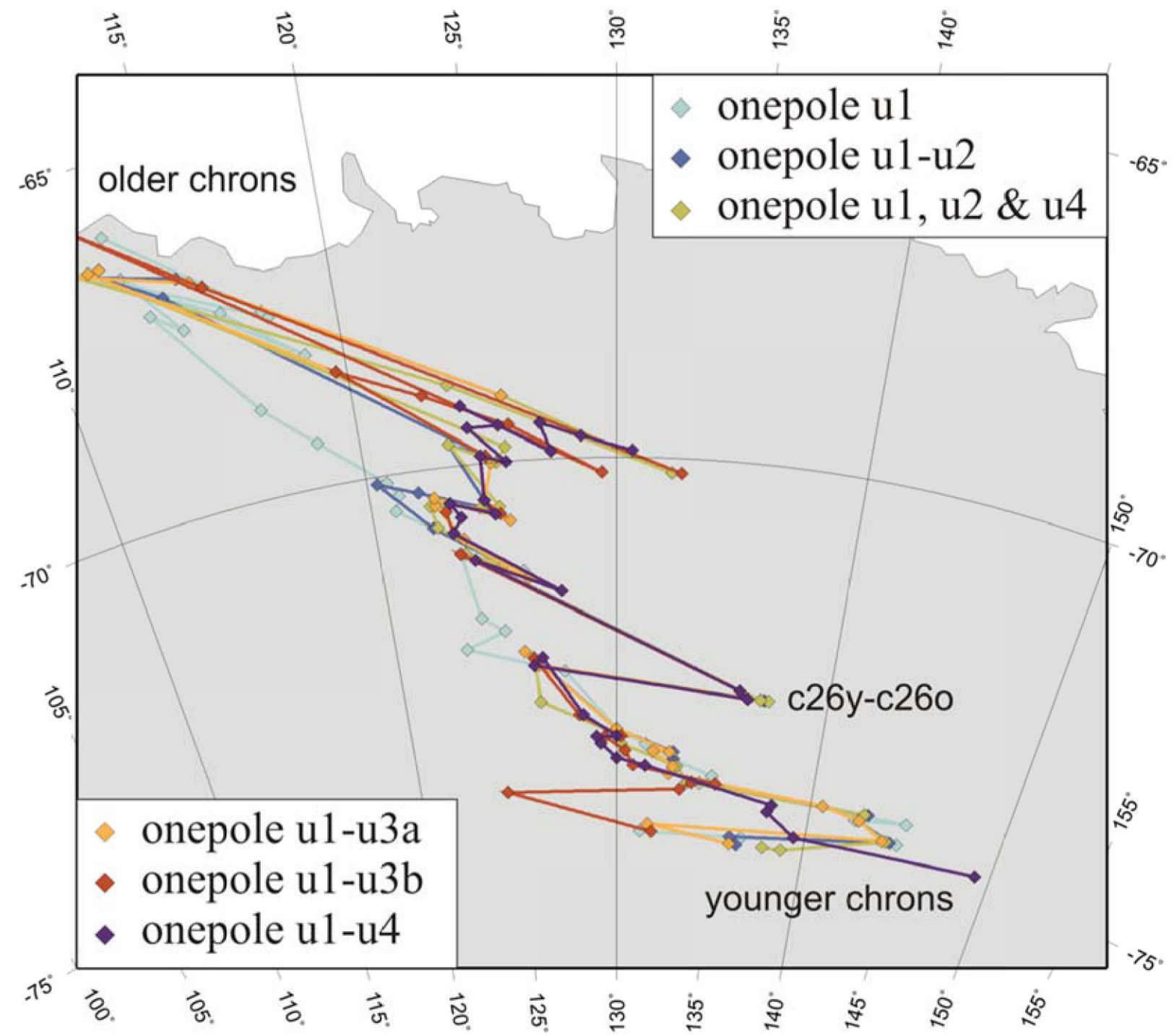

Figure V-1: onepole finite rotation poles, calculated including magnetic anomaly data with progressively higher identification uncertainty category: only the very best (u1); anomalies whose identification uncertainty is low $(\mathrm{u} 1-\mathrm{u} 2)$; anomalies whose identification uncertainty is either low or the highest (who have a 0.1 probability of being correctly identified; solution $\mathrm{u} 1$, $\mathrm{u} 2 \& \mathrm{u} 4)$; all of the anomalies (u1-u4). In cases where multiple plausible identifications of the same anomaly were made on a profile, I consider two types of solution: just the preferred identifications (u1-u3a) or all plausible interpretations (u1-u3b).

Solution u1, which contains a small number of the most certain magnetic anomaly identifications, differs significantly from all other solutions. For instance, it does not show the eastward excursion at c26y-c26o that affects other solutions.

The finite rotations calculated for c20y to c21o vary substantially between each solution. Only solution $\mathrm{u} 1 \mathrm{-u} 3 \mathrm{~b}$ reproduces the change of spreading direction around that time quantified by [Cande et al., 1995], which is identifiable using gravity and swath bathymetry data as a change of fracture zone azimuth. However, rotations calculated for c20y-c21o also have large 
95\% confidence regions, which might account for this discrepancy. Such large error ellipses are mainly due to the small number of datapoints.

For chrons older than c29o and c31y respectively, the rotations calculated for solutions u1 and $\mathrm{u} 1-\mathrm{u} 4$ differ from the other solutions. The number of data with identification uncertainty category $\mathrm{u} 1$ is small. This likely accounts for the inconsistency between model $\mathrm{u} 1$ and the others. Conversely, solution $\mathrm{u} 1-\mathrm{u} 4$ utilises the largest amount of magnetic data of all the solutions. This in turn accounts for the rotations older than $\mathrm{c} 31 \mathrm{y}$ being more precisely defined than for other models (smaller 95\% ellipses, better residuals; see Figure V-3). Rotations with reasonable $95 \%$ confidence ellipses were calculated for model u1-u 4 for c32.1y-c33y.

I conclude that inclusion of data with higher uncertainty categories (with appropriate weighting; models $\mathrm{u} 1-\mathrm{u} 3 \mathrm{a}$ and $\mathrm{u} 1-\mathrm{u} 4)$ gives consistent results and leads to higher precision. The gains of using more data outweigh the risk of including erroneous identifications. In particular, I was able to calculate older rotations (c32.1y to c33y) using the u1-u4 solution. The rotations below were all calculated using $\mathrm{u} 1-\mathrm{u} 4$ data.

\section{Two-plate solution}

The first set of rotations calculated in this study is that of a two-plate, Pacific-Antarctica system (Table V-1). It corresponds to the plate configuration first considered in plate tectonic studies of the Southwest Pacific [Molnar et al., 1975; Weissel et al., 1977]. It is the simplest configuration possible for the creation of seafloor. The analysis of [Cande et al., 1995] is the most reliable published two-plate solution, because it is the only published analysis to use swath bathymetry mapping of fracture zones. 


\begin{tabular}{|c|c|c|c|c|c|c|}
\hline \multirow{2}{*}{ Chron } & \multicolumn{3}{|c|}{ onepole } & \multicolumn{3}{|c|}{ JaMBES } \\
\hline & longitude & latitude & angle $\left({ }^{\circ}\right)$ & Iongitude & latitude & angle $\left({ }^{\circ}\right)$ \\
\hline$c 20 y$ & 149.2 & -74.9 & 43.0868 & 144.8 & -74.9 & 40.7572 \\
\hline $\mathrm{c} 20 \mathrm{o}$ & 139.4 & -75.0 & 38.7419 & 142.0 & -74.9 & 39.9051 \\
\hline c21y & 137.8 & -74.7 & 40.4354 & 138.2 & -74.8 & 40.3961 \\
\hline c21o & 138.0 & -74.6 & 41.0233 & 135.8 & -74.6 & 39.8719 \\
\hline$c 22 y$ & 131.4 & -74.2 & 38.9019 & 134.0 & -74.4 & 40.1296 \\
\hline c22o & 130.0 & -74.1 & 38.6533 & 133.2 & -74.3 & 40.0870 \\
\hline$c 23 y$ & 129.2 & -73.9 & 39.0004 & 132.0 & -74.1 & 40.3161 \\
\hline c23o & 129.0 & -73.8 & 39.4515 & 130.8 & -73.9 & 40.2945 \\
\hline c24y & 130.0 & -73.8 & 39.8796 & 130.4 & -73.8 & 40.1617 \\
\hline$c 240$ & 128.4 & -73.5 & 40.3570 & 129.4 & -73.6 & 40.8267 \\
\hline c25y & 126.6 & -72.7 & 41.4797 & 127.6 & -72.9 & 42.0107 \\
\hline c25o & 126.2 & -72.8 & 41.7495 & 127.4 & -72.8 & 42.2844 \\
\hline c26y & 136.2 & -73.2 & 46.5793 & 127.2 & -72.4 & 41.1067 \\
\hline$c 26 \circ$ & 135.8 & -73.1 & 46.5817 & 127.2 & -72.3 & 41.6928 \\
\hline c27y & 124.0 & -71.3 & 44.4224 & 125.4 & -71.5 & 45.0864 \\
\hline c27o & 127.6 & -71.8 & 46.7067 & 125.4 & -71.5 & 45.5830 \\
\hline c28y & 123.2 & -70.9 & 45.2033 & 125.2 & -71.2 & 45.9566 \\
\hline c28o & 123.6 & -70.7 & 46.6743 & 125.2 & -70.9 & 47.2779 \\
\hline c29y & 123.2 & -70.5 & 46.7578 & 125.0 & -70.8 & 47.4131 \\
\hline c29o & 125.0 & -70.7 & 48.1519 & 125.0 & -70.6 & 47.8844 \\
\hline c30y & 124.6 & -70.5 & 48.5475 & 125.2 & -70.5 & 48.6517 \\
\hline$c 30 \circ$ & 124.6 & -69.9 & 49.7916 & 125.4 & -70.1 & 50.4806 \\
\hline c31y & 125.6 & -70.0 & 50.1687 & 125.4 & -70.1 & 50.5055 \\
\hline c310 & 124.2 & -69.5 & 50.4840 & 125.6 & -69.9 & 51.8467 \\
\hline c32.1y & 125.4 & -69.5 & 53.6038 & 126.4 & -69.7 & 54.0469 \\
\hline c32.10 & 124.0 & -69.2 & 53.7628 & 126.6 & -69.6 & 54.7505 \\
\hline c32.2y & 127.4 & -69.9 & 53.9640 & 126.6 & -69.6 & 53.7947 \\
\hline c32.20 & 127.0 & -69.5 & 56.6283 & 127.2 & -69.5 & 56.6856 \\
\hline$c 32 r$ & 128.6 & -69.7 & 57.7902 & 127.4 & -69.5 & 57.4605 \\
\hline c33y & 130.6 & -69.9 & 59.9035 & 127.6 & -69.5 & 58.3377 \\
\hline c33o & 348.0 & 64.7 & 89.2303 & & & \\
\hline
\end{tabular}

Table V-1: Coordinates of the finite rotation poles calculated with onepole and JaMBES for a two plate model.

Figure V-2 compares the finite rotation poles calculated with JaMBES to published analyses of the Southwest Pacific two-plate system (Pacific-Antarctica). Rotation poles of Mayes et al. (1990) are farthest from those of Cande et al. (1995), but are based on a less precise dataset. There is a strong similarity between the location of the JaMBES rotation poles and those of Cande [Cande et al., 1995] and some other previous studies [Molnar et al., 1975; Weissel et al., 1977] (Figure V-2). 


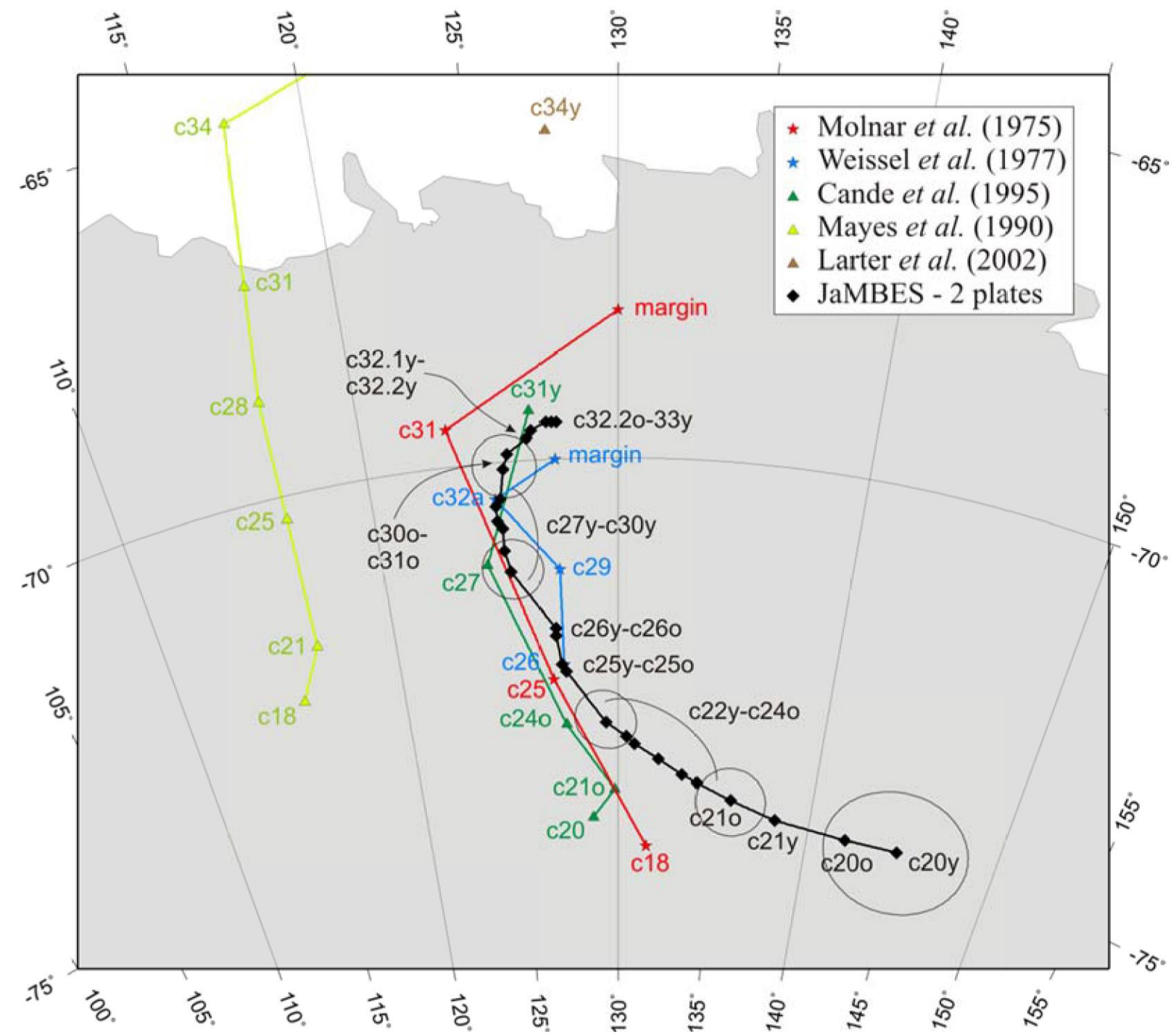

Figure V-2: Finite rotation poles calculated using JaMBES (two plate system) compared to previous studies. JaMBES 95\% confidence regions are included for c20y, c21o, c24o, c27o and c31y.

The rotation poles calculated for chrons younger than c22y diverge from the Cande [Cande et al., 1995] rotation poles, and the observed fracture zone trend in the southwest Pacific. These rotation poles have relatively large onepole 95\% error ellipses (Figure V-3), and the difference is significant for the youngest rotations. The onepole solution for c20y is doubtful and as it is the youngest chron considered its influence on the JaMBES calculation is strong. The divergence is also likely due to my interpretation of the seafloor and magnetic data being different from that of [Cande et al., 1995]. As the period c20y-c24o is not the focus of this work, I decided not to investigate further. 

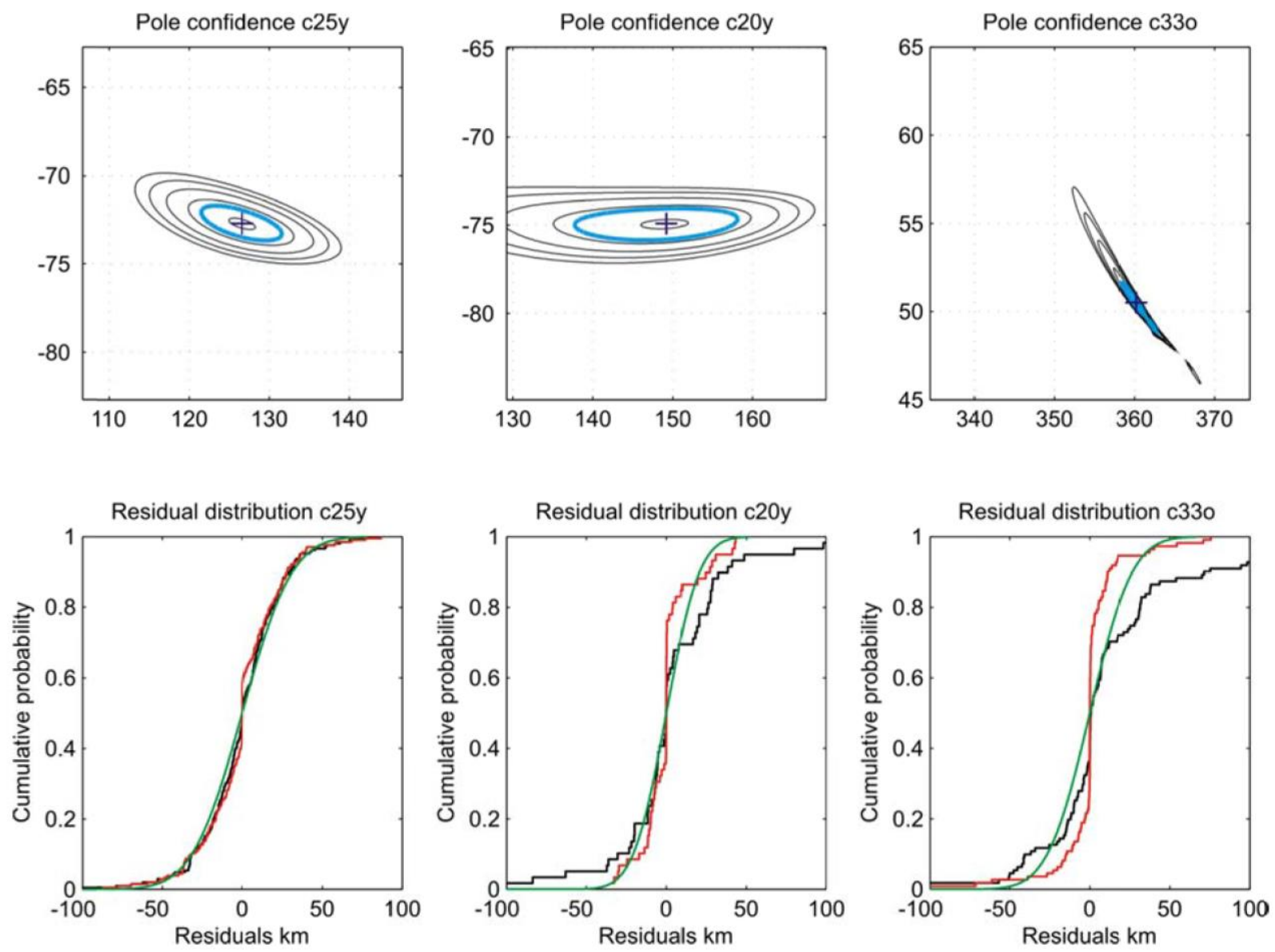

Figure V-3: Rotation poles confidence ellipses calculated with onepole and residual distribution, for $\mathrm{c} 25 \mathrm{y}, \mathrm{c} 20 \mathrm{y}$ and c33o. Top row) For each chron, best fit rotation pole (onepole) in dark blue, 95\% confidence ellipse in cyan. Bottom row) For each chron, raw residuals in black, weighted residuals (Jeffrey's weighting) in red and Gaussian model in green. Note the coordinates of the $\mathrm{c} 33 \mathrm{o}$ finite rotation pole.

A key question concerning the plate tectonic history of the Southwest Pacific that I aim to address is the existence and motion of an independent Bellingshausen plate [Stock and Molnar, 1987; Stock et al., 1996].

The existence of the Bellingshausen plate is evident from inspection of the alignment of fracture zone data and their rotated conjugates (Figure V-4). If fracture zone segments and their conjugates can be aligned with a single rotation, then a two plate model is satisfactory (c25y in Figure V-4). However if adjacent fracture zones are perfectly aligned with their conjugates using a rotation, but the alignment of a sector of fracture zones with their conjugates presents a systematic offset (c29o in Figure V-4), then more than two plates are required to match the fracture zone conjugates. Based on the plate tectonic reconstructions produced from the 
rotations in Table $\mathrm{V}-1$, the existence of a third plate in the southwest Pacific (Bellingshausen) for times older than c28y is unequivocal. 

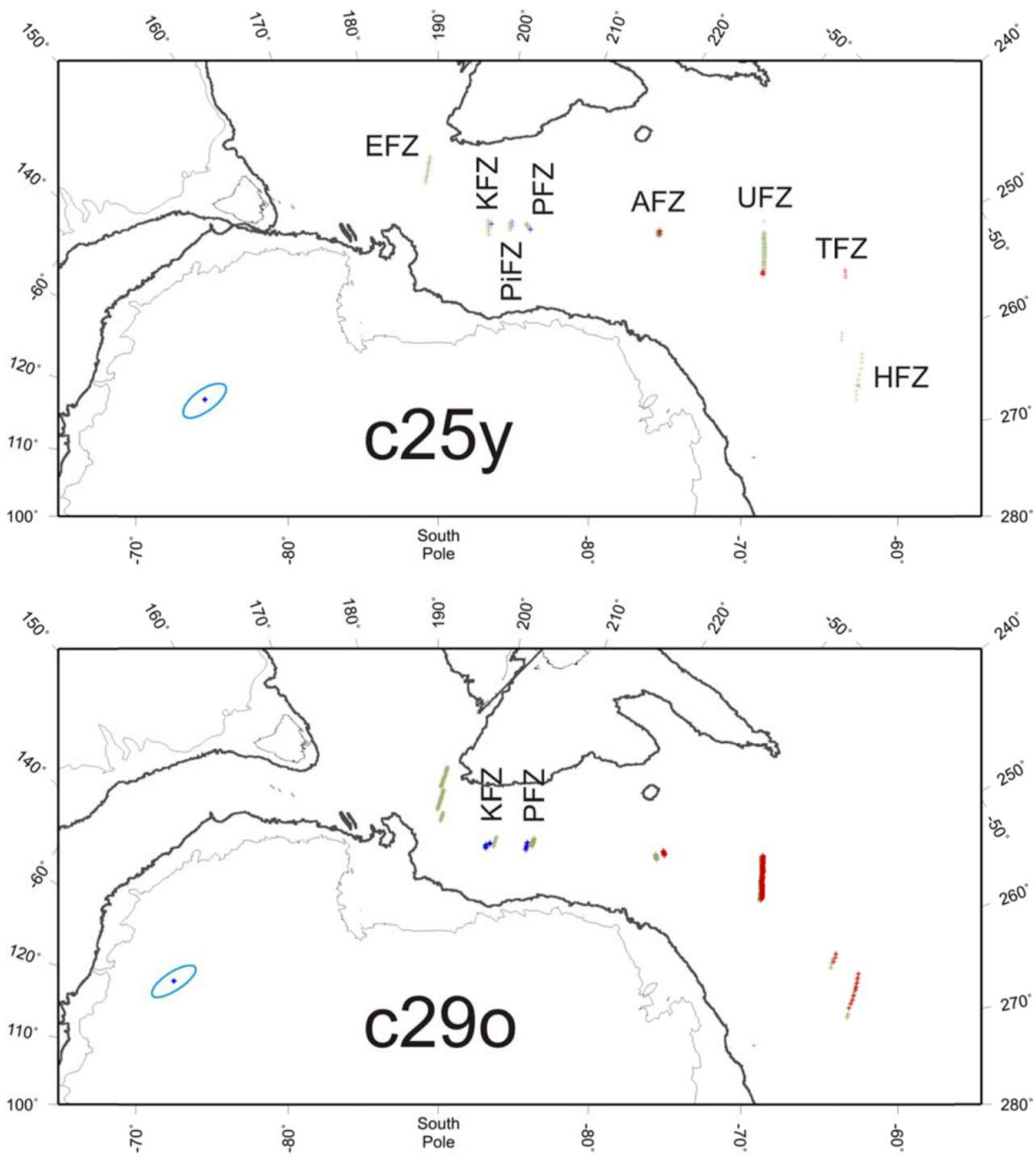

Figure V-4: Tectonic reconstructions for the Southwest Pacific using a two plate system (Pacific-Antarctica). Magnetic data were removed from the figure for clarity. Pacific-Antarctica finite rotation pole used and 95\% ellipse are plotted in cyan. Fracture zone points for each chron: blue for Antarctica south of the Antipodes FZ, red for Antarctica north of and including the Antipodes FZ, green for the Pacific plate. The systematic mismatch of conjugate Antipodes FZ segments is likely due to inaccurate picking of the Antarctica fracture zone data: the gravity signal of the Antipodes FZ is less strong near Antarctica, and the presence of the Marie Byrd seamounts complicates interpretation of the gravity signal. 


\section{Three-plate solution}

To quantify Bellingshausen plate motion, I calculated finite rotations independently for the southern and northern parts of the Southwest Pacific, with the boundary between the two systems placed along the Antipodes FZ, following [Stock et al., 1996] and [Heinemann et al., 1999]. Antipodes FZ data were included in the calculation of both the Pacific-Marie Byrd Land and the Pacific-Bellingshausen finite rotations. The results of the calculations are grouped in Table V-2 and Table V-3, and in Figure V-5 and Figure V-6. 


\begin{tabular}{|c|c|c|c|c|c|c|}
\hline \multirow{2}{*}{ Chron } & \multicolumn{3}{|c|}{ onepole } & \multicolumn{3}{|c|}{ JaMBES } \\
\hline & longitude & latitude & angle $\left({ }^{\circ}\right)$ & \begin{tabular}{|l} 
longitude \\
\end{tabular} & latitude & angle $\left({ }^{\circ}\right)$ \\
\hline$c 20 y$ & 152.4 & -74.8 & 44.9163 & 151.2 & -74.8 & 43.1635 \\
\hline $\mathrm{c} 20 \mathrm{o}$ & 144.4 & -75.1 & 40.9617 & 146.4 & -75.0 & 41.8585 \\
\hline c21y & 145.2 & -75.0 & 44.2727 & 140.2 & -75.0 & 41.3261 \\
\hline c21o & 142.2 & -74.8 & 43.1917 & 137.0 & -74.9 & 40.4126 \\
\hline$c 22 y$ & 131.6 & -74.5 & 39.0293 & 134.6 & -74.7 & 40.4096 \\
\hline c22o & 130.4 & -74.4 & 38.8913 & 133.8 & -74.6 & 40.3712 \\
\hline c23y & 128.8 & -74.2 & 38.8937 & 132.6 & -74.4 & 40.6359 \\
\hline c23o & 128.0 & -74.0 & 39.0633 & 131.2 & -74.2 & 40.5359 \\
\hline c24y & 126.8 & -73.8 & 38.7895 & 131.6 & -74.1 & 40.6810 \\
\hline c24o & 128.6 & -73.7 & 40.4999 & 130.0 & -73.8 & 41.1534 \\
\hline$c 25 y$ & 132.8 & -73.3 & 44.8630 & 130.2 & -73.2 & 43.3887 \\
\hline c25o & 131.0 & -73.1 & 44.2661 & 130.0 & -73.1 & 43.6537 \\
\hline$c 26 y$ & 140.6 & -73.1 & 49.8318 & 130.2 & -72.7 & 42.8231 \\
\hline c26o & 139.2 & -73.0 & 49.0457 & 130.4 & -72.6 & 42.9572 \\
\hline c27y & 123.8 & -71.4 & 44.4566 & 127.0 & -71.6 & 45.8010 \\
\hline c27o & 124.6 & -71.4 & 44.9234 & 126.6 & -71.5 & 46.0535 \\
\hline c28y & 121.6 & -70.7 & 44.4398 & 125.2 & -71.0 & 45.8553 \\
\hline c28o & 124.2 & -70.7 & 47.1009 & 124.0 & -70.5 & 46.6201 \\
\hline c29y & 123.8 & -70.5 & 47.0902 & 123.4 & -70.3 & 46.5384 \\
\hline c29o & 123.6 & -70.3 & 47.2113 & 122.4 & -70.0 & 46.4311 \\
\hline c30y & 120.0 & -69.6 & 46.0823 & 120.8 & -69.5 & 46.2962 \\
\hline $\mathrm{c} 30 \mathrm{o}$ & 112.6 & -66.8 & 42.6223 & 117.0 & -68.1 & 45.9091 \\
\hline c31y & 115.8 & -67.5 & 44.2716 & 116.6 & -68.0 & 45.6010 \\
\hline c31o & 114.2 & -66.5 & 44.2754 & 114.4 & -67.3 & 45.7993 \\
\hline c32.1y & 113.4 & -67.3 & 46.5530 & 108.0 & -65.4 & 43.6618 \\
\hline c32.10 & 105.2 & -64.7 & 42.7735 & 107.2 & -65.1 & 43.8778 \\
\hline c32.2y & 82.8 & -50.0 & 29.8759 & & & \\
\hline$c 32.20$ & 83.6 & -50.1 & 33.3293 & & & \\
\hline$c 32 r$ & 83.4 & -50.0 & 33.5284 & & & \\
\hline c33y & 86.6 & -52.8 & 35.2809 & & & \\
\hline c33o & 295.6 & 41.3 & 35.4101 & & & \\
\hline
\end{tabular}

Table V-2: Pacific-Marie Byrd Land finite rotations calculated with onepole and JaMBES for a three plate model. onepole rotations for $\mathrm{c} 32.2 \mathrm{y}-\mathrm{c} 33 \mathrm{o}$ were inconclusive, and were not included in the JaMBES calculations. 


\begin{tabular}{|c|c|c|c|c|c|c|}
\hline \multirow{2}{*}{ Chron } & \multicolumn{3}{|c|}{ onepole } & \multicolumn{3}{|c|}{ JaMBES } \\
\hline & longitude & latitude & angle $\left({ }^{\circ}\right)$ & longitude & latitude & angle $\left(^{\circ}\right)$ \\
\hline c20y & 90.0 & -65.5 & 26.4330 & & & \\
\hline c20o & 90.2 & -65.4 & 26.4604 & & & \\
\hline c21y & 129.2 & -73.9 & 36.7068 & 132.8 & -74.2 & 37.9295 \\
\hline c210 & 133.6 & -74.2 & 38.6698 & 133.0 & -74.2 & 38.5700 \\
\hline c22y & 138.8 & -74.5 & 40.8916 & 132.6 & -74.1 & 39.4654 \\
\hline c22o & 130.0 & -74.0 & 38.4257 & 131.6 & -74.1 & 39.4269 \\
\hline c23y & 129.6 & -73.8 & 38.7622 & 130.8 & -73.9 & 39.7254 \\
\hline c23o & 128.4 & -73.5 & 38.9791 & 130.0 & -73.7 & 39.8794 \\
\hline c24y & 131.0 & -73.8 & 40.0579 & 129.8 & -73.6 & 39.8810 \\
\hline c24o & 133.8 & -74.1 & 41.5236 & 129.2 & -73.5 & 40.7073 \\
\hline c25y & 127.6 & -72.8 & 41.5705 & 126.8 & -72.7 & 41.5586 \\
\hline c25o & 126.6 & -73.0 & 41.7510 & 126.2 & -72.8 & 41.7230 \\
\hline c26y & 125.4 & -72.3 & 42.2060 & 125.4 & -72.3 & 40.1447 \\
\hline c26o & 125.6 & -72.3 & 42.5544 & 125.4 & -72.3 & 41.0803 \\
\hline c27y & 125.8 & -71.7 & 44.9713 & 125.0 & -71.6 & 44.9716 \\
\hline c27o & 117.4 & -69.9 & 42.7794 & 124.8 & -71.6 & 45.3998 \\
\hline c28y & 124.6 & -71.3 & 45.7432 & 125.4 & -71.5 & 46.1769 \\
\hline $\mathrm{c} 280$ & 125.2 & -71.2 & 47.1331 & 126.2 & -71.4 & 47.9157 \\
\hline$c 29 y$ & 123.2 & -70.7 & 46.7817 & 126.4 & -71.4 & 48.2669 \\
\hline c29o & 126.6 & -71.3 & 49.1415 & 127.4 & -71.4 & 49.3825 \\
\hline c30y & 128.4 & -71.5 & 50.1719 & 128.6 & -71.5 & 50.6690 \\
\hline c30o & 129.6 & -71.3 & 52.7263 & 132.8 & -71.8 & 54.9000 \\
\hline c31y & 146.2 & -73.0 & 59.0872 & 134.0 & -71.8 & 55.7121 \\
\hline c310 & 143.6 & -72.7 & 58.9766 & 136.8 & -72.0 & 58.6663 \\
\hline c32.1y & 162.6 & -72.2 & 72.4029 & & & \\
\hline c32.10 & 159.0 & -72.3 & 69.7998 & & & \\
\hline c32.2y & 163.4 & -72.1 & 73.5440 & & & \\
\hline c32.2o & 151.8 & -72.2 & 69.3984 & & & \\
\hline c32r & 165.2 & -71.8 & 77.6446 & & & \\
\hline c33y & 167.6 & -71.6 & 80.3935 & & & \\
\hline c33o & 348.0 & 64.7 & 89.2303 & & & \\
\hline
\end{tabular}

Table V-3: Pacific-Bellingshausen finite rotations calculated with onepole and JaMBES for a three plate model. onepole rotations for $\mathrm{c} 20 \mathrm{y}-\mathrm{c} 20 \mathrm{o}$ and $\mathrm{c} 32.1 \mathrm{y}-\mathrm{c} 33 \mathrm{o}$ were inconclusive, and were not included in the JaMBES calculations. 


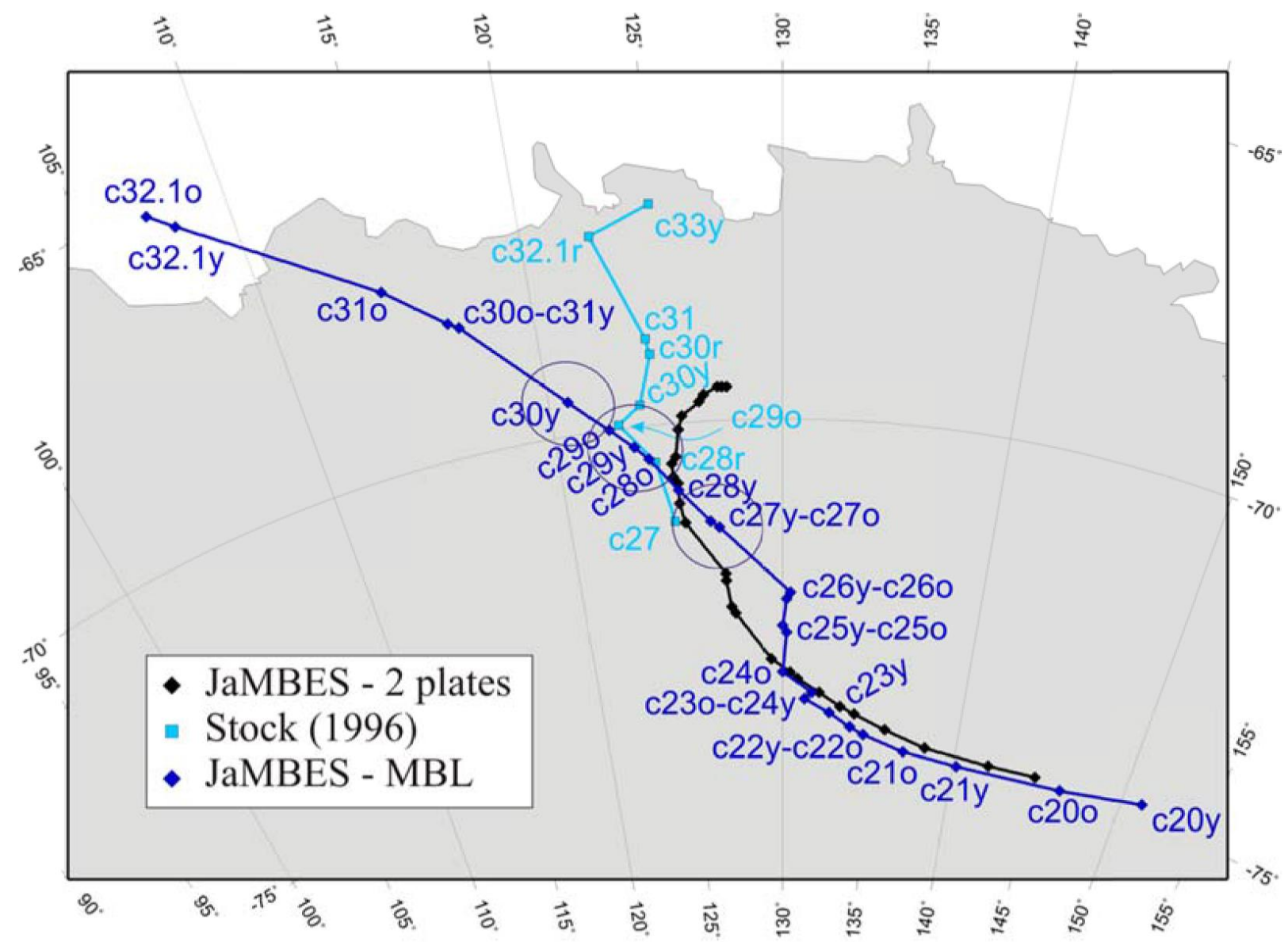

Figure V-5: Pacific-Marie Byrd Land finite rotation poles (JaMBES and [Stock et al., 1996]). Equivalent rotation poles for a two plate system are included as a reference. JaMBES 95\% confidence regions are included for c27y, c29y and c30y. 


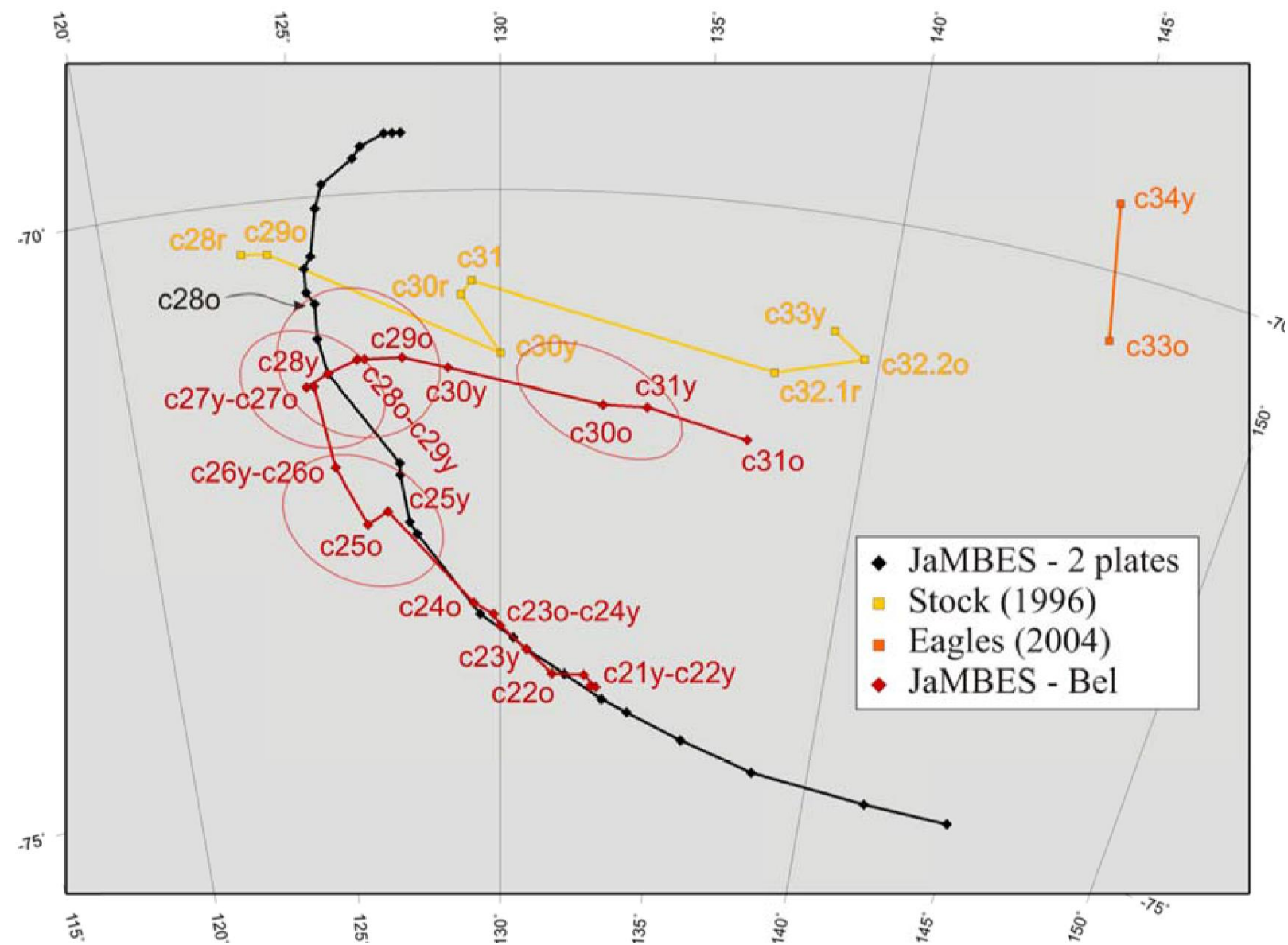

Figure V-6: Pacific-Bellingshausen finite rotation poles (JaMBES and [Stock et al., 1996]). Equivalent rotation poles for a two plate system are included as a reference. JaMBES 95\% confidence regions are included for c25o, c27y, c28o and c30o.

Figure V-7 is a different representation of the same rotation poles. The Antarctica section of the Antipodes FZ is represented twice as a marker: first attached to Marie Byrd Land (dark blue), then moving with Bellingshausen (red). The displacement between the two representations illustrates the Marie Byrd Land-Bellingshausen relative motion. For times younger than $\mathrm{c} 25 \mathrm{o}$, the two representations are superimposed, indicating no motion (within uncertainties) between Marie Byrd Land and Bellingshausen. For times older than c27y however, the Bellingshausen section is rotated west of the Marie Byrd Land one. The distance between the two sections also increases with time. This shows there was extension between Marie Byrd Land and Bellingshausen between c31o and c27y. These observations are in agreement with published works [Davy, 2006; Eagles et al., 2004a; Larter et al., 2002; Stock et al., 1996]. 

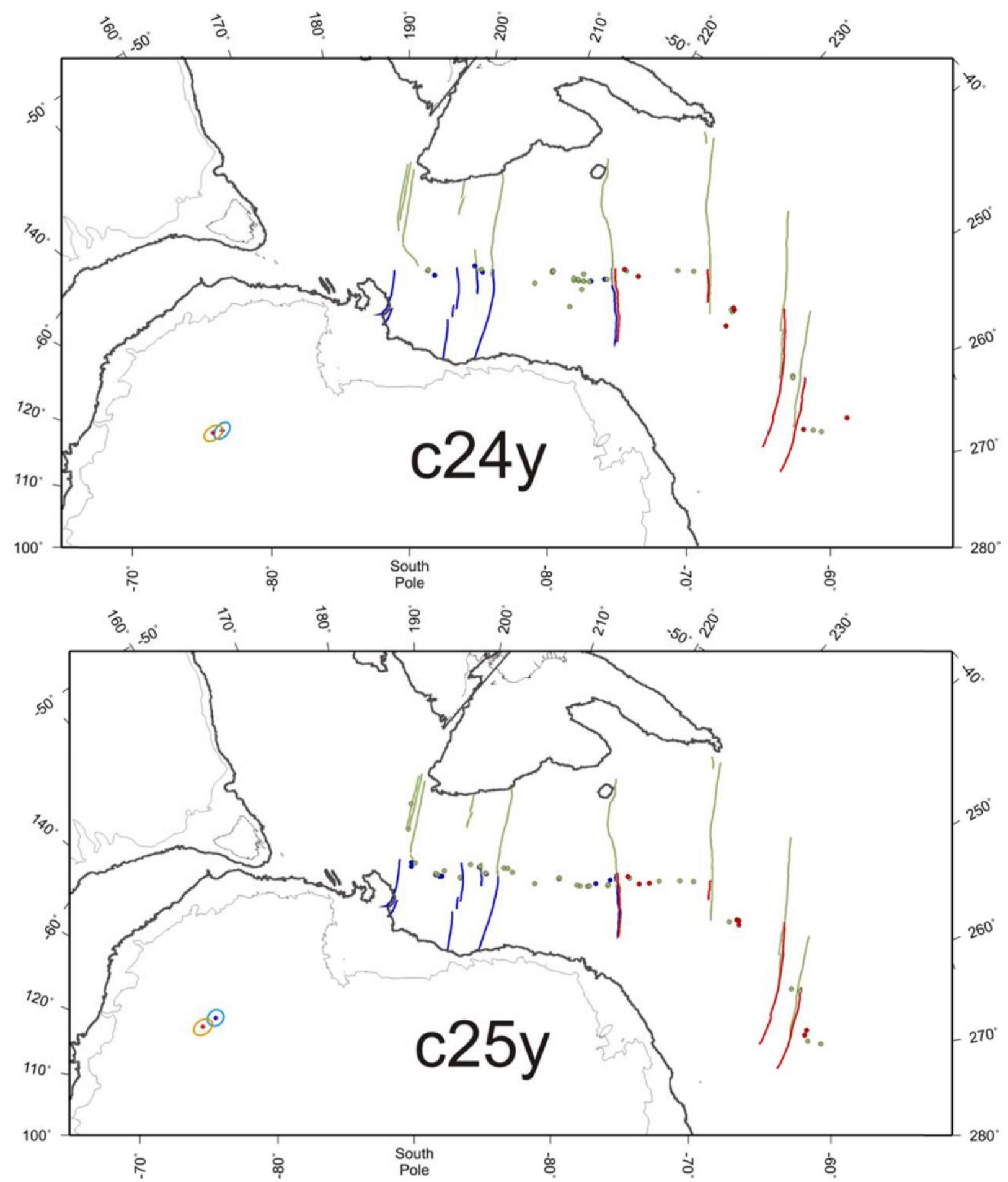

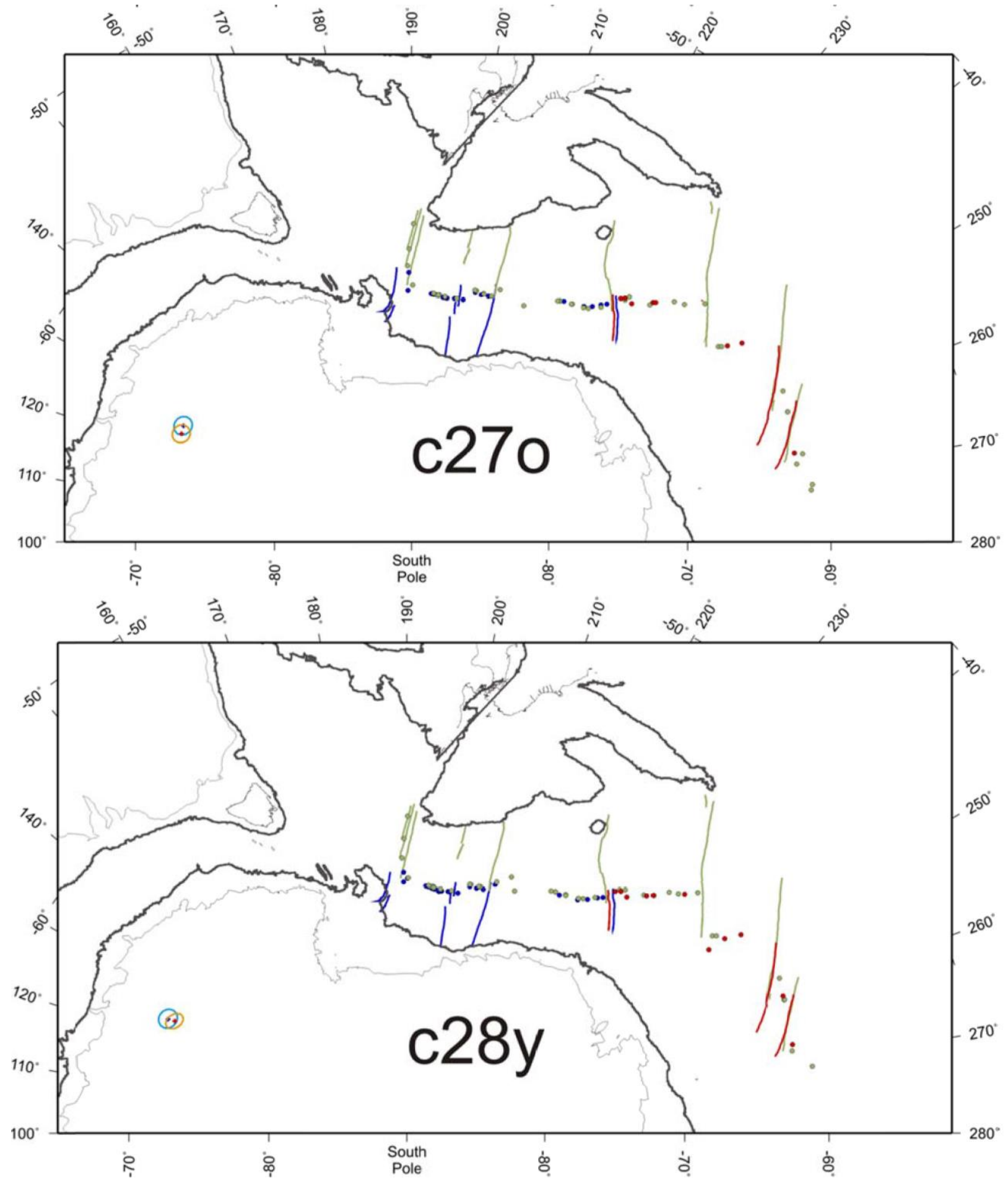

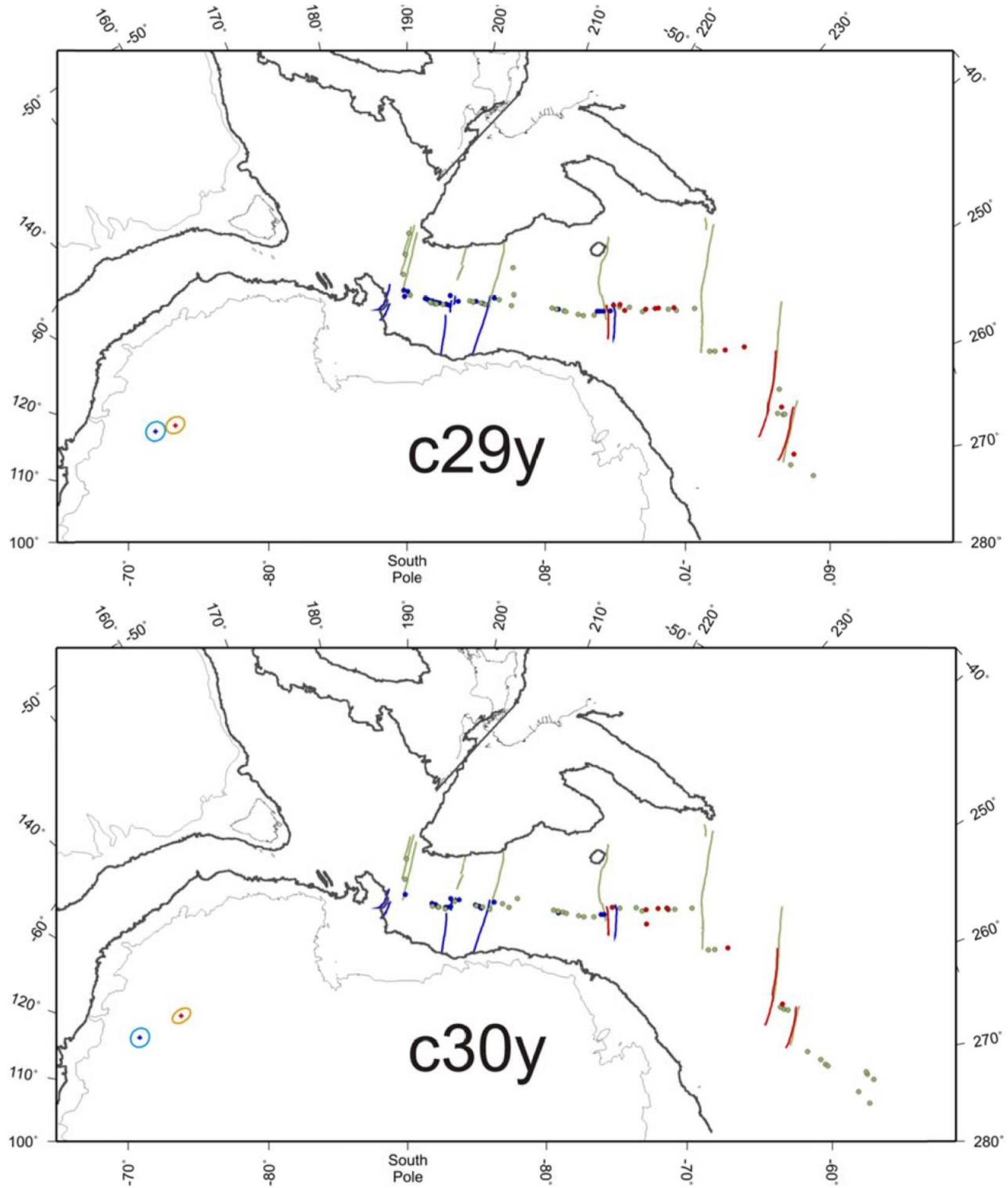

Figure V-7: Tectonic reconstructions for the Southwest Pacific using a three plate system. The paleoposition of each block is calculated using a three plate model (Pacific-Marie Byrd LandBellingshausen, JaMBES rotations from Table V-2 and Table V-3). Dark blue = Marie Byrd Land; green $=$ Pacific; red = Bellingshausen. Marie Byrd Land (Antarctica) is considered fixed. Lord Howe Rise-Australia rotations from [Gaina et al., 1998], Pacific-Lord Howe Rise rotation from [Keller, 2003]. Same fracture zones as in Figure V-4. Note the discrepancy between the Marie Byrd Land-rotated Antipodes FZ (blue) and the Bellingshausen-rotated Antipodes FZ (red) 
From c20y to c24o, the Pacific-Marie Byrd Land JaMBES rotations are very similar to those calculated using a two plate system (Figure V-5). The c25y-c26o rotation poles diverge from the two-plate solutions. The $\mathrm{c} 27 \mathrm{y}-\mathrm{c} 28 \mathrm{y}$ rotation poles are very similar to those calculated using a two-plate system. For chrons older than c28o, Pacific-Marie Byrd Land plate motions differ significantly from the two plate solutions. My Pacific-Marie Byrd Land poles for c28oc29o are nearly identical to those of [Stock et al., 1996].

Similar observations can be made regarding the Pacific-Bellingshausen rotations (Figure V-6). The trend and location of Pacific-Bellingshausen and Pacific-Antarctica (two-plate system) rotation poles are very close for chrons younger than c25o. The c27y-c27o PacificBellingshausen rotation poles are located close to their Pacific-Antarctica counterparts. The Pacific-Bellingshausen pole track diverges from that of the two-plate system for times older than c28y. The rate of Pacific-Bellingshausen pole migration is larger for c30y-c31o than it is for $\mathrm{c} 27 \mathrm{o}-\mathrm{c} 30 \mathrm{y}$ (taking the non-linear age increase into account).

To further quantify the timing of existence of the Bellingshausen plate, I plotted the Pacific-Marie Byrd Land and the Pacific-Bellingshausen rotation pole locations (Figure V-8). The results confirm that the Bellingshausen plate motion is significantly different at the $95 \%$ confidence level for times older than c28o. My best estimate for cessation of movement at the Marie Byrd Land-Bellingshausen plate boundary is between c27o and c28y, based on extrapolation of trends in the rotation data (Figure V-8). 


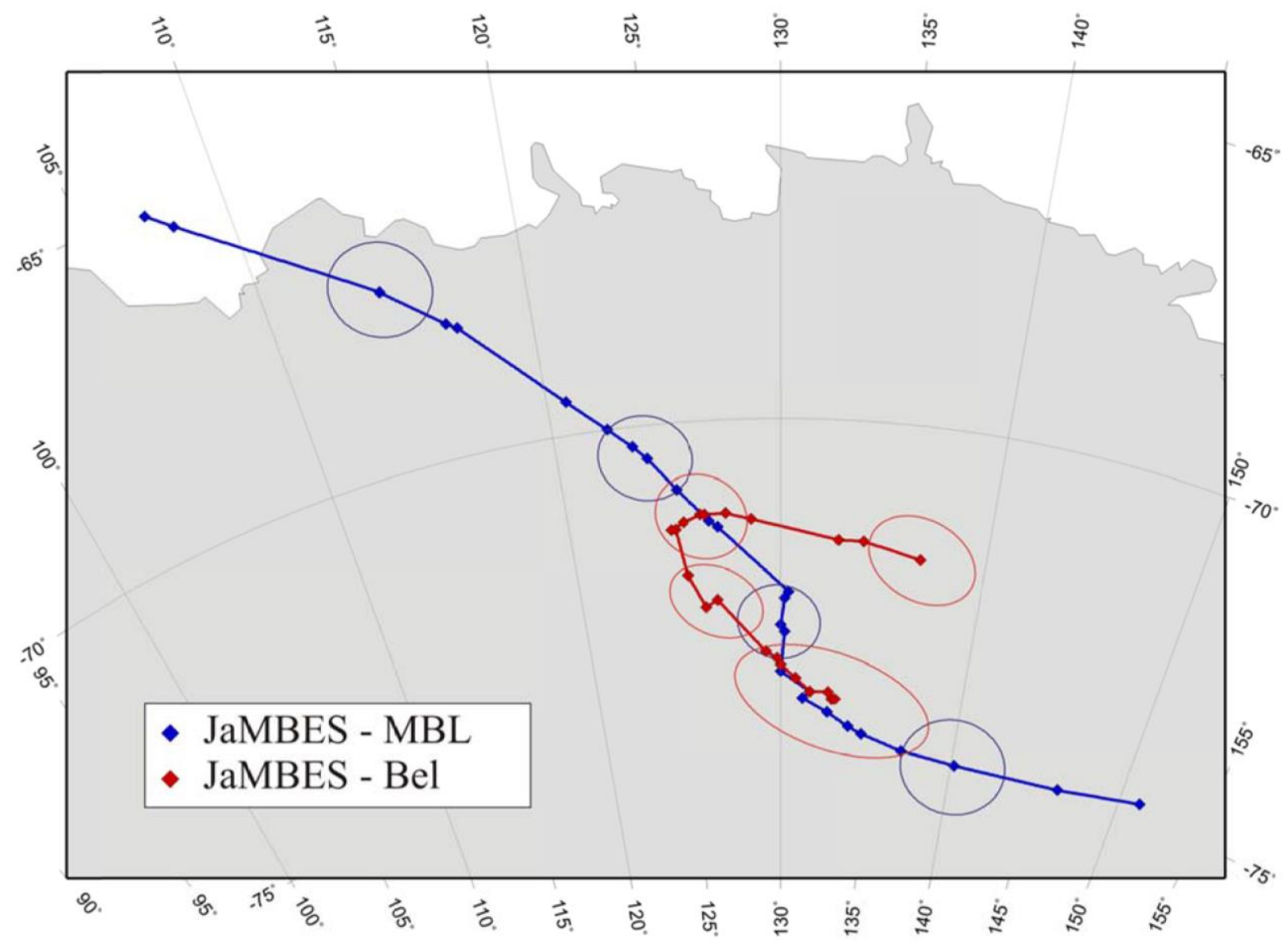

Figure V-8: Comparison between the Pacific-Marie Byrd Land and the Pacific-Bellingshausen rotation poles (both JaMBES results) with selected 95\% confidence ellipses (c21y, c25y, c28o and c31o). See Appendix G for all uncertainty results.

The lack of suitable data near the continental margin of Antarctica meant that it was not possible for me to calculate Pacific-Bellingshausen rotations for times older than c31o, making it impossible to verify the date at which the Bellingshausen plate started moving independently (usually taken to be anomaly 32 or since the breakup, see Chapter I).

Although I have shown that it is possible to improve our understanding of the tectonics of the southwest Pacific using data available today, there is no doubt in my mind that additional magnetic data from the Antarctica sector are needed to refine the plate rotations in the region, particularly for chrons older than c31o. During this study I had the opportunity to compare airborne magnetic profiles (helimag) to shiptrack ones of similar quality (polarstern). My understanding is that airborne magnetic data collection is more cost effective than shipborne one, so I believe that systematic airborne magnetic data collection might be a solution to counteract the sparse and clustered data distribution near Antarctica. 
The $\mathrm{c} 20 \mathrm{y}-\mathrm{c} 24 \mathrm{o}$ change in spreading direction on the Pacific-Antarctica ridge has been linked in publications to the formation of the $\mathrm{H}$ bend [Stock, 2006]. Because for c20y-c24o my identification uncertainties are relatively high, the rotations I calculated do not reproduce this change of motion. I expect that a similar study focused on the c24 change of plate motion (for instance, c7y-c26o) could significantly improve the quantification of the c20y-c24o change by lowering the identification uncertainty.

\section{Conclusions}

The objective of this thesis was to determine a quantitative plate motion history for the region between New Zealand and Antarctica for the Late Cretaceous to Middle Eocene period (Chapter I). I used a compilation of existing magnetic profiles to re-interpret the pattern of magnetic anomalies in terms of isochrons, and I evaluated the uncertainty in this primary interpretation in terms of identification uncertainty category (Chapter II). I used bathymetry and gravity data to locate fracture zones (Chapter III).

The primary difficulty that I had to overcome in this study was the sparse data coverage. New data had become available since prior published studies [Cande et al., 1995; Heinemann et al., 1999], but there was still insufficient spatial coverage to use the Hellinger fitting criterion (hellinger1, [Kirkwood et al., 1999]). In collaboration with my supervisors, we developed a new quantitative reconstruction method that has simpler assumptions and requires fewer parameters (Chapter IV). The method solves for both the best-fitting reconstruction and the uncertainty associated with each finite rotation.

Based on results described earlier in this chapter, the existence of separate Bellingshausen and Marie Byrd Land plates [Stock and Molnar, 1987] is demonstrated at the 95\% confidence level for times older than $\mathrm{c} 29 \mathrm{y}$, and the best estimate for cessation of movement at the Marie Byrd Land-Bellingshausen plate boundary is during anomaly $27 \mathrm{r}$. There are insufficient data to quantify plate motions in the three-plate circuit before c31o. My conclusions are similar to those of [Cande et al., 1995] and [Stock et al., 1996], but are based upon a more robust analysis of a larger dataset.

The quantitative plate reconstructions that I have made during this thesis represent a significant contribution to understanding global plate motions, because the Pacific-Antarctica ridge is the only non-destructive plate boundary linking the Pacific hemisphere to the rest of the 
world [Gordon, 2000]. Better tectonic reconstructions are needed for the period c20y-c34y in order to improve our understanding of deeper geological processes, like the prediction of hotspot trails [Steinberger et al., 2004] and the initiation of subduction (Mariana trench, [Sharp and Clague, 2006]). 


\section{REFERENCES}


Bullard, E., J. E. Everett, and A. G. Smith (1965), The fit of continents around the atlantic, Philosophical Transactions of the Royal Society of London, Series A: Mathematical and Physical Sciences, 258(1088), 41-51.

Cande, S. C., and J. C. Mutter (1982), A revised identification of the oldest sea-floor spreading anomalies between Australia and Antarctica, Earth and Planetary Science Letters, 58(2), 151160.

Cande, S. C., and D. V. Kent (1995), Revised calibration of the geomagnetic polarity timescale for the Late Cretaceous and Cenozoic, Journal of Geophysical Research, 100(B4), 6093-6095.

Cande, S. C., and J. M. Stock (2004), Pacific-Antarctic-Australia motion and the formation of the Macquarie Plate, Geophysical Journal International, 157(1), 399-414.

Cande, S. C., E. M. Herron, and B. R. Hall (1982), The early Cenozoic tectonic history of the Southeast Pacific, Earth and Planetary Science Letters, 57(1), 63-74.

Cande, S. C., C. A. Raymond, J. Stock, and W. F. Haxby (1995), Geophysics of the Pitman fracture zone and Pacific-Antarctic Plate motions during the Cenozoic, Science, 270(5238), 947-953.

Cande, S. C., J. M. Stock, R. D. Mueller, and T. Ishihara (2000), Cenozoic motion between East and West Antarctica, Nature (London), 404(6774), 145-150.

Chang, T. (1988), Estimating the relative rotation of two tectonic plates from boundary crossings, J. Am. stat. Assoc., 83, 1178-1183.

Channell, J. E. T., E. Erba, M. Nakanishi, and K. Tamaki (1995), Late Jurassic-Early Cretaceous time scales and oceanic magnetic anomaly block models, in Geochronology, time scales and global stratigraphic correlation, edited by W. A. Berggren, D. V. Kent, M.-P. Aubry and J. Hardenbol, pp. 51-63, Society for Sedimentary Geology.

Cordier, C. (2006), Approches pétrogéochimique et géomathématique de la cyclicité magmatique en contexte d'accrétion océation océanique: de la fusion partielle aux séquences de réalimentation/vidange des réservoirs crustaux., Université de Bretagne Occidentale, Brest.

Courtillot, V., A. Davaille, J. Besse, and J. Stock (2003), Three distinct types of hotspots in the Earth's mantle, Earth and Planetary Science Letters, 205(3-4), 295-308.

Croon, M. B., S. C. Cande, and J. M. Stock (2008), Revised Pacific-Antarctic plate motions and geophysics of the Menard fracture zone, Geochemistry, Geophysics, Geosystems, Q07001.

Croon, M. B., S. C. Cande, and J. M. Stock (2010), Abyssal hill deflections at Pacific-Antarctic ridge-transform intersections, Geochemistry, Geophysics, Geosystems, Q11004.

Cunningham, A. P., R. D. Larter, P. F. Barker, K. Gohl, and F. O. Nitsche (2002), Tectonic evolution of the Pacific margin of Antarctica; 2, Structure of Late Cretaceous-early Tertiary plate boundaries in the Bellingshausen Sea from seismic reflection and gravity data, Journal of Geophysical Research, 107(B12), 20. 
Davy, B. (2006), Bollons Seamount and early New Zealand-Antarctic seafloor spreading, Geochemistry, Geophysics, Geosystems, Q06021.

Davy, B. (2014), Rotation and offset of the Gondwana convergent margin in the New Zealand region following Cretaceous jamming of Hikurangi Plateau large igneous province subduction, Tectonics, 33(8), 1577-1595.

Davy, B., K. Hoernle, and R. Werner (2008), Hikurangi Plateau; crustal structure, rifted formation, and Gondwana subduction history, Geochemistry, Geophysics, Geosystems, Q07004.

Dick, H. J. B., J. Lin, and H. Schouten (2003), An ultraslow-spreading class of ocean ridge, Nature (London), 426(6965), 405-412.

Duncan, R. A., and D. A. Clague (1985), Pacific plate motion recorded by linear volcanic chains, Ocean Basins and Margins, 7, 89-121.

Eagles, G. (2004), Tectonic evolution of the Antarctic-Phoenix plate system since 15 Ma, Earth and Planetary Science Letters, 217(1-2), 97-109.

Eagles, G., K. Gohl, and R. D. Larter (2004a), High-resolution animated tectonic reconstruction of the South Pacific and West Antarctic margin, Geochemistry, Geophysics, Geosystems, 5(7), 21-21.

Eagles, G., K. Gohl, and R. D. Larter (2004b), Life of the Bellingshausen Plate, Geophysical Research Letters, 31(7), 4.

Faccenna, C., T. W. Becker, S. Lallemand, and B. Steinberger (2012), On the role of slab pull in the Cenozoic motion of the Pacific plate, Geophysical Research Letters, 39.

Gac, S., J. Dyment, C. Tisseau, and J. Goslin (2003), Axial magnetic anomalies over slowspreading ridge segments; insights from numerical 3-D thermal and physical modelling, Geophysical Journal International, 154(3), 618-632.

Gaina, C., D. R. Mueller, J.-Y. Royer, J. Stock, J. L. Hardebeck, and P. Symonds (1998), The tectonic history of the Tasman Sea; a puzzle with 13 pieces, Journal of Geophysical Research, 103(B6), 12-12,433.

Gohl, K., and C. participants (2010), The Expedition of the Research Vessel "Polarstern" to the Amundsen Sea, Antarctica, in 2010 (ANT-XXVI/3)Rep., ALFRED-WEGENER-INSTITUT FÜR POLAR- UND MEERESFORSCHUNG. Gohl, K., F. Nitsche, and H. Miller (1997), Seismic and gravity data reveal Tertiary interplate subduction in the Bellingshausen Sea, southeast Pacific, Geology, 25(4), 371-374.

Gordon, R. G. (2000), The Antarctic connection, Nature (London), 404(6774), 139-140.

Grobys, J. W. G., K. Gohl, G. Uenzelmann-Neben, B. Davy, and D. Barker (2009), Extensional and magmatic nature of the Campbell Plateau and Great South Basin from deep crustal studies, Tectonophysics, 472(1-4), 213-225. 
Grobys, J. W. G., K. Gohl, B. Davy, G. Uenzelmann-Neben, T. Deen, and D. Barker (2007), Is the Bounty Trough off eastern New Zealand an aborted rift?, Journal of Geophysical Research, 112(B3), B03103.

Gurnis, M., M. Turner, S. Zahirovic, L. DiCaprio, S. Spasojevic, R. D. Muller, J. Boyden, M. Seton, V. C. Manea, and D. J. Bower (2012), Plate tectonic reconstructions with continuously closing plates, Computers \& Geosciences, 38(1), 35-42.

Hall, C. E., and M. Gurnis (2005), Strength of fracture zones from their bathymetric and gravitational evolution, Journal of Geophysical Research-Solid Earth, 110(B1).

Hayes, D. E., and J. Ringis (1973), Seafloor spreading in the Tasman Sea, Nature, 243, 454458 .

Heinemann, J., J. Stock, R. Clayton, K. Hafner, S. Cande, and C. Raymond (1999), Constraints on the proposed Marie Byrd Land-Bellingshausen plate boundary from seismic reflection data, Journal of Geophysical Research, 104(B11), 25-25,330.

Hellinger, S. J. (1981), The uncertainties of finite rotations in plate tectonics, Journal of Geophysical Research, 86(B10), 9312-9318.

Hoernle, K., F. Hauff, P. van den Bogaard, R. Werner, N. Mortimer, J. Geldmacher, D. GarbeSchonberg, and B. Davy (2010), Age and geochemistry of volcanic rocks from the Hikurangi and Manihiki oceanic Plateaus, Geochimica Et Cosmochimica Acta, 74(24), 7196-7219.

Hoernle, K., J. D. L. White, P. van den Bogaard, F. Hauff, D. S. Coombs, R. Werner, C. Timm, D. Garbe-Schonberg, A. Reay, and A. F. Cooper (2006), Cenozoic intraplate volcanism on New Zealand: Upwelling induced by lithospheric removal, Earth and Planetary Science Letters, 248(1-2), 350-367.

Iaffaldano, G., R. Hawkins, T. Bodin, and M. Sambridge (2014), REDBACK: Open-source software for efficient noise-reduction in plate kinematic reconstructions, Geochemistry, Geophysics, Geosystems, 15(4), 1663-1670.

Keller, W. R. (2003), Cenozoic plate tectonic reconstructions and plate boundary processes in the Southwest Pacific, PhD thesis, 129 pp, California Institute of Technology.

Kipf, A., Hauff, F., Werner, R., Gohl, K., van den Bogaard, P., Hoernle, K., ... \& Klügel, A. (2014). Seamounts off the West Antarctic margin: A case for non-hotspot driven intraplate volcanism. Gondwana Research, 25(4), 1660-1679.

Kirkwood, B. H., J. Y. Royer, T. C. Chang, and R. G. Gordon (1999), Statistical tools for estimating and combining finite rotations and their uncertainties, Geophysical Journal International, 137(2), 408-428.

Larter, R. D., A. P. Cunningham, P. F. Barker, K. Gohl, and F. O. Nitsche (2002), Tectonic evolution of the Pacific margin of Antarctica; 1, Late Cretaceous tectonic reconstructions, Journal of Geophysical Research, 107(B12), 19. 
Le Pichon, X., and J. R. Heirtzler (1968), Magnetic anomalies in the Indian Ocean and sea-floor spreading, Journal of Geophysical Research, 73(6), 2101-2117.

Lowrie, W., and D. V. Kent (2004), Geomagnetic polarity timescales and reversal frequency regimes, in Timescales of the Paleomagnetic Field, edited by J. E. T. Channell, D. V. Kent, W. Lowrie and J. G. Meert, pp. 117-129.

Marks, K. M., and J. M. Stock (1997), Early Tertiary gravity field reconstructions of the Southwest Pacific, Earth and Planetary Science Letters, 152(1-4), 267-274.

Maus, S., et al. (2005), The 10th-Generation International Geomagnetic Reference Field, Geophysical Journal International, 161(3), 561-565.

Mayes, C. L., L. A. Lawver, and D. T. Sandwell (1990), Tectonic History and New Isochron Chart of the South Pacific, J. Geophys. Res., 95(B6), 8543-8567.

McCarron, J. J., and R. D. Larter (1998), Late Cretaceous to early Tertiary subduction history of the Antarctic peninsula, J. Geol. Soc. London, 155, 255-268.

Mendel, V., M. Munschy, and D. Sauter (2005), MODMAG, a MATLAB program to model marine magnetic anomalies, Computers \& Geosciences, 31(5), 589-597.

Molnar, P., T. Atwater, J. Mammerickx, and S. M. Smith (1975), Magnetic anomalies, bathymetry and tectonic evolution of South Pacific since Late Cretaceous, Geophysical Journal of the Royal Astronomical Society, 40(3), 383.

Morgan, W. J. (1968), Rises, trenches, great faults, and crustal blocks, Journal of Geophysical Research, 73(6), 1959-1982.

Mutter, J. C., K. A. Hegarty, S. C. Cande, and J. K. Weissel (1985), Breakup between Australia and Antarctica: A brief review in the light of new data, Tectonophysics, 114(1-4), 255-279.

Richards, M. A., and C. Lithgow-Bertelloni (1996), Plate motion changes, the HawaiianEmperor bend, and the apparent success and failure of geodynamic models, Earth and Planetary Science Letters, 137(1-4), 19-27.

Royer, J.-Y., and N. Rollet (1997), Plate-tectonic setting of the Tasmanian region, Australian Journal of Earth Sciences, 44(5), 543-560.

Sandwell, D. T., and W. H. F. Smith (1997), Marine gravity anomaly from Geosat and ERS 1 satellite altimetry, Journal of Geophysical Research, 102(B5), 10-10,054.

Schellart, W. P., G. S. Lister, and V. G. Toy (2006), A Late Cretaceous and Cenozoic reconstruction of the Southwest Pacific region; tectonics controlled by subduction and slab rollback processes, Earth-Science Reviews, 76(3-4), 191-233.

Sharp, W. D., and D. A. Clague (2006), 50-Ma initiation of Hawaiian-Emperor bend records major change in Pacific plate motion, Science, 313(5791), 1281-1284. 
Smith, W. H. F., and D. T. Sandwell (1997), Global sea floor topography from satellite altimetry and ship depth soundings, Science, 277(5334), 1956-1962.

Steinberger, B., R. Sutherland, and R. J. O'Connell (2004), Prediction of Emperor-Hawaii seamount locations from a revised model of global plate motion and mantle flow, Nature, 430(6996), 167-173.

Stock, J. M. (2006), The Hawaiian-Emperor Bend: Older than expected, Science, 313(5791), 1250-1251.

Stock, J. M., and P. Molnar (1982), Uncertainties in the relative positions of the Australia, Antarctica, Lord Howe, and Pacific plates since the Late Cretaceous, Journal of Geophysical Research, 87(B6), 4697-4714.

Stock, J. M., and P. Molnar (1987), Revised history of Early Tertiary plate motion in the Southwest Pacific, Nature, 325(6104), 495-499.

Stock, J. M., S. C. Cande, and C. A. Raymond (1996), Updated history of the Bellingshausen plate, Geology, submitted.

Sutherland, R. (1995), The Australia-Pacific boundary and Cenozoic plate motions in the SW Pacific: some constraints from Geosat data, Tectonics, 14(4), 819-831.

Sutherland, R. (1999), Basement geology and tectonic development of the greater New Zealand region; an interpretation from regional magnetic data, Tectonophysics, 308(3), 341-362.

Taylor, B. (2006), The single largest oceanic plateau: Ontong Java-Manihiki-Hikurangi, Earth and Planetary Science Letters, 241(3-4), 372-380.

Tikku, A. A., and S. C. Cande (1999), The oldest magnetic anomalies in the AustralianAntarctic Basin; are they isochrons?, Journal of Geophysical Research, 104(B1), 661-677.

Veevers, J. J. (1986), Breakup of Australia and Antarctica estimated as MidCretaceous (95+/$5 \mathrm{Ma}$ ) from magnetic and seismic data at the continental-margin, Earth and Planetary Science Letters, 77(1), 91-99.

Veevers, J. J., and Z. X. Li (1991), Review of seafloor spreading around Australia; II, Marine magnetic anomaly modelling, Australian Journal of Earth Sciences, 38(4), 391-408.

Veevers, J. J., C. M. Powell, and S. R. Roots (1991), Review of seafloor spreading around Australia; I, Synthesis of the patterns of spreading, Australian Journal of Earth Sciences, 38(4), 373-389.

Weissel, J. K., and D. E. Hayes (1972), Magnetic anomalies in the Southeast Indian Ocean, in Antarctic oceanology II: The Australian-New Zealand sector, edited by D. E. Hayes, pp. 165196.

Weissel, J. K., and D. E. Hayes (1977), Evolution of the Tasman Sea reappraised, Earth and Planetary Science Letters, 36(1), 77-84. 
Weissel, J. K., D. E. Hayes, and E. M. Herron (1977), Plate tectonics synthesis: The displacements between Australia, New Zealand, and Antarctica since the Late Cretaceous, Marine Geology, 25(1-3), 231-277.

Whittaker, J. M., and R. D. Mueller (2006), Seismic stratigraphy of the Adare Trough area, Antarctica, Marine Geology, 230(3-4), 179-197.

Whittaker, J. M., R. D. Mueller, G. Leitchenkov, H. Stagg, M. Sdrolias, C. Gaina, and A. Goncharov (2007), Major Australian-Antarctic Plate reorganization at Hawaiian-Emperor bend time, Science, 318(5847), 83-86. 
APPENDICES 

Appendix A: Magnetic profiles and their interpretation

All the magnetic profiles studied during this project are represented here. Each profile displays the shiptrack magnetic and (where available) bathymetric data, as well as the interpretation of the magnetic wiggles. An annotated generic synthetic magnetic profile is included on each page.

For each profile, each magnetic anomaly is pointed out by a symbol indicating its age. For instance, an upside-down triangle indicates anomaly 27 . If the outline of the symbol is white, the magnetic chron is c27y (young edge of the anomaly); if it is black, the magnetic chron is c27o (old edge of the anomaly). The identification uncertainty for each anomaly is colourcoded.

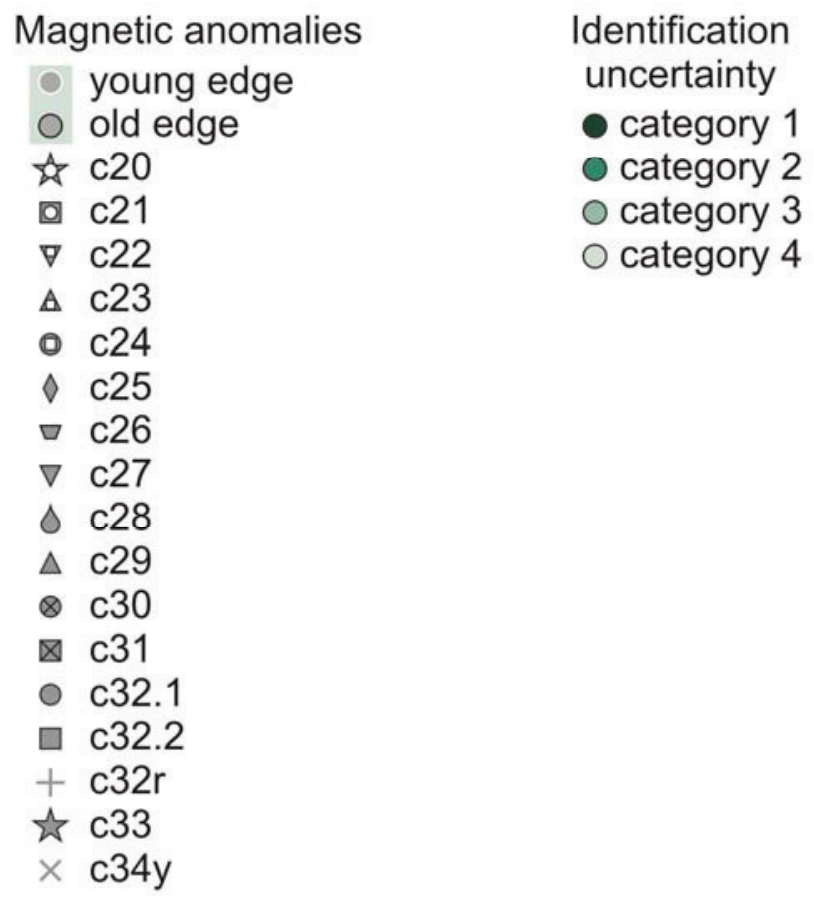




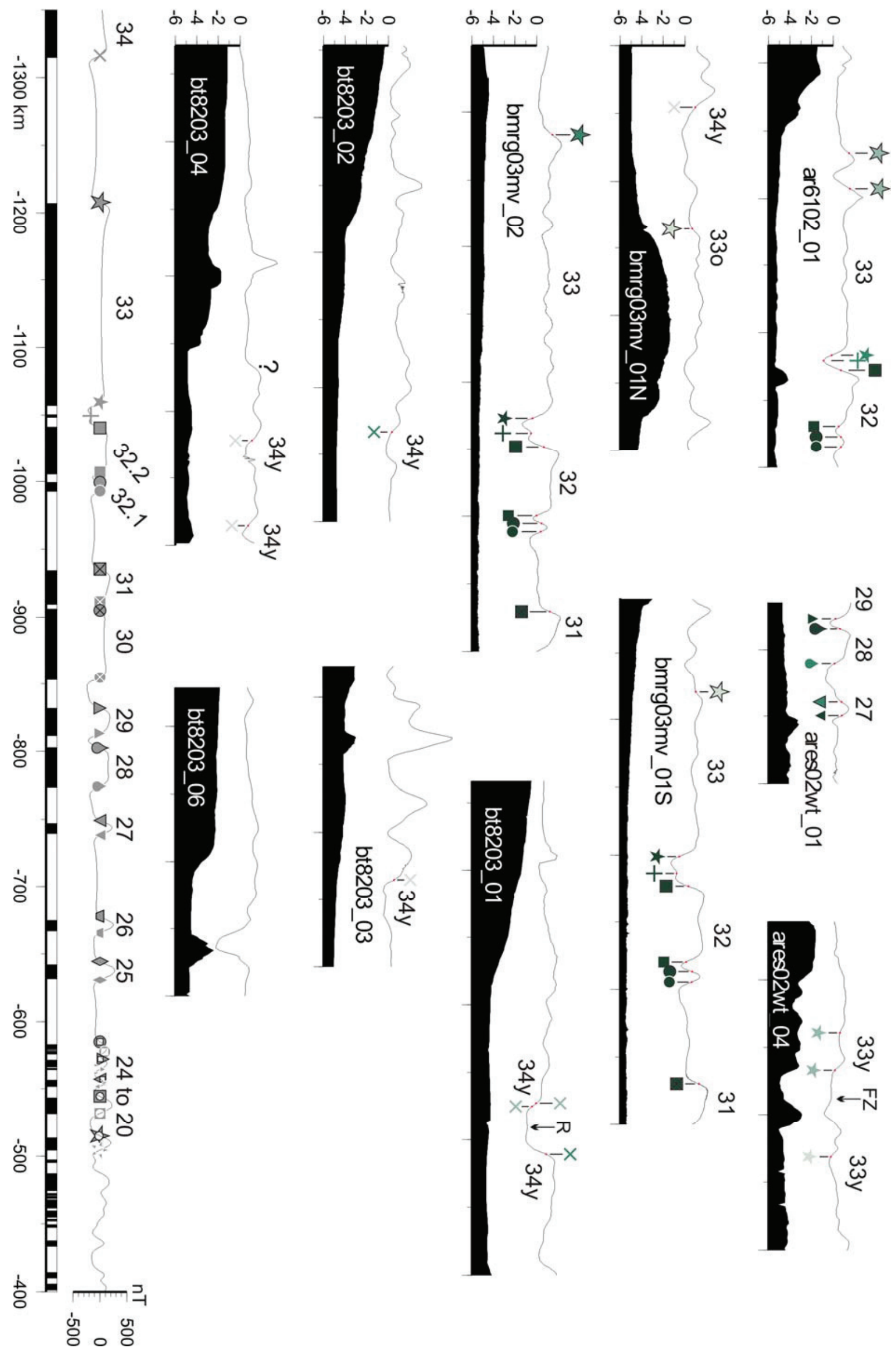




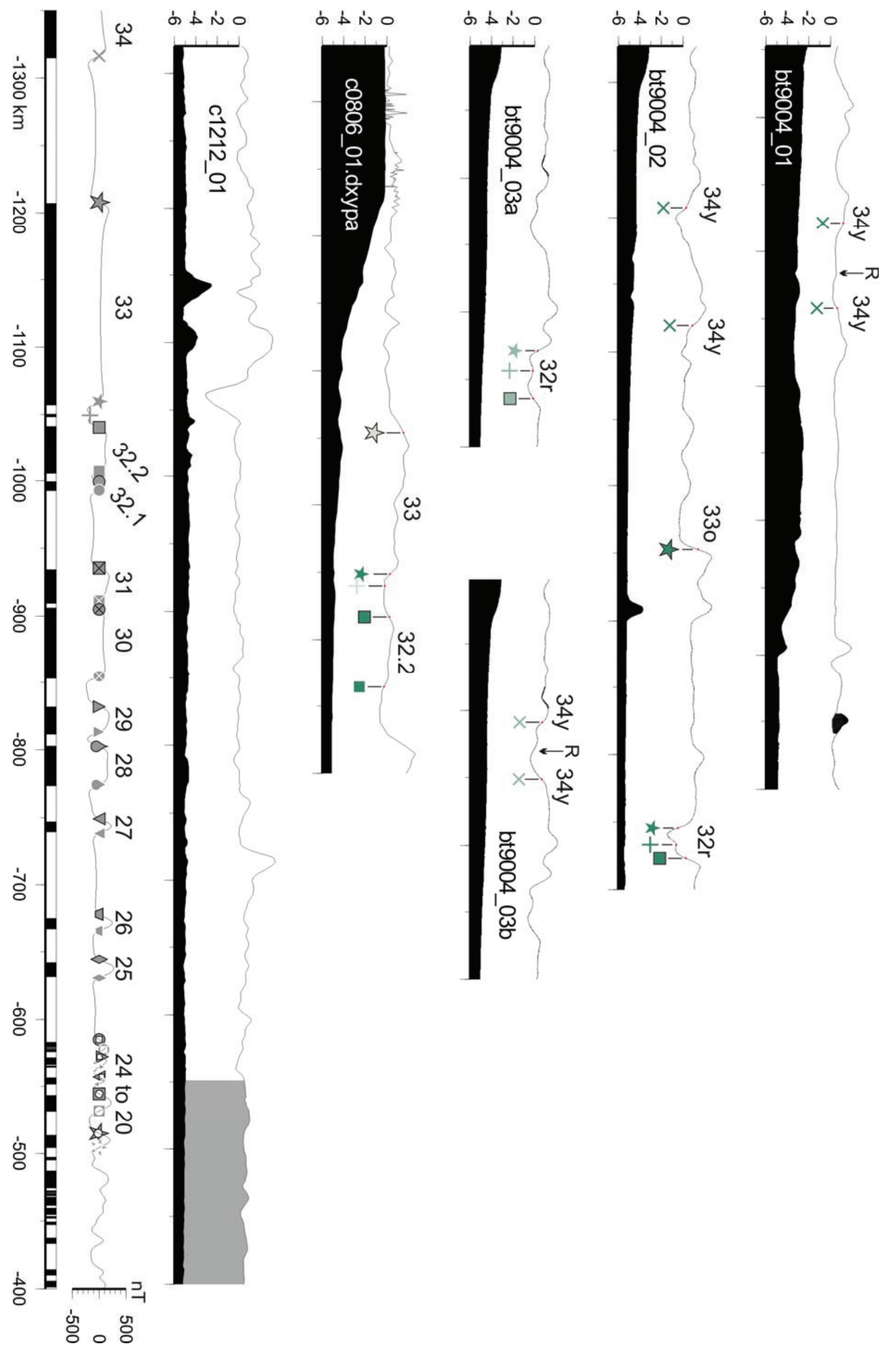




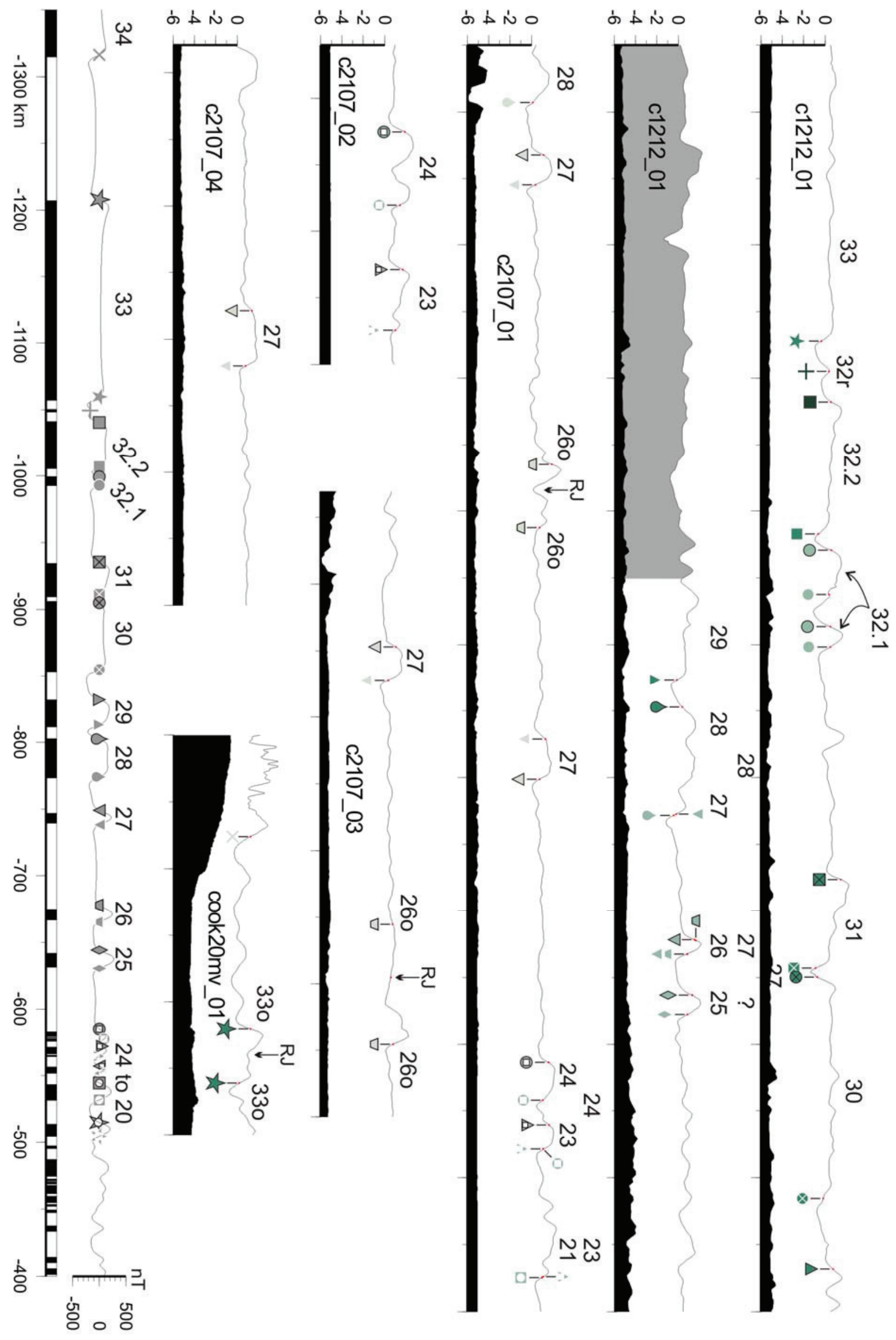



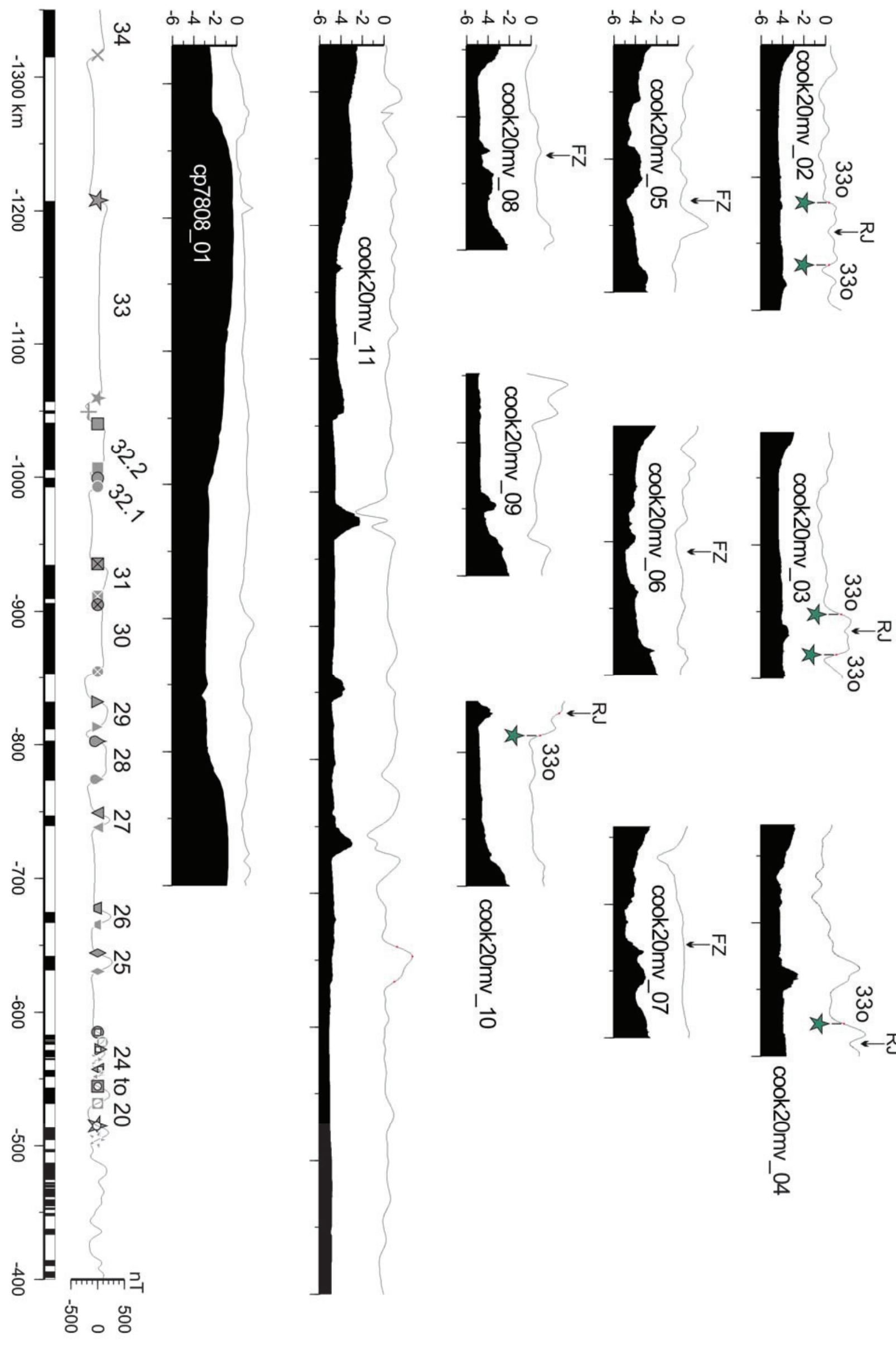


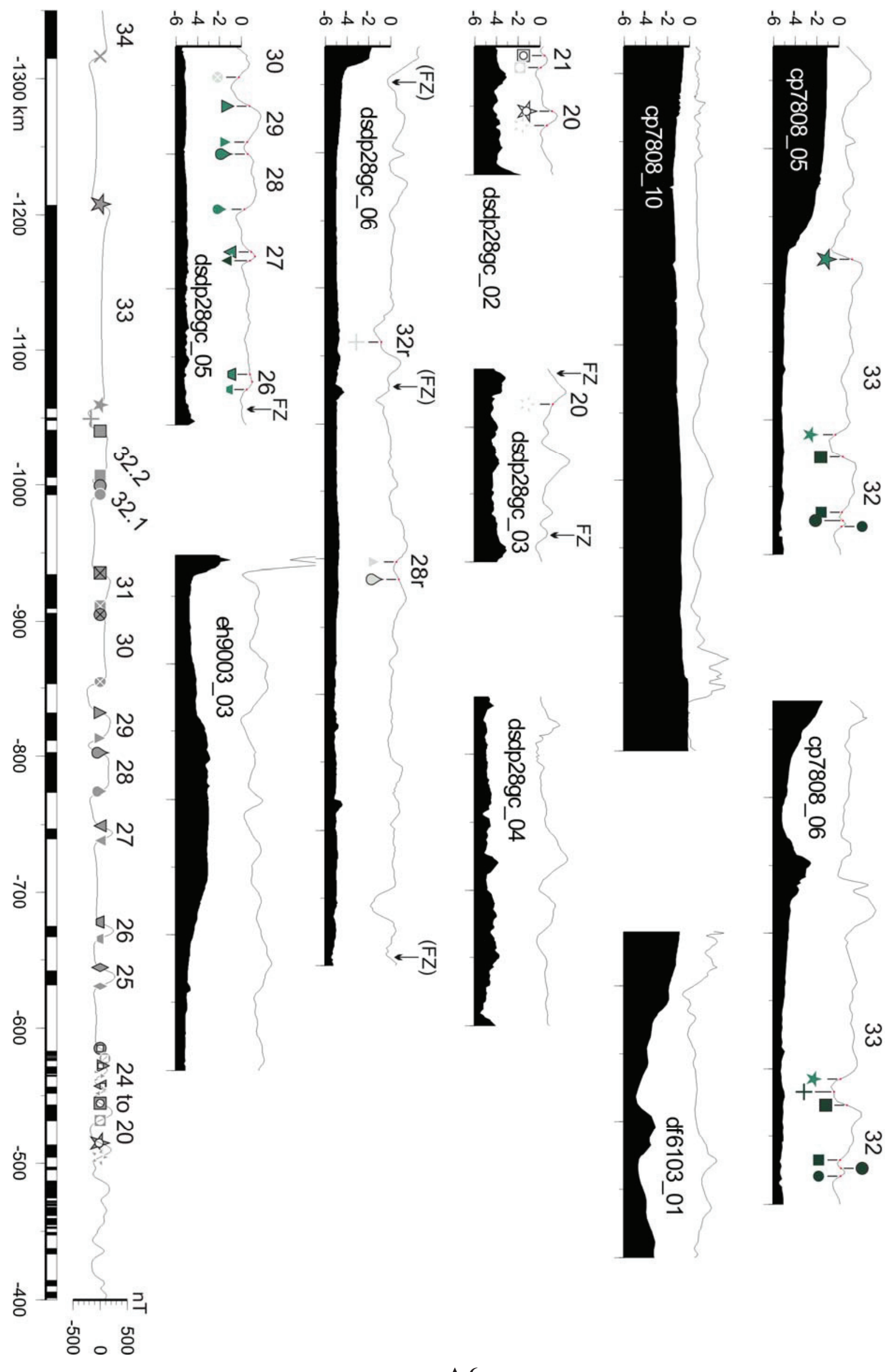



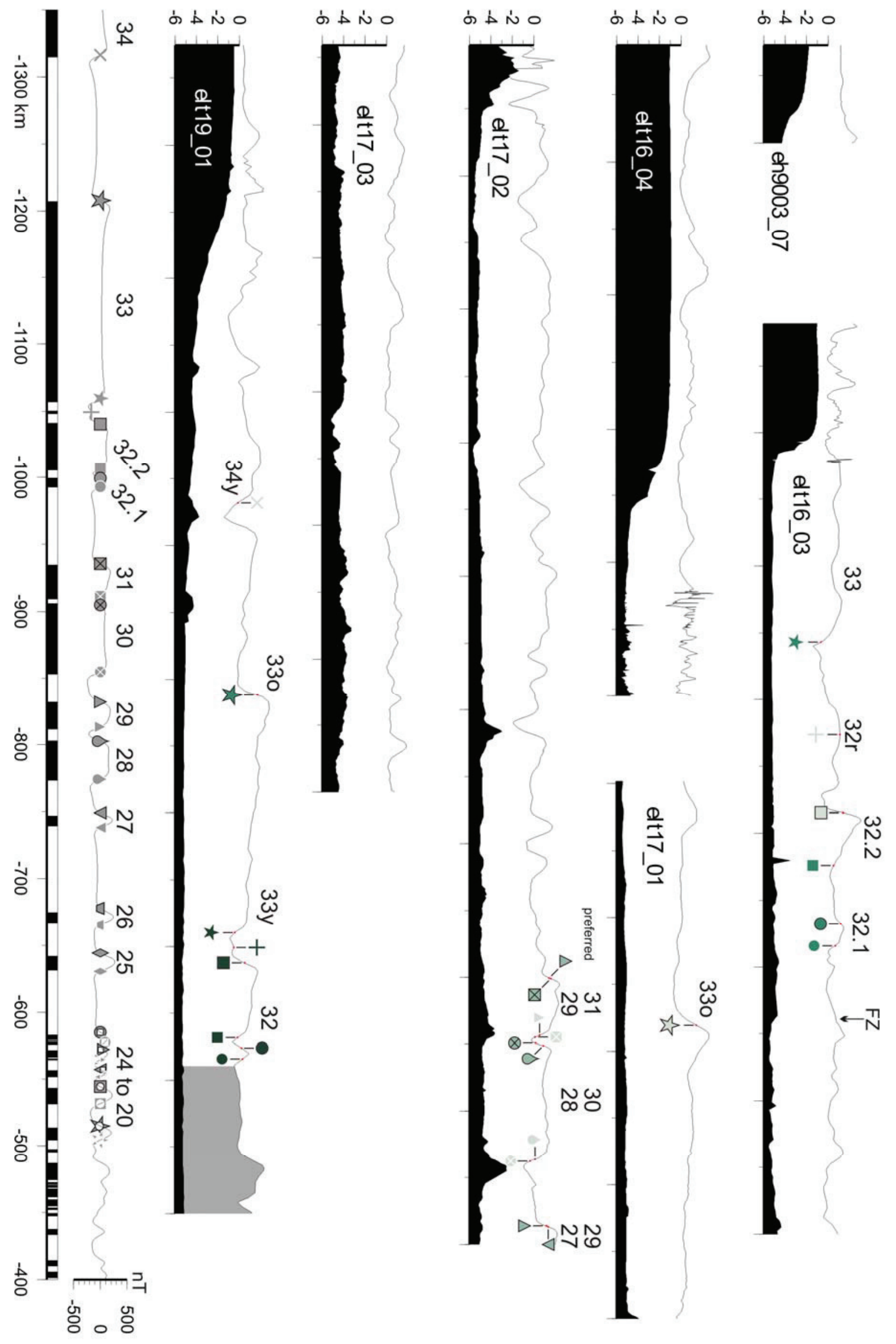


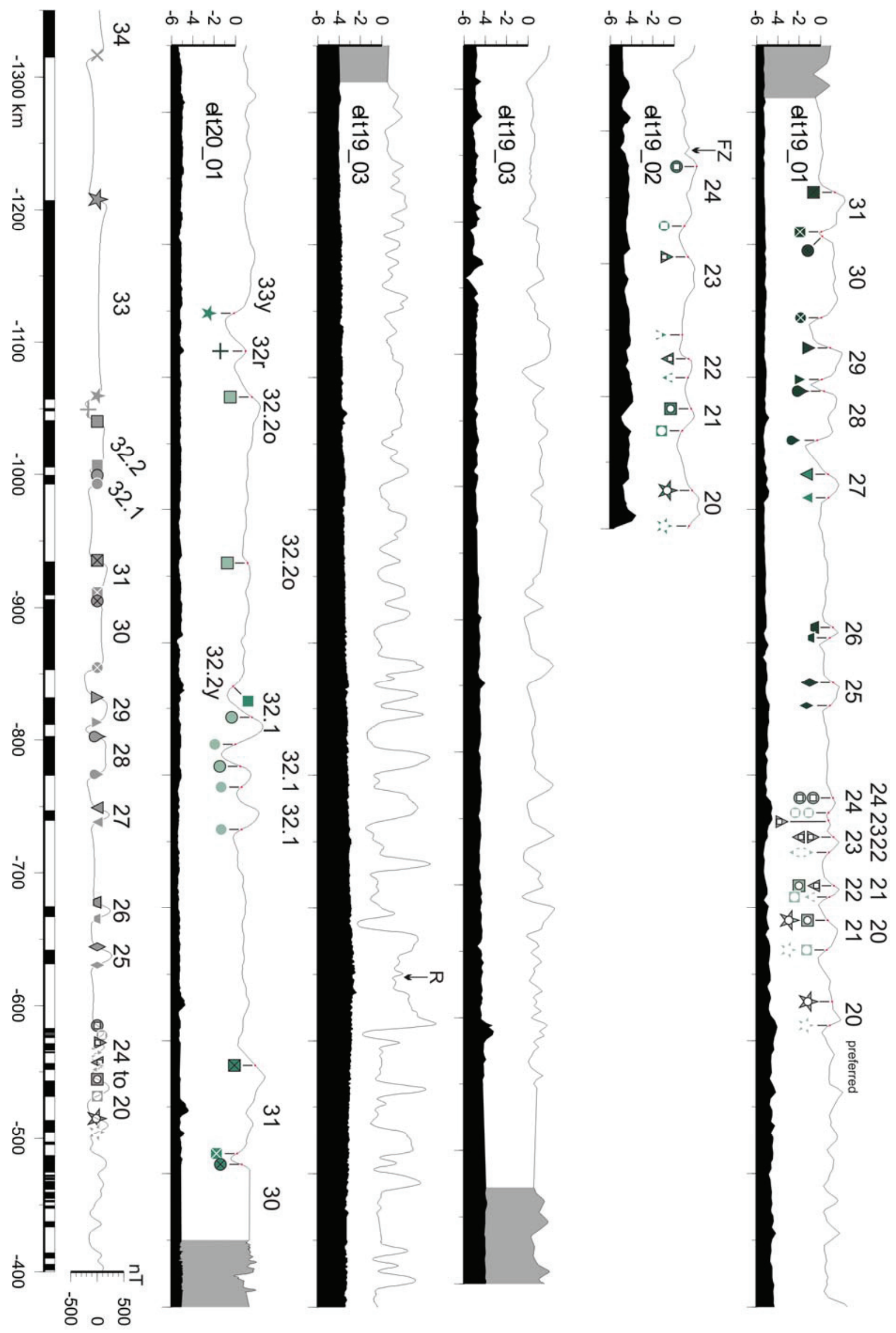




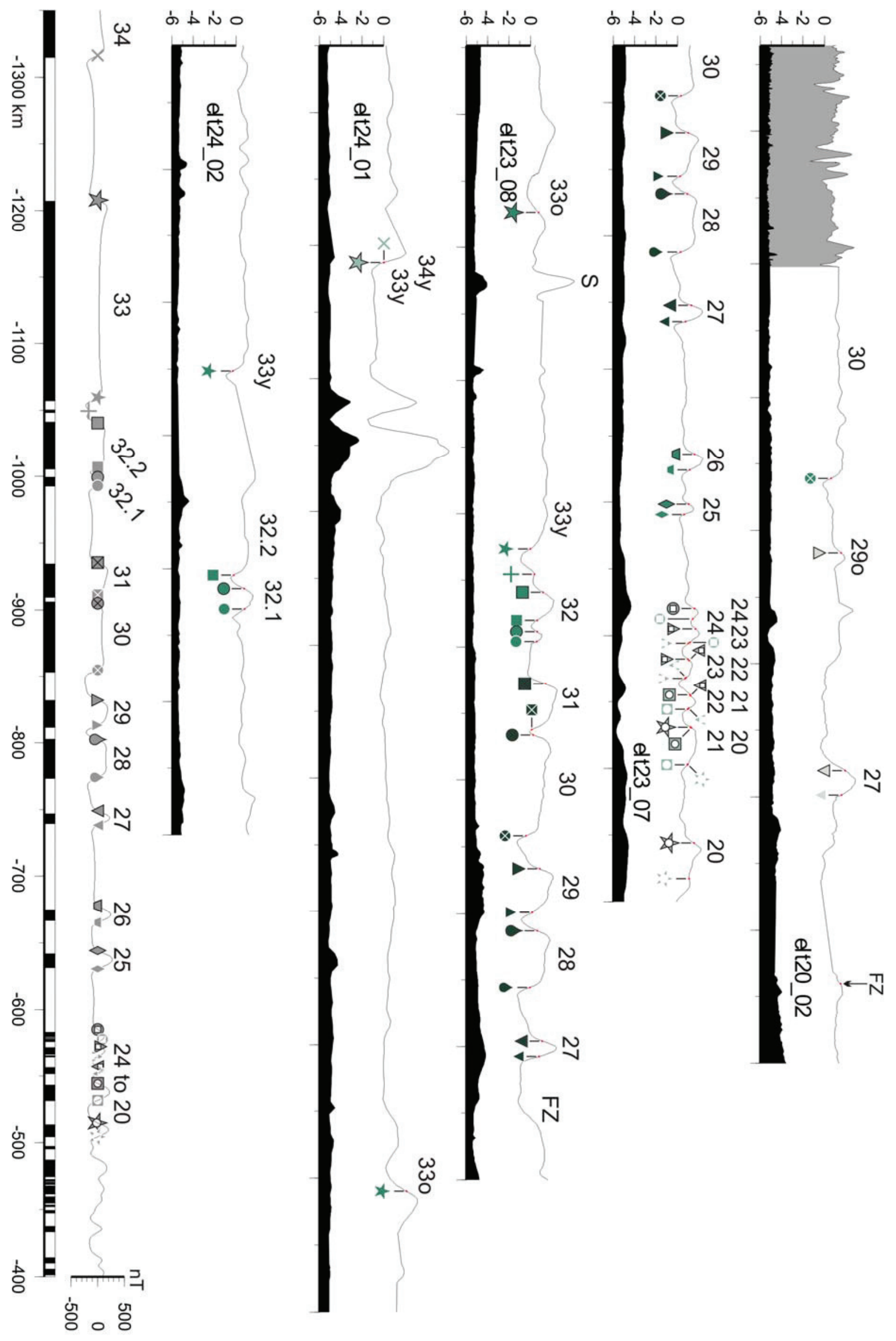




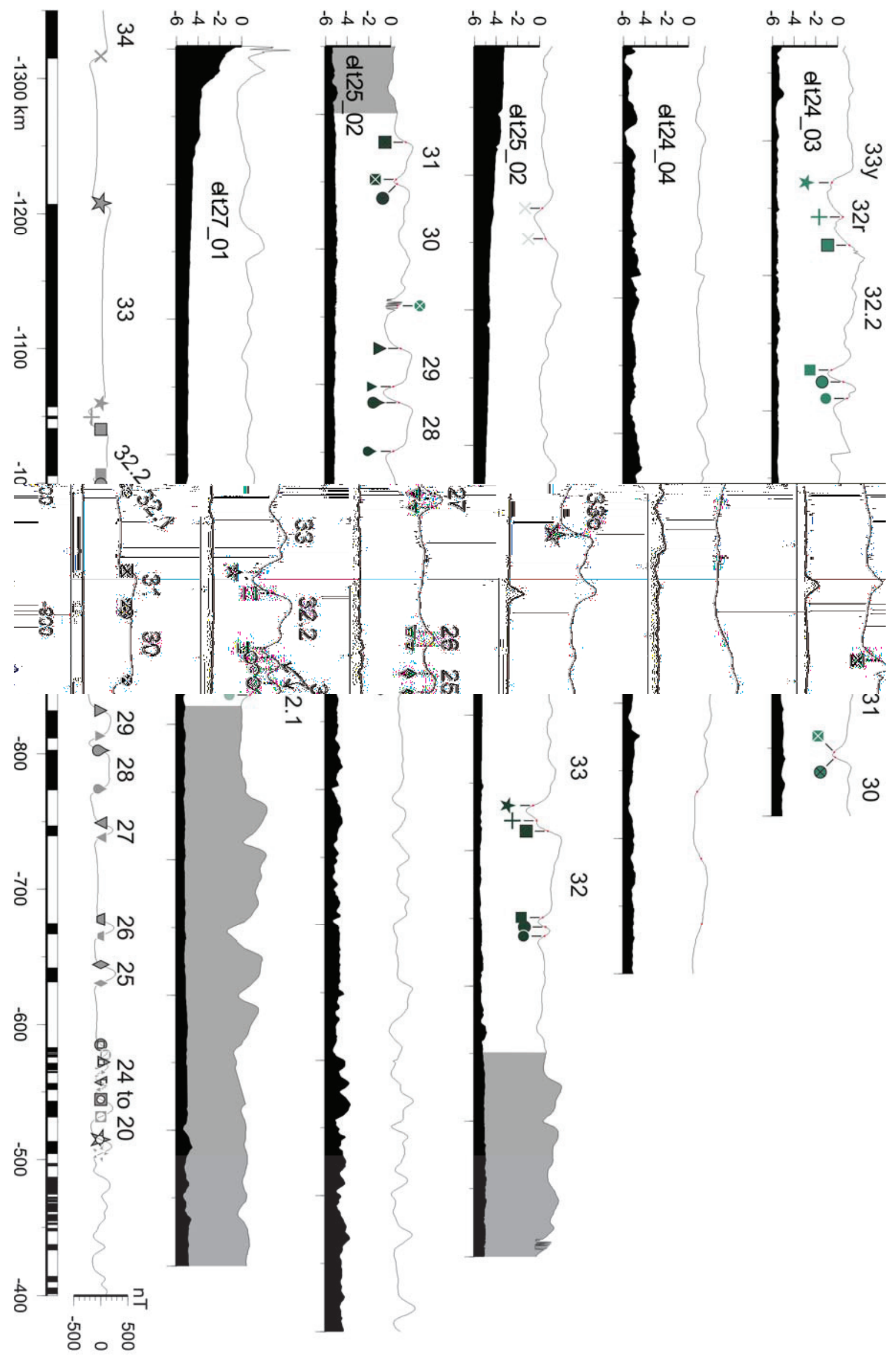




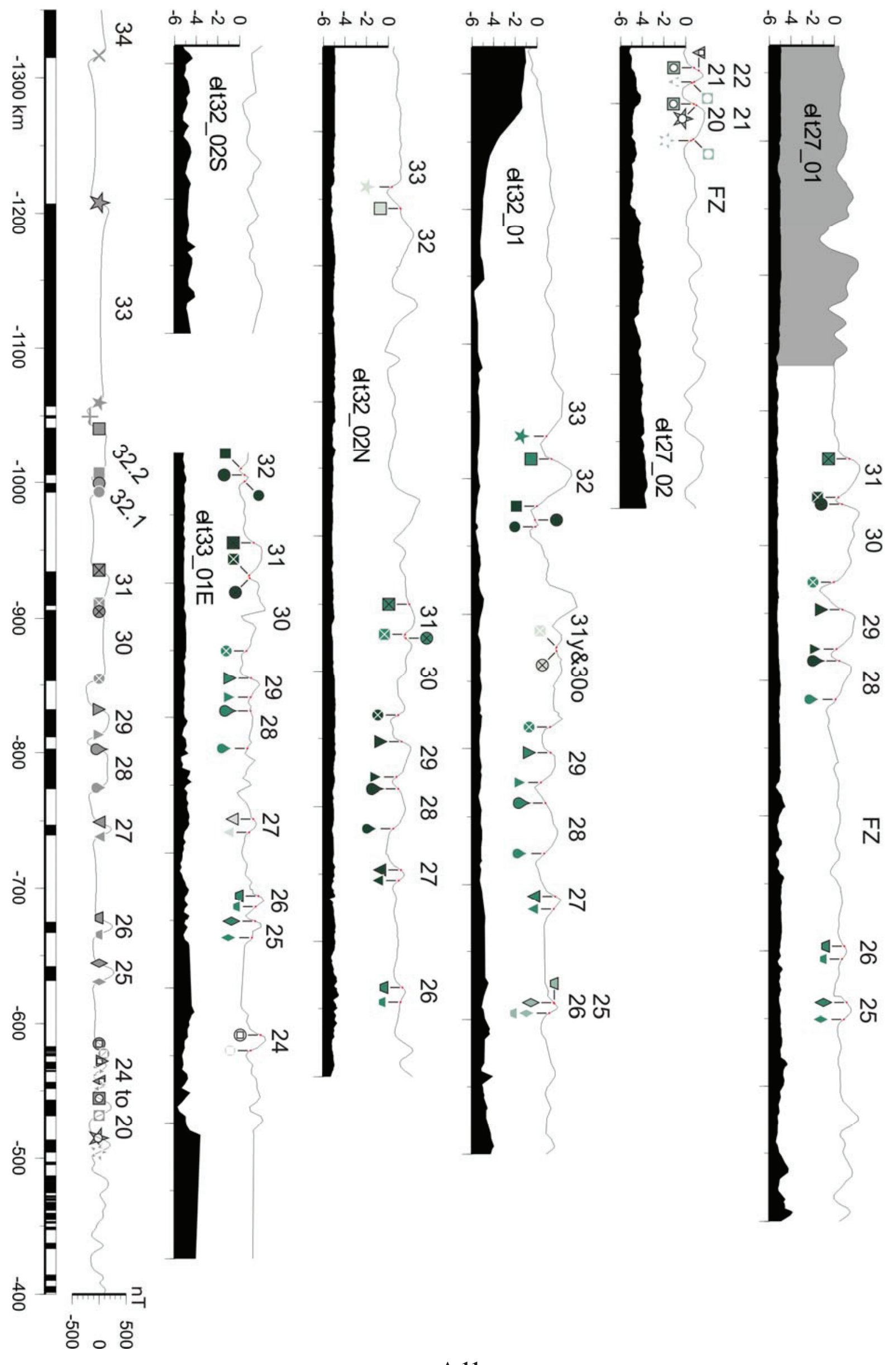




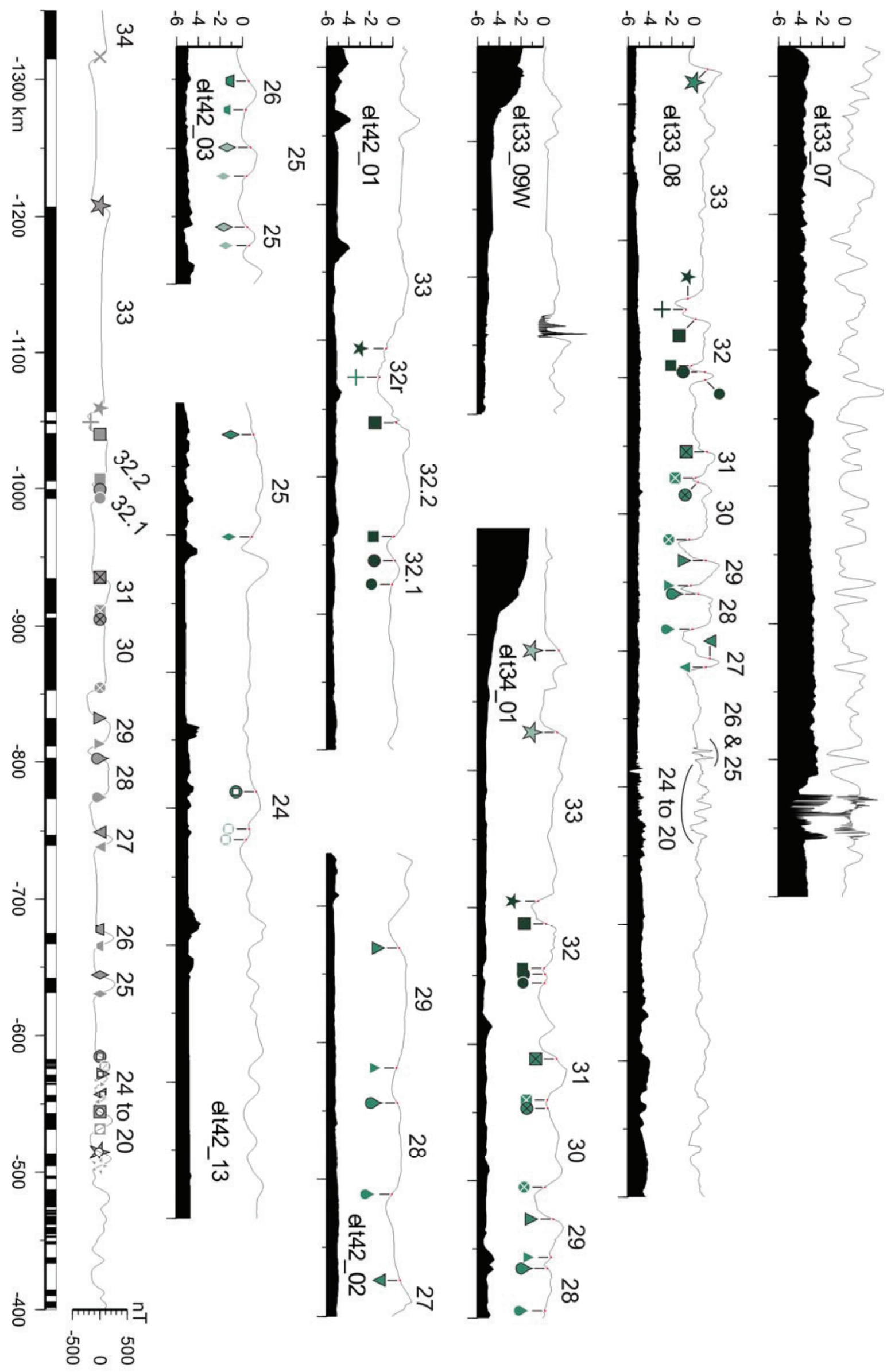




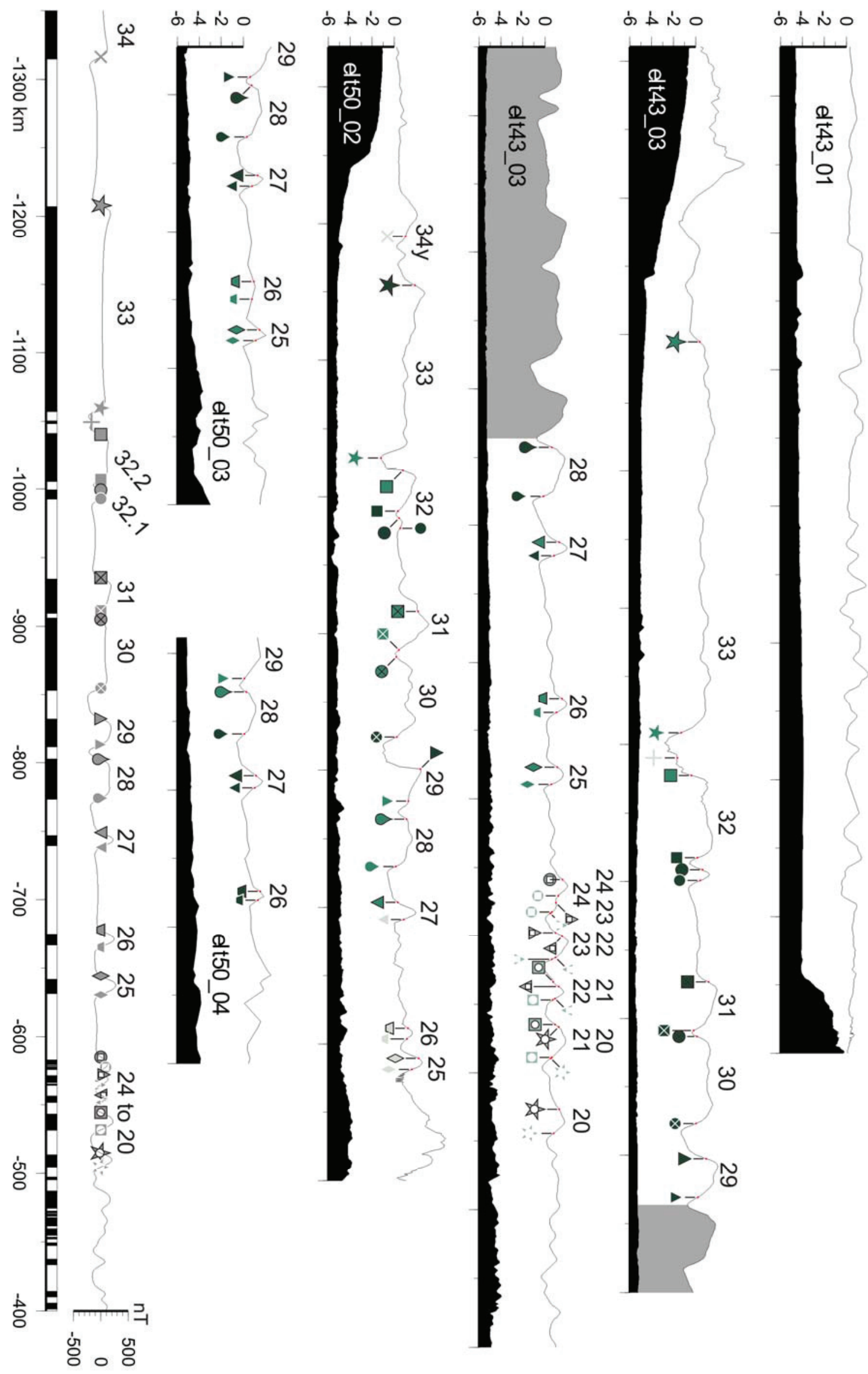




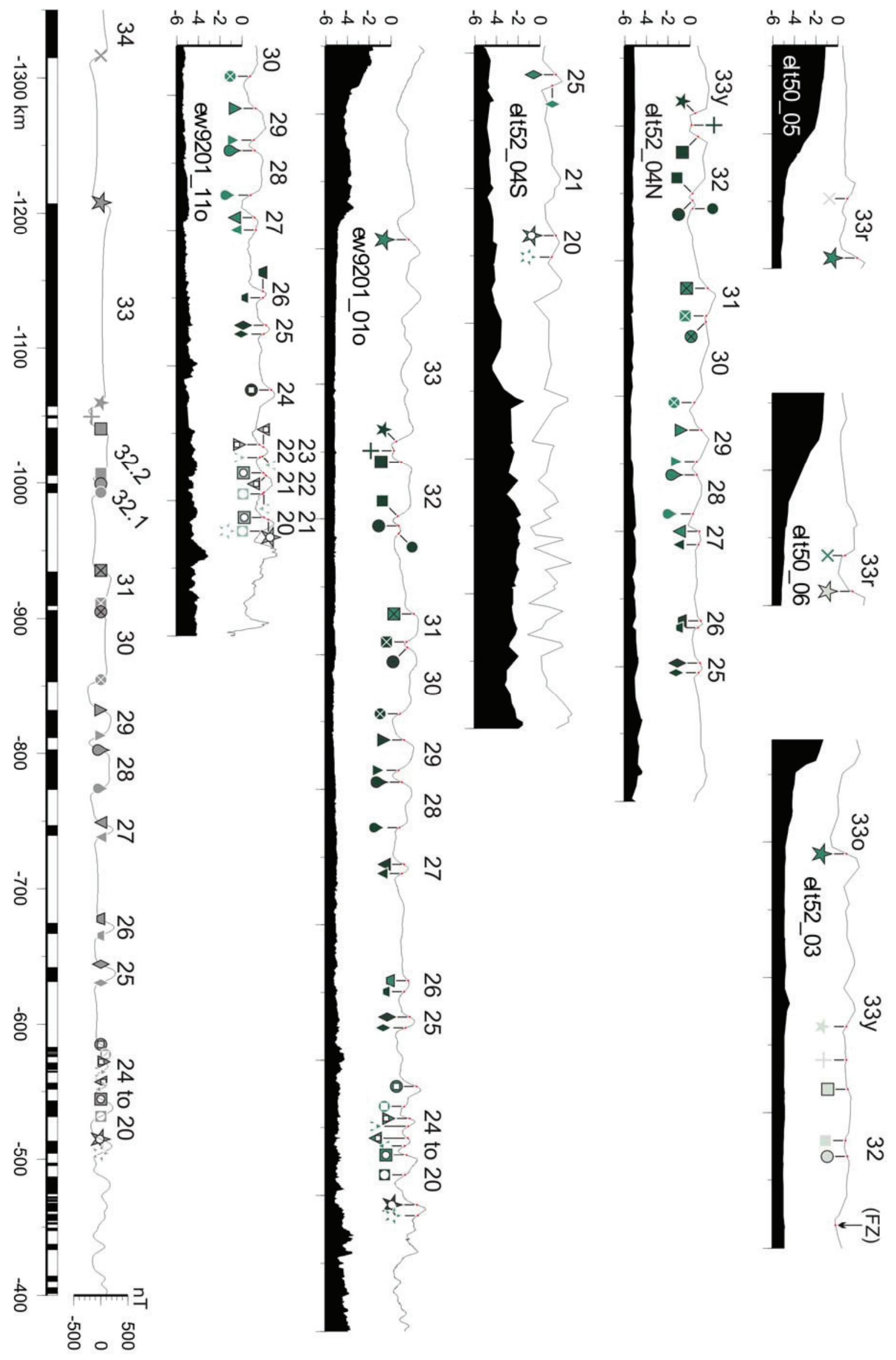




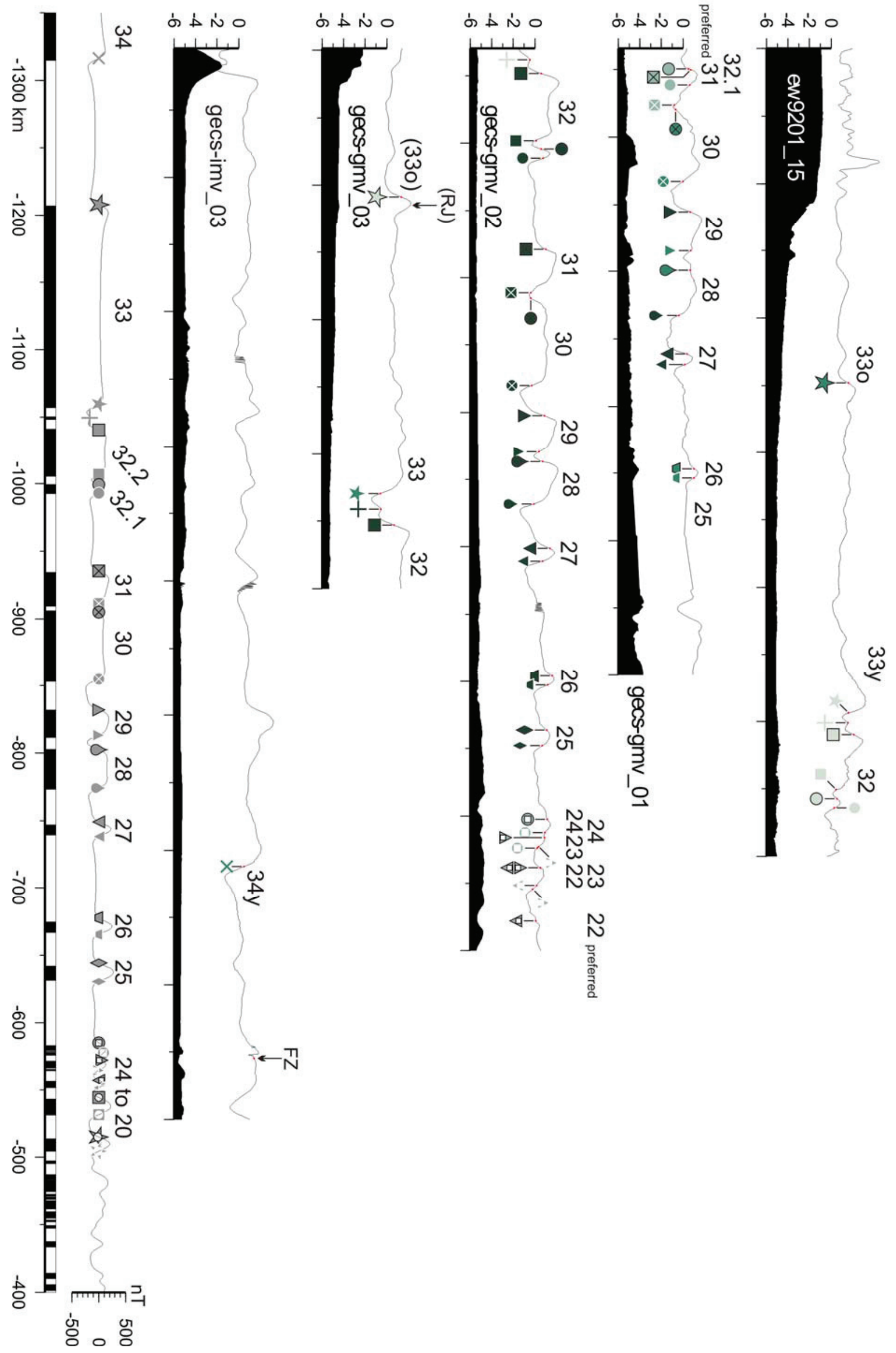




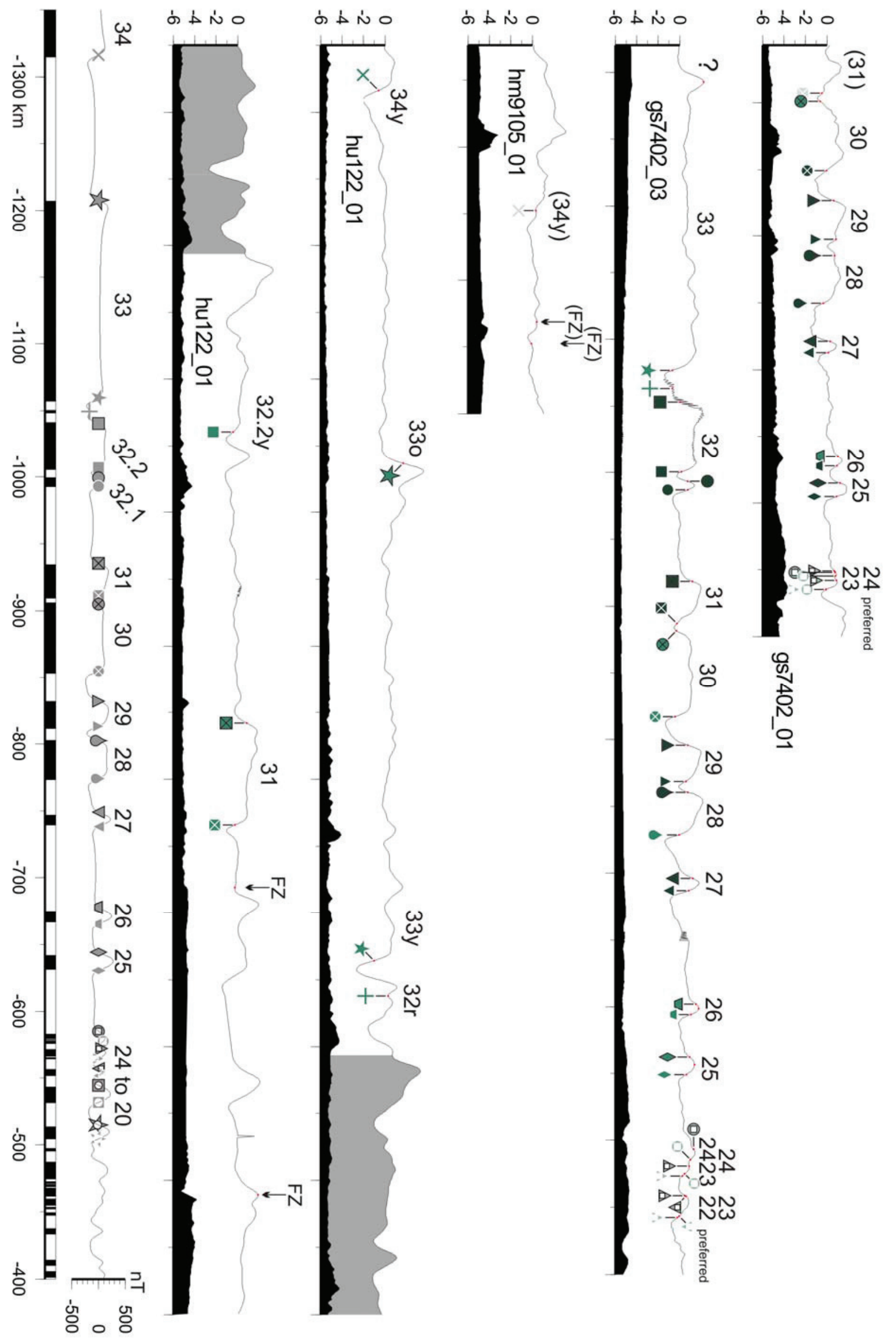




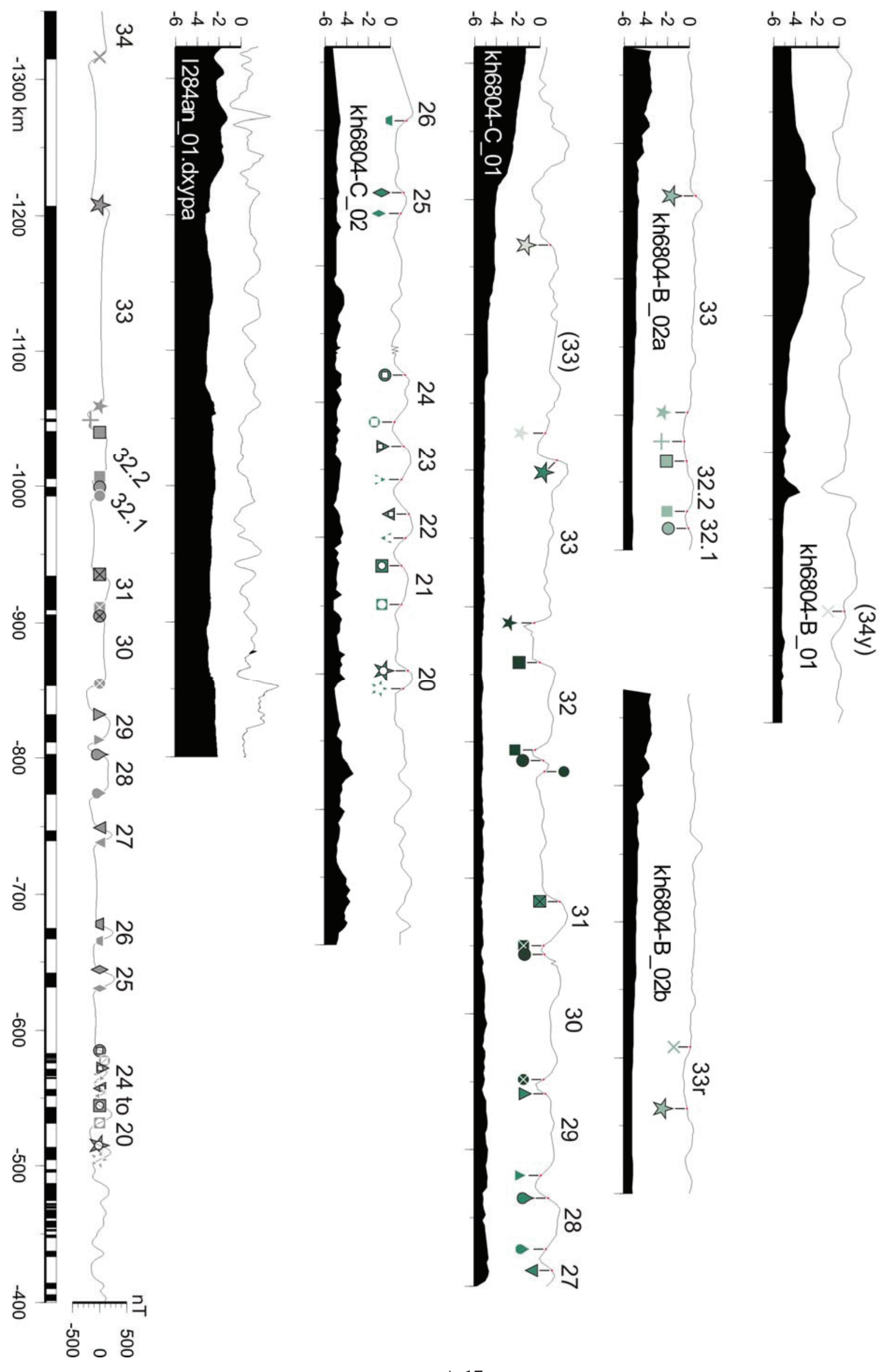




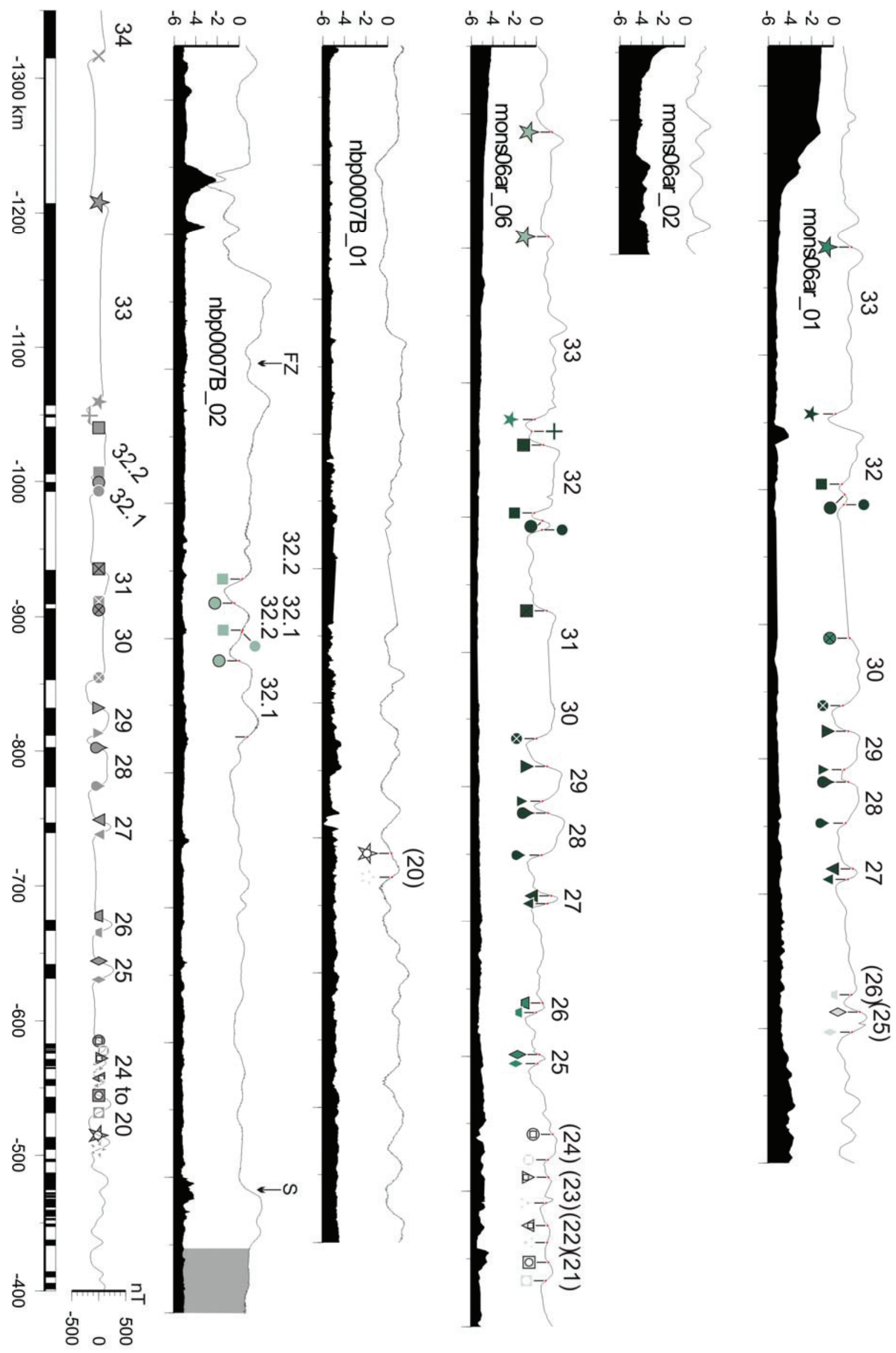




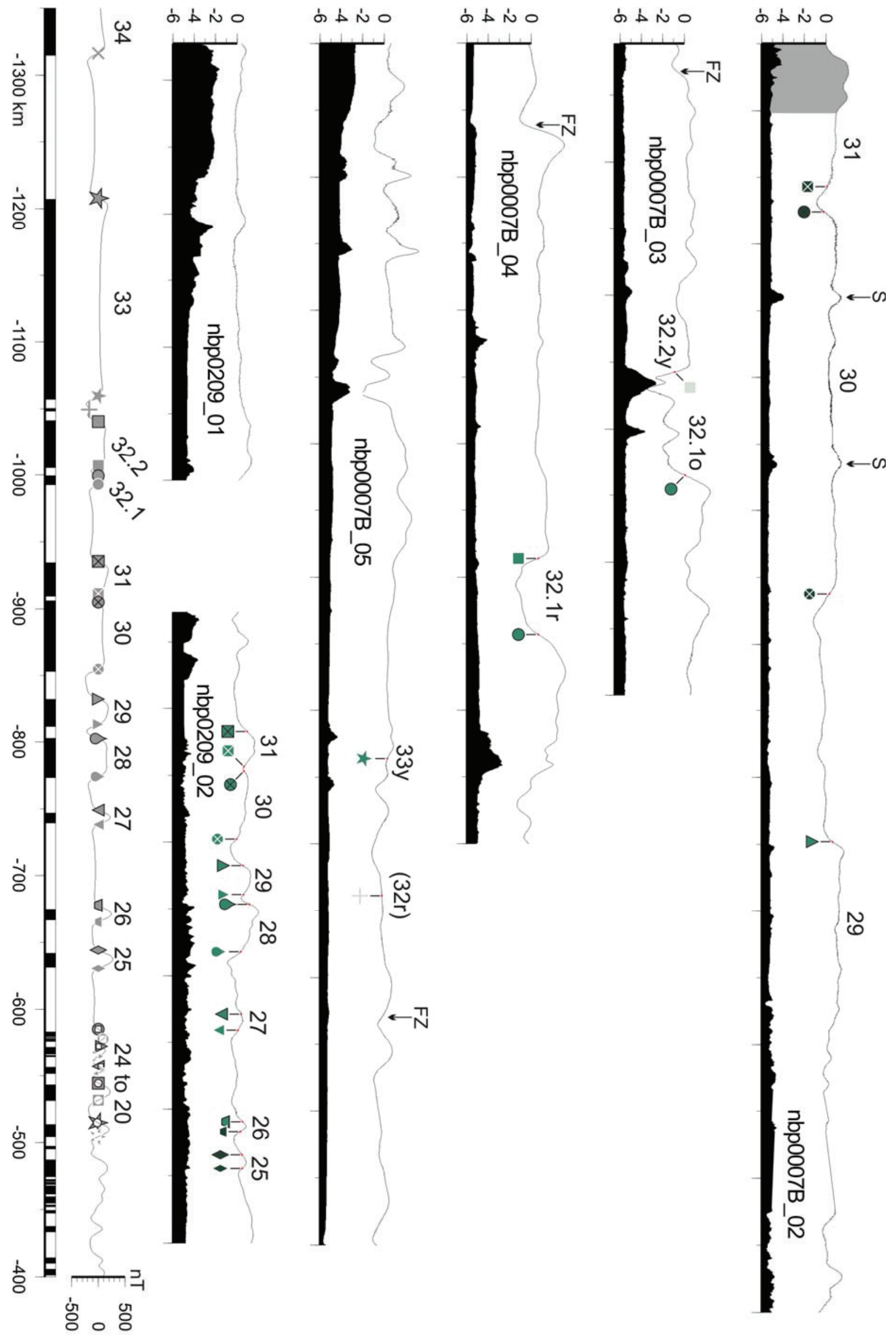



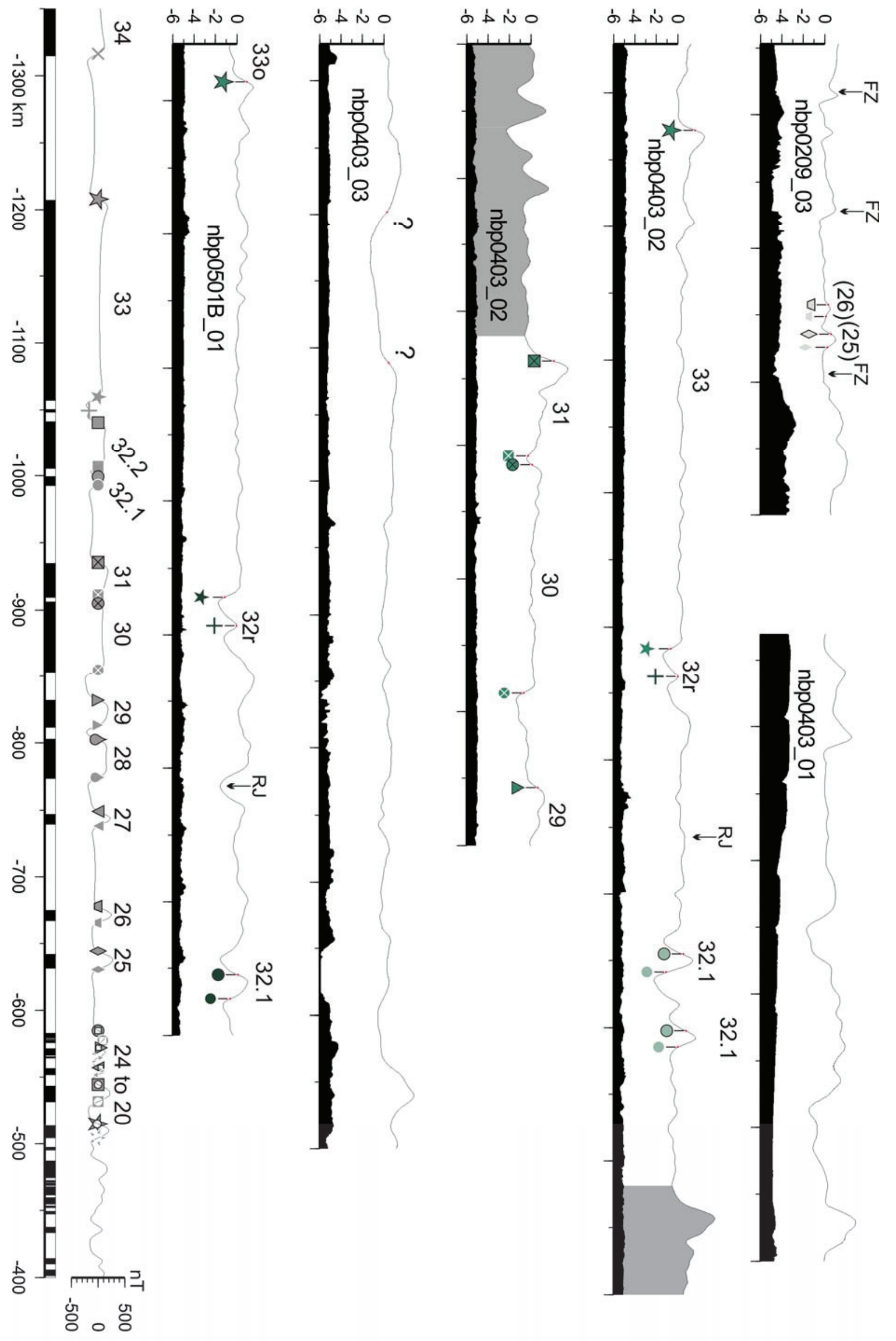


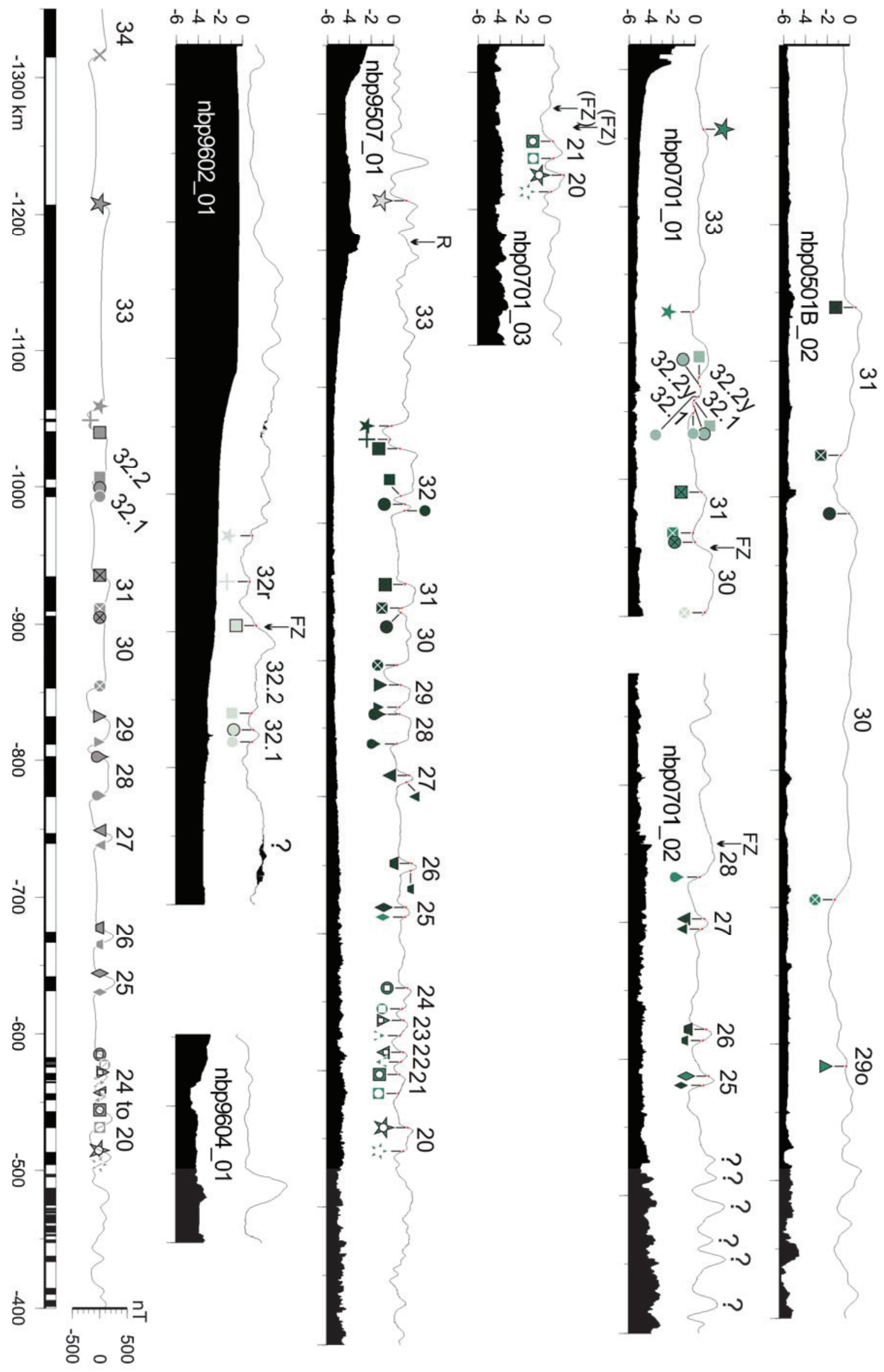



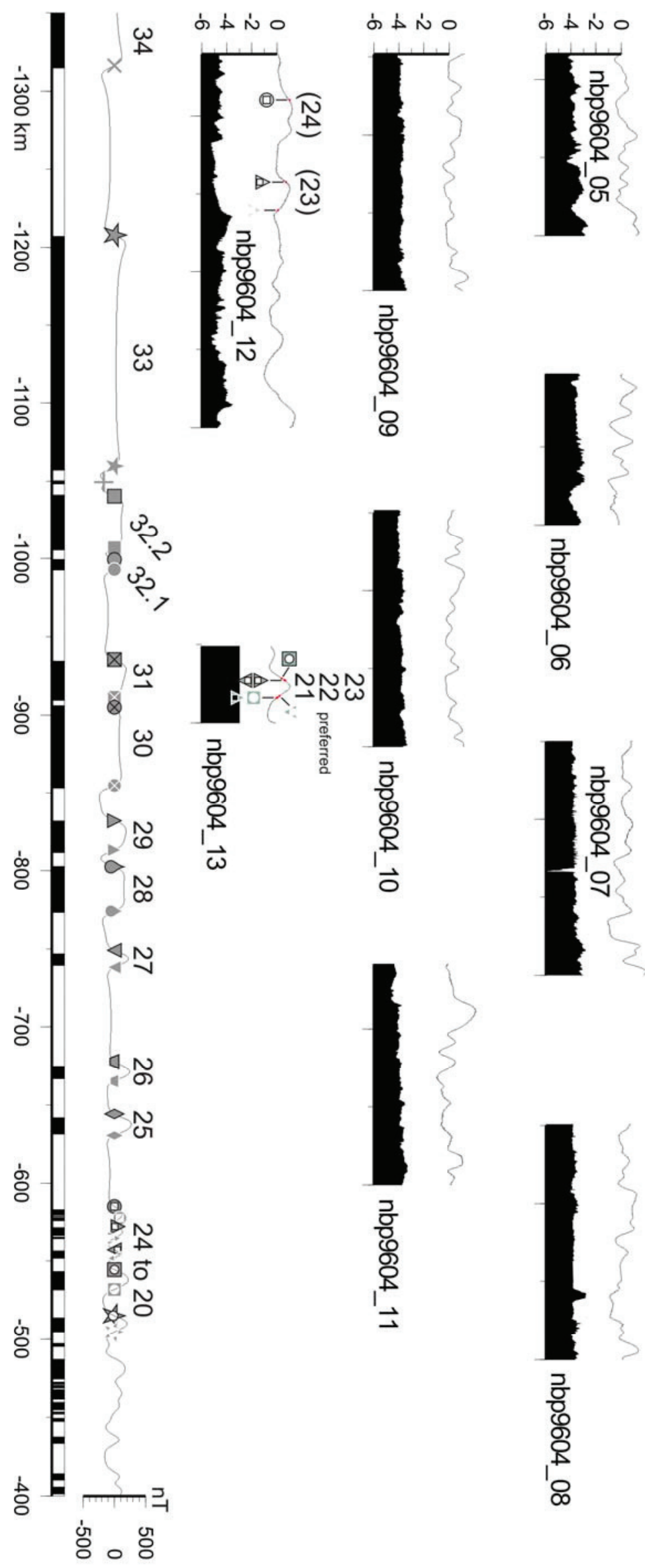

को

बमํㅇ
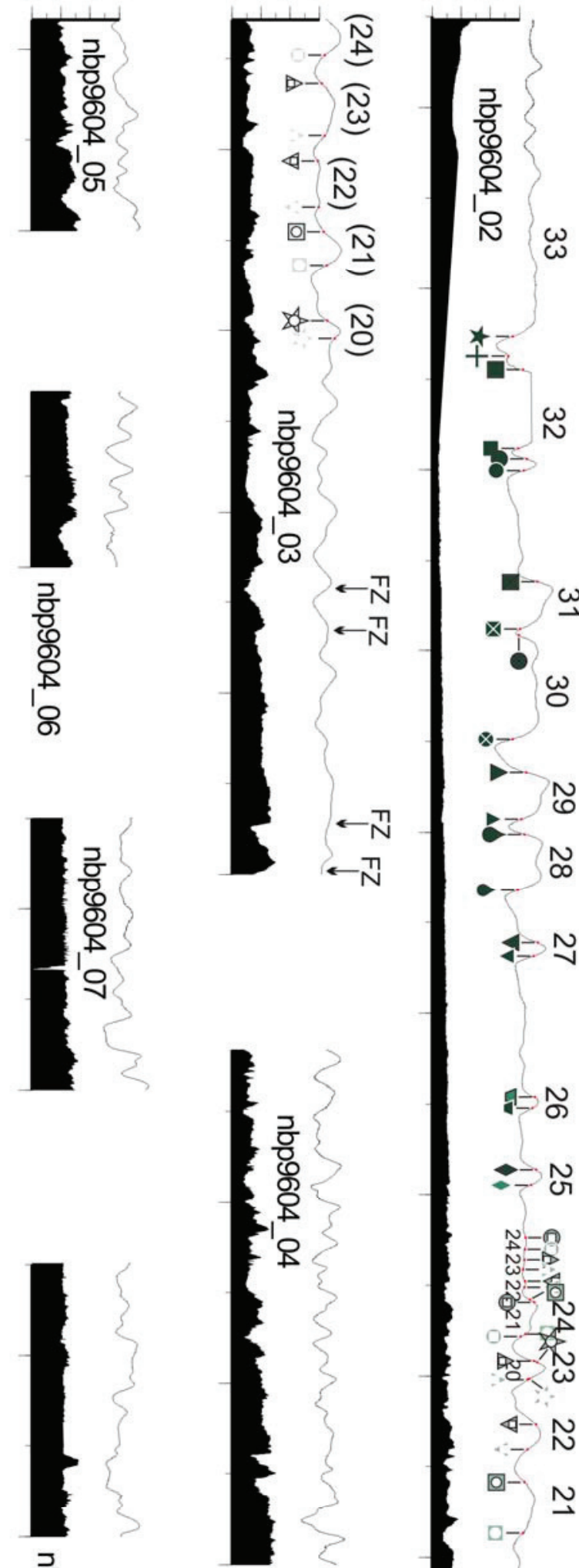

$\Leftrightarrow N$

$\because$ N
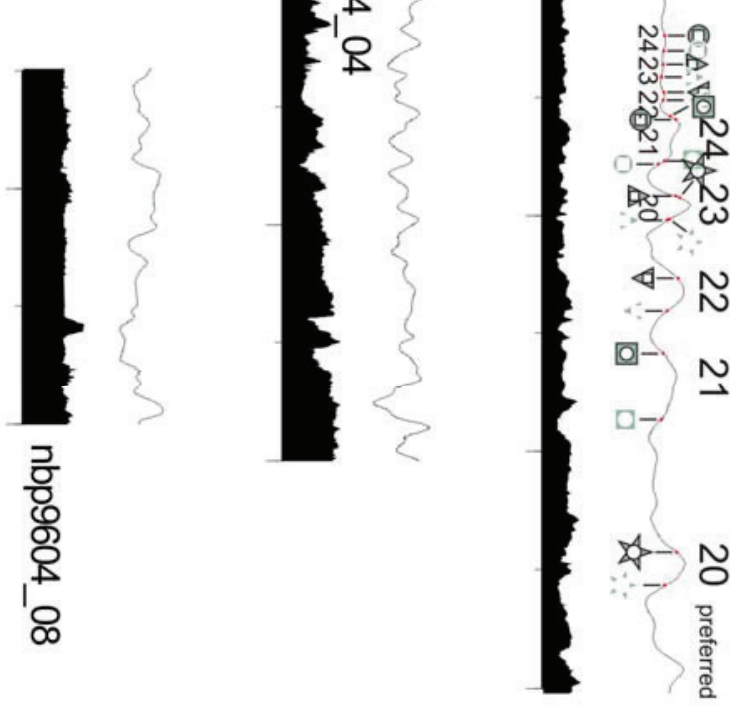


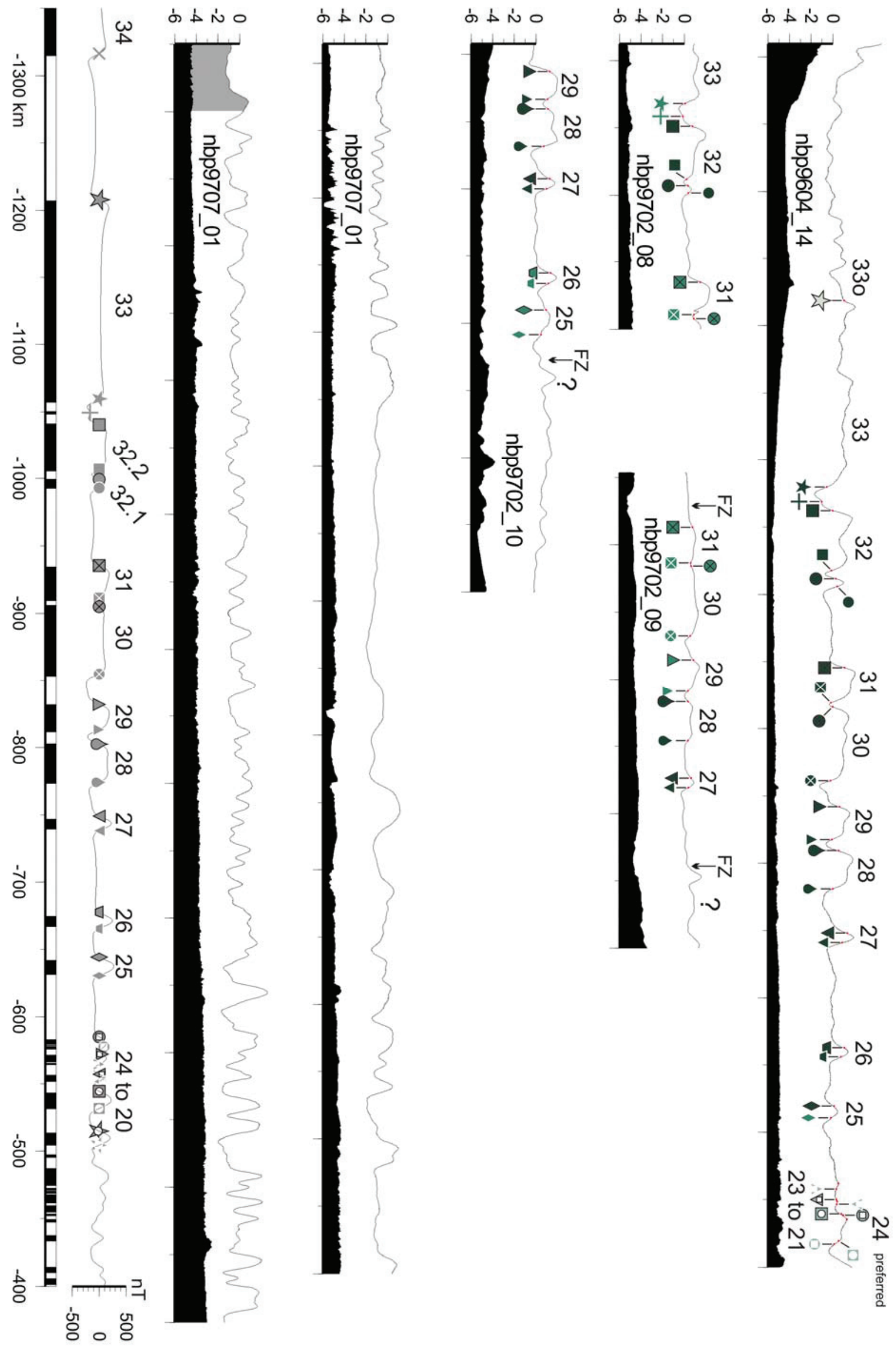




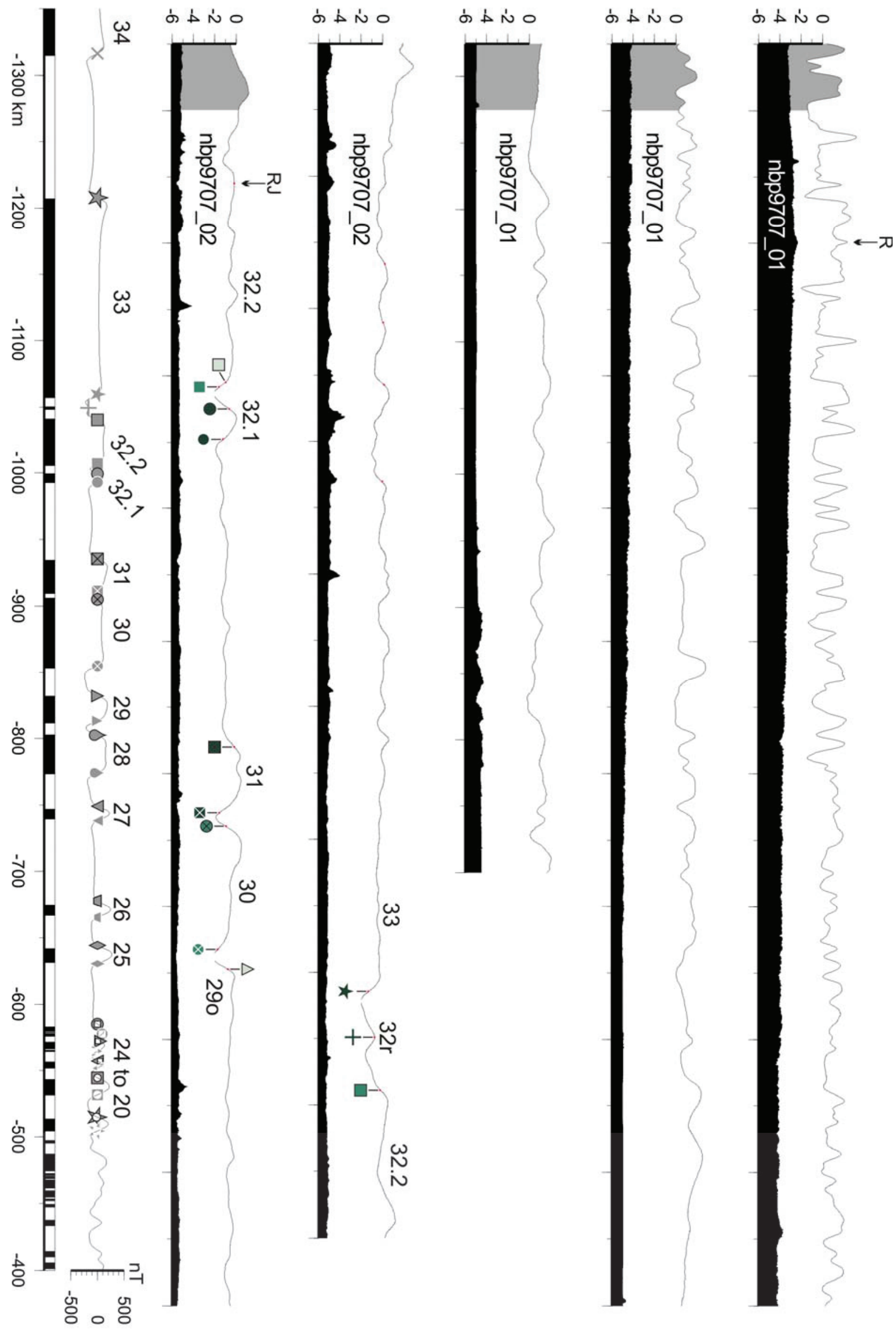




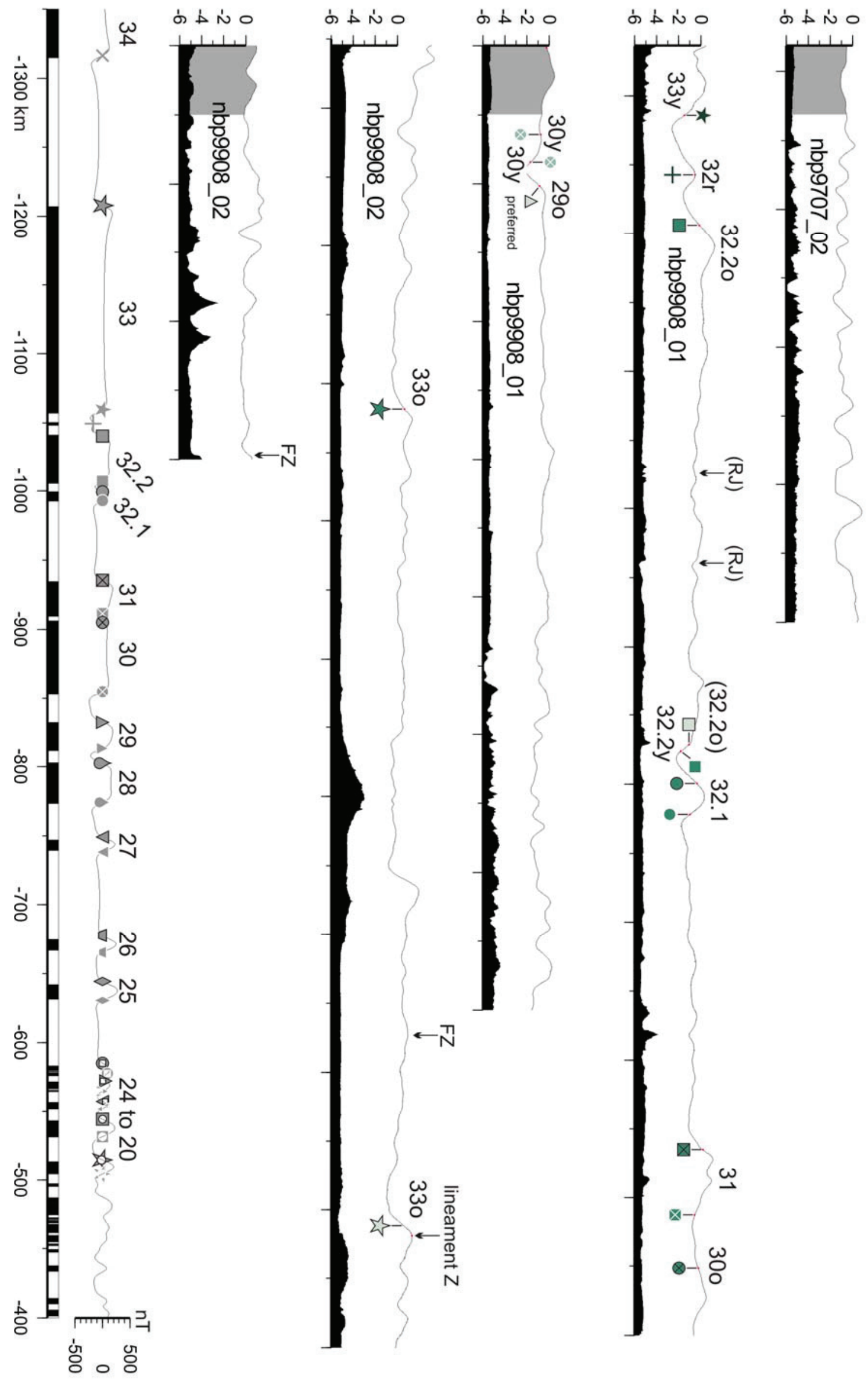




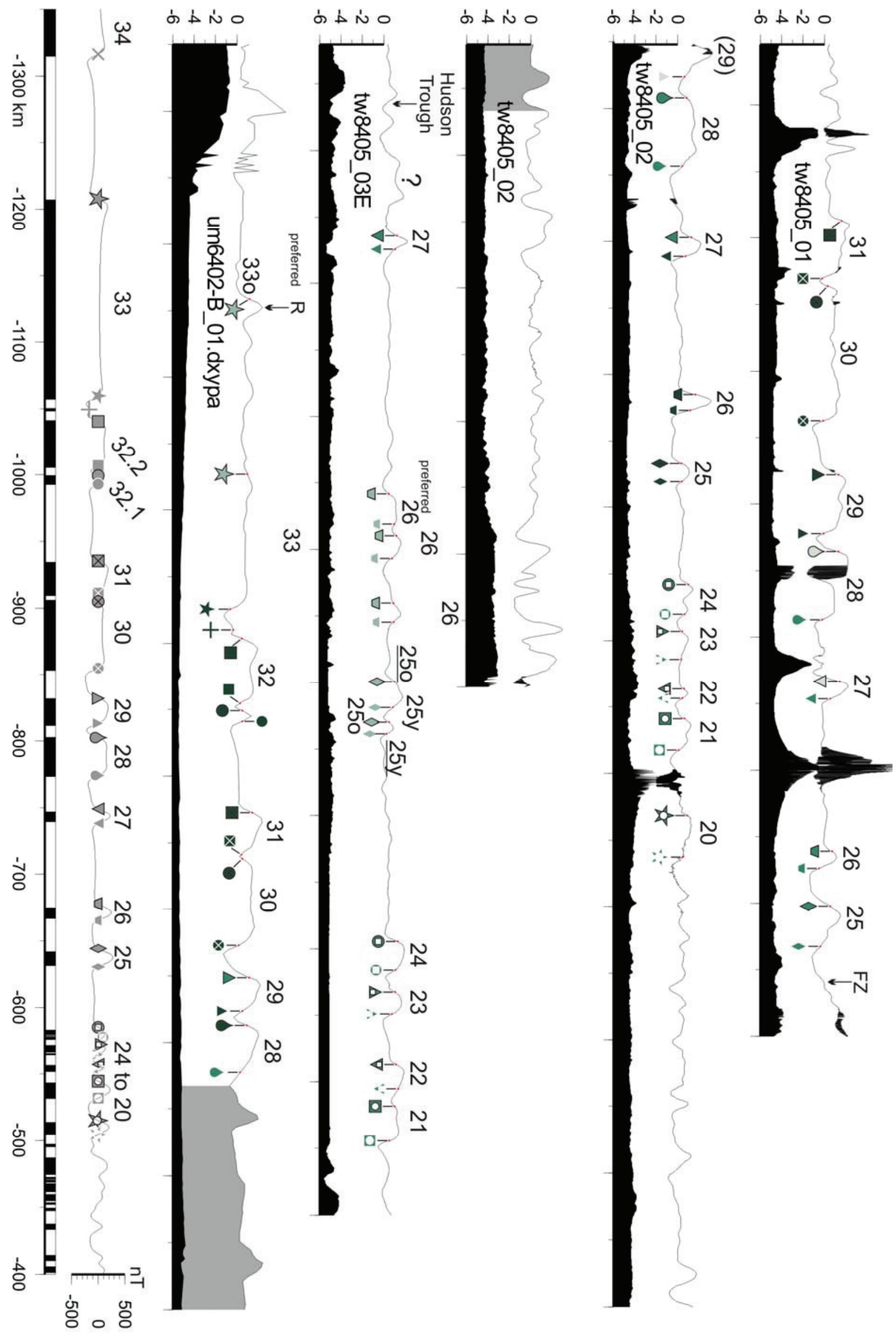




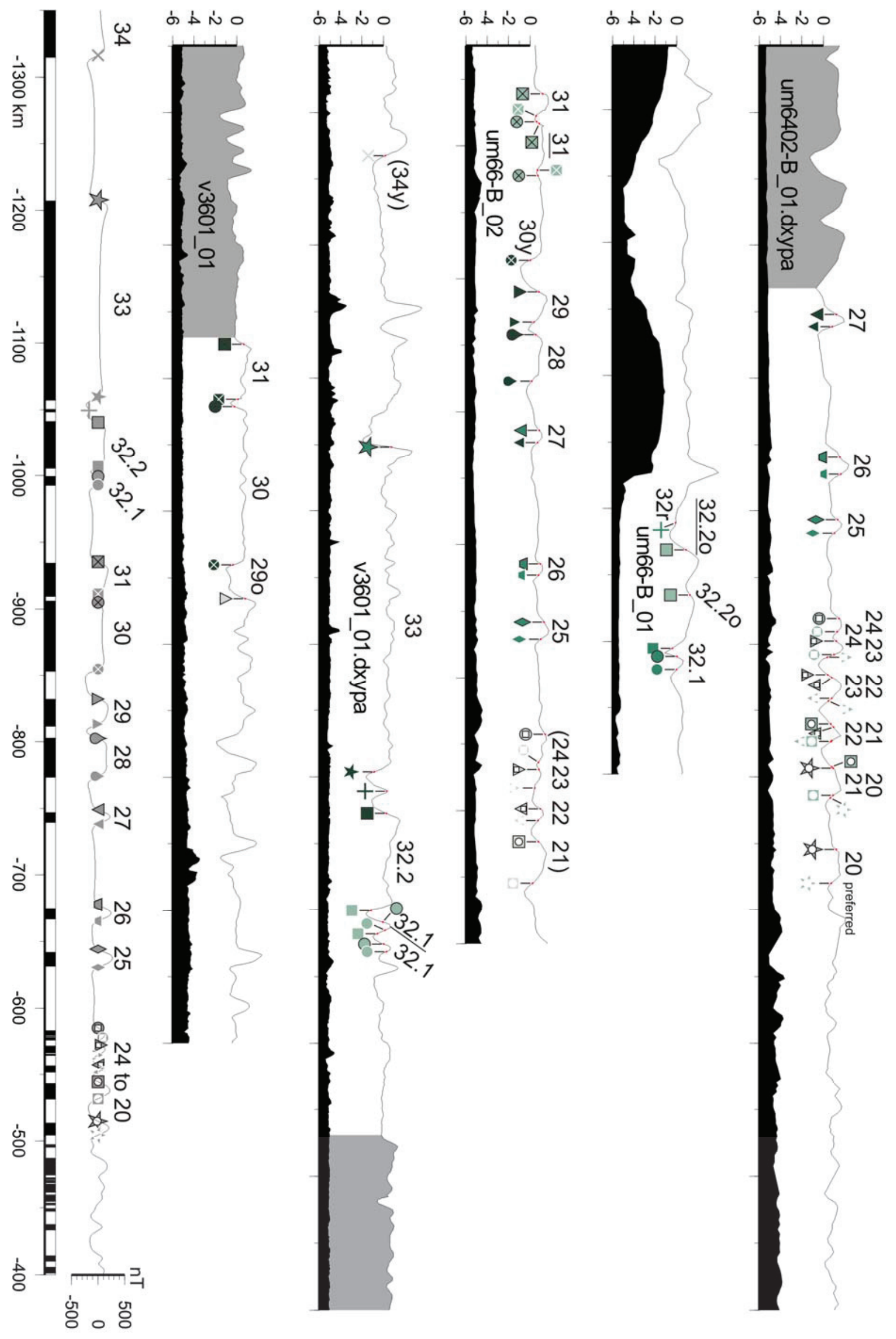




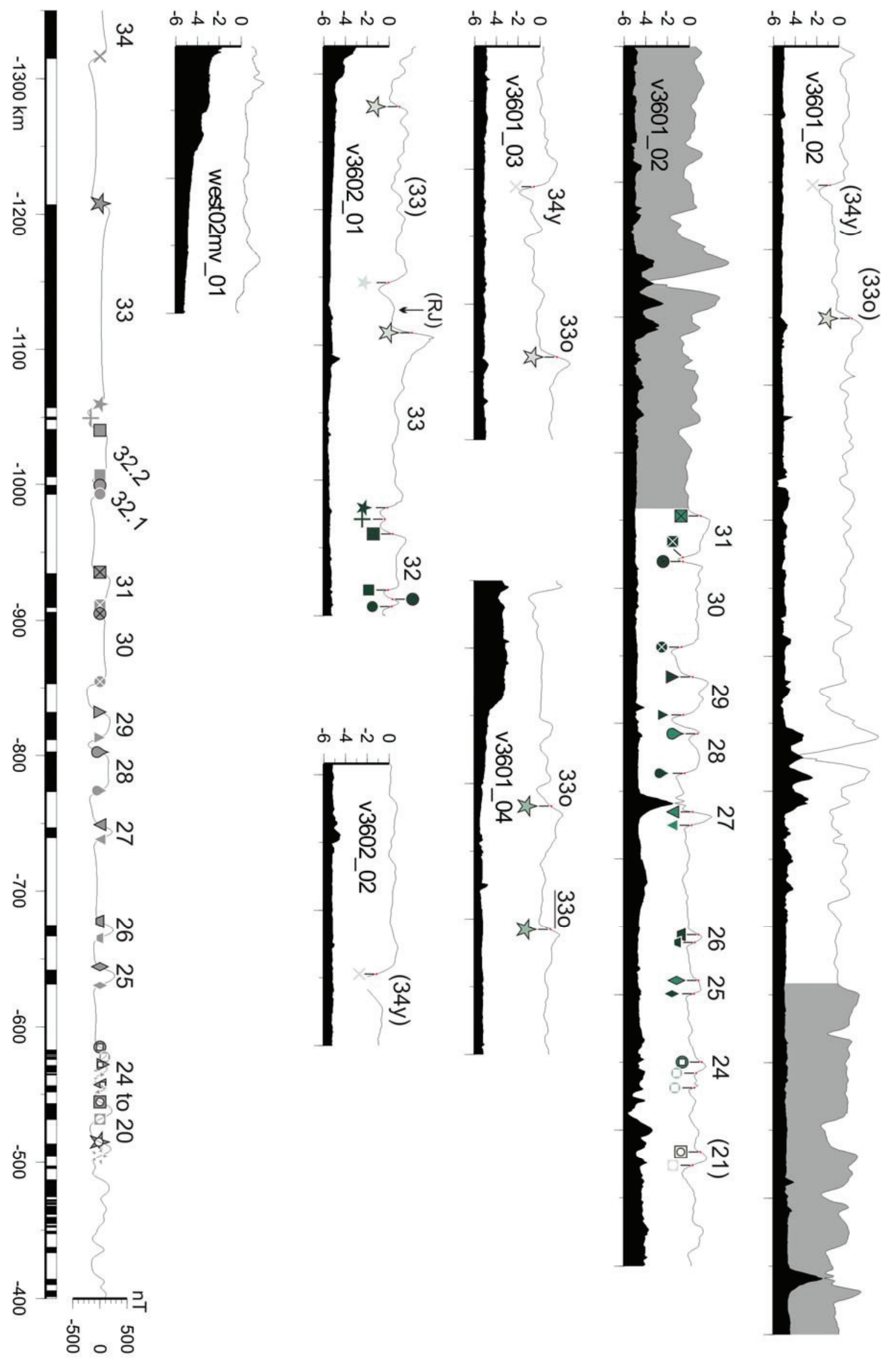




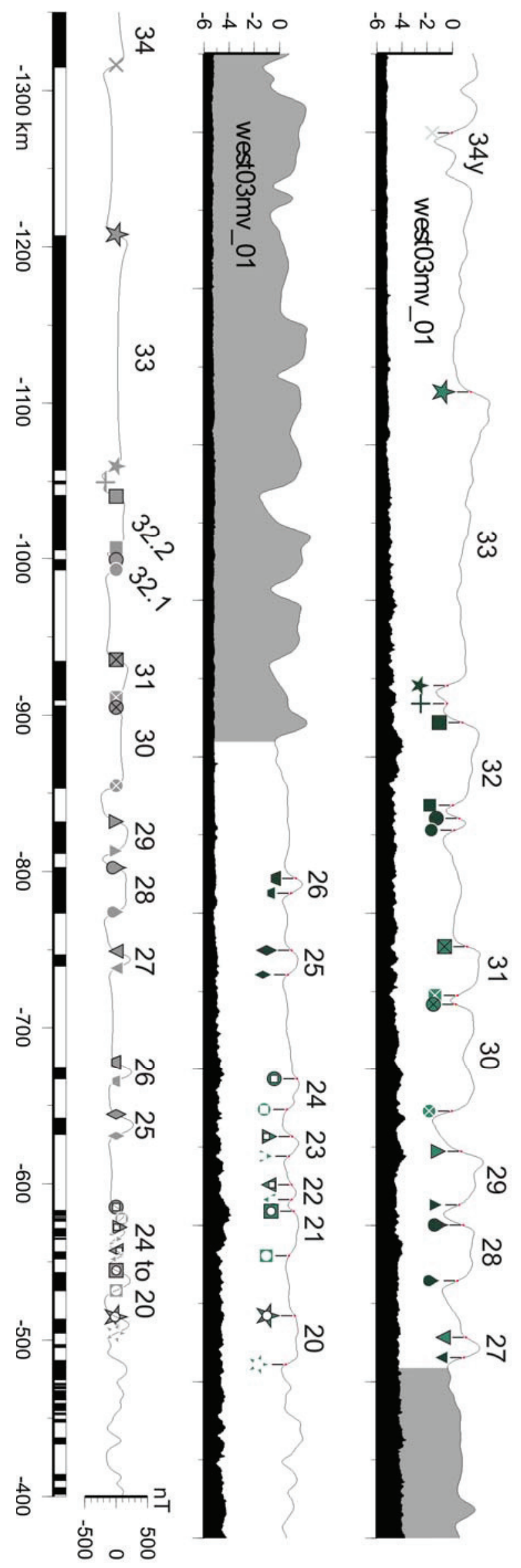




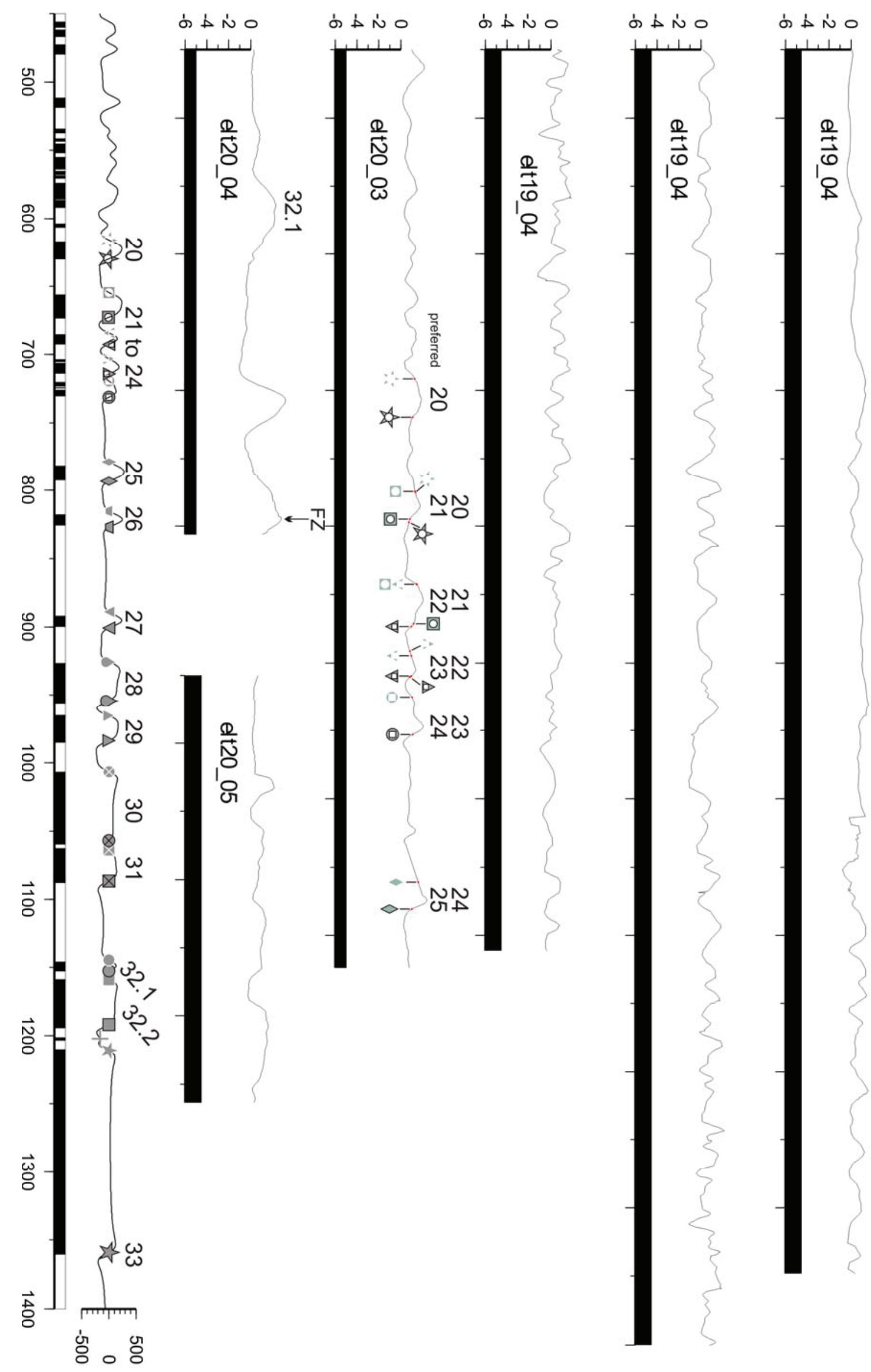




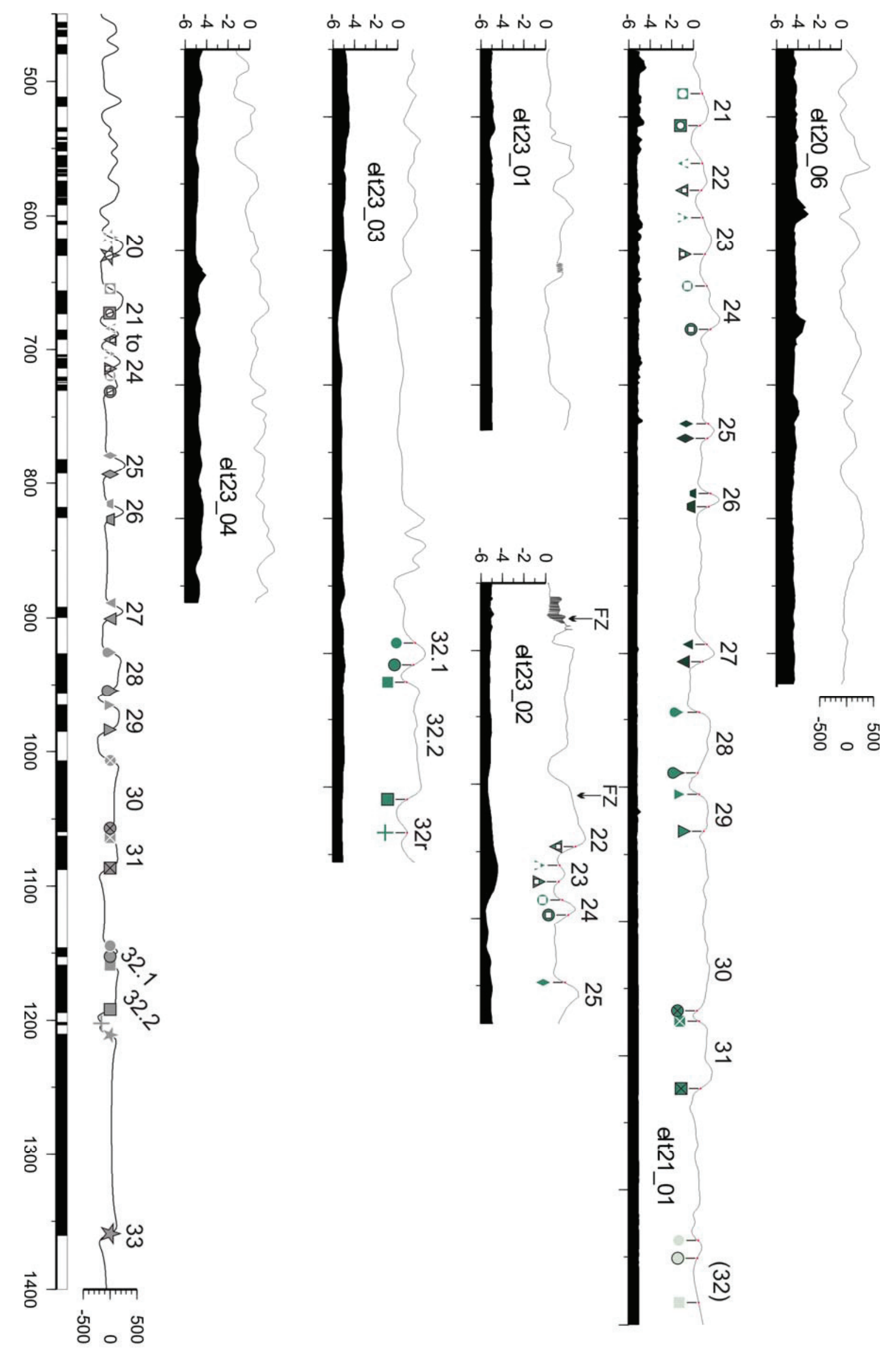




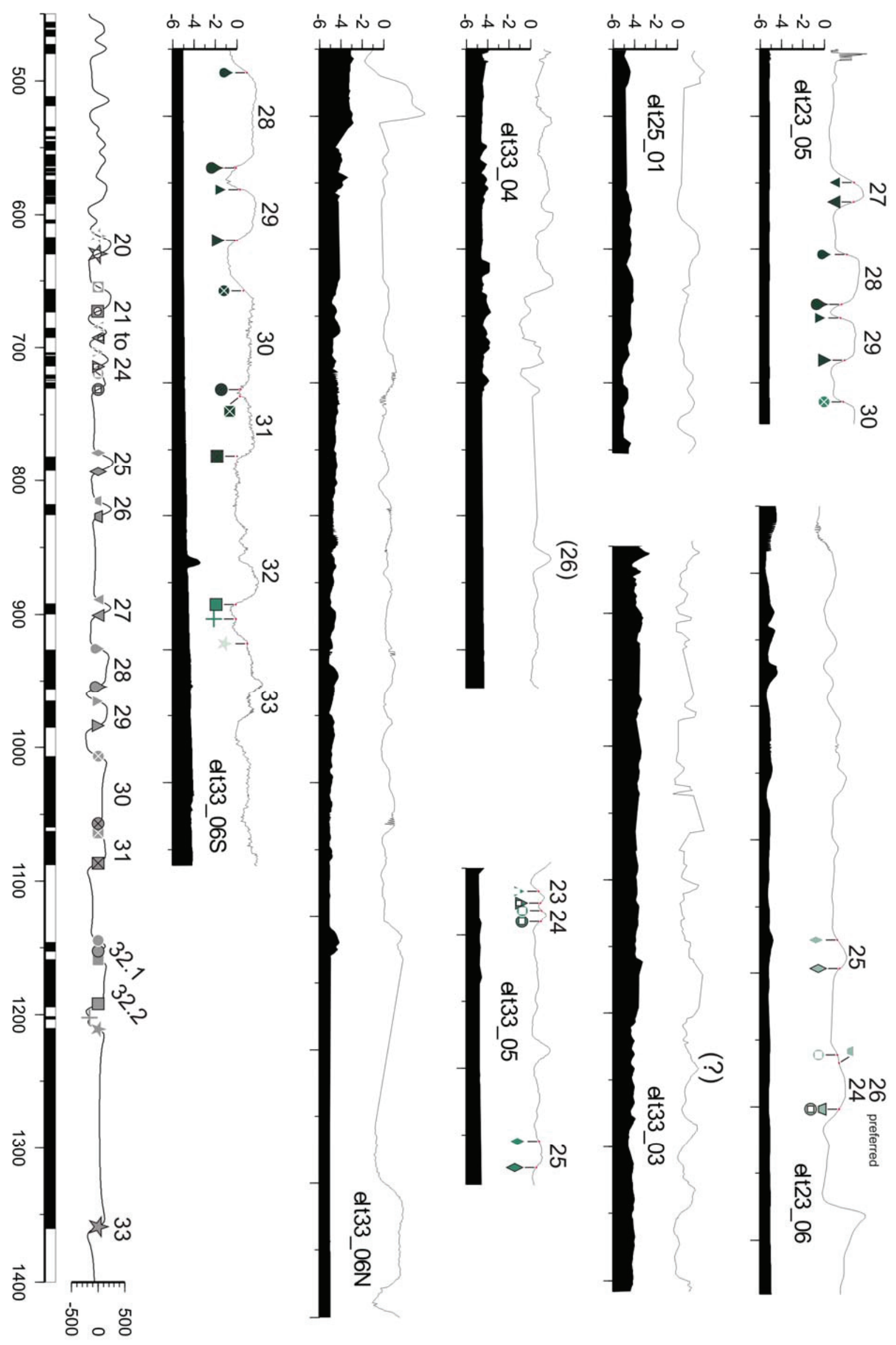




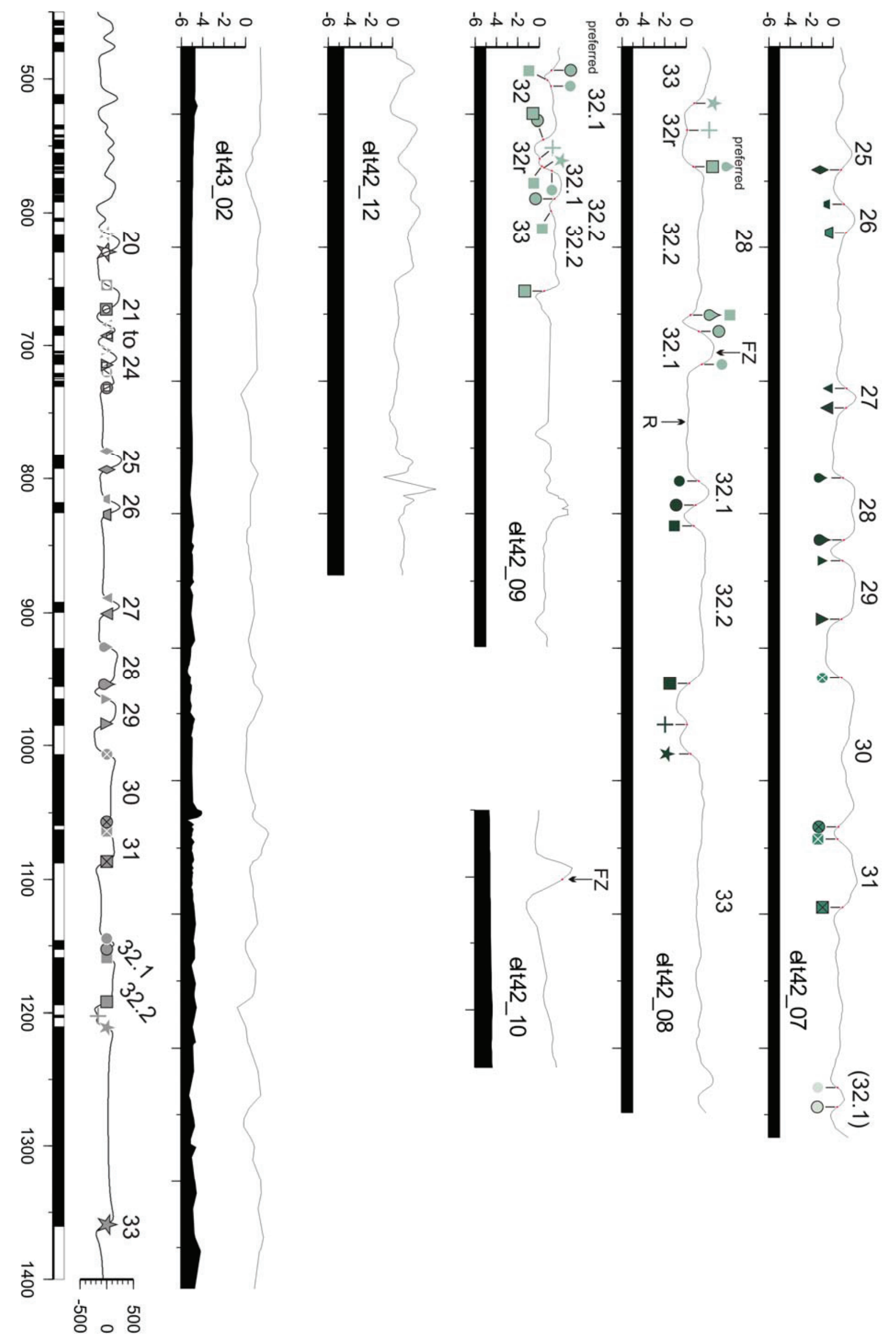




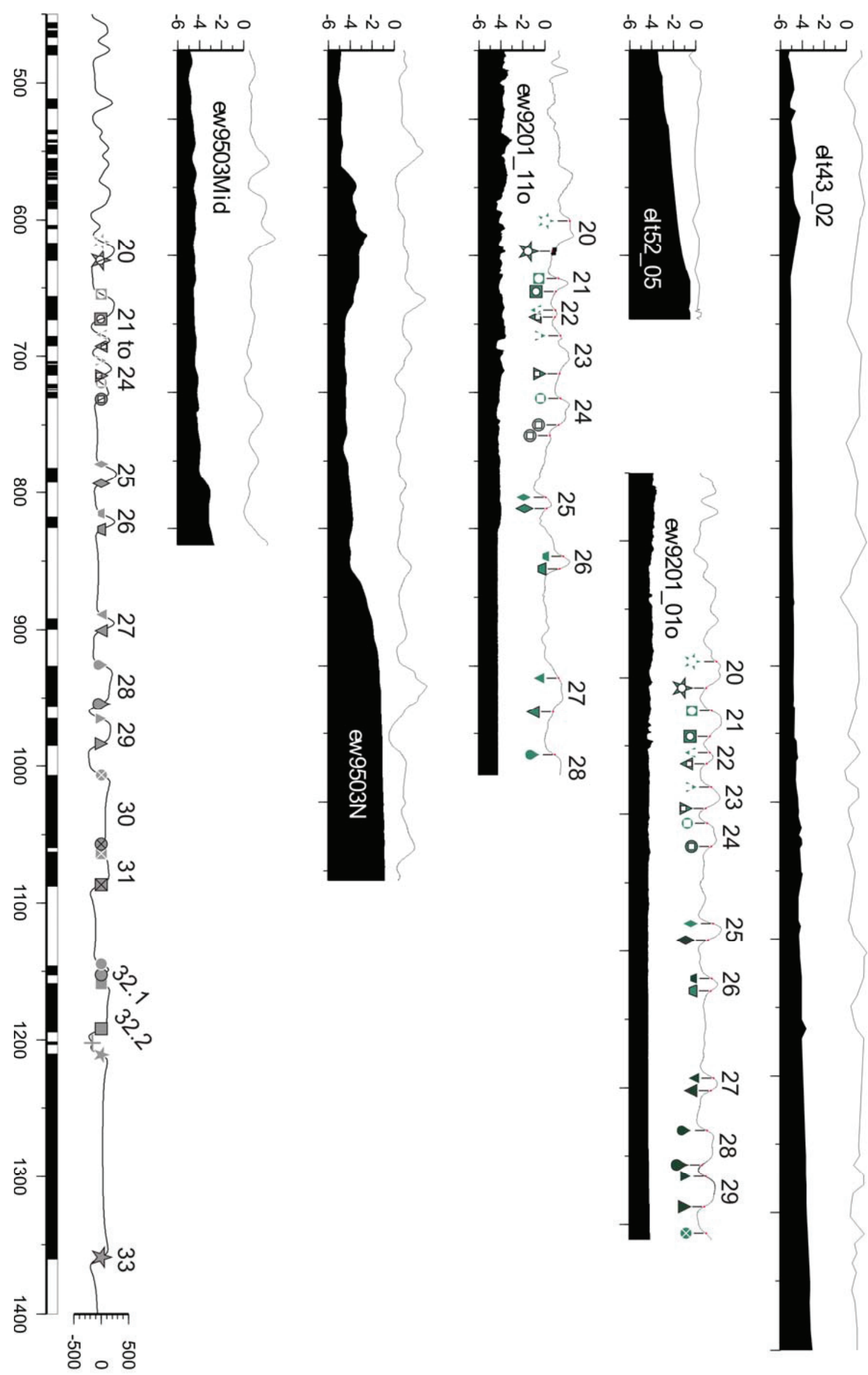




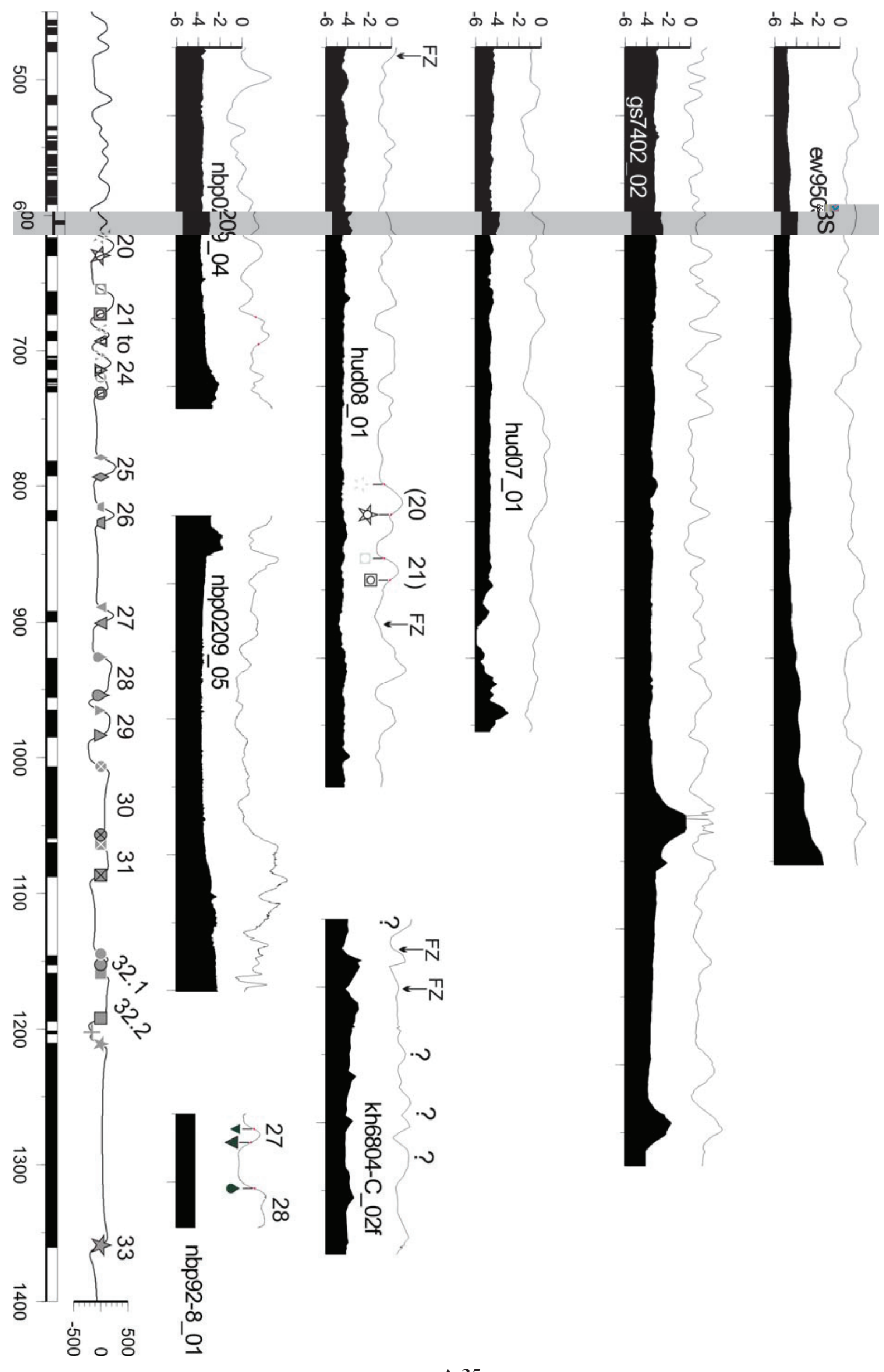




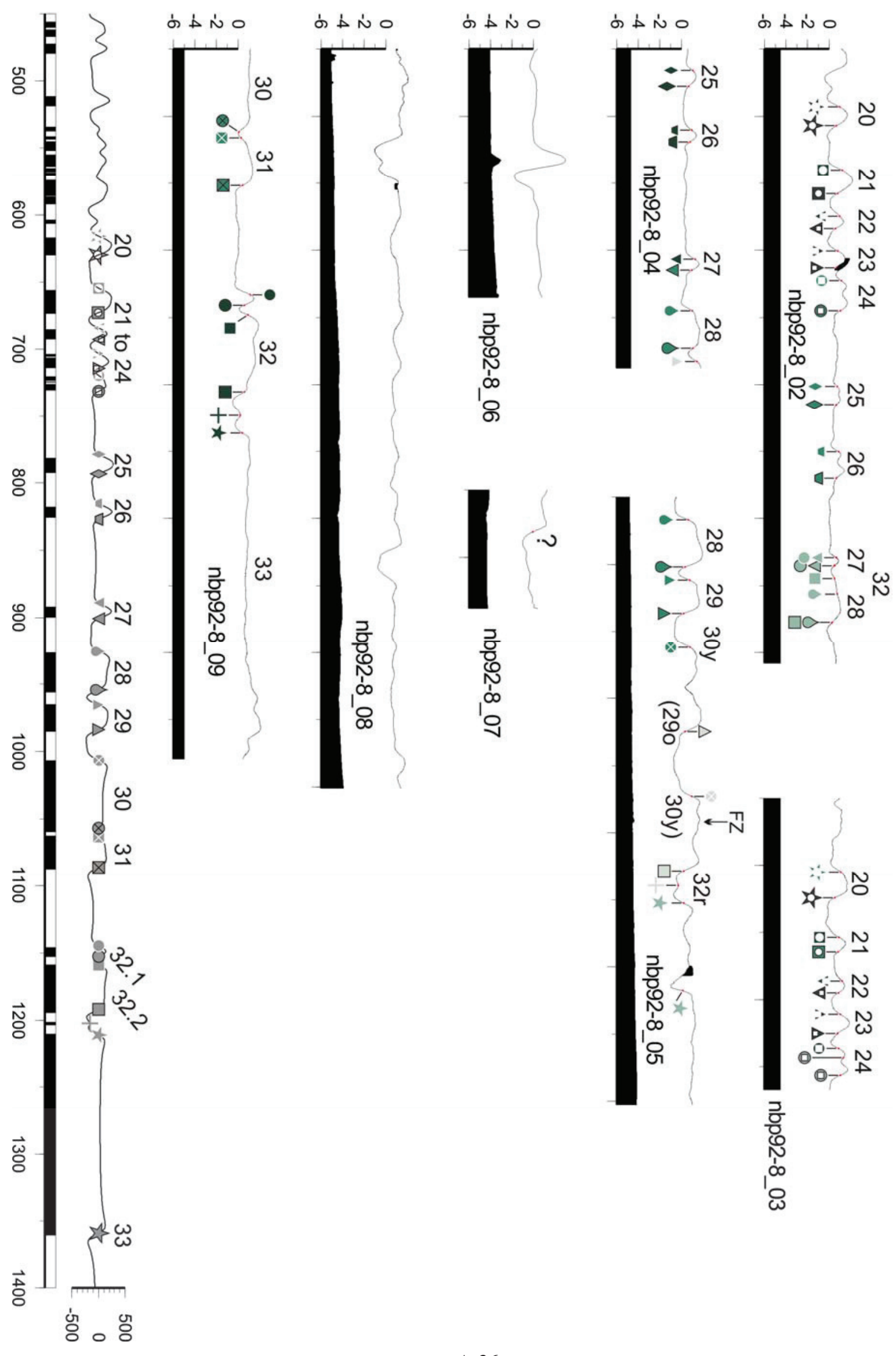




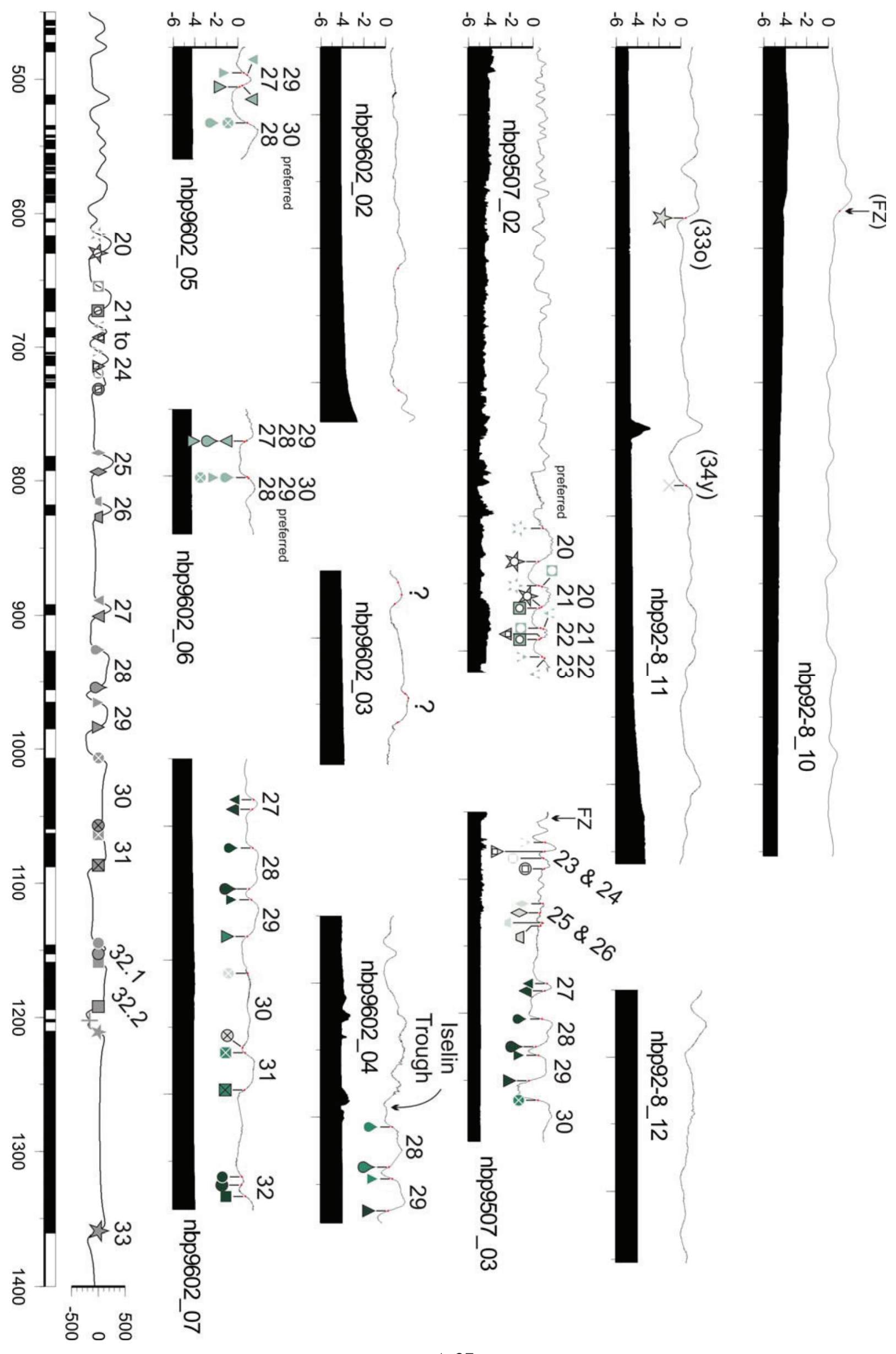




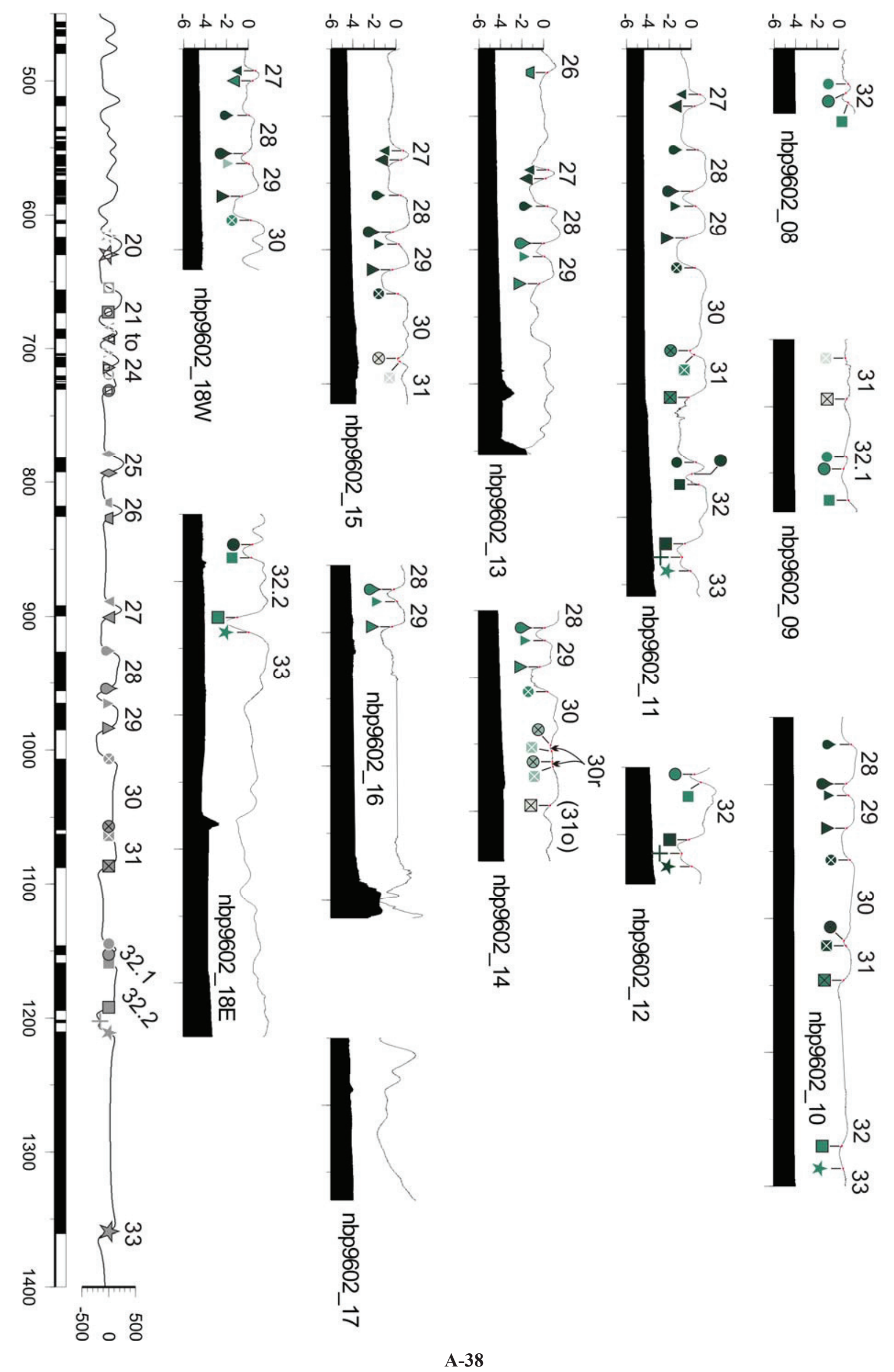




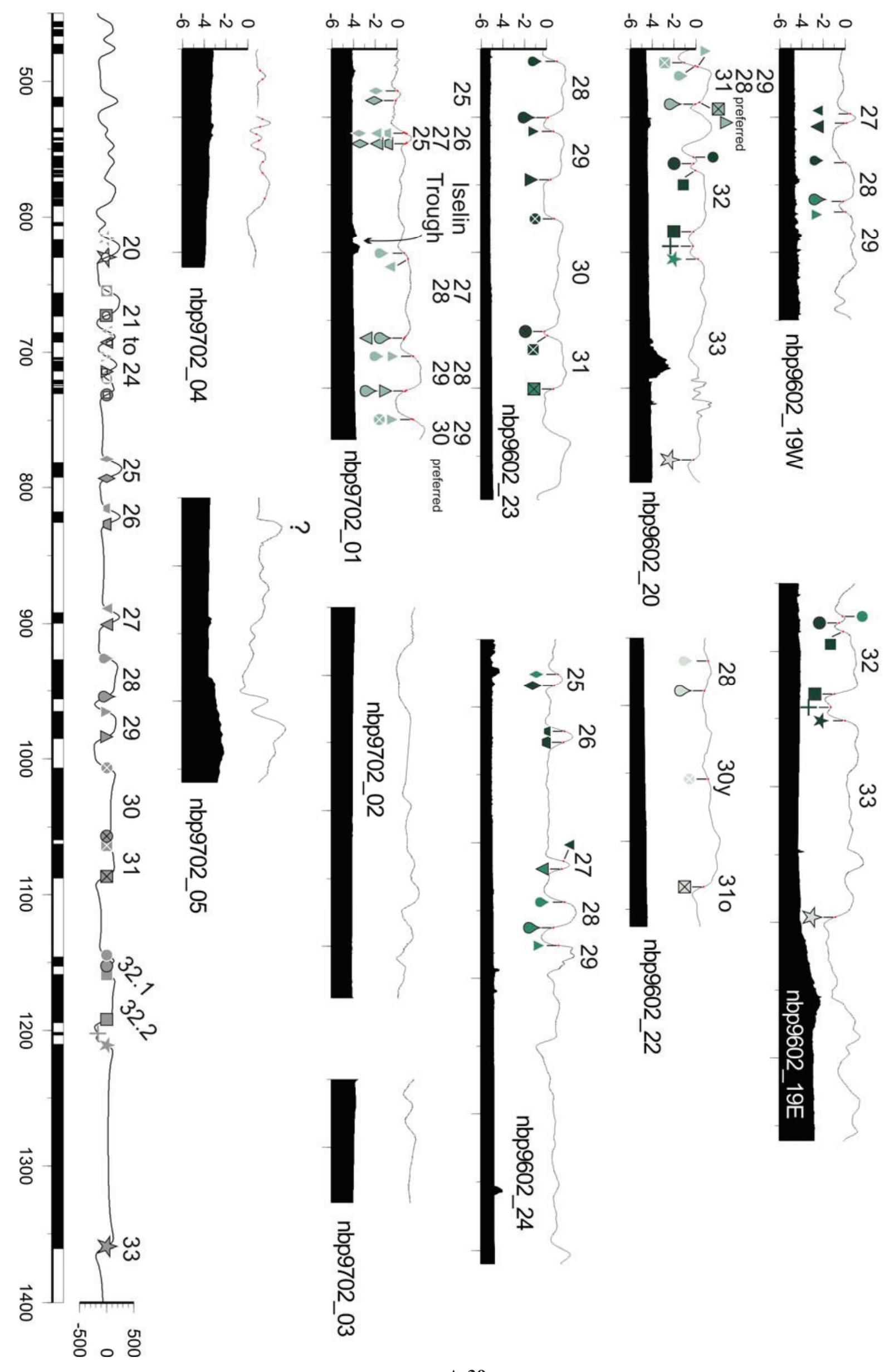




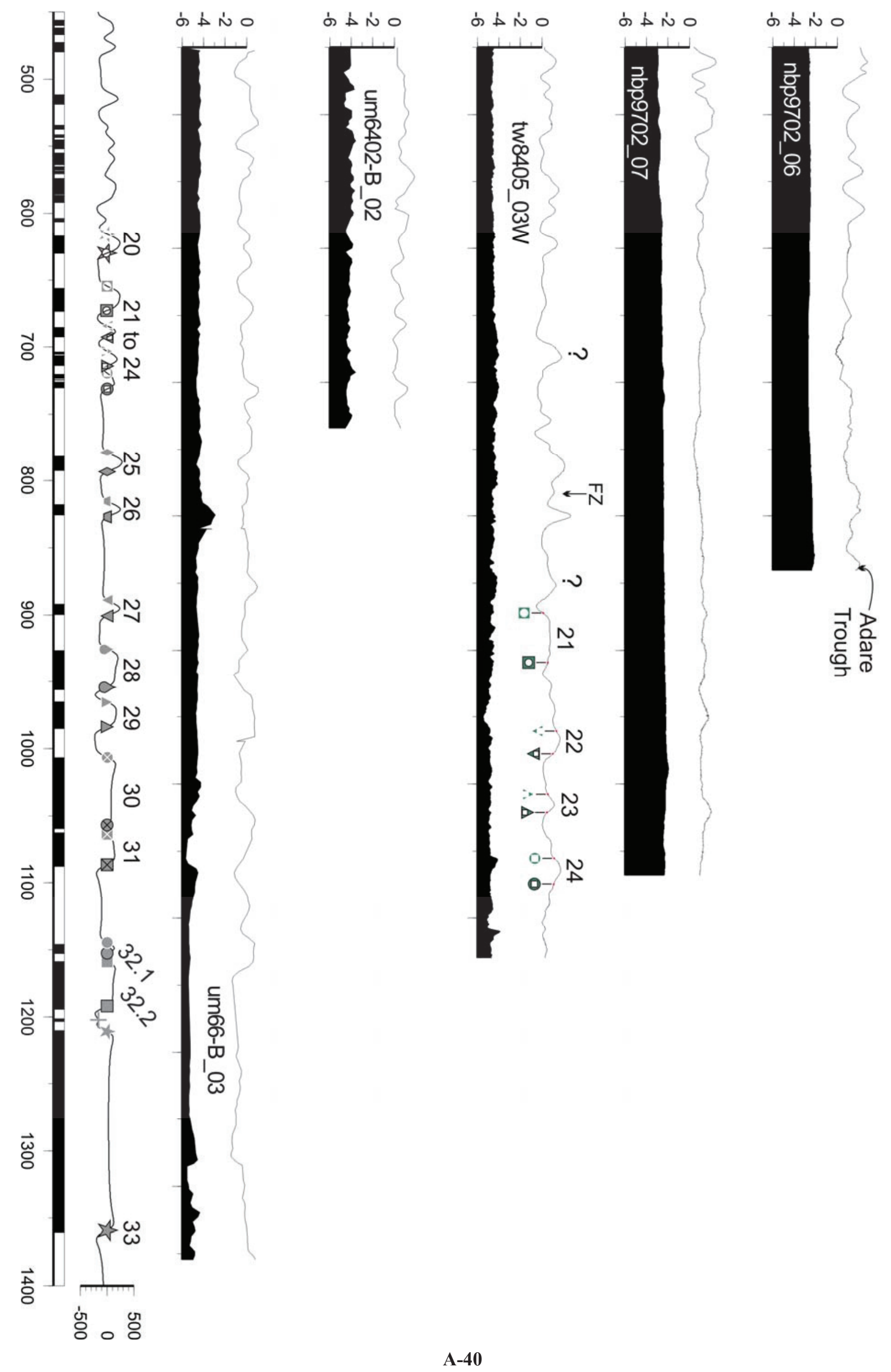




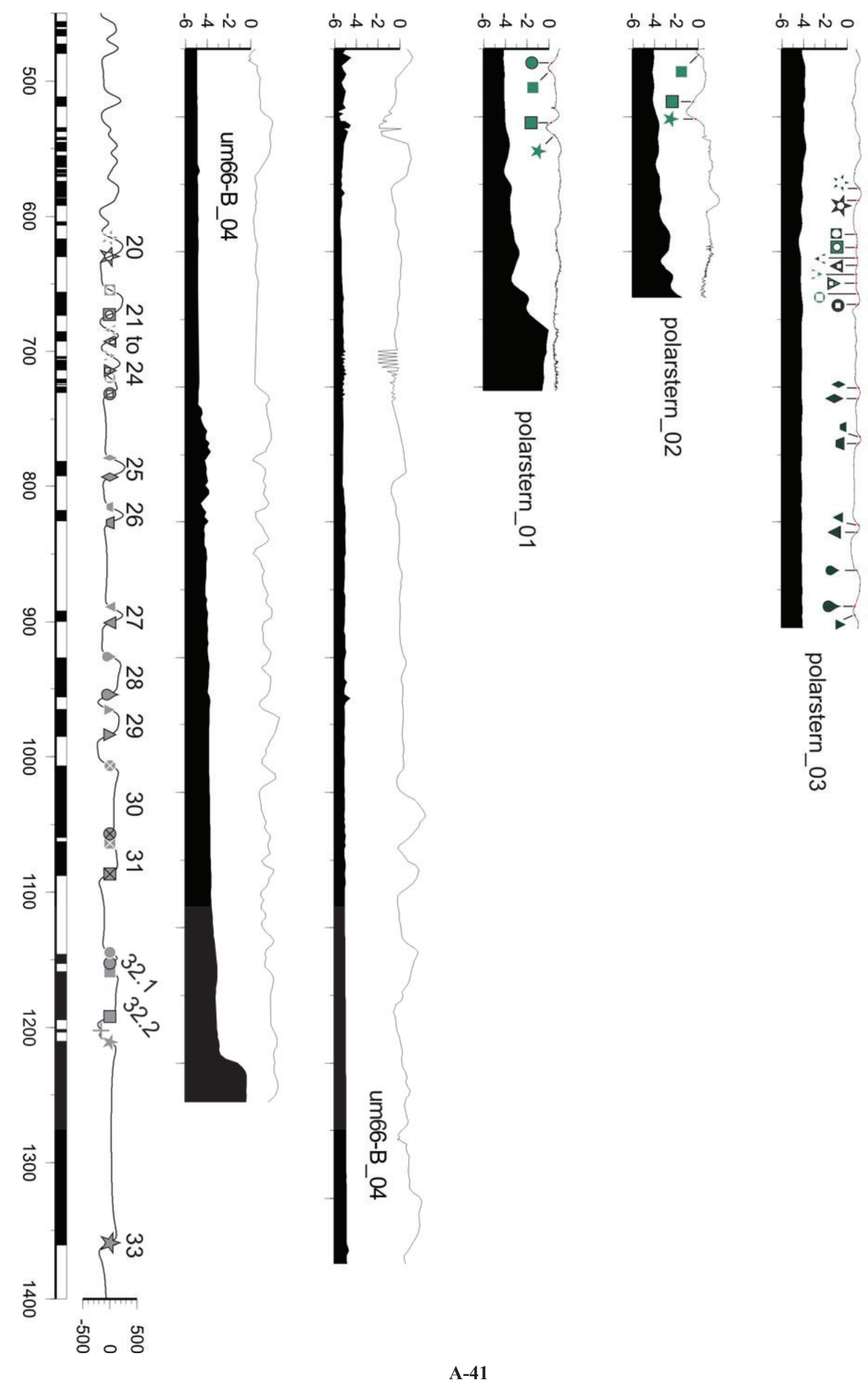




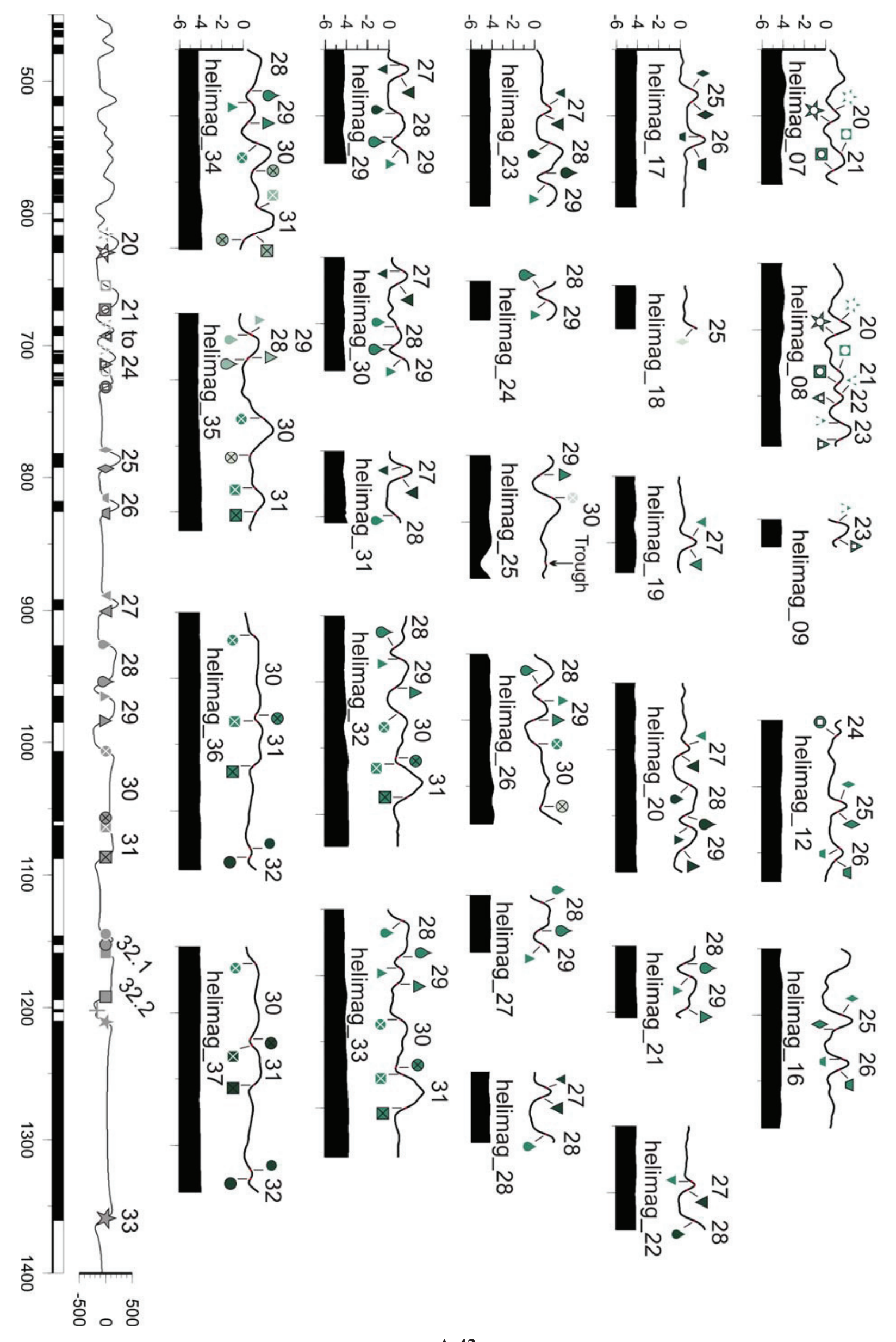




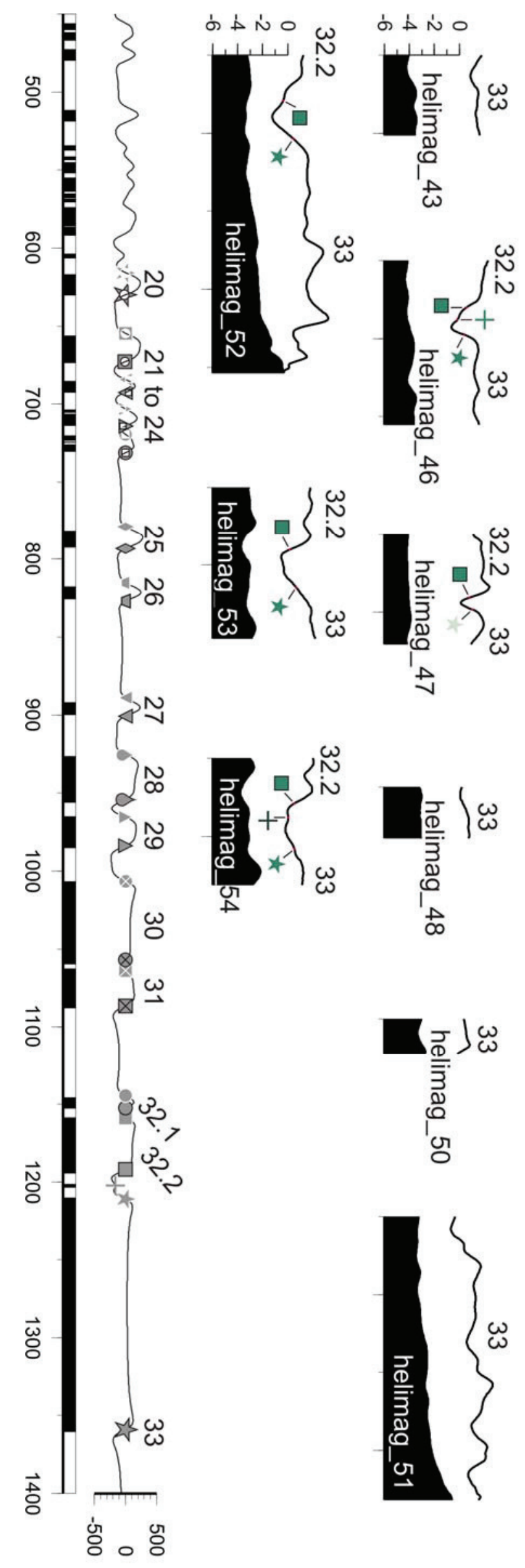



Appendix B: Location of the magnetic profiles interpreted in this study

The A0 map enclosed at the end of the appendices shows the location of all the magnetic anomalies and magnetic profiles presented in Appendix A. The helimag profiles are colourcoded (red, green, cyan and orange) and the number of the profile is written on the map. The shiptrack profiles are drawn in dark blue. The name of each shiptrack profile can be found using the table below.

\begin{tabular}{|c|c|}
\hline 1 & nbp90702_10 \\
\hline 2 & nbp0209_02 \\
\hline 3 & nbp9702_09 \\
\hline 4 & ares02wt_04 \\
\hline 5 & elt16_03 \\
\hline 6 & nbp9702_08 \\
\hline 7 & ares $02 w t \_01$ \\
\hline 8 & nbp0209_03 \\
\hline 9 & cp7808_06 \\
\hline 10 & cp7808_05 \\
\hline 11 & elt50_04 \\
\hline 12 & elt50_05 \\
\hline 13 & elt42_01 \\
\hline 14 & elt50_02 \\
\hline 15 & elt50_03 \\
\hline 16 & gs $74 \overline{0} 2 \_01$ \\
\hline 16 & gecs-gmv_01 \\
\hline 17 & mons06ar_01 \\
\hline 17 & ar6102_01 \\
\hline 18 & elt34_01 \\
\hline 19 & elt32_01 \\
\hline 20 & elt52_04S \\
\hline 21 & nbp0701_02 \\
\hline 22 & dsdp28gc_02 \\
\hline 23 & dsdp28gc_03 \\
\hline 24 & nbp0701_03 \\
\hline 25 & nbp0701_01 \\
\hline 26 & elt42_02 \\
\hline 27 & elt52_04N \\
\hline 28 & elt27_02 \\
\hline 29 & elt50_06 \\
\hline 30 & ew9201_110 \\
\hline 31 & dsdp28ge_05 \\
\hline 32 & ew9201_010 \\
\hline 33 & ew9201_15 \\
\hline 34 & elt52_03 \\
\hline 35 & elt33_01E \\
\hline 36 & dsdp $\overline{2} 8 \mathrm{gc} \_06$ \\
\hline 37 & elt32_02N \\
\hline 38 & elt27_01 \\
\hline 39 & elt42_03 \\
\hline 40 & elt43_03 \\
\hline 41 & mons06ar_06 \\
\hline 42 & gs7402_03 \\
\hline $42 \mathrm{~N}$ & gecs-gmv_03 \\
\hline $42 \mathrm{~S}$ & gecs-gmv_02 \\
\hline 43 & bmrg03mv_01s \\
\hline 44 & bmrg03mv_02 \\
\hline 45 & um6402-B_01 \\
\hline 46 & nbp9604_02 \\
\hline 47 & nbp9604_14 \\
\hline 48 & nbp9604_13 \\
\hline 49 & nbp9604_12 \\
\hline
\end{tabular}

\begin{tabular}{|l|l|}
\hline 50 & kh6804-C_02 \\
\hline 51 & nbp9604_03 \\
\hline 52 & nbp9507_01 \\
\hline 53 & cook20mv_01 \\
\hline 54 & cook20mv_02 \\
\hline 56 & cook20mv_03 \\
\hline 57 & um66-B_01 \\
\hline 58 & cook20mv_10 \\
\hline 59 & bmrg03mv_01N \\
\hline 60 & um66-B_02 \\
\hline 61 & bt9004_01 \\
\hline 62 & bt8203_01 \\
\hline 63 & elt25_02 \\
\hline 64 & bt9004_02 \\
\hline 65 & bt9004_03 a \& b \\
\hline 66 & bt8203_02 \\
\hline 67 & bt8203_03 \\
\hline 68 & west03mv_01 \\
\hline 69 & kh6804-C_01 \\
\hline 70 & elt19_01 \\
\hline 71 & c0806_01 \\
\hline 72 & kh6804-B_02a \\
\hline 73 & bt8203_04 \\
\hline 74 & elt23_08 \\
\hline 75 & nbp0007B_05 \\
\hline 76 & elt33_08 \\
\hline 77 & gecs-imv_03 \\
\hline 78 & v3601_02 \\
\hline 79 & v3601_04 \\
\hline 80 & nbp0007B_04 \\
\hline 81 & elt17_01 \\
\hline 82 & hm9105_01 \\
\hline 83 & nbp9908_02 \\
\hline 84 & v3601_03 \\
\hline 85 & elt17_02 \\
\hline 86 & v3601_02 \\
\hline 87 & tw8405_01 \\
\hline 88 & nbp0007B_03 \\
\hline 89 & nbp9707_02 \\
\hline 90 & v3601_01 \\
\hline 91 & nbp9908_01 \\
\hline 92 & elt20_02 \\
\hline 93 & tw8405_02 \\
\hline 94 & nbp0007B_02 \\
\hline 95 & c1212_01 \\
\hline 96 & nbp0403_02 \\
\hline 98 & nbp0501B_01 \\
\hline 99 & hu122_01 \\
\hline 100 & elt24_02 \\
\hline 101 & nbpo-0301B_02 \\
\hline & \\
\hline
\end{tabular}

\begin{tabular}{|c|c|}
\hline 102 & lélt19_02 \\
\hline 103 & nbp0007B_01 \\
\hline 104 & elt23_07 \\
\hline 105 & elt20_01 \\
\hline 106 & nbp97̄02_01 \\
\hline 107 & nbp9602_04 \\
\hline 108 & polarstern_03 \\
\hline 109 & nbp9602_05 \\
\hline 110 & nbp9602_06 \\
\hline 111 & nbp9602_07 \\
\hline 112 & nbp9602_08 \\
\hline 113 & nbp9602_09 \\
\hline 114 & nbp9602_10 \\
\hline 115 & polarstern_02 \\
\hline 116 & polarstern_01 \\
\hline 117 & nbp9602_-11 \\
\hline 118 & nbp9602_12 \\
\hline 119 & nbp92-8_01 \\
\hline 120 & nbp92-8_02 \\
\hline 121 & nbp9602_13 \\
\hline 122 & nbp9602_14 \\
\hline 123 & nbp92-8_03 \\
\hline 124 & nbp9602_15 \\
\hline 125 & nbp9602_16 \\
\hline 126 & elt33_05 \\
\hline 127 & nbp9602_18E \& W \\
\hline 128 & nbp92-8_04 \\
\hline 129 & nbp9602_19 E \& W \\
\hline 130 & nbp9602_20 \\
\hline 131 & nbp92-8_05 \\
\hline 132 & elt33_06S \\
\hline 133 & nbp9507_02 \\
\hline 134 & elt42_07 \\
\hline 135 & nbp950707_03 \\
\hline 136 & nbp92-8_09 \\
\hline 137 & nbp9602_22 \\
\hline 138 & nbp92-8_11 \\
\hline 139 & elt42_08 \\
\hline 140 & elt23_05 \\
\hline 141 & elt23_06 \\
\hline 142 & elt20_03 \\
\hline 143 & elt21_01 \\
\hline 144 & elt23_03 \\
\hline 145 & elt42_09 \\
\hline 146 & nbp9602_23 \\
\hline 147 & elt23_02 \\
\hline 148 & nbp9602_24 \\
\hline 149 & hud08_01 \\
\hline 150 & tw8405_03W \\
\hline 151 & c2107_01 \\
\hline 152 & c2107_03 \\
\hline 153 & tw8405_03E \\
\hline
\end{tabular}




\begin{tabular}{|c|c|}
\hline ar6102_01 & 17 \\
\hline ares02wt_01 & 7 \\
\hline ares02wt_04 & 4 \\
\hline bmrg03mv_01N & 59 \\
\hline bmrg03mv_01S & 43 \\
\hline bmrg03mv_02 & 44 \\
\hline bt8203_01 & 62 \\
\hline bt8203_02 & 66 \\
\hline bt8203_03 & 67 \\
\hline bt8203_04 & 73 \\
\hline bt9004_01 & 61 \\
\hline bt9004_02 & 64 \\
\hline bt9004_03 a \& b & 65 \\
\hline c0806_01 & 71 \\
\hline c1212_01 & 95 \\
\hline c2107_01 & 151 \\
\hline c2107_03 & 152 \\
\hline cook20mv_01 & 53 \\
\hline cook20mv_02 & 54 \\
\hline cook20mv_03 & 55 \\
\hline cook20mv_04 & 56 \\
\hline cook20mv_10 & 58 \\
\hline cp7808_05 & 10 \\
\hline cp7808_06 & 9 \\
\hline dsdp28gc_02 & 22 \\
\hline dsdp28gc_03 & 23 \\
\hline dsdp28gc_05 & 31 \\
\hline dsdp28gc_06 & 36 \\
\hline elt16_03 & 5 \\
\hline elt17_01 & 81 \\
\hline elt17_02 & 85 \\
\hline elt19_01 & 70 \\
\hline elt19_02 & 102 \\
\hline elt20_01 & 105 \\
\hline elt20_02 & 92 \\
\hline elt20_03 & 142 \\
\hline elt21_01 & 143 \\
\hline elt23_02 & 147 \\
\hline elt23_03 & 144 \\
\hline elt23_05 & 140 \\
\hline elt23_06 & 141 \\
\hline elt23_07 & 104 \\
\hline elt23_08 & 74 \\
\hline elt24_02 & 99 \\
\hline elt24_03 & 100 \\
\hline elt25_02 & 63 \\
\hline elt27_01 & 38 \\
\hline elt27_02 & 28 \\
\hline elt32_01 & 19 \\
\hline elt32_02N & 37 \\
\hline elt33_01E & 35 \\
\hline elt33_05 & 126 \\
\hline elt33_06s & 132 \\
\hline elt33_08 & 76 \\
\hline elt34_01 & 18 \\
\hline elt42_01 & 13 \\
\hline elt42_02 & 26 \\
\hline
\end{tabular}

\begin{tabular}{|c|c|}
\hline elt42_03 & 39 \\
\hline elt42_07 & 134 \\
\hline elt42_08 & 139 \\
\hline elt42_09 & 145 \\
\hline elt43_03 & 40 \\
\hline elt50_02 & 14 \\
\hline elt50_03 & 15 \\
\hline elt50_04 & 11 \\
\hline elt50_05 & 12 \\
\hline elt50_06 & 29 \\
\hline elt52_03 & 34 \\
\hline elt52_04N & 27 \\
\hline elt52_04S & 20 \\
\hline ew9201_010 & 32 \\
\hline ew9201_110 & 30 \\
\hline ew9201_15 & 33 \\
\hline gecs-gmv_01 & 16 \\
\hline gecs-gmv_02 & $42 \mathrm{~S}$ \\
\hline gecs-gmv_03 & $42 \mathrm{~N}$ \\
\hline gecs-imv_03 & 77 \\
\hline gs7402_01 & 16 \\
\hline gs7402_03 & 42 \\
\hline hm9105_01 & 82 \\
\hline hu122_01 & 98 \\
\hline hud08_01 & 149 \\
\hline kh6804-B_02a & 72 \\
\hline kh6804-C_01 & 69 \\
\hline kh6804-C_02 & 50 \\
\hline mons06ar_01 & 17 \\
\hline mons06ar_06 & 41 \\
\hline nbp0007B_01 & 103 \\
\hline nbp0007B_02 & 94 \\
\hline nbp0007B_03 & 88 \\
\hline nbp0007B_04 & 80 \\
\hline nbp0007B_05 & 75 \\
\hline nbp0209_02 & 2 \\
\hline nbp0209_03 & 8 \\
\hline nbpo403_02 & 96 \\
\hline nbp0501B_01 & 97 \\
\hline nbp0501B_02 & 101 \\
\hline nbp0701_01 & 25 \\
\hline nbp0701_02 & 21 \\
\hline nbp0701_03 & 24 \\
\hline nbp92-8_01 & 119 \\
\hline nbp92-8_02 & 120 \\
\hline nbp92-8_03 & 123 \\
\hline nbp92-8_04 & 128 \\
\hline nbp92-8_05 & 131 \\
\hline nbp92-8_09 & 136 \\
\hline nbp92-8_11 & 138 \\
\hline nbp9507_01 & 52 \\
\hline nbp9507_02 & 133 \\
\hline nbp9507_03 & 135 \\
\hline nbp9602_04 & 107 \\
\hline nbp9602_05 & 109 \\
\hline nbp9602_06 & 110 \\
\hline nbp9602_07 & 111 \\
\hline
\end{tabular}

\begin{tabular}{|c|c|}
\hline nbp9602_08 & 112 \\
\hline nbp9602 09 & 113 \\
\hline $\mathrm{nbp} 9602 \_10$ & 114 \\
\hline nbp9602 11 & 117 \\
\hline nbp9602_12 & 118 \\
\hline nbp9602_13 & 121 \\
\hline nbp9602_14 & 122 \\
\hline nbp9602_15 & 124 \\
\hline nbp9602_16 & 125 \\
\hline nbp9602_18E \& W & 127 \\
\hline nbp9602_19E \& W & 129 \\
\hline nbp9602_20 & 130 \\
\hline nbp9602_22 & 137 \\
\hline nbp9602_23 & 146 \\
\hline nbp9602_24 & 148 \\
\hline nbp9604_02 & 46 \\
\hline nbp9604_03 & 51 \\
\hline nbp9604_12 & 49 \\
\hline nbp9604_13 & 48 \\
\hline nbp9604 14 & 47 \\
\hline nbp9702_01 & 106 \\
\hline nbp9702_08 & 6 \\
\hline nbp9702_09 & 3 \\
\hline nbp9702_10 & 1 \\
\hline nbp9707_02 & 89 \\
\hline nbp9908_01 & 91 \\
\hline nbp9908_02 & 83 \\
\hline polarstern_01 & 116 \\
\hline polarstern_02 & 115 \\
\hline polarstern_03 & 108 \\
\hline tw8405_01 & 87 \\
\hline tw8405_02 & 93 \\
\hline tw8405_03E & 153 \\
\hline tw8405_03W & 150 \\
\hline um6402-B_01 & 45 \\
\hline um66-B_01 & 57 \\
\hline um66-B_02 & 60 \\
\hline v3601_01 & 90 \\
\hline v3601_02 & 78 \\
\hline v3601_02 & 86 \\
\hline v3601_03 & 84 \\
\hline v3601_04 & 79 \\
\hline west03mv_01 & 68 \\
\hline
\end{tabular}


Appendix C: Magnetic reversals and fracture zones database

Magnetic data

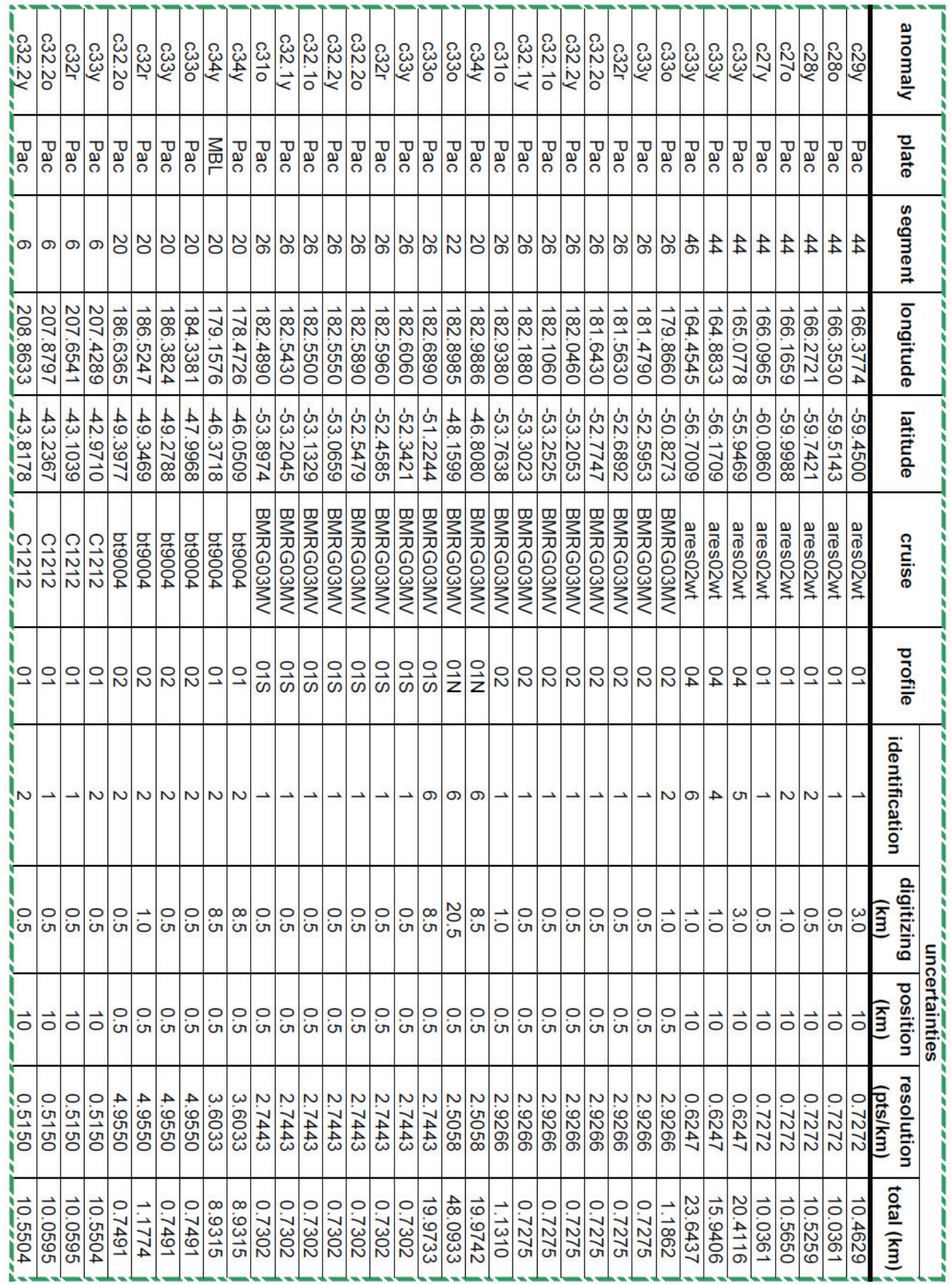




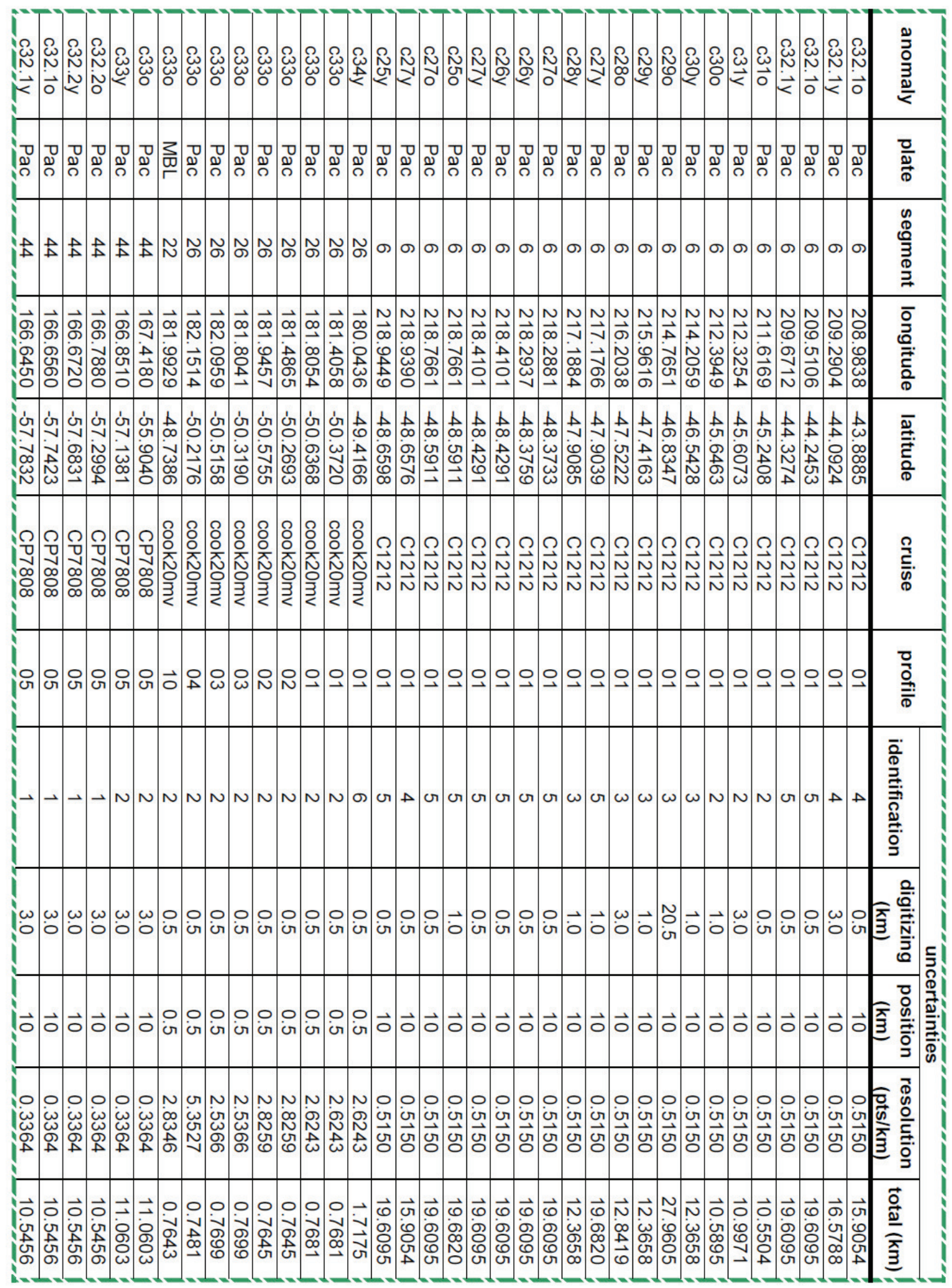




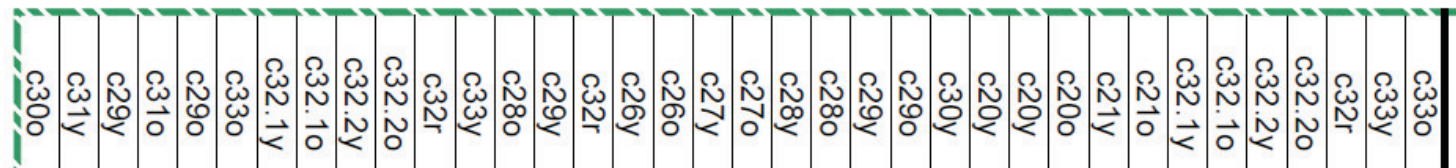

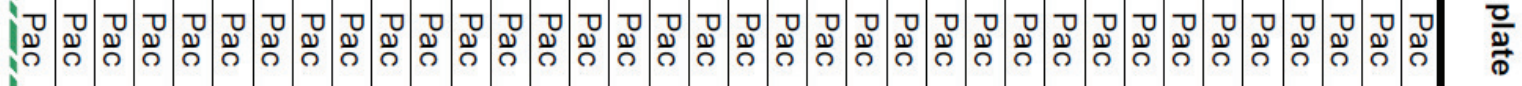

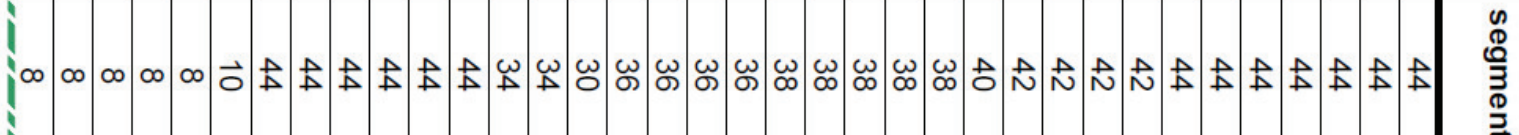

î̉ హె

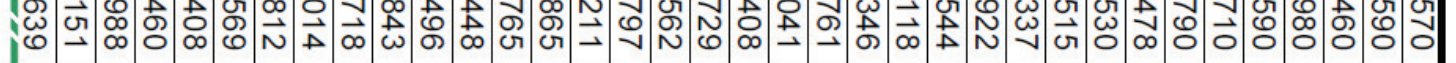

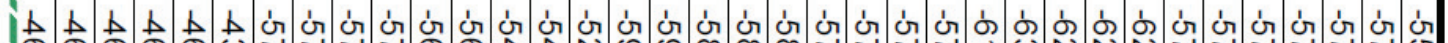
$1 \%$ o

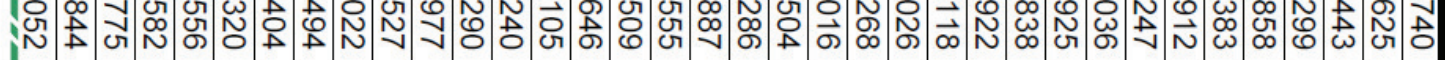

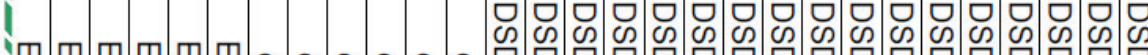

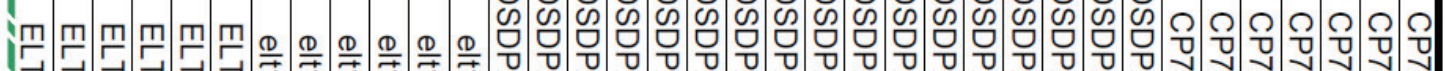

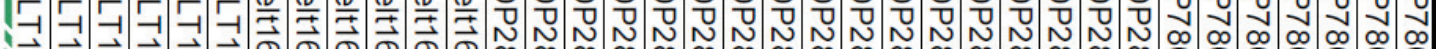

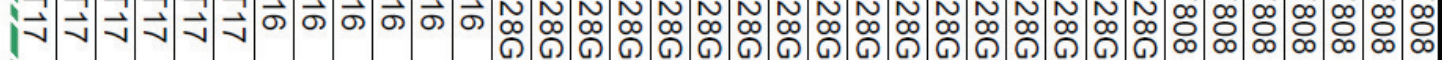

iN (

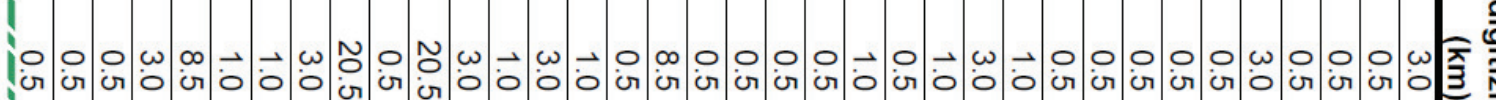

1

iN ON

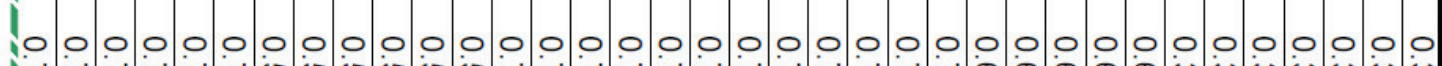
ปี

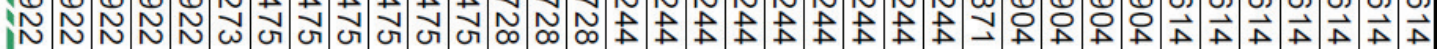
1

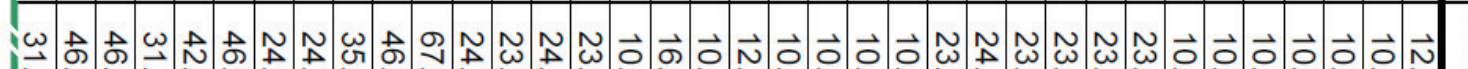
\% 6 i

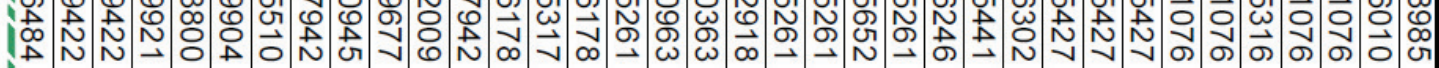




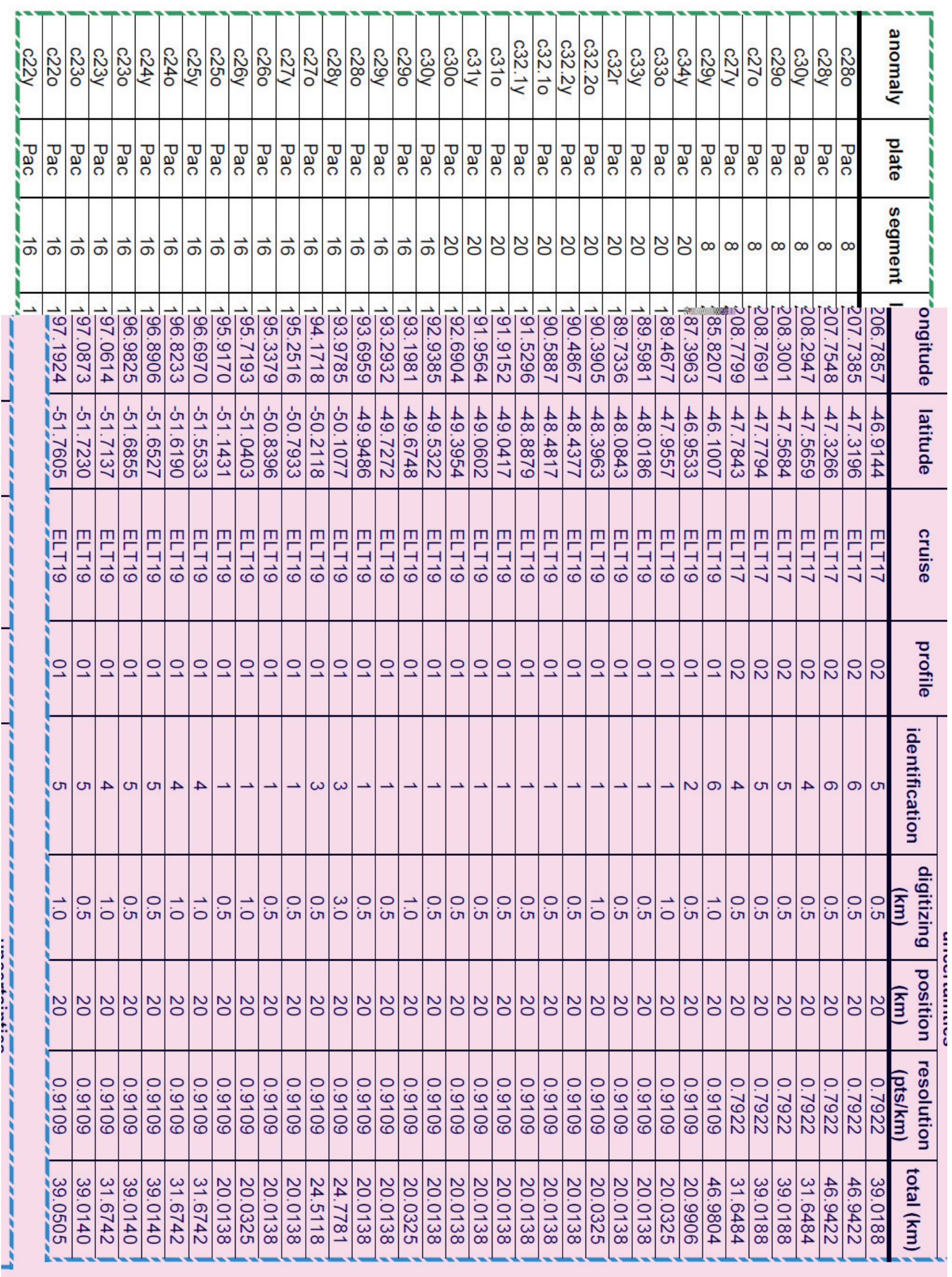




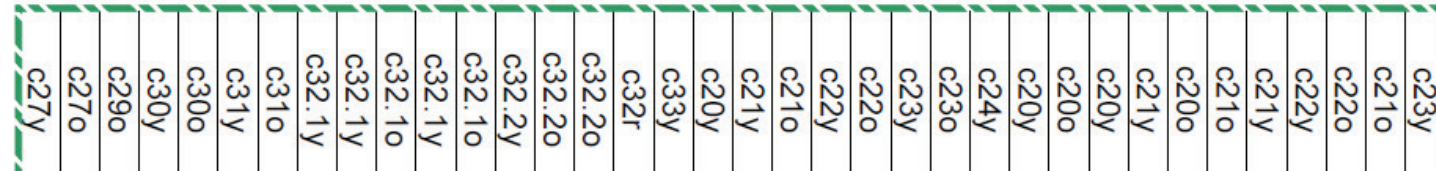

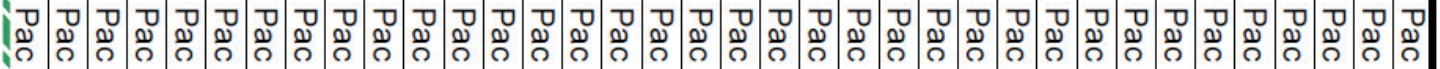

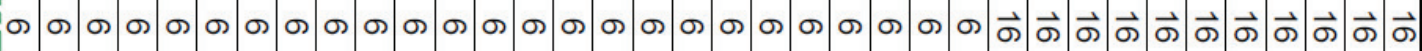

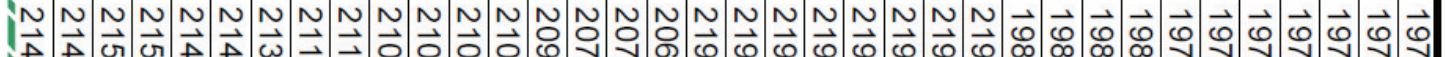

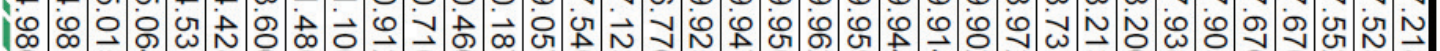

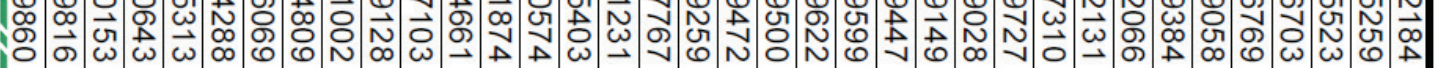

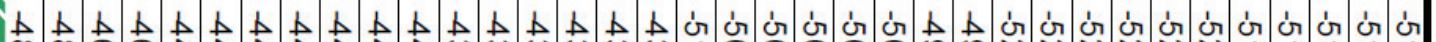

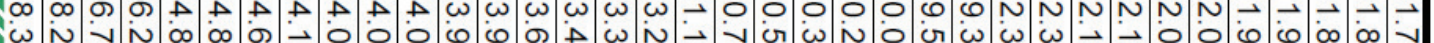

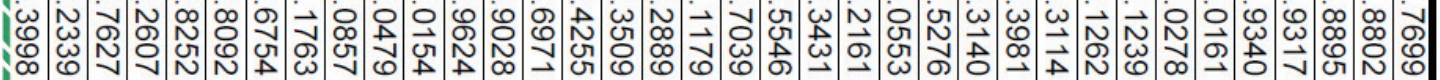
$\frac{\text { 믈 }}{\stackrel{0}{D}}$

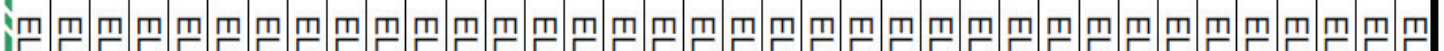

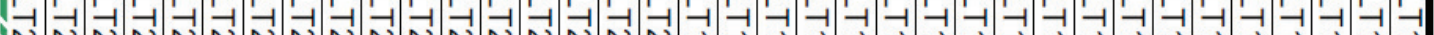

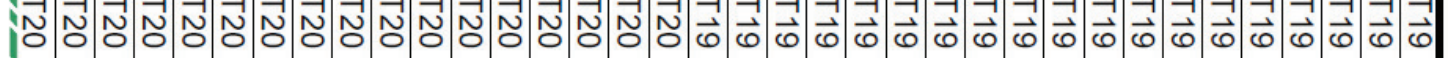

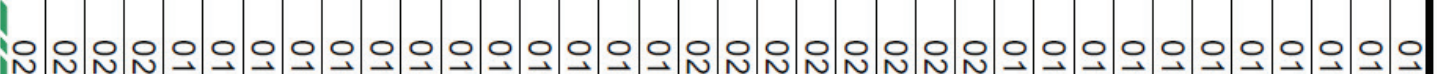

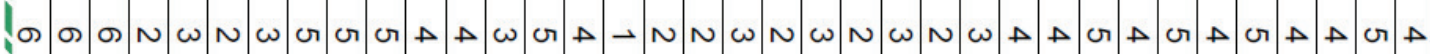

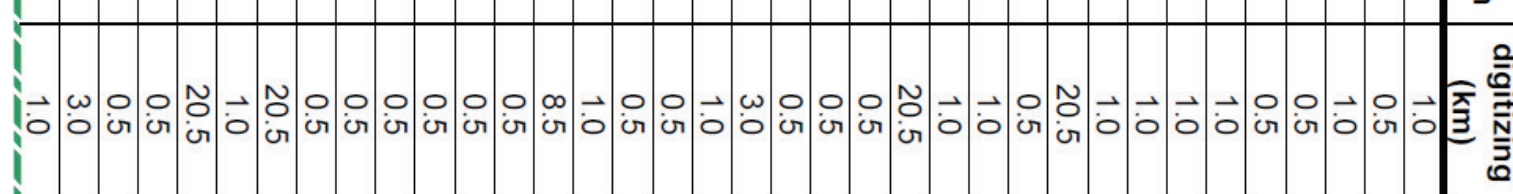

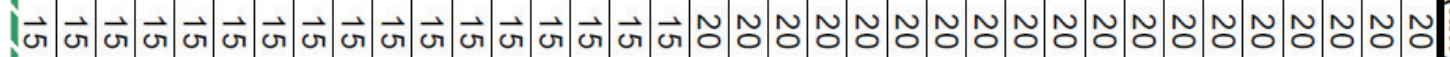

$0000000000000000000000000000000000000 \overline{0}$

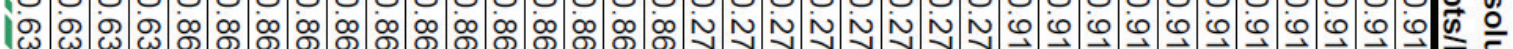

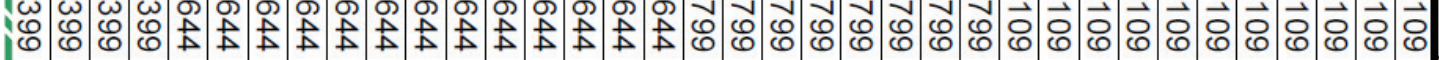

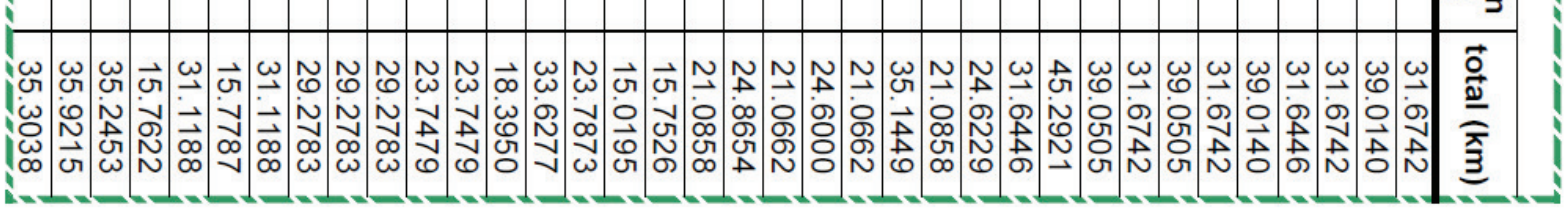




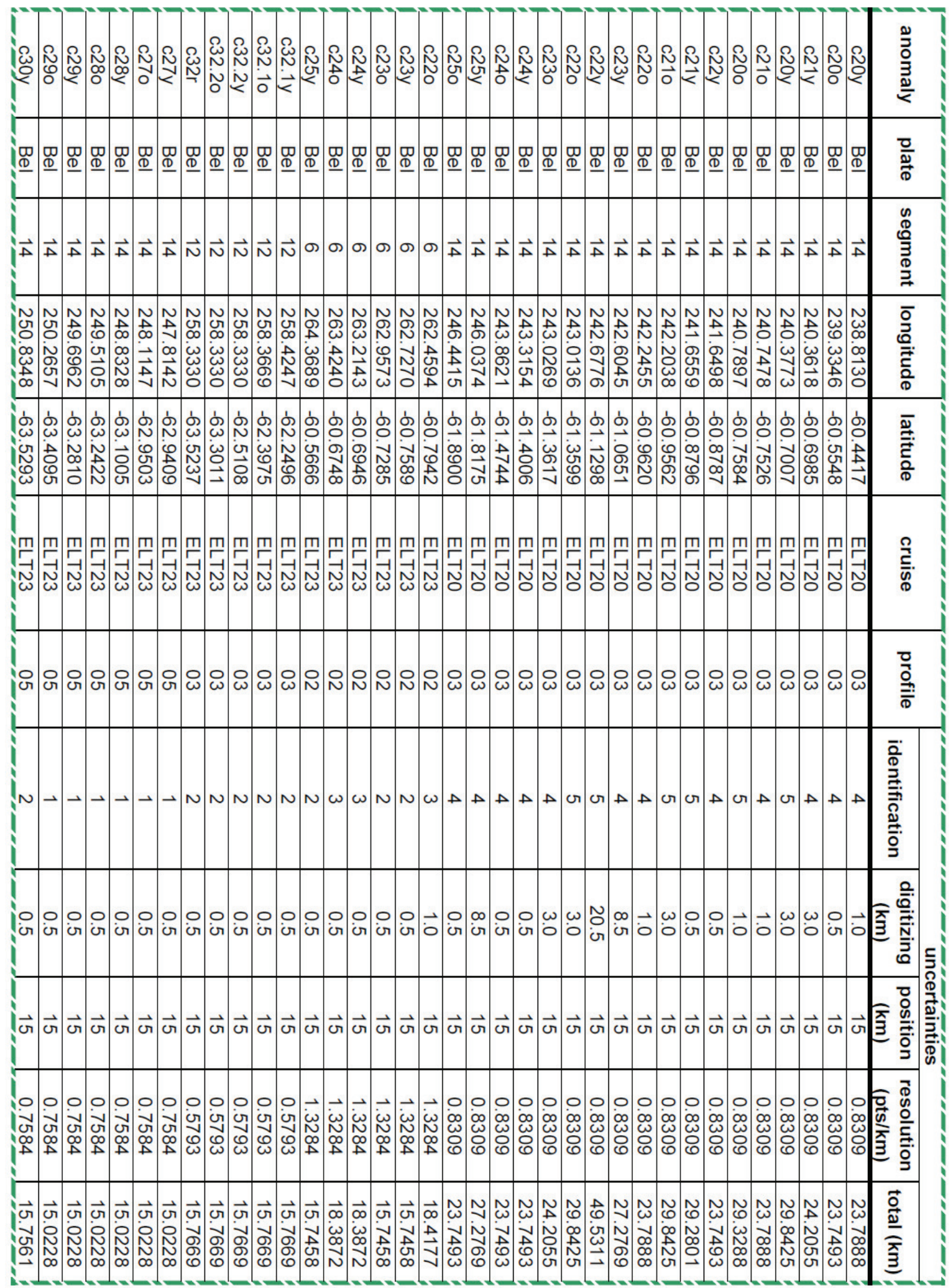




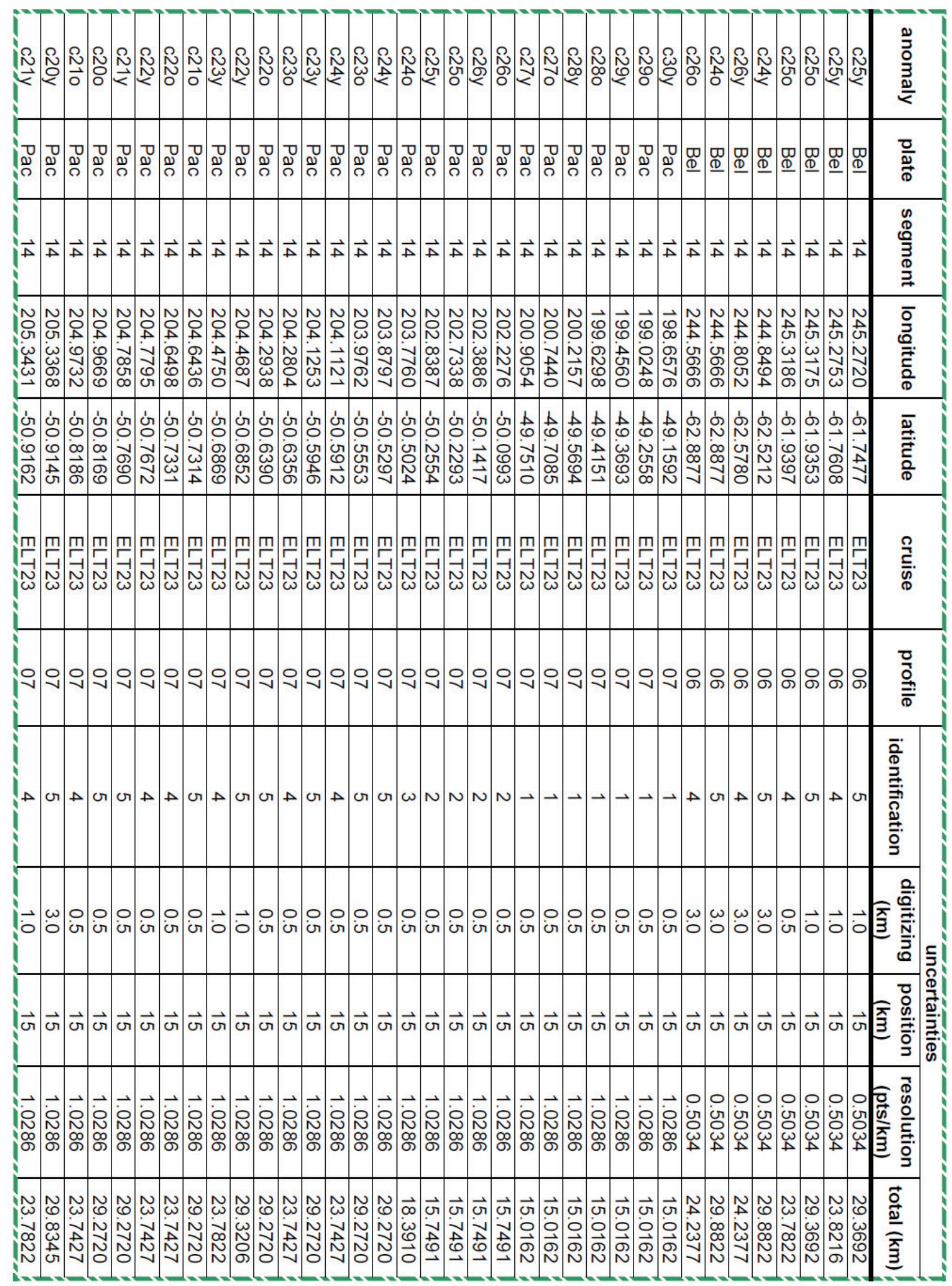




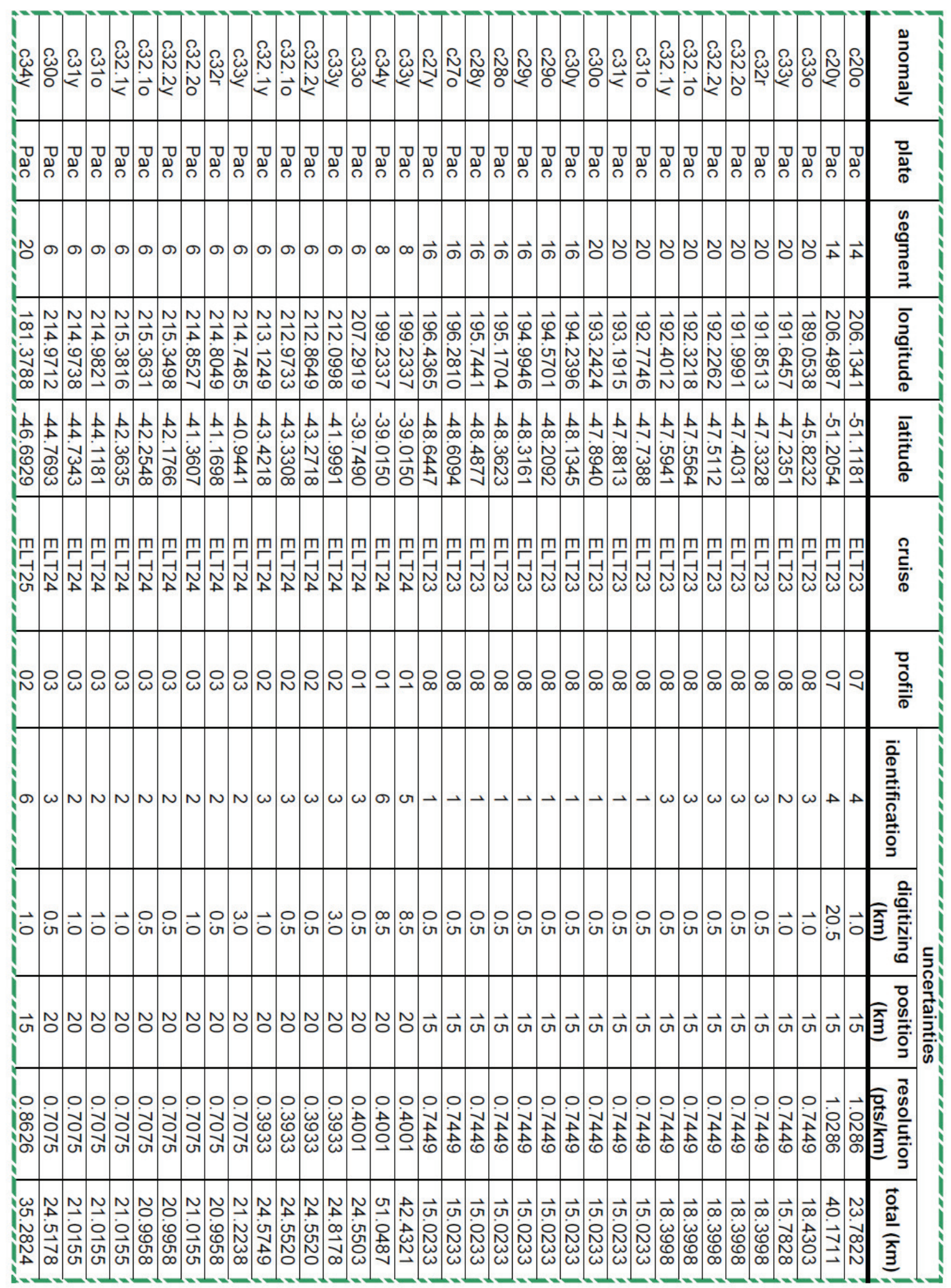




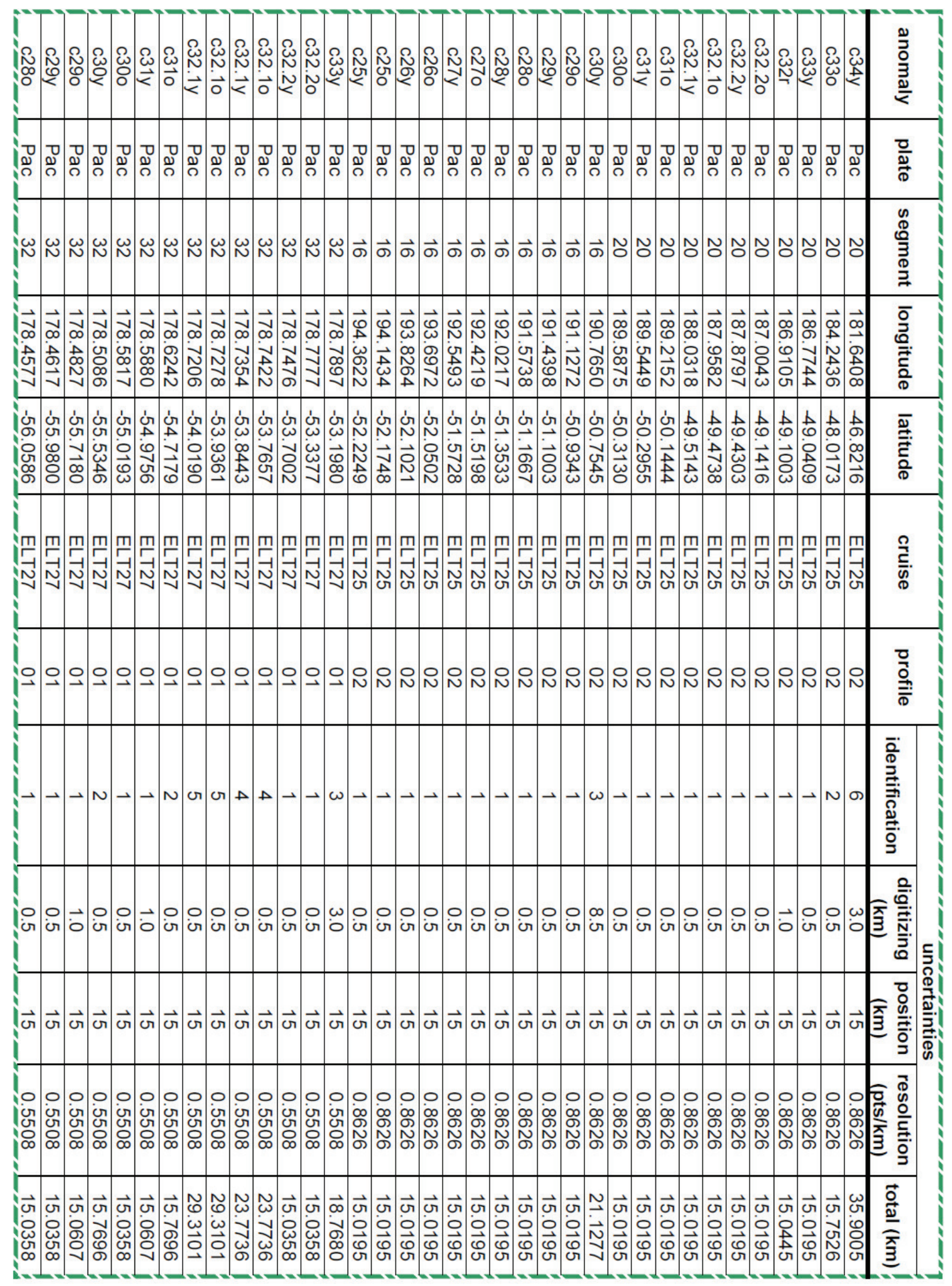




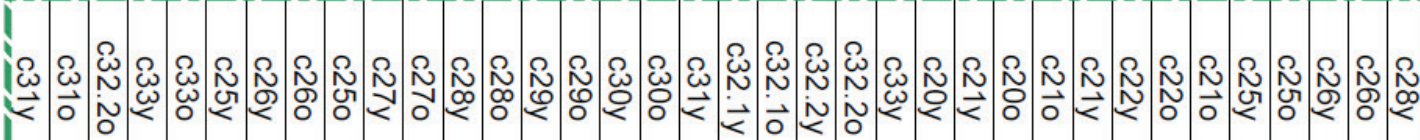

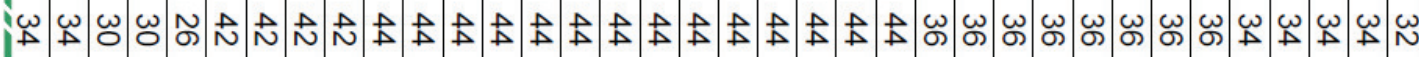

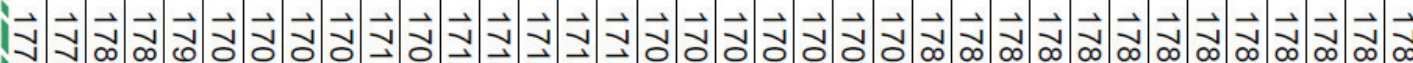
-

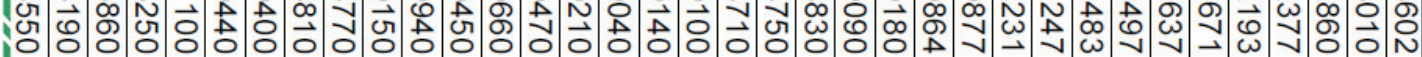

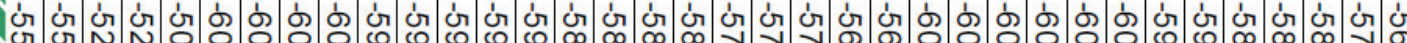

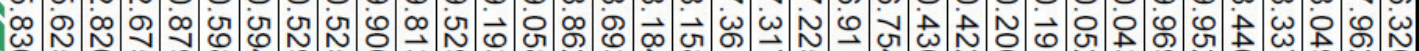

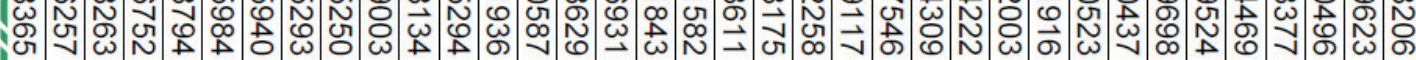

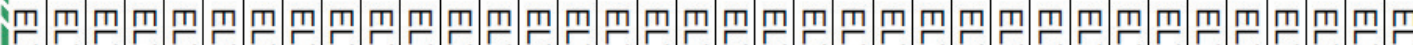

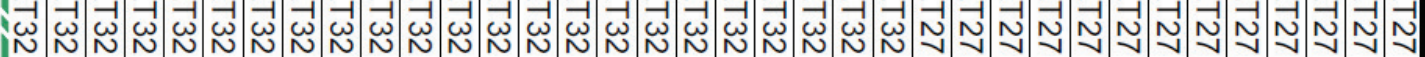

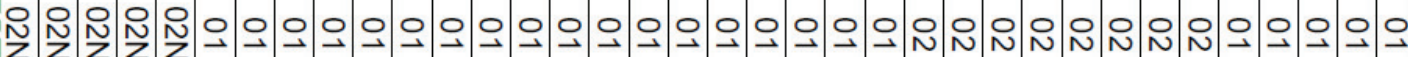

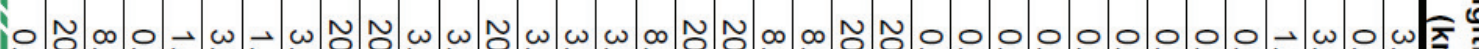

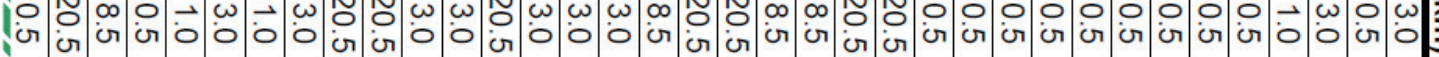

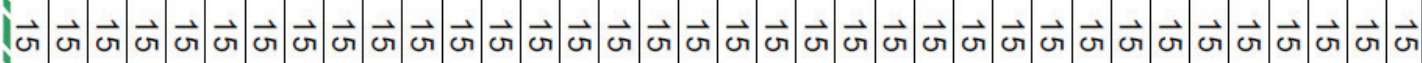

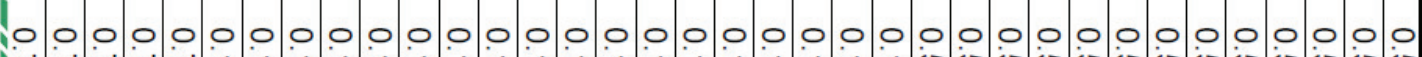
సै

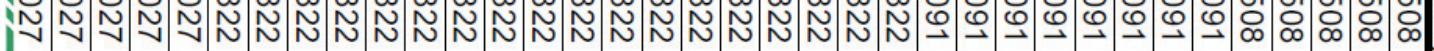

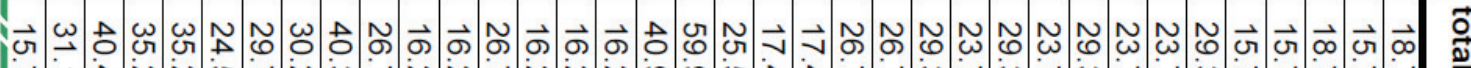

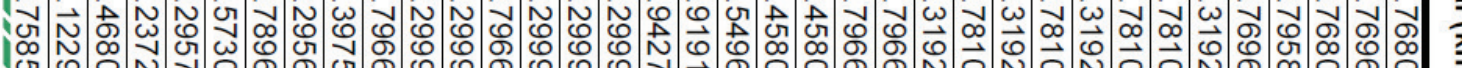
G

产




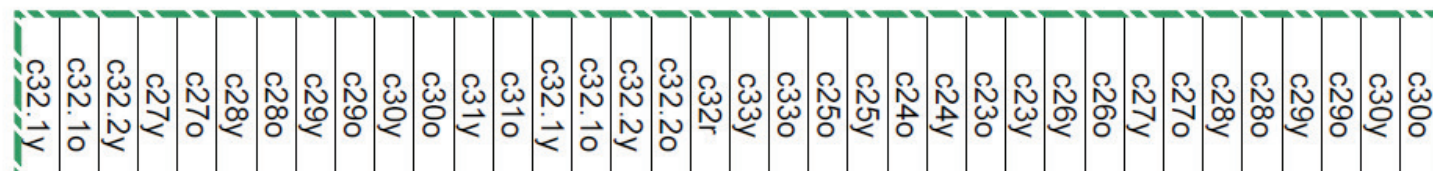

i

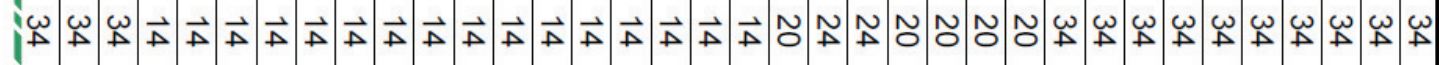

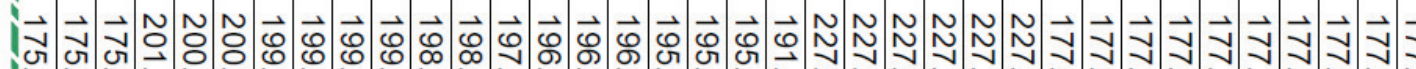

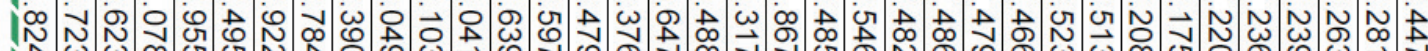

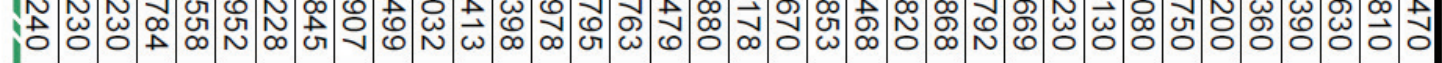
ín

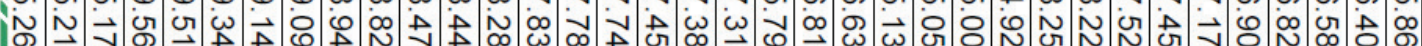
全

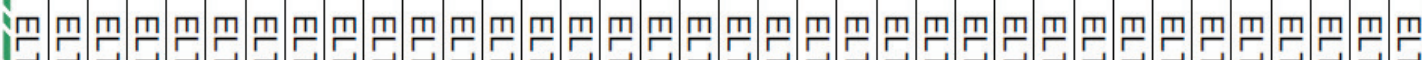

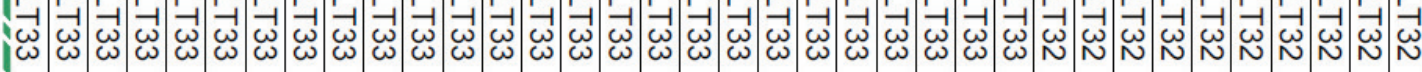

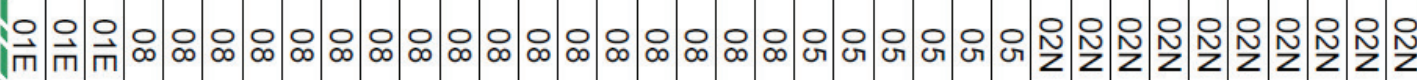

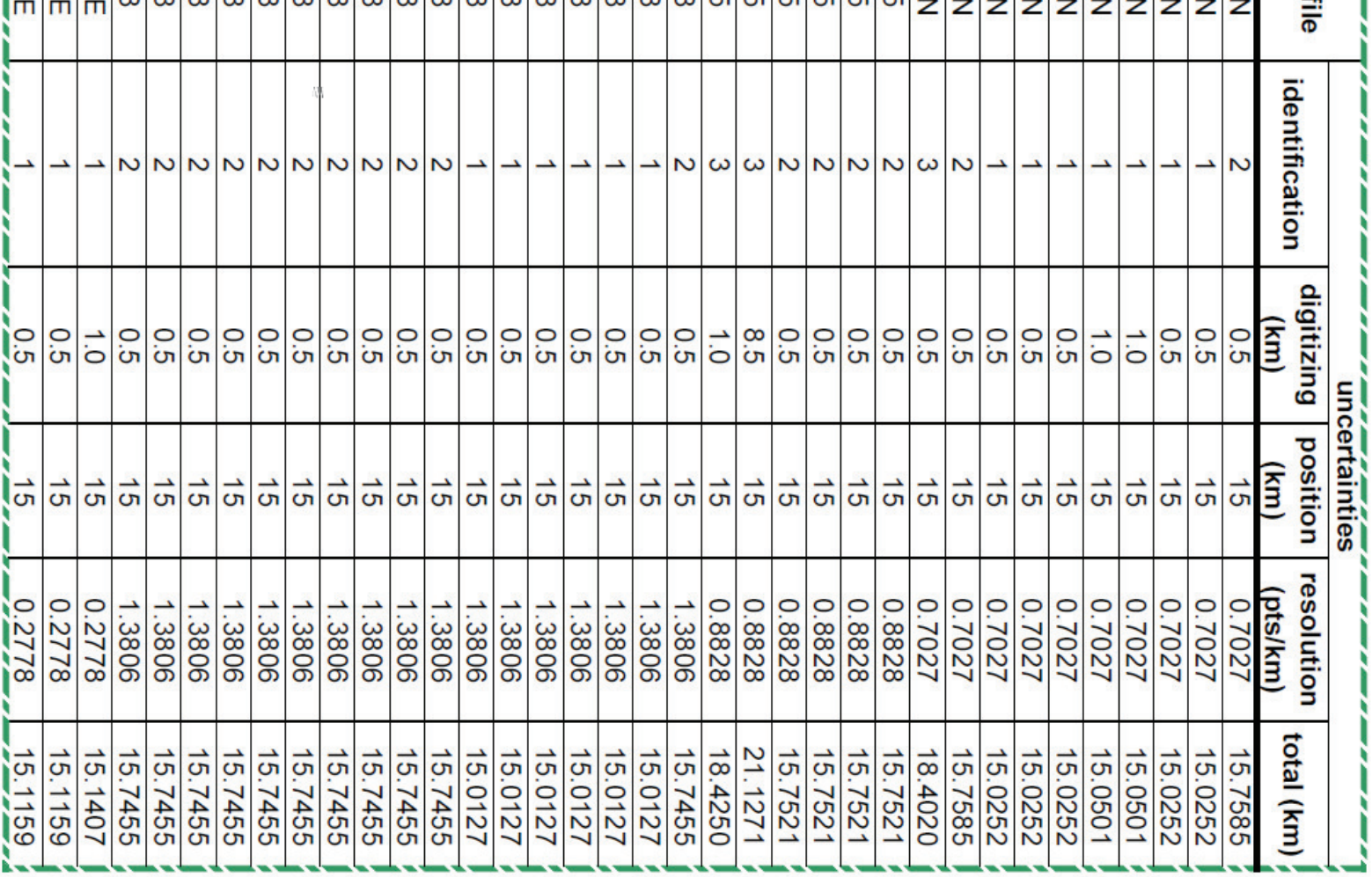




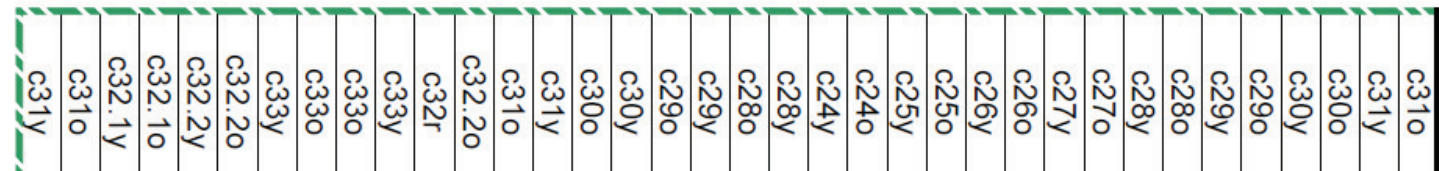

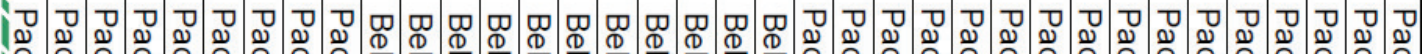

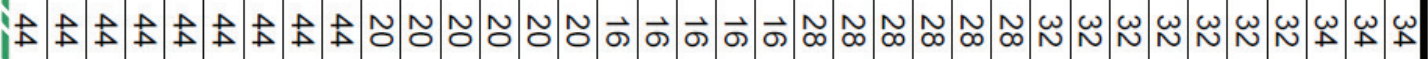

-

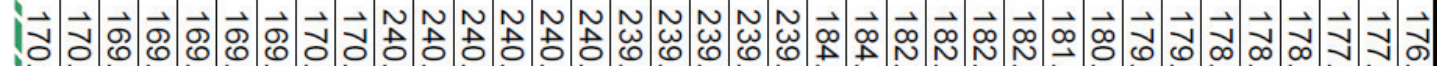

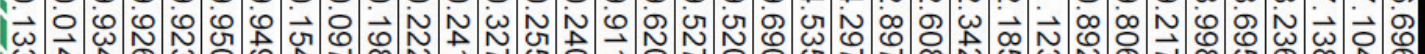

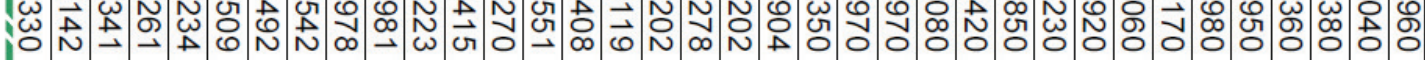

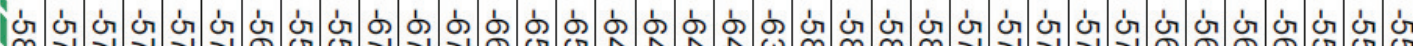

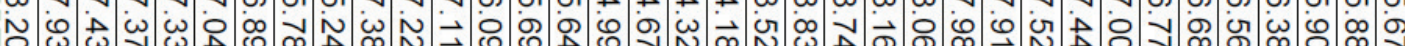
等

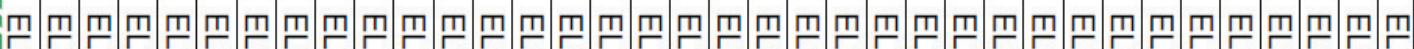

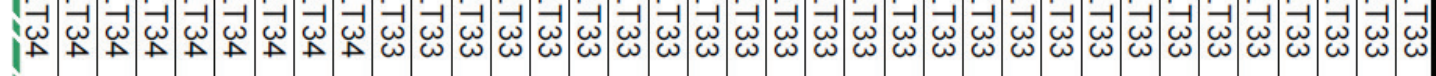

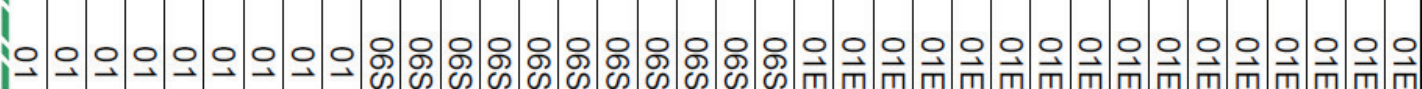

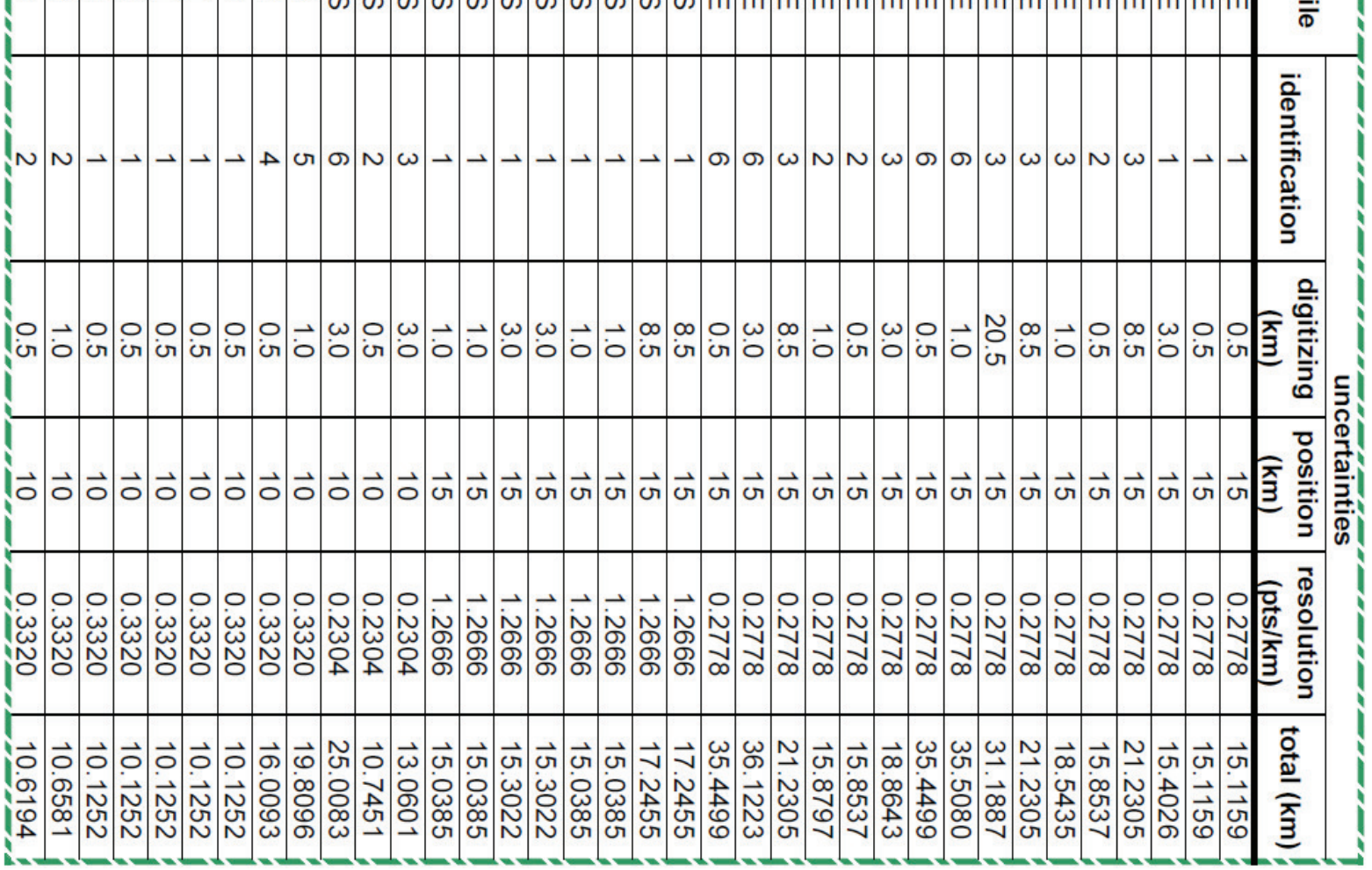




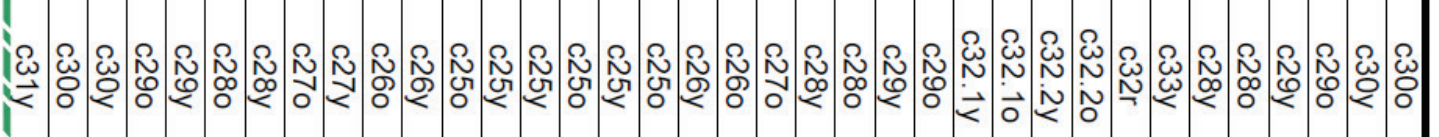

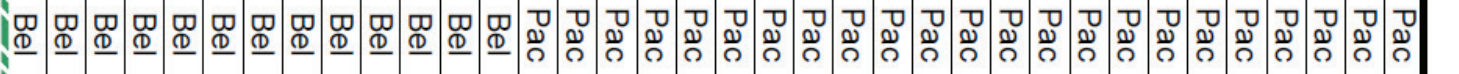

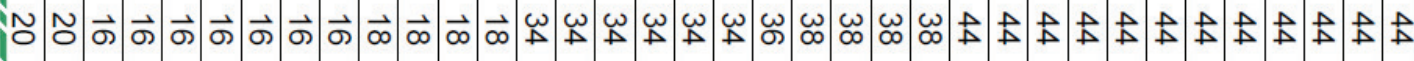

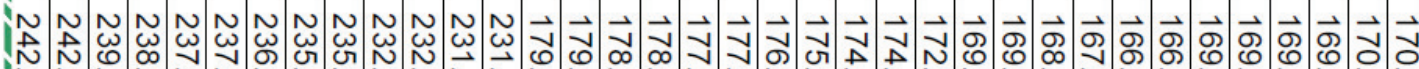

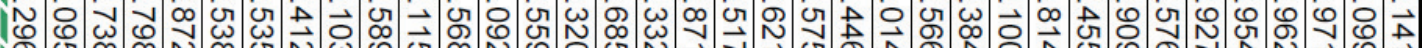

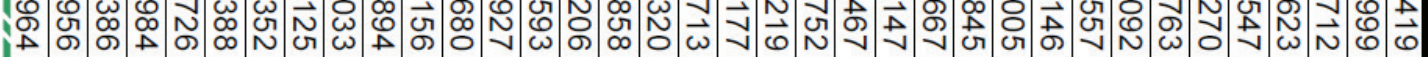

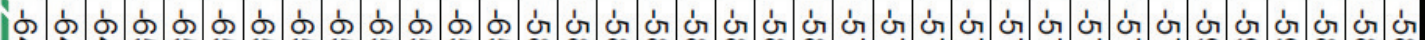

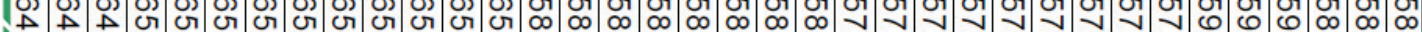

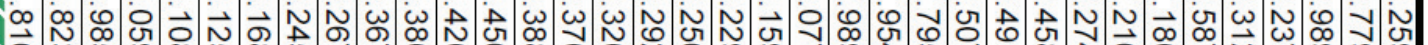

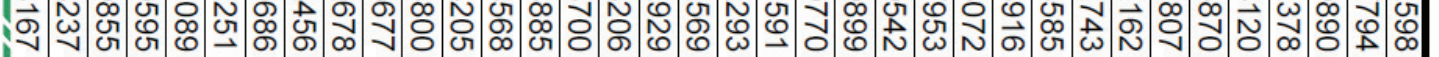

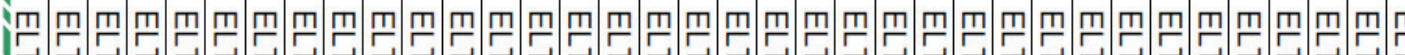

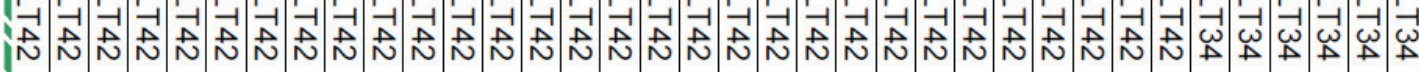

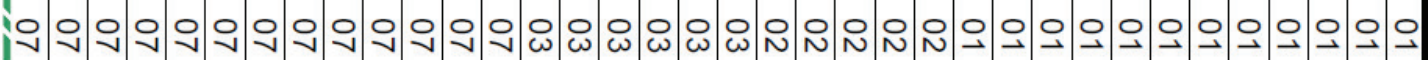

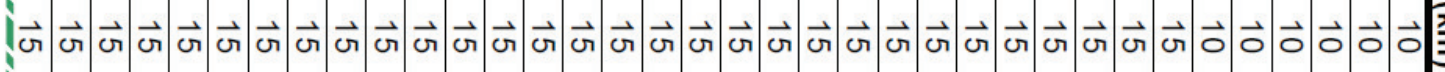

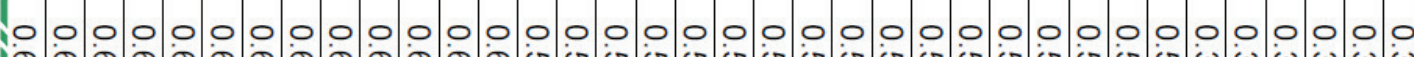
இ৪

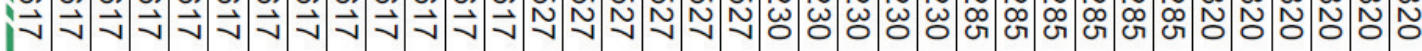




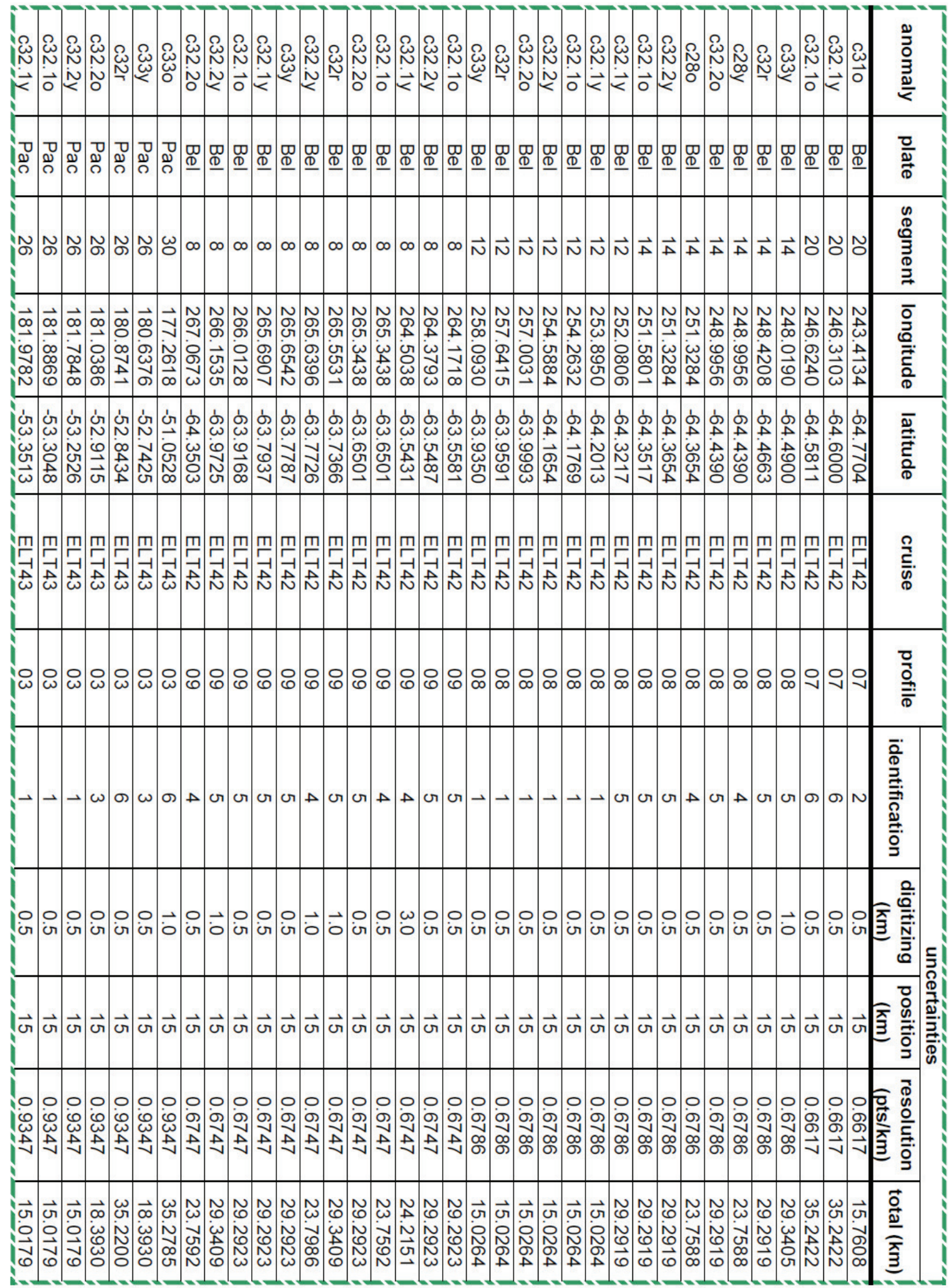




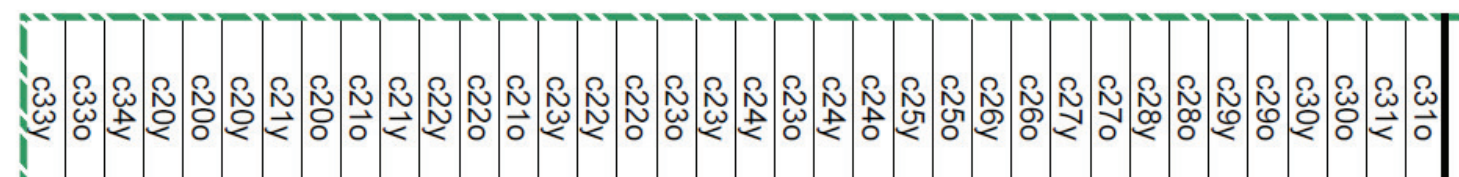

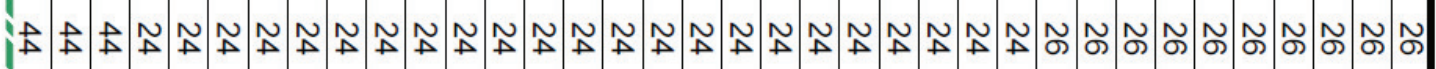

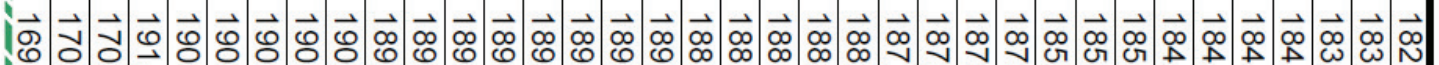

1\%

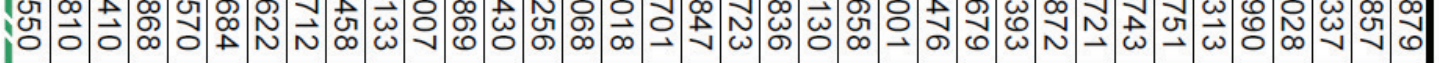

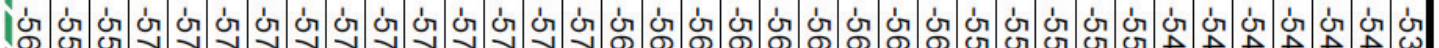

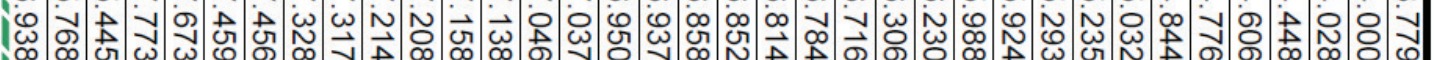

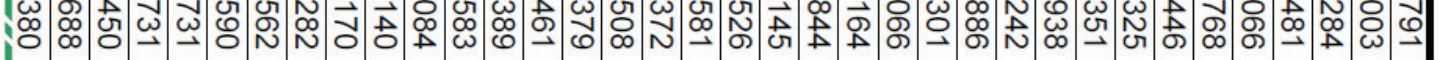

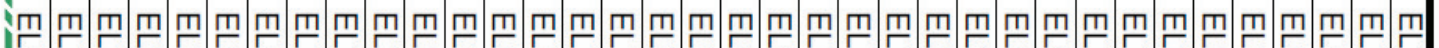

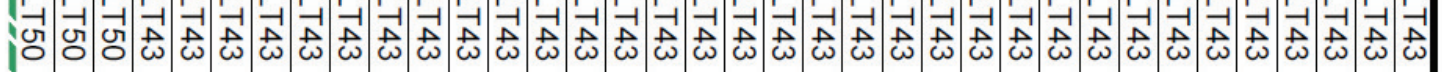

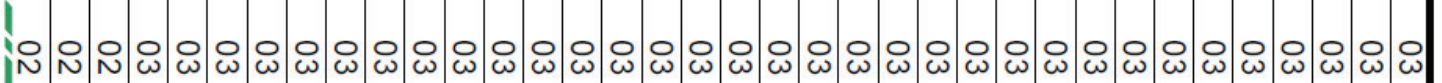

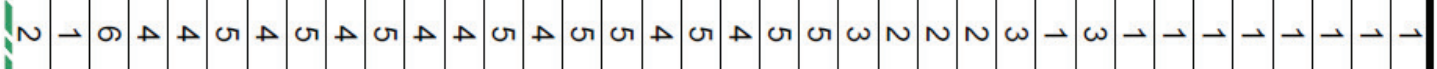

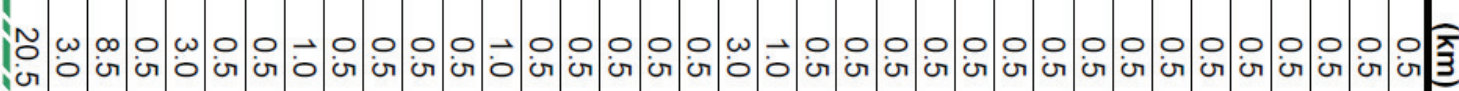

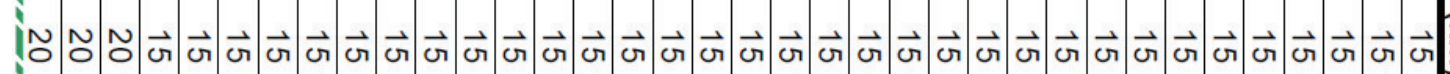
1

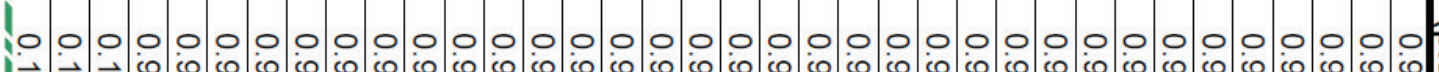

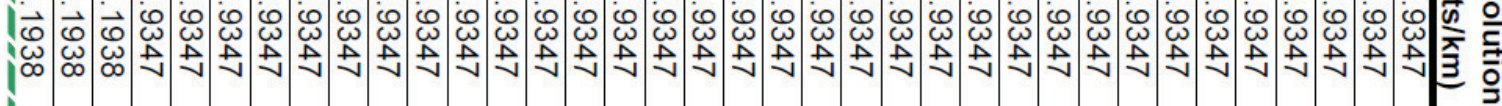

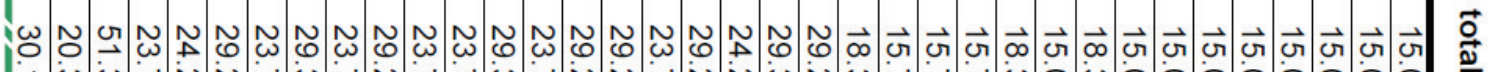

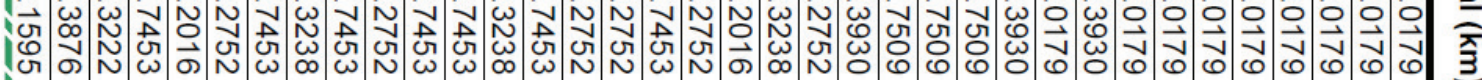




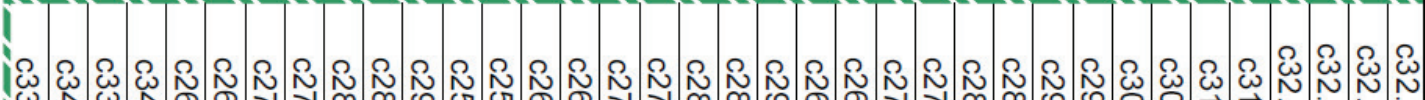

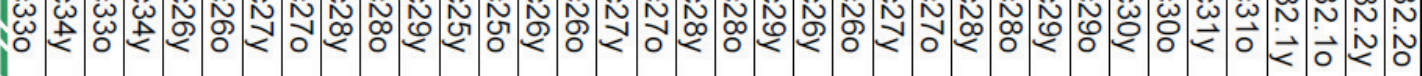

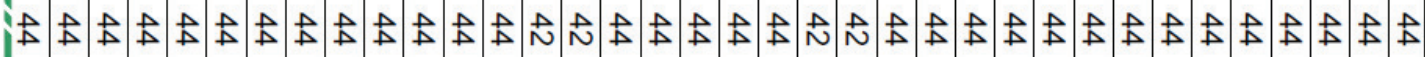

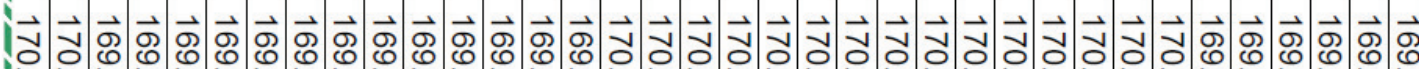

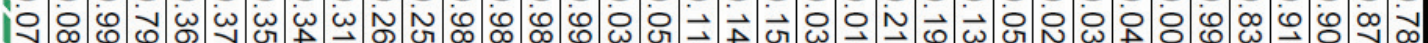

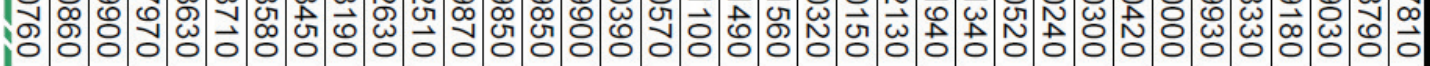

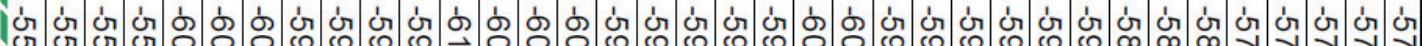

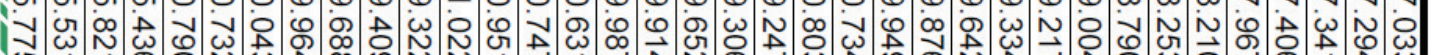

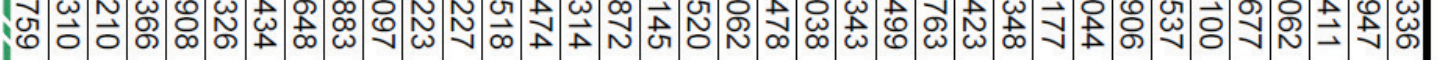

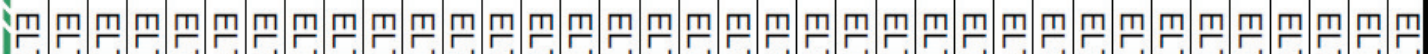

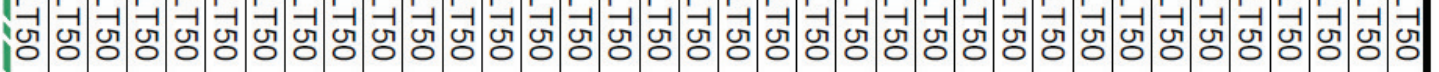

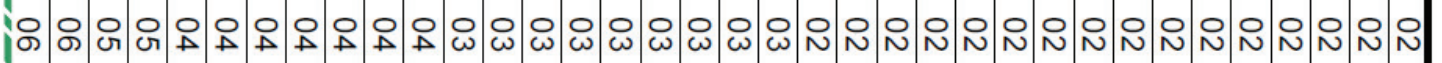

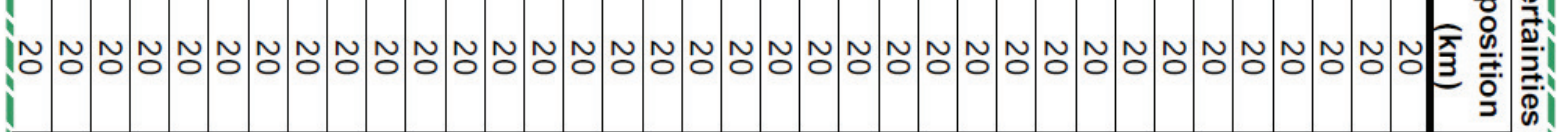

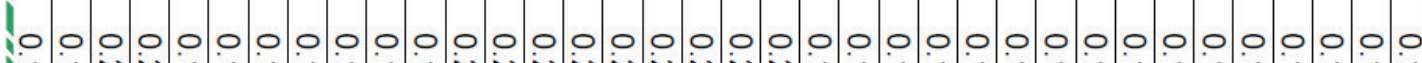

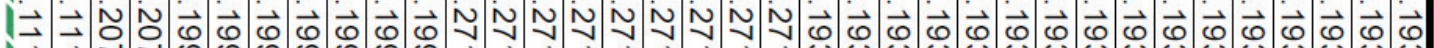

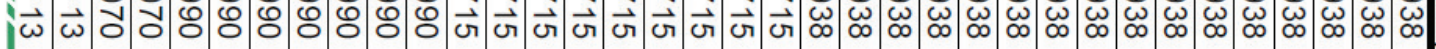




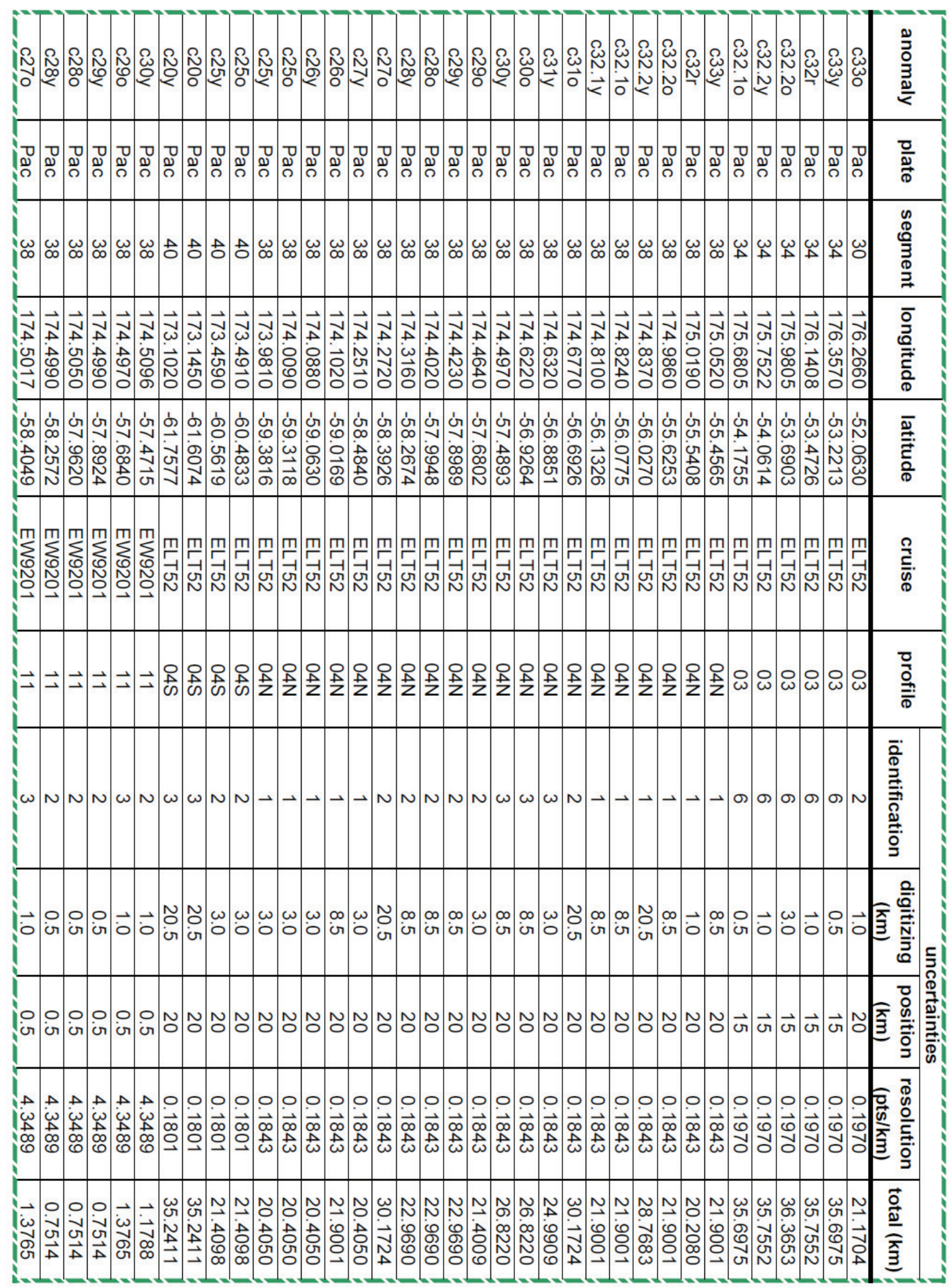




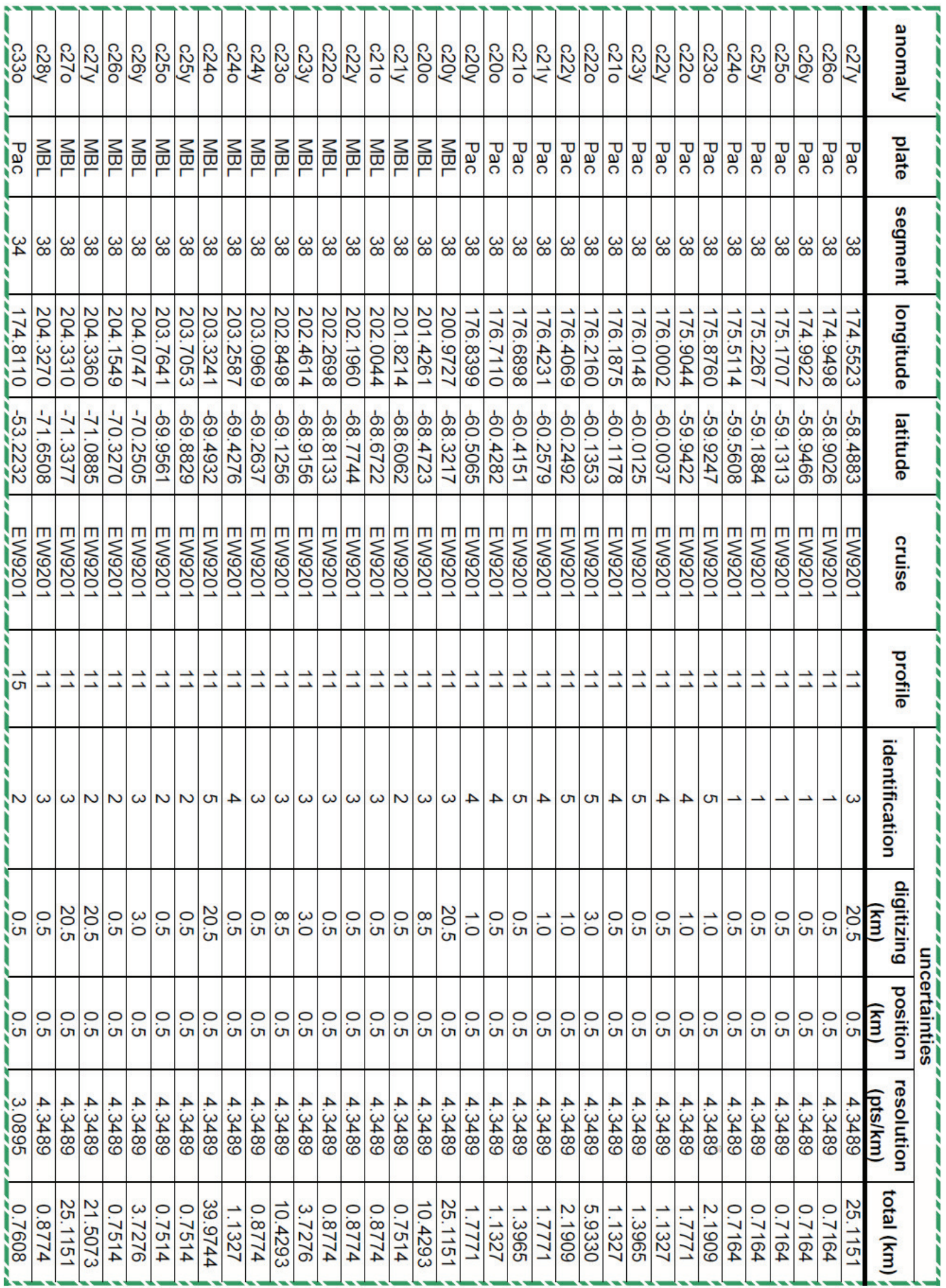




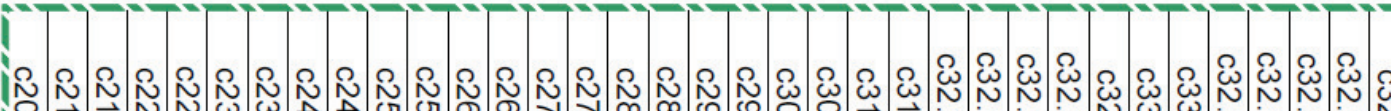

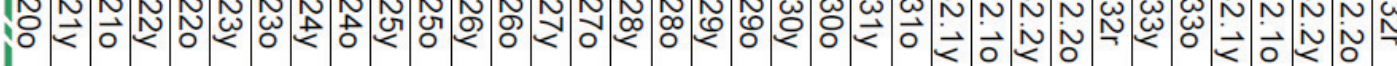

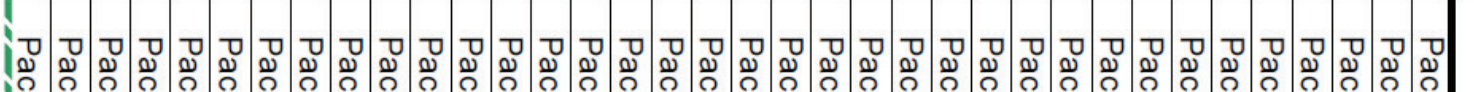

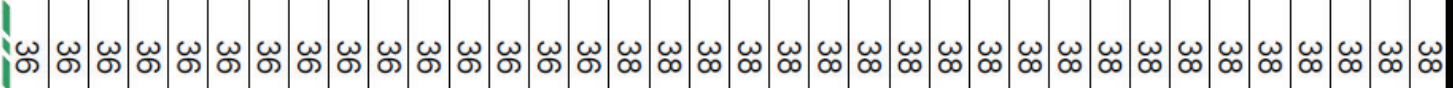

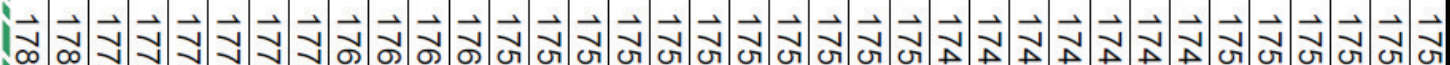

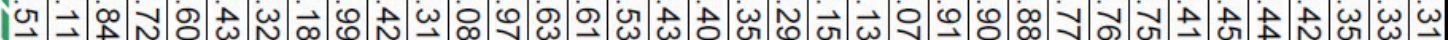

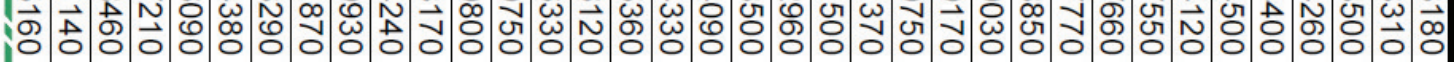

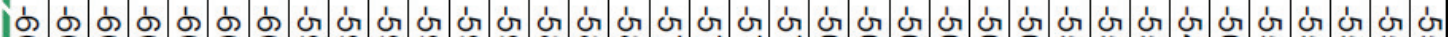

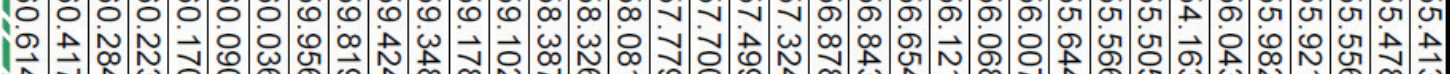

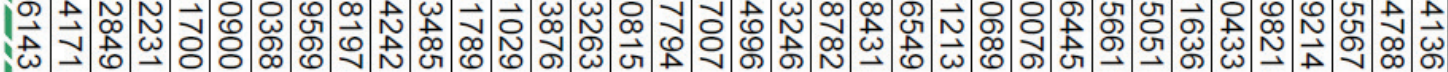

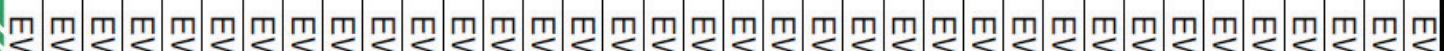

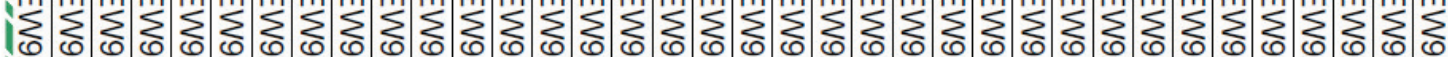

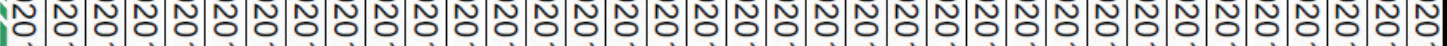

li

1

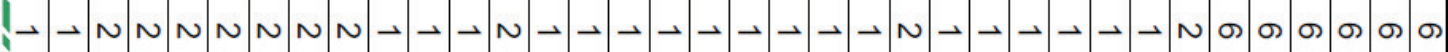

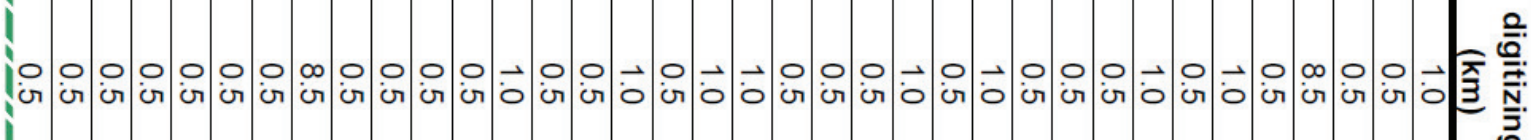

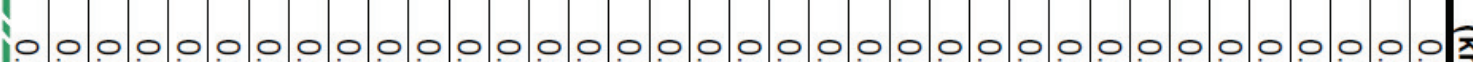

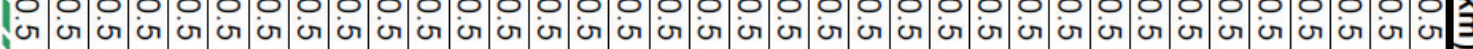

$\omega \omega \omega \omega \omega \omega \omega \omega \omega \omega \omega \omega \omega \omega \omega \omega \omega \omega \omega \omega \omega \omega \omega \omega \omega \omega \omega \omega \omega \omega \omega \omega \omega \omega \omega \hat{\mathbb{D}}$

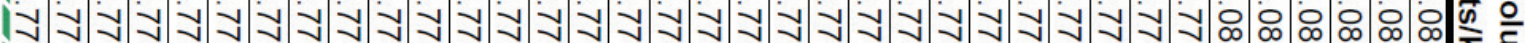

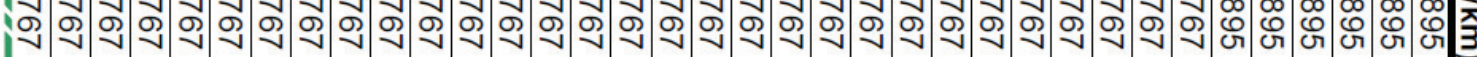

(

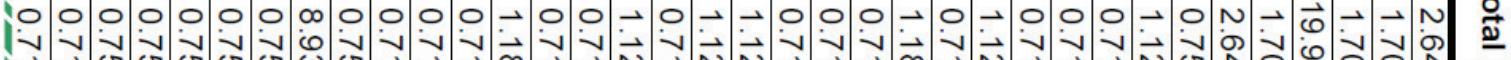

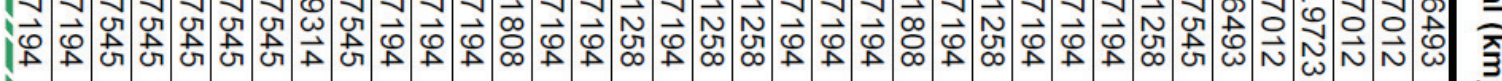




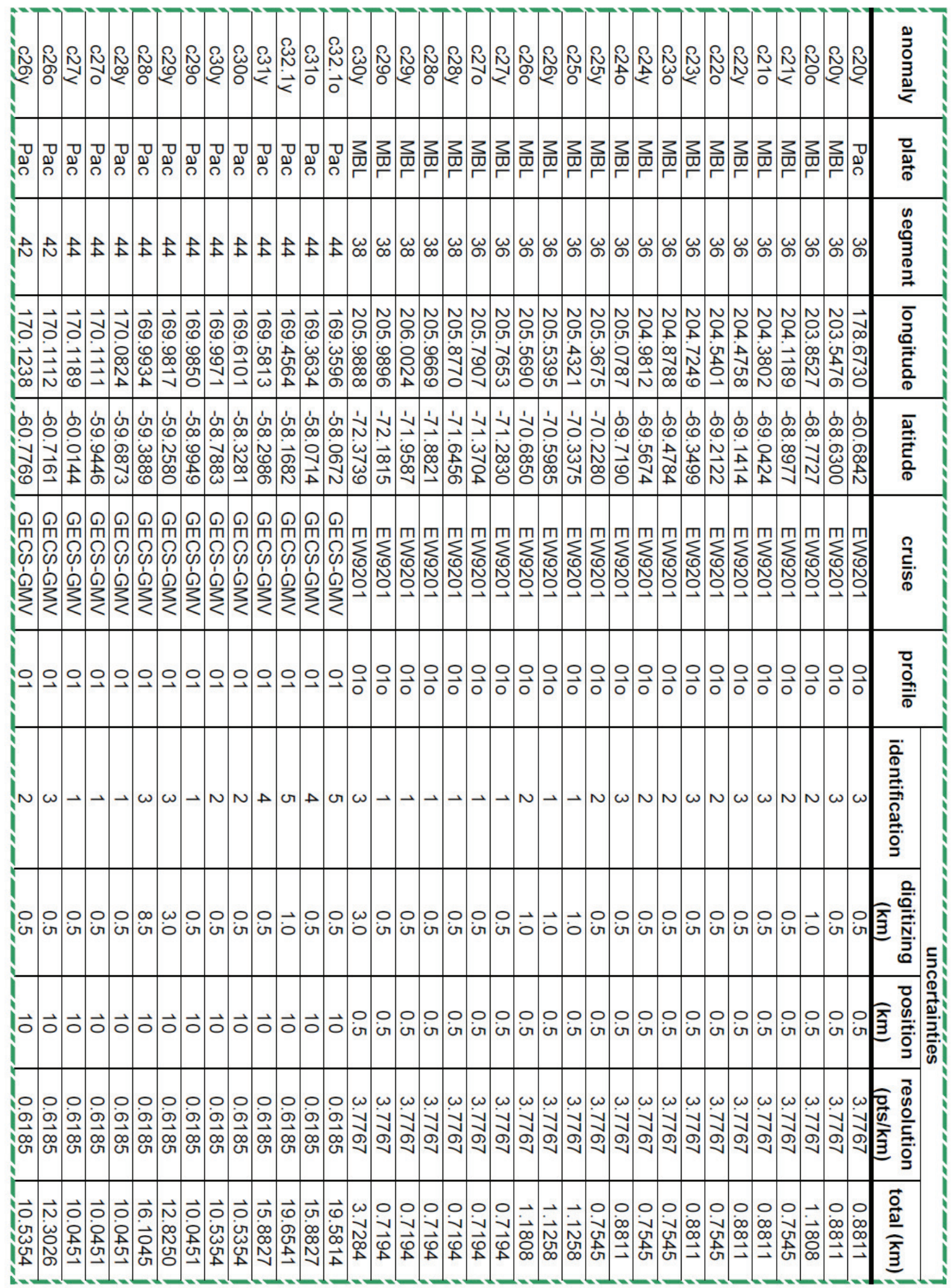




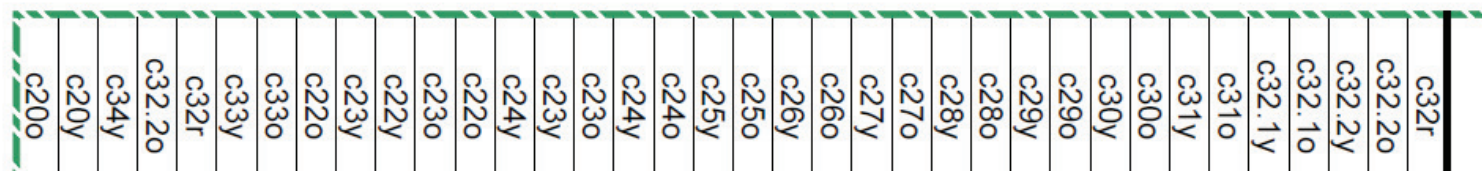
일

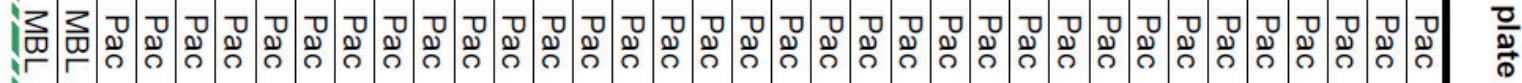

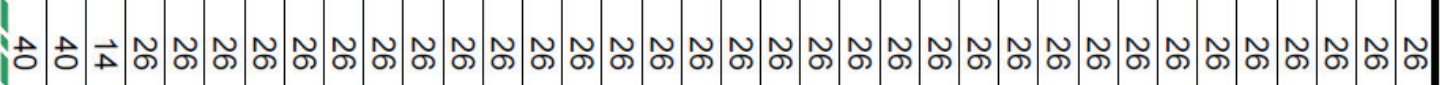

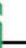

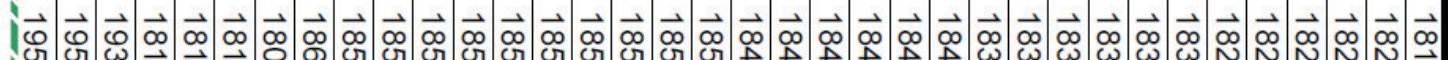
10

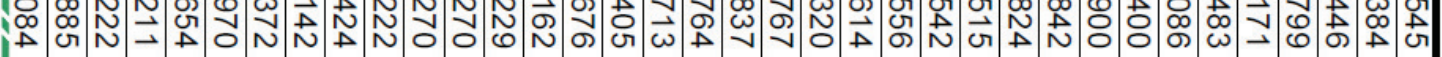

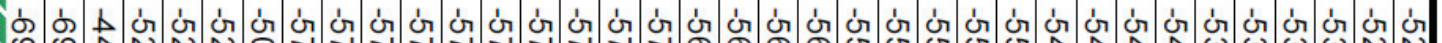

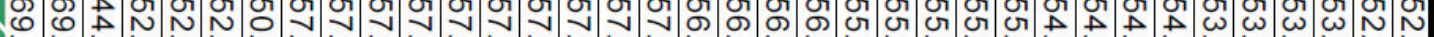
J

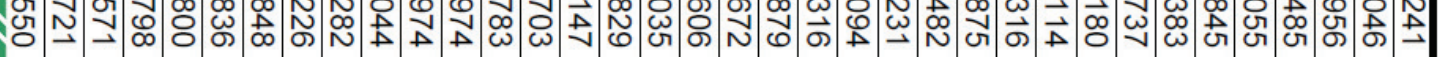

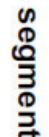

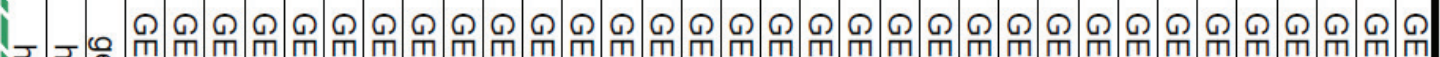
•

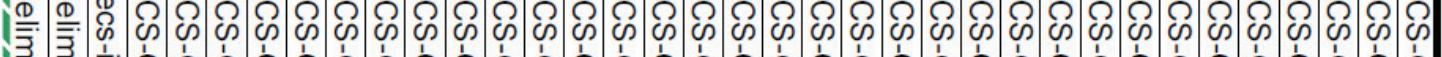

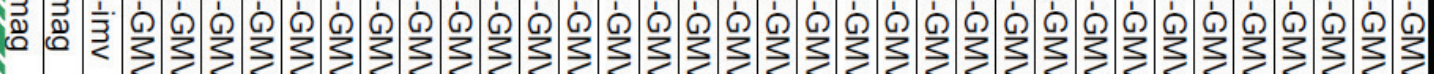

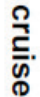

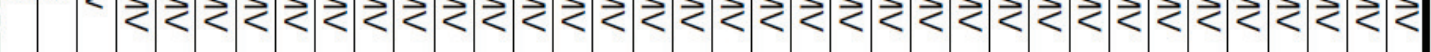

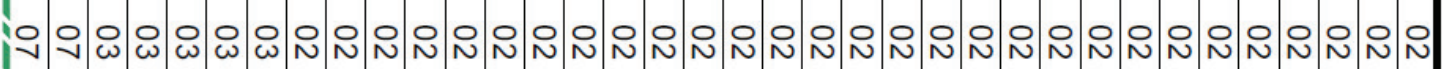

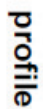

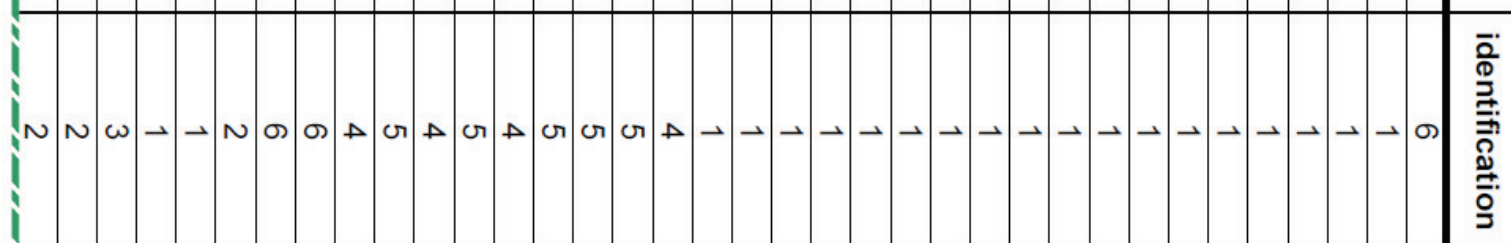
í 1

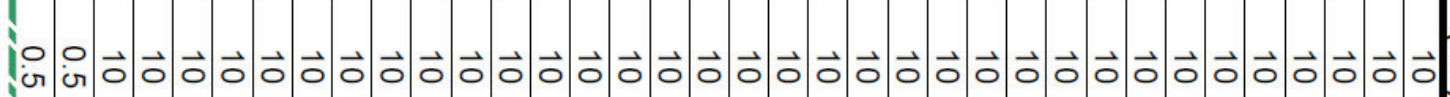
1 1

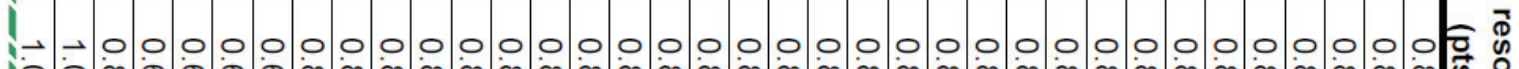
Nمن

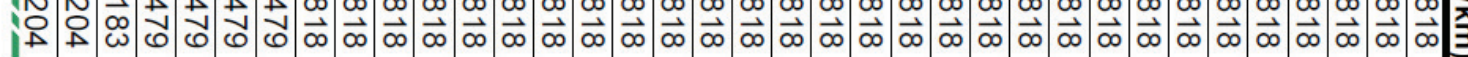

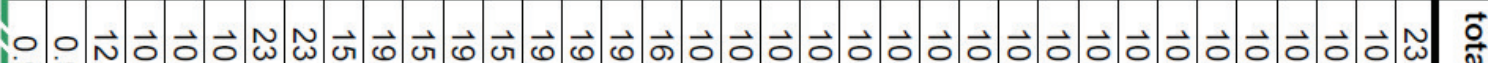
6 i

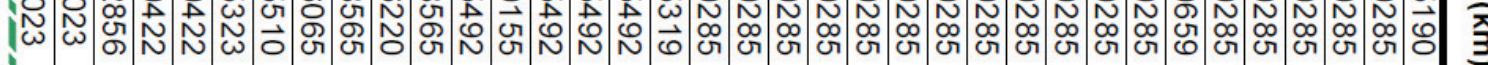
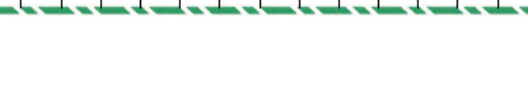


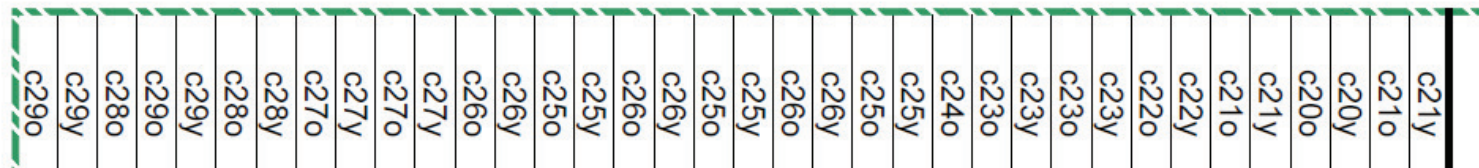

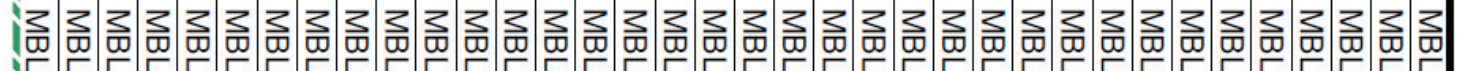

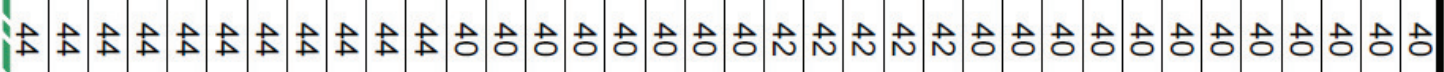

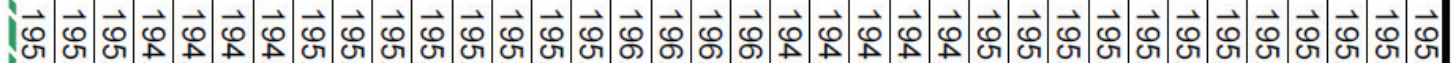

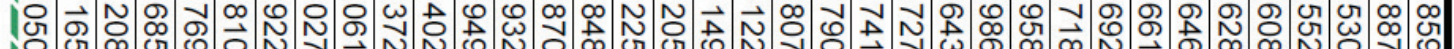
:

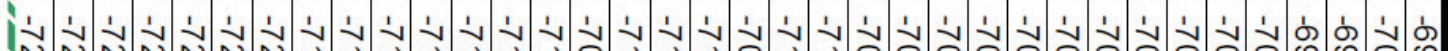

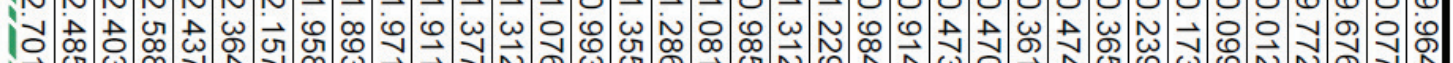

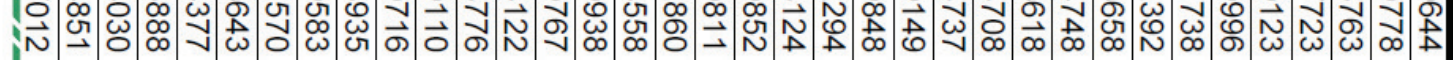

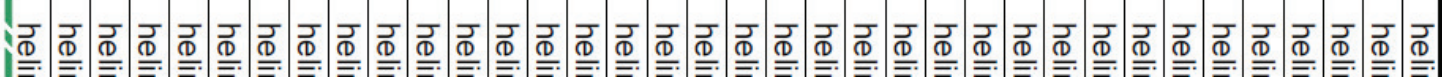

豙 每

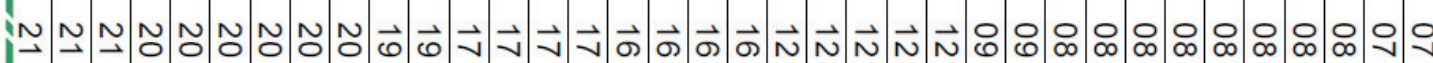

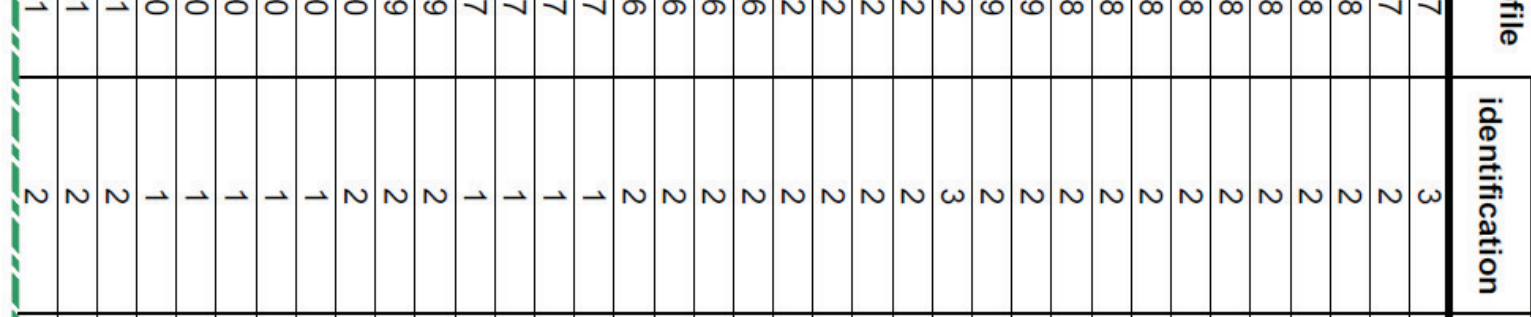

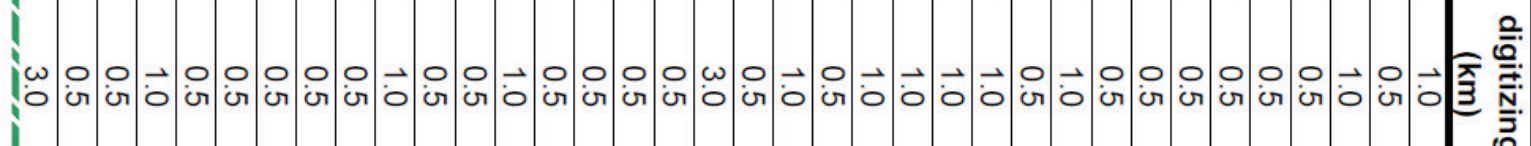

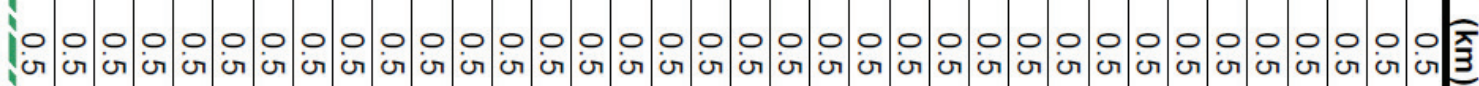

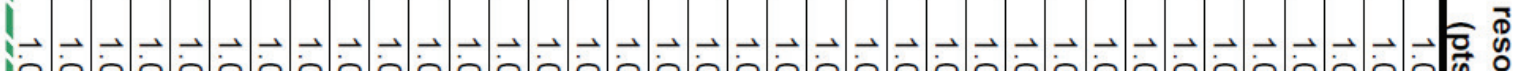

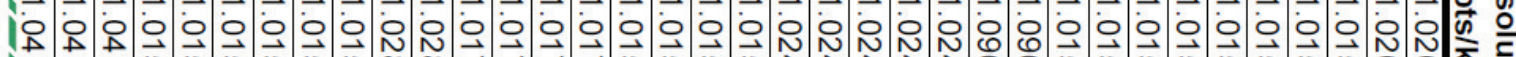

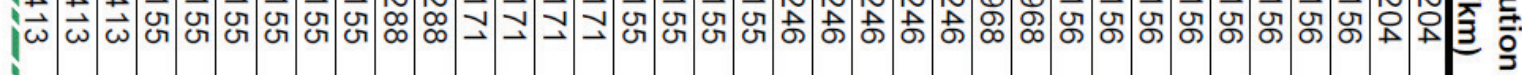

$\omega \circ \circ \rightarrow \quad$ 이 li

i 


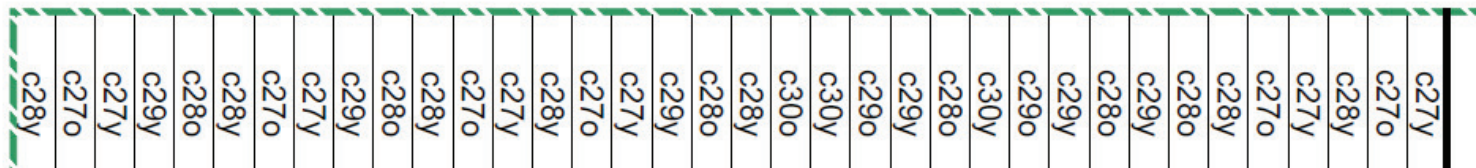
을

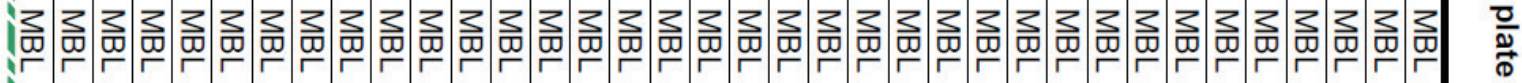

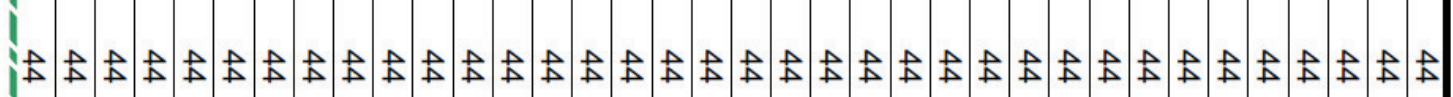

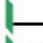

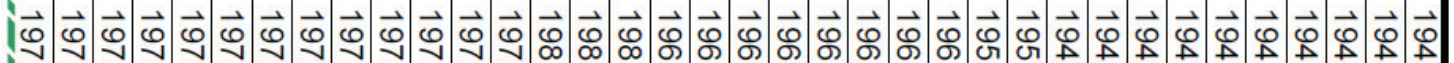

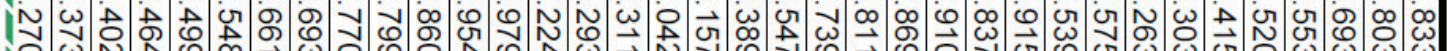

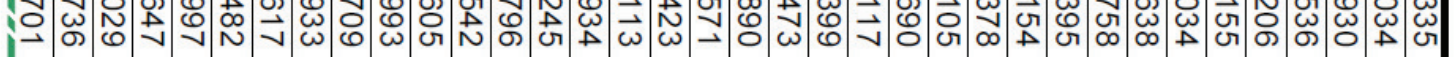

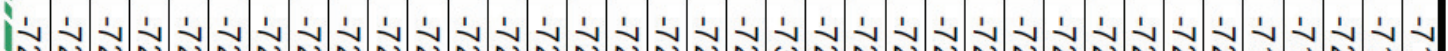

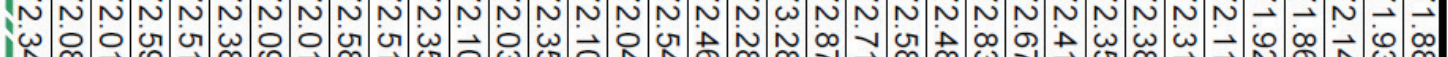

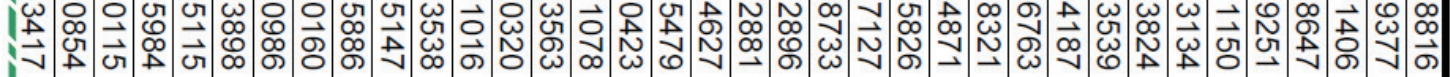

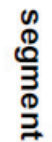

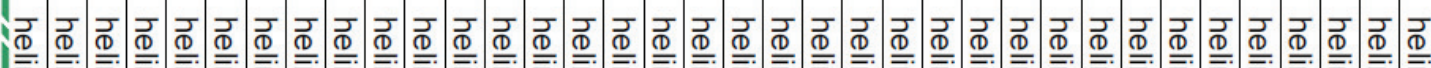

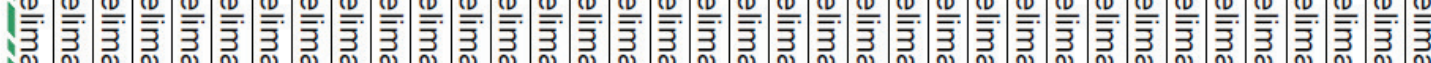

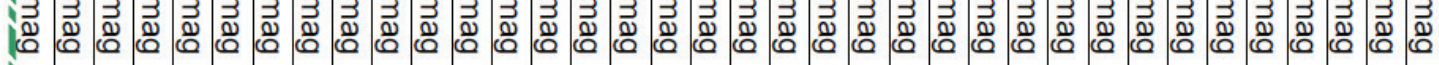

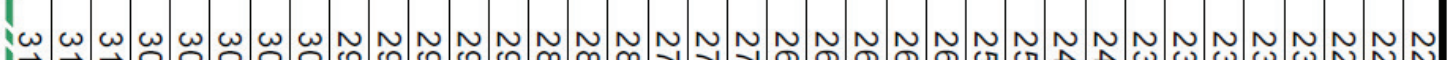

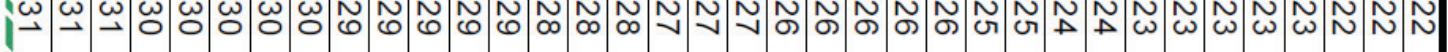

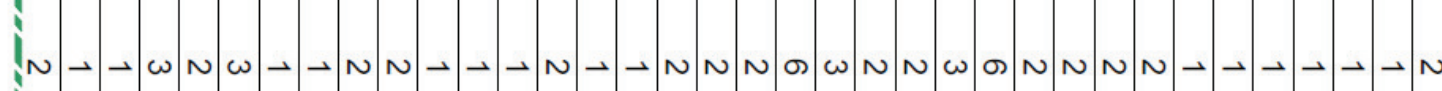

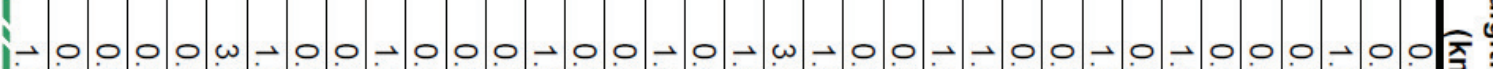

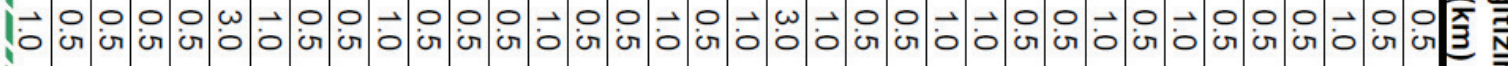

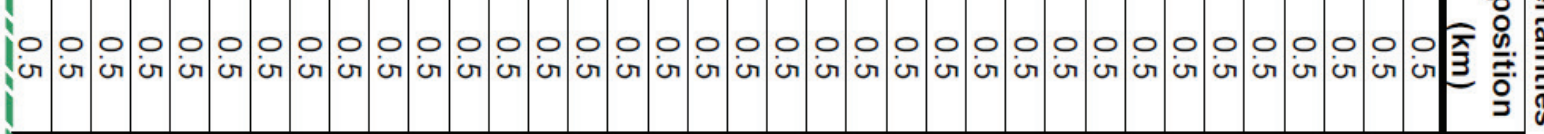

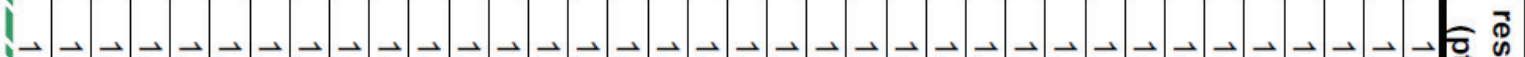

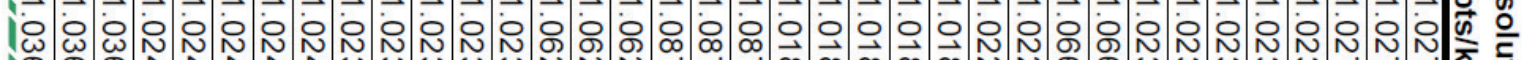

i.

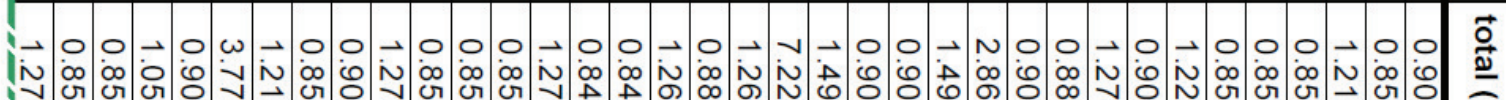

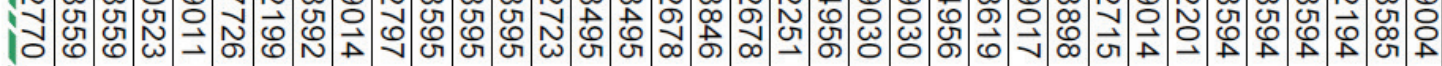




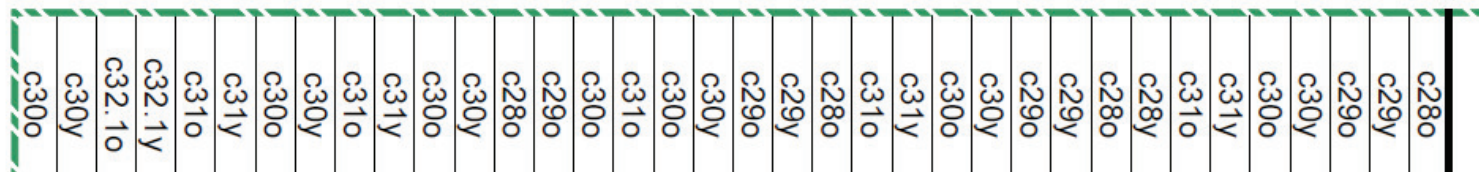

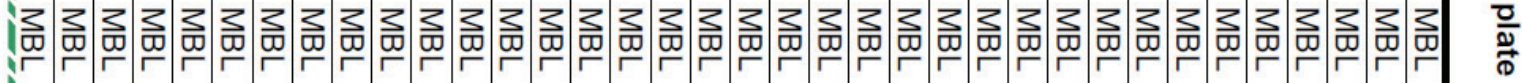

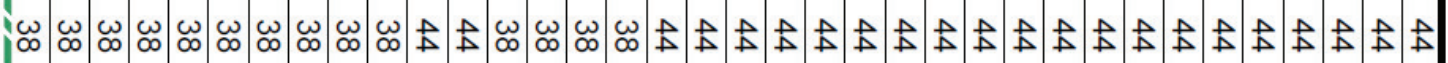

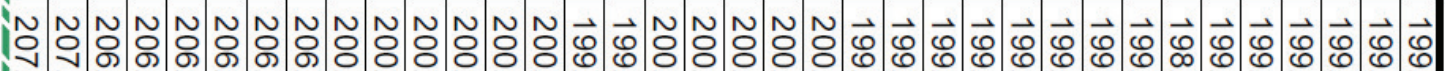

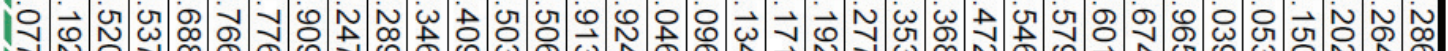

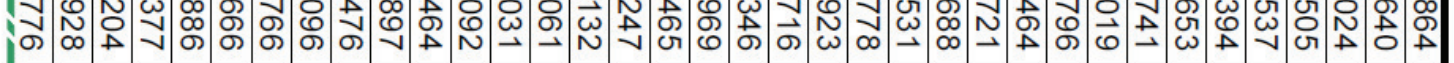

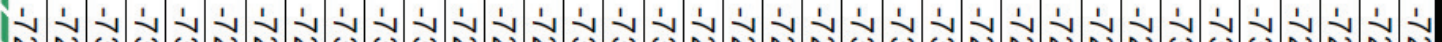

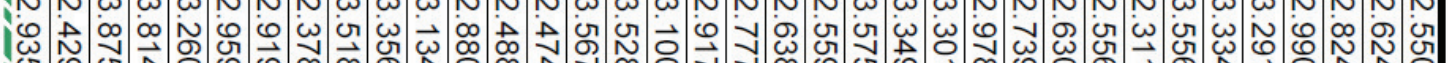

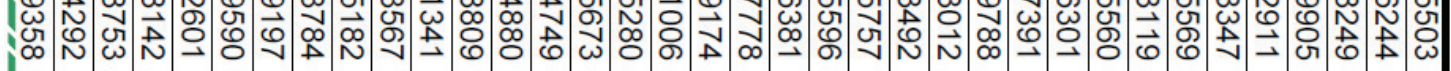

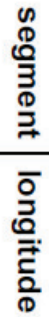

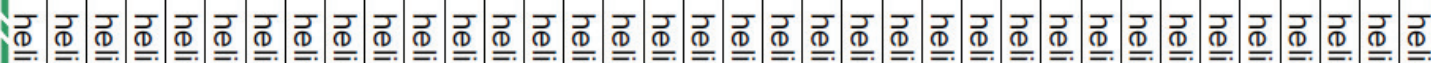

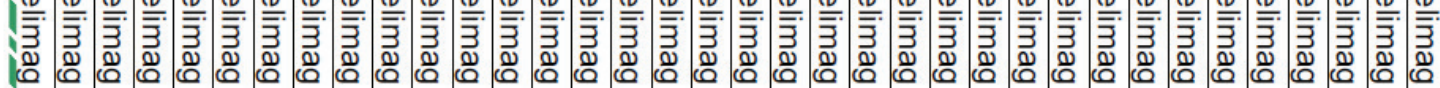

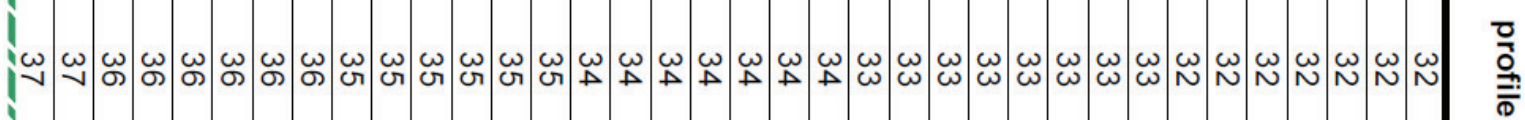

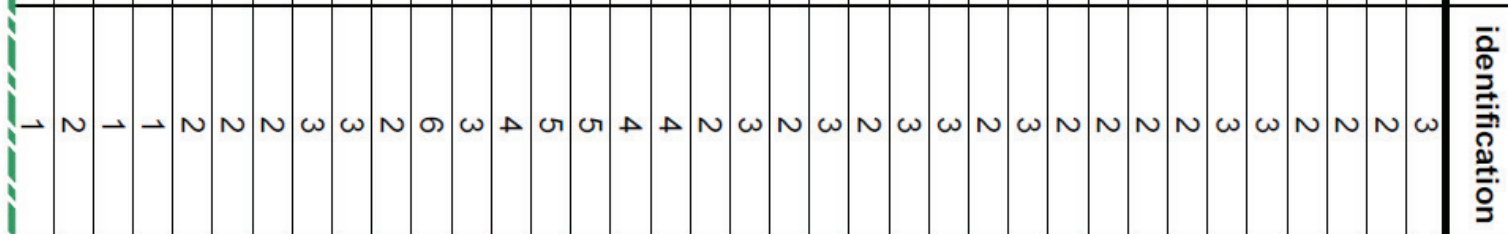

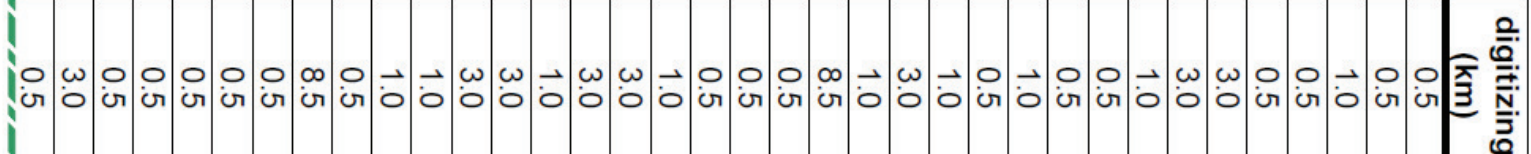

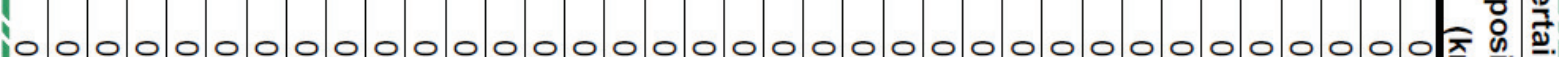

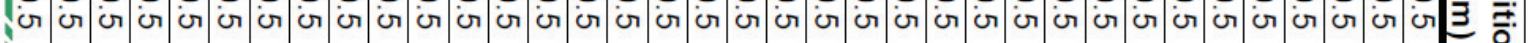

\begin{tabular}{llllll}
\hline & & & \\
\hline
\end{tabular}

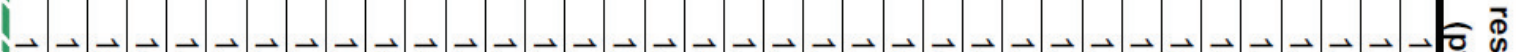

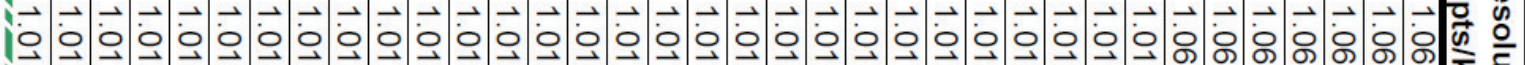

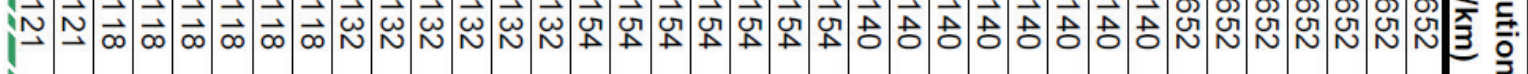

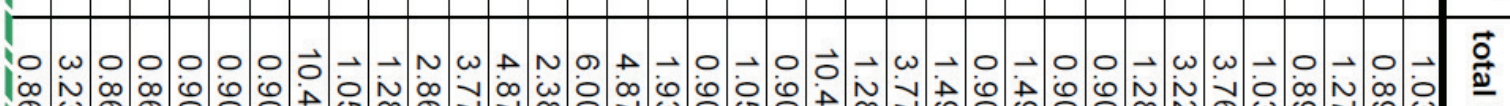

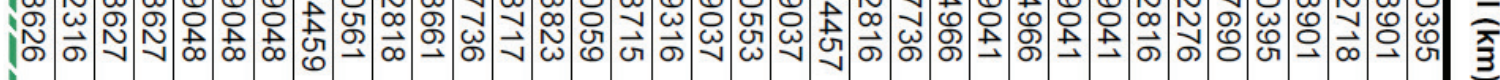




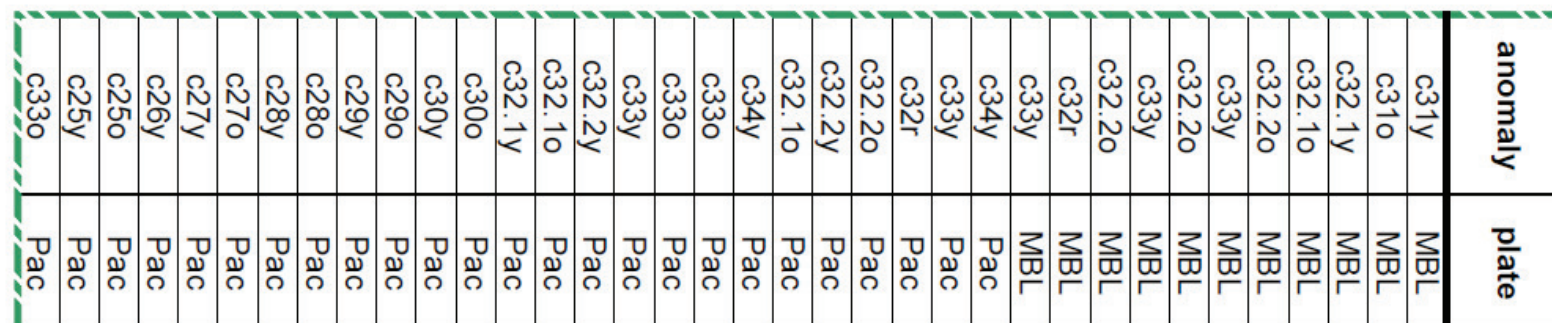

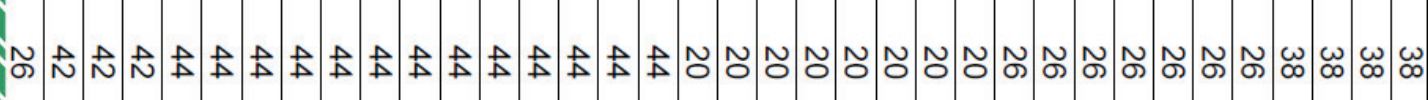

-

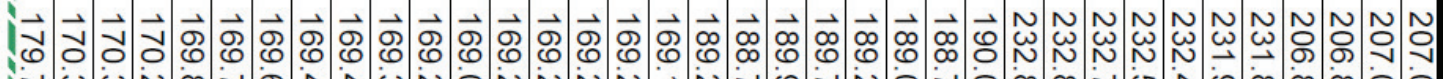

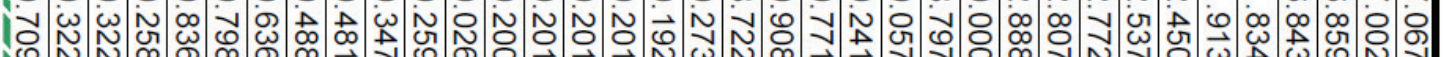

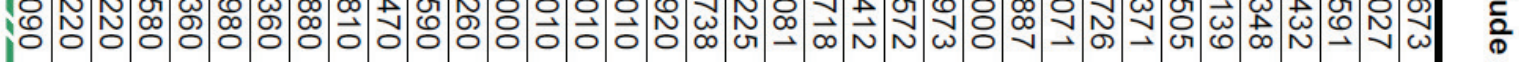

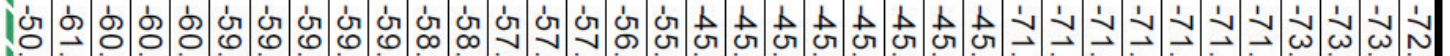

1\%

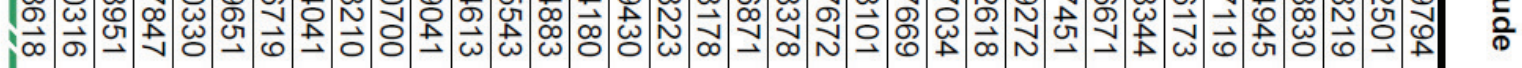

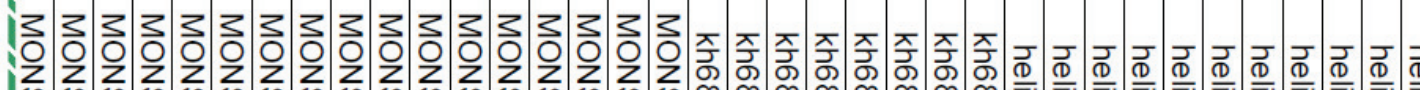

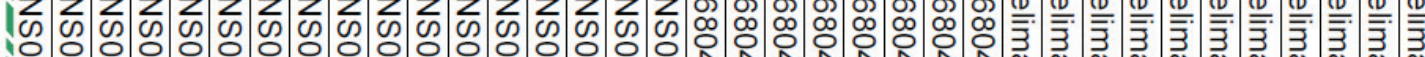

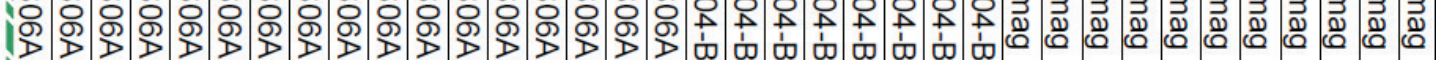

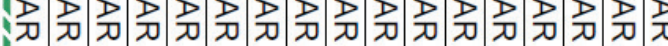

10 000000000000 o o o e er er $\omega \omega \omega \omega$

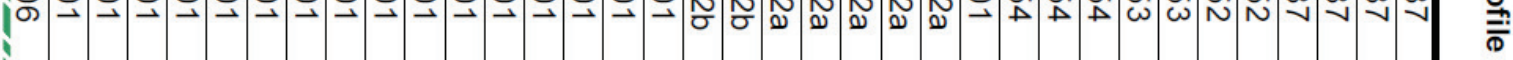

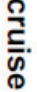

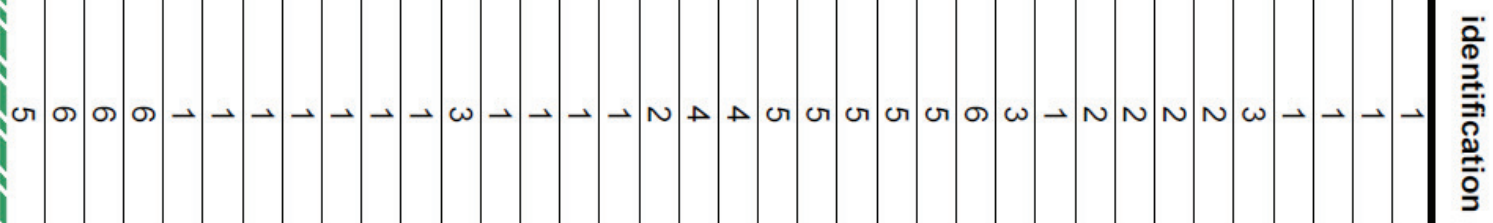

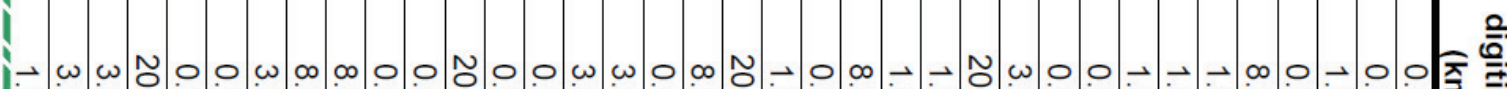

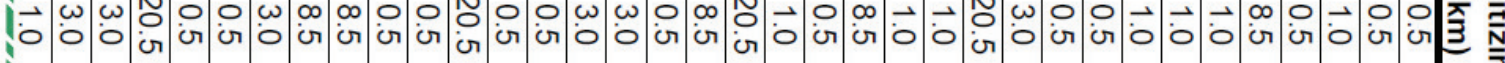

in

1

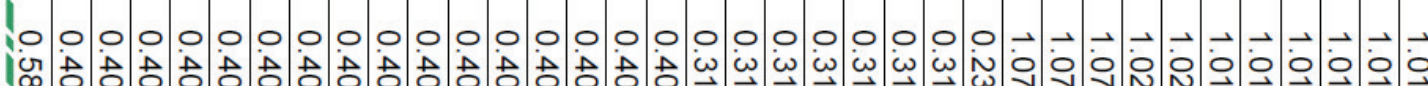

N

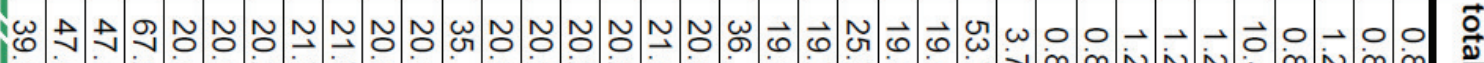

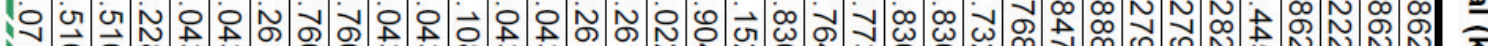

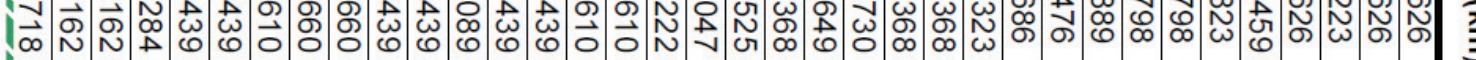




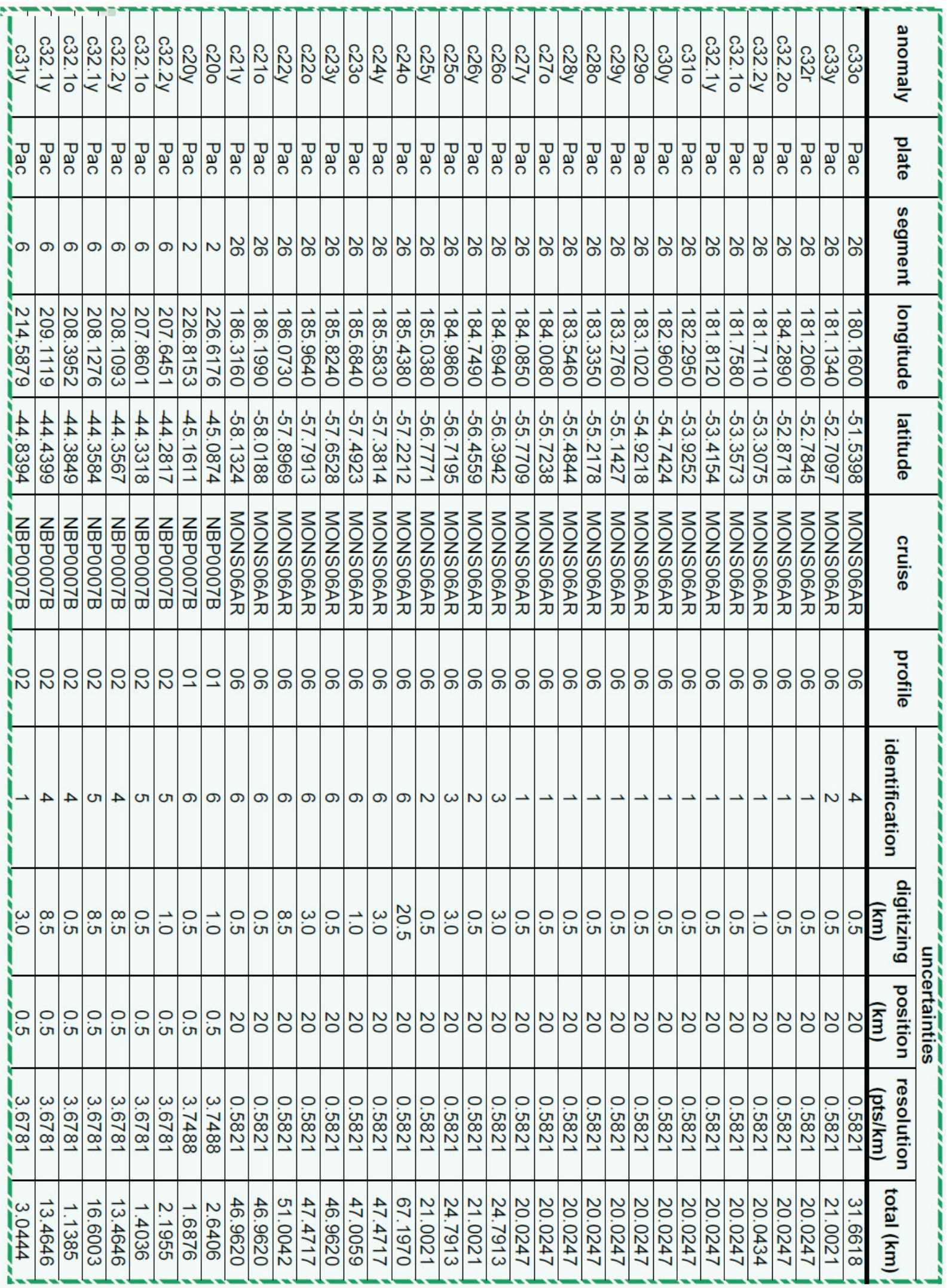




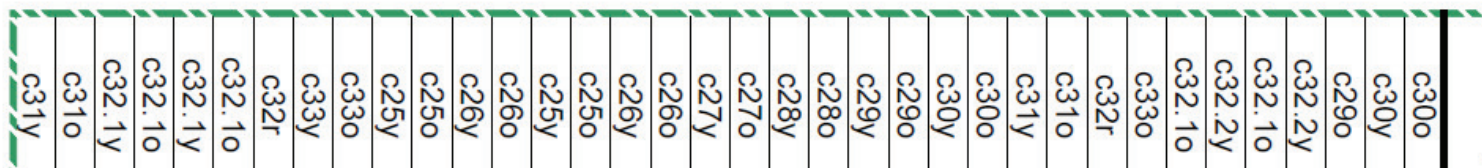

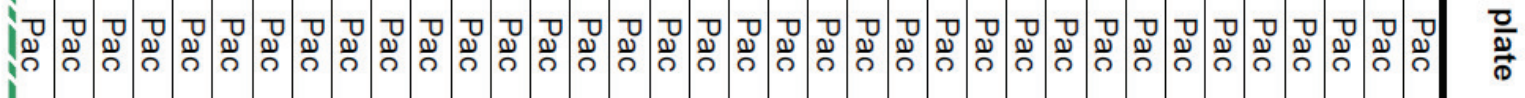

சのののののののの

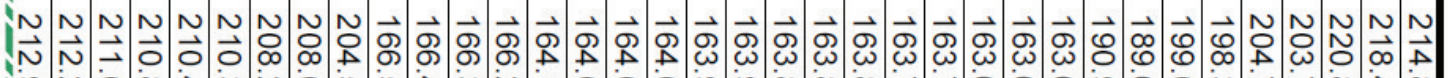

i

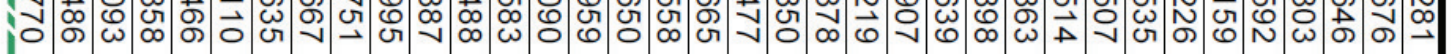

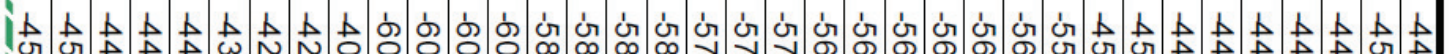

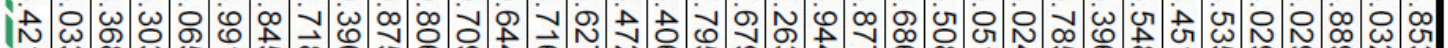

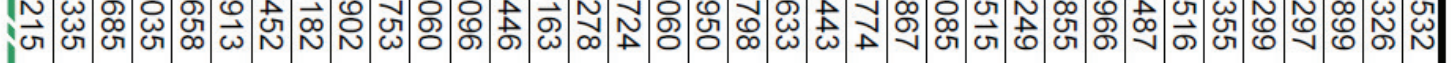

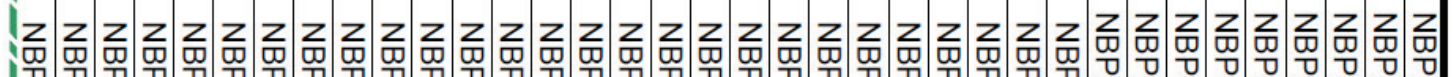

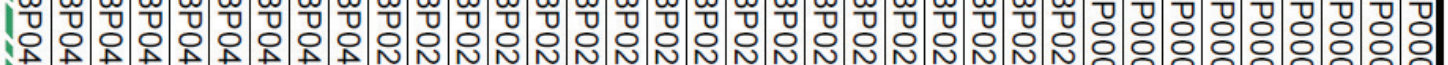

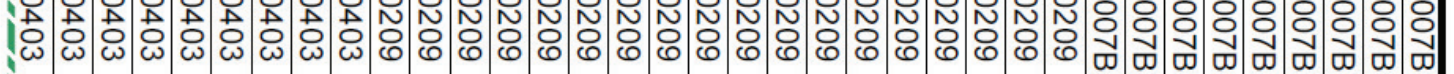

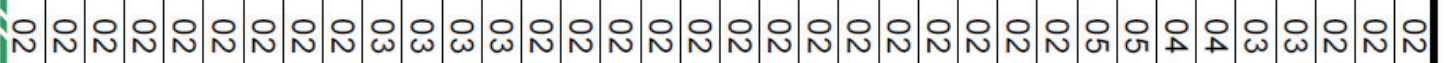

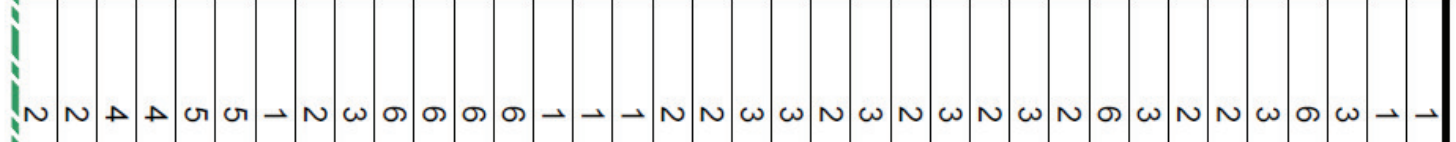

-

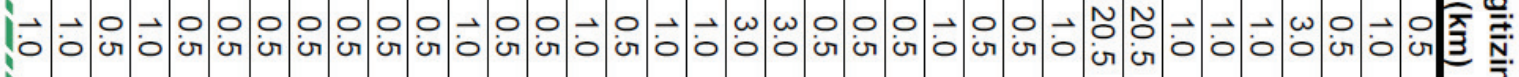

1

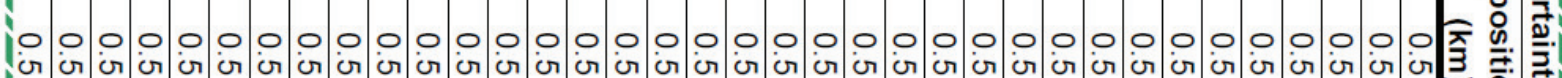
(1)

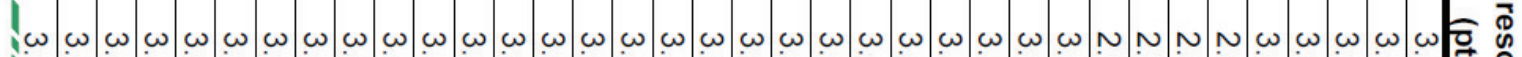

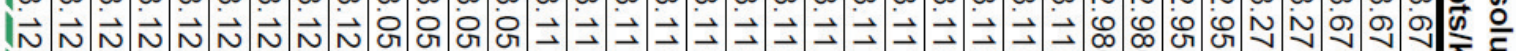

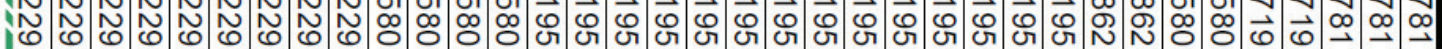

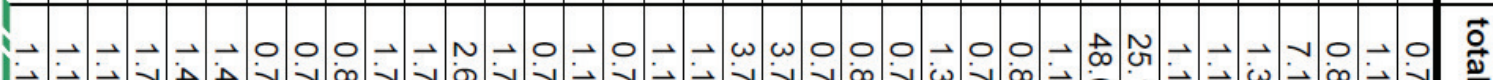

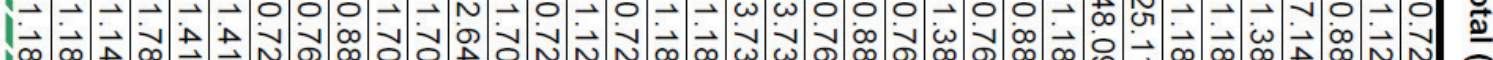

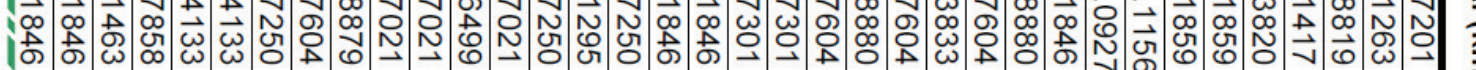




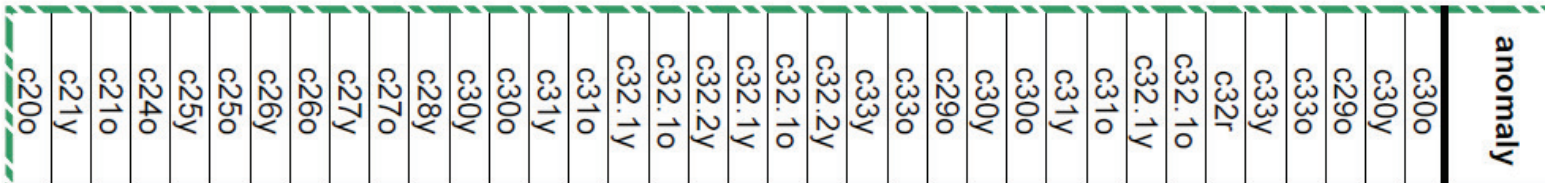

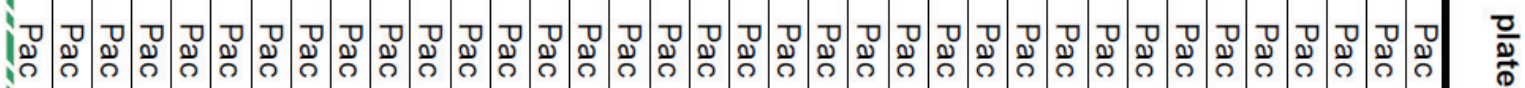

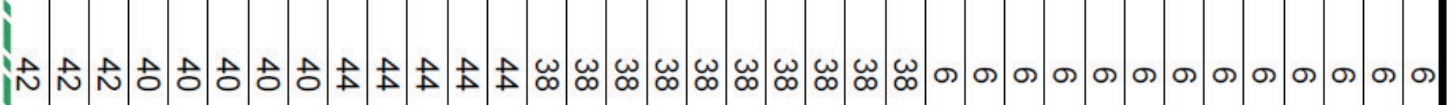

-

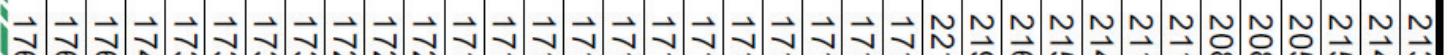

ने के

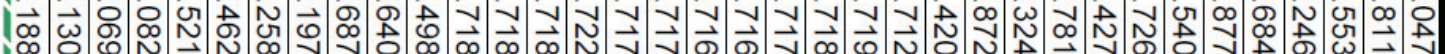

N

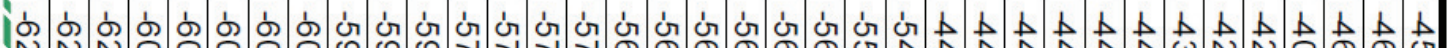

भᄀ

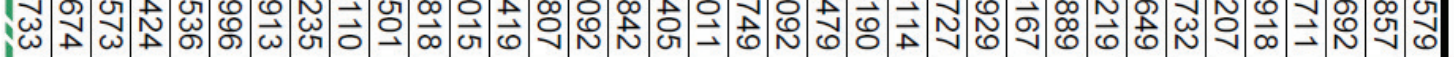

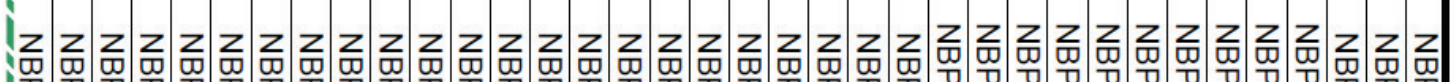

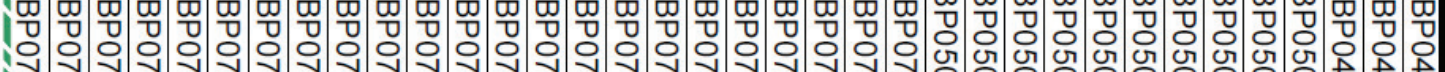

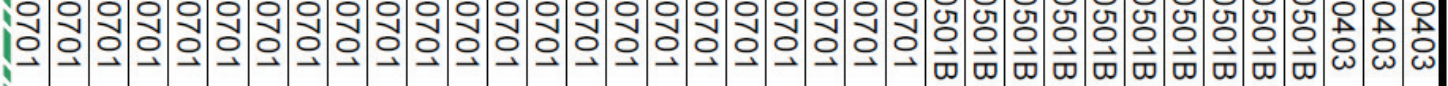

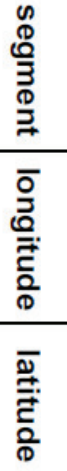

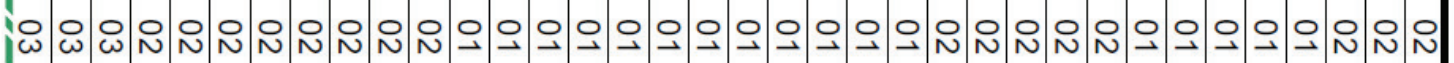

高.

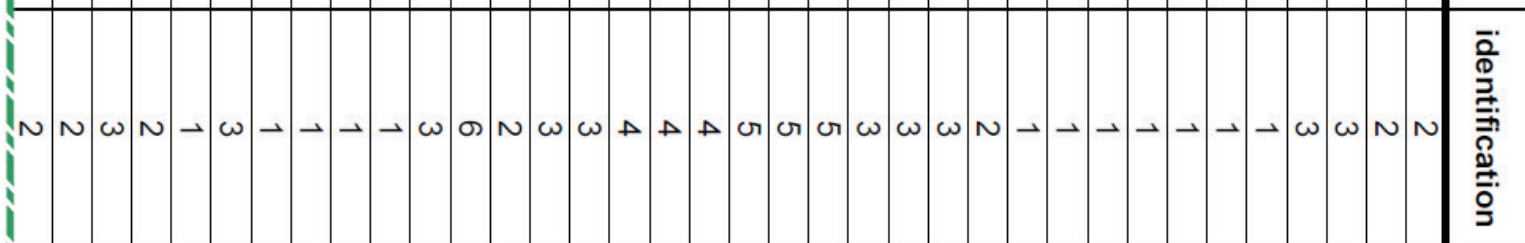

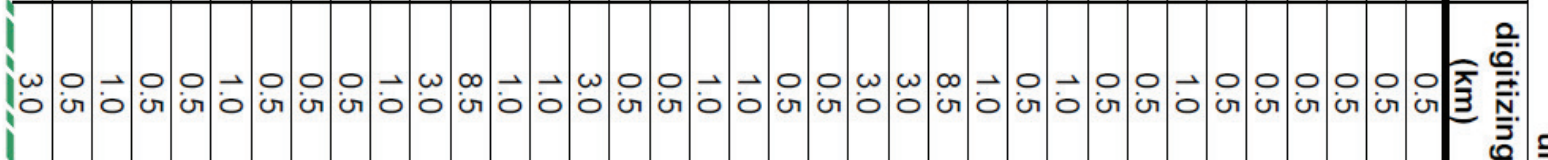

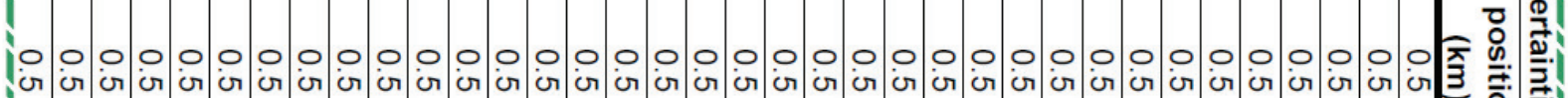

1 (1)

$\omega \omega \omega \omega \omega \omega \omega \omega \omega \omega \omega \omega \omega \omega \omega \omega \omega \omega \omega \omega \omega \omega \omega N N N N N N N N N N \omega \omega \omega \frac{0}{0} \overline{0}$

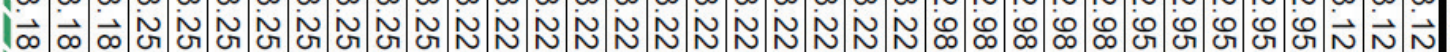

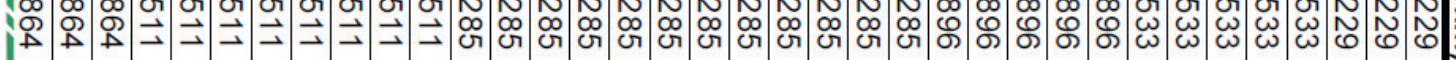

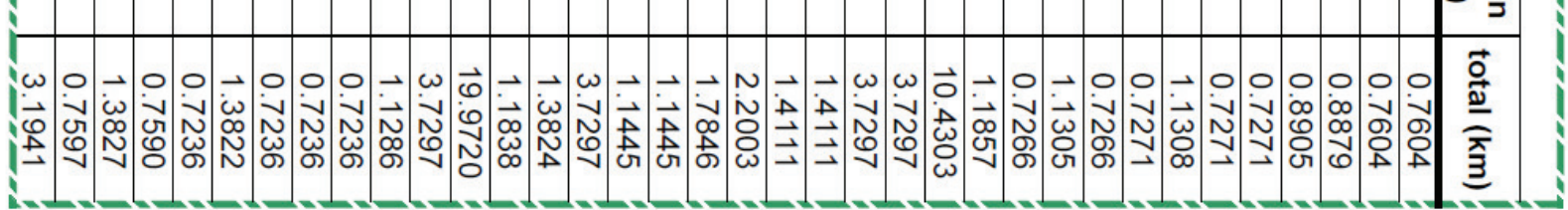




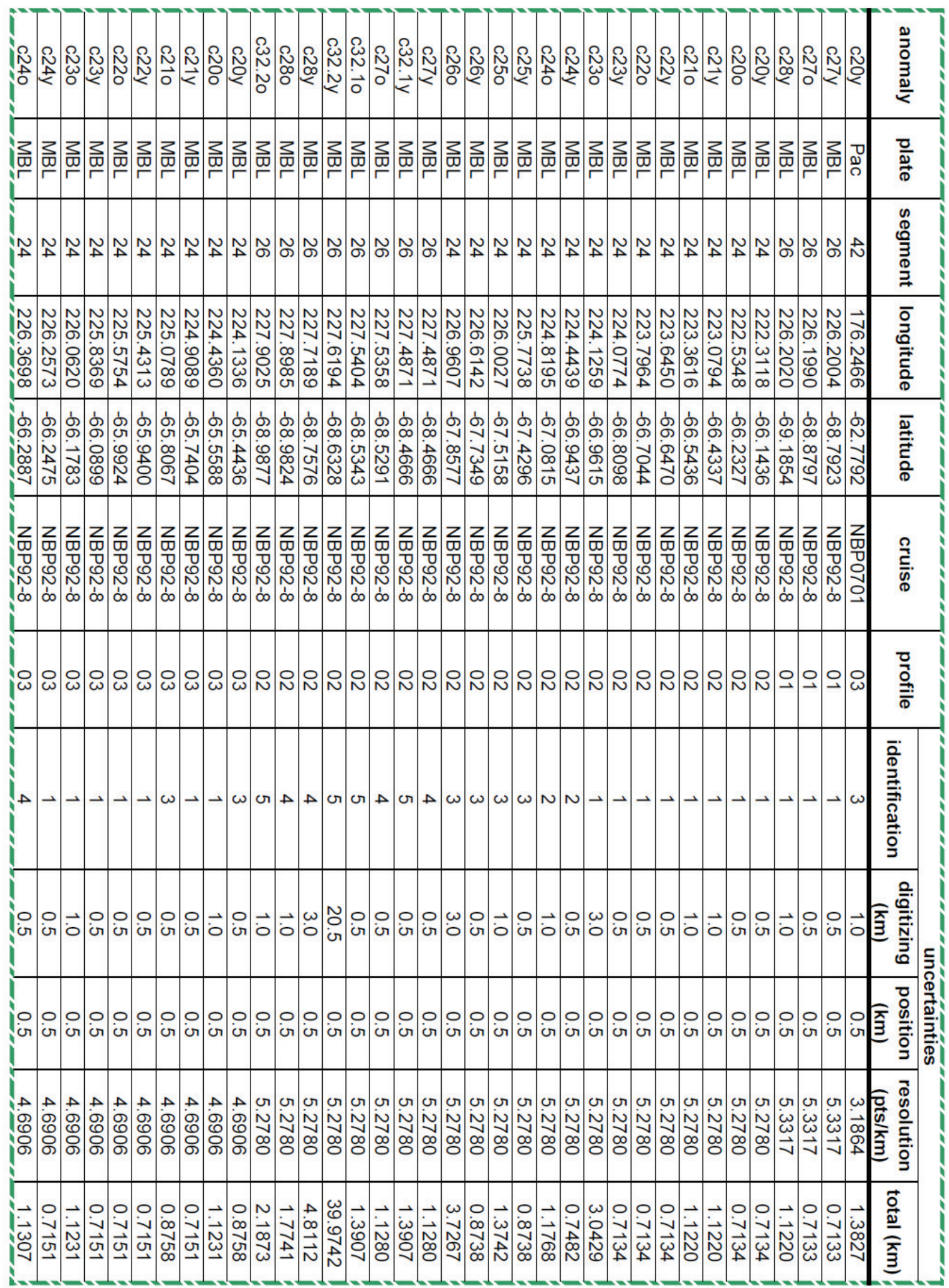


i心

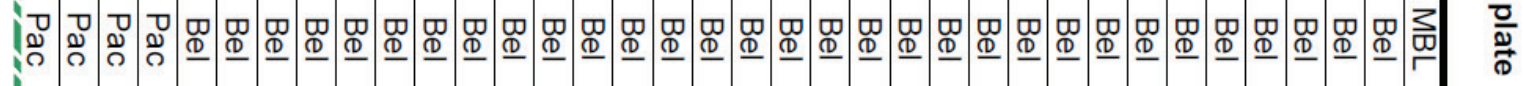

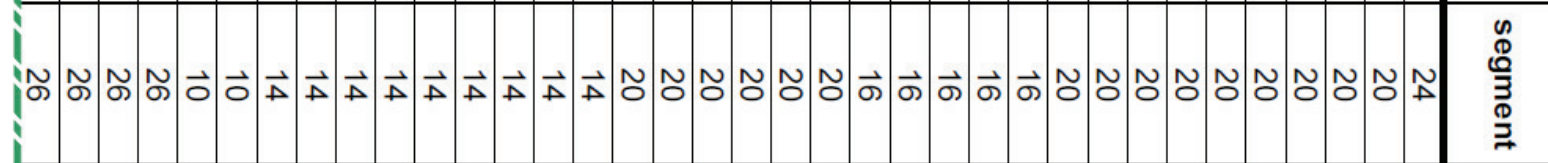

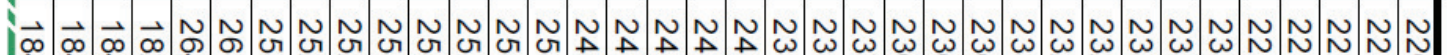

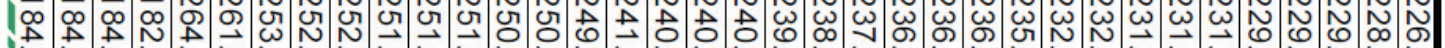
1ౌ้

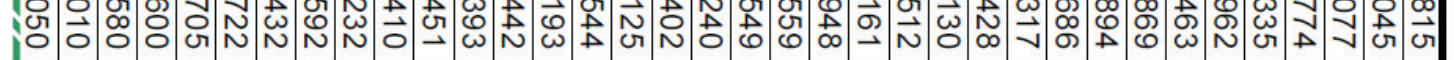
$\frac{\overline{0}}{\frac{0}{2}}$

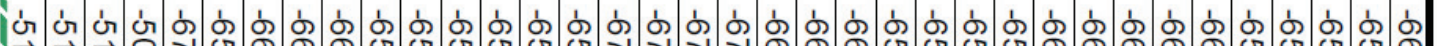

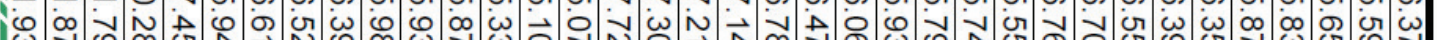

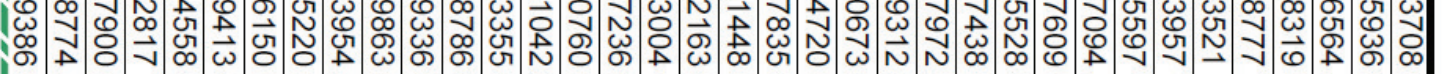
高

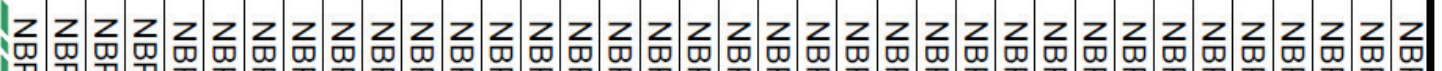

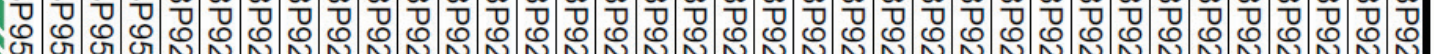

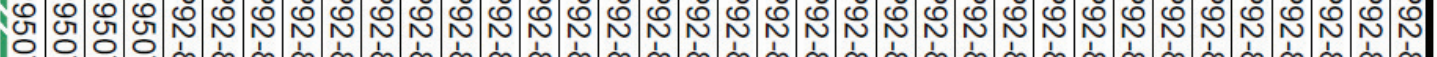

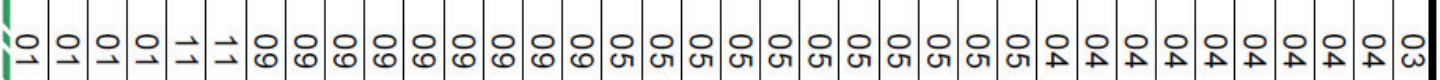

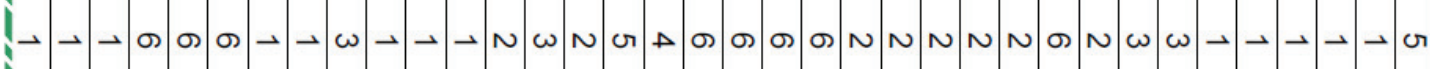

1

io

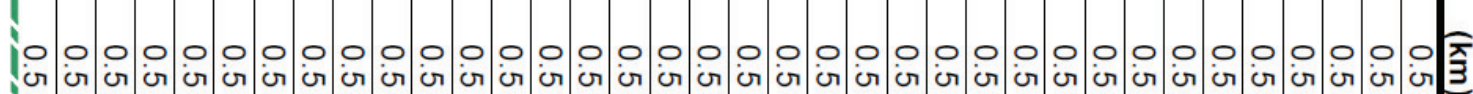

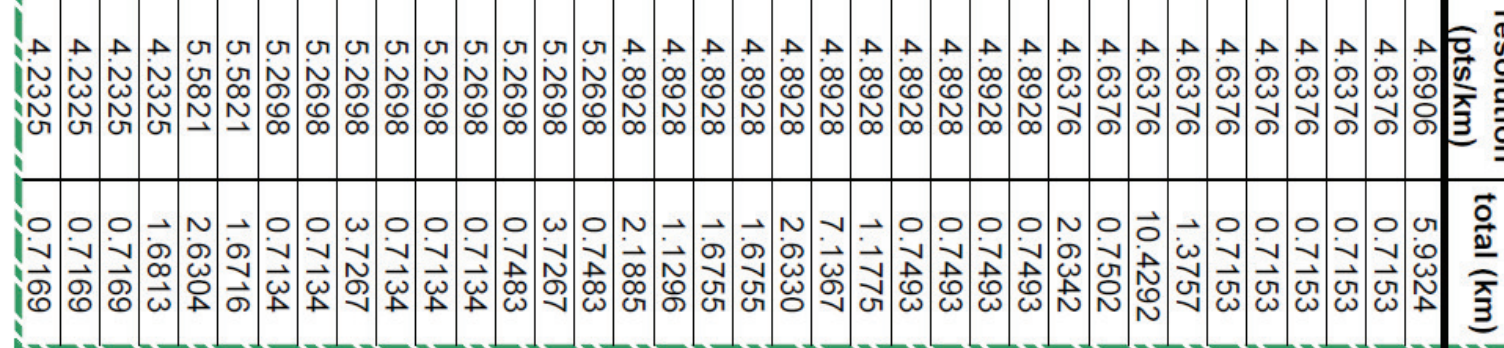




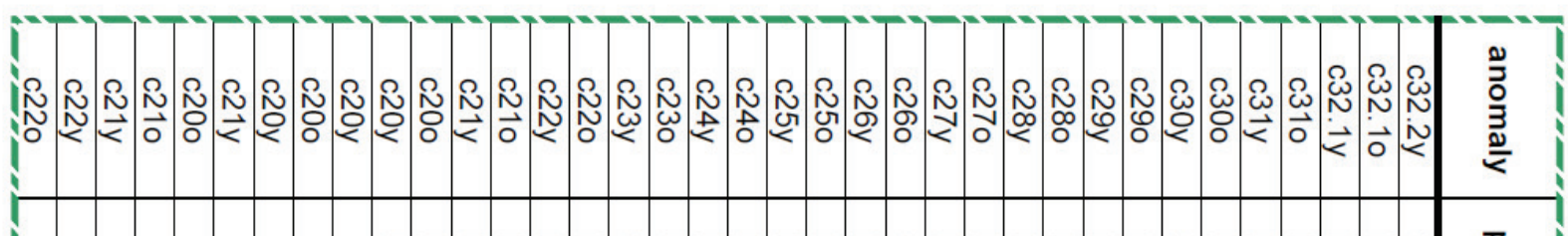

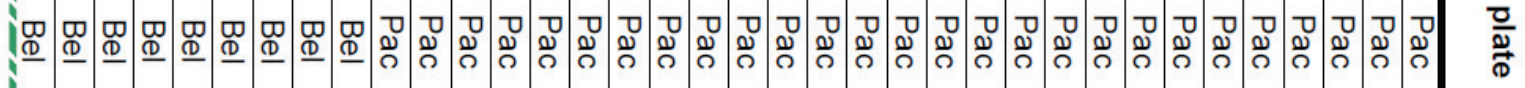

in. 要

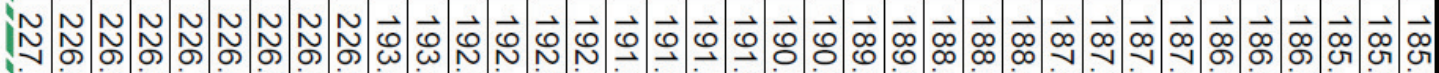

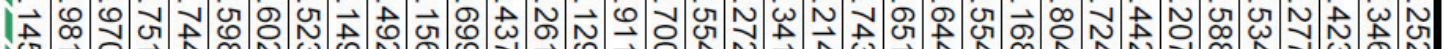

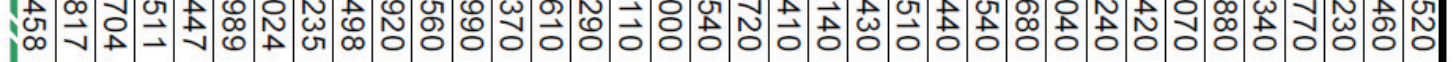

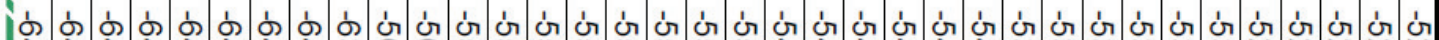
DA

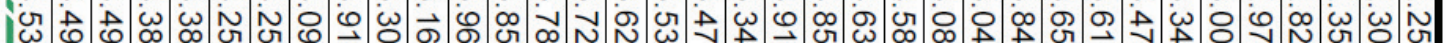

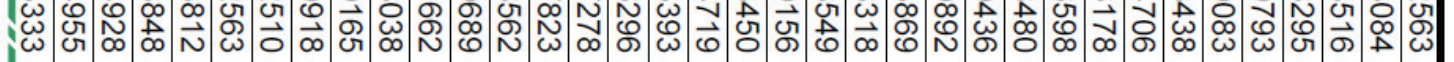

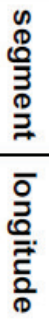

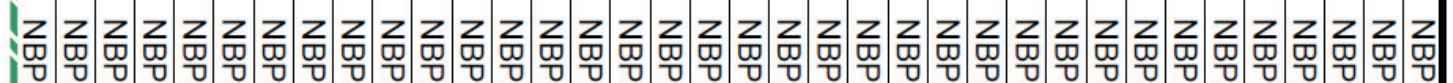
C

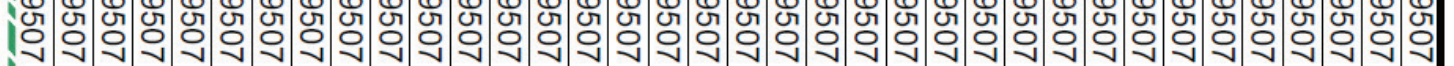

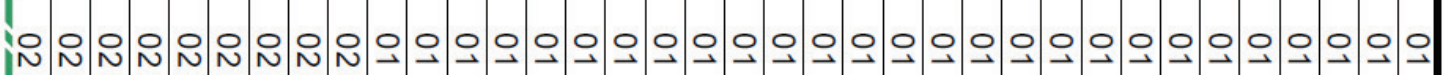

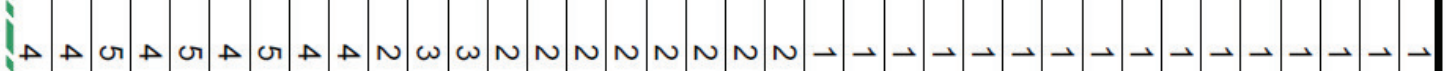

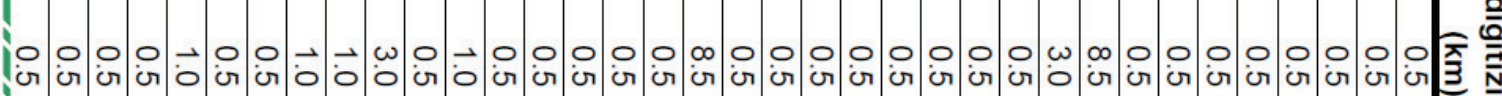
I

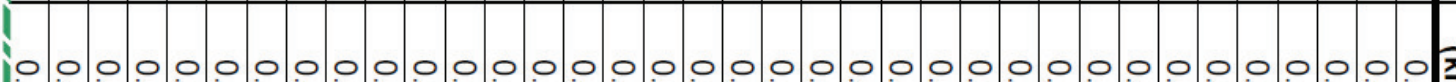

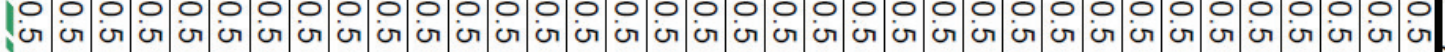

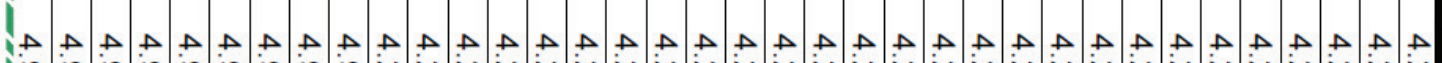
$18 \% 8 \%$

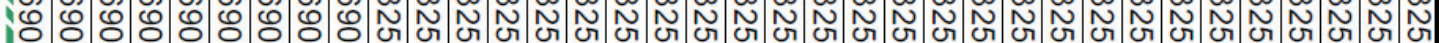




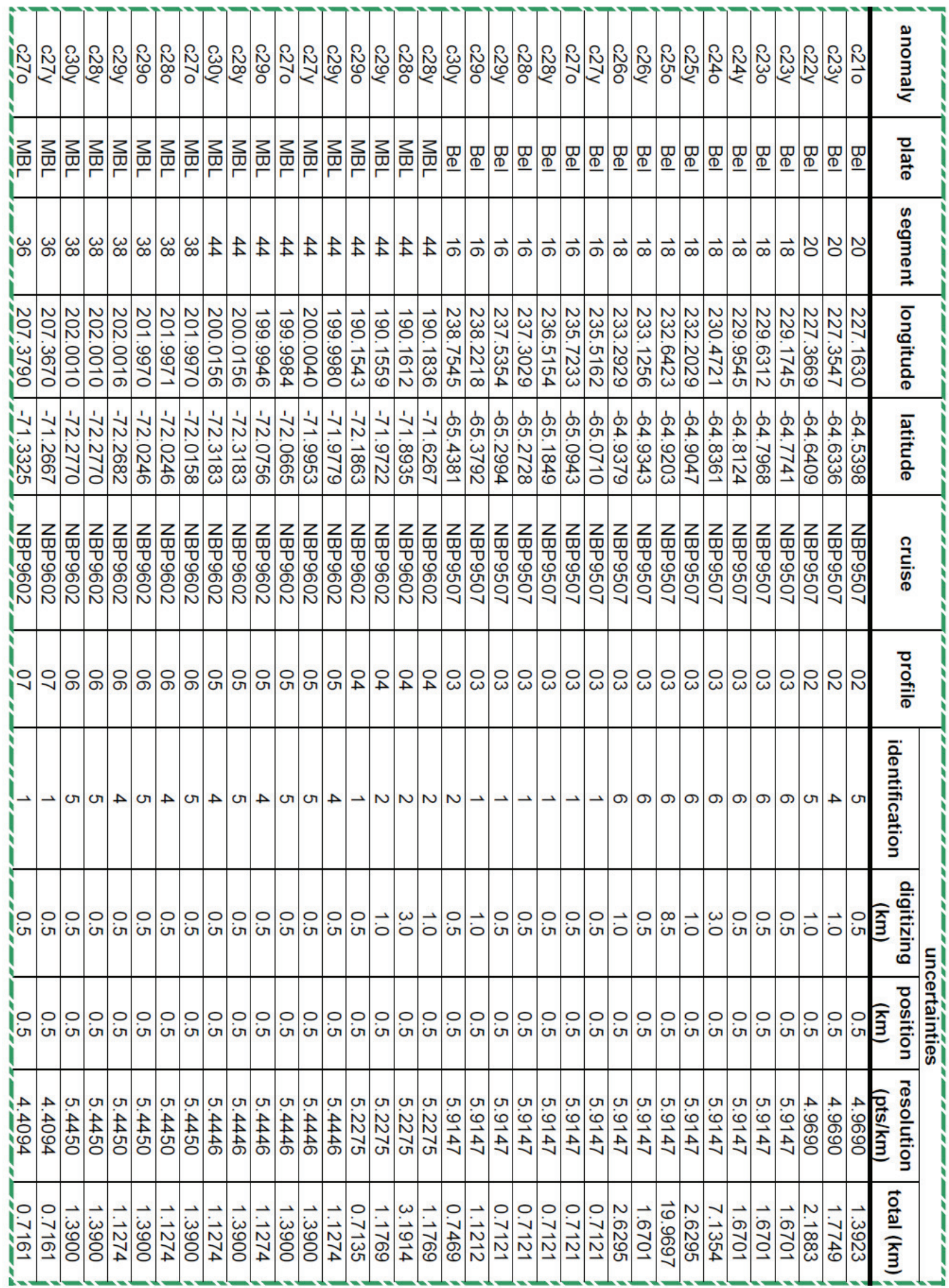




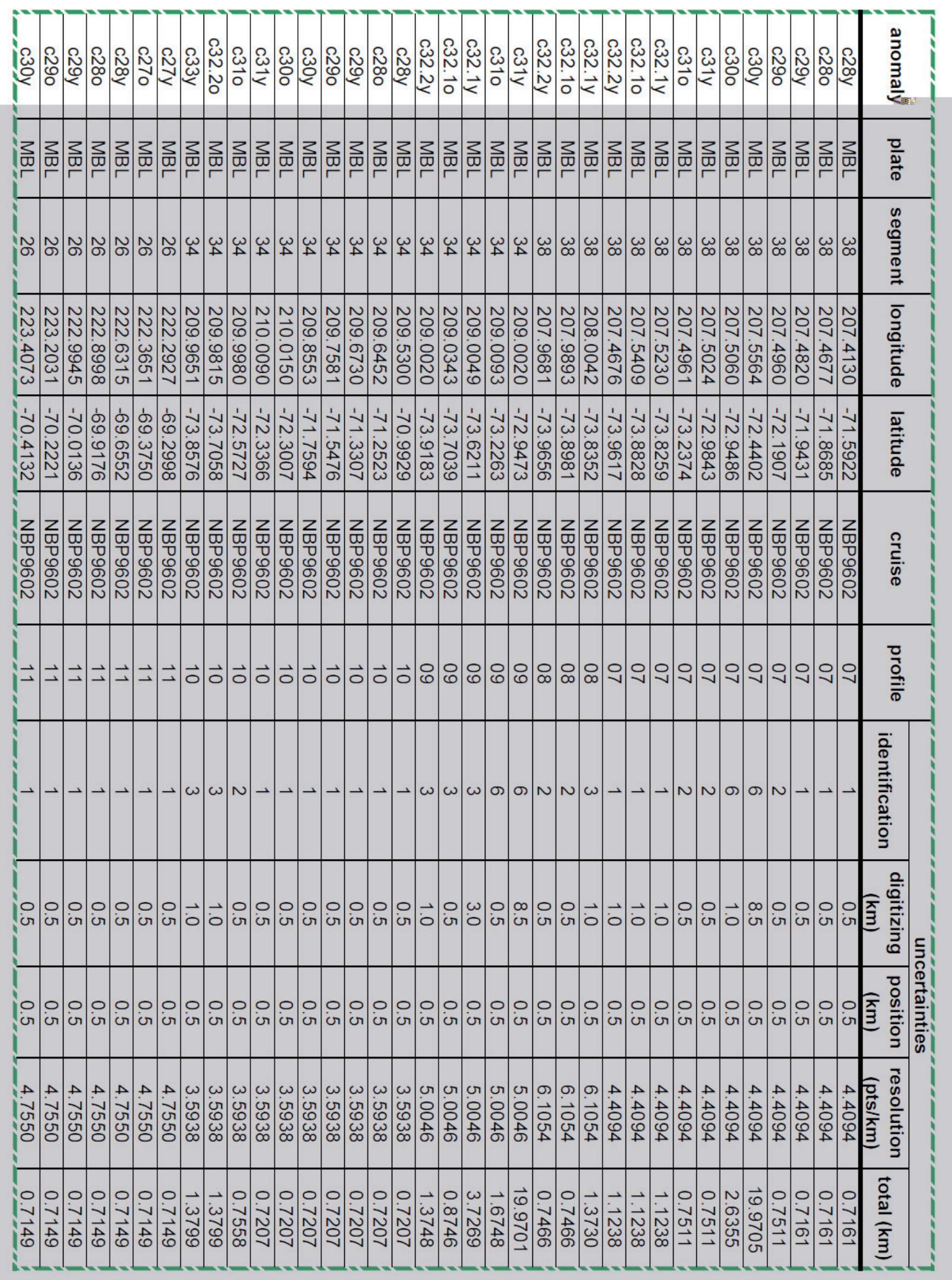




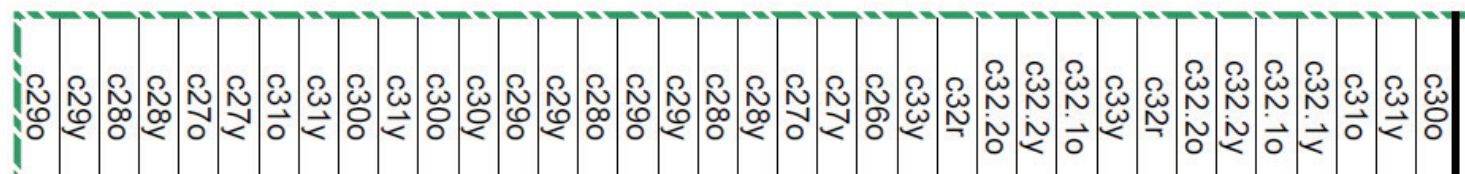

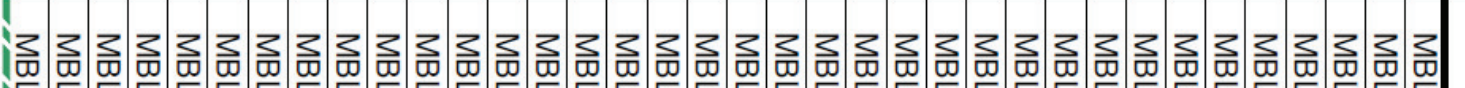

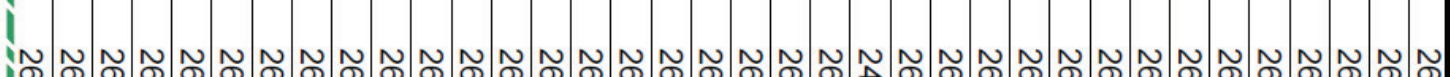

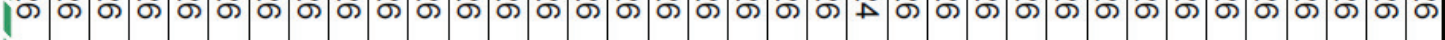

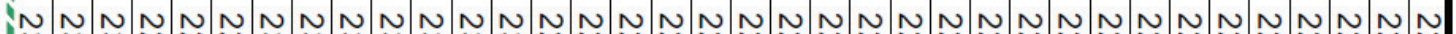

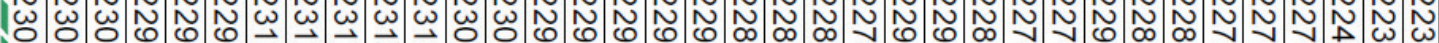

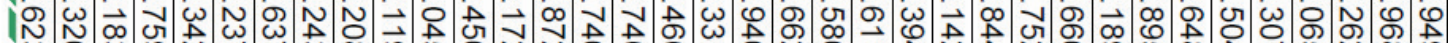

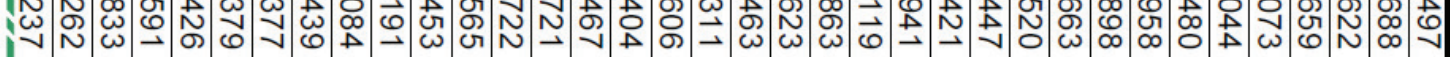

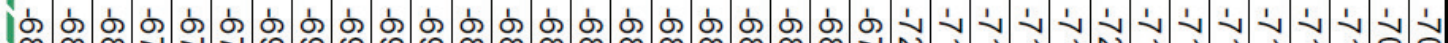

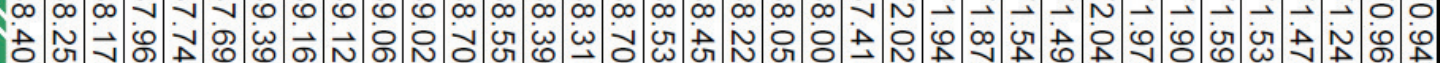

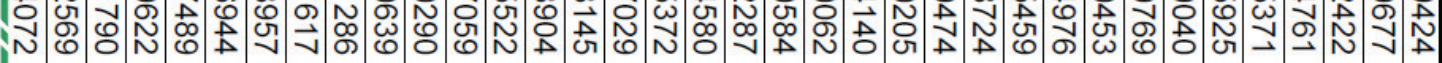

苟 |

z z z z z z z z z z z z z z z z z z z z z z z z z z z z z z z z z z z

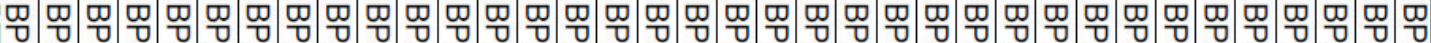

மூ

I N

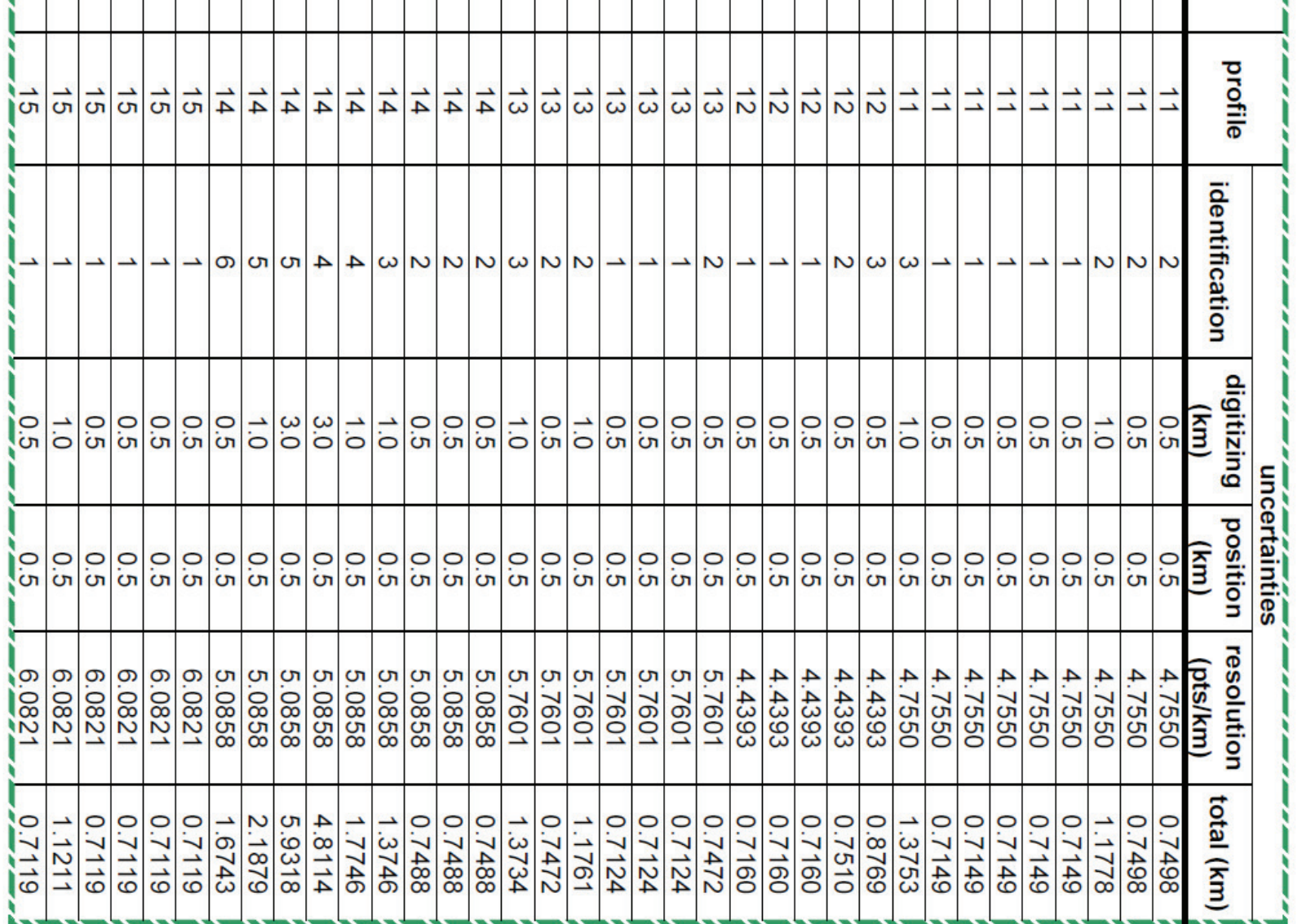




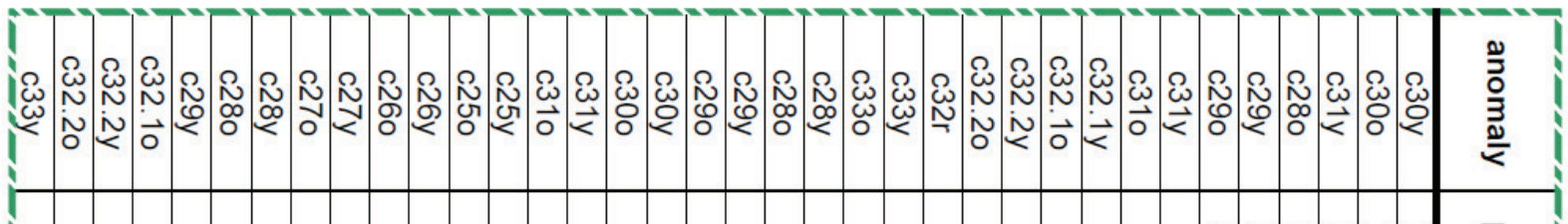

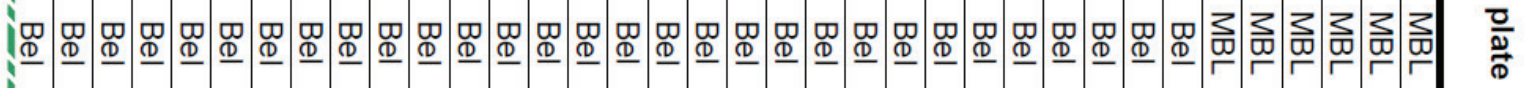

in. กิก

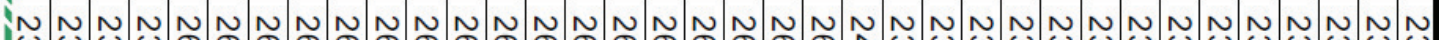

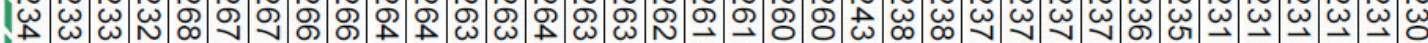

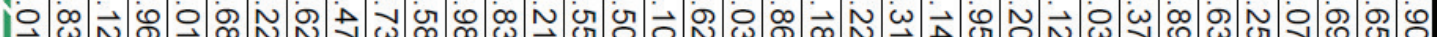

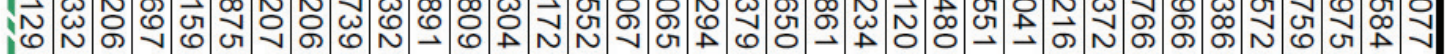

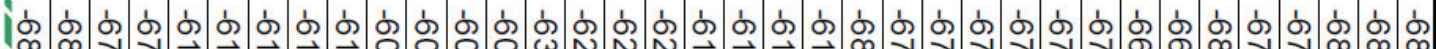

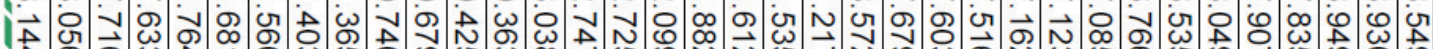

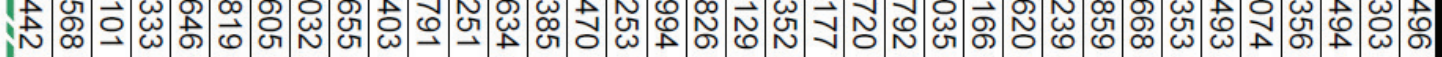

足

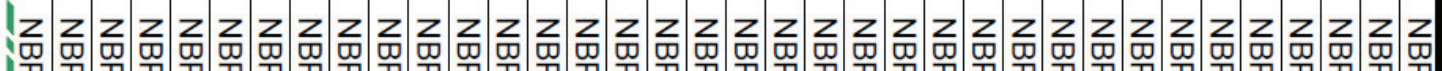

足

骨.

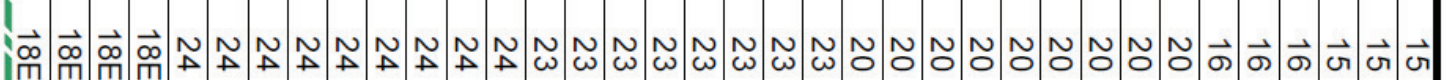

(

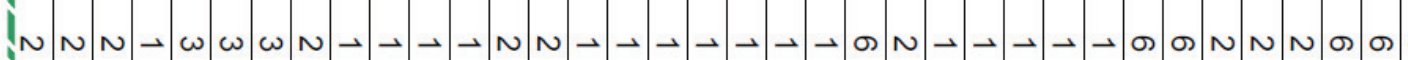

I

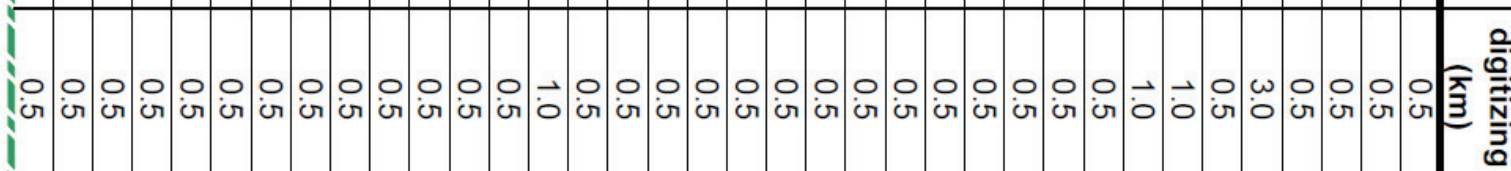

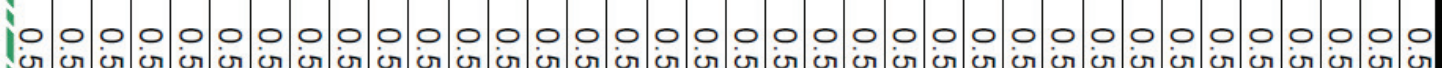

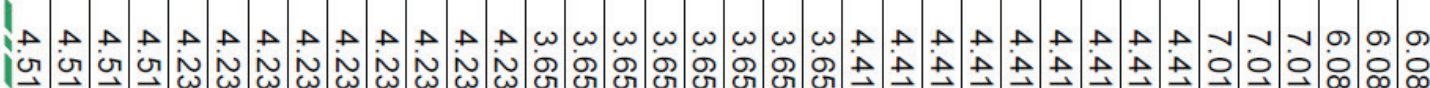

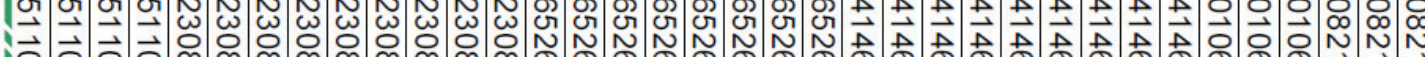

○ 이

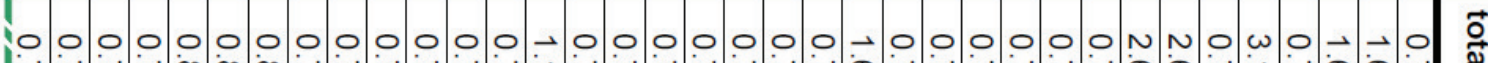

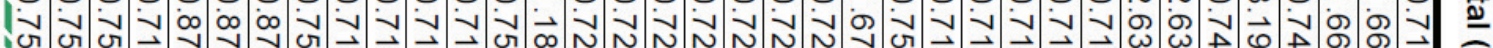

付 


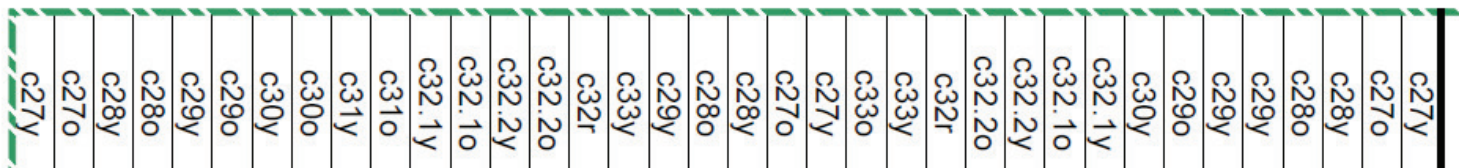

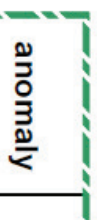

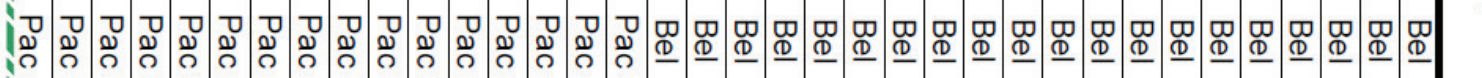

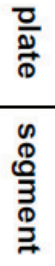

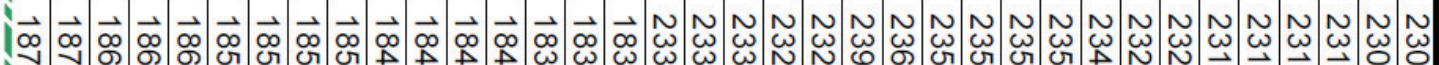

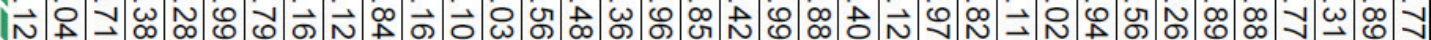

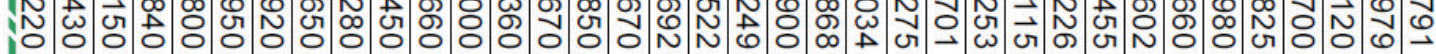

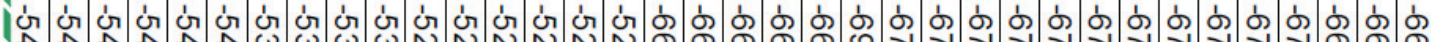

il

N $\begin{gathered}\vec{N} \\ \omega\end{gathered}$

$\frac{\overline{\mathrm{g}}}{\mathrm{\vec {E }}}$

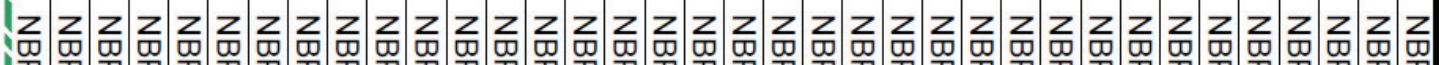

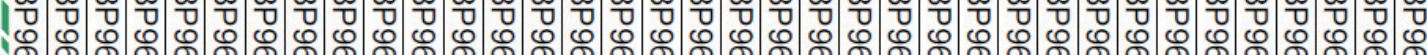

:

章.

íN

;

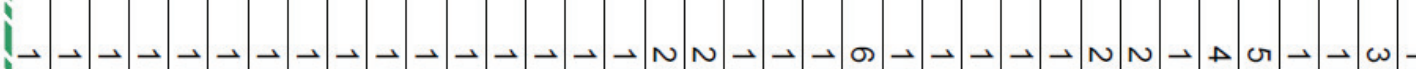

!

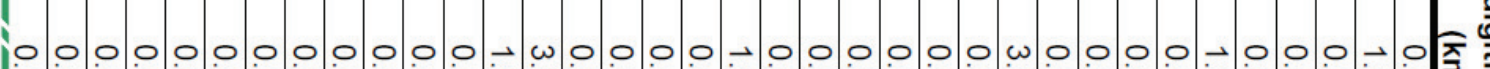

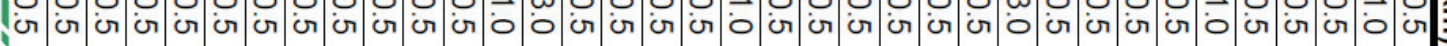

I

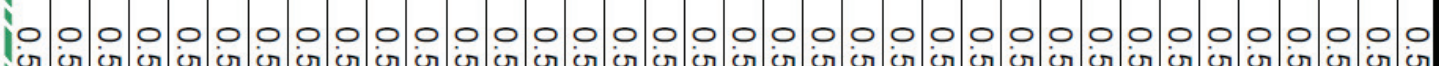

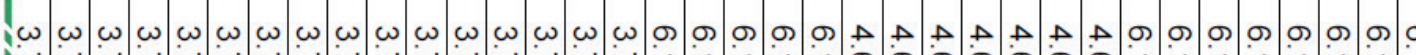

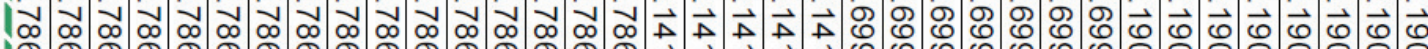

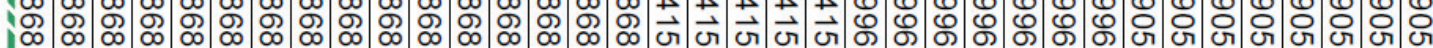

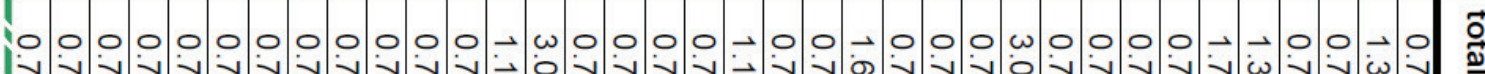

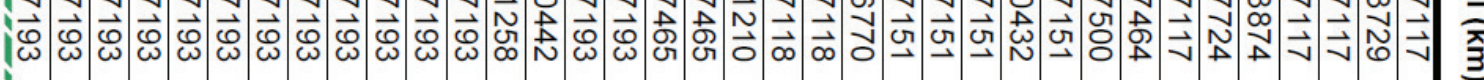




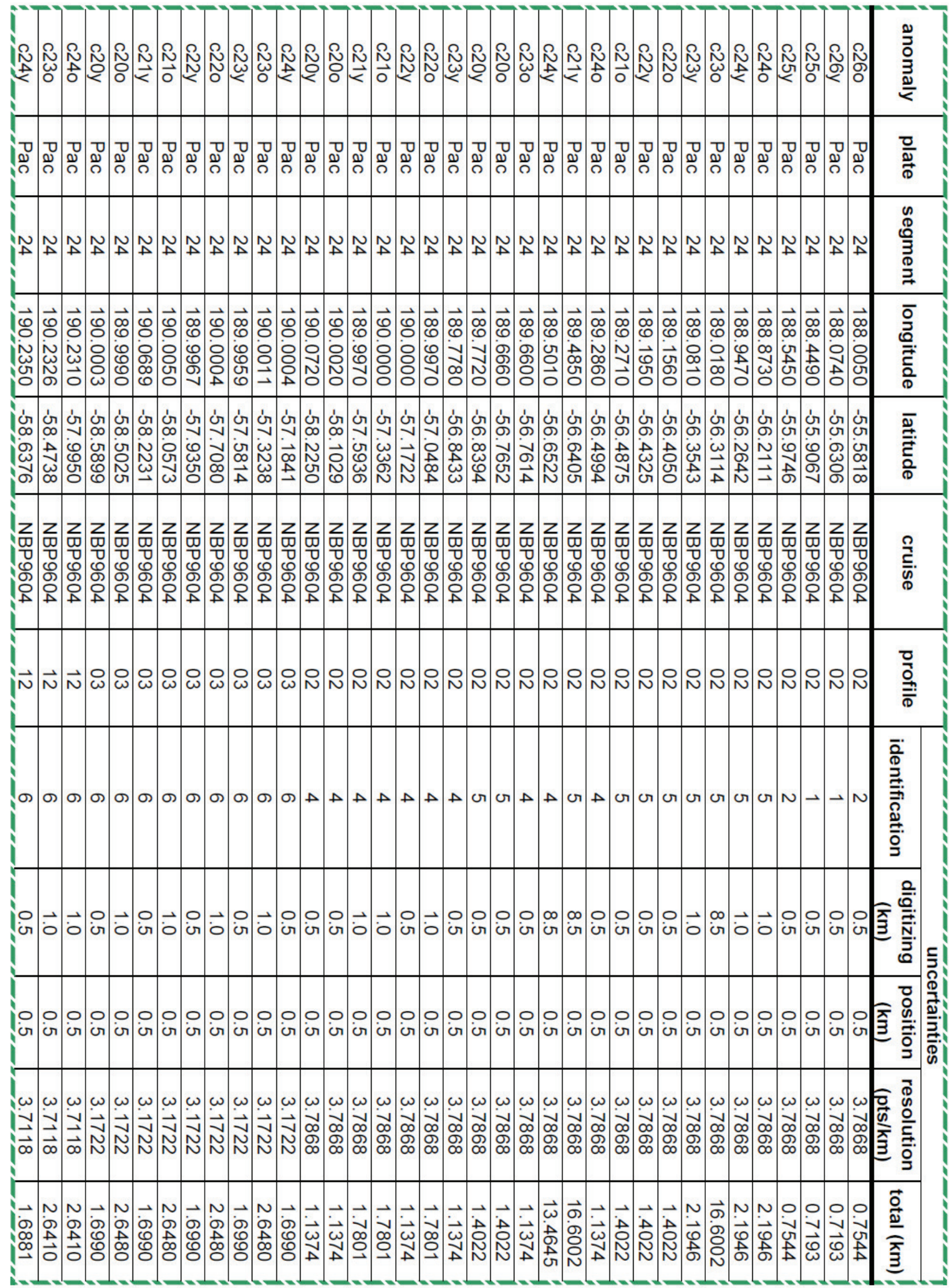




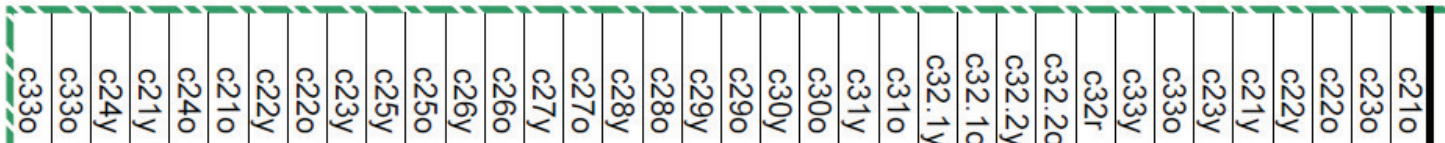

గొ๊

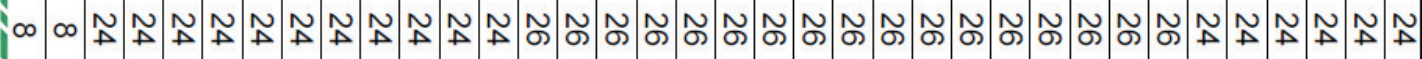

三

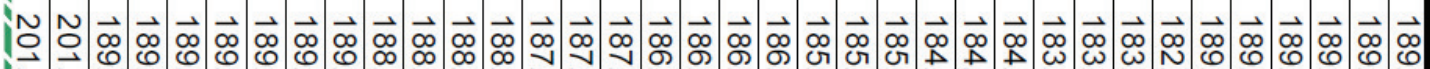

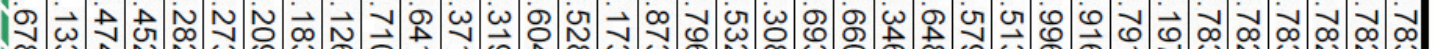

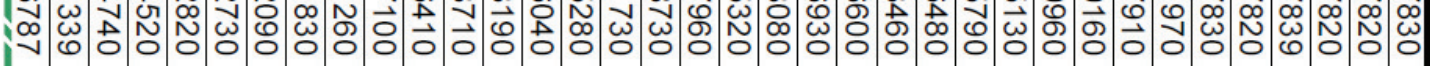
Â

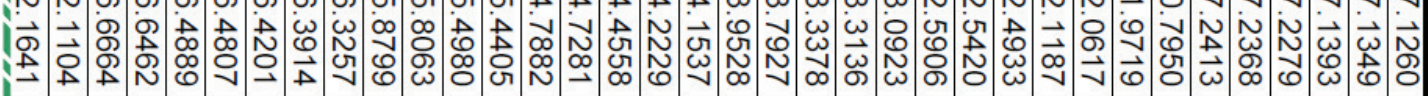

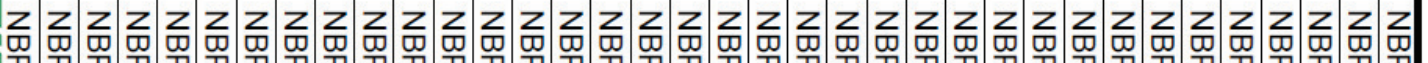
0 .

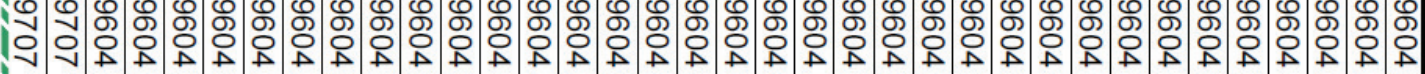

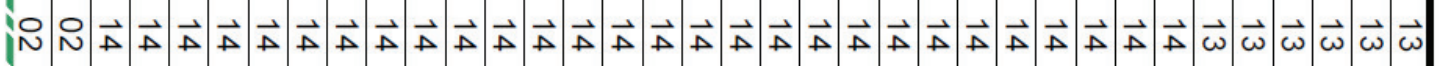

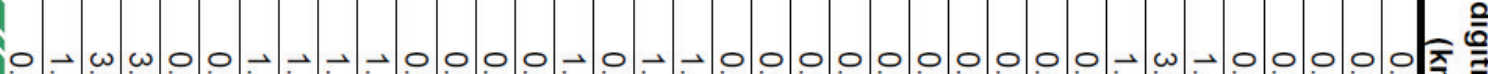
앙 $\overrightarrow{0} 0$ o

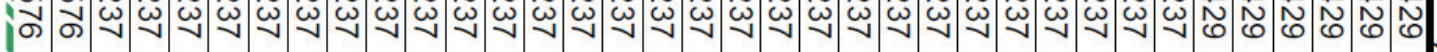




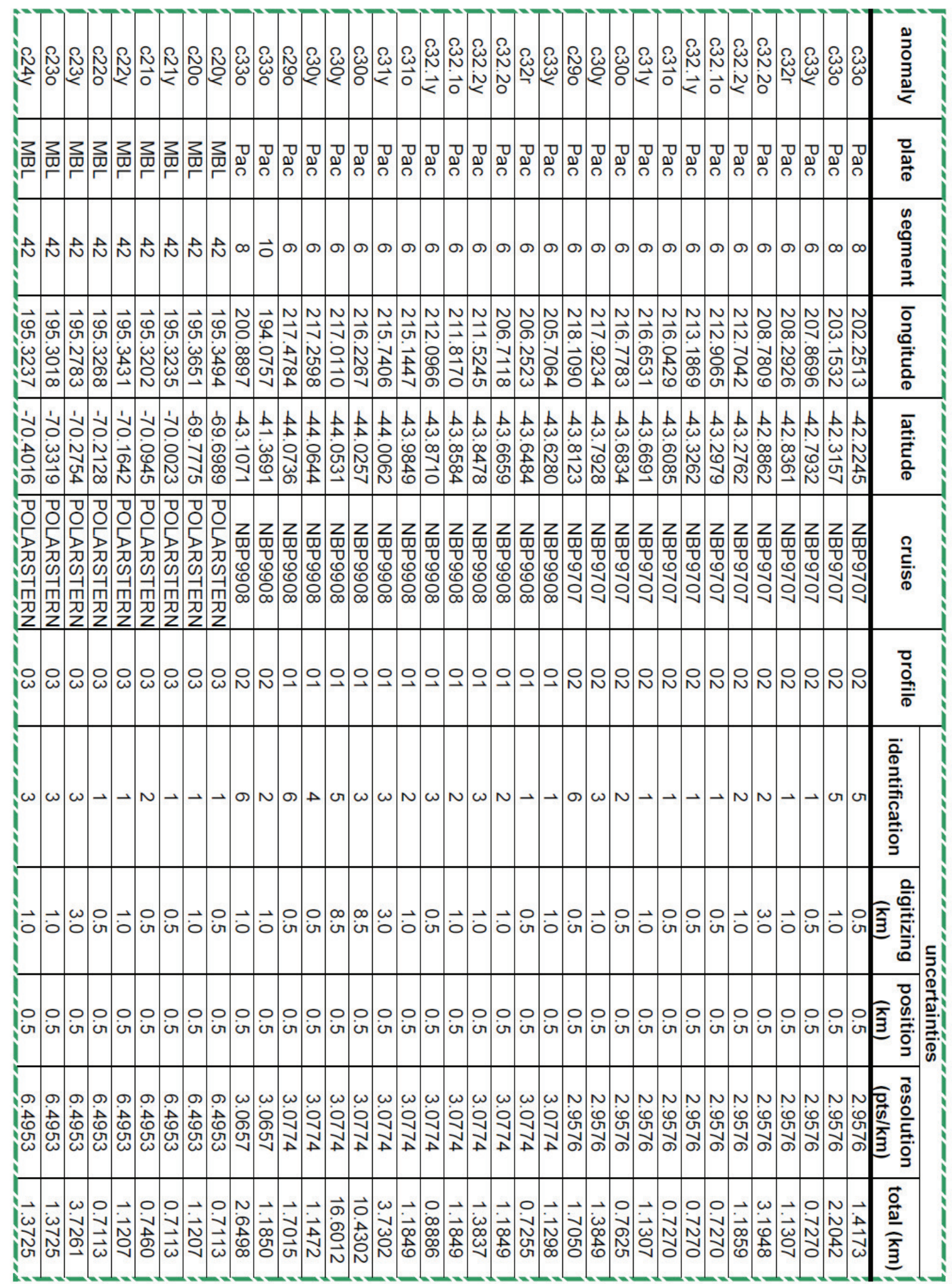




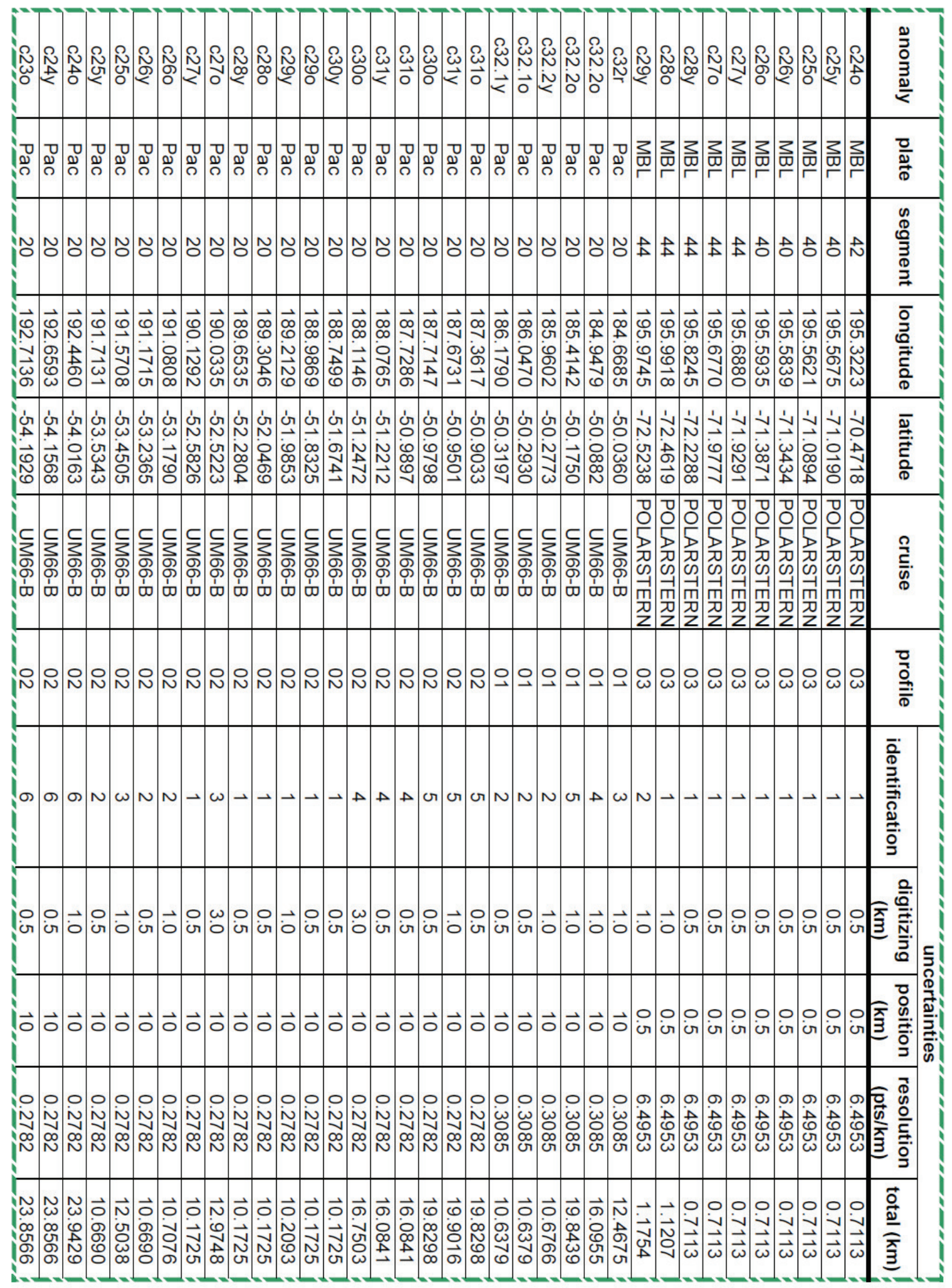




\begin{tabular}{|c|c|c|c|c|c|c|c|c|c|c|c|c|c|c|c|c|c|c|c|c|c|c|c|c|c|}
\hline 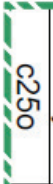 & 㣽 & & 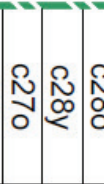 & 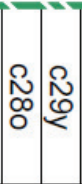 & $\mid \begin{array}{l}\mathfrak{W} \\
\varnothing\end{array}$ & 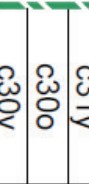 & 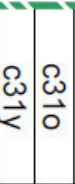 & . & & స్ట్ర & $\hat{\mathscr{E}}$ & $\stackrel{\mathscr{E}}{\gtrless} \mid \stackrel{\varrho}{\varnothing}$ & $\stackrel{\stackrel{N}{N}}{\gtrless}$ & $\mid \begin{array}{c}\mathcal{E} \\
N \\
\overrightarrow{0}\end{array}$ & & 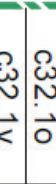 & & & 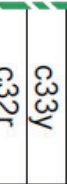 & & & 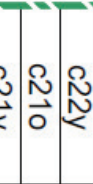 & 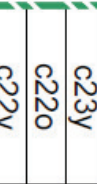 & $\begin{array}{l}\stackrel{0}{0} \\
\stackrel{0}{3} \\
\stackrel{0}{*}\end{array}$ & \\
\hline & Љ & ڤె & ڤె & 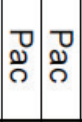 & ڤొ & ڤొ & $\begin{array}{lll}0 \\
\end{array}$ & ڤ & & 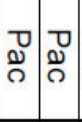 & ڤై & ڤై & ڤె & ڤొ & & $\begin{array}{lll}0 \\
0 \\
\end{array}$ & & & & ڤ̊ & है & $\begin{array}{lll}0 \\
\end{array}$ & 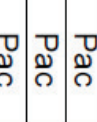 & $\frac{\overline{\frac{D}{2}}}{\frac{\overrightarrow{0}}{0}}$ & \\
\hline & $\infty \infty^{\infty}$ & & \begin{tabular}{l|l|l|l|l|l|l|l|l|l|l|l|} 
& 0
\end{tabular} & $\infty$ & $\infty \infty$ & 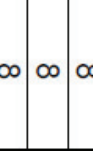 & $\infty \mid \infty$ & $\infty$ & $\infty$ & の & & の) 0 & $\$ 0$ & () & の & 0 & & (ब) & & $\infty$ & $\infty$ & $\mathcal{D} N \tilde{O}$ & $\widetilde{O} \mid \sim N$ & 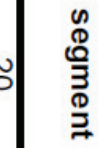 & \\
\hline & 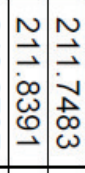 & \begin{tabular}{|l}
$N$ \\
$\overrightarrow{0}$ \\
0 \\
0 \\
0 \\
$\omega$ \\
$\omega$
\end{tabular} & 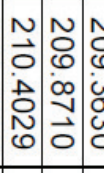 & 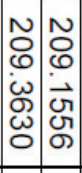 & 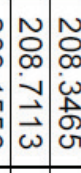 & 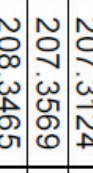 & 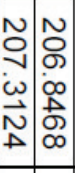 & \begin{tabular}{|l|}
$\overrightarrow{0}$ \\
$\vdots$ \\
$\vec{N}$ \\
$\vec{\omega}$ \\
$\omega$ \\
\end{tabular} & & 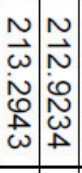 & 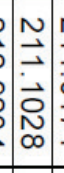 & 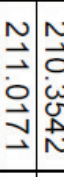 & & $\begin{array}{l}N \\
0 \\
-1 \\
6 \\
\mathcal{N} \\
0\end{array}$ & & 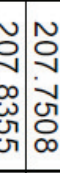 & & 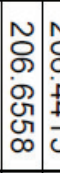 & & $\mid \begin{array}{l}n \\
0 \\
n \\
0 \\
0 \\
0 \\
0 \\
0 \\
0\end{array}$ & 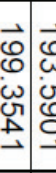 & 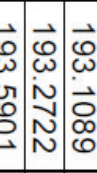 & 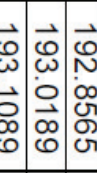 & $\begin{array}{l}\overline{0} \\
\frac{0}{\vec{*}} \\
\frac{0}{0}\end{array}$ & \\
\hline & 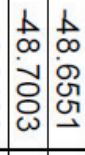 & 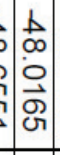 & 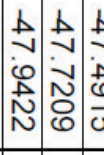 & 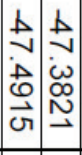 & 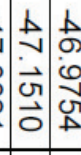 & 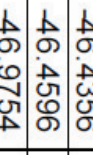 & 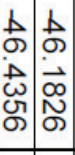 & $\vec{b}$ & & 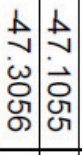 & $\begin{array}{l}b \\
0 \\
\vec{b} \\
0 \\
0 \\
\vdots\end{array}$ & 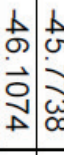 & & 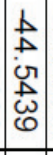 & & 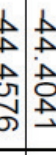 & & 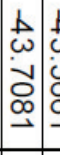 & 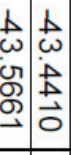 & 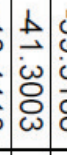 & 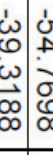 & 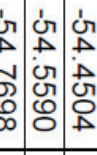 & 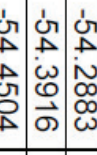 & $\begin{array}{l}\overline{9} \\
\overline{\vec{E}} \\
\frac{0}{0}\end{array}$ & \\
\hline & 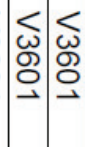 & 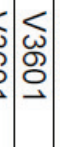 & 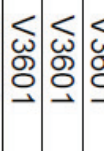 & & 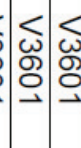 & 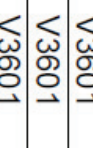 & 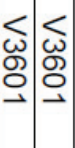 & 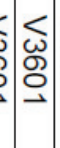 & 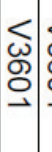 & 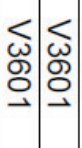 & & 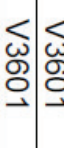 & & \begin{tabular}{l}
$\vec{\omega}$ \\
0 \\
0 \\
\hdashline
\end{tabular} & 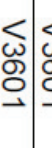 & 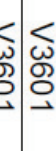 & & 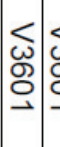 & 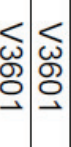 & $\begin{array}{l}\mid \\
0 \\
0 \\
\vdots\end{array}$ & & 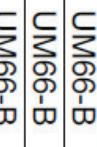 & 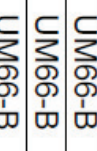 & 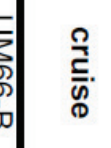 & \\
\hline & N N & N & NOR & & N & సิ & ని & N & N. & $\stackrel{\circ}{\circ}$ 으 & & $\stackrel{\circ}{\circ}$ & $\stackrel{\circ}{\rightarrow}$ & 오. & 이 & & & 오엉 & $\stackrel{0}{\circ} 0$ & 오 & & & Q & 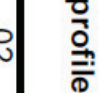 & \\
\hline & & & & & & & & ब & ब & の $\rightarrow$ & & & & $G$ & & $A$ & & $\rightarrow-$ & & $N$ & ब $\sigma$ & $\circlearrowleft$ の & の & 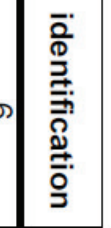 & \\
\hline & 이잉 & & 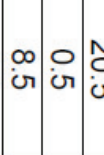 & $\begin{array}{ll}N & 0 \\
\text { ov } & \text { or }\end{array}$ & 이이 & $\begin{array}{llll}0 & 0 \\
\text { vi vi }\end{array}$ & $\begin{array}{lll}\circ & 0 \\
\text { iv } & 0 \\
\end{array}$ & ن & & $\begin{array}{lll}0 & 0 \\
\text { iv } & \text { or }\end{array}$ & $\overrightarrow{0}$ & $\overrightarrow{0}$ o & & Or & & & & 일 & & & 은 & $\vec{b}$ bi & $\begin{array}{c}0 \\
ن\end{array}$ & r. & \\
\hline & & & & & $\vec{v} \overrightarrow{\mathrm{v}}$ & & & & & $\vec{v} \vec{v}$ & & $\vec{v} \mid \vec{v}$ & & $\vec{v}$ & & & & $\vec{v} \vec{c}$ & & $\overrightarrow{\mathrm{v}} \overrightarrow{\mathrm{c}}$ & & $\overrightarrow{0} \overrightarrow{0} \overrightarrow{0}$ & $\overrightarrow{0} \overrightarrow{0}$ & 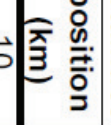 & 离 \\
\hline & $\begin{array}{lll}0 & 0 \\
0 & 0 \\
0 & 0 \\
0 & 0 \\
0 & 0 \\
0 & 0\end{array}$ & 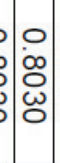 & 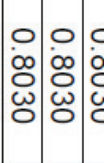 & 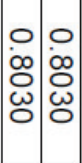 & 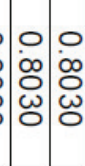 & 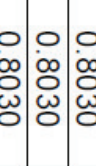 & 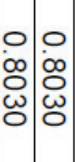 & : & & \begin{tabular}{l|l}
0 & 0 \\
on & 0 \\
0 & 0 \\
0 & 0 \\
& 0 \\
$N$
\end{tabular} & 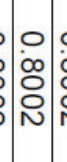 & 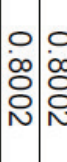 & & $\begin{array}{l}0 \\
0 \\
0 \\
0 \\
N\end{array}$ & & $\begin{array}{lll}0 & 0 \\
0 \\
0\end{array}$ & & 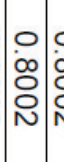 & 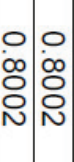 & 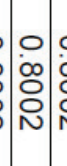 & 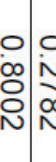 & 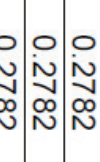 & 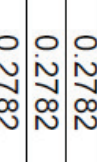 & 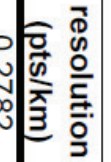 & \\
\hline & \begin{tabular}{|l|l}
$\vec{v}$ & $\vec{v}$ \\
$\dot{2}$ & 0 \\
$\vec{N}$ & $\vec{N}$ \\
\end{tabular} & 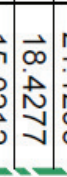 & 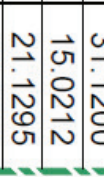 & \begin{tabular}{|l|l}
$\omega$ & $\vec{\omega}$ \\
$\overrightarrow{\vec{N}}$ & 0 \\
$\tilde{N}$ & $\vec{N}$ \\
0 & $\vec{N}$ \\
\end{tabular} & $\begin{array}{ll}\vec{u} & \vec{u} \\
\dot{D} & \vec{N} \\
N & \vec{N} \\
\vec{N} & \vec{N}\end{array}$ & 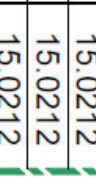 & 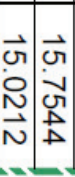 & 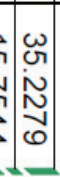 & 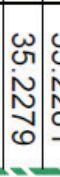 & 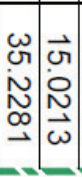 & 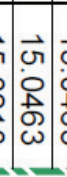 & 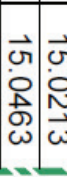 & 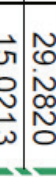 & 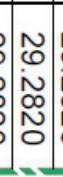 & 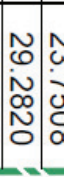 & 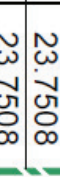 & 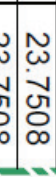 & 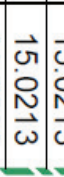 & 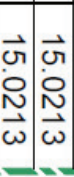 & 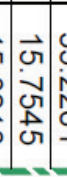 & 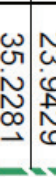 & 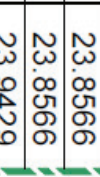 & 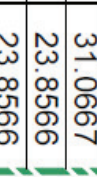 & 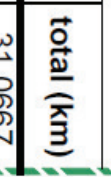 & \\
\hline
\end{tabular}


i

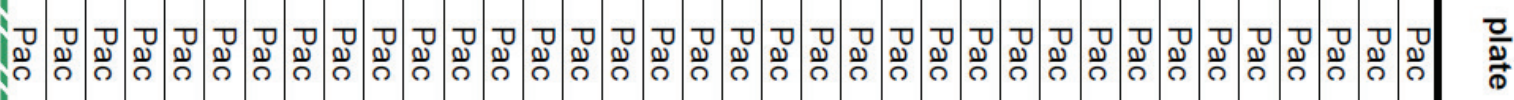

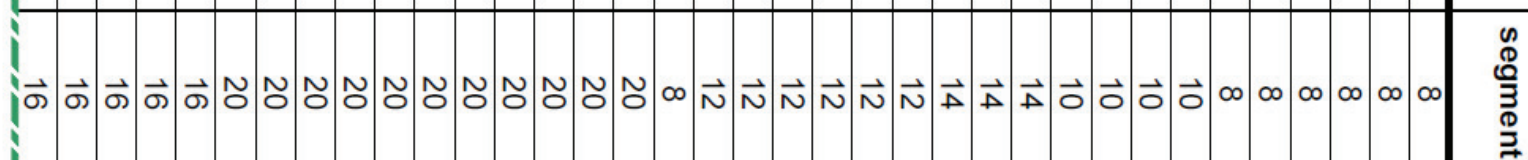

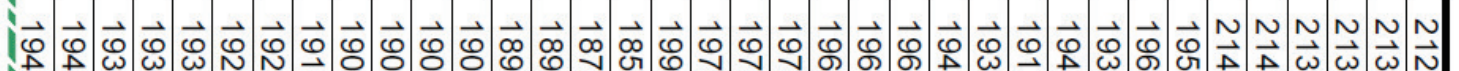
I

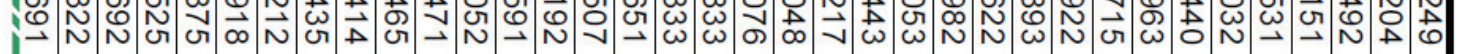

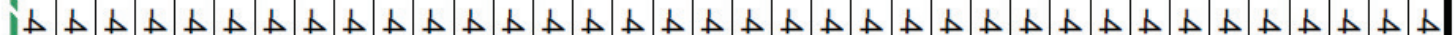

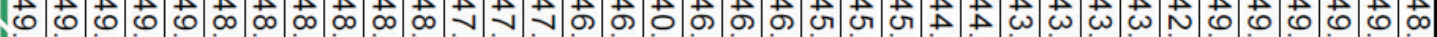

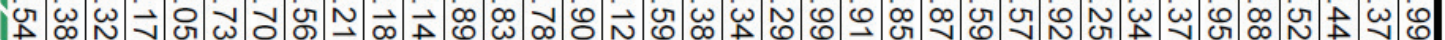

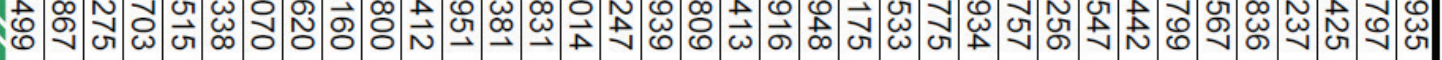

$\sum_{\mathrm{m}} \sum_{\mathrm{m}} \sum_{\mathrm{m}} \sum_{\mathrm{m}} \sum_{\mathrm{m}} \sum_{\mathrm{m}} \sum_{\mathrm{m}} \sum_{\mathrm{m}} \sum_{\mathrm{m}} \sum_{\mathrm{m}} \sum_{\mathrm{m}} \sum_{\mathrm{m}} \sum_{\mathrm{m}} \sum_{\mathrm{m}} \sum_{\mathrm{m}} \sum_{\mathrm{m}}$

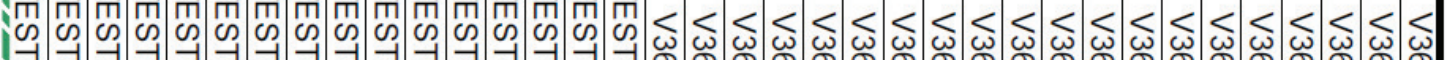

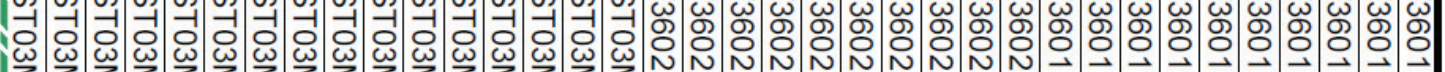
২)

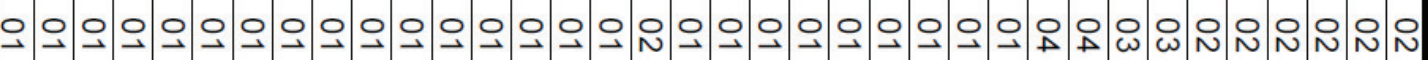

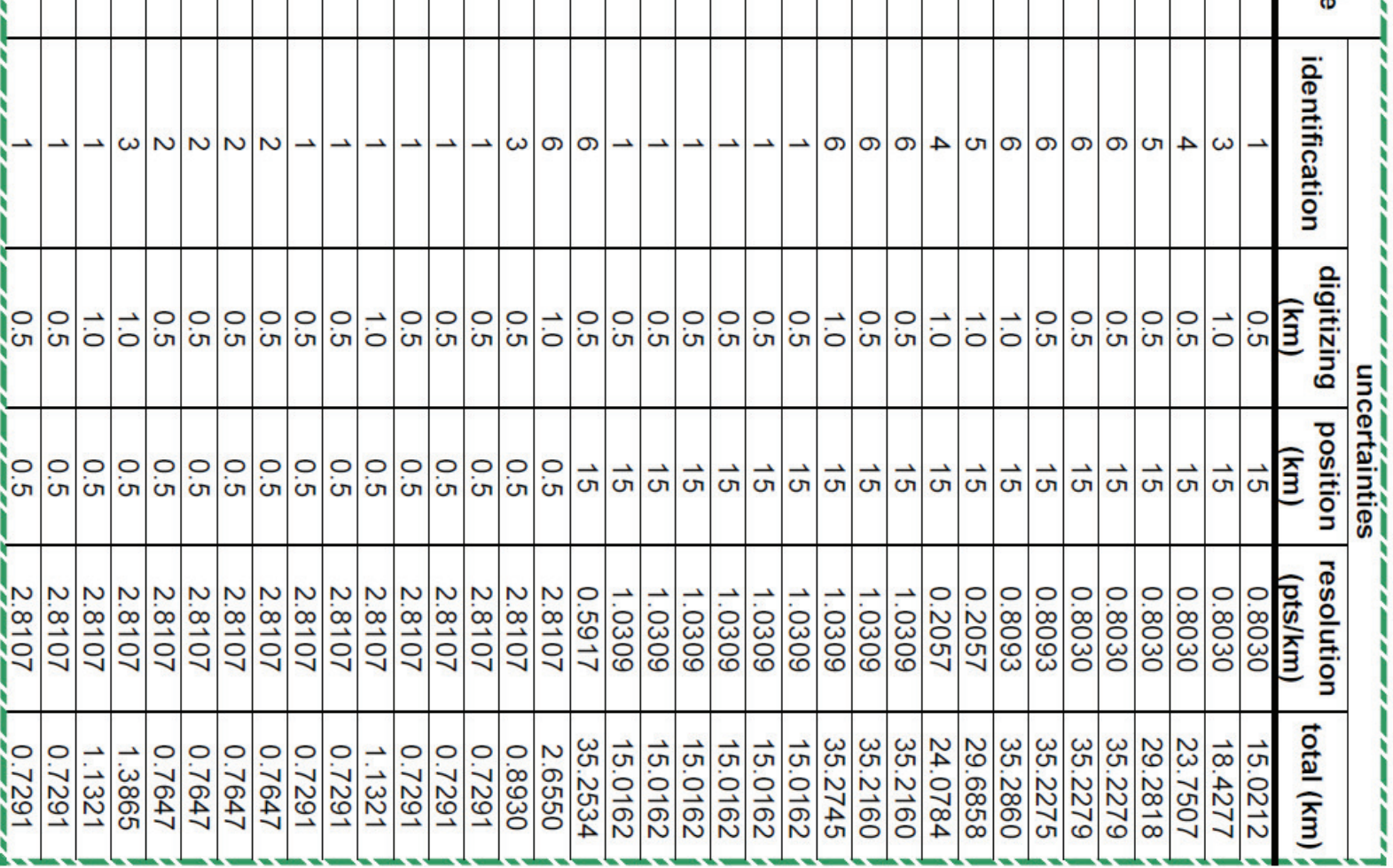




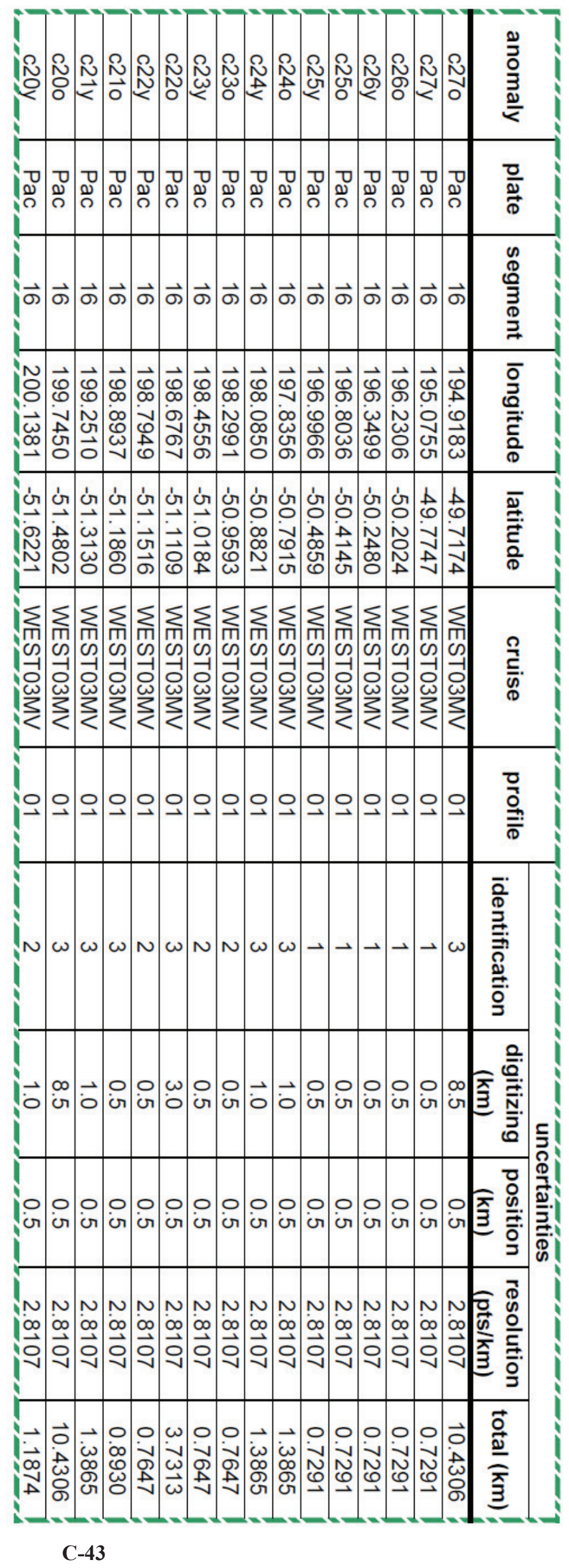


Fracture zone data 


\begin{tabular}{|c|c|c|c|c|c|}
\hline age (chron) & plate & segment & longitude & latitude & uncertainty $(\mathrm{km})$ \\
\hline c22o & $\mathrm{Bel}$ & 7 & -98.5907 & -60.7835 & 1.5 \\
\hline$c 220-c 240$ & $\mathrm{Bel}$ & 7 & -98.1565 & -60.9322 & 1.5 \\
\hline$c 230-c 24 y$ & Bel & 7 & -98.5907 & -60.7835 & 1.5 \\
\hline$c 23 y-c 24 o$ & Bel & 7 & -97.7054 & -61.0612 & 1.5 \\
\hline c24o-c26o & Bel & 7 & -97.3032 & -61.1956 & 1.5 \\
\hline$c 26 y-c 26 o$ & Bel & 7 & -96.7238 & -61.3467 & 1.5 \\
\hline$c 27 y-c 29 o$ & Bel & 7 & -95.7042 & -61.5657 & 1.5 \\
\hline$c 27 y-c 290$ & Bel & 7 & -95.3402 & -61.6751 & 1.5 \\
\hline$c 27 y-c 29 o$ & Bel & 7 & -94.6442 & -61.8690 & 1.5 \\
\hline$c 27 y-c 30 y$ & Bel & 7 & -96.2004 & -61.4550 & 1.5 \\
\hline $\mathrm{c} 280-\mathrm{c} 290$ & Bel & 7 & -93.6960 & -62.1700 & 1.5 \\
\hline c28y & Bel & 7 & -98.9655 & -60.6467 & 1.5 \\
\hline c28y & Bel & 7 & -98.5907 & -60.7835 & 1.5 \\
\hline$c 28 y-c 280$ & Bel & 7 & -98.1565 & -60.9322 & 1.5 \\
\hline$c 28 y-c 290$ & Bel & 7 & -97.3032 & -61.1956 & 1.5 \\
\hline$c 28 y-c 29 o$ & Bel & 7 & -94.1112 & -62.0332 & 1.5 \\
\hline$c 28 y-c 29 y$ & Bel & 7 & -97.7054 & -61.0612 & 1.5 \\
\hline$c 28 y-c 30 y$ & Bel & 7 & -96.7238 & -61.3467 & 1.5 \\
\hline$c 29 y-c 29 o$ & Bel & 7 & -93.1761 & -62.3402 & 1.5 \\
\hline c30o & Bel & 7 & -95.3402 & -61.6751 & 1.5 \\
\hline c30o-c31y & Bel & 7 & -94.6442 & -61.8690 & 1.5 \\
\hline c30o-c31y & Bel & 7 & -94.1112 & -62.0332 & 1.5 \\
\hline c310 & Bel & 7 & -93.6960 & -62.1700 & 1.5 \\
\hline c310 & $\mathrm{Bel}$ & 7 & -93.1761 & -62.3402 & 1.5 \\
\hline c310 & Bel & 7 & -92.5265 & -62.5436 & 1.5 \\
\hline$c 20 \circ$ & Pac & 7 & -140.0493 & -51.5845 & 1.5 \\
\hline c20y & $\mathrm{Pac}$ & 7 & -141.5186 & -51.0135 & 1.5 \\
\hline $\mathrm{c} 20 \mathrm{y}-\mathrm{c} 20 \mathrm{o}$ & $\mathrm{Pac}$ & 7 & -140.3705 & -51.4477 & 1.5 \\
\hline$c 20 y-c 220$ & $\mathrm{Pac}$ & 7 & -140.9951 & -51.1919 & 1.5 \\
\hline $\mathrm{c} 20 \mathrm{y}-\mathrm{c} 220$ & $\mathrm{Pac}$ & 7 & -140.7334 & -51.2871 & 1.5 \\
\hline$c 20 y-c 23 y$ & $\mathrm{Pac}$ & 7 & -141.2390 & -51.1086 & 1.5 \\
\hline$c 210$ & Pac & 7 & -141.7625 & -50.9183 & 1.5 \\
\hline c210 & Pac & 7 & -141.5186 & -51.0135 & 1.5 \\
\hline $\mathrm{c} 230-\mathrm{c} 240$ & Pac & 7 & -142.3811 & -50.6565 & 1.5 \\
\hline c23o-c24o & Pac & 7 & -142.0956 & -50.7517 & 1.5 \\
\hline c23y & Pac & 7 & -141.5186 & -51.0135 & 1.5 \\
\hline$c 23 y-c 24 y$ & Pac & 7 & -141.7625 & -50.9183 & 1.5 \\
\hline c24o-c27o & Pac & 7 & -145.2900 & -49.4252 & 1.5 \\
\hline c24o-c28o & Pac & 7 & -146.4917 & -48.9493 & 1.5 \\
\hline c24o-c28y & Pac & 7 & -146.0931 & -49.0682 & 1.5 \\
\hline$c 240-c 28 y$ & Pac & 7 & -145.7600 & -49.2051 & 1.5 \\
\hline$c 24 y-c 25 o$ & Pac & 7 & -143.5649 & -50.2937 & 1.5 \\
\hline$c 24 y-c 25 \circ$ & Pac & 7 & -143.1783 & -50.4007 & 1.5 \\
\hline$c 24 y-c 25 y$ & Pac & 7 & -142.7856 & -50.5316 & 1.5 \\
\hline$c 24 y-c 26 o$ & $\mathrm{Pac}$ & 7 & -144.8373 & -49.6738 & 1.5 \\
\hline$c 24 y-c 26 o$ & $\mathrm{Pac}$ & 7 & -144.4536 & -49.8820 & 1.5 \\
\hline$c 24 y-c 26 o$ & Pac & 7 & -144.0527 & -50.0884 & 1.5 \\
\hline c26o-c29o & Pac & 7 & -147.7052 & -48.3782 & 1.5 \\
\hline$c 26 y-c 290$ & Pac & 7 & -147.4494 & -48.5031 & 1.5 \\
\hline c27o-c30y & Pac & 7 & -149.1001 & -47.5573 & 1.5 \\
\hline$c 27 y-c 29 o$ & Pac & 7 & -148.4726 & -47.9321 & 1.5 \\
\hline$c 27 y-c 30 y$ & Pac & 7 & -148.8265 & -47.6941 & 1.5 \\
\hline c28o-c30y & Pac & 7 & -149.9151 & -47.1290 & 1.5 \\
\hline$c 28 y-c 30 y$ & Pac & 7 & -149.6177 & -47.2837 & 1.5 \\
\hline$c 28 y-c 30 y$ & Pac & 7 & -149.3321 & -47.4383 & 1.5 \\
\hline$c 290-c 30 y$ & Pac & 7 & -150.2066 & -46.9565 & 1.5 \\
\hline c29o-c31y & Pac & 7 & -150.4564 & -46.8078 & 1.5 \\
\hline c30o-c31o & Pac & 7 & -151.4261 & -46.1594 & 1.5 \\
\hline
\end{tabular}




\begin{tabular}{|c|c|c|c|c|c|}
\hline age (chron) & plate & segment & longitude & latitude & uncertainty $(\mathrm{km})$ \\
\hline $\mathrm{c} 300-\mathrm{c} 310$ & $\mathrm{Pac}$ & 7 & -151.2179 & -46.3140 & 1.5 \\
\hline $\mathrm{c} 300-\mathrm{c} 310$ & $\mathrm{Pac}$ & 7 & -150.9561 & -46.5044 & 1.5 \\
\hline$c 300-c 310$ & $\mathrm{Pac}$ & 7 & -150.7360 & -46.6352 & 1.5 \\
\hline$c 220-c 230$ & $\mathrm{Bel}$ & 9 & -112.6414 & -58.5605 & 1.5 \\
\hline$c 220-c 23 y$ & Bel & 9 & -112.8716 & -58.4570 & 1.5 \\
\hline$c 220-c 24 y$ & Bel & 9 & -112.3380 & -58.6557 & 1.5 \\
\hline c23o-c24y & Bel & 9 & -112.0643 & -58.7568 & 1.5 \\
\hline$c 240$ & $\mathrm{Bel}$ & 9 & -111.4814 & -58.9056 & 1.5 \\
\hline$c 240-c 25 y$ & Bel & 9 & -111.2494 & -58.9710 & 1.5 \\
\hline$c 24 y-c 240$ & Bel & 9 & -111.8205 & -58.8104 & 1.5 \\
\hline$c 25 y-c 250$ & Bel & 9 & -110.9579 & -59.0364 & 1.5 \\
\hline$c 25 y-c 25 o$ & $\mathrm{Bel}$ & 9 & -110.6455 & -59.1305 & 1.5 \\
\hline$c 25 y-c 250$ & Bel & 9 & -110.4169 & -59.1975 & 1.5 \\
\hline c26o & Bel & 9 & -109.6331 & -59.4324 & 1.5 \\
\hline c26y & $\mathrm{Bel}$ & 9 & -110.1540 & -59.2579 & 1.5 \\
\hline$c 26 y-c 26 o$ & Bel & 9 & -109.9623 & -59.3257 & 1.5 \\
\hline$c 26 y-c 26 o$ & Bel & 9 & -109.7767 & -59.3876 & 1.5 \\
\hline $\mathrm{c} 280-c 290$ & Bel & 9 & -100.7477 & -62.1415 & 1.5 \\
\hline c28o-c29o & Bel & 9 & -100.3194 & -62.2772 & 1.5 \\
\hline$c 280-c 29 y$ & Bel & 9 & -101.1403 & -61.9916 & 1.5 \\
\hline c28y & Bel & 9 & -101.9398 & -61.7132 & 1.5 \\
\hline c28y & $\mathrm{Bel}$ & 9 & -101.5258 & -61.8631 & 1.5 \\
\hline c29o & Bel & 9 & -99.8554 & -62.4342 & 1.5 \\
\hline$c 30 \circ$ & Bel & 9 & -100.7477 & -62.1415 & 1.5 \\
\hline$c 30 \circ$ & Bel & 9 & -100.3194 & -62.2772 & 1.5 \\
\hline $\mathrm{c} 300-c 310$ & Bel & 9 & -103.3883 & -61.2543 & 1.5 \\
\hline$c 300-c 310$ & Bel & 9 & -102.9005 & -61.3911 & 1.5 \\
\hline$c 300-c 310$ & Bel & 9 & -102.6328 & -61.4803 & 1.5 \\
\hline c30o-c31o & $\mathrm{Bel}$ & 9 & -102.2825 & -61.6133 & 1.5 \\
\hline$c 300-c 310$ & Bel & 9 & -101.9398 & -61.7132 & 1.5 \\
\hline$c 300-c 310$ & Bel & 9 & -101.5258 & -61.8631 & 1.5 \\
\hline c30o-c31o & $\mathrm{Bel}$ & 9 & -99.8554 & -62.4342 & 1.5 \\
\hline $\mathrm{c} 300-\mathrm{c} 310$ & Bel & 9 & -98.2493 & -62.8697 & 1.5 \\
\hline$c 300-c 310$ & Bel & 9 & -97.7210 & -63.0624 & 1.5 \\
\hline c30o-c31o & Bel & 9 & -97.3498 & -63.1766 & 1.5 \\
\hline c30o-c31y & $\mathrm{Bel}$ & 9 & -103.8939 & -61.1174 & 1.5 \\
\hline$c 30 y-c 310$ & Bel & 9 & -99.2843 & -62.5056 & 1.5 \\
\hline$c 30 y-c 310$ & Bel & 9 & -98.7561 & -62.6912 & 1.5 \\
\hline c31o & $\mathrm{Bel}$ & 9 & -96.9073 & -63.3622 & 1.5 \\
\hline c310 & $\mathrm{Bel}$ & 9 & -96.6089 & -63.4593 & 1.5 \\
\hline c24o & Pac & 9 & -148.1714 & -49.8006 & 1.5 \\
\hline $\mathrm{c} 240$ & Pac & 9 & -147.6431 & -50.0505 & 1.5 \\
\hline $\mathrm{c} 240-\mathrm{c} 250$ & Pac & 9 & -148.5640 & -49.6364 & 1.5 \\
\hline$c 25 y-c 25 o$ & Pac & 9 & -148.8638 & -49.5294 & 1.5 \\
\hline$c 25 y-c 260$ & Pac & 9 & -149.1850 & -49.3866 & 1.5 \\
\hline$c 26 y-c 26 o$ & Pac & 9 & -149.9060 & -49.0939 & 1.5 \\
\hline$c 26 y-c 26 o$ & Pac & 9 & -149.5562 & -49.2153 & 1.5 \\
\hline c27y & Pac & 9 & -150.5085 & -48.8369 & 1.5 \\
\hline$c 27 y-c 27 o$ & Pac & 9 & -151.3651 & -48.3872 & 1.5 \\
\hline$c 27 y-c 27 o$ & $\mathrm{Pac}$ & 9 & -150.8654 & -48.6656 & 1.5 \\
\hline $\mathrm{c} 280-c 290$ & $\mathrm{Pac}$ & 9 & -152.3787 & -48.0589 & 1.5 \\
\hline c28o-c29y & Pac & 9 & -152.0575 & -48.2516 & 1.5 \\
\hline $\mathrm{c} 28 \mathrm{y}$ & Pac & 9 & -151.4293 & -48.5728 & 1.5 \\
\hline c28y & Pac & 9 & -151.2865 & -48.6513 & 1.5 \\
\hline$c 28 y-c 280$ & Pac & 9 & -151.7791 & -48.3872 & 1.5 \\
\hline c29o-c30y & $\mathrm{Pac}$ & 9 & -152.9905 & -47.7191 & 1.5 \\
\hline$c 29 y-c 29 o$ & $\mathrm{Pac}$ & 9 & -152.6907 & -47.8833 & 1.5 \\
\hline$c 300-c 310$ & Pac & 9 & -154.2591 & -46.8913 & 1.5 \\
\hline
\end{tabular}




\begin{tabular}{|c|c|c|c|c|c|}
\hline age (chron) & plate & segment & longitude & latitude & uncertainty $(\mathrm{km})$ \\
\hline $\mathrm{c} 300-\mathrm{c} 31 \mathrm{y}$ & $\mathrm{Pac}$ & 9 & -153.9664 & -47.0412 & 1.5 \\
\hline c30o-c31y & Pac & 9 & -153.6951 & -47.2125 & 1.5 \\
\hline $\mathrm{c} 30 \mathrm{y}$ & Pac & 9 & -153.3953 & -47.4124 & 1.5 \\
\hline c30y & Pac & 9 & -153.1954 & -47.5766 & 1.5 \\
\hline c310 & Pac & 9 & -154.6588 & -46.6486 & 1.5 \\
\hline$c 310$ & Pac & 9 & -154.4732 & -46.7557 & 1.5 \\
\hline c32.10 & Pac & 9 & -159.4060 & -43.9433 & 1.5 \\
\hline c32.10 & Pac & 9 & -158.9134 & -44.2503 & 1.5 \\
\hline c32.10 & Pac & 9 & -158.6422 & -44.3859 & 1.5 \\
\hline c32.10 & Pac & 9 & -158.2995 & -44.6215 & 1.5 \\
\hline c32.10 & Pac & 9 & -158.1066 & -44.7640 & 1.5 \\
\hline c32.10 & Pac & 9 & -157.9068 & -44.8783 & 1.5 \\
\hline c32.10 & Pac & 9 & -157.7283 & -44.9782 & 1.5 \\
\hline c32.10 & Pac & 9 & -157.4856 & -45.0924 & 1.5 \\
\hline c32.10 & Pac & 9 & -157.2500 & -45.2209 & 1.5 \\
\hline c32.10 & Pac & 9 & -157.0287 & -45.3565 & 1.5 \\
\hline $\mathrm{c} 32.10-\mathrm{c} 32.2 \mathrm{y}$ & Pac & 9 & -159.6804 & -43.7598 & 1.5 \\
\hline$c 32.1 y-c 32.10$ & Pac & 9 & -156.8003 & -45.4779 & 1.5 \\
\hline $\mathrm{c} 32.2 \mathrm{y}$ & Pac & 9 & -160.1841 & -43.3830 & 1.5 \\
\hline c32.2y & Pac & 9 & -159.9398 & -43.5738 & 1.5 \\
\hline$c 210$ & Bel & 15 & 240.0063 & -62.0901 & 1.5 \\
\hline c210 & Bel & 15 & 240.0501 & -62.1096 & 1.5 \\
\hline c21o & $\mathrm{Bel}$ & 15 & 240.0964 & -62.1266 & 1.5 \\
\hline c210 & $\mathrm{Bel}$ & 15 & 240.1572 & -62.1534 & 1.5 \\
\hline c21y & $\mathrm{Bel}$ & 15 & 239.5415 & -61.9149 & 1.5 \\
\hline c21y & Bel & 15 & 239.5731 & -61.9270 & 1.5 \\
\hline c21y & Bel & 15 & 239.6120 & -61.9416 & 1.5 \\
\hline c21y & $\mathrm{Bel}$ & 15 & 239.6461 & -61.9635 & 1.5 \\
\hline c21y & $\mathrm{Bel}$ & 15 & 239.6899 & -61.9830 & 1.5 \\
\hline c21y & $\mathrm{Bel}$ & 15 & 239.7532 & -62.0122 & 1.5 \\
\hline c22o & $\mathrm{Bel}$ & 15 & 240.7462 & -62.2897 & 1.5 \\
\hline$c 220$ & $\mathrm{Bel}$ & 15 & 240.8120 & -62.3189 & 1.5 \\
\hline$c 22 y$ & $\mathrm{Bel}$ & 15 & 240.5759 & -62.2361 & 1.5 \\
\hline$c 22 y-c 220$ & $\mathrm{Bel}$ & 15 & 240.6391 & -62.2556 & 1.5 \\
\hline$c 22 y-c 220$ & $\mathrm{Bel}$ & 15 & 240.6976 & -62.2702 & 1.5 \\
\hline $\mathrm{c} 230$ & $\mathrm{Bel}$ & 15 & 241.1405 & -62.5014 & 1.5 \\
\hline c23o & Bel & 15 & 241.1916 & -62.5233 & 1.5 \\
\hline c23o & Bel & 15 & 241.2501 & -62.5574 & 1.5 \\
\hline$c 230$ & Bel & 15 & 241.3206 & -62.5939 & 1.5 \\
\hline c23y & $\mathrm{Bel}$ & 15 & 240.9142 & -62.3870 & 1.5 \\
\hline c23y & Bel & 15 & 240.9556 & -62.4162 & 1.5 \\
\hline$c 23 y$ & Bel & 15 & 241.0140 & -62.4479 & 1.5 \\
\hline c24o & $\mathrm{Bel}$ & 15 & 241.8147 & -62.8555 & 1.5 \\
\hline c24o & Bel & 15 & 241.8512 & -62.8775 & 1.5 \\
\hline c24o & Bel & 15 & 241.8902 & -62.8896 & 1.5 \\
\hline c24y & Bel & 15 & 241.3961 & -62.6475 & 1.5 \\
\hline c24y & $\mathrm{Bel}$ & 15 & 241.4569 & -62.6864 & 1.5 \\
\hline c24y & Bel & 15 & 241.5153 & -62.7217 & 1.5 \\
\hline$c 25 y$ & Bel & 15 & 242.9002 & -63.2839 & 1.5 \\
\hline c25y & Bel & 15 & 242.9611 & -63.3010 & 1.5 \\
\hline c25y & $\mathrm{Bel}$ & 15 & 243.0122 & -63.3326 & 1.5 \\
\hline c25y & Bel & 15 & 243.0779 & -63.3618 & 1.5 \\
\hline c25y & Bel & 15 & 243.1412 & -63.3764 & 1.5 \\
\hline $\mathrm{c} 25 \mathrm{y}$ & Bel & 15 & 243.1923 & -63.3788 & 1.5 \\
\hline c25y & Bel & 15 & 243.2410 & -63.3886 & 1.5 \\
\hline$c 25 y$ & Bel & 15 & 243.2848 & -63.3983 & 1.5 \\
\hline c25y & $\mathrm{Bel}$ & 15 & 243.3408 & -63.4129 & 1.5 \\
\hline$c 25 y$ & Bel & 15 & 243.4089 & -63.4567 & 1.5 \\
\hline
\end{tabular}




\begin{tabular}{|c|c|c|c|c|c|}
\hline age (chron) & plate & segment & longitude & latitude & uncertainty $(\mathrm{km})$ \\
\hline $\mathrm{c} 260-\mathrm{c} 30 \mathrm{y}$ & $\mathrm{Bel}$ & 15 & 243.7910 & -63.6028 & 1.5 \\
\hline$c 260-c 30 y$ & $\mathrm{Bel}$ & 15 & 243.8373 & -63.6174 & 1.5 \\
\hline$c 260-c 30 y$ & $\mathrm{Bel}$ & 15 & 243.8835 & -63.6295 & 1.5 \\
\hline c26y & Bel & 15 & 243.5306 & -63.5176 & 1.5 \\
\hline$c 26 y$ & Bel & 15 & 243.5939 & -63.5516 & 1.5 \\
\hline c26y & $\mathrm{Bel}$ & 15 & 243.6474 & -63.5638 & 1.5 \\
\hline c26y & $\mathrm{Bel}$ & 15 & 243.6864 & -63.5808 & 1.5 \\
\hline c27y & Bel & 15 & 241.0140 & -62.4479 & 1.5 \\
\hline c27y & $\mathrm{Bel}$ & 15 & 241.0894 & -62.4844 & 1.5 \\
\hline$c 27 y-c 27 o$ & $\mathrm{Bel}$ & 15 & 241.1405 & -62.5014 & 1.5 \\
\hline$c 27 y-c 27 o$ & Bel & 15 & 241.1916 & -62.5233 & 1.5 \\
\hline$c 27 y-c 280$ & Bel & 15 & 241.7198 & -62.8166 & 1.5 \\
\hline$c 27 y-c 280$ & Bel & 15 & 241.7685 & -62.8507 & 1.5 \\
\hline$c 27 y-c 280$ & Bel & 15 & 241.8147 & -62.8555 & 1.5 \\
\hline$c 27 y-c 280$ & Bel & 15 & 241.8512 & -62.8775 & 1.5 \\
\hline$c 27 y-c 280$ & $\mathrm{Bel}$ & 15 & 241.8902 & -62.8896 & 1.5 \\
\hline$c 27 y-c 280$ & Bel & 15 & 241.9340 & -62.9018 & 1.5 \\
\hline$c 27 y-c 280$ & $\mathrm{Bel}$ & 15 & 241.9827 & -62.9213 & 1.5 \\
\hline$c 27 y-c 280$ & $\mathrm{Bel}$ & 15 & 242.0119 & -62.9383 & 1.5 \\
\hline$c 27 y-c 280$ & Bel & 15 & 242.0435 & -62.9651 & 1.5 \\
\hline$c 27 y-c 280$ & Bel & 15 & 242.0727 & -62.9821 & 1.5 \\
\hline$c 27 y-c 280$ & Bel & 15 & 242.0970 & -62.9967 & 1.5 \\
\hline$c 27 y-c 280$ & Bel & 15 & 242.1530 & -63.0089 & 1.5 \\
\hline$c 27 y-c 28 y$ & Bel & 15 & 241.2501 & -62.5574 & 1.5 \\
\hline$c 27 y-c 28 y$ & $\mathrm{Bel}$ & 15 & 241.3206 & -62.5939 & 1.5 \\
\hline$c 27 y-c 28 y$ & Bel & 15 & 241.3961 & -62.6475 & 1.5 \\
\hline$c 27 y-c 28 y$ & Bel & 15 & 241.4569 & -62.6864 & 1.5 \\
\hline$c 27 y-c 28 y$ & Bel & 15 & 241.5153 & -62.7217 & 1.5 \\
\hline$c 27 y-c 28 y$ & Bel & 15 & 241.5835 & -62.7558 & 1.5 \\
\hline$c 27 y-c 28 y$ & Bel & 15 & 241.6200 & -62.7728 & 1.5 \\
\hline$c 27 y-c 28 y$ & Bel & 15 & 241.6687 & -62.7898 & 1.5 \\
\hline$c 27 y-c 29 o$ & Bel & 15 & 242.5351 & -63.1574 & 1.5 \\
\hline$c 27 y-c 290$ & Bel & 15 & 242.5984 & -63.1793 & 1.5 \\
\hline$c 27 y-c 290$ & Bel & 15 & 242.6593 & -63.2036 & 1.5 \\
\hline$c 27 y-c 290$ & Bel & 15 & 242.7299 & -63.2279 & 1.5 \\
\hline$c 27 y-c 290$ & $\mathrm{Bel}$ & 15 & 242.7810 & -63.2401 & 1.5 \\
\hline$c 27 y-c 29 o$ & $\mathrm{Bel}$ & 15 & 242.8418 & -63.2498 & 1.5 \\
\hline$c 27 y-c 290$ & Bel & 15 & 242.9002 & -63.2839 & 1.5 \\
\hline$c 27 y-c 29 o$ & Bel & 15 & 242.9611 & -63.3010 & 1.5 \\
\hline$c 27 y-c 29 o$ & Bel & 15 & 243.0122 & -63.3326 & 1.5 \\
\hline$c 27 y-c 290$ & Bel & 15 & 243.0779 & -63.3618 & 1.5 \\
\hline$c 27 y-c 290$ & Bel & 15 & 243.1412 & -63.3764 & 1.5 \\
\hline$c 27 y-c 290$ & Bel & 15 & 243.1923 & -63.3788 & 1.5 \\
\hline$c 27 y-c 29 y$ & Bel & 15 & 242.2285 & -63.0381 & 1.5 \\
\hline$c 27 y-c 29 y$ & Bel & 15 & 242.2869 & -63.0576 & 1.5 \\
\hline$c 27 y-c 29 y$ & Bel & 15 & 242.3283 & -63.0673 & 1.5 \\
\hline$c 27 y-c 29 y$ & Bel & 15 & 242.3769 & -63.0819 & 1.5 \\
\hline$c 27 y-c 29 y$ & Bel & 15 & 242.4159 & -63.1062 & 1.5 \\
\hline$c 27 y-c 29 y$ & Bel & 15 & 242.4719 & -63.1257 & 1.5 \\
\hline$c 27 y-c 30 o$ & Bel & 15 & 244.4336 & -63.7987 & 1.5 \\
\hline$c 27 y-c 30 y$ & Bel & 15 & 243.2410 & -63.3886 & 1.5 \\
\hline$c 27 y-c 30 y$ & $\mathrm{Bel}$ & 15 & 243.2848 & -63.3983 & 1.5 \\
\hline c27y-c30y & $\mathrm{Bel}$ & 15 & 243.3408 & -63.4129 & 1.5 \\
\hline$c 27 y-c 30 y$ & Bel & 15 & 243.4089 & -63.4567 & 1.5 \\
\hline$c 27 y-c 30 y$ & Bel & 15 & 243.4649 & -63.4908 & 1.5 \\
\hline$c 27 y-c 30 y$ & Bel & 15 & 243.5306 & -63.5176 & 1.5 \\
\hline$c 27 y-c 30 y$ & Bel & 15 & 243.5939 & -63.5516 & 1.5 \\
\hline$c 27 y-c 30 y$ & $\mathrm{Bel}$ & 15 & 243.6474 & -63.5638 & 1.5 \\
\hline
\end{tabular}




\begin{tabular}{|c|c|c|c|c|c|}
\hline age (chron) & plate & segment & Iongitude & latitude & uncertainty $(\mathrm{km})$ \\
\hline$c 27 y-c 30 y$ & $\mathrm{Bel}$ & 15 & 243.6864 & -63.5808 & 1.5 \\
\hline c27y-c30y & $\mathrm{Bel}$ & 15 & 243.7448 & -63.5930 & 1.5 \\
\hline$c 27 y-c 30 y$ & Bel & 15 & 243.9541 & -63.6441 & 1.5 \\
\hline$c 27 y-c 30 y$ & $\mathrm{Bel}$ & 15 & 243.9930 & -63.6514 & 1.5 \\
\hline$c 27 y-c 30 y$ & $\mathrm{Bel}$ & 15 & 244.0369 & -63.6660 & 1.5 \\
\hline$c 27 y-c 30 y$ & Bel & 15 & 244.0855 & -63.6758 & 1.5 \\
\hline$c 27 y-c 30 y$ & Bel & 15 & 244.1342 & -63.6855 & 1.5 \\
\hline$c 27 y-c 30 y$ & Bel & 15 & 244.1829 & -63.6977 & 1.5 \\
\hline$c 27 y-c 30 y$ & $\mathrm{Bel}$ & 15 & 244.2316 & -63.7147 & 1.5 \\
\hline$c 27 y-c 30 y$ & Bel & 15 & 244.2754 & -63.7342 & 1.5 \\
\hline$c 27 y-c 30 y$ & $\mathrm{Bel}$ & 15 & 244.3143 & -63.7512 & 1.5 \\
\hline$c 27 y-c 30 y$ & $\mathrm{Bel}$ & 15 & 244.3727 & -63.7792 & 1.5 \\
\hline$c 27 y-c 310$ & $\mathrm{Bel}$ & 15 & 245.1394 & -64.0494 & 1.5 \\
\hline$c 27 y-c 310$ & Bel & 15 & 245.1759 & -64.0761 & 1.5 \\
\hline c27y-c310 & $\mathrm{Bel}$ & 15 & 245.2270 & -64.1029 & 1.5 \\
\hline$c 27 y-c 310$ & $\mathrm{Bel}$ & 15 & 245.2976 & -64.1224 & 1.5 \\
\hline$c 27 y-c 310$ & $\mathrm{Bel}$ & 15 & 245.3585 & -64.1492 & 1.5 \\
\hline$c 27 y-c 310$ & $\mathrm{Bel}$ & 15 & 245.3925 & -64.1589 & 1.5 \\
\hline$c 27 y-c 310$ & $\mathrm{Bel}$ & 15 & 245.4753 & -64.1857 & 1.5 \\
\hline$c 27 y-c 310$ & $\mathrm{Bel}$ & 15 & 245.5580 & -64.2051 & 1.5 \\
\hline$c 27 y-c 310$ & Bel & 15 & 245.6408 & -64.2343 & 1.5 \\
\hline c27y-c310 & Bel & 15 & 245.6919 & -64.2416 & 1.5 \\
\hline$c 27 y-c 310$ & Bel & 15 & 245.7430 & -64.2489 & 1.5 \\
\hline$c 27 y-c 31 y$ & $\mathrm{Bel}$ & 15 & 244.4993 & -63.8182 & 1.5 \\
\hline$c 27 y-c 31 y$ & $\mathrm{Bel}$ & 15 & 244.5504 & -63.8352 & 1.5 \\
\hline$c 27 y-c 31 y$ & $\mathrm{Bel}$ & 15 & 244.6356 & -63.8766 & 1.5 \\
\hline$c 27 y-c 31 y$ & Bel & 15 & 244.7329 & -63.9252 & 1.5 \\
\hline c27y-c31y & Bel & 15 & 244.8011 & -63.9423 & 1.5 \\
\hline c27y-c31y & Bel & 15 & 244.8595 & -63.9593 & 1.5 \\
\hline c27y-c31y & Bel & 15 & 244.9082 & -63.9715 & 1.5 \\
\hline$c 27 y-c 31 y$ & $\mathrm{Bel}$ & 15 & 244.9642 & -63.9764 & 1.5 \\
\hline c27y-c31y & $\mathrm{Bel}$ & 15 & 245.0128 & -63.9910 & 1.5 \\
\hline c27y-c31y & $\mathrm{Bel}$ & 15 & 245.0664 & -64.0080 & 1.5 \\
\hline c28o-c31o & Bel & 15 & 246.7409 & -64.5398 & 1.5 \\
\hline$c 280-c 310$ & Bel & 15 & 246.7969 & -64.5617 & 1.5 \\
\hline c28o-c31o & Bel & 15 & 246.8529 & -64.5739 & 1.5 \\
\hline c28o-c31o & $\mathrm{Bel}$ & 15 & 246.9088 & -64.5812 & 1.5 \\
\hline$c 280-c 310$ & $\mathrm{Bel}$ & 15 & 246.9624 & -64.6006 & 1.5 \\
\hline$c 280-c 310$ & Bel & 15 & 247.0135 & -64.6152 & 1.5 \\
\hline c28o-c31o & $\mathrm{Bel}$ & 15 & 247.0719 & -64.6274 & 1.5 \\
\hline c28o-c31o & Bel & 15 & 247.1157 & -64.6323 & 1.5 \\
\hline$c 280-c 310$ & Bel & 15 & 247.1741 & -64.6445 & 1.5 \\
\hline$c 28 y-c 310$ & Bel & 15 & 245.8014 & -64.2636 & 1.5 \\
\hline$c 28 y-c 310$ & Bel & 15 & 245.8623 & -64.2855 & 1.5 \\
\hline$c 28 y-c 310$ & $\mathrm{Bel}$ & 15 & 245.9329 & -64.3074 & 1.5 \\
\hline$c 28 y-c 310$ & Bel & 15 & 245.9888 & -64.3220 & 1.5 \\
\hline$c 28 y-c 310$ & Bel & 15 & 246.0545 & -64.3414 & 1.5 \\
\hline$c 28 y-c 310$ & Bel & 15 & 246.1105 & -64.3463 & 1.5 \\
\hline$c 28 y-c 310$ & Bel & 15 & 246.1568 & -64.3633 & 1.5 \\
\hline$c 28 y-c 310$ & Bel & 15 & 246.2054 & -64.3682 & 1.5 \\
\hline$c 28 y-c 310$ & Bel & 15 & 246.2468 & -64.3852 & 1.5 \\
\hline$c 28 y-c 310$ & Bel & 15 & 246.2882 & -64.3950 & 1.5 \\
\hline$c 28 y-c 310$ & Bel & 15 & 246.3563 & -64.4193 & 1.5 \\
\hline$c 28 y-c 310$ & Bel & 15 & 246.4196 & -64.4339 & 1.5 \\
\hline$c 28 y-c 310$ & Bel & 15 & 246.4732 & -64.4485 & 1.5 \\
\hline$c 28 y-c 310$ & $\mathrm{Bel}$ & 15 & 246.5097 & -64.4607 & 1.5 \\
\hline$c 28 y-c 310$ & Bel & 15 & 246.5657 & -64.4777 & 1.5 \\
\hline$c 28 y-c 310$ & Bel & 15 & 246.6216 & -64.4948 & 1.5 \\
\hline
\end{tabular}




\begin{tabular}{|c|c|c|c|c|c|}
\hline age (chron) & plate & segment & longitude & latitude & uncertainty $(\mathrm{km})$ \\
\hline$c 28 y-c 310$ & $\mathrm{Bel}$ & 15 & 246.6752 & -64.5118 & 1.5 \\
\hline$c 290-c 310$ & Bel & 15 & 247.2642 & -64.6664 & 1.5 \\
\hline c29o-c31o & Bel & 15 & 247.3153 & -64.6858 & 1.5 \\
\hline$c 290-c 310$ & Bel & 15 & 247.3859 & -64.7126 & 1.5 \\
\hline$c 290-c 310$ & Bel & 15 & 247.4346 & -64.7442 & 1.5 \\
\hline c29o-c31o & Bel & 15 & 247.5027 & -64.7881 & 1.5 \\
\hline$c 290-c 310$ & Bel & 15 & 247.5709 & -64.8173 & 1.5 \\
\hline c29o-c31o & Bel & 15 & 247.6341 & -64.8440 & 1.5 \\
\hline$c 300$ & Bel & 15 & 247.6999 & -64.8732 & 1.5 \\
\hline c30o-c31o & Bel & 15 & 248.2134 & -65.0850 & 1.5 \\
\hline$c 300-c 310$ & Bel & 15 & 248.2645 & -65.1069 & 1.5 \\
\hline c30o-c31o & Bel & 15 & 248.3132 & -65.1288 & 1.5 \\
\hline$c 300-c 310$ & Bel & 15 & 248.3667 & -65.1604 & 1.5 \\
\hline c30o-c310 & Bel & 15 & 248.4300 & -65.1799 & 1.5 \\
\hline c30o-c31o & Bel & 15 & 248.4836 & -65.2018 & 1.5 \\
\hline$c 300-c 310$ & Bel & 15 & 248.5468 & -65.2286 & 1.5 \\
\hline$c 300-c 310$ & Bel & 15 & 248.6320 & -65.2578 & 1.5 \\
\hline$c 300-c 310$ & Bel & 15 & 248.6831 & -65.2846 & 1.5 \\
\hline c30o-c31o & Bel & 15 & 248.7245 & -65.2967 & 1.5 \\
\hline$c 300-c 310$ & Bel & 15 & 248.7805 & -65.3235 & 1.5 \\
\hline c30o-c310 & Bel & 15 & 248.8243 & -65.3430 & 1.5 \\
\hline c30o-c31o & Bel & 15 & 248.8925 & -65.3722 & 1.5 \\
\hline c30o-c31o & Bel & 15 & 248.9265 & -65.3868 & 1.5 \\
\hline c30o-c31o & $\mathrm{Bel}$ & 15 & 248.9752 & -65.4087 & 1.5 \\
\hline$c 300-c 310$ & Bel & 15 & 249.0288 & -65.4379 & 1.5 \\
\hline c30o-c31o & Bel & 15 & 249.0799 & -65.4647 & 1.5 \\
\hline$c 30 y-c 310$ & Bel & 15 & 247.6999 & -64.8732 & 1.5 \\
\hline$c 30 y-c 310$ & Bel & 15 & 247.7753 & -64.9000 & 1.5 \\
\hline$c 30 y-c 310$ & Bel & 15 & 247.8361 & -64.9219 & 1.5 \\
\hline$c 30 y-c 310$ & Bel & 15 & 247.9092 & -64.9487 & 1.5 \\
\hline$c 30 y-c 310$ & Bel & 15 & 247.9773 & -64.9706 & 1.5 \\
\hline$c 30 y-c 310$ & Bel & 15 & 248.0503 & -64.9974 & 1.5 \\
\hline$c 30 y-c 310$ & Bel & 15 & 248.1209 & -65.0314 & 1.5 \\
\hline$c 30 y-c 310$ & Bel & 15 & 248.1647 & -65.0607 & 1.5 \\
\hline c310 & Bel & 15 & 249.2454 & -65.5267 & 1.5 \\
\hline c310 & Bel & 15 & 249.2965 & -65.5462 & 1.5 \\
\hline c310 & Bel & 15 & 249.3646 & -65.5681 & 1.5 \\
\hline c310 & Bel & 15 & 249.4182 & -65.5852 & 1.5 \\
\hline c310 & Bel & 15 & 249.4571 & -65.5925 & 1.5 \\
\hline c310 & Bel & 15 & 249.5277 & -65.6144 & 1.5 \\
\hline$c 310$ & Bel & 15 & 249.5861 & -65.6411 & 1.5 \\
\hline c310 & Bel & 15 & 249.6421 & -65.6874 & 1.5 \\
\hline c310 & Bel & 15 & 249.6908 & -65.7263 & 1.5 \\
\hline c31y-c31o & Bel & 15 & 249.1285 & -65.4866 & 1.5 \\
\hline c31y-c310 & Bel & 15 & 249.1894 & -65.5073 & 1.5 \\
\hline c32.10 & Bel & 15 & 250.7398 & -66.2350 & 1.5 \\
\hline c32.10 & Bel & 15 & 250.7885 & -66.2569 & 1.5 \\
\hline c32.10 & Bel & 15 & 250.8371 & -66.2788 & 1.5 \\
\hline$c 32.10-c 32.20$ & Bel & 15 & 250.8882 & -66.2910 & 1.5 \\
\hline c32.1y & Bel & 15 & 250.5597 & -66.1401 & 1.5 \\
\hline c32.1y & Bel & 15 & 250.6327 & -66.1742 & 1.5 \\
\hline c32.1y & Bel & 15 & 250.6814 & -66.2058 & 1.5 \\
\hline c32.2o & Bel & 15 & 251.0148 & -66.3494 & 1.5 \\
\hline c32.20 & Bel & 15 & 251.0708 & -66.3737 & 1.5 \\
\hline c32.2o & Bel & 15 & 251.1292 & -66.4029 & 1.5 \\
\hline c32.2o & Bel & 15 & 251.1779 & -66.4321 & 1.5 \\
\hline c32.20 & Bel & 15 & 251.2412 & -66.4687 & 1.5 \\
\hline$c 32.20$ & Bel & 15 & 251.2971 & -66.4954 & 1.5 \\
\hline
\end{tabular}




\begin{tabular}{|c|c|c|c|c|c|}
\hline age (chron) & plate & segment & longitude & latitude & uncertainty $(\mathrm{km})$ \\
\hline $\mathrm{c} 32.20$ & $\mathrm{Bel}$ & 15 & 251.3604 & -66.5368 & 1.5 \\
\hline c32.20 & Bel & 15 & 251.4188 & -66.5660 & 1.5 \\
\hline c32.20 & $\mathrm{Bel}$ & 15 & 251.4626 & -66.5903 & 1.5 \\
\hline c32.20 & Bel & 15 & 251.5308 & -66.6208 & 1.5 \\
\hline c32.20 & Bel & 15 & 251.5892 & -66.6573 & 1.5 \\
\hline c32.20 & $\mathrm{Bel}$ & 15 & 251.6403 & -66.6792 & 1.5 \\
\hline$c 32.2 y-c 32.20$ & Bel & 15 & 250.9199 & -66.3105 & 1.5 \\
\hline$c 32.2 y-c 32.20$ & Bel & 15 & 250.9661 & -66.3226 & 1.5 \\
\hline $\mathrm{c} 32 \mathrm{r}$ & $\mathrm{Bel}$ & 15 & 251.7377 & -66.7522 & 1.5 \\
\hline$c 32 r$ & $\mathrm{Bel}$ & 15 & 251.7790 & -66.7838 & 1.5 \\
\hline$c 32 r-c 33 y$ & $\mathrm{Bel}$ & 15 & 251.8204 & -66.8130 & 1.5 \\
\hline$c 33 y$ & Bel & 15 & 251.8594 & -66.8471 & 1.5 \\
\hline c33y & $\mathrm{Bel}$ & 15 & 251.9007 & -66.8861 & 1.5 \\
\hline c20o & Pac & 15 & 200.8969 & -50.9368 & 1.5 \\
\hline c20o & Pac & 15 & 200.9544 & -50.9594 & 1.5 \\
\hline c20o & Pac & 15 & 200.9955 & -50.9778 & 1.5 \\
\hline c20o & Pac & 15 & 201.0553 & -51.0137 & 1.5 \\
\hline c20o & Pac & 15 & 201.1343 & -51.0541 & 1.5 \\
\hline$c 20 \circ$ & $\mathrm{Pac}$ & 15 & 201.1709 & -51.0836 & 1.5 \\
\hline $\mathrm{c} 20 \mathrm{o}$ & $\mathrm{Pac}$ & 15 & 201.2263 & -51.1119 & 1.5 \\
\hline c20o & Pac & 15 & 201.2818 & -51.1384 & 1.5 \\
\hline c20o & Pac & 15 & 201.3350 & -51.1687 & 1.5 \\
\hline c20o & Pac & 15 & 201.3860 & -51.1919 & 1.5 \\
\hline c20o & Pac & 15 & 201.4374 & -51.2175 & 1.5 \\
\hline c20o & Pac & 15 & 201.5024 & -51.2555 & 1.5 \\
\hline c210 & $\mathrm{Pac}$ & 15 & 199.7366 & -50.2684 & 1.5 \\
\hline c21o & Pac & 15 & 199.7772 & -50.2989 & 1.5 \\
\hline c210 & $\mathrm{Pac}$ & 15 & 199.8245 & -50.3256 & 1.5 \\
\hline c21o & Pac & 15 & 199.8729 & -50.3540 & 1.5 \\
\hline c210 & Pac & 15 & 199.9240 & -50.3772 & 1.5 \\
\hline c210 & $\mathrm{Pac}$ & 15 & 199.9704 & -50.4067 & 1.5 \\
\hline c21y & Pac & 15 & 200.0615 & -50.4567 & 1.5 \\
\hline c21y & Pac & 15 & 200.1020 & -50.4780 & 1.5 \\
\hline c21y & Pac & 15 & 200.1482 & -50.5086 & 1.5 \\
\hline c21y-c21o & Pac & 15 & 200.0152 & -50.4305 & 1.5 \\
\hline $\mathrm{c} 220$ & Pac & 15 & 199.8245 & -50.3256 & 1.5 \\
\hline c22o & Pac & 15 & 199.8729 & -50.3540 & 1.5 \\
\hline c22y & Pac & 15 & 199.6827 & -50.2424 & 1.5 \\
\hline$c 22 y$ & Pac & 15 & 199.7366 & -50.2684 & 1.5 \\
\hline$c 22 y-c 220$ & Pac & 15 & 199.7772 & -50.2989 & 1.5 \\
\hline $\mathrm{c} 230$ & Pac & 15 & 199.1772 & -49.9832 & 1.5 \\
\hline c23o & Pac & 15 & 199.2264 & -50.0124 & 1.5 \\
\hline c23o & Pac & 15 & 199.2792 & -50.0417 & 1.5 \\
\hline c23o & Pac & 15 & 199.3268 & -50.0629 & 1.5 \\
\hline$c 23 y$ & Pac & 15 & 199.5848 & -50.2017 & 1.5 \\
\hline c23y & Pac & 15 & 199.6286 & -50.2236 & 1.5 \\
\hline c23y & Pac & 15 & 199.6827 & -50.2424 & 1.5 \\
\hline c24o & Pac & 15 & 199.3609 & -50.0873 & 1.5 \\
\hline c24o & Pac & 15 & 201.3860 & -51.1919 & 1.5 \\
\hline c24o & Pac & 15 & 201.4374 & -51.2175 & 1.5 \\
\hline c24o & Pac & 15 & 201.5024 & -51.2555 & 1.5 \\
\hline c24o & Pac & 15 & 201.5550 & -51.2883 & 1.5 \\
\hline c24o & Pac & 15 & 201.6012 & -51.3123 & 1.5 \\
\hline c24o & Pac & 15 & 201.6411 & -51.3243 & 1.5 \\
\hline c24o & Pac & 15 & 201.6971 & -51.3559 & 1.5 \\
\hline c24o & Pac & 15 & 201.7312 & -51.3754 & 1.5 \\
\hline c24o & Pac & 15 & 201.7774 & -51.3973 & 1.5 \\
\hline c24o & Pac & 15 & 201.8358 & -51.4289 & 1.5 \\
\hline
\end{tabular}




\begin{tabular}{|c|c|c|c|c|c|}
\hline age (chron) & plate & segment & longitude & latitude & uncertainty $(\mathrm{km})$ \\
\hline c24o & $\mathrm{Pac}$ & 15 & 201.8845 & -51.4460 & 1.5 \\
\hline c24o & Pac & 15 & 201.9287 & -51.4749 & 1.5 \\
\hline c24o & Pac & 15 & 201.9798 & -51.5017 & 1.5 \\
\hline c24o & Pac & 15 & 202.0309 & -51.5285 & 1.5 \\
\hline$c 24 o-c 25 o$ & Pac & 15 & 201.0553 & -51.0137 & 1.5 \\
\hline$c 240-c 250$ & Pac & 15 & 201.1343 & -51.0541 & 1.5 \\
\hline$c 240-c 250$ & Pac & 15 & 201.1709 & -51.0836 & 1.5 \\
\hline$c 240-c 25 o$ & Pac & 15 & 201.2263 & -51.1119 & 1.5 \\
\hline$c 240-c 25 y$ & Pac & 15 & 201.2818 & -51.1384 & 1.5 \\
\hline$c 240-c 25 y$ & Pac & 15 & 201.3350 & -51.1687 & 1.5 \\
\hline$c 240-c 260$ & Pac & 15 & 200.1020 & -50.4780 & 1.5 \\
\hline $\mathrm{c} 240-c 260$ & Pac & 15 & 200.1482 & -50.5086 & 1.5 \\
\hline$c 240-c 26 o$ & Pac & 15 & 200.1854 & -50.5303 & 1.5 \\
\hline c24o-c26o & Pac & 15 & 200.2364 & -50.5640 & 1.5 \\
\hline c24o-c26o & Pac & 15 & 200.2891 & -50.5936 & 1.5 \\
\hline c24o-c26o & Pac & 15 & 200.3318 & -50.6172 & 1.5 \\
\hline$c 240-c 260$ & Pac & 15 & 200.3877 & -50.6442 & 1.5 \\
\hline c24o-c26o & Pac & 15 & 200.4472 & -50.6774 & 1.5 \\
\hline $\mathrm{c} 240-c 260$ & Pac & 15 & 200.4980 & -50.7110 & 1.5 \\
\hline$c 240-c 260$ & Pac & 15 & 200.5643 & -50.7564 & 1.5 \\
\hline $\mathrm{c} 240-c 260$ & Pac & 15 & 200.6224 & -50.7908 & 1.5 \\
\hline$c 240-c 26 o$ & Pac & 15 & 200.6838 & -50.8248 & 1.5 \\
\hline $\mathrm{c} 240-c 260$ & Pac & 15 & 200.7166 & -50.8422 & 1.5 \\
\hline$c 240-c 26 o$ & Pac & 15 & 200.7509 & -50.8552 & 1.5 \\
\hline c24o-c26o & Pac & 15 & 200.7885 & -50.8772 & 1.5 \\
\hline$c 240-c 260$ & Pac & 15 & 200.8412 & -50.9018 & 1.5 \\
\hline$c 240-c 26 o$ & Pac & 15 & 200.8969 & -50.9368 & 1.5 \\
\hline$c 240-c 26 y$ & Pac & 15 & 200.9544 & -50.9594 & 1.5 \\
\hline$c 240-c 26 y$ & Pac & 15 & 200.9955 & -50.9778 & 1.5 \\
\hline c24o-c27o & Pac & 15 & 199.5848 & -50.2017 & 1.5 \\
\hline c24o-c27o & Pac & 15 & 199.6286 & -50.2236 & 1.5 \\
\hline c24o-c27o & Pac & 15 & 199.6827 & -50.2424 & 1.5 \\
\hline c24o-c27o & Pac & 15 & 199.7366 & -50.2684 & 1.5 \\
\hline$c 240-c 270$ & Pac & 15 & 199.7772 & -50.2989 & 1.5 \\
\hline c24o-c27o & Pac & 15 & 199.8245 & -50.3256 & 1.5 \\
\hline c24o-c27o & Pac & 15 & 199.8729 & -50.3540 & 1.5 \\
\hline c24o-c27y & Pac & 15 & 199.9240 & -50.3772 & 1.5 \\
\hline c24o-c27y & Pac & 15 & 199.9704 & -50.4067 & 1.5 \\
\hline$c 240-c 27 y$ & Pac & 15 & 200.0152 & -50.4305 & 1.5 \\
\hline$c 240-c 27 y$ & Pac & 15 & 200.0615 & -50.4567 & 1.5 \\
\hline$c 240-c 28 y$ & Pac & 15 & 199.1772 & -49.9832 & 1.5 \\
\hline c24o-c28y & Pac & 15 & 199.2264 & -50.0124 & 1.5 \\
\hline$c 240-c 28 y$ & Pac & 15 & 199.2792 & -50.0417 & 1.5 \\
\hline$c 240-c 28 y$ & Pac & 15 & 199.3268 & -50.0629 & 1.5 \\
\hline$c 24 y-c 27 o$ & Pac & 15 & 199.4655 & -50.1384 & 1.5 \\
\hline$c 24 y-c 270$ & Pac & 15 & 199.5020 & -50.1603 & 1.5 \\
\hline$c 24 y-c 27 o$ & Pac & 15 & 199.5434 & -50.1749 & 1.5 \\
\hline$c 24 y-c 28 y$ & Pac & 15 & 199.3609 & -50.0873 & 1.5 \\
\hline$c 24 y-c 28 y$ & Pac & 15 & 199.3998 & -50.1068 & 1.5 \\
\hline c25o-c29o & Pac & 15 & 198.2881 & -49.4602 & 1.5 \\
\hline$c 250-c 290$ & Pac & 15 & 198.3323 & -49.4849 & 1.5 \\
\hline c25o-c29o & Pac & 15 & 198.3848 & -49.5186 & 1.5 \\
\hline c25o-c29o & Pac & 15 & 198.4446 & -49.5481 & 1.5 \\
\hline$c 25 y-c 280$ & Pac & 15 & 198.9008 & -49.8360 & 1.5 \\
\hline$c 25 y-c 280$ & Pac & 15 & 198.9352 & -49.8498 & 1.5 \\
\hline$c 25 y-c 280$ & Pac & 15 & 198.9844 & -49.8816 & 1.5 \\
\hline$c 25 y-c 28 y$ & Pac & 15 & 197.7533 & -49.1724 & 1.5 \\
\hline$c 25 y-c 28 y$ & $\mathrm{Pac}$ & 15 & 199.0266 & -49.9029 & 1.5 \\
\hline
\end{tabular}




\begin{tabular}{|c|c|c|c|c|c|}
\hline age (chron) & plate & segment & longitude & latitude & uncertainty $(\mathrm{km})$ \\
\hline$c 25 y-c 28 y$ & $\mathrm{Pac}$ & 15 & 199.0674 & -49.9318 & 1.5 \\
\hline$c 25 y-c 28 y$ & $\mathrm{Pac}$ & 15 & 199.1220 & -49.9515 & 1.5 \\
\hline$c 25 y-c 290$ & $\mathrm{Pac}$ & 15 & 198.4786 & -49.5773 & 1.5 \\
\hline$c 25 y-c 290$ & $\mathrm{Pac}$ & 15 & 198.5298 & -49.6162 & 1.5 \\
\hline$c 25 y-c 29 y$ & $\mathrm{Pac}$ & 15 & 198.5882 & -49.6503 & 1.5 \\
\hline$c 25 y-c 29 y$ & Pac & 15 & 198.6417 & -49.6746 & 1.5 \\
\hline$c 25 y-c 29 y$ & Pac & 15 & 198.6928 & -49.7160 & 1.5 \\
\hline$c 25 y-c 29 y$ & Pac & 15 & 198.7447 & -49.7558 & 1.5 \\
\hline$c 25 y-c 29 y$ & Pac & 15 & 198.7812 & -49.7826 & 1.5 \\
\hline$c 25 y-c 29 y$ & $\mathrm{Pac}$ & 15 & 198.8153 & -49.7972 & 1.5 \\
\hline$c 25 y-c 29 y$ & Pac & 15 & 198.8646 & -49.8157 & 1.5 \\
\hline$c 260-c 30 y$ & Pac & 15 & 197.8690 & -49.2285 & 1.5 \\
\hline c26o-c30y & Pac & 15 & 197.9140 & -49.2531 & 1.5 \\
\hline c26o-c30y & $\mathrm{Pac}$ & 15 & 197.9578 & -49.2802 & 1.5 \\
\hline$c 260-c 30 y$ & Pac & 15 & 198.0003 & -49.3032 & 1.5 \\
\hline$c 260-c 30 y$ & Pac & 15 & 198.0439 & -49.3261 & 1.5 \\
\hline$c 260-c 30 y$ & Pac & 15 & 198.0706 & -49.3432 & 1.5 \\
\hline$c 260-c 30 y$ & $\mathrm{Pac}$ & 15 & 198.1248 & -49.3704 & 1.5 \\
\hline$c 260-c 30 y$ & $\mathrm{Pac}$ & 15 & 198.1760 & -49.3950 & 1.5 \\
\hline$c 260-c 30 y$ & Pac & 15 & 198.2325 & -49.4240 & 1.5 \\
\hline c27o-c31o & Pac & 15 & 196.5164 & -48.4686 & 1.5 \\
\hline c27o-c31o & Pac & 15 & 196.5773 & -48.4978 & 1.5 \\
\hline$c 27 y-c 30 o$ & $\mathrm{Pac}$ & 15 & 197.4092 & -48.9758 & 1.5 \\
\hline$c 27 y-c 300$ & Pac & 15 & 197.4498 & -49.0001 & 1.5 \\
\hline$c 27 y-c 30 y$ & $\mathrm{Pac}$ & 15 & 197.5028 & -49.0284 & 1.5 \\
\hline$c 27 y-c 30 y$ & $\mathrm{Pac}$ & 15 & 197.5558 & -49.0568 & 1.5 \\
\hline c27y-c30y & Pac & 15 & 197.6038 & -49.0830 & 1.5 \\
\hline$c 27 y-c 30 y$ & Pac & 15 & 197.6422 & -49.1123 & 1.5 \\
\hline$c 27 y-c 30 y$ & Pac & 15 & 197.6952 & -49.1407 & 1.5 \\
\hline c27y-c30y & $\mathrm{Pac}$ & 15 & 197.8110 & -49.1980 & 1.5 \\
\hline$c 27 y-c 310$ & Pac & 15 & 196.6187 & -48.5173 & 1.5 \\
\hline$c 27 y-c 310$ & Pac & 15 & 196.6576 & -48.5465 & 1.5 \\
\hline$c 27 y-c 310$ & Pac & 15 & 196.7014 & -48.5733 & 1.5 \\
\hline$c 27 y-c 310$ & Pac & 15 & 196.7477 & -48.6000 & 1.5 \\
\hline$c 27 y-c 310$ & Pac & 15 & 196.7842 & -48.6219 & 1.5 \\
\hline$c 27 y-c 310$ & $\mathrm{Pac}$ & 15 & 196.8450 & -48.6511 & 1.5 \\
\hline$c 27 y-c 310$ & $\mathrm{Pac}$ & 15 & 196.8915 & -48.7272 & 1.5 \\
\hline$c 27 y-c 310$ & $\mathrm{Pac}$ & 15 & 196.9402 & -48.7467 & 1.5 \\
\hline$c 27 y-c 310$ & Pac & 15 & 196.9937 & -48.7832 & 1.5 \\
\hline$c 27 y-c 310$ & Pac & 15 & 197.0424 & -48.8027 & 1.5 \\
\hline c27y-c31y & Pac & 15 & 197.0845 & -48.8212 & 1.5 \\
\hline c27y-c31y & $\mathrm{Pac}$ & 15 & 197.1276 & -48.8341 & 1.5 \\
\hline$c 27 y-c 31 y$ & Pac & 15 & 197.1689 & -48.8609 & 1.5 \\
\hline c27y-c31y & Pac & 15 & 197.2225 & -48.8852 & 1.5 \\
\hline c27y-c31y & Pac & 15 & 197.2677 & -48.9011 & 1.5 \\
\hline c27y-c31y & $\mathrm{Pac}$ & 15 & 197.3139 & -48.9230 & 1.5 \\
\hline$c 27 y-c 31 y$ & Pac & 15 & 197.3738 & -48.9561 & 1.5 \\
\hline $\mathrm{c} 280-\mathrm{c} 32.10$ & Pac & 15 & 195.9615 & -48.1583 & 1.5 \\
\hline c28o-c32.10 & Pac & 15 & 196.0078 & -48.1826 & 1.5 \\
\hline$c 280-c 32.1 y$ & $\mathrm{Pac}$ & 15 & 196.0589 & -48.2191 & 1.5 \\
\hline c28o-c32.1y & $\mathrm{Pac}$ & 15 & 196.1124 & -48.2556 & 1.5 \\
\hline$c 280-c 32.2 y$ & $\mathrm{Pac}$ & 15 & 195.7206 & -48.0366 & 1.5 \\
\hline$c 280-c 32.2 y$ & Pac & 15 & 195.7765 & -48.0707 & 1.5 \\
\hline$c 280-c 32.2 y$ & Pac & 15 & 195.8276 & -48.0999 & 1.5 \\
\hline c28o-c32.2y & $\mathrm{Pac}$ & 15 & 195.8982 & -48.1315 & 1.5 \\
\hline$c 28 y-c 310$ & Pac & 15 & 196.1416 & -48.2727 & 1.5 \\
\hline$c 28 y-c 310$ & Pac & 15 & 196.1830 & -48.2946 & 1.5 \\
\hline$c 28 y-c 310$ & Pac & 15 & 196.2292 & -48.3214 & 1.5 \\
\hline
\end{tabular}




\begin{tabular}{|c|c|c|c|c|c|}
\hline age (chron) & plate & segment & longitude & latitude & uncertainty $(\mathrm{km})$ \\
\hline$c 28 y-c 310$ & $\mathrm{Pac}$ & 15 & 196.2730 & -48.3360 & 1.5 \\
\hline$c 28 y-c 310$ & Pac & 15 & 196.3290 & -48.3652 & 1.5 \\
\hline$c 28 y-c 310$ & Pac & 15 & 196.3826 & -48.3968 & 1.5 \\
\hline$c 28 y-c 310$ & Pac & 15 & 196.4312 & -48.4260 & 1.5 \\
\hline$c 28 y-c 310$ & Pac & 15 & 196.4678 & -48.4443 & 1.5 \\
\hline $\mathrm{c} 290-\mathrm{c} 32.2 \mathrm{o}$ & Pac & 15 & 195.2240 & -47.8102 & 1.5 \\
\hline c29o-c32.2y & Pac & 15 & 195.2946 & -47.8492 & 1.5 \\
\hline c29o-c32.2y & Pac & 15 & 195.3457 & -47.8760 & 1.5 \\
\hline$c 290-c 32.2 y$ & Pac & 15 & 195.4017 & -47.8930 & 1.5 \\
\hline c29o-c32.2y & Pac & 15 & 195.4528 & -47.9271 & 1.5 \\
\hline$c 29 y-c 32.2 y$ & Pac & 15 & 195.5356 & -47.9636 & 1.5 \\
\hline$c 29 y-c 32.2 y$ & Pac & 15 & 195.5891 & -47.9855 & 1.5 \\
\hline$c 29 y-c 32.2 y$ & Pac & 15 & 195.6402 & -48.0122 & 1.5 \\
\hline$c 30 o-c 32 r$ & Pac & 15 & 194.9587 & -47.6472 & 1.5 \\
\hline$c 300-c 33 y$ & Pac & 15 & 194.1556 & -47.1665 & 1.5 \\
\hline$c 300-c 33 y$ & Pac & 15 & 194.1945 & -47.1981 & 1.5 \\
\hline$c 300-c 33 y$ & Pac & 15 & 194.2383 & -47.2273 & 1.5 \\
\hline$c 300-c 33 y$ & Pac & 15 & 194.2821 & -47.2687 & 1.5 \\
\hline c30o-c33y & Pac & 15 & 194.3211 & -47.3003 & 1.5 \\
\hline$c 300-c 33 y$ & Pac & 15 & 194.3551 & -47.3222 & 1.5 \\
\hline c30o-c33y & Pac & 15 & 194.4014 & -47.3417 & 1.5 \\
\hline$c 30 o-c 33 y$ & Pac & 15 & 194.4574 & -47.3807 & 1.5 \\
\hline$c 300-c 33 y$ & Pac & 15 & 194.4890 & -47.3953 & 1.5 \\
\hline c30o-c33y & Pac & 15 & 194.5304 & -47.4245 & 1.5 \\
\hline$c 300-c 33 y$ & Pac & 15 & 194.5645 & -47.4537 & 1.5 \\
\hline c30o-c33y & Pac & 15 & 194.6010 & -47.4683 & 1.5 \\
\hline$c 300-c 33 y$ & Pac & 15 & 194.6496 & -47.4902 & 1.5 \\
\hline$c 300-c 33 y$ & Pac & 15 & 194.7105 & -47.5230 & 1.5 \\
\hline$c 300-c 33 y$ & Pac & 15 & 194.7640 & -47.5498 & 1.5 \\
\hline c30o-c33y & Pac & 15 & 194.8054 & -47.5644 & 1.5 \\
\hline c30o-c33y & Pac & 15 & 194.8590 & -47.6009 & 1.5 \\
\hline c30o-c33y & Pac & 15 & 194.9028 & -47.6204 & 1.5 \\
\hline$c 30 y-c 32.20$ & Pac & 15 & 195.1437 & -47.7689 & 1.5 \\
\hline$c 30 y-c 32 r$ & Pac & 15 & 195.0099 & -47.6764 & 1.5 \\
\hline$c 30 y-c 32 r$ & Pac & 15 & 195.0731 & -47.7104 & 1.5 \\
\hline c310-c33y & Pac & 15 & 193.7783 & -46.8622 & 1.5 \\
\hline c310-c33y & Pac & 15 & 193.8148 & -46.8866 & 1.5 \\
\hline c310-c33y & Pac & 15 & 193.8586 & -46.9377 & 1.5 \\
\hline c310-c33y & Pac & 15 & 193.9000 & -46.9742 & 1.5 \\
\hline c310-c33y & Pac & 15 & 193.9438 & -46.9985 & 1.5 \\
\hline c310-c33y & Pac & 15 & 193.9852 & -47.0448 & 1.5 \\
\hline c310-c33y & Pac & 15 & 194.0241 & -47.0764 & 1.5 \\
\hline$c 31 y-c 33 y$ & Pac & 15 & 194.0728 & -47.1081 & 1.5 \\
\hline$c 31 y-c 33 y$ & Pac & 15 & 194.1118 & -47.1421 & 1.5 \\
\hline c32.10-c33y & Pac & 15 & 193.7004 & -46.7941 & 1.5 \\
\hline c32.10-c33y & Pac & 15 & 193.7394 & -46.8160 & 1.5 \\
\hline$c 32.20-c 33 y$ & Pac & 15 & 193.4570 & -46.5410 & 1.5 \\
\hline$c 32.20-c 33 y$ & Pac & 15 & 193.4911 & -46.5677 & 1.5 \\
\hline c32.2o-c33y & Pac & 15 & 193.5228 & -46.5945 & 1.5 \\
\hline$c 32.2 y-c 33 y$ & Pac & 15 & 193.5544 & -46.6188 & 1.5 \\
\hline$c 32.2 y-c 33 y$ & Pac & 15 & 193.5860 & -46.6456 & 1.5 \\
\hline$c 32.2 y-c 33 y$ & Pac & 15 & 193.6128 & -46.6748 & 1.5 \\
\hline$c 32.2 y-c 33 y$ & Pac & 15 & 193.6396 & -46.7138 & 1.5 \\
\hline$c 32.2 y-c 33 y$ & Pac & 15 & 193.6688 & -46.7503 & 1.5 \\
\hline $\mathrm{c} 330$ & Pac & 15 & 192.4304 & -45.5416 & 1.5 \\
\hline c33o & Pac & 15 & 192.4712 & -45.5787 & 1.5 \\
\hline c33o & Pac & 15 & 192.5069 & -45.6140 & 1.5 \\
\hline c33o & $\mathrm{Pac}$ & 15 & 192.5598 & -45.6466 & 1.5 \\
\hline
\end{tabular}




\begin{tabular}{|c|c|c|c|c|c|}
\hline age (chron) & plate & segment & longitude & latitude & uncertainty $(\mathrm{km})$ \\
\hline c33o & $\mathrm{Pac}$ & 15 & 192.5962 & -45.6847 & 1.5 \\
\hline c33o & Pac & 15 & 192.6341 & -45.7175 & 1.5 \\
\hline c33o & Pac & 15 & 192.6697 & -45.7508 & 1.5 \\
\hline c33o & Pac & 15 & 192.7079 & -45.7896 & 1.5 \\
\hline c33o & Pac & 15 & 192.7451 & -45.8240 & 1.5 \\
\hline c33o & Pac & 15 & 192.7775 & -45.8595 & 1.5 \\
\hline c33o & Pac & 15 & 192.8148 & -45.9063 & 1.5 \\
\hline c33o & Pac & 15 & 192.8535 & -45.9478 & 1.5 \\
\hline c33o & Pac & 15 & 192.8906 & -45.9822 & 1.5 \\
\hline c33o & Pac & 15 & 192.9217 & -46.0163 & 1.5 \\
\hline c33o & Pac & 15 & 192.9508 & -46.0554 & 1.5 \\
\hline c33o & Pac & 15 & 192.9776 & -46.0822 & 1.5 \\
\hline c33o & Pac & 15 & 193.0043 & -46.1138 & 1.5 \\
\hline c33o & Pac & 15 & 193.0408 & -46.1479 & 1.5 \\
\hline c33o & Pac & 15 & 193.0774 & -46.1893 & 1.5 \\
\hline c33o & Pac & 15 & 193.1285 & -46.2185 & 1.5 \\
\hline c33o & Pac & 15 & 193.1820 & -46.2501 & 1.5 \\
\hline c33o & Pac & 15 & 193.2185 & -46.2842 & 1.5 \\
\hline c33y & Pac & 15 & 193.2575 & -46.3256 & 1.5 \\
\hline c33y & Pac & 15 & 193.2842 & -46.3596 & 1.5 \\
\hline c33y & Pac & 15 & 193.3207 & -46.3961 & 1.5 \\
\hline c33y & Pac & 15 & 193.3524 & -46.4278 & 1.5 \\
\hline c33y & Pac & 15 & 193.3840 & -46.4594 & 1.5 \\
\hline c33y & Pac & 15 & 193.4205 & -46.5045 & 1.5 \\
\hline c20o & Bel-MBL & 21 & 225.5374 & -65.0656 & 1.5 \\
\hline c20o & Bel-MBL & 21 & 225.6029 & -65.0951 & 1.5 \\
\hline$c 20 \circ$ & Bel-MBL & 21 & 225.6553 & -65.1213 & 1.5 \\
\hline $\mathrm{c} 200$ & Bel-MBL & 21 & 225.7110 & -65.1508 & 1.5 \\
\hline$c 20 y$ & Bel-MBL & 21 & 225.3703 & -64.9804 & 1.5 \\
\hline $\mathrm{c} 20 \mathrm{y}$ & Bel-MBL & 21 & 225.4227 & -65.0001 & 1.5 \\
\hline $\mathrm{c} 20 \mathrm{y}$ & Bel-MBL & 21 & 225.4752 & -65.0263 & 1.5 \\
\hline c21o & Bel-MBL & 21 & 226.1533 & -65.3785 & 1.5 \\
\hline c210 & Bel-MBL & 21 & 226.2090 & -65.4047 & 1.5 \\
\hline c210 & Bel-MBL & 21 & 226.2712 & -65.4243 & 1.5 \\
\hline c210 & Bel-MBL & 21 & 226.2975 & -65.4407 & 1.5 \\
\hline c210 & Bel-MBL & 21 & 226.3531 & -65.4735 & 1.5 \\
\hline c21y & Bel-MBL & 21 & 225.9273 & -65.2769 & 1.5 \\
\hline c21y & Bel-MBL & 21 & 225.9993 & -65.3097 & 1.5 \\
\hline c21y & Bel-MBL & 21 & 226.0550 & -65.3392 & 1.5 \\
\hline c22o & Bel-MBL & 21 & 226.2090 & -65.4047 & 1.5 \\
\hline c22o & Bel-MBL & 21 & 226.2712 & -65.4243 & 1.5 \\
\hline c22o-c23o & Bel-MBL & 21 & 226.3990 & -65.5128 & 1.5 \\
\hline$c 220-c 23 y$ & Bel-MBL & 21 & 226.2975 & -65.4407 & 1.5 \\
\hline$c 220-c 23 y$ & Bel-MBL & 21 & 226.3531 & -65.4735 & 1.5 \\
\hline c22o-c24y & Bel-MBL & 21 & 226.4481 & -65.5390 & 1.5 \\
\hline$c 22 y$ & Bel-MBL & 21 & 226.5923 & -65.5980 & 1.5 \\
\hline$c 22 y-c 240$ & Bel-MBL & 21 & 226.5399 & -65.5816 & 1.5 \\
\hline$c 22 y-c 24 y$ & Bel-MBL & 21 & 226.4973 & -65.5619 & 1.5 \\
\hline c23o-c24o & Bel-MBL & 21 & 226.5923 & -65.5980 & 1.5 \\
\hline c23o-c24o & Bel-MBL & 21 & 226.7987 & -65.7159 & 1.5 \\
\hline c23o-c24o & Bel-MBL & 21 & 226.8446 & -65.7454 & 1.5 \\
\hline $\mathrm{c} 230-\mathrm{c} 240$ & Bel-MBL & 21 & 226.8937 & -65.7683 & 1.5 \\
\hline $\mathrm{c} 230-c 240$ & Bel-MBL & 21 & 226.9592 & -65.8076 & 1.5 \\
\hline$c 23 y-c 240$ & Bel-MBL & 21 & 226.6414 & -65.6143 & 1.5 \\
\hline$c 23 y-c 240$ & Bel-MBL & 21 & 226.6971 & -65.6406 & 1.5 \\
\hline$c 23 y-c 240$ & Bel-MBL & 21 & 226.7495 & -65.6864 & 1.5 \\
\hline c24o & Bel-MBL & 21 & 227.1591 & -65.9026 & 1.5 \\
\hline c24o & Bel-MBL & 21 & 227.2377 & -65.9354 & 1.5 \\
\hline
\end{tabular}




\begin{tabular}{|c|c|c|c|c|c|}
\hline age (chron) & plate & segment & longitude & latitude & uncertainty $(\mathrm{km})$ \\
\hline $\mathrm{c} 240$ & Bel-MBL & 21 & 227.3229 & -65.9813 & 1.5 \\
\hline$c 24 y-c 24 o$ & Bel-MBL & 21 & 227.0116 & -65.8404 & 1.5 \\
\hline$c 24 y-c 24 o$ & Bel-MBL & 21 & 227.0509 & -65.8633 & 1.5 \\
\hline$c 24 y-c 24 o$ & Bel-MBL & 21 & 227.0935 & -65.8895 & 1.5 \\
\hline $\mathrm{c} 250$ & Bel-MBL & 21 & 228.2565 & -66.3875 & 1.5 \\
\hline$c 25 o-c 26 y$ & Bel-MBL & 21 & 228.3483 & -66.4006 & 1.5 \\
\hline c25y & Bel-MBL & 21 & 227.7193 & -66.1451 & 1.5 \\
\hline c25y & Bel-MBL & 21 & 227.7815 & -66.1745 & 1.5 \\
\hline$c 25 y-c 25 o$ & Bel-MBL & 21 & 227.8437 & -66.2008 & 1.5 \\
\hline$c 25 y-c 25 \circ$ & Bel-MBL & 21 & 227.9224 & -66.2630 & 1.5 \\
\hline$c 25 y-c 25 o$ & Bel-MBL & 21 & 228.0010 & -66.2990 & 1.5 \\
\hline$c 25 y-c 25 \circ$ & Bel-MBL & 21 & 228.0927 & -66.3318 & 1.5 \\
\hline$c 25 y-c 25 o$ & Bel-MBL & 21 & 228.1877 & -66.3646 & 1.5 \\
\hline $\mathrm{c} 26 \mathrm{o}$ & Bel-MBL & 21 & 229.0297 & -66.5775 & 1.5 \\
\hline c26o & Bel-MBL & 21 & 229.1116 & -66.6070 & 1.5 \\
\hline c26o & Bel-MBL & 21 & 229.1640 & -66.6234 & 1.5 \\
\hline$c 260$ & Bel-MBL & 21 & 229.2295 & -66.6496 & 1.5 \\
\hline c26y & Bel-MBL & 21 & 228.4138 & -66.4235 & 1.5 \\
\hline c26y & Bel-MBL & 21 & 228.4990 & -66.4399 & 1.5 \\
\hline c26y & Bel-MBL & 21 & 228.5809 & -66.4563 & 1.5 \\
\hline$c 26 y-c 26 o$ & Bel-MBL & 21 & 228.6365 & -66.4727 & 1.5 \\
\hline$c 26 y-c 26 o$ & Bel-MBL & 21 & 228.7512 & -66.5087 & 1.5 \\
\hline$c 26 y-c 26 o$ & Bel-MBL & 21 & 228.8298 & -66.5284 & 1.5 \\
\hline$c 26 y-c 26 o$ & Bel-MBL & 21 & 228.8954 & -66.5447 & 1.5 \\
\hline$c 26 y-c 26 o$ & Bel-MBL & 21 & 228.9609 & -66.5644 & 1.5 \\
\hline$c 270-c 28 y$ & Bel-MBL & 21 & 230.4679 & -67.1918 & 1.5 \\
\hline$c 270-c 28 y$ & Bel-MBL & 21 & 230.5268 & -67.2147 & 1.5 \\
\hline c27o-c28y & Bel-MBL & 21 & 230.5923 & -67.2442 & 1.5 \\
\hline c27y & Bel-MBL & 21 & 229.7930 & -66.9428 & 1.5 \\
\hline c27y & Bel-MBL & 21 & 229.8683 & -66.9723 & 1.5 \\
\hline$c 27 y-c 27 o$ & Bel-MBL & 21 & 229.9470 & -67.0083 & 1.5 \\
\hline$c 27 y-c 27 o$ & Bel-MBL & 21 & 230.0125 & -67.0345 & 1.5 \\
\hline$c 27 y-c 27 o$ & Bel-MBL & 21 & 230.0715 & -67.0607 & 1.5 \\
\hline$c 27 y-c 27 o$ & Bel-MBL & 21 & 230.1337 & -67.0738 & 1.5 \\
\hline$c 27 y-c 27 o$ & Bel-MBL & 21 & 230.2123 & -67.0935 & 1.5 \\
\hline$c 27 y-c 27 o$ & Bel-MBL & 21 & 230.2746 & -67.1230 & 1.5 \\
\hline$c 27 y-c 27 o$ & Bel-MBL & 21 & 230.3335 & -67.1557 & 1.5 \\
\hline$c 27 y-c 28 y$ & Bel-MBL & 21 & 230.3991 & -67.1754 & 1.5 \\
\hline $\mathrm{c} 280$ & Bel-MBL & 21 & 231.1493 & -67.5128 & 1.5 \\
\hline c28o & Bel-MBL & 21 & 231.2312 & -67.5374 & 1.5 \\
\hline $\mathrm{c} 280$ & Bel-MBL & 21 & 231.3196 & -67.5603 & 1.5 \\
\hline c28o & Bel-MBL & 21 & 231.4015 & -67.5832 & 1.5 \\
\hline c28o & Bel-MBL & 21 & 231.5064 & -67.6029 & 1.5 \\
\hline $\mathrm{c} 280-c 290$ & Bel-MBL & 21 & 231.6046 & -67.6127 & 1.5 \\
\hline c28y & Bel-MBL & 21 & 230.6644 & -67.2802 & 1.5 \\
\hline c28y & Bel-MBL & 21 & 230.7299 & -67.3064 & 1.5 \\
\hline c28y & Bel-MBL & 21 & 230.7856 & -67.3392 & 1.5 \\
\hline c28y & Bel-MBL & 21 & 230.8610 & -67.3785 & 1.5 \\
\hline$c 28 y-c 280$ & Bel-MBL & 21 & 230.9331 & -67.4211 & 1.5 \\
\hline$c 28 y-c 280$ & Bel-MBL & 21 & 230.9887 & -67.4407 & 1.5 \\
\hline$c 28 y-c 280$ & Bel-MBL & 21 & 231.0313 & -67.4669 & 1.5 \\
\hline$c 28 y-c 280$ & Bel-MBL & 21 & 231.0969 & -67.4833 & 1.5 \\
\hline$c 290-c 30 y$ & Bel-MBL & 21 & 231.9454 & -67.7208 & 1.5 \\
\hline$c 290-c 30 y$ & Bel-MBL & 21 & 232.0436 & -67.7438 & 1.5 \\
\hline$c 290-c 30 y$ & Bel-MBL & 21 & \multicolumn{2}{|c|}{$227.13 ? 1,1,9.7-2$} & $15, .0$ \\
\hline \multicolumn{2}{|c|}{\begin{tabular}{|l|l|}
$c 29 o-c 30 y$ & Bel-MB
\end{tabular}} & 21 & \begin{tabular}{l|l} 
& 232.2205 \\
\end{tabular} & -67.8027 & 1.5 \\
\hline \multicolumn{2}{|c|}{$\begin{array}{l}c 29 o-c 30 y \\
c 29 y-c 29 o\end{array}$} & 21 & \multicolumn{2}{|c|}{\begin{tabular}{|l|l|}
231.6964 & -67.6488 \\
\end{tabular}} & 1.5 \\
\hline \multicolumn{2}{|c|}{$c 29 y-c 290$} & 21 & 231.775 & -67.675 & 1.5 \\
\hline
\end{tabular}




\begin{tabular}{|c|c|c|c|c|c|}
\hline age (chron) & plate & segment & longitude & latitude & uncertainty $(\mathrm{km})$ \\
\hline$c 29 y-c 290$ & Bel-MBL & 21 & 231.8569 & -67.6979 & 1.5 \\
\hline c30y & Bel-MBL & 21 & 232.2959 & -67.8486 & 1.5 \\
\hline$c 30 y$ & Bel-MBL & 21 & 232.3942 & -67.8781 & 1.5 \\
\hline $\mathrm{c} 30 \mathrm{y}$ & Bel-MBL & 21 & 232.4720 & -67.9129 & 1.5 \\
\hline$c 32.10-c 32.2 y$ & Bel-MBL & 21 & 232.5531 & -67.9547 & 1.5 \\
\hline$c 32.10-c 32.2 y$ & Bel-MBL & 21 & 232.6047 & -67.9768 & 1.5 \\
\hline c32.1y & Bel-MBL & 21 & 232.2959 & -67.8486 & 1.5 \\
\hline c32.1y & Bel-MBL & 21 & 232.3942 & -67.8781 & 1.5 \\
\hline$c 32.1 y-c 32.2 y$ & Bel-MBL & 21 & 232.4720 & -67.9129 & 1.5 \\
\hline$c 32.2 y$ & Bel-MBL & 21 & 232.6685 & -68.0136 & 1.5 \\
\hline c32.2y & Bel-MBL & 21 & 232.7496 & -68.0505 & 1.5 \\
\hline c32.2y & Bel-MBL & 21 & 232.8110 & -68.0751 & 1.5 \\
\hline c32.2y & Bel-MBL & 21 & 232.8626 & -68.1070 & 1.5 \\
\hline c32.2y & Bel-MBL & 21 & 232.9216 & -68.1316 & 1.5 \\
\hline c32.2y & Bel-MBL & 21 & 232.9953 & -68.1635 & 1.5 \\
\hline c32.2y & Bel-MBL & 21 & 233.0494 & -68.1856 & 1.5 \\
\hline $\mathrm{c} 32.2 \mathrm{y}$ & Bel-MBL & 21 & 233.1182 & -68.2077 & 1.5 \\
\hline$c 32.2 y-c 32.20$ & Bel-MBL & 21 & 233.1845 & -68.2421 & 1.5 \\
\hline$c 32.2 y-c 32.20$ & Bel-MBL & 21 & 233.2533 & -68.2692 & 1.5 \\
\hline$c 32.2 y-c 32.20$ & Bel-MBL & 21 & 233.3221 & -68.2864 & 1.5 \\
\hline$c 32.2 y-c 32 r$ & Bel-MBL & 21 & 233.3983 & -68.3159 & 1.5 \\
\hline$c 32 r$ & Bel-MBL & 21 & 233.4646 & -68.3503 & 1.5 \\
\hline$c 32 r-c 33 y$ & Bel-MBL & 21 & 233.5334 & -68.3797 & 1.5 \\
\hline$c 33 y$ & Bel-MBL & 21 & 233.5948 & -68.4068 & 1.5 \\
\hline c33y & Bel-MBL & 21 & 233.6415 & -68.4313 & 1.5 \\
\hline c20o & Pac & 21 & 193.4129 & -55.8823 & 1.5 \\
\hline c20o & Pac & 21 & 193.4693 & -55.9120 & 1.5 \\
\hline$c 200$ & Pac & 21 & 193.5375 & -55.9505 & 1.5 \\
\hline$c 20 \circ$ & Pac & 21 & 193.6206 & -55.9802 & 1.5 \\
\hline$c 20 y$ & Pac & 21 & 193.6799 & -55.9950 & 1.5 \\
\hline$c 20 y$ & Pac & 21 & 193.7481 & -56.0099 & 1.5 \\
\hline $\mathrm{c} 20 \mathrm{y}$ & Pac & 21 & 193.8194 & -56.0484 & 1.5 \\
\hline$c 20 y$ & Pac & 21 & 193.8698 & -56.0751 & 1.5 \\
\hline c21o & Pac & 21 & 192.7187 & -55.5678 & 1.5 \\
\hline c210 & Pac & 21 & 192.7810 & -55.6034 & 1.5 \\
\hline c21o & Pac & 21 & 192.8225 & -55.6301 & 1.5 \\
\hline$c 210-c 22 y$ & Pac & 21 & 192.6534 & -55.5426 & 1.5 \\
\hline c21y & Pac & 21 & 193.0124 & -55.7043 & 1.5 \\
\hline c21y & Pac & 21 & 193.0628 & -55.7280 & 1.5 \\
\hline c21y & Pac & 21 & 193.1281 & -55.7547 & 1.5 \\
\hline c22o & Pac & 21 & 192.4546 & -55.4417 & 1.5 \\
\hline c22y & Pac & 21 & 192.6059 & -55.5100 & 1.5 \\
\hline$c 22 y-c 220$ & Pac & 21 & 192.4961 & -55.4625 & 1.5 \\
\hline$c 22 y-c 220$ & Pac & 21 & 192.5288 & -55.4803 & 1.5 \\
\hline$c 22 y-c 220$ & Pac & 21 & 192.5674 & -55.4981 & 1.5 \\
\hline c23o & Pac & 21 & 192.1016 & -55.2340 & 1.5 \\
\hline$c 230$ & Pac & 21 & 192.1431 & -55.2607 & 1.5 \\
\hline c23o-c24y & Pac & 21 & 192.0571 & -55.2044 & 1.5 \\
\hline$c 23 y$ & Pac & 21 & 192.2410 & -55.3112 & 1.5 \\
\hline c23y & Pac & 21 & 192.2825 & -55.3230 & 1.5 \\
\hline c23y & Pac & 21 & 192.3211 & -55.3527 & 1.5 \\
\hline c24o & Pac & 21 & 191.7099 & -54.9967 & 1.5 \\
\hline c24o & Pac & 21 & 191.7722 & -55.0442 & 1.5 \\
\hline c24o & Pac & 21 & 191.8345 & -55.0798 & 1.5 \\
\hline c24y & Pac & 21 & 191.9592 & -55.1421 & 1.5 \\
\hline c24y & Pac & 21 & 192.0037 & -55.1717 & 1.5 \\
\hline c25o-c26o & Pac & 21 & 190.4105 & -54.1081 & 1.5 \\
\hline c25o-c26o & Pac & 21 & 190.4787 & -54.1408 & 1.5 \\
\hline
\end{tabular}




\begin{tabular}{|c|c|c|c|c|c|}
\hline age (chron) & plate & segment & longitude & latitude & uncertainty $(\mathrm{km})$ \\
\hline$c 25 y$ & $\mathrm{Pac}$ & 21 & 190.9890 & -54.4790 & 1.5 \\
\hline$c 25 y$ & $\mathrm{Pac}$ & 21 & 191.0276 & -54.5057 & 1.5 \\
\hline$c 25 y$ & Pac & 21 & 191.0691 & -54.5264 & 1.5 \\
\hline$c 25 y-c 250$ & $\mathrm{Pac}$ & 21 & 190.5825 & -54.2149 & 1.5 \\
\hline$c 25 y-c 25 o$ & Pac & 21 & 190.6330 & -54.2416 & 1.5 \\
\hline$c 25 y-c 250$ & Pac & 21 & 190.6745 & -54.2624 & 1.5 \\
\hline$c 25 y-c 25 o$ & Pac & 21 & 190.7012 & -54.2891 & 1.5 \\
\hline$c 25 y-c 250$ & Pac & 21 & 190.7398 & -54.3158 & 1.5 \\
\hline$c 25 y-c 250$ & Pac & 21 & 190.7724 & -54.3455 & 1.5 \\
\hline$c 25 y-c 250$ & Pac & 21 & 190.8110 & -54.3692 & 1.5 \\
\hline$c 25 y-c 25 o$ & $\mathrm{Pac}$ & 21 & 190.8555 & -54.3989 & 1.5 \\
\hline$c 25 y-c 25 o$ & Pac & 21 & 190.9059 & -54.4226 & 1.5 \\
\hline$c 25 y-c 250$ & Pac & 21 & 190.9475 & -54.4463 & 1.5 \\
\hline$c 25 y-c 26 y$ & Pac & 21 & 190.5410 & -54.1823 & 1.5 \\
\hline c26o & Pac & 21 & 189.9684 & -53.8233 & 1.5 \\
\hline c26o & $\mathrm{Pac}$ & 21 & 190.0426 & -53.8737 & 1.5 \\
\hline$c 26 y-c 26 o$ & $\mathrm{Pac}$ & 21 & 190.0989 & -53.9093 & 1.5 \\
\hline$c 26 y-c 26 o$ & $\mathrm{Pac}$ & 21 & 190.1553 & -53.9390 & 1.5 \\
\hline$c 26 y-c 26 o$ & Pac & 21 & 190.1880 & -53.9628 & 1.5 \\
\hline$c 26 y-c 26 o$ & Pac & 21 & 190.2414 & -53.9924 & 1.5 \\
\hline$c 26 y-c 260$ & Pac & 21 & 190.2829 & -54.0221 & 1.5 \\
\hline$c 26 y-c 26 o$ & Pac & 21 & 190.3155 & -54.0518 & 1.5 \\
\hline$c 26 y-c 26 o$ & Pac & 21 & 190.3630 & -54.0785 & 1.5 \\
\hline$c 270-c 28 y$ & Pac & 21 & 188.9478 & -53.1988 & 1.5 \\
\hline c27o-c28y & Pac & 21 & 188.9983 & -53.2196 & 1.5 \\
\hline c27y & Pac & 21 & 189.5442 & -53.5800 & 1.5 \\
\hline c27y & Pac & 21 & 189.5916 & -53.6186 & 1.5 \\
\hline$c 27 y-c 270$ & Pac & 21 & 189.1852 & -53.3620 & 1.5 \\
\hline$c 27 y-c 270$ & Pac & 21 & 189.2237 & -53.3916 & 1.5 \\
\hline$c 27 y-c 27 o$ & Pac & 21 & 189.2742 & -53.4272 & 1.5 \\
\hline$c 27 y-c 27 o$ & Pac & 21 & 189.3454 & -53.4673 & 1.5 \\
\hline$c 27 y-c 270$ & Pac & 21 & 189.4255 & -53.5118 & 1.5 \\
\hline$c 27 y-c 27 o$ & $\mathrm{Pac}$ & 21 & 189.4878 & -53.5444 & 1.5 \\
\hline$c 27 y-c 28 y$ & Pac & 21 & 189.0695 & -53.2700 & 1.5 \\
\hline$c 27 y-c 28 y$ & Pac & 21 & 189.1318 & -53.3175 & 1.5 \\
\hline c28o-c29o & Pac & 21 & 188.2061 & -52.8131 & 1.5 \\
\hline c28o-c29o & Pac & 21 & 188.2565 & -52.8398 & 1.5 \\
\hline c28o-c29o & Pac & 21 & 188.3129 & -52.8754 & 1.5 \\
\hline $\mathrm{c} 280-\mathrm{c} 290$ & Pac & 21 & 188.3871 & -52.9021 & 1.5 \\
\hline c28o-c29y & Pac & 21 & 188.4435 & -52.9377 & 1.5 \\
\hline c28o-c29y & Pac & 21 & 188.5028 & -52.9614 & 1.5 \\
\hline$c 280-c 29 y$ & Pac & 21 & 188.5325 & -52.9822 & 1.5 \\
\hline c28y & Pac & 21 & 188.8232 & -53.1484 & 1.5 \\
\hline c28y & Pac & 21 & 188.8885 & -53.1721 & 1.5 \\
\hline$c 28 y-c 280$ & Pac & 21 & 188.6927 & -53.0890 & 1.5 \\
\hline$c 28 y-c 280$ & Pac & 21 & 188.7550 & -53.1217 & 1.5 \\
\hline$c 28 y-c 29 y$ & Pac & 21 & 188.5859 & -53.0149 & 1.5 \\
\hline$c 28 y-c 29 y$ & Pac & 21 & 188.6452 & -53.0623 & 1.5 \\
\hline c29o-c30y & Pac & 21 & 187.9035 & -52.6915 & 1.5 \\
\hline$c 290-c 30 y$ & Pac & 21 & 187.9806 & -52.7182 & 1.5 \\
\hline$c 290-c 30 y$ & Pac & 21 & 188.0518 & -52.7419 & 1.5 \\
\hline c29o-c30y & Pac & 21 & 188.1349 & -52.7834 & 1.5 \\
\hline c30o-c31o & Pac & 21 & 186.6248 & -52.2405 & 1.5 \\
\hline c30o-c31o & Pac & 21 & 186.6990 & -52.2613 & 1.5 \\
\hline $\mathrm{c} 30 \mathrm{o}-\mathrm{c} 310$ & Pac & 21 & 186.7731 & -52.2731 & 1.5 \\
\hline$c 300-c 310$ & Pac & 21 & 186.8295 & -52.2731 & 1.5 \\
\hline$c 300-c 310$ & Pac & 21 & 186.8948 & -52.2731 & 1.5 \\
\hline c30o-c31o & Pac & 21 & 186.9719 & -52.2731 & 1.5 \\
\hline
\end{tabular}




\begin{tabular}{|c|c|c|c|c|c|}
\hline age (chron) & plate & segment & longitude & latitude & uncertainty $(\mathrm{km})$ \\
\hline $\mathrm{c} 300-\mathrm{c} 310$ & $\mathrm{Pac}$ & 21 & 187.0461 & -52.2909 & 1.5 \\
\hline $\mathrm{c} 30 \mathrm{o}-\mathrm{c} 31 \mathrm{o}$ & Pac & 21 & 187.1025 & -52.3354 & 1.5 \\
\hline c30o-c31o & Pac & 21 & 187.1559 & -52.3592 & 1.5 \\
\hline$c 300-c 310$ & Pac & 21 & 187.2330 & -52.3888 & 1.5 \\
\hline$c 300-c 310$ & Pac & 21 & 187.3190 & -52.4126 & 1.5 \\
\hline$c 30 o-c 310$ & Pac & 21 & 187.3932 & -52.4512 & 1.5 \\
\hline c30y & Pac & 21 & 187.5920 & -52.5342 & 1.5 \\
\hline$c 30 y$ & Pac & 21 & 187.6483 & -52.5669 & 1.5 \\
\hline c30y & Pac & 21 & 187.7107 & -52.5936 & 1.5 \\
\hline c30y & Pac & 21 & 187.7670 & -52.6292 & 1.5 \\
\hline$c 30 y$ & Pac & 21 & 187.8204 & -52.6618 & 1.5 \\
\hline$c 30 y-c 310$ & Pac & 21 & 187.4644 & -52.4719 & 1.5 \\
\hline$c 30 y-c 31 y$ & Pac & 21 & 187.5208 & -52.4927 & 1.5 \\
\hline c310 & Pac & 21 & 186.3251 & -52.0862 & 1.5 \\
\hline c310 & Pac & 21 & 186.3815 & -52.1189 & 1.5 \\
\hline c310 & Pac & 21 & 186.4646 & -52.1545 & 1.5 \\
\hline c310 & Pac & 21 & 186.5150 & -52.1841 & 1.5 \\
\hline c31o-c32.1y & Pac & 21 & 186.2035 & -52.0358 & 1.5 \\
\hline c31o-c32.1y & Pac & 21 & 186.2717 & -52.0536 & 1.5 \\
\hline c310-c32.2y & Pac & 21 & 186.1412 & -51.9972 & 1.5 \\
\hline c31y & Pac & 21 & 187.5920 & -52.5342 & 1.5 \\
\hline c31y & Pac & 21 & 187.6483 & -52.5669 & 1.5 \\
\hline c31y & Pac & 21 & 187.7107 & -52.5936 & 1.5 \\
\hline c31y-c31o & Pac & 21 & 186.5595 & -52.2197 & 1.5 \\
\hline c32.10 & Pac & 21 & 184.8061 & -50.8446 & 1.5 \\
\hline$c 32.1 y-c 32.10$ & Pac & 21 & 184.8655 & -50.9099 & 1.5 \\
\hline$c 32.1 y-c 32.10$ & Pac & 21 & 184.9218 & -50.9663 & 1.5 \\
\hline$c 32.1 y-c 32.20$ & Pac & 21 & 185.0909 & -51.1176 & 1.5 \\
\hline c32.1y-c32.2y & Pac & 21 & 185.5834 & -51.6516 & 1.5 \\
\hline$c 32.1 y-c 32.2 y$ & Pac & 21 & 185.6398 & -51.6931 & 1.5 \\
\hline c32.1y-c32.2y & Pac & 21 & 185.6962 & -51.7317 & 1.5 \\
\hline$c 32.1 y-c 32.2 y$ & Pac & 21 & 185.7525 & -51.7703 & 1.5 \\
\hline$c 32.1 y-c 32.2 y$ & Pac & 21 & 185.8237 & -51.8044 & 1.5 \\
\hline$c 32.1 y-c 32.2 y$ & Pac & 21 & 185.8950 & -51.8489 & 1.5 \\
\hline$c 32.1 y-c 32.2 y$ & Pac & 21 & 185.9632 & -51.8756 & 1.5 \\
\hline$c 32.1 y-c 32.2 y$ & Pac & 21 & 186.0255 & -51.9171 & 1.5 \\
\hline c32.1y-c32.2y & Pac & 21 & 186.0819 & -51.9527 & 1.5 \\
\hline c32.1yc32r & Pac & 21 & 185.5300 & -51.6012 & 1.5 \\
\hline$c 32.1 y-c 32 r$ & Pac & 21 & 185.4796 & -51.5537 & 1.5 \\
\hline$c 32.1 y-c 33 y$ & Pac & 21 & 184.9782 & -51.0137 & 1.5 \\
\hline$c 32.1 y-c 33 y$ & Pac & 21 & 185.0316 & -51.0701 & 1.5 \\
\hline$c 32.1 y-c 33 y$ & Pac & 21 & 185.1325 & -51.1680 & 1.5 \\
\hline$c 32.1 y-c 33 y$ & Pac & 21 & 185.1740 & -51.2392 & 1.5 \\
\hline c32.1y-c33y & Pac & 21 & 185.2422 & -51.3134 & 1.5 \\
\hline$c 32.1 y-c 33 y$ & Pac & 21 & 185.2986 & -51.3608 & 1.5 \\
\hline$c 32.1 y-c 33 y$ & Pac & 21 & 185.3520 & -51.4320 & 1.5 \\
\hline$c 32.1 y-c 33 y$ & Pac & 21 & 185.4114 & -51.4884 & 1.5 \\
\hline$c 32.20-c 33 y$ & Pac & 21 & 184.7082 & -50.7348 & 1.5 \\
\hline$c 32.20-c 33 y$ & Pac & 21 & 184.7557 & -50.7912 & 1.5 \\
\hline$c 32.20-c 33 y$ & Pac & 21 & 184.8061 & -50.8446 & 1.5 \\
\hline$c 32.20-c 33 y$ & Pac & 21 & 184.8655 & -50.9099 & 1.5 \\
\hline$c 32.20-c 33 y$ & Pac & 21 & 184.9218 & -50.9663 & 1.5 \\
\hline$c 32 r-c 33 y$ & Pac & 21 & 184.5510 & -50.5628 & 1.5 \\
\hline$c 32 r-c 33 y$ & Pac & 21 & 184.5925 & -50.6132 & 1.5 \\
\hline$c 32 r-c 33 y$ & Pac & 21 & 184.6459 & -50.6814 & 1.5 \\
\hline c33y & Pac & 21 & 184.4946 & -50.4500 & 1.5 \\
\hline c33y & Pac & 21 & 184.5213 & -50.5064 & 1.5 \\
\hline c20o & MBL & 35 & 204.9890 & -68.4459 & 1.5 \\
\hline
\end{tabular}




\begin{tabular}{|c|c|c|c|c|c|}
\hline age (chron) & plate & segment & longitude & latitude & uncertainty $(\mathrm{km})$ \\
\hline $\mathrm{c} 200$ & $\mathrm{MBL}$ & 35 & 205.0584 & -68.4791 & 1.5 \\
\hline$c 20 \circ$ & $\mathrm{MBL}$ & 35 & 205.1187 & -68.5122 & 1.5 \\
\hline$c 20 \circ$ & MBL & 35 & 205.1971 & -68.5575 & 1.5 \\
\hline $\mathrm{c} 20 \mathrm{y}$ & $\mathrm{MBL}$ & 35 & 204.6392 & -68.3223 & 1.5 \\
\hline $\mathrm{c} 20 \mathrm{y}$ & $\mathrm{MBL}$ & 35 & 204.6965 & -68.3434 & 1.5 \\
\hline c210 & $\mathrm{MBL}$ & 35 & 205.7218 & -68.7384 & 1.5 \\
\hline c210 & MBL & 35 & 205.7972 & -68.7686 & 1.5 \\
\hline$c 210-c 22 y$ & $\mathrm{MBL}$ & 35 & 205.8635 & -68.7836 & 1.5 \\
\hline$c 220$ & MBL & 35 & 206.1892 & -68.9012 & 1.5 \\
\hline$c 220$ & MBL & 35 & 206.2706 & -68.9389 & 1.5 \\
\hline c22o & MBL & 35 & 206.3490 & -68.9661 & 1.5 \\
\hline$c 22 y$ & MBL & 35 & 205.9540 & -68.8168 & 1.5 \\
\hline $\mathrm{c} 22 \mathrm{y}$ & $\mathrm{MBL}$ & 35 & 206.0263 & -68.8409 & 1.5 \\
\hline$c 230$ & MBL & 35 & 206.8134 & -69.1470 & 1.5 \\
\hline $\mathrm{c} 230$ & MBL & 35 & 206.8616 & -69.1892 & 1.5 \\
\hline c23o-c24y & MBL & 35 & 206.8978 & -69.2314 & 1.5 \\
\hline$c 23 y$ & MBL & 35 & 206.4847 & -69.0324 & 1.5 \\
\hline$c 23 y$ & MBL & 35 & 206.5480 & -69.0535 & 1.5 \\
\hline c23y & MBL & 35 & 206.6355 & -69.0837 & 1.5 \\
\hline c24o & $\mathrm{MBL}$ & 35 & 207.0627 & -69.4290 & 1.5 \\
\hline c24o & MBL & 35 & 207.1350 & -69.4933 & 1.5 \\
\hline c24o & $\mathrm{MBL}$ & 35 & 207.1994 & -69.5375 & 1.5 \\
\hline c24y & MBL & 35 & 206.9370 & -69.2887 & 1.5 \\
\hline c24y & MBL & 35 & 206.9913 & -69.3581 & 1.5 \\
\hline$c 250$ & MBL & 35 & 207.9311 & -70.1527 & 1.5 \\
\hline$c 250$ & $\mathrm{MBL}$ & 35 & 207.9794 & -70.2250 & 1.5 \\
\hline c25y & MBL & 35 & 207.7180 & -69.9918 & 1.5 \\
\hline c25y & MBL & 35 & 207.7824 & -70.0361 & 1.5 \\
\hline$c 25 y-c 25 o$ & MBL & 35 & 207.8467 & -70.0883 & 1.5 \\
\hline$c 26 o$ & MBL & 35 & 208.3895 & -70.6653 & 1.5 \\
\hline c26y & MBL & 35 & 208.1804 & -70.4100 & 1.5 \\
\hline c26y & MBL & 35 & 208.2488 & -70.4864 & 1.5 \\
\hline$c 26 y-c 26 o$ & MBL & 35 & 208.2930 & -70.5346 & 1.5 \\
\hline$c 26 y-c 26 o$ & MBL & 35 & 208.3453 & -70.5949 & 1.5 \\
\hline c27o-c28o & MBL & 35 & 208.6790 & -71.2925 & 1.5 \\
\hline$c 27 y-c 28 y$ & MBL & 35 & 208.6026 & -71.1116 & 1.5 \\
\hline$c 27 y-c 28 y$ & MBL & 35 & 208.6347 & -71.1920 & 1.5 \\
\hline c28o-c29o & $\mathrm{MBL}$ & 35 & 208.7715 & -71.6464 & 1.5 \\
\hline $\mathrm{c} 280-c 290$ & $\mathrm{MBL}$ & 35 & 208.7956 & -71.7187 & 1.5 \\
\hline$c 280-c 30 y$ & $\mathrm{MBL}$ & 35 & 208.8358 & -71.8112 & 1.5 \\
\hline$c 28 y-c 29 y$ & MBL & 35 & 208.7111 & -71.4011 & 1.5 \\
\hline$c 28 y-c 29 y$ & MBL & 35 & 208.7192 & -71.4694 & 1.5 \\
\hline$c 28 y-c 29 y$ & MBL & 35 & 208.7433 & -71.5458 & 1.5 \\
\hline$c 290-c 30 y$ & $\mathrm{MBL}$ & 35 & 208.9001 & -72.0042 & 1.5 \\
\hline c29o-c30y & $\mathrm{MBL}$ & 35 & 208.9283 & -72.0766 & 1.5 \\
\hline c29o-c30y & MBL & 35 & 208.9484 & -72.1650 & 1.5 \\
\hline$c 29 y-c 30 y$ & MBL & 35 & 208.8639 & -71.9117 & 1.5 \\
\hline $\mathrm{c} 30 \mathrm{o}-\mathrm{c} 310$ & MBL & 35 & 209.0810 & -72.6857 & 1.5 \\
\hline c30o-c31o & $\mathrm{MBL}$ & 35 & 209.0891 & -72.7661 & 1.5 \\
\hline c30o-c31o & MBL & 35 & 209.1092 & -72.9390 & 1.5 \\
\hline$c 300-c 310$ & $\mathrm{MBL}$ & 35 & 209.1092 & -72.8626 & 1.5 \\
\hline c30o-c31y & MBL & 35 & 209.0368 & -72.4967 & 1.5 \\
\hline c30o-c31y & MBL & 35 & 209.0529 & -72.5731 & 1.5 \\
\hline c30y & MBL & 35 & 208.9765 & -72.2655 & 1.5 \\
\hline c30y-c31y & MBL & 35 & 209.0127 & -72.3841 & 1.5 \\
\hline c31o & MBL & 35 & 209.1212 & -73.1240 & 1.5 \\
\hline c31o & MBL & 35 & 209.1253 & -73.2164 & 1.5 \\
\hline c31y-c31o & MBL & 35 & 209.1172 & -73.0194 & 1.5 \\
\hline
\end{tabular}




\begin{tabular}{|c|c|c|c|c|c|}
\hline age (chron) & plate & segment & longitude & latitude & uncertainty $(\mathrm{km})$ \\
\hline$c 32.10$ & MBL & 35 & 209.1574 & -73.6245 & 1.5 \\
\hline c32.1y & MBL & 35 & 209.1454 & -73.3732 & 1.5 \\
\hline$c 32.1 y-c 32.10$ & MBL & 35 & 209.1494 & -73.4175 & 1.5 \\
\hline c32.1y-c32.10 & MBL & 35 & 209.1574 & -73.4939 & 1.5 \\
\hline$c 32.2 y-c 32 r$ & MBL & 35 & 209.1574 & -73.8256 & 1.5 \\
\hline$c 32.2 y-c 32 r$ & MBL & 35 & 209.1574 & -73.7331 & 1.5 \\
\hline$c 32.2 y-c 33 y$ & MBL & 35 & 209.1534 & -73.9140 & 1.5 \\
\hline c33y & MBL & 35 & 209.1414 & -74.1593 & 1.5 \\
\hline c33y & MBL & 35 & 209.1494 & -74.0346 & 1.5 \\
\hline c210 & Pac & 35 & 178.6995 & -59.8977 & 1.5 \\
\hline c210 & Pac & 35 & 178.7412 & -59.9590 & 1.5 \\
\hline c210-c22y & Pac & 35 & 178.6774 & -59.8535 & 1.5 \\
\hline $\mathrm{c} 22 \mathrm{o}$ & Pac & 35 & 178.5841 & -59.7037 & 1.5 \\
\hline c22o-c23o & Pac & 35 & 178.5473 & -59.6387 & 1.5 \\
\hline$c 22 y$ & Pac & 35 & 178.6406 & -59.8093 & 1.5 \\
\hline$c 22 y-c 22 o$ & Pac & 35 & 178.6160 & -59.7602 & 1.5 \\
\hline$c 230-c 24 y$ & Pac & 35 & 178.4466 & -59.4815 & 1.5 \\
\hline$c 23 y-c 230$ & Pac & 35 & 178.5178 & -59.5994 & 1.5 \\
\hline$c 23 y-c 24 y$ & Pac & 35 & 178.4834 & -59.5429 & 1.5 \\
\hline $\mathrm{c} 240$ & Pac & 35 & 178.2821 & -59.2508 & 1.5 \\
\hline c24o & Pac & 35 & 178.3116 & -59.2950 & 1.5 \\
\hline c24o & Pac & 35 & 178.3361 & -59.3318 & 1.5 \\
\hline c24o & Pac & 35 & 178.3681 & -59.3833 & 1.5 \\
\hline$c 24 y$ & Pac & 35 & 178.4024 & -59.4251 & 1.5 \\
\hline$c 250-c 26 o$ & Pac & 35 & 177.3517 & -58.5892 & 1.5 \\
\hline c25o-c26o & Pac & 35 & 177.3762 & -58.6186 & 1.5 \\
\hline$c 250-c 260$ & Pac & 35 & 177.4081 & -58.6628 & 1.5 \\
\hline$c 25 y$ & Pac & 35 & 177.7052 & -58.8482 & 1.5 \\
\hline$c 25 y$ & Pac & 35 & 177.7592 & -58.8850 & 1.5 \\
\hline$c 25 y-c 26 o$ & Pac & 35 & 177.4572 & -58.6947 & 1.5 \\
\hline$c 25 y-c 26 o$ & Pac & 35 & 177.5211 & -58.7377 & 1.5 \\
\hline$c 25 y-c 26 y$ & Pac & 35 & 177.5726 & -58.7671 & 1.5 \\
\hline$c 25 y-c 26 y$ & Pac & 35 & 177.6119 & -58.7892 & 1.5 \\
\hline$c 25 y-c 26 y$ & Pac & 35 & 177.6487 & -58.8187 & 1.5 \\
\hline $\mathrm{c} 26 \mathrm{o}$ & Pac & 35 & 177.1508 & -58.3382 & 1.5 \\
\hline$c 26 y-c 26 o$ & Pac & 35 & 177.1775 & -58.3872 & 1.5 \\
\hline$c 26 y-c 26 o$ & Pac & 35 & 177.2167 & -58.4247 & 1.5 \\
\hline$c 26 y-c 26 o$ & Pac & 35 & 177.2584 & -58.4664 & 1.5 \\
\hline$c 26 y-c 26 o$ & Pac & 35 & 177.2854 & -58.5130 & 1.5 \\
\hline$c 26 y-c 26 o$ & Pac & 35 & 177.3222 & -58.5548 & 1.5 \\
\hline c27o-c28o & Pac & 35 & 176.6299 & -57.5691 & 1.5 \\
\hline c27o-c28y & Pac & 35 & 176.6667 & -57.6059 & 1.5 \\
\hline c27y & Pac & 35 & 176.9791 & -58.0843 & 1.5 \\
\hline c27y & Pac & 35 & 177.0040 & -58.1331 & 1.5 \\
\hline$c 27 y-c 270$ & Pac & 35 & 176.8460 & -57.8527 & 1.5 \\
\hline$c 27 y-c 27 o$ & Pac & 35 & 176.8852 & -57.8993 & 1.5 \\
\hline$c 27 y-c 270$ & Pac & 35 & 176.9147 & -57.9509 & 1.5 \\
\hline$c 27 y-c 27 o$ & Pac & 35 & 176.9494 & -58.0214 & 1.5 \\
\hline$c 27 y-c 28 y$ & Pac & 35 & 176.7183 & -57.6661 & 1.5 \\
\hline$c 27 y-c 28 y$ & Pac & 35 & 176.7600 & -57.7201 & 1.5 \\
\hline$c 27 y-c 28 y$ & Pac & 35 & 176.7870 & -57.7569 & 1.5 \\
\hline$c 27 y-c 28 y$ & Pac & 35 & 176.8116 & -57.8011 & 1.5 \\
\hline$c 280-c 290$ & Pac & 35 & 176.3279 & -57.1665 & 1.5 \\
\hline $\mathrm{c} 280-\mathrm{c} 290$ & Pac & 35 & 176.3574 & -57.2352 & 1.5 \\
\hline c28o-c29o & Pac & 35 & 176.3844 & -57.2843 & 1.5 \\
\hline c28o-c30y & Pac & 35 & 176.2936 & -57.1223 & 1.5 \\
\hline$c 28 y-c 280$ & Pac & 35 & 176.5882 & -57.5323 & 1.5 \\
\hline$c 28 y-c 290$ & $\mathrm{Pac}$ & 35 & 176.4065 & -57.3310 & 1.5 \\
\hline
\end{tabular}




\begin{tabular}{|c|c|c|c|c|c|}
\hline age (chron) & plate & segment & longitude & latitude & uncertainty $(\mathrm{km})$ \\
\hline$c 28 y-c 29 y$ & $\mathrm{Pac}$ & 35 & 176.4458 & -57.3850 & 1.5 \\
\hline$c 28 y-c 29 y$ & Pac & 35 & 176.4728 & -57.4267 & 1.5 \\
\hline$c 28 y-c 29 y$ & Pac & 35 & 176.5121 & -57.4709 & 1.5 \\
\hline$c 28 y-c 29 y$ & Pac & 35 & 176.5514 & -57.4979 & 1.5 \\
\hline c29o-c30y & Pac & 35 & 176.1119 & -56.7933 & 1.5 \\
\hline$c 290-c 30 y$ & Pac & 35 & 176.1340 & -56.8351 & 1.5 \\
\hline c29o-c30y & Pac & 35 & 176.1610 & -56.8891 & 1.5 \\
\hline c29o-c30y & Pac & 35 & 176.1905 & -56.9504 & 1.5 \\
\hline$c 29 y-c 30 y$ & Pac & 35 & 176.2248 & -57.0094 & 1.5 \\
\hline$c 29 y-c 30 y$ & Pac & 35 & 176.2592 & -57.0683 & 1.5 \\
\hline$c 300-c 310$ & Pac & 35 & 175.7658 & -56.1366 & 1.5 \\
\hline $\mathrm{c} 300-\mathrm{c} 310$ & Pac & 35 & 175.7878 & -56.1931 & 1.5 \\
\hline$c 300-c 310$ & Pac & 35 & 175.8124 & -56.2422 & 1.5 \\
\hline c30o-c31o & Pac & 35 & 175.8320 & -56.3011 & 1.5 \\
\hline $\mathrm{c} 30 \mathrm{o}-\mathrm{c} 31 \mathrm{o}$ & Pac & 35 & 175.8492 & -56.3453 & 1.5 \\
\hline c30o-c31o & Pac & 35 & 175.8738 & -56.4042 & 1.5 \\
\hline$c 300-c 310$ & Pac & 35 & 175.9131 & -56.4680 & 1.5 \\
\hline c30o-c31o & Pac & 35 & 175.9450 & -56.5269 & 1.5 \\
\hline $\mathrm{c} 30 \mathrm{o}-\mathrm{c} 310$ & Pac & 35 & 175.9769 & -56.5736 & 1.5 \\
\hline c30o-c31y & Pac & 35 & 175.9965 & -56.6006 & 1.5 \\
\hline c30o-c31y & Pac & 35 & 176.0211 & -56.6448 & 1.5 \\
\hline c30y-c31y & Pac & 35 & 176.0554 & -56.6890 & 1.5 \\
\hline$c 30 y-c 31 y$ & Pac & 35 & 176.0751 & -56.7442 & 1.5 \\
\hline c31o-c32.1y & Pac & 35 & 175.7142 & -56.0175 & 1.5 \\
\hline c31o-c32.1y & Pac & 35 & 175.7216 & -56.0642 & 1.5 \\
\hline c310-c32.2y & $\mathrm{Pac}$ & 35 & 175.6381 & -55.8113 & 1.5 \\
\hline c310-c32.2y & Pac & 35 & 175.6602 & -55.8825 & 1.5 \\
\hline c310-c32.2y & Pac & 35 & 175.6749 & -55.9193 & 1.5 \\
\hline c310-c32.2y & Pac & 35 & 175.6946 & -55.9586 & 1.5 \\
\hline c32.10-c32.2o & Pac & 35 & 175.4294 & -55.2663 & 1.5 \\
\hline $\mathrm{c} 32.10-\mathrm{c} 32.20$ & Pac & 35 & 175.4515 & -55.3203 & 1.5 \\
\hline$c 32.1 y-c 32.20$ & $\mathrm{Pac}$ & 35 & 175.5448 & -55.5143 & 1.5 \\
\hline$c 32.1 y-c 32.20$ & $\mathrm{Pac}$ & 35 & 175.5644 & -55.5535 & 1.5 \\
\hline c32.1y-c32.2y & Pac & 35 & 175.5914 & -55.5977 & 1.5 \\
\hline$c 32.1 y-c 32.2 y$ & Pac & 35 & 175.5988 & -55.6542 & 1.5 \\
\hline$c 32.1 y-c 32.2 y$ & Pac & 35 & 175.6062 & -55.6984 & 1.5 \\
\hline c32.1y-c32.2y & Pac & 35 & 175.6209 & -55.7426 & 1.5 \\
\hline$c 32.1 y-c 32 r$ & Pac & 35 & 175.5006 & -55.4332 & 1.5 \\
\hline$c 32.1 y-c 32 r$ & Pac & 35 & 175.5178 & -55.4676 & 1.5 \\
\hline c32.1y-c33y & Pac & 35 & 175.4785 & -55.3792 & 1.5 \\
\hline$c 32.2 y-c 32.20$ & Pac & 35 & 175.4098 & -55.2344 & 1.5 \\
\hline c33o & Pac & 35 & 174.3148 & -53.4177 & 1.5 \\
\hline c33o & Pac & 35 & 174.3468 & -53.4570 & 1.5 \\
\hline c33o & Pac & 35 & 174.3787 & -53.4938 & 1.5 \\
\hline c33o & Pac & 35 & 174.4351 & -53.5282 & 1.5 \\
\hline c33o & Pac & 35 & 174.4793 & -53.5674 & 1.5 \\
\hline c33o & Pac & 35 & 174.5211 & -53.6165 & 1.5 \\
\hline c33o & Pac & 35 & 174.5653 & -53.6583 & 1.5 \\
\hline c33o & Pac & 35 & 174.6217 & -53.7123 & 1.5 \\
\hline c33o & Pac & 35 & 174.6610 & -53.7749 & 1.5 \\
\hline$c 330$ & Pac & 35 & 174.6831 & -53.8289 & 1.5 \\
\hline c33o & Pac & 35 & 174.7052 & -53.8805 & 1.5 \\
\hline c33o & Pac & 35 & 174.7248 & -53.9246 & 1.5 \\
\hline c33o & Pac & 35 & 174.7494 & -53.9713 & 1.5 \\
\hline c33o & Pac & 35 & 174.7715 & -54.0155 & 1.5 \\
\hline c33o & Pac & 35 & 174.8034 & -54.0769 & 1.5 \\
\hline c33y & Pac & 35 & 175.4294 & -55.2663 & 1.5 \\
\hline c33y & $\mathrm{Pac}$ & 35 & 175.4515 & -55.3203 & 1.5 \\
\hline
\end{tabular}




\begin{tabular}{|c|c|c|c|c|c|}
\hline age (chron) & plate & segment & longitude & latitude & uncertainty $(\mathrm{km})$ \\
\hline c20y & MBL & 37 & 201.4858 & -68.1922 & 1.5 \\
\hline $\mathrm{c} 20 \mathrm{y}$ & MBL & 37 & 201.7211 & -68.2606 & 1.5 \\
\hline c20y & MBL & 37 & 201.9292 & -68.3274 & 1.5 \\
\hline$c 20 y-c 20 \circ$ & MBL & 37 & 201.9929 & -68.3562 & 1.5 \\
\hline$c 20 y-c 20 \circ$ & MBL & 37 & 202.2268 & -68.4526 & 1.5 \\
\hline$c 20 y-c 210$ & MBL & 37 & 202.6321 & -68.6363 & 1.5 \\
\hline$c 20 y-c 21 y$ & MBL & 37 & 202.5031 & -68.5695 & 1.5 \\
\hline$c 20 y-c 220$ & MBL & 37 & 202.7992 & -68.7259 & 1.5 \\
\hline c21o-c23o & MBL & 37 & 203.2243 & -69.0941 & 1.5 \\
\hline$c 21 y-c 23 y$ & MBL & 37 & 203.0269 & -68.9218 & 1.5 \\
\hline$c 220-c 230$ & MBL & 37 & 203.3442 & -69.2141 & 1.5 \\
\hline $\mathrm{c} 230-c 240$ & MBL & 37 & 203.6787 & -69.4779 & 1.5 \\
\hline$c 230-c 24 y$ & MBL & 37 & 203.5143 & -69.3340 & 1.5 \\
\hline c24o & MBL & 37 & 203.8271 & -69.6230 & 1.5 \\
\hline$c 24 y-c 240$ & $\mathrm{MBL}$ & 37 & 203.7679 & -69.5709 & 1.5 \\
\hline$c 250-c 26 o$ & MBL & 37 & 204.4619 & -70.3363 & 1.5 \\
\hline$c 25 y$ & MBL & 37 & 204.0393 & -69.8876 & 1.5 \\
\hline$c 25 y-c 25 o$ & MBL & 37 & 204.1498 & -70.0177 & 1.5 \\
\hline$c 25 y-c 25 o$ & MBL & 37 & 204.2929 & -70.1867 & 1.5 \\
\hline$c 26 \circ$ & MBL & 37 & 204.7935 & -70.6549 & 1.5 \\
\hline$c 26 y-c 26 o$ & MBL & 37 & 204.5660 & -70.4468 & 1.5 \\
\hline$c 26 y-c 26 o$ & MBL & 37 & 204.7025 & -70.5638 & 1.5 \\
\hline c27y & $\mathrm{MBL}$ & 37 & 205.0552 & -71.1677 & 1.5 \\
\hline $\mathrm{c} 20 \mathrm{o}$ & Pac & 37 & 177.8919 & -60.7606 & 1.5 \\
\hline$c 200$ & Pac & 37 & 177.9375 & -60.7805 & 1.5 \\
\hline $\mathrm{c} 20 \mathrm{y}$ & Pac & 37 & 178.1002 & -60.8497 & 1.5 \\
\hline c20y & Pac & 37 & 178.2192 & -60.8858 & 1.5 \\
\hline$c 20 y-c 20 \circ$ & Pac & 37 & 178.0246 & -60.8183 & 1.5 \\
\hline c210 & Pac & 37 & 177.4187 & -60.5056 & 1.5 \\
\hline $\mathrm{c} 210-\mathrm{c} 220$ & Pac & 37 & 177.1927 & -60.3489 & 1.5 \\
\hline$c 210-c 22 y$ & Pac & 37 & 177.3147 & -60.4321 & 1.5 \\
\hline c21y & Pac & 37 & 177.5161 & -60.5544 & 1.5 \\
\hline c21y & Pac & 37 & 177.5808 & -60.5964 & 1.5 \\
\hline c21y & Pac & 37 & 177.6438 & -60.6356 & 1.5 \\
\hline c22o-c23o & Pac & 37 & 176.9366 & -60.1887 & 1.5 \\
\hline$c 22 y-c 23 y$ & Pac & 37 & 177.0467 & -60.2518 & 1.5 \\
\hline c23o-c24y & Pac & 37 & 176.7950 & -60.1063 & 1.5 \\
\hline$c 23 y-c 230$ & Pac & 37 & 176.8731 & -60.1564 & 1.5 \\
\hline c24o & $\mathrm{Pac}$ & 37 & 176.0804 & -59.6297 & 1.5 \\
\hline c24o & Pac & 37 & 176.1630 & -59.7091 & 1.5 \\
\hline c24o & Pac & 37 & 176.2626 & -59.7821 & 1.5 \\
\hline c24o & Pac & 37 & 176.3489 & -59.8475 & 1.5 \\
\hline c24o & $\mathrm{Pac}$ & 37 & 176.4351 & -59.8981 & 1.5 \\
\hline c24o & Pac & 37 & 176.5050 & -59.9391 & 1.5 \\
\hline c24o & Pac & 37 & 176.5640 & -59.9813 & 1.5 \\
\hline$c 240-c 25 y$ & Pac & 37 & 175.9702 & -59.5425 & 1.5 \\
\hline$c 24 y$ & Pac & 37 & 176.6344 & -60.0259 & 1.5 \\
\hline c24y & Pac & 37 & 176.7088 & -60.0676 & 1.5 \\
\hline c25o-c26o & Pac & 37 & 175.4122 & -59.1158 & 1.5 \\
\hline c25y & Pac & 37 & 175.8905 & -59.4875 & 1.5 \\
\hline$c 25 y-c 25 o$ & Pac & 37 & 175.6407 & -59.3022 & 1.5 \\
\hline$c 25 y-c 250$ & Pac & 37 & 175.7834 & -59.4113 & 1.5 \\
\hline$c 25 y-c 26 y$ & Pac & 37 & 175.5050 & -59.1927 & 1.5 \\
\hline c26o & $\mathrm{Pac}$ & 37 & 175.1410 & -58.8842 & 1.5 \\
\hline$c 26 y-c 26 o$ & Pac & 37 & 175.2481 & -58.9579 & 1.5 \\
\hline$c 26 y-c 26 o$ & Pac & 37 & 175.3337 & -59.0388 & 1.5 \\
\hline $\mathrm{c} 20 \mathrm{o}$ & MBL & 39 & 199.2849 & -69.2516 & 1.5 \\
\hline c20o & MBL & 39 & 199.3260 & -69.2901 & 1.5 \\
\hline
\end{tabular}




\begin{tabular}{|c|c|c|c|c|c|c|c|c|}
\hline age (chron) & \multicolumn{2}{|c|}{ plate } & segment & $\frac{\text { longitude }}{1993593}$ & \multicolumn{2}{|c|}{ latitude } & \multicolumn{2}{|c|}{ uncertainty $(\mathrm{km})$} \\
\hline $\mathrm{c} 200$ & \multicolumn{2}{|c|}{$\mathrm{MBL}$} & 39 & 199.3593 & \multirow{2}{*}{\multicolumn{2}{|c|}{$\begin{array}{l}-69.3287 \\
-690936\end{array}$}} & \multirow{2}{*}{\multicolumn{2}{|c|}{$\begin{array}{l}1.5 \\
15\end{array}$}} \\
\hline$c 20 y$ & \multicolumn{2}{|c|}{$\mathrm{MBL}$} & 39 & 198.9484 & & & & \\
\hline$c 20 y$ & & & 39 & 199.0434 & -69 & 1334 & & $\frac{1.5}{1.5}$ \\
\hline $\mathrm{c} 20 \mathrm{y}$ & & & 39 & 199.1230 & -69 & 1668 & & 1.5 \\
\hline c21o-c22o & & & 39 & 199.5751 & -69 & 5804 & & 1.5 \\
\hline$c 210-c 22 y$ & & & 39 & 199.5392 & -69 & 5470 & & 1.5 \\
\hline c21y & & & 39 & 199.4107 & -69 & 3877 & & 1.5 \\
\hline c21y & & & 39 & 199.4570 & -69 & 4417 & & 1.5 \\
\hline$c 21 y-c 210$ & & & 39 & 199.5058 & -69 & 4982 & & 1.5 \\
\hline$c 220-c 230$ & & & 39 & 199.6624 & -69 & 6831 & & 1.5 \\
\hline$c 22 y-c 23 y$ & & & 39 & 199.6188 & -69 & 6343 & & 1.5 \\
\hline c23o-c24y & & & 39 & 199.7472 & -69 & 7730 & & 1.5 \\
\hline$c 23 y-c 230$ & & & 39 & 199.6933 & -69 & 7114 & & 1.5 \\
\hline c24y & & & 39 & 199.8063 & -69 & 8321 & & 1.5 \\
\hline$c 24 y$ & & & 39 & 199.8525 & -69 & 8809 & & 1.5 \\
\hline c25o & & & 39 & 200.3740 & -70 & 4139 & & 1.5 \\
\hline c25o & & & 39 & 200.4074 & -70 & 4550 & & 1.5 \\
\hline c25y & & & 39 & 200.2455 & -70 & 2778 & & 1.5 \\
\hline c25y & & & 39 & 200.3020 & -70 & 3343 & & 1.5 \\
\hline$c 25 y-c 25 o$ & & & 39 & 200.3406 & -70 & 3805 & & 1.5 \\
\hline $\mathrm{c} 260$ & & & 39 & 200.7104 & -70 & 8198 & & 1.5 \\
\hline c26o & & & 39 & 200.7593 & -70 & 8609 & & 1.5 \\
\hline c26y & & & 39 & 200.6000 & -70 & 6605 & & 1.5 \\
\hline c26y & & & 39 & 200.6385 & -70 & 7042 & & 1.5 \\
\hline$c 26 y-c 26 o$ & & & 39 & 200.6822 & -70 & 7581 & & 1.5 \\
\hline$c 270$ & & & 39 & 201.0521 & -71 & 5685 & & 1.5 \\
\hline c27o & & & 39 & 201.0546 & -71 & 5146 & & 1.5 \\
\hline c27y & & & 39 & 201.0315 & -71 & 3373 & & 1.5 \\
\hline c27y & & & 39 & 201.0495 & -71 & 3913 & & 1.5 \\
\hline$c 27 y-c 27 \circ$ & & & 39 & 201.0546 & -71 & 4529 & & 1.5 \\
\hline $\mathrm{c} 280-c 290$ & & & 39 & 201.1651 & -72 & 3558 & & 1.5 \\
\hline $\mathrm{c} 280-\mathrm{c} 290$ & & & 39 & 201.1754 & -72 & 4072 & & 1.5 \\
\hline$c 280-c 30 y$ & & & 39 & 200.4125 & -72 & 4637 & & 1.5 \\
\hline c28o-c30y & & & 39 & 200.4151 & -72 & 5356 & & 1.5 \\
\hline c28y & & & 39 & 201.0726 & -71 & 8279 & & 1.5 \\
\hline c28y & & & 39 & 201.0726 & -71 & 7663 & & 1.5 \\
\hline c28y & & & 39 & 201.0752 & -71 & 3870 & & 1.5 \\
\hline c28y & & & 39 & 201.0778 & -71 & 9358 & & 1.5 \\
\hline$c 28 y-c 280$ & & & 39 & 201.0855 & -71 & 9821 & & 1.5 \\
\hline$c 28 y-c 29 o$ & & & 39 & 201.1523 & -72 & 2967 & & 1.5 \\
\hline$c 28 y-c 29 y$ & & & 39 & 201.0906 & -72 & 0411 & & 1.5 \\
\hline$c 28 y-c 29 y$ & & & 39 & 201.1035 & -72 & 0771 & & 1.5 \\
\hline$c 28 y-c 29 y$ & & & 39 & 201.1240 & -72 & 1683 & & 1.5 \\
\hline$c 28 y-c 29 y$ & & & 39 & 201.1394 & -72 & 2325 & & 1.5 \\
\hline$c 290-c 30 y$ & & & 39 & $200.425^{2}$ & $7 ?$ & 844.1 & דדיט & $15 \quad 1.4$ \\
\hline $\mathrm{c} 290-\mathrm{c} 3$ & & MBL & L & \begin{tabular}{|l|l}
9 & 200.4 \\
\end{tabular} & 510 & -72.6 & 512 & 1.5 \\
\hline$c 29 o-c 3$ & & MBL & L & 200.4 & 690 & -72.7 & 308 & 1.5 \\
\hline$c 300-c 3$ & & MBL & 3 & 200.5 & 281 & -72.9 & 594 & 1.5 \\
\hline$c 300-c 3$ & & MBL & 3 & 200.5 & 486 & -73.0 & 262 & 1.5 \\
\hline $\mathrm{c} 30 \mathrm{o}-\mathrm{c} 3$ & & MBL & 3 & 200.5 & 820 & -73.0 & 930 & 1.5 \\
\hline$c 30 o-c 3$ & & MBL & 3 & 200.6 & 000 & -73.1 & 418 & 1.5 \\
\hline $\mathrm{c} 30 \mathrm{y}$ & & MBL & 3 & 200.4 & 793 & -72.7 & 950 & 1.5 \\
\hline c30y & & $\mathrm{MBL}$ & 3 & 200.4 & 947 & -72.8 & 541 & 1.5 \\
\hline c310 & & MBL & 3 & 199.7 & 623 & -73.5 & 824 & 1.5 \\
\hline $\mathrm{c} 240$ & & $\mathrm{Pac}$ & 3 & 174.2 & 483 & -60.7 & 971 & 1.5 \\
\hline $\mathrm{c} 24 \mathrm{o}$ & & Pac & 3 & 174.1 & 650 & -60.7 & 317 & 1.5 \\
\hline $\mathrm{c} 24 \mathrm{o}$ & & Pac & 3 & 174.0 & 520 & -60.6 & 484 & 1.5 \\
\hline c24o & & $\mathrm{Pac}$ & 3 & 173.9 & 806 & -60.6 & 008 & 1.5 \\
\hline
\end{tabular}




\begin{tabular}{|c|c|c|c|c|c|}
\hline age (chron) & plate & segment & longitude & latitude & uncertainty $(\mathrm{km})$ \\
\hline $\mathrm{c} 240$ & $\mathrm{Pac}$ & 39 & 173.8795 & -60.5294 & 1.5 \\
\hline c24o-c25o & Pac & 39 & 173.7129 & -60.3986 & 1.5 \\
\hline $\mathrm{c} 240-\mathrm{c} 250$ & Pac & 39 & 173.6118 & -60.2915 & 1.5 \\
\hline$c 240-c 25 y$ & Pac & 39 & 173.7902 & -60.4640 & 1.5 \\
\hline$c 240-c 26 y$ & Pac & 39 & 173.5404 & -60.2320 & 1.5 \\
\hline$c 250-c 27 y$ & $\mathrm{Pac}$ & 39 & 172.8741 & -59.5360 & 1.5 \\
\hline$c 25 y-c 26 o$ & Pac & 39 & 173.4274 & -60.1368 & 1.5 \\
\hline$c 25 y-c 26 o$ & Pac & 39 & 173.3500 & -60.0595 & 1.5 \\
\hline$c 25 y-c 26 o$ & Pac & 39 & 173.2667 & -59.9762 & 1.5 \\
\hline$c 25 y-c 26 o$ & Pac & 39 & 173.2073 & -59.9167 & 1.5 \\
\hline$c 25 y-c 26 o$ & Pac & 39 & 173.1478 & -59.8572 & 1.5 \\
\hline$c 25 y-c 26 o$ & Pac & 39 & 173.0883 & -59.7859 & 1.5 \\
\hline$c 25 y-c 26 o$ & Pac & 39 & 173.0228 & -59.7026 & 1.5 \\
\hline$c 25 y-c 26 o$ & Pac & 39 & 172.9753 & -59.6490 & 1.5 \\
\hline$c 25 y-c 26 o$ & Pac & 39 & 172.9277 & -59.5895 & 1.5 \\
\hline c26o-c27o & Pac & 39 & 172.7135 & -59.3219 & 1.5 \\
\hline$c 26 y-c 27 \circ$ & $\mathrm{Pac}$ & 39 & 172.8206 & -59.4646 & 1.5 \\
\hline$c 26 y-c 270$ & Pac & 39 & 172.7611 & -59.3932 & 1.5 \\
\hline c27o-c29o & Pac & 39 & 172.3804 & -58.6378 & 1.5 \\
\hline$c 27 y-c 280$ & Pac & 39 & 172.4947 & -58.9516 & 1.5 \\
\hline$c 27 y-c 280$ & Pac & 39 & 172.4577 & -58.8638 & 1.5 \\
\hline$c 27 y-c 28 y$ & Pac & 39 & 172.6659 & -59.2445 & 1.5 \\
\hline$c 27 y-c 28 y$ & Pac & 39 & 172.6302 & -59.1672 & 1.5 \\
\hline$c 27 y-c 28 y$ & Pac & 39 & 172.5826 & -59.0899 & 1.5 \\
\hline$c 27 y-c 28 y$ & Pac & 39 & 172.5470 & -59.0244 & 1.5 \\
\hline$c 27 y-c 29 y$ & Pac & 39 & 172.4220 & -58.7805 & 1.5 \\
\hline$c 27 y-c 29 y$ & Pac & 39 & 172.4042 & -58.7032 & 1.5 \\
\hline$c 280-c 30 y$ & Pac & 39 & 172.2852 & -58.3998 & 1.5 \\
\hline c28o-c30y & Pac & 39 & 172.2376 & -58.2571 & 1.5 \\
\hline$c 28 y-c 30 y$ & Pac & 39 & 172.3387 & -58.5307 & 1.5 \\
\hline $\mathrm{c} 290-\mathrm{c} 30 \mathrm{o}$ & Pac & 39 & 172.1186 & -58.0132 & 1.5 \\
\hline$c 290-c 30 y$ & Pac & 39 & 172.1781 & -58.1321 & 1.5 \\
\hline $\mathrm{c} 30 \mathrm{o}-\mathrm{c} 310$ & Pac & 39 & 171.9759 & -57.6741 & 1.5 \\
\hline$c 300-c 310$ & Pac & 39 & 171.9164 & -57.5611 & 1.5 \\
\hline$c 300-c 310$ & Pac & 39 & 171.7176 & -57.4770 & 1.5 \\
\hline$c 300-c 310$ & Pac & 39 & 171.8748 & -57.4718 & 1.5 \\
\hline c30o-c31y & $\mathrm{Pac}$ & 39 & 172.0294 & -57.7931 & 1.5 \\
\hline c30y-c31y & Pac & 39 & 172.0651 & -57.8882 & 1.5 \\
\hline$c 310$ & Pac & 39 & 171.7439 & -57.2458 & 1.5 \\
\hline c310 & Pac & 39 & 171.6606 & -57.1149 & 1.5 \\
\hline c32.2o-c33y & Pac & 39 & 171.1496 & -56.4364 & 1.5 \\
\hline c32.20-c33y & Pac & 39 & 171.1496 & -56.3927 & 1.5 \\
\hline c32.20-c33y & $\mathrm{Pac}$ & 39 & 171.1496 & -56.3362 & 1.5 \\
\hline$c 32.20-c 33 y$ & Pac & 39 & 171.1496 & -56.2925 & 1.5 \\
\hline c32.20-c33y & Pac & 39 & 171.1496 & -56.2206 & 1.5 \\
\hline c32.2y & $\mathrm{Pac}$ & 39 & 171.3089 & -57.1209 & 1.5 \\
\hline c32.2y & Pac & 39 & 171.2935 & -57.0798 & 1.5 \\
\hline$c 32.2 y$ & Pac & 39 & 171.2704 & -57.0361 & 1.5 \\
\hline$c 32.2 y$ & Pac & 39 & 171.2601 & -56.9796 & 1.5 \\
\hline c32.2y & Pac & 39 & 171.2293 & -56.9308 & 1.5 \\
\hline c32.2y & Pac & 39 & 171.2062 & -56.8846 & 1.5 \\
\hline$c 32.2 y-c 32.20$ & Pac & 39 & 171.1984 & -56.8294 & 1.5 \\
\hline $\mathrm{c} 32.2 \mathrm{y}-\mathrm{c} 32.20$ & Pac & 39 & 171.1753 & -56.7857 & 1.5 \\
\hline$c 32.2 y-c 32 r$ & Pac & 39 & 171.1702 & -56.7446 & 1.5 \\
\hline$c 32.2 y-c 33 y$ & Pac & 39 & 171.1625 & -56.6881 & 1.5 \\
\hline$c 32.2 y-c 33 y$ & Pac & 39 & 171.1625 & -56.6444 & 1.5 \\
\hline$c 32.2 y-c 33 y$ & Pac & 39 & 171.1625 & -56.5879 & 1.5 \\
\hline$c 32.2 y-c 33 y$ & $\mathrm{Pac}$ & 39 & 171.1548 & -56.5365 & 1.5 \\
\hline
\end{tabular}




\begin{tabular}{|c|c|c|c|c|c|}
\hline age (chron) & plate & segment & longitude & latitude & uncertainty $(\mathrm{km})$ \\
\hline$c 32.2 y-c 33 y$ & $\mathrm{Pac}$ & 39 & 171.1548 & -56.4723 & 1.5 \\
\hline c32r-c33y & $\mathrm{Pac}$ & 39 & 171.1368 & -56.0819 & 1.5 \\
\hline$c 32 r-c 33 y$ & Pac & 39 & 171.1394 & -56.1487 & 1.5 \\
\hline c33o & Pac & 39 & 170.5280 & -55.0275 & 1.5 \\
\hline c33o & $\mathrm{Pac}$ & 39 & 170.5511 & -55.0891 & 1.5 \\
\hline c33o & Pac & 39 & 170.5794 & -55.1405 & 1.5 \\
\hline c33o & Pac & 39 & 170.6128 & -55.1996 & 1.5 \\
\hline c33o & $\mathrm{Pac}$ & 39 & 170.6308 & -55.2535 & 1.5 \\
\hline c33o & $\mathrm{Pac}$ & 39 & 170.6436 & -55.3229 & 1.5 \\
\hline c33o & $\mathrm{Pac}$ & 39 & 170.6667 & -55.3961 & 1.5 \\
\hline c33o & Pac & 39 & 170.6976 & -55.4629 & 1.5 \\
\hline c33o & Pac & 39 & 170.7335 & -55.5296 & 1.5 \\
\hline c33o & Pac & 39 & 170.7566 & -55.5964 & 1.5 \\
\hline c33y & $\mathrm{Pac}$ & 39 & 171.1291 & -56.0280 & 1.5 \\
\hline$c 270-c 29 y$ & $\mathrm{Pac}$ & 45 & 165.0644 & -58.8628 & 1.5 \\
\hline c27y & $\mathrm{Pac}$ & 45 & 165.4060 & -60.1012 & 1.5 \\
\hline$c 27 y-c 27 o$ & Pac & 45 & 165.2542 & -59.8450 & 1.5 \\
\hline$c 27 y-c 270$ & $\mathrm{Pac}$ & 45 & 165.3174 & -59.9272 & 1.5 \\
\hline$c 27 y-c 27 o$ & Pac & 45 & 165.3680 & -60.0190 & 1.5 \\
\hline$c 27 y-c 28 y$ & $\mathrm{Pac}$ & 45 & 165.1972 & -59.5730 & 1.5 \\
\hline$c 27 y-c 28 y$ & Pac & 45 & 165.2004 & -59.6647 & 1.5 \\
\hline$c 27 y-c 28 y$ & Pac & 45 & 165.2130 & -59.7501 & 1.5 \\
\hline$c 27 y-c 29 y$ & $\mathrm{Pac}$ & 45 & 165.0928 & -58.9641 & 1.5 \\
\hline$c 27 y-c 29 y$ & Pac & 45 & 165.1150 & -59.0621 & 1.5 \\
\hline$c 27 y-c 29 y$ & Pac & 45 & 165.1308 & -59.2187 & 1.5 \\
\hline$c 27 y-c 29 y$ & Pac & 45 & 165.1687 & -59.3231 & 1.5 \\
\hline$c 27 y-c 29 y$ & $\mathrm{Pac}$ & 45 & 165.1909 & -59.4686 & 1.5 \\
\hline c28o-c31y & $\mathrm{Pac}$ & 45 & 164.9663 & -58.4168 & 1.5 \\
\hline c28o-c31y & $\mathrm{Pac}$ & 45 & 164.9790 & -58.5212 & 1.5 \\
\hline$c 28 y-c 29 y$ & Pac & 45 & 165.0327 & -58.7585 & 1.5 \\
\hline$c 28 y-c 30 o$ & Pac & 45 & 164.9979 & -58.6446 & 1.5 \\
\hline c29o-c31o & $\mathrm{Pac}$ & 45 & 164.9220 & -58.0910 & 1.5 \\
\hline$c 290-c 310$ & Pac & 45 & 164.9284 & -58.1986 & 1.5 \\
\hline$c 29 y-c 310$ & $\mathrm{Pac}$ & 45 & 164.9473 & -58.3030 & 1.5 \\
\hline c30y-c31o & Pac & 45 & 164.8841 & -57.9772 & 1.5 \\
\hline c31o-c32.2o & $\mathrm{Pac}$ & 45 & 164.7164 & -57.3525 & 1.5 \\
\hline $\mathrm{c} 310-c 32 r$ & Pac & 45 & 164.7069 & -57.2512 & 1.5 \\
\hline c31y & Pac & 45 & 164.8145 & -57.7637 & 1.5 \\
\hline$c 31 y-c 310$ & Pac & 45 & 164.8335 & -57.8617 & 1.5 \\
\hline c31y-c32.2y & Pac & 45 & 164.7322 & -57.4505 & 1.5 \\
\hline$c 31 y-c 32.2 y$ & Pac & 45 & 164.7670 & -57.5707 & 1.5 \\
\hline c31y-c32.2y & Pac & 45 & 164.7892 & -57.6719 & 1.5 \\
\hline c32.1y & Pac & 45 & 164.8145 & -57.7637 & 1.5 \\
\hline$c 32.1 y-c 33 y$ & Pac & 45 & 164.6974 & -57.1279 & 1.5 \\
\hline c32r-c33y & Pac & 45 & 164.6785 & -57.0235 & 1.5 \\
\hline c33y & Pac & 45 & 164.6563 & -56.9223 & 1.5 \\
\hline$c 260-c 28 y$ & $\mathrm{Pac}$ & 47 & 164.1555 & -58.4491 & 1.5 \\
\hline $\mathrm{c} 26 \mathrm{y}-\mathrm{c} 270$ & Pac & 47 & 164.2124 & -58.6483 & 1.5 \\
\hline c26y-c27o & Pac & 47 & 164.2441 & -58.7369 & 1.5 \\
\hline c26y-c27o & Pac & 47 & 164.2662 & -58.8318 & 1.5 \\
\hline c26y-c28y & Pac & 47 & 164.1840 & -58.5408 & 1.5 \\
\hline c27o-c30y & Pac & 47 & 164.0701 & -57.7247 & 1.5 \\
\hline$c 27 y-c 280$ & Pac & 47 & 164.1365 & -58.3352 & 1.5 \\
\hline$c 27 y-c 29 o$ & Pac & 47 & 164.0891 & -58.0252 & 1.5 \\
\hline$c 27 y-c 290$ & $\mathrm{Pac}$ & 47 & 164.0922 & -57.9271 & 1.5 \\
\hline$c 27 y-c 29 y$ & Pac & 47 & 164.0986 & -58.1296 & 1.5 \\
\hline$c 27 y-c 29 y$ & Pac & 47 & 164.1049 & -58.2308 & 1.5 \\
\hline$c 27 y-c 300$ & Pac & 47 & 164.0638 & -57.4353 & 1.5 \\
\hline
\end{tabular}




\begin{tabular}{|c|c|c|c|c|c|}
\hline age (chron) & plate & segment & longitude & latitude & uncertainty $(\mathrm{km})$ \\
\hline$c 27 y-c 30 y$ & $\mathrm{Pac}$ & 47 & 164.0669 & -57.5302 & 1.5 \\
\hline$c 27 y-c 30 y$ & Pac & 47 & 164.0764 & -57.6361 & 1.5 \\
\hline$c 27 y-c 30 y$ & Pac & 47 & 164.0796 & -57.8323 & 1.5 \\
\hline c27y-c31y & Pac & 47 & 164.0448 & -57.3404 & 1.5 \\
\hline$c 280-c 310$ & Pac & 47 & 163.9973 & -56.9671 & 1.5 \\
\hline c28o-c31o & Pac & 47 & 164.0100 & -57.0620 & 1.5 \\
\hline$c 280-c 310$ & Pac & 47 & 164.0195 & -57.1538 & 1.5 \\
\hline$c 28 y-c 31 y$ & Pac & 47 & 164.0290 & -57.2550 & 1.5 \\
\hline c29o-c31o & Pac & 47 & 163.9088 & -56.6951 & 1.5 \\
\hline$c 290-c 310$ & Pac & 47 & 163.9436 & -56.7932 & 1.5 \\
\hline$c 29 y-c 310$ & Pac & 47 & 163.9720 & -56.8691 & 1.5 \\
\hline c30o-c31o & Pac & 47 & 163.8423 & -56.0941 & 1.5 \\
\hline $\mathrm{c} 30 \mathrm{o}-\mathrm{c} 31 \mathrm{o}$ & Pac & 47 & 163.8518 & -56.1922 & 1.5 \\
\hline $\mathrm{c} 30 \mathrm{o}-\mathrm{c} 310$ & Pac & 47 & 163.8582 & -56.2839 & 1.5 \\
\hline c30o-c31o & Pac & 47 & 163.8708 & -56.3946 & 1.5 \\
\hline$c 30 y-c 310$ & Pac & 47 & 163.8835 & -56.4990 & 1.5 \\
\hline$c 30 y-c 310$ & Pac & 47 & 163.8961 & -56.6002 & 1.5 \\
\hline c31o & Pac & 47 & 163.7759 & -55.7889 & 1.5 \\
\hline c310 & Pac & 47 & 163.7981 & -55.8901 & 1.5 \\
\hline c310 & Pac & 47 & 163.8139 & -55.9660 & 1.5 \\
\hline c25o-c28o & Pac & 49 & 163.5577 & -56.9023 & 1.5 \\
\hline$c 25 o-c 29 y$ & Pac & 49 & 163.5608 & -56.8200 & 1.5 \\
\hline c25y & Pac & 49 & 163.9151 & -58.7100 & 1.5 \\
\hline$c 25 y-c 25 o$ & Pac & 49 & 163.8740 & -58.5993 & 1.5 \\
\hline$c 25 y-c 25 o$ & Pac & 49 & 163.8360 & -58.5107 & 1.5 \\
\hline$c 25 y-c 26 o$ & Pac & 49 & 163.8139 & -58.4064 & 1.5 \\
\hline$c 25 y-c 26 o$ & Pac & 49 & 163.8044 & -58.3020 & 1.5 \\
\hline$c 25 y-c 26 o$ & Pac & 49 & 163.7981 & -58.1944 & 1.5 \\
\hline$c 25 y-c 26 o$ & Pac & 49 & 163.7917 & -58.0932 & 1.5 \\
\hline$c 25 y-c 26 o$ & Pac & 49 & 163.7696 & -57.9778 & 1.5 \\
\hline$c 25 y-c 26 o$ & Pac & 49 & 163.7475 & -57.8734 & 1.5 \\
\hline$c 25 y-c 27 o$ & Pac & 49 & 163.7000 & -57.6646 & 1.5 \\
\hline$c 25 y-c 27 \circ$ & Pac & 49 & 163.6589 & -57.5760 & 1.5 \\
\hline$c 25 y-c 270$ & Pac & 49 & 163.6399 & -57.4622 & 1.5 \\
\hline$c 25 y-c 27 o$ & Pac & 49 & 163.6367 & -57.3546 & 1.5 \\
\hline$c 25 y-c 27 y$ & Pac & 49 & 163.7316 & -57.7627 & 1.5 \\
\hline$c 25 y-c 28 y$ & Pac & 49 & 163.6304 & -57.2534 & 1.5 \\
\hline$c 25 y-c 28 y$ & Pac & 49 & 163.6209 & -57.1617 & 1.5 \\
\hline$c 25 y-c 28 y$ & Pac & 49 & 163.5830 & -57.0699 & 1.5 \\
\hline$c 25 y-c 28 y$ & Pac & 49 & 163.5672 & -56.9750 & 1.5 \\
\hline c26o-c29o & Pac & 49 & 163.5260 & -56.5322 & 1.5 \\
\hline c26y-c29o & Pac & 49 & 163.5260 & -56.6208 & 1.5 \\
\hline$c 26 y-c 29 y$ & Pac & 49 & 163.5387 & -56.7252 & 1.5 \\
\hline c27o-c29o & Pac & 49 & 163.4786 & -55.9423 & 1.5 \\
\hline c27y-c29o & Pac & 49 & 163.5134 & -56.4515 & 1.5 \\
\hline$c 27 y-c 29 o$ & Pac & 49 & 163.5039 & -56.3883 & 1.5 \\
\hline$c 27 y-c 29 o$ & Pac & 49 & 163.4944 & -56.2902 & 1.5 \\
\hline$c 27 y-c 29 o$ & Pac & 49 & 163.4881 & -56.1890 & 1.5 \\
\hline$c 27 y-c 29 o$ & Pac & 49 & 163.4849 & -56.1163 & 1.5 \\
\hline$c 27 y-c 29 o$ & Pac & 49 & 163.4849 & -56.0372 & 1.5 \\
\hline c28o-c29o & Pac & 49 & 163.4185 & -55.5247 & 1.5 \\
\hline c28o-c29o & Pac & 49 & 163.4311 & -55.6102 & 1.5 \\
\hline$c 28 y-c 29 o$ & Pac & 49 & 163.4628 & -55.7304 & 1.5 \\
\hline$c 28 y-c 29 o$ & Pac & 49 & 163.4817 & -55.8347 & 1.5 \\
\hline $\mathrm{c} 290$ & Pac & 49 & 163.3932 & -55.2590 & 1.5 \\
\hline c29o & Pac & 49 & 163.3963 & -55.3445 & 1.5 \\
\hline$c 29 y-c 290$ & Pac & 49 & 163.4153 & -55.4267 & 1.5 \\
\hline
\end{tabular}



Appendix D: Magnetic reversals timescale, after [Cande \& Kent, 1995]

\begin{tabular}{|c|c|c|}
\hline \multicolumn{2}{|c|}{ Chron } & Date (Ma) \\
\hline \multicolumn{2}{|l|}{$c 20 y$} & 42.536 \\
\hline \multicolumn{2}{|l|}{ c20o } & 43.789 \\
\hline \multicolumn{2}{|l|}{ c21y } & 46.264 \\
\hline \multicolumn{2}{|l|}{$c 210$} & 47.906 \\
\hline \multirow{2}{*}{\multicolumn{2}{|c|}{$\begin{array}{l}c 22 y \\
c 220\end{array}$}} & 49.037 \\
\hline & & 49.714 \\
\hline \multirow[t]{3}{*}{ c23y } & c23.1y & 50.778 \\
\hline & c23.10 & 50.946 \\
\hline & $c 23.2 y$ & 51.047 \\
\hline c23o & c23.2o & 51.743 \\
\hline \multirow[t]{5}{*}{ c24y } & c24.1y & 52.364 \\
\hline & c24.10 & 52.663 \\
\hline & $c 24.2 y$ & 52.757 \\
\hline & c24.2o & 52.801 \\
\hline & c24.3y & 52.903 \\
\hline c24o & c24.3o & 53.347 \\
\hline \multirow{2}{*}{\multicolumn{2}{|c|}{$\begin{array}{l}\text { c25y } \\
\text { c25o }\end{array}$}} & 55.904 \\
\hline & & 56.391 \\
\hline \multirow{2}{*}{\multicolumn{2}{|c|}{ c26y }} & 57.554 \\
\hline & & 57.911 \\
\hline \multicolumn{2}{|l|}{ c27y } & 60.920 \\
\hline \multicolumn{2}{|l|}{ c27o } & 61.276 \\
\hline \multirow{2}{*}{\multicolumn{2}{|c|}{$\begin{array}{l}\text { c28y } \\
\text { c28o }\end{array}$}} & 62.499 \\
\hline & & 63.634 \\
\hline \multicolumn{2}{|l|}{ c29y } & 63.976 \\
\hline \multicolumn{2}{|l|}{$\mathrm{c} 290$} & 64.745 \\
\hline \multicolumn{2}{|l|}{ c30y } & 65.578 \\
\hline \multicolumn{2}{|l|}{$\mathrm{c} 30 \mathrm{o}$} & 67.610 \\
\hline \multirow{2}{*}{\multicolumn{2}{|c|}{$\begin{array}{l}\text { c31y } \\
\text { c31o }\end{array}$}} & 67.735 \\
\hline & & 68.737 \\
\hline \multirow[t]{3}{*}{ c32y } & c32.1y & 71.071 \\
\hline & c32.10 & 71.338 \\
\hline & c32.2y & 71.587 \\
\hline c32o & c32.2o & 73.004 \\
\hline \multirow{2}{*}{$c 32 r$} & c32r_y & 73.291 \\
\hline & c32r_o & 73.374 \\
\hline \multirow{2}{*}{\multicolumn{2}{|c|}{$\begin{array}{l}\text { c33y } \\
\text { c33o }\end{array}$}} & 73.619 \\
\hline & & 79.075 \\
\hline \multicolumn{2}{|l|}{ c34y } & 83.000 \\
\hline \multicolumn{2}{|l|}{ c34o } & 118.000 \\
\hline
\end{tabular}



Appendix E: Finite rotations calculated during this study: solutions from Figure V-1 and hellinger 1

u1:

\begin{tabular}{|c|c|c|c|c|c|c|}
\hline \multirow{2}{*}{ Chron } & \multicolumn{3}{|c|}{ onepole } & \multicolumn{3}{|c|}{ JaMBES } \\
\hline & longitude & latitude & angle $\left({ }^{\circ}\right)$ & longitude & latitude & angle $\left({ }^{\circ}\right)$ \\
\hline c20y & 131.2 & -75.1 & 34.6389 & 133.6 & -75.2 & 36.6916 \\
\hline c20o & 136.6 & -75.1 & 37.4804 & 137.4 & -75.1 & 38.0501 \\
\hline c21y & 144.8 & -74.8 & 41.9859 & 138.6 & -74.8 & 40.5880 \\
\hline c21o & 142.4 & -74.6 & 41.3132 & 138.2 & -74.6 & 41.0348 \\
\hline$c 22 y$ & 145.0 & -74.5 & 44.7162 & 137.4 & -74.5 & 41.7531 \\
\hline c22o & 134.2 & -74.4 & 40.5328 & 136.4 & -74.5 & 41.4119 \\
\hline$c 23 y$ & 134.8 & -74.3 & 41.1940 & 135.6 & -74.3 & 42.0838 \\
\hline c23o & 132.8 & -74.1 & 40.9352 & 134.4 & -74.1 & 42.0665 \\
\hline$c 24 y$ & 131.4 & -73.9 & 40.8343 & 133.8 & -74.0 & 41.4204 \\
\hline c24o & 130.0 & -73.7 & 40.9892 & 132.6 & -73.8 & 42.3993 \\
\hline c25y & 127.6 & -72.9 & 41.0668 & 129.2 & -73.1 & 42.8567 \\
\hline c25o & 123.2 & -72.5 & 40.3513 & 128.4 & -72.9 & 42.7994 \\
\hline c26y & 125.0 & -72.3 & 40.9796 & 126.8 & -72.5 & 40.9165 \\
\hline c26o & 124.0 & -72.1 & 40.7173 & 126.4 & -72.4 & 41.4530 \\
\hline c27y & 123.4 & -71.2 & 44.2068 & 122.2 & -71.2 & 43.6796 \\
\hline c27o & 126.0 & -71.5 & 45.5194 & 121.8 & -71.0 & 44.0047 \\
\hline c28y & 121.0 & -70.5 & 43.8205 & 120.2 & -70.4 & 43.8382 \\
\hline c28o & 121.2 & -70.3 & 45.0655 & 118.8 & -69.9 & 44.5276 \\
\hline c29y & 120.8 & -70.1 & 44.9926 & 118.4 & -69.7 & 44.4637 \\
\hline c29o & 118.4 & -69.4 & 43.8038 & 117.6 & -69.3 & 44.1427 \\
\hline c30y & 116.6 & -68.8 & 43.2645 & 116.8 & -68.8 & 44.4211 \\
\hline$c 300$ & 113.6 & -67.2 & 45.0227 & 115.0 & -67.6 & 44.9037 \\
\hline c31y & 114.6 & -67.5 & 45.6908 & 114.8 & -67.5 & 44.6479 \\
\hline c310 & 113.0 & -66.6 & 44.8312 & 114.2 & -66.9 & 45.4810 \\
\hline c32.1y & 118.6 & -68.2 & 49.4285 & & & \\
\hline c32.10 & 116.0 & -67.4 & 48.4610 & & & \\
\hline c32.2y & 111.0 & -66.2 & 46.2242 & & & \\
\hline c32.2o & 117.6 & -67.6 & 51.1187 & & & \\
\hline c32r & 117.4 & -67.5 & 50.4547 & & & \\
\hline c33y & 112.8 & -66.0 & 52.6621 & & & \\
\hline c33o & 350.0 & 62.9 & 76.2957 & & & \\
\hline
\end{tabular}


u1-u2:

\begin{tabular}{|c|c|c|c|c|c|c|}
\hline \multirow{2}{*}{ Chron } & \multicolumn{3}{|c|}{ onepole } & \multicolumn{3}{|c|}{ JaMBES } \\
\hline & longitude & latitude & angle $\left({ }^{\circ}\right)$ & longitude & latitude & angle $\left({ }^{\circ}\right)$ \\
\hline $\mathrm{c} 20 \mathrm{y}$ & 136.4 & -75.2 & 37.1944 & 144.4 & -75.4 & 40.4813 \\
\hline$c 200$ & 136.0 & -75.1 & 39.6420 & 143.0 & -75.3 & 40.2872 \\
\hline c21y & 144.4 & -74.8 & 41.4477 & 140.8 & -74.8 & 41.6762 \\
\hline c21o & 142.6 & -74.6 & 41.2593 & 138.8 & -74.6 & 41.3362 \\
\hline c22y & 143.0 & -74.5 & 43.5479 & 137.2 & -74.5 & 41.6541 \\
\hline $\mathrm{c} 220$ & 133.6 & -74.4 & 39.8827 & 135.6 & -74.4 & 41.0684 \\
\hline c23y & 133.0 & -74.2 & 40.8534 & 134.4 & 74.2 & 41.4787 \\
\hline c23o & 132.8 & -74.1 & 41.2988 & 133.4 & -74.0 & 41.5557 \\
\hline c24y & 132.8 & -74.0 & 41.8060 & 132.8 & -73.8 & 40.9902 \\
\hline c24o & 130.0 & -73.7 & 41.1552 & 131.6 & -73.6 & 41.8671 \\
\hline c25y & 6.2 & 2.7 & 41.2981 & 129.0 & 2.8 & 42.6629 \\
\hline$c 250$ & 126.4 & -72.8 & 41.8467 & 128.6 & -72.6 & 42.7940 \\
\hline c26y & 137.0 & -73.2 & 47.1003 & 128.2 & -72.3 & 41.6266 \\
\hline $\mathrm{c} 260$ & 6.8 & & 47.2 & 128.2 & 2.2 & 42.0106 \\
\hline c27y & 123.6 & -71.2 & 44.2057 & 124.8 & -71.3 & 44.7449 \\
\hline c27o & 125.2 & -71.4 & 45.8311 & 124.4 & -71.1 & 45.0069 \\
\hline c28y & 122.4 & -70.8 & 44.6662 & 123.4 & -70.8 & 45.1148 \\
\hline c28o & 0.4 & & 44.9764 & 122.6 & 0.4 & 46.0697 \\
\hline$c 29 y$ & 122.0 & -70.3 & 46.3220 & 122.2 & -70.3 & 46.1010 \\
\hline c29o & 124.6 & -70.6 & 48.0628 & 121.8 & -70.1 & 46.2379 \\
\hline c30y & 4.6 & -70.5 & 48.5718 & 121.2 & -69.8 & 46.6231 \\
\hline$c 300$ & 3.4 & & 49.3 & 120.0 & -69.1 & 47.6364 \\
\hline c31y & 124.8 & -69.9 & 50.7559 & 120.0 & -69.1 & 47.5800 \\
\hline c310 & 114.2 & -67.0 & 46.9892 & 119.0 & -68.7 & 48.3418 \\
\hline c32.1y & 112.0 & -66.4 & 45.1057 & 117.4 & -68.0 & 48.7535 \\
\hline c32.10 & 112.4 & & 45.6923 & 117.4 & -67.8 & 49.2811 \\
\hline c32.2y & 1.6 & -66.4 & 45.8145 & 117.0 & -67.9 & 49.1012 \\
\hline c32.20 & 114.8 & -66.8 & 49.0756 & 116.4 & -67.3 & 50.7810 \\
\hline c32r & 115.6 & -67.0 & 53.6206 & 116.2 & -67.2 & 51.3381 \\
\hline$c 33 y$ & 125.6 & & 57.8333 & 116.2 & -67.1 & 51.6830 \\
\hline c33o & 322.0 & 52.2 & 44.1761 & & & \\
\hline
\end{tabular}


u1, u2 \& u4:

\begin{tabular}{|c|c|c|c|c|c|c|}
\hline \multirow{2}{*}{ Chron } & \multicolumn{3}{|c|}{ onepole } & \multicolumn{3}{|c|}{ JaMBES } \\
\hline & longitude & latitude & angle $\left({ }^{\circ}\right)$ & longitude & latitude & angle $\left({ }^{\circ}\right)$ \\
\hline$c 20 y$ & 137.8 & -75.2 & 34.6280 & & & \\
\hline $\mathrm{c} 200$ & 138.8 & -75.2 & 35.7473 & & & \\
\hline c21y & 144.2 & -74.8 & 39.0610 & & & \\
\hline c21o & 142.6 & -74.6 & 38.7699 & & & \\
\hline$c 22 y$ & 142.8 & -74.5 & 43.3625 & 139.6 & -74.5 & 42.8737 \\
\hline $\mathrm{c} 220$ & 133.6 & -74.4 & 39.8518 & 135.8 & -74.5 & 41.1627 \\
\hline c23y & 133.0 & -74.2 & 40.8534 & 133.6 & -74.2 & 41.0886 \\
\hline c23o & 130.2 & -73.9 & 40.0252 & 131.6 & -74.0 & 40.6880 \\
\hline c24y & 130.2 & -73.8 & 40.2995 & 130.8 & -73.8 & 40.2964 \\
\hline c24o & 126.4 & -73.3 & 39.3851 & 129.4 & -73.6 & 40.8267 \\
\hline c25y & 126.2 & -72.7 & 41.2987 & 127.8 & -72.9 & 42.1070 \\
\hline c25o & 126.4 & -72.8 & 41.8466 & 127.6 & -72.9 & 42.4139 \\
\hline c26y & 137.2 & -73.2 & 47.2389 & 128.8 & -72.5 & 41.9996 \\
\hline $\mathrm{c} 260$ & 136.8 & -73.2 & 47.2405 & 129.2 & -72.5 & 42.4778 \\
\hline c27y & 123.6 & -71.2 & 44.2057 & 126.8 & -71.8 & 45.8086 \\
\hline c27o & 125.2 & -71.4 & 45.8312 & 126.8 & -71.7 & 46.2253 \\
\hline c28y & 122.6 & -70.8 & 44.7513 & 126.4 & -71.4 & 46.4959 \\
\hline $\mathrm{c} 280$ & 122.4 & -70.5 & 45.7386 & 126.0 & -71.0 & 47.6306 \\
\hline c29y & 123.0 & -70.5 & 46.7111 & 125.8 & -70.9 & 47.7766 \\
\hline c29o & 124.4 & -70.6 & 47.9309 & 125.2 & -70.7 & 48.0263 \\
\hline c30y & 125.2 & -70.6 & 48.7977 & 124.6 & -70.4 & 48.3413 \\
\hline c30o & 123.4 & -69.7 & 49.3115 & 122.2 & -69.5 & 48.7587 \\
\hline c31y & 125.2 & -70.0 & 50.9666 & 122.4 & -69.5 & 48.8206 \\
\hline c310 & 125.6 & -69.8 & 50.9646 & 120.6 & -68.9 & 49.0968 \\
\hline c32.1y & 112.0 & -66.4 & 45.1218 & 115.2 & -67.3 & 47.4147 \\
\hline c32.10 & 112.4 & -66.4 & 45.6834 & & & \\
\hline c32.2y & 111.6 & -66.4 & 45.8072 & & & \\
\hline c32.2o & 123.6 & -68.9 & 55.9028 & & & \\
\hline c32r & 132.2 & -70.2 & 58.9956 & & & \\
\hline c33y & 125.6 & -69.1 & 57.8333 & & & \\
\hline c33o & 332.8 & 52.8 & 2.6925 & & & \\
\hline
\end{tabular}


u1-u3a:

\begin{tabular}{|c|c|c|c|c|c|c|}
\hline \multirow{2}{*}{ Chron } & \multicolumn{3}{|c|}{ onepole } & \multicolumn{3}{|c|}{ JaMBES } \\
\hline & longitude & latitude & angle $\left({ }^{\circ}\right)$ & longitude & latitude & angle $\left({ }^{\circ}\right)$ \\
\hline c20y & 136.0 & -75.2 & 37.6069 & 136.2 & -75.2 & 37.2218 \\
\hline c20o & 131.6 & -75.0 & 36.7375 & 136.8 & -75.1 & 37.8239 \\
\hline c21y & 144.0 & -74.8 & 43.9417 & 138.0 & -74.7 & 40.3131 \\
\hline c21o & 142.6 & -74.6 & 41.8922 & 137.6 & -74.6 & 40.7378 \\
\hline$c 22 y$ & 140.6 & -74.5 & 43.6598 & 136.6 & -74.5 & 41.3600 \\
\hline c22o & 132.6 & -74.3 & 39.9718 & 135.8 & -74.4 & 41.1512 \\
\hline c23y & 132.8 & -74.2 & 40.7571 & 135.2 & -74.3 & 41.8828 \\
\hline c23o & 131.8 & -74.0 & 40.7990 & 134.4 & -74.1 & 42.0665 \\
\hline c24y & 132.6 & -74.0 & 41.7067 & 133.8 & -74.0 & 41.4204 \\
\hline c24o & 130.0 & -73.7 & 41.1552 & 132.8 & -73.8 & 42.4987 \\
\hline c25y & 125.8 & -72.6 & 41.0713 & 130.4 & -73.1 & 43.4645 \\
\hline c25o & 126.4 & -72.8 & 41.8466 & 129.8 & -73.0 & 43.5242 \\
\hline c26y & 136.2 & -73.2 & 46.5499 & 129.6 & -72.7 & 42.4805 \\
\hline c26o & 135.8 & -73.1 & 46.5713 & 129.6 & -72.6 & 42.6576 \\
\hline c27y & 123.4 & -71.2 & 44.2264 & 125.8 & -71.7 & 45.3454 \\
\hline c27o & 126.2 & -71.6 & 46.1453 & 125.8 & -71.6 & 45.7850 \\
\hline$c 28 y$ & 123.6 & -71.0 & 45.4681 & 124.8 & -71.2 & 45.8127 \\
\hline c28o & 122.6 & -70.5 & 46.3098 & 124.2 & -70.8 & 46.8580 \\
\hline c29y & 122.6 & -70.4 & 46.5538 & 124.0 & -70.7 & 46.9797 \\
\hline c29o & 125.6 & -70.8 & 48.4997 & 123.8 & -70.5 & 47.3024 \\
\hline c30y & 124.6 & -70.5 & 48.5713 & 123.4 & -70.2 & 47.7299 \\
\hline c30o & 125.0 & -70.0 & 50.3619 & 123.2 & -69.6 & 49.2372 \\
\hline c31y & 124.8 & -69.9 & 50.7559 & 123.2 & -69.6 & 49.2278 \\
\hline c310 & 119.6 & -68.5 & 49.4998 & 122.4 & -69.2 & 50.0228 \\
\hline c32.1y & 112.0 & -66.4 & 45.1057 & & & \\
\hline c32.10 & 112.4 & -66.4 & 45.6925 & & & \\
\hline c32.2y & 111.6 & -66.4 & 45.8132 & & & \\
\hline c32.20 & 115.2 & -66.9 & 49.3404 & & & \\
\hline c32r & 115.6 & -67.0 & 53.6206 & & & \\
\hline c33y & 125.6 & -69.1 & 57.8333 & & & \\
\hline c33o & 321.8 & 52.2 & 44.8269 & & & \\
\hline
\end{tabular}


u1-u3b:

\begin{tabular}{|c|c|c|c|c|c|c|}
\hline \multirow{2}{*}{ Chron } & \multicolumn{3}{|c|}{ onepole } & \multicolumn{3}{|c|}{ JaMBES 1} \\
\hline & longitude & latitude & angle $\left({ }^{\circ}\right)$ & longitude & latitude & angle $\left({ }^{\circ}\right)$ \\
\hline c20y & 131.8 & -75.1 & 35.8964 & 129.4 & -75.0 & 34.8792 \\
\hline c20o & 124.4 & -74.5 & 33.8574 & 129.6 & -74.8 & 35.2769 \\
\hline c21y & 133.2 & -74.5 & 38.4348 & 131.4 & -74.5 & 37.3697 \\
\hline c21o & 133.8 & -74.4 & 39.0654 & 131.8 & -74.4 & 38.0611 \\
\hline c22y & 135.0 & -74.4 & 40.6344 & 131.8 & -74.3 & 39.1340 \\
\hline c22o & 130.8 & -74.2 & 39.0502 & 131.6 & -74.2 & 39.4498 \\
\hline c23y & 130.4 & -74.0 & 39.5673 & 131.4 & -74.0 & 40.0205 \\
\hline $\mathrm{c} 230$ & 129.4 & -73.8 & 39.6348 & 131.2 & -73.9 & 40.4808 \\
\hline c24y & 130.2 & -73.8 & 40.4010 & 131.0 & -73.8 & 40.3643 \\
\hline c24o & 128.2 & -73.5 & 40.2593 & 130.4 & -73.6 & 41.2922 \\
\hline c25y & 126.2 & -72.7 & 41.2967 & 129.4 & -73.1 & 42.9567 \\
\hline c25o & 126.2 & -72.8 & 41.7527 & 129.2 & -73.0 & 43.2241 \\
\hline c26y & 136.2 & -73.2 & 46.5499 & 129.2 & -72.7 & 42.2552 \\
\hline$c 260$ & 135.8 & -73.1 & 46.5712 & 129.2 & -72.6 & 42.5103 \\
\hline c27y & 123.4 & -71.2 & 44.2268 & 126.6 & -71.8 & 45.7244 \\
\hline c27o & 127.6 & -71.8 & 46.7069 & 126.4 & -71.7 & 46.0665 \\
\hline c28y & 123.2 & -70.9 & 45.2080 & 125.6 & -71.3 & 46.1518 \\
\hline c28o & 123.0 & -70.6 & 46.4424 & 125.0 & -70.9 & 47.2041 \\
\hline c29y & 123.2 & -70.5 & 46.7231 & 124.8 & -70.8 & 47.3364 \\
\hline c29o & 125.2 & -70.7 & 48.2760 & 124.4 & -70.6 & 47.6178 \\
\hline c30y & 124.6 & -70.5 & 48.5666 & 123.8 & -70.3 & 47.9505 \\
\hline$c 300$ & 124.6 & -69.9 & 50.1996 & 122.8 & -69.5 & 49.0102 \\
\hline c31y & 124.8 & -69.9 & 50.7559 & 122.8 & -69.5 & 48.9948 \\
\hline c310 & 119.6 & -68.5 & 49.4998 & 121.8 & -69.0 & 49.6489 \\
\hline c32.1y & 122.6 & -69.0 & 51.9188 & & & \\
\hline c32.10 & 125.8 & -69.5 & 53.9977 & & & \\
\hline c32.2y & 129.4 & -70.2 & 54.6781 & & & \\
\hline c32.20 & 111.8 & -65.8 & 47.6404 & & & \\
\hline c32r & 115.6 & -67.0 & 53.6203 & & & \\
\hline c33y & 132.6 & -70.2 & 61.5636 & & & \\
\hline c33o & 321.0 & 52.1 & 44.9223 & & & \\
\hline
\end{tabular}


Appendix F: JaMBES and associated software - documentation

JaMBES is a MatLab (C) code that produces Euler poles for some consecutive suite of seafloor magnetic anomalies. Here JaMBES and the pre-processing codes that must be run first are described.

The three codes which have to be run to produce the set of Euler poles are:

- poledatain, which processes database data into a simplified format for subsequent analysis

- polefit which produces single Euler poles for a set of ridge segment (RS)and fracture zone (FZ) data for a given chron.

- JaMBES itself.

\section{Operating controls}

The suite of codes is managed by altering commands in a .xlsx workbook, 'JaMBES_controls.xlsx', which is read by all three codes.

The first sheet is structured thus:

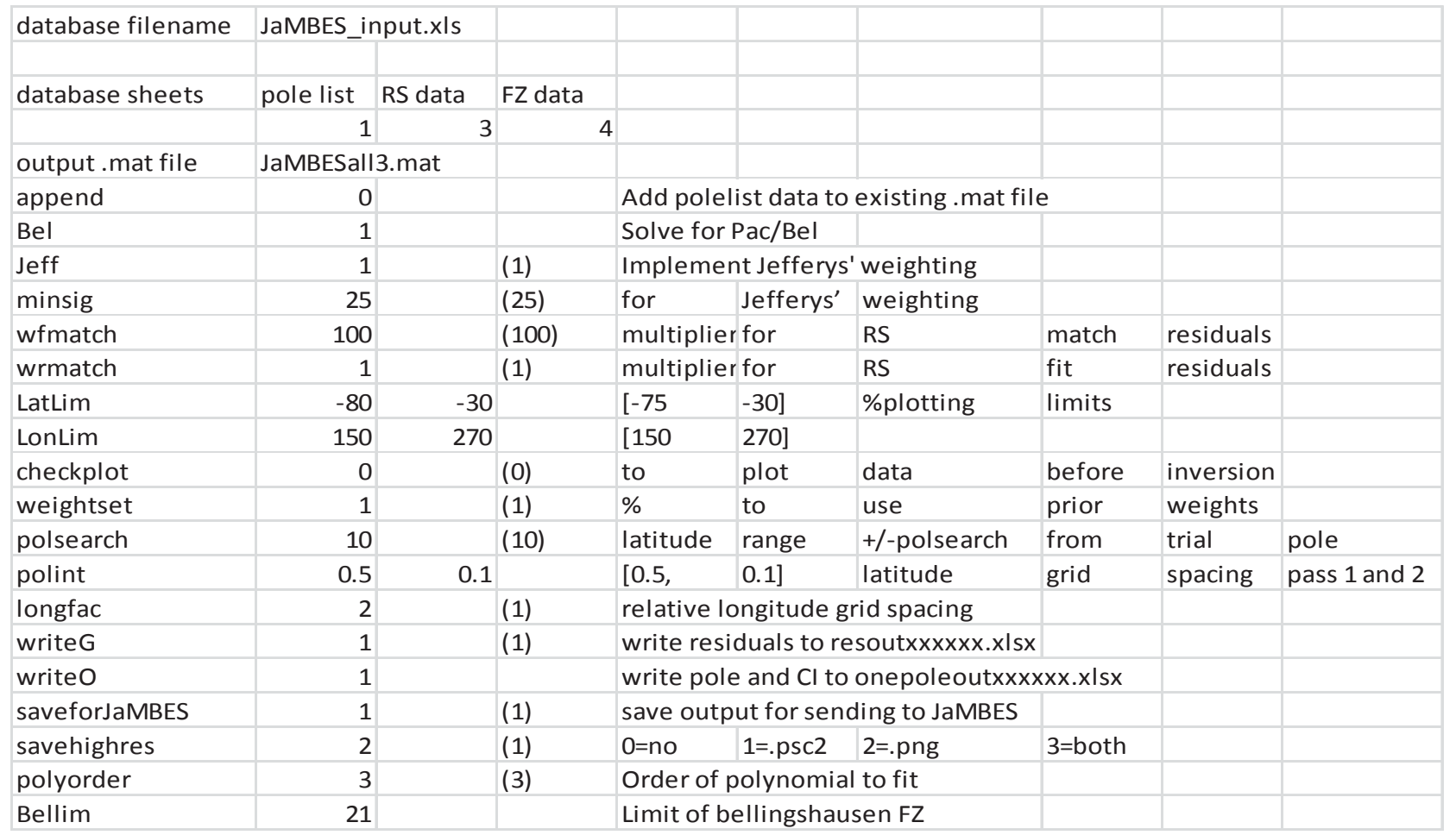

The first 5 lines are used by poledatain.

The name of the database file (.xls or .xlsx) is in position B1.

The sheets from the database to be read for the list of chrons, for magnetic (RS) and fracture zone (FZ) data are in B4, C4 and D4 (1, 3, 4 above).

The name of the MatLab .mat file for the output is in position B5.

The remaining lines are the parameters for onepole, whose effects are briefly described by their accompanying annotations. 
The second sheet has the following structure:

\begin{tabular}{|l|l|l|l|}
\hline Ridge segments not use & 6 & 12 & 13 \\
\hline FZ not use & 0 & & \\
\hline exclude Ids & 5 & 6 & \\
\hline
\end{tabular}

The rows are lists of ridge segment, FZ and uncertainty Id identifiers not to be used in the inversion. The code assigns these an uncertainty of 9999 , ensuring a weight of very near zero. A zero is placed in column B if there are no exclusions e.g. for FZ in the example above.

The third sheet contains the list of chrons to be processed by onepole or JaMBES. It has the structure:

\begin{tabular}{|l|}
\hline c32.1y \\
c32.1o \\
c32.2y \\
c32.2o \\
\hline c32r \\
\hline
\end{tabular}

\section{poledatain}

poledatain reads the .xls workbook named in 'JaMBES_controls.xls' sheet 1 B1 e.g. "Jambes_input.xls" above.

Sheet 1 has a list of chrons to be processed, with this structure:

\begin{tabular}{lrrr} 
Pole list & trial pole & \multicolumn{2}{c}{ Geomagnet } \\
c20y & -70 & 130 & 42.536 \\
c20o & -70 & 130 & 43.789 \\
c21y & -70 & 130 & 46.264 \\
c21o & -70 & 130 & 47.906 \\
c22y & -70 & 130 & 49.037 \\
c22o & -70 & 130 & 49.714 \\
c23y & -70 & 130 & 50.778 \\
c23o & -70 & 130 & 51.743 \\
c24y & -70 & 130 & 52.364 \\
c24o & -70 & 130 & 53.347
\end{tabular}

Each chron to be processed has an accompanying trial Euler pole and geomagnetic age.

For each chron in the 'pole list', poledatain reads the RS data and finds all the matching RS data and stores the required data in an array (Gall) indexed by the position of the chron on the list.

It then reads the FZ data, finds the data for the chron being processed, and appends it to the RS data.

Finally, poledatain saves its output to a MatLab .mat file specified in controls sheet 1 . The following are saved:

$\mathrm{Xp}$ - the numeric data accompanying the chron list (sheet columns 2-4)

Hp- the chron list

Gall - data for the Euler pole inversion

Call - supplementary data giving, for each chron, the cruise name and profile number of each ridge segment

ID - The qualitative descriptor for the RS error

npole - the number of chrons processed

$\mathrm{nr}-\mathrm{a}$ vector giving the number of RS for each chron. 
Gall is indexed by the chron and has the following structure for each chron:

\begin{tabular}{|r|r|r|r|r|r|}
\hline 1 & 20 & -64.251 & 226.6024 & 2.300549 & 0 \\
\hline 2 & 24 & -56.8394 & 189.772 & 2.307211 & 0 \\
\hline 2 & 24 & -58.225 & 190.072 & 2.029572 & 0 \\
\hline 2 & 24 & -58.5899 & 190.0003 & 2.566456 & 0 \\
\hline 2 & 6 & -50.7856 & 221.4934 & 62.62899 & 0 \\
\hline 2 & 24 & -57.7361 & 189.8194 & 3.92665 & 0 \\
\hline 2 & 24 & -58.1562 & 190.6105 & 4.656291 & 0 \\
\hline 2 & 16 & -51.6221 & 200.1381 & 2.534259 & 0 \\
\hline 1 & 39 & -68.8162 & 198.4706 & 1.5 & 1 \\
\hline 1 & 39 & -68.8676 & 198.5348 & 1.5 & 1 \\
\hline 1 & 39 & -68.9087 & 198.5888 & 1.5 & 1 \\
\hline 1 & 39 & -68.9421 & 198.6324 & 1.5 & 1 \\
\hline 1 & 39 & -68.9678 & 198.6838 & 1.5 & 1 \\
\hline
\end{tabular}

Column 1 is a plate identifier. In the reconstructions, plate 2 is held fixed and plate 1 backrotated.

Column 2 is a segment identifier.

Columns 3 and 4 are the position (latitude, longitude) of the datapoint.

Column 5 is an estimate of the uncertainty of the datapoint; an estimated standard deviation in $\mathrm{km}$. This is described in chapter 2.

Column 6 is a RS/FZ identifier: 0 for RS and 1 for FZ.

\section{polefit}

polefit finds best-fitting Euler poles independently for each of specified number of chrons. It does this by computing the misfit on a grid of latitude and longitude followed by a grid search for the best fit, which has the advantage that $95 \%$ confidence intervals for the poles' locations are generated directly from the contoured grid values.

For each grid-point, a best-fitting finite rotation is calculated, as described below. The misclosure from back-rotation contributes to the overall misfit. The misfit at each grid-point has three components:

\section{Matching RS}

It is assumed that the spreading direction is (approximately) normal to the RS axis direction.

First a ridge centre is calculated for each RS, separately for each plate, as a weighted mean of the position of each datapoint, which is a 3 vector on a unit sphere, the weights coming from the prior standard deviation estimates (see 'Gall' above). An overall weight is also calculated. Segments consisting of a single point are not excluded, but they are assigned zero contribution to the degrees of freedom of the residuals.

Then the scalar product is calculated of each datapoint vector with the normal, $n$, to the great circle plane between the trial pole and the ridge centre. This is the distance (on a unit sphere) of the datapoint from the great circle plane, converted to weighted surface misfit in $\mathrm{km}$ by multiplying by its weight and the Earth's radius $(6371 \mathrm{~km})$.

\section{$\underline{\mathrm{FZ}}$}

It is assumed that the FZ are parallel to the plate motion in the sense that they lie on a small circle around the trial pole. If this were so, the angular distance of each FZ point, on both plates, from the pole would be the same. Therefore, the spread of these angles about the mean angle 
gives a measure of pole misfit. The angle differences are converted to weighted surface misfit in km by multiplying by its weight and the Earth's radius.

\section{Rotation angle}

For the trial pole, the rotation angle is determined from the weighted mean cosine of the angle between the normals $\mathrm{n}$ for corresponding RS on the two plates (figure 1).

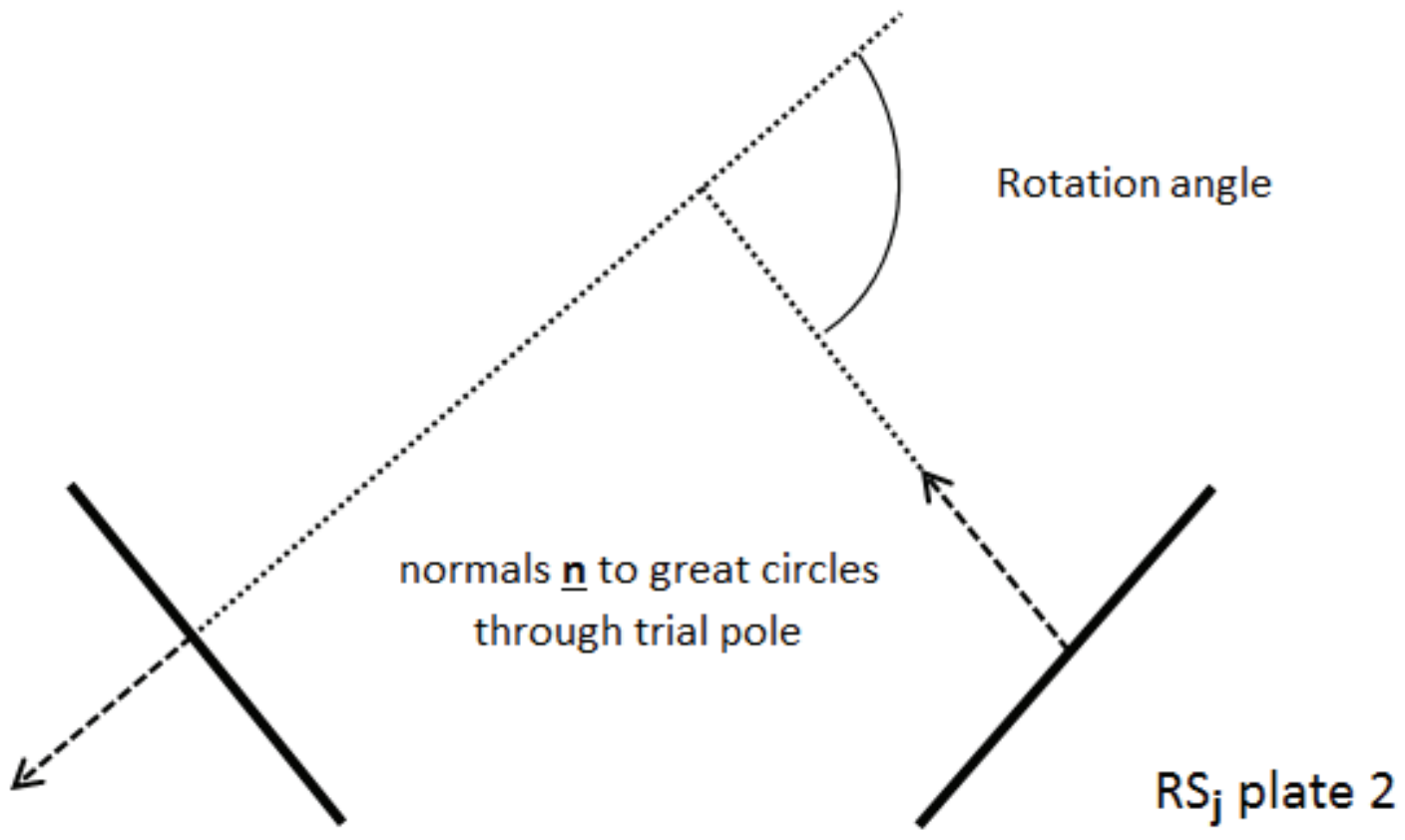

\section{$\mathrm{RS}_{\mathrm{j}}$ plate 1}

\section{+ Trial pole}

Figure 1: cartoon showing calculation of the rotation angle for a pair of RS.

Misclosure

There is no reason to assume that the ridge centres of a matching pair of RS will coincide after back rotation. However the component of the back-rotated point in the direction of rotation from the true Euler pole should be small (see figure 2).

\section{Back-rotated centre $\mathrm{RS}_{\mathrm{j}}$ plate 2}

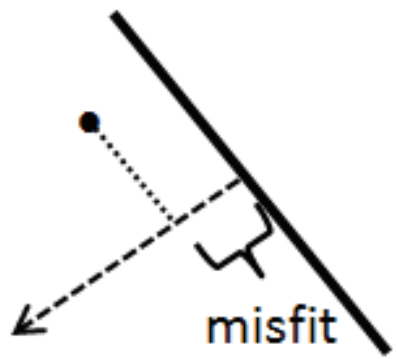

$\mathrm{RS}_{\mathrm{j}}$ plate 1

normal to great circle

through pole

+ Trial pole

Figure 2: cartoon showing calculation of back-rotation misfit of a RS. 
So the misclosure is calculated as the scalar product of the back-rotated RS with the normal to the "fixed" plate RS. This is converted to a weighted surface displacement by multiplying by its weight and the Earth's radius.

NB: the user-provided uncertainties can be bypassed, and all weights made 1, if controls parameter "weightset" is 0 . This is not recommended for final processing.

\section{Iterations}

The gridsearch is repeated three times. In the first iteration, a coarse grid is used to refine the pole estimate provided in JaMBES_controls.xls sheet 1. Subsequently, a finer grid is used. At each iteration the search grid is re-centred on the new trail pole of the previous iteration. The latitude grid spacing is specified in JaMBES_controls.xls sheet 1, parameter "polint". The relative longitude spacing is set by parameter "longfac", so that e.g. longfac $=2$ doubles the latitude grid spacing.

\section{Jeffreys weighting}

After the first iteration, if controls parameter "Jeff" = 1, then Jeffreys' weighting (Jeffreys, 1961) is applied. It is commonly observed that the distributions of RS residuals are long tailed, implying that the error distributions are not Gaussian. It is well known that these "outliers" have excessive influence on a least-squares solution. Jeffreys' weighting attempts to assign a weight to each residual according to its probability that it belongs to a central Gaussian distribution with standard deviation, $\sigma$, smaller that the rms residual which is "contaminated" by the outliers. A limiting minimum value for $\sigma$ is set by controls parameter "minsig" $(25 \mathrm{~km}$ in the example). This weighting is only applied to RS residuals, as FZ residuals are found not to have outliers. Because of possible skewness, the Jeffreys weights are separately calculated for the negative and positive residuals.

The Jeffreys weights are applied in the second and 3rd iterations. A cumulative distribution of the final residuals is displayed in a MatLab figure. Three curves are plotted:

- The final residuals (black)

- The final weighted residuals (blue)

- A Gaussian model fitted to the weighted residuals (cyan).

If the data are uncontaminated by outliers, the three curves should be quite similar.

\section{Uncertainty estimation}

If it can be assumed that the data-points, for RS, FZ and misclosure, are independent and that their relative uncertainties are normal and that their standard deviations have been correctly estimated a priori, then at the best estimate of the true pole, the mean sum of weighted squared errors from the three sources of misfit are an estimate of the variance $\sigma^{2}$ of the now commonly distributed error. At any nearby point, the mean sum of weighted squared errors divided by $\sigma^{2}$ has an $\mathrm{F}$ distribution with degrees of freedom $\mathrm{df}$ of the numerator and denominator equal to the number of independent data minus the number of estimated parameters.

Therefore if the mean of the sum of squared residuals (MSSR) at the gridpoints is divided by the minimum MSSR, the contour of the F0.95(df, df) estimates the $95 \%$ confidence area for the pole. The estimated $95 \%$ contour is displayed in the same figure as the residuals.

We have found that the projection of the contour onto a sphere gives a shape which is well approximated by an ellipse, implying Gaussian errors. In particular, there are no secondary least-squares minima. A best-fitting Gaussian distribution for the uncertainties is found by finding the ellipse that best matches the observed $95 \%$ contour. 
However, some of the assumptions may not be valid.

While it is reasonable to treat every RS measurement as independent, it is not valid to treat each datapoint defining an FZ position as independent of the rest. The FZ are assumed to lie on a small circle. If the number of datapoints defining the arc of the circle is, say, doubled from a large number, the accuracy of the resulting arc model is not much improved. The problem of fairly assigning degrees of freedom to FZ segments remains to be resolved. In onepole the problem is dealt with by:

- Each FZ on each plate is assigned $2 \mathrm{df}$;

- The (weighted) contributions from RS and FZ matching are re-weighted so the RS and FZ make equal contribution to the overall misfit.

\section{JaMBES}

$J a M B E S$ refines the individual Euler pole estimates from onepole as follows.

First, a smooth track for the Euler pole through time is calculated. It is assumed that the track may be modelled as a polynomial function of pole position, in three dimensions, in which time (from a geomagnetic timescale) is used to parameterise the track. Polynomial orders from 1 (linear) to 3 generally give satisfactory results. The smooth track assumption is reasonable for most times, but becomes invalid if, for example, one plate breaks into two (e.g. Antarctica to Marie Byrd Land and Bellingshausen), or some other event causes a discontinuous change in plate motion.

JaMBES calculates a posterior Gaussian probability distribution for the "smooth-track" pole estimates, which is then used in the second refinement. The second refinement involves calculating a maximum likelihood (ML) estimate of the Euler pole, where the likelihood function is the product of the posterior probability densities from onepole and smoothtrack.

The effect of this is that when the onepole uncertainties are large, the ML estimate is close to the smooth-track, but where the onepole solution is well determined and distant from the smooth track the ML pole deviates away from the smooth track towards to onepole solution.

A 95\% confidence region is calculated for the ML estimate by assuming that twice the log of the likelihood ratio at any point to the maximum likelihood is approximately a chi-squared distribution with $2 \mathrm{df}$ (e.g. Williams (2001) p 229).

onepole and JaMBES can be run in two-plate or three-plate modes. If in JaMBES_controls.xls $\mathrm{Bel}=1$, solutions for Pacific-Bellingshausen and Pacific-Marie Byrd Land are separately calculated, with Bellim (21 in the example) specifying the FZ change-over point between Bellingshausen and Marie Byrd Land.

\section{$\underline{\text { References }}$}

Jeffresys, H. 1961. Ch. 4 Approximate methods and simplifications. Theory of Probability. 3rd ed. Oxford: Clarendon Press.

Williams. D. 2001. Weighing the odds. Cambridge UP 547pp. 
Appendix G: Figures from the main text including all 95\% confidence regions

Figure $\mathrm{V}-2$ :

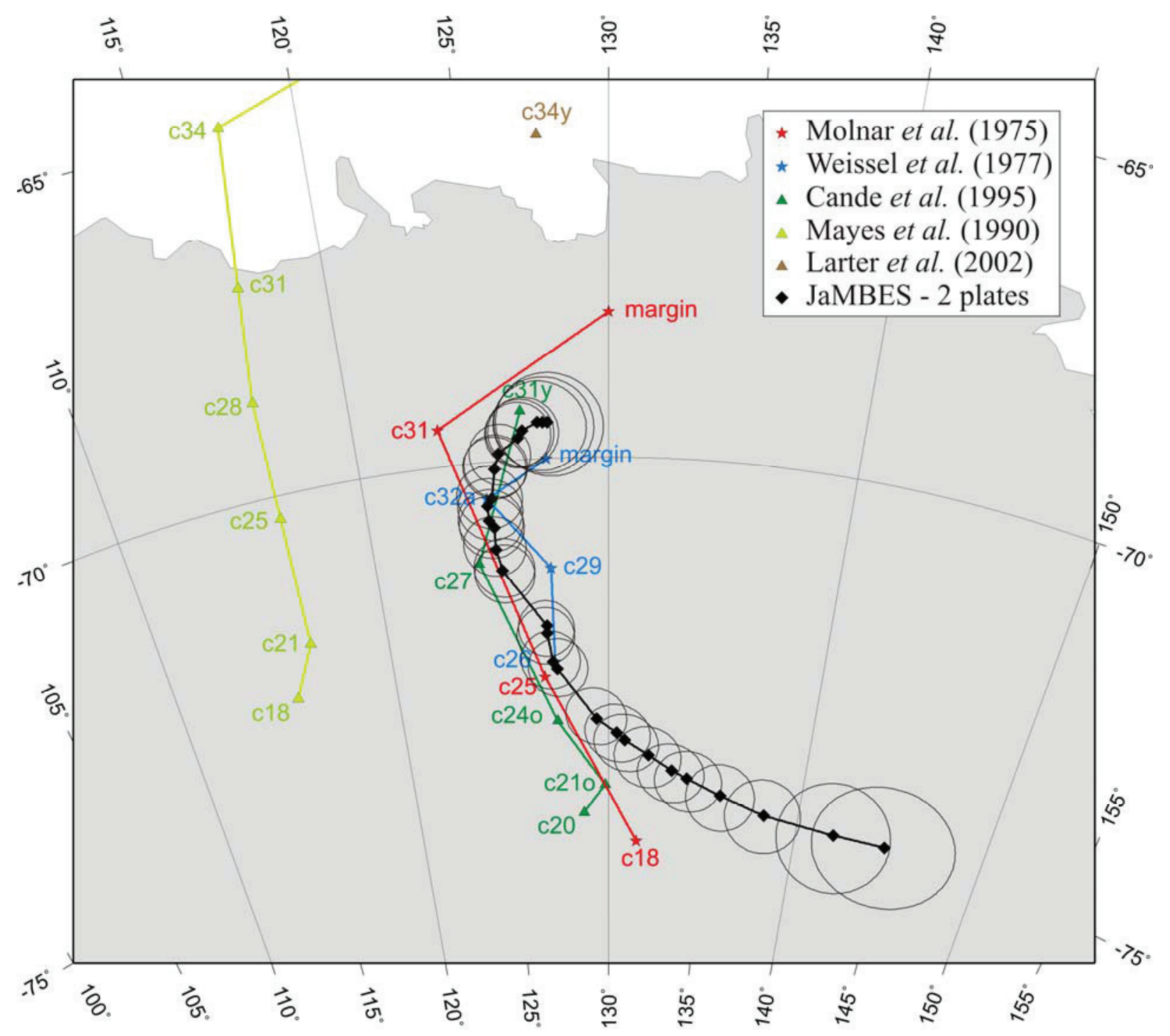


Figure V-5:

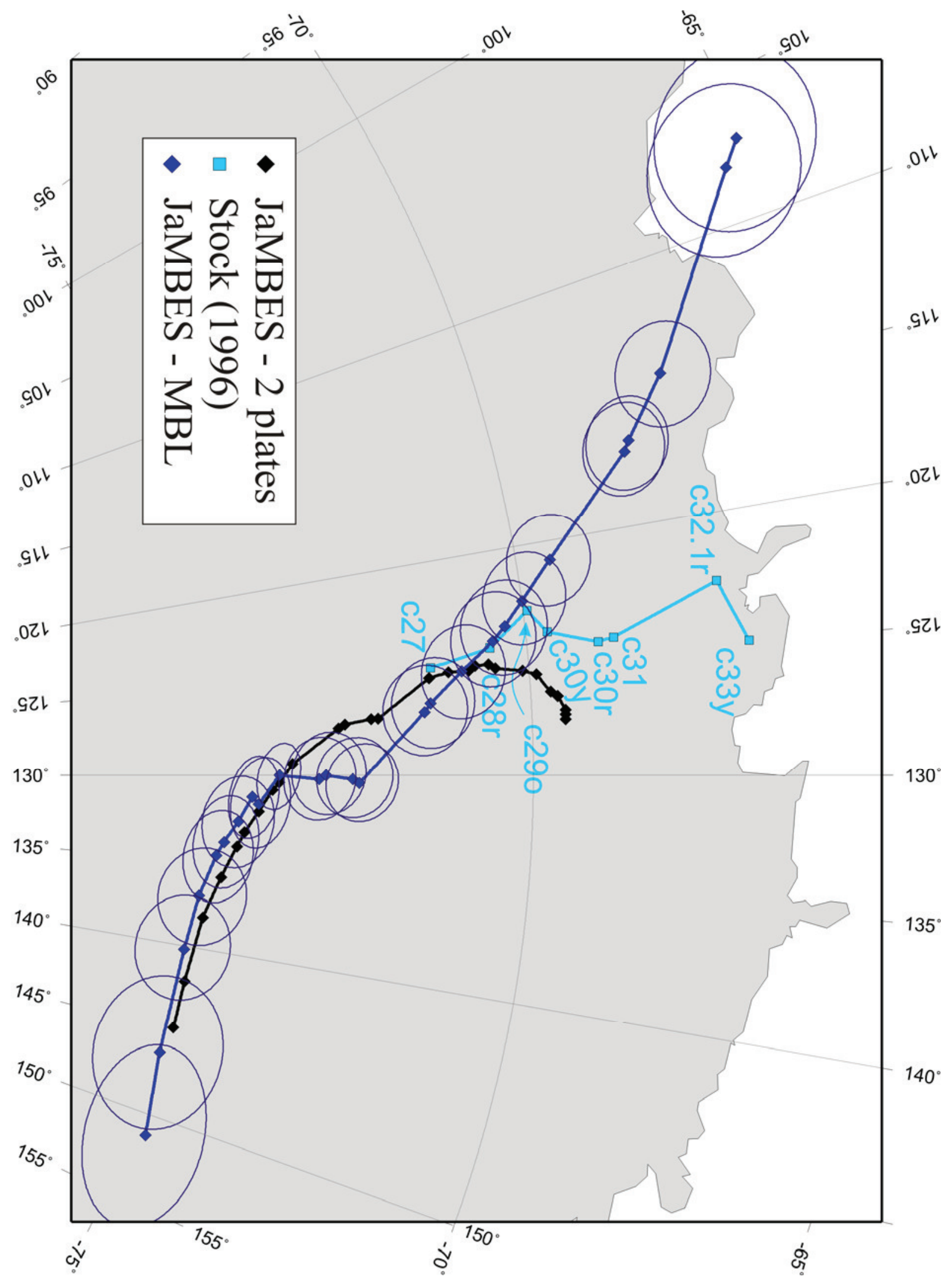


Figure V-6:

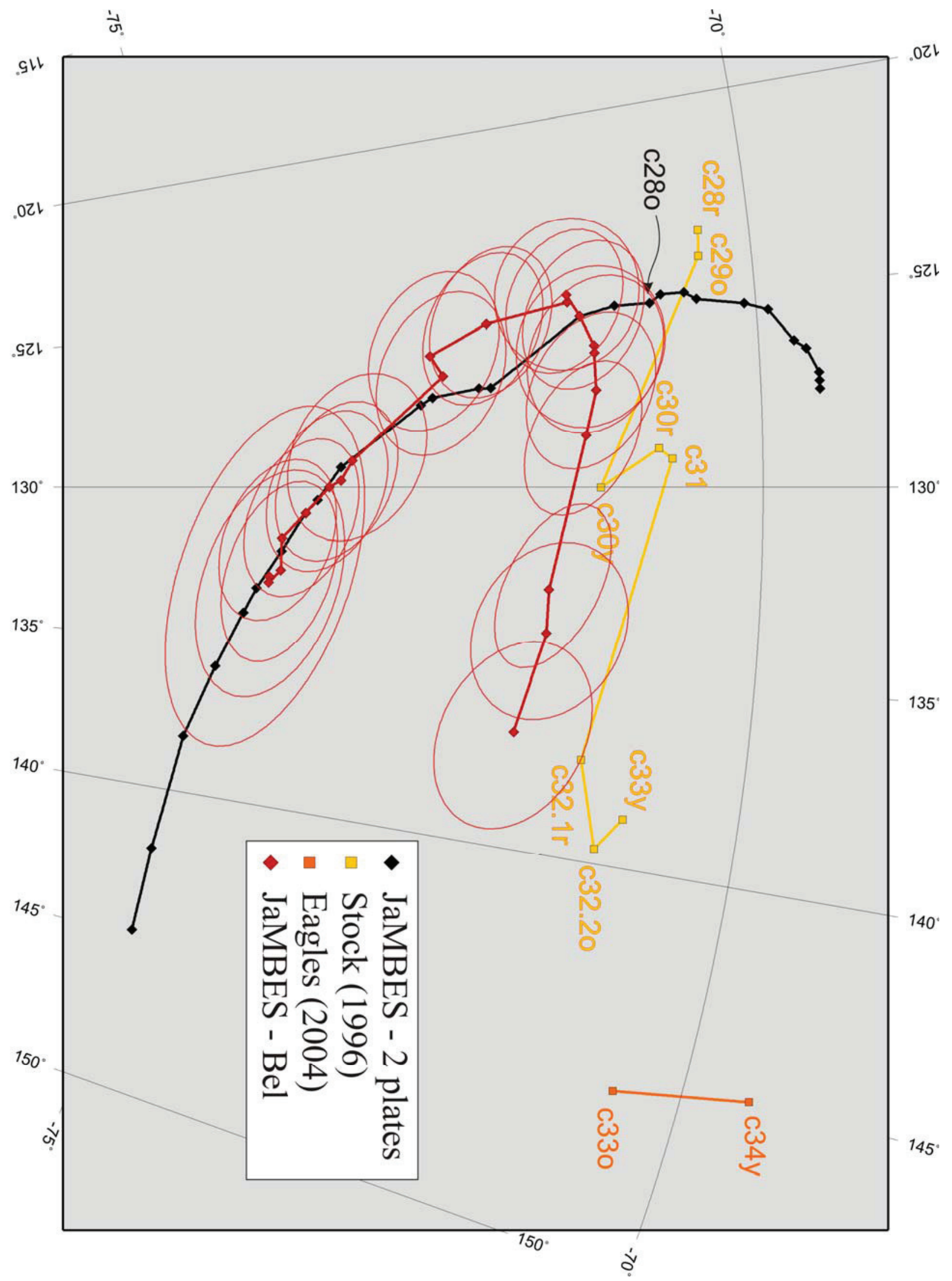


Figure V-8:

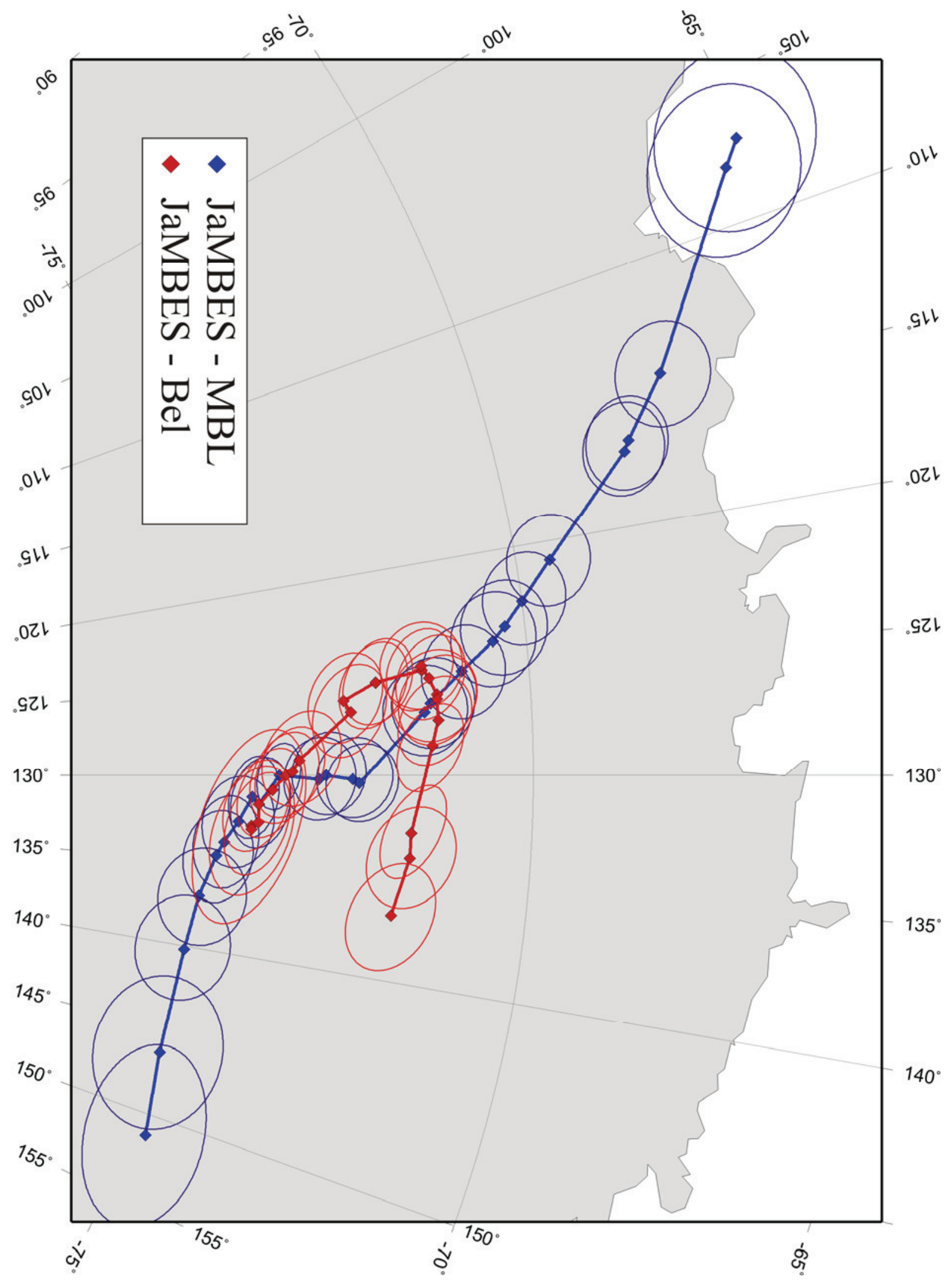


Appendix H: Full output from hellinger

Covariance matrices are listed as terms

$$
\frac{1}{\hat{K}}\left(\begin{array}{lll}
a & b & c \\
b & d & e \\
c & e & f
\end{array}\right) \quad \text { [Kirkwood et al., 1999]. }
$$

Table H-1A. Finite rotations using all data, and fracture zone uncertainty assigned as $1.5 \mathrm{~km}$.

\begin{tabular}{|c|c|c|c|c|c|c|c|}
\hline \multirow{2}{*}{ Chron } & \multicolumn{7}{|c|}{ Hellinger - rotations } \\
\hline & Iongitude & latitude & angle $(\stackrel{\circ}{)})$ & rmin & df & ndat & nsect \\
\hline c20y & 176.4581 & \begin{tabular}{|c|}
-69.2698 \\
\end{tabular} & 71.5187 & 1283.8575 & 26 & 43 & 7 \\
\hline c20o & 131.0320 & -74.9059 & 36.7219 & 2484.3074 & 23 & 40 & 7 \\
\hline c21y & 130.3143 & -74.5989 & 37.5278 & 834.6496 & 32 & 51 & 8 \\
\hline c210 & 104.0467 & -70.3020 & 30.3157 & 2725.7428 & 41 & 64 & 10 \\
\hline c22y & 124.8968 & -73.8976 & 36.9116 & 2628.0125 & 32 & 53 & 9 \\
\hline $\mathrm{c} 220$ & 129.0815 & -74.1139 & 38.6169 & 1781.2169 & 40 & 61 & 9 \\
\hline c23y & 130.1706 & -74.1552 & 39.3772 & 1431.9776 & 36 & 55 & 8 \\
\hline $\mathrm{c} 230$ & 128.2227 & -73.8098 & 39.2640 & 1385.6744 & 43 & 60 & 7 \\
\hline c24y & 128.9282 & -73.6471 & 40.8312 & 3303.7624 & 51 & 70 & 8 \\
\hline c24o & 129.2694 & -73.6633 & 40.3999 & 1732.9771 & 117 & 140 & 10 \\
\hline c25y & 130.3226 & -73.1510 & 43.0864 & 800.7541 & 143 & 172 & 13 \\
\hline c250 & 127.3579 & -72.8446 & 42.4543 & 238.1173 & 132 & 161 & 13 \\
\hline c26y & 128.7294 & -72.7038 & 44.0460 & 787.9690 & 138 & 169 & 14 \\
\hline c26o & 128.6037 & -72.6960 & 44.1610 & 667.0427 & 143 & 174 & 14 \\
\hline c27y & 124.8711 & -71.4199 & 45.0004 & 5893.9692 & 248 & 275 & 12 \\
\hline$c 270$ & 125.3171 & -71.4266 & 45.4760 & 6452.6495 & 249 & 276 & 12 \\
\hline$c 28 y$ & 122.3163 & -70.8243 & 44.5332 & 4690.6926 & 295 & 324 & 13 \\
\hline c28o & 122.1058 & -70.5781 & 45.2462 & 5076.3816 & 292 & 323 & 14 \\
\hline c29y & 122.8966 & -70.6359 & 46.0210 & 5710.3536 & 270 & 301 & 14 \\
\hline c29o & 124.1605 & -70.6855 & 47.5285 & 7211.1036 & 260 & 289 & 13 \\
\hline c30y & 125.8718 & -70.8514 & 49.1753 & 7516.9001 & 239 & 268 & 13 \\
\hline$c 300$ & 125.0401 & -70.3467 & 50.7921 & 9172.3845 & 226 & 253 & 12 \\
\hline c31y & 126.0783 & -70.4801 & 51.6504 & 8438.8419 & 222 & 249 & 12 \\
\hline c310 & 129.7517 & -70.8697 & 54.4753 & 7001.0225 & 218 & 245 & 12 \\
\hline c32.1y & 120.7690 & -69.0434 & 51.3224 & 24345.6753 & 115 & 132 & 7 \\
\hline c32.10 & 119.7568 & -68.5784 & 51.0075 & 26191.6689 & 122 & 145 & 10 \\
\hline c32.2y & 125.6254 & -69.5099 & 55.5125 & 12363.0204 & 133 & 156 & 10 \\
\hline$c 32.20$ & 114.0328 & -66.4265 & 49.2549 & 18704.1787 & 112 & 129 & 7 \\
\hline$c 32 r$ & 119.3172 & -67.7705 & 52.8974 & 7099.6165 & 86 & 103 & 7 \\
\hline$c 33 y$ & 116.7153 & -67.1287 & 51.4554 & 16764.1019 & 92 & 107 & 6 \\
\hline c33o & 134.0173 & -70.4553 & 66.6918 & 60.9334 & 11 & 18 & 2 \\
\hline
\end{tabular}


Table H-1B. Covariance matrices, using all data, and fracture zone uncertainty assigned as $1.5 \mathrm{~km}$.

\begin{tabular}{|c|c|c|c|c|c|c|c|}
\hline \multirow{2}{*}{ Chron } & \multicolumn{7}{|c|}{ Hellinger - covariance } \\
\hline & kappa hat & a & b & c & d & e & $f$ \\
\hline c20y & 0.0203 & $7.83 \mathrm{E}-05$ & $4.29 \mathrm{E}-05$ & $1.60 \mathrm{E}-04$ & $2.46 \mathrm{E}-05$ & $8.89 \mathrm{E}-05$ & 3.34E-04 \\
\hline $\mathrm{c} 20 \mathrm{o}$ & & $3.12 \mathrm{E}-06$ & $2.68 \mathrm{E}-06$ & $9.03 \mathrm{E}-06$ & $2.37 \mathrm{E}-06$ & $7.83 \mathrm{E}-06$ & $2.64 \mathrm{E}-05$ \\
\hline c21y & 0.0383 & $8.69 \mathrm{E}-06$ & 7.84E-06 & 2.57E-05 & $7.22 \mathrm{E}-06$ & 2.33E-05 & 7.66E-05 \\
\hline c21o & 0.0150 & 3.66E-05 & 3.57E-05 & 1.15E-04 & $3.50 \mathrm{E}-05$ & $1.12 \mathrm{E}-04$ & 3.61E-04 \\
\hline$c 22 y$ & 0.0122 & 1.08E-05 & 1.04E-05 & 3.38E-05 & 1.00E-05 & 3.25E-05 & 1.06E-04 \\
\hline c22o & 0.0225 & 1.95E-07 & 1.95E-07 & 7.06E-07 & $2.66 \mathrm{E}-07$ & $8.38 \mathrm{E}-07$ & $2.98 \mathrm{E}-06$ \\
\hline$c 23 y$ & 0.0251 & $1.95 \mathrm{E}-07$ & $1.84 \mathrm{E}-07$ & $6.62 \mathrm{E}-07$ & $2.31 \mathrm{E}-07$ & $7.10 \mathrm{E}-07$ & $2.55 \mathrm{E}-06$ \\
\hline$c 230$ & 0.0310 & $3.72 E-07$ & $2.92 E-07$ & $1.28 \mathrm{E}-06$ & $3.75 \mathrm{E}-07$ & $1.18 \mathrm{E}-06$ & $4.79 \mathrm{E}-06$ \\
\hline c24y & 0.0154 & $1.90 \mathrm{E}-07$ & $1.51 \mathrm{E}-07$ & $8.44 \mathrm{E}-07$ & 7.22E-07 & 1.32E-06 & 4.77E-06 \\
\hline c24o & 0.0675 & $1.11 \mathrm{E}-07$ & 8.67E-08 & 3.77E-07 & $1.49 \mathrm{E}-07$ & $3.46 \mathrm{E}-07$ & $1.39 \mathrm{E}-06$ \\
\hline c25y & & 1.92E-07 & 1.63E-07 & $6.60 \mathrm{E}-07$ & 2.13E-07 & $6.05 \mathrm{E}-07$ & $2.34 \mathrm{E}-06$ \\
\hline c25o & & $2.68 \mathrm{E}-07$ & $1.62 \mathrm{E}-07$ & 8.72E-07 & $2.25 \mathrm{E}-07$ & $5.75 \mathrm{E}-07$ & $2.93 \mathrm{E}-06$ \\
\hline c26y & 0.1751 & $1.40 \mathrm{E}-07$ & 1.03E-07 & $4.82 E-07$ & $1.31 \mathrm{E}-07$ & $3.92 \mathrm{E}-07$ & $1.73 \mathrm{E}-06$ \\
\hline c26o & 0.2144 & $1.25 \mathrm{E}-07$ & $9.95 \mathrm{E}-08$ & 4.36E-07 & 1.43E-07 & 3.93E-07 & $1.60 \mathrm{E}-06$ \\
\hline$c 27 y$ & 0.0421 & $3.97 \mathrm{E}-08$ & $2.87 \mathrm{E}-08$ & $1.46 \mathrm{E}-07$ & $3.06 \mathrm{E}-08$ & $1.13 \mathrm{E}-07$ & $5.55 \mathrm{E}-07$ \\
\hline c27o & 0.0386 & $4.29 \mathrm{E}-08$ & $3.15 \mathrm{E}-08$ & $1.59 \mathrm{E}-07$ & 3.45E-08 & $1.26 \mathrm{E}-07$ & $6.08 \mathrm{E}-07$ \\
\hline$c 28 y$ & 0.0629 & $4.27 \mathrm{E}-08$ & $3.45 \mathrm{E}-08$ & $1.67 \mathrm{E}-07$ & 3.97E-08 & $1.45 \mathrm{E}-07$ & $6.72 \mathrm{E}-07$ \\
\hline $\mathrm{c} 280$ & 0.0575 & 5.98E-08 & $4.58 \mathrm{E}-08$ & 2.38E-07 & 4.76E-08 & $1.94 \mathrm{E}-07$ & $9.69 \mathrm{E}-07$ \\
\hline$c 29 y$ & 0.0473 & 7.10E-08 & $5.43 E-08$ & 2.87E-07 & $5.52 E-08$ & 2.32E-07 & $1.19 \mathrm{E}-06$ \\
\hline$c 290$ & 0.0361 & 3.97E-08 & $3.23 E-08$ & $1.62 \mathrm{E}-07$ & 3.75E-08 & $1.43 \mathrm{E}-07$ & $6.91 \mathrm{E}-07$ \\
\hline c30y & 0.0318 & 4.80E-08 & 5.13E-08 & 2.05E-07 & 7.27E-08 & 2.36E-07 & $9.11 \mathrm{E}-07$ \\
\hline$c 300$ & 0.0246 & 8.90E-08 & $6.28 E-08$ & 3.72E-07 & 7.32E-08 & 2.87E-07 & $1.60 \mathrm{E}-06$ \\
\hline c31y & 0.0263 & 4.07E-08 & $3.72 \mathrm{E}-08$ & $1.74 \mathrm{E}-07$ & $6.08 \mathrm{E}-08$ & $1.84 \mathrm{E}-07$ & $7.85 \mathrm{E}-07$ \\
\hline c310 & & $1.38 \mathrm{E}-07$ & $1.08 \mathrm{E}-07$ & $5.77 \mathrm{E}-07$ & 1.39E-07 & $5.04 \mathrm{E}-07$ & $2.48 \mathrm{E}-06$ \\
\hline c32.1y & 0.0047 & $1.59 \mathrm{E}-07$ & $1.39 \mathrm{E}-07$ & 6.24E-07 & $1.59 \mathrm{E}-07$ & $6.21 \mathrm{E}-07$ & $2.78 \mathrm{E}-06$ \\
\hline c32.10 & 0.0047 & $1.16 \mathrm{E}-07$ & $9.97 \mathrm{E}-08$ & 4.47E-07 & $1.12 \mathrm{E}-07$ & $4.44 \mathrm{E}-07$ & $2.02 \mathrm{E}-06$ \\
\hline$c 32.2 y$ & 0.0108 & $1.77 \mathrm{E}-07$ & $1.63 \mathrm{E}-07$ & $7.54 \mathrm{E}-07$ & 1.83E-07 & $7.54 \mathrm{E}-07$ & $3.51 \mathrm{E}-06$ \\
\hline c32.20 & 0.0060 & 2.38E-07 & 3.29E-07 & $1.21 \mathrm{E}-06$ & 4.87E-07 & 1.76E-06 & $6.54 \mathrm{E}-06$ \\
\hline c32r & 0.0121 & 4.20E-07 & 5.42E-07 & 2.05E-06 & 7.39E-07 & $2.75 \mathrm{E}-06$ & $1.05 \mathrm{E}-05$ \\
\hline c33y & 0.0055 & 2.66E-07 & 3.66E-07 & 1.29E-06 & 5.47E-07 & $1.89 \mathrm{E}-06$ & $6.77 \mathrm{E}-06$ \\
\hline c33o & 0.1805 & 2.76E-05 & 4.23E-05 & 9.51E-05 & 6.92E-05 & 1.72E-04 & $4.91 \mathrm{E}-04$ \\
\hline
\end{tabular}


Table H-2A. Finite rotations using all data, and fracture zone uncertainty assigned as $5 \mathrm{~km}$.

\begin{tabular}{|c|c|c|c|c|c|c|c|}
\hline \multirow{2}{*}{ Chron } & \multicolumn{7}{|c|}{ Hellinger - rotations } \\
\hline & Iongitude & latitude & angle ( $\left.{ }^{\circ}\right)$ & rmin & df & ndat & nsect \\
\hline c20y & 306.9952 & -53.7752 & 94.9113 & 1445.9635 & 26 & 43 & 7 \\
\hline c20o & 134.4829 & -75.0163 & 37.7697 & 2482.4870 & 23 & 40 & 7 \\
\hline c21y & 133.1424 & -74.7507 & 38.4313 & 829.5779 & 32 & 51 & 8 \\
\hline c21o & 96.8041 & -69.1596 & 28.8836 & 2692.3736 & 41 & 64 & 10 \\
\hline c22y & 124.6106 & -73.9472 & 36.8682 & 2619.0114 & 32 & 53 & 9 \\
\hline c220 & 109.0384 & -71.2539 & 32.3235 & 1670.6542 & 40 & 61 & 9 \\
\hline$c 23 y$ & 129.2884 & -74.0616 & 39.0655 & 1409.1271 & 36 & 55 & 8 \\
\hline c23o & 130.1275 & -74.0746 & 39.7844 & 1283.8496 & 43 & 60 & 7 \\
\hline c24y & 190.6916 & -69.4811 & 98.6971 & 805.0067 & 51 & 70 & 8 \\
\hline c240 & 128.8138 & -73.6097 & 40.2564 & 1337.8746 & 117 & 140 & 10 \\
\hline c25y & 127.9500 & -72.8867 & 42.1370 & 172.7677 & 143 & 172 & 13 \\
\hline c25o & 126.5673 & -72.7675 & 42.0340 & 87.8992 & 132 & 161 & 13 \\
\hline c26y & 129.2328 & -72.7534 & 44.2396 & 134.4609 & 138 & 169 & 14 \\
\hline c26o & 128.9217 & -72.7342 & 44.2833 & 93.7893 & 143 & 174 & 14 \\
\hline c27y & 123.7916 & -71.2812 & 44.3554 & 4463.1187 & 248 & 275 & 12 \\
\hline c27o & 124.3258 & -71.3097 & 44.8614 & 4926.0380 & 249 & 276 & 12 \\
\hline c28y & 122.0546 & -70.7320 & 44.5832 & 1982.0912 & 295 & 324 & 13 \\
\hline $\mathrm{c} 280$ & 120.8609 & -70.2583 & 44.8657 & 1645.9685 & 292 & 323 & 14 \\
\hline c29y & 121.0798 & -70.2306 & 45.2763 & 3021.5864 & 270 & 301 & 14 \\
\hline c29o & 122.6132 & -70.3710 & 46.8207 & 3681.9170 & 260 & 289 & 13 \\
\hline c30y & 123.2205 & -70.3828 & 47.9038 & 3097.7119 & 239 & 268 & 13 \\
\hline$c 300$ & 117.7190 & -68.9012 & 46.7746 & 1900.5733 & 226 & 253 & 12 \\
\hline c31y & 117.6047 & -68.8488 & 46.8687 & 1637.5190 & 222 & 249 & 12 \\
\hline$c 310$ & 119.6305 & -69.1174 & 48.8195 & 1727.1850 & 218 & 245 & 12 \\
\hline c32.1y & 108.7633 & -66.4488 & 44.7969 & 14134.1370 & 115 & 132 & 7 \\
\hline$c 32.10$ & 114.1282 & -67.0652 & 47.3871 & 16689.2206 & 122 & 145 & 10 \\
\hline$c 32.2 y$ & 120.3240 & -68.5185 & 52.1823 & 6357.3307 & 133 & 156 & 10 \\
\hline$c 32.20$ & 109.9214 & -65.0027 & 46.8372 & 15916.8840 & 112 & 129 & 7 \\
\hline$c 32 r$ & 23.4562 & -58.1510 & 98.0170 & 9250.8948 & 86 & 103 & 7 \\
\hline c33y & 112.1495 & -65.9375 & 49.0461 & 14396.8733 & 92 & 107 & 6 \\
\hline c33o & & & & & & & \\
\hline
\end{tabular}


Table H-2B. Covariance matrices, using all data, and fracture zone uncertainty assigned as $5 \mathrm{~km}$.

\begin{tabular}{|c|c|c|c|c|c|c|c|}
\hline \multirow{2}{*}{ Chron } & \multicolumn{7}{|c|}{ Hellinger - covariance } \\
\hline & kappa hat & $\mathbf{a}$ & $\mathbf{b}$ & C & d & e & $f$ \\
\hline c20y & 0.0180 & $1.53 \mathrm{E}+01$ & $-4.75 \mathrm{E}-01$ & $-4.85 E+00$ & $1.50 \mathrm{E}-02$ & $1.51 \mathrm{E}-01$ & $1.54 \mathrm{E}+00$ \\
\hline c20o & 0.0093 & $9.20 \mathrm{E}-06$ & 7.70E-06 & $2.56 \mathrm{E}-05$ & $6.73 E-06$ & 2.23E-05 & 7.41E-05 \\
\hline c21y & 0.0386 & 4.03E-06 & $4.18 \mathrm{E}-06$ & $1.44 \mathrm{E}-05$ & $4.74 \mathrm{E}-06$ & $1.58 \mathrm{E}-05$ & $5.35 \mathrm{E}-05$ \\
\hline c210 & 0.0152 & 5.62E-05 & 4.51E-05 & $1.44 \mathrm{E}-04$ & 3.85E-05 & 1.23E-04 & 3.92E-04 \\
\hline c22y & 0.0122 & 1.97E-05 & 1.74E-05 & $5.65 \mathrm{E}-05$ & 1.57E-05 & $5.11 \mathrm{E}-05$ & 1.66E-04 \\
\hline c22o & 0.0239 & 2.14E-05 & 2.05E-05 & 6.67E-05 & $2.00 \mathrm{E}-05$ & 6.49E-05 & 2.12E-04 \\
\hline$c 23 y$ & 0.0255 & $2.56 \mathrm{E}-06$ & $2.53 E-06$ & $8.35 \mathrm{E}-06$ & $2.84 \mathrm{E}-06$ & $9.29 \mathrm{E}-06$ & $3.08 \mathrm{E}-05$ \\
\hline$c 230$ & 0.0335 & $1.93 \mathrm{E}-06$ & $1.90 \mathrm{E}-06$ & $6.32 \mathrm{E}-06$ & $2.20 \mathrm{E}-06$ & $6.85 \mathrm{E}-06$ & $2.26 \mathrm{E}-05$ \\
\hline c24y & 0.0634 & 3.54E-05 & 2.37E-05 & 7.76E-05 & 2.50E-05 & $8.35 \mathrm{E}-05$ & 2.81E-04 \\
\hline c24o & 0.0875 & $1.07 \mathrm{E}-06$ & $1.12 \mathrm{E}-06$ & $3.72 \mathrm{E}-06$ & $1.34 \mathrm{E}-06$ & $4.21 \mathrm{E}-06$ & 1.40E-05 \\
\hline c25y & 0.8277 & 6.42E-07 & $5.40 \mathrm{E}-07$ & 2.03E-06 & $5.38 \mathrm{E}-07$ & $1.82 \mathrm{E}-06$ & $6.85 \mathrm{E}-06$ \\
\hline c25o & 1.5017 & $8.64 \mathrm{E}-07$ & $6.12 \mathrm{E}-07$ & $2.62 \mathrm{E}-06$ & $6.01 \mathrm{E}-07$ & $2.03 \mathrm{E}-06$ & $8.68 \mathrm{E}-06$ \\
\hline c26y & 1.0263 & $5.62 E-07$ & $4.78 \mathrm{E}-07$ & $1.80 \mathrm{E}-06$ & $4.95 \mathrm{E}-07$ & $1.68 \mathrm{E}-06$ & $6.38 \mathrm{E}-06$ \\
\hline$c 260$ & 1.5247 & $3.99 \mathrm{E}-07$ & $3.30 \mathrm{E}-07$ & $1.25 \mathrm{E}-06$ & $3.62 E-07$ & $1.19 \mathrm{E}-06$ & $4.49 E-06$ \\
\hline c27y & 0.0556 & 9.02E-08 & $6.08 \mathrm{E}-08$ & $2.74 \mathrm{E}-07$ & $5.22 \mathrm{E}-08$ & $2.01 \mathrm{E}-07$ & $9.70 \mathrm{E}-07$ \\
\hline c27o & 0.0505 & 9.80E-08 & 6.64E-08 & 3.06E-07 & 5.83E-08 & 2.27E-07 & 1.10E-06 \\
\hline$c 28 y$ & 0.1488 & $9.95 \mathrm{E}-08$ & $7.67 \mathrm{E}-08$ & $3.50 \mathrm{E}-07$ & 7.63E-08 & $3.02 E-07$ & $1.40 \mathrm{E}-06$ \\
\hline$c 280$ & 0.1774 & $1.37 \mathrm{E}-07$ & $1.04 \mathrm{E}-07$ & $5.02 E-07$ & 9.96E-08 & $4.16 \mathrm{E}-07$ & $2.02 E-06$ \\
\hline$c 29 y$ & 0.0894 & $1.48 \mathrm{E}-07$ & $1.17 \mathrm{E}-07$ & $5.58 \mathrm{E}-07$ & $1.17 \mathrm{E}-07$ & $4.88 \mathrm{E}-07$ & $2.33 \mathrm{E}-06$ \\
\hline c29o & 0.0706 & 7.09E-08 & $5.59 E-08$ & 2.39E-07 & 6.12E-08 & $2.25 \mathrm{E}-07$ & $1.01 \mathrm{E}-06$ \\
\hline $\mathrm{c} 30 \mathrm{y}$ & 0.0772 & $9.85 \mathrm{E}-08$ & 1.01E-07 & $3.63 E-07$ & 1.32E-07 & 4.32E-07 & $1.60 \mathrm{E}-06$ \\
\hline c30o & 0.1189 & $1.90 \mathrm{E}-07$ & 1.47E-07 & 7.43E-07 & $1.49 \mathrm{E}-07$ & 6.32E-07 & $3.25 E-06$ \\
\hline c31y & 0.1356 & 2.37E-07 & $1.93 \mathrm{E}-07$ & $9.51 \mathrm{E}-07$ & $1.90 \mathrm{E}-07$ & 8.35E-07 & 4.17E-06 \\
\hline$c 310$ & 0.1262 & $3.97 \mathrm{E}-07$ & $3.70 \mathrm{E}-07$ & $1.69 \mathrm{E}-06$ & $4.12 \mathrm{E}-07$ & $1.67 \mathrm{E}-06$ & $7.58 \mathrm{E}-06$ \\
\hline c32.1y & 0.0081 & $6.66 \mathrm{E}-07$ & 4.13E-07 & 1.57E-06 & $3.34 \mathrm{E}-07$ & $1.25 \mathrm{E}-06$ & 5.17E-06 \\
\hline$c 32.10$ & 0.0073 & $3.68 \mathrm{E}-07$ & 2.12E-07 & 7.06E-07 & $1.67 \mathrm{E}-07$ & $5.91 \mathrm{E}-07$ & $2.49 E-06$ \\
\hline c32.2y & 0.0209 & 3.22E-07 & $2.56 \mathrm{E}-07$ & $8.40 \mathrm{E}-07$ & 2.51E-07 & $8.85 \mathrm{E}-07$ & 4.24E-06 \\
\hline c32.20 & 0.0070 & $4.18 \mathrm{E}-07$ & $4.22 \mathrm{E}-07$ & $1.24 \mathrm{E}-06$ & $5.58 \mathrm{E}-07$ & $1.90 \mathrm{E}-06$ & 7.16E-06 \\
\hline$c 32 r$ & 0.0093 & 4.42E-02 & $3.42 \mathrm{E}-02$ & $-1.94 \mathrm{E}-02$ & 2.64E-02 & $-1.50 \mathrm{E}-02$ & 8.62E-03 \\
\hline c33y & 0.0064 & $4.23 E-07$ & $4.41 E-07$ & $1.24 \mathrm{E}-06$ & $6.28 \mathrm{E}-07$ & $2.11 \mathrm{E}-06$ & $8.10 \mathrm{E}-06$ \\
\hline c33o & & & & & & & \\
\hline
\end{tabular}


Table H-3A. Finite rotations using select data (high confidence), and fracture zone uncertainty assigned as $5 \mathrm{~km}$.

\begin{tabular}{|c|c|c|c|c|c|c|c|}
\hline \multirow{2}{*}{ Chron } & \multicolumn{7}{|c|}{ Hellinger - rotations } \\
\hline & \begin{tabular}{|l|} 
Iongitude \\
\end{tabular} & latitude & angle $(\stackrel{\circ}{)})$ & rmin & df & ndat & nsect \\
\hline c20y & 179.6707 & -67.5787 & 84.5905 & 101.7054 & 21 & 36 & 6 \\
\hline c20o & 133.7073 & -75.0628 & 37.1940 & 48.5275 & 18 & 33 & 6 \\
\hline c21y & 139.4452 & -74.8114 & 40.4553 & 625.3028 & 25 & 42 & 7 \\
\hline c210 & 126.5634 & -74.2866 & 36.8197 & 274.1180 & 36 & 59 & 10 \\
\hline c22y & 133.6332 & -74.5264 & 39.5341 & 438.9227 & 26 & 47 & 9 \\
\hline c22o & 129.9852 & -74.3012 & 38.6958 & 121.6887 & 36 & 57 & 9 \\
\hline c23y & 130.8379 & -74.2427 & 39.4771 & 393.3227 & 29 & 46 & 7 \\
\hline c23o & 130.0725 & -74.0733 & 39.7380 & 205.2175 & 37 & 54 & 7 \\
\hline c24y & 130.4038 & -74.1867 & 39.4785 & 72.3037 & 44 & 63 & 8 \\
\hline c24o & 128.7451 & -73.6061 & 40.2125 & 86.8932 & 113 & 136 & 10 \\
\hline c25y & 130.8061 & -73.1739 & 43.2623 & 121.7770 & 142 & 171 & 13 \\
\hline c25o & 127.9594 & -72.9278 & 42.5909 & 73.0483 & 131 & 160 & 13 \\
\hline c26y & 129.3193 & -72.7620 & 44.2746 & 132.9404 & 137 & 168 & 14 \\
\hline$c 260$ & 128.9662 & -72.7392 & 44.3015 & 93.0662 & 142 & 173 & 14 \\
\hline c27y & 124.9967 & -71.4573 & 44.9787 & 621.2527 & 235 & 262 & 12 \\
\hline c27o & 125.0212 & -71.4012 & 45.2595 & 540.0729 & 240 & 267 & 12 \\
\hline c28y & 122.8819 & -70.8397 & 45.0935 & 948.1802 & 282 & 311 & 13 \\
\hline c28o & 121.3847 & -70.3449 & 45.1610 & 1049.4563 & 277 & 306 & 13 \\
\hline$c 29 y$ & 121.5039 & -70.3270 & 45.4287 & 2167.2223 & 257 & 286 & 13 \\
\hline c29o & 122.2245 & -70.3409 & 46.5095 & 2392.5536 & 245 & 272 & 12 \\
\hline c30y & 121.1286 & -70.0510 & 46.6152 & 2201.4771 & 226 & 253 & 12 \\
\hline$c 300$ & 129.0469 & -70.8856 & 53.5411 & 649.0013 & 207 & 232 & 11 \\
\hline c31y & 129.7834 & -70.9548 & 54.1646 & 585.3567 & 203 & 230 & 12 \\
\hline c310 & 126.5486 & -70.3295 & 53.0442 & 1207.8618 & 202 & 227 & 11 \\
\hline c32.1y & 119.2528 & -68.5735 & 51.0905 & 1160.3170 & 105 & 122 & 7 \\
\hline c32.10 & 118.1956 & -68.1618 & 50.5897 & 1449.0228 & 109 & 128 & 8 \\
\hline c32.2y & 118.5753 & -68.3020 & 51.1267 & 1032.9940 & 118 & 137 & 8 \\
\hline c32.20 & 100.4248 & -61.6213 & 42.3956 & 2712.4418 & 97 & 112 & 6 \\
\hline$c 32 r$ & 121.9982 & -68.3499 & 54.7157 & 539.5081 & 73 & 88 & 6 \\
\hline c33y & 105.6354 & -63.5018 & 45.2278 & 3118.8564 & 80 & 93 & 5 \\
\hline c33o & 7 & $\sqrt{3}$ & 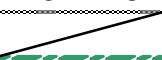 & 7 & & & \\
\hline
\end{tabular}


Table H-3B. Covariance matrices, select data (high confidence), and fracture zone uncertainty assigned as $5 \mathrm{~km}$.

\begin{tabular}{|c|c|c|c|c|c|c|c|}
\hline \multirow{2}{*}{ Chron } & \multicolumn{7}{|c|}{ Hellinger - covariance } \\
\hline & kappa hat & a & b & C & d & $\mathrm{e}$ & $f$ \\
\hline$c 20 y$ & 0.2065 & $1.11 \mathrm{E}-04$ & 4.39E-05 & $1.72 E-04$ & $2.39 \mathrm{E}-05$ & $8.99 E-05$ & $3.66 \mathrm{E}-04$ \\
\hline $\mathrm{c} 200$ & 0.3709 & $1.14 \mathrm{E}-05$ & $9.30 \mathrm{E}-06$ & $3.07 E-05$ & $7.82 E-06$ & $2.57 \mathrm{E}-05$ & $8.52 E-05$ \\
\hline c21y & 0.0400 & 4.70E-05 & $3.85 \mathrm{E}-05$ & $1.24 \mathrm{E}-04$ & $3.18 \mathrm{E}-05$ & $1.02 \mathrm{E}-04$ & $3.30 \mathrm{E}-04$ \\
\hline c21o & 0.1313 & 1.38E-05 & 1.19E-05 & 3.84E-05 & 1.05E-05 & 3.35E-05 & $1.08 \mathrm{E}-04$ \\
\hline$c 22 y$ & 0.0592 & 1.64E-05 & 1.37E-05 & 4.43E-05 & 1.15E-05 & 3.73E-05 & $1.21 \mathrm{E}-04$ \\
\hline c22o & 0.2958 & $1.10 \mathrm{E}-06$ & $9.03 \mathrm{E}-07$ & 3.04E-06 & $8.53 \mathrm{E}-07$ & $2.77 \mathrm{E}-06$ & $9.40 \mathrm{E}-06$ \\
\hline$c 23 y$ & 0.0737 & $1.82 E-06$ & $1.82 E-06$ & $5.98 \mathrm{E}-06$ & $2.13 \mathrm{E}-06$ & $6.84 \mathrm{E}-06$ & $2.24 \mathrm{E}-05$ \\
\hline c23o & 0.1803 & $1.99 \mathrm{E}-06$ & $1.96 \mathrm{E}-06$ & $6.50 \mathrm{E}-06$ & $2.25 \mathrm{E}-06$ & 7.01E-06 & $2.32 \mathrm{E}-05$ \\
\hline$c 24 y$ & 0.6085 & $1.65 \mathrm{E}-06$ & 1.57E-06 & 5.77E-06 & $2.02 E-06$ & 6.32E-06 & 2.27E-05 \\
\hline$c 240$ & 1.3004 & $1.08 \mathrm{E}-06$ & 1.13E-06 & 3.76E-06 & $1.35 \mathrm{E}-06$ & 4.26E-06 & 1.42E-05 \\
\hline c25y & 1.1661 & 1.47E-06 & $1.48 \mathrm{E}-06$ & $5.18 \mathrm{E}-06$ & $1.60 \mathrm{E}-06$ & 5.38E-06 & $1.87 \mathrm{E}-05$ \\
\hline c25o & 1.7933 & $8.55 \mathrm{E}-07$ & $5.04 \mathrm{E}-07$ & $2.54 \mathrm{E}-06$ & $3.71 \mathrm{E}-07$ & $1.59 \mathrm{E}-06$ & $8.25 \mathrm{E}-06$ \\
\hline c26y & 1.0305 & $5.59 \mathrm{E}-07$ & 4.78E-07 & $1.81 \mathrm{E}-06$ & $5.00 \mathrm{E}-07$ & 1.70E-06 & $6.46 \mathrm{E}-06$ \\
\hline c26o & 1.5258 & 4.04E-07 & 3.37E-07 & $1.27 \mathrm{E}-06$ & $3.73 \mathrm{E}-07$ & $1.22 \mathrm{E}-06$ & 4.62E-06 \\
\hline$c 27 y$ & 0.3783 & $9.42 E-08$ & $5.86 \mathrm{E}-08$ & $2.89 \mathrm{E}-07$ & $5.03 E-08$ & $2.02 \mathrm{E}-07$ & $1.04 \mathrm{E}-06$ \\
\hline c27o & 0.4444 & 1.05E-07 & $6.80 \mathrm{E}-08$ & $3.30 \mathrm{E}-07$ & $6.04 \mathrm{E}-08$ & 2.39E-07 & 1.19E-06 \\
\hline$c 28 y$ & 0.2974 & $9.57 \mathrm{E}-08$ & $6.84 \mathrm{E}-08$ & $3.32 E-07$ & 7.14E-08 & 2.73E-07 & $1.32 \mathrm{E}-06$ \\
\hline$c 280$ & 0.2639 & 2.04E-07 & $1.30 \mathrm{E}-07$ & $7.51 \mathrm{E}-07$ & 1.13E-07 & $5.12 \mathrm{E}-07$ & $2.94 \mathrm{E}-06$ \\
\hline$c 29 y$ & 0.1186 & 2.33E-07 & $1.49 \mathrm{E}-07$ & $8.70 \mathrm{E}-07$ & $1.34 \mathrm{E}-07$ & $6.00 \mathrm{E}-07$ & 3.47E-06 \\
\hline c29o & 0.1024 & 1.24E-07 & $8.03 \mathrm{E}-08$ & $4.48 \mathrm{E}-07$ & 7.88E-08 & $3.27 \mathrm{E}-07$ & $1.83 \mathrm{E}-06$ \\
\hline c30y & 0.1027 & 1.76E-07 & 1.57E-07 & $6.83 \mathrm{E}-07$ & 2.01E-07 & $6.97 \mathrm{E}-07$ & $2.98 \mathrm{E}-06$ \\
\hline c30o & 0.3190 & $1.45 \mathrm{E}-06$ & $1.37 \mathrm{E}-06$ & $6.30 \mathrm{E}-06$ & $1.57 \mathrm{E}-06$ & $6.23 \mathrm{E}-06$ & $2.79 \mathrm{E}-05$ \\
\hline c31y & 0.3468 & $1.69 E-06$ & $1.57 \mathrm{E}-06$ & $7.33 E-06$ & $1.74 \mathrm{E}-06$ & 7.09E-06 & $3.24 \mathrm{E}-05$ \\
\hline c31o & 0.1672 & $4.85 \mathrm{E}-07$ & $4.96 \mathrm{E}-07$ & $2.14 \mathrm{E}-06$ & $6.85 \mathrm{E}-07$ & 2.39E-06 & $9.91 \mathrm{E}-06$ \\
\hline c32.1y & 0.0905 & 1.19E-06 & $1.31 \mathrm{E}-06$ & $5.13 \mathrm{E}-06$ & $1.57 \mathrm{E}-06$ & 6.17E-06 & $2.56 \mathrm{E}-05$ \\
\hline c32.10 & 0.0752 & $2.86 \mathrm{E}-07$ & $2.06 \mathrm{E}-07$ & $6.87 \mathrm{E}-07$ & 1.86E-07 & $6.62 \mathrm{E}-07$ & 3.37E-06 \\
\hline c32.2y & 0.1142 & $9.08 \mathrm{E}-07$ & $1.01 \mathrm{E}-06$ & $3.88 \mathrm{E}-06$ & $1.26 \mathrm{E}-06$ & 4.93E-06 & $2.04 \mathrm{E}-05$ \\
\hline c32.20 & 0.0358 & 7.57E-07 & $9.74 \mathrm{E}-07$ & $3.56 \mathrm{E}-06$ & $1.49 \mathrm{E}-06$ & $5.82 E-06$ & 2.39E-05 \\
\hline c32r & 0.1353 & $1.82 E-06$ & $2.20 \mathrm{E}-06$ & $8.52 E-06$ & $2.75 \mathrm{E}-06$ & $1.08 \mathrm{E}-05$ & 4.37E-05 \\
\hline c33y & 0.0257 & $6.28 \mathrm{E}-07$ & $5.65 \mathrm{E}-07$ & $1.58 \mathrm{E}-06$ & $7.48 \mathrm{E}-07$ & $2.52 E-06$ & $9.42 \mathrm{E}-06$ \\
\hline c33o & & & & & & & \\
\hline
\end{tabular}




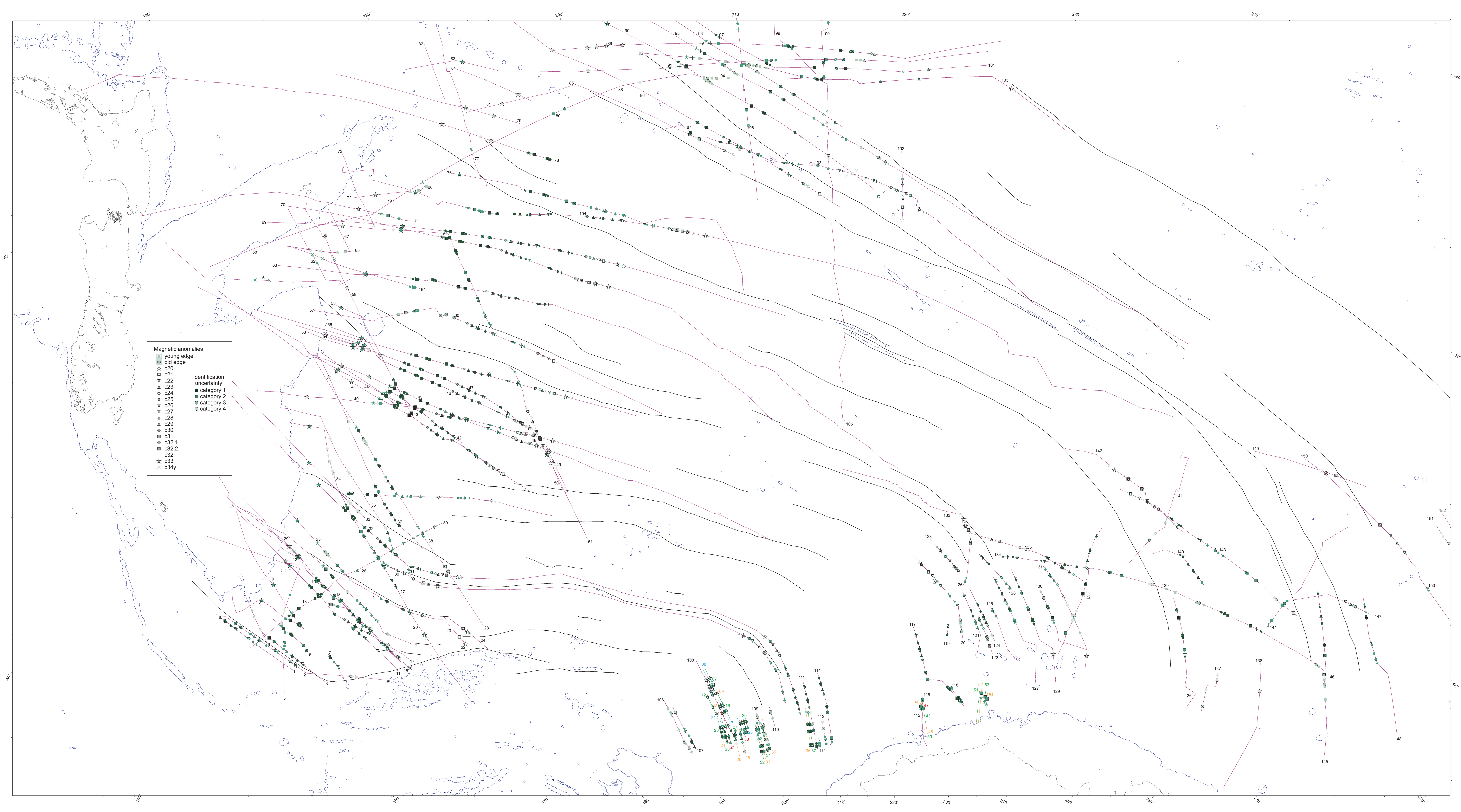

